\title{
TOXICOLOGICAL PROFILE FOR ARSENIC
}

\author{
U.S. DEPARTMENT OF HEALTH AND HUMAN SERVICES \\ Public Health Service \\ Agency for Toxic Substances and Disease Registry
}

August 2007 


\section{DISCLAIMER}

The use of company or product name(s) is for identification only and does not imply endorsement by the Agency for Toxic Substances and Disease Registry. 


\section{UPDATE STATEMENT}

A Toxicological Profile for Arsenic, Draft for Public Comment was released in September 2005. This edition supersedes any previously released draft or final profile.

Toxicological profiles are revised and republished as necessary. For information regarding the update status of previously released profiles, contact ATSDR at:

Agency for Toxic Substances and Disease Registry

Division of Toxicology and Environmental Medicine/Applied Toxicology Branch

1600 Clifton Road NE

Mailstop F-32

Atlanta, Georgia 30333 
This page is intentionally blank. 


\section{FOREWORD}

This toxicological profile is prepared in accordance with guidelines developed by the Agency for Toxic Substances and Disease Registry (ATSDR) and the Environmental Protection Agency (EPA). The original guidelines were published in the Federal Register on April 17, 1987. Each profile will be revised and republished as necessary.

The ATSDR toxicological profile succinctly characterizes the toxicologic and adverse health effects information for the hazardous substance described therein. Each peer-reviewed profile identifies and reviews the key literature that describes a hazardous substance's toxicologic properties. Other pertinent literature is also presented, but is described in less detail than the key studies. The profile is not intended to be an exhaustive document; however, more comprehensive sources of specialty information are referenced.

The focus of the profiles is on health and toxicologic information; therefore, each toxicological profile begins with a public health statement that describes, in nontechnical language, a substance's relevant toxicological properties. Following the public health statement is information concerning levels of significant human exposure and, where known, significant health effects. The adequacy of information to determine a substance's health effects is described in a health effects summary. Data needs that are of significance to protection of public health are identified by ATSDR and EPA.

Each profile includes the following:

(A) The examination, summary, and interpretation of available toxicologic information and epidemiologic evaluations on a hazardous substance to ascertain the levels of significant human exposure for the substance and the associated acute, subacute, and chronic health effects;

(B) A determination of whether adequate information on the health effects of each substance is available or in the process of development to determine levels of exposure that present a significant risk to human health of acute, subacute, and chronic health effects; and

(C) Where appropriate, identification of toxicologic testing needed to identify the types or levels of exposure that may present significant risk of adverse health effects in humans.

The principal audiences for the toxicological profiles are health professionals at the Federal, State, and local levels; interested private sector organizations and groups; and members of the public.

This profile reflects ATSDR's assessment of all relevant toxicologic testing and information that has been peer-reviewed. Staff of the Centers for Disease Control and Prevention and other Federal scientists have also reviewed the profile. In addition, this profile has been peer-reviewed by a nongovernmental panel and is being made available for public review. Final responsibility for the contents and views expressed in this toxicological profile resides with ATSDR.

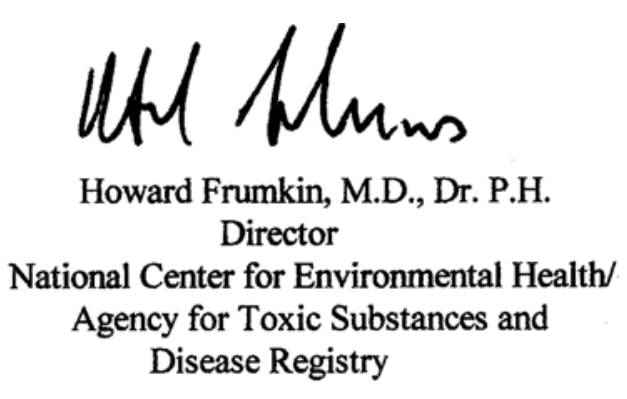

Howard Frumkin, M.D., Dr. P.H. Director National Center for Environmental Health/
Agency for Toxic Substances and
Disease Registry

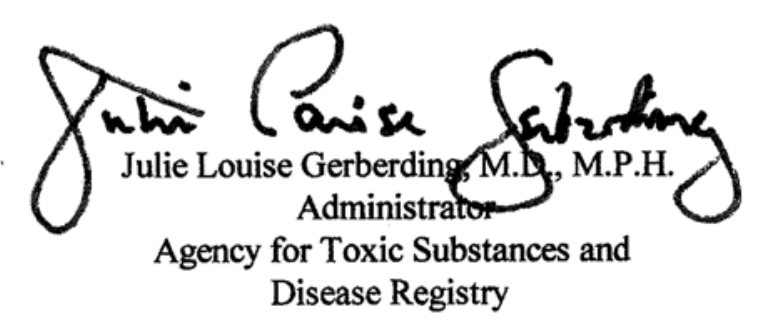


The toxicological profiles are developed in response to the Superfund Amendments and Reauthorization Act (SARA) of 1986 (Public Law 99-499) which amended the Comprehensive Environmental Response, Compensation, and Liability Act of 1980 (CERCLA or Superfund). This public law directed ATSDR to prepare toxicological profiles for hazardous substances most commonly found at facilities on the CERCLA National Priorities List and that pose the most significant potential threat to human health, as determined by ATSDR and the EPA. The availability of the revised priority list of 275 hazardous substances was announced in the Federal Register on December 7, 2005 (70 FR 72840). For prior versions of the list of substances, see Federal Register notices dated April 17, 1987 (52 FR 12866); October 20, 1988 (53 FR 41280); October 26, 1989 (54 FR 43619); October 17, 1990 (55 FR 42067); October 17, 1991 (56 FR 52166); October 28, 1992 (57 FR 48801); February 28, 1994 (59 FR 9486); April 29, 1996 (61 FR 18744); November 17, 1997 (62 FR 61332); October 21, 1999 (64 FR 56792); October 25, 2001 (66 FR 54014); and November 7, 2003 (68 FR 63098). Section 104(i)(3) of CERCLA, as amended, directs the Administrator of ATSDR to prepare a toxicological profile for each substance on the list. 


\section{QUICK REFERENCE FOR HEALTH CARE PROVIDERS}

Toxicological Profiles are a unique compilation of toxicological information on a given hazardous substance. Each profile reflects a comprehensive and extensive evaluation, summary, and interpretation of available toxicologic and epidemiologic information on a substance. Health care providers treating patients potentially exposed to hazardous substances will find the following information helpful for fast answers to often-asked questions.

\section{Primary Chapters/Sections of Interest}

Chapter 1: Public Health Statement: The Public Health Statement can be a useful tool for educating patients about possible exposure to a hazardous substance. It explains a substance's relevant toxicologic properties in a nontechnical, question-and-answer format, and it includes a review of the general health effects observed following exposure.

Chapter 2: Relevance to Public Health: The Relevance to Public Health Section evaluates, interprets, and assesses the significance of toxicity data to human health.

Chapter 3: Health Effects: Specific health effects of a given hazardous compound are reported by type of health effect (death, systemic, immunologic, reproductive), by route of exposure, and by length of exposure (acute, intermediate, and chronic). In addition, both human and animal studies are reported in this section.

NOTE: Not all health effects reported in this section are necessarily observed in the clinical setting. Please refer to the Public Health Statement to identify general health effects observed following exposure.

Pediatrics: Four new sections have been added to each Toxicological Profile to address child health issues:

Section 1.6 How Can (Chemical X) Affect Children?

Section 1.7 How Can Families Reduce the Risk of Exposure to (Chemical X)?

Section 3.7 Children's Susceptibility

Section 6.6 Exposures of Children

Other Sections of Interest:

Section 3.8 Biomarkers of Exposure and Effect

Section 3.11 Methods for Reducing Toxic Effects

\section{ATSDR Information Center}

Phone: 1-800-CDC-INFO (800-232-4636) or 1-888-232-6348 (TTY) Fax: (770) 488-4178

E-mail: cdcinfo@cdc.gov Internet: http://www.atsdr.cdc.gov

The following additional material can be ordered through the ATSDR Information Center:

Case Studies in Environmental Medicine: Taking an Exposure History-The importance of taking an exposure history and how to conduct one are described, and an example of a thorough exposure history is provided. Other case studies of interest include Reproductive and Developmental 
Hazards; Skin Lesions and Environmental Exposures; Cholinesterase-Inhibiting Pesticide Toxicity; and numerous chemical-specific case studies.

Managing Hazardous Materials Incidents is a three-volume set of recommendations for on-scene (prehospital) and hospital medical management of patients exposed during a hazardous materials incident. Volumes I and II are planning guides to assist first responders and hospital emergency department personnel in planning for incidents that involve hazardous materials. Volume IIIMedical Management Guidelines for Acute Chemical Exposures - is a guide for health care professionals treating patients exposed to hazardous materials.

Fact Sheets (ToxFAQs) provide answers to frequently asked questions about toxic substances.

\section{Other Agencies and Organizations}

The National Center for Environmental Health (NCEH) focuses on preventing or controlling disease, injury, and disability related to the interactions between people and their environment outside the workplace. Contact: NCEH, Mailstop F-29, 4770 Buford Highway, NE, Atlanta, GA 30341-3724 • Phone: 770-488-7000 • FAX: 770-488-7015.

The National Institute for Occupational Safety and Health (NIOSH) conducts research on occupational diseases and injuries, responds to requests for assistance by investigating problems of health and safety in the workplace, recommends standards to the Occupational Safety and Health Administration (OSHA) and the Mine Safety and Health Administration (MSHA), and trains professionals in occupational safety and health. Contact: NIOSH, 200 Independence Avenue, SW, Washington, DC 20201 • Phone: 800-356-4674 or NIOSH Technical Information Branch, Robert A. Taft Laboratory, Mailstop C-19, 4676 Columbia Parkway, Cincinnati, OH 45226$1998 \cdot$ Phone: 800-35-NIOSH.

The National Institute of Environmental Health Sciences (NIEHS) is the principal federal agency for biomedical research on the effects of chemical, physical, and biologic environmental agents on human health and well-being. Contact: NIEHS, PO Box 12233, 104 T.W. Alexander Drive, Research Triangle Park, NC 27709 • Phone: 919-541-3212.

\section{Referrals}

The Association of Occupational and Environmental Clinics (AOEC) has developed a network of clinics in the United States to provide expertise in occupational and environmental issues. Contact: AOEC, 1010 Vermont Avenue, NW, \#513, Washington, DC 20005 • Phone: 202-3474976•FAX: 202-347-4950•e-mail: AOEC@AOEC.ORG・Web Page: http://www.aoec.org/.

The American College of Occupational and Environmental Medicine (ACOEM) is an association of physicians and other health care providers specializing in the field of occupational and environmental medicine. Contact: ACOEM, 25 Northwest Point Boulevard, Suite 700, Elk Grove Village, IL 60007-1030 • Phone: 847-818-1800•FAX: 847-818-9266. 


\section{CONTRIBUTORS}

\section{CHEMICAL MANAGER(S)/AUTHOR(S):}

Selene Chou, Ph.D.

Carolyn Harper, Ph.D.

ATSDR, Division of Toxicology and Environmental Medicine, Atlanta, GA

Lisa Ingerman, Ph.D., DABT

Fernando Llados, Ph.D.

Joan Colman, Ph.D.

Lara Chappell, Ph.D.

Mark Osier, Ph.D., DABT

Marc Odin, M.S.

Gloria Sage, Ph.D.

Syracuse Research Corporation, North Syracuse, NY

\section{THE PROFILE HAS UNDERGONE THE FOLLOWING ATSDR INTERNAL REVIEWS:}

1. Health Effects Review. The Health Effects Review Committee examines the health effects chapter of each profile for consistency and accuracy in interpreting health effects and classifying end points.

2. Minimal Risk Level Review. The Minimal Risk Level Workgroup considers issues relevant to substance-specific Minimal Risk Levels (MRLs), reviews the health effects database of each profile, and makes recommendations for derivation of MRLs.

3. Data Needs Review. The Applied Toxicology Branch reviews data needs sections to assure consistency across profiles and adherence to instructions in the Guidance.

4. Green Border Review. Green Border review assures the consistency with ATSDR policy. 
This page is intentionally blank. 


\section{PEER REVIEW}

A peer review panel was assembled for arsenic. The panel consisted of the following members:

1. Toby Rossman, Ph.D., Professor of Environmental Medicine, New York University School of Medicine, Nelson Institute of Environmental Medicine, Tuxedo, New York;

2. Rosalind Schoof, Ph.D., DABT, Integral Consulting, Inc., Mercer Island, Washington; and

3. Allan Smith, M.D., Ph.D., Professor of Epidemiology, School of Public Health, University of California, Berkeley, California.

Draft for Public Comment:

1. Alan Hall, M.D., Toxicology Consulting and Medical Translating Services, Inc. (TCMTS, Inc.), Elk Mountain, Wyoming;

2. Gary Pascoe, Ph.D., Pascoe Environmental Consulting, Port Townsend, Washington; and

3. Toby Rossman, Ph.D., Professor of Environmental Medicine, New York University School of Medicine, Nelson Institute of Environmental Medicine, Tuxedo, New York.

These experts collectively have knowledge of arsenic's physical and chemical properties, toxicokinetics, key health end points, mechanisms of action, human and animal exposure, and quantification of risk to humans. All reviewers were selected in conformity with the conditions for peer review specified in Section 104(I)(13) of the Comprehensive Environmental Response, Compensation, and Liability Act, as amended.

Scientists from the Agency for Toxic Substances and Disease Registry (ATSDR) have reviewed the peer reviewers' comments and determined which comments will be included in the profile. A listing of the peer reviewers' comments not incorporated in the profile, with a brief explanation of the rationale for their exclusion, exists as part of the administrative record for this compound.

The citation of the peer review panel should not be understood to imply its approval of the profile's final content. The responsibility for the content of this profile lies with the ATSDR. 
This page is intentionally blank. 


\section{CONTENTS}

DISCLAIMER

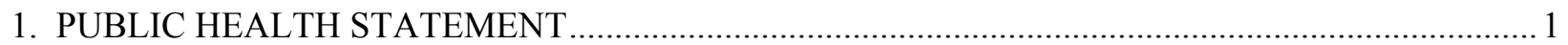

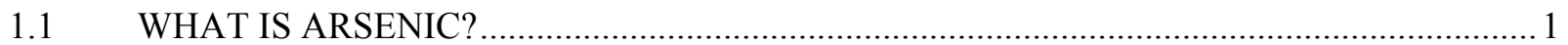

1.2 WHAT HAPPENS TO ARSENIC WHEN IT ENTERS THE ENVIRONMENT?.................... 3

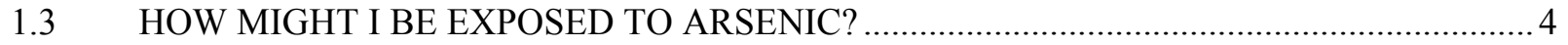

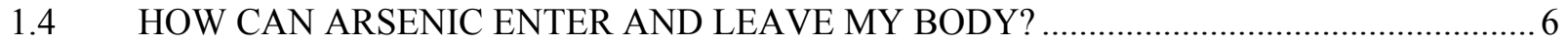

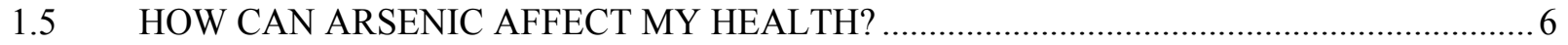

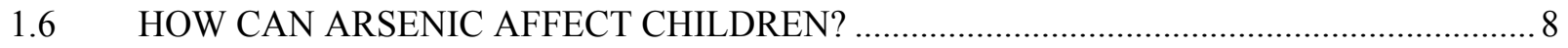

1.7 HOW CAN FAMILIES REDUCE THE RISK OF EXPOSURE TO ARSENIC? ................... 10

1.8 IS THERE A MEDICAL TEST TO DETERMINE WHETHER I HAVE BEEN

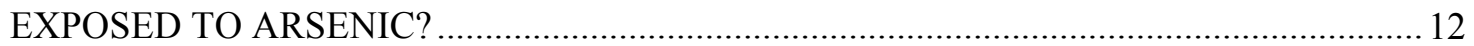

1.9 WHAT RECOMMENDATIONS HAS THE FEDERAL GOVERNMENT MADE TO

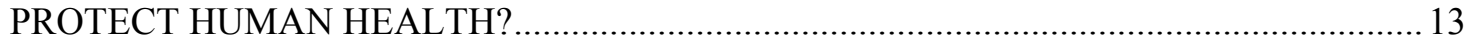

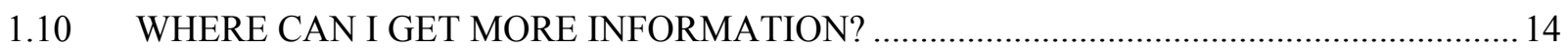

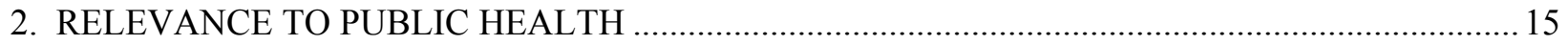

2.1 BACKGROUND AND ENVIRONMENTAL EXPOSURES TO ARSENIC IN THE

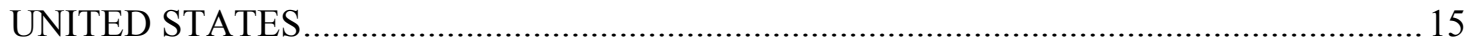

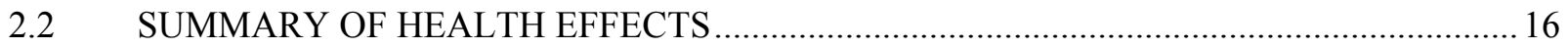

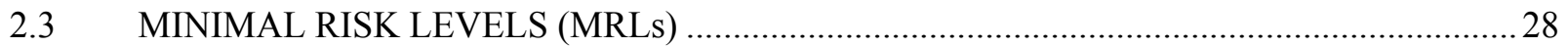

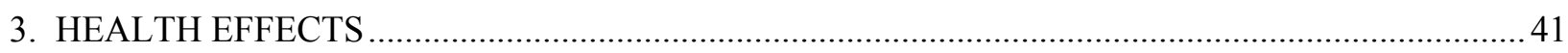

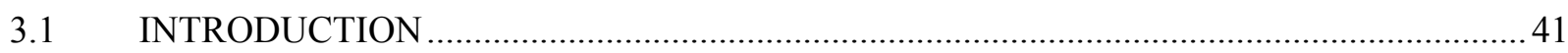

3.2 DISCUSSION OF HEALTH EFFECTS BY ROUTE OF EXPOSURE …........................... 41

3.2.1 Inhalation Exposure ……......................................................................... 44

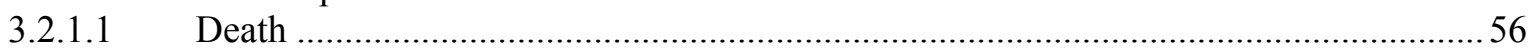

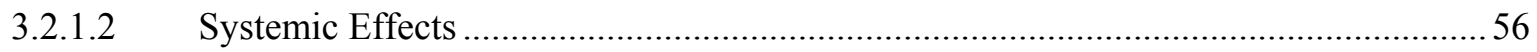

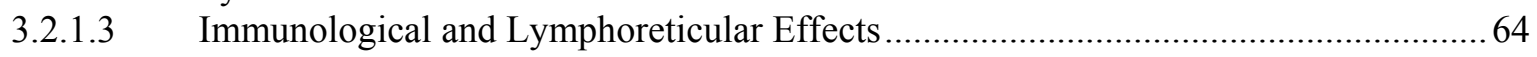

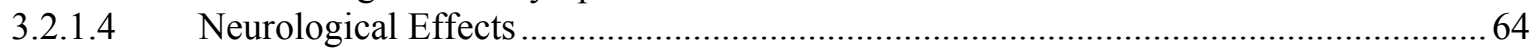

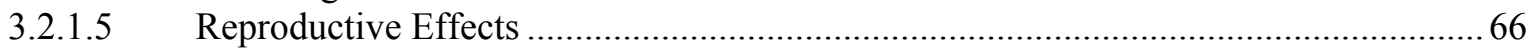

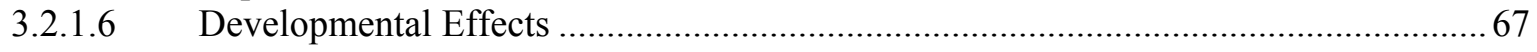

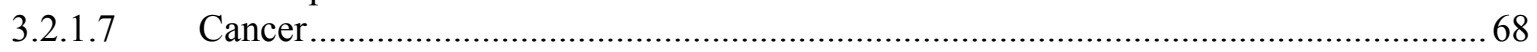

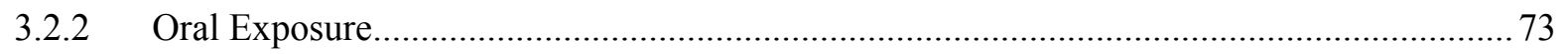

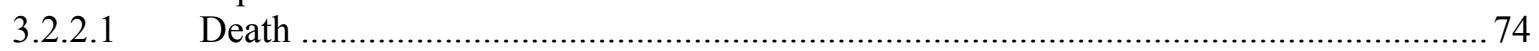

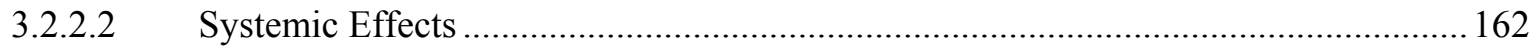

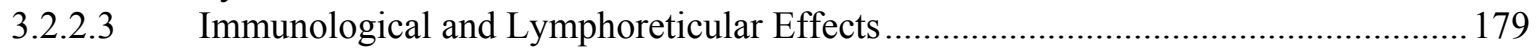

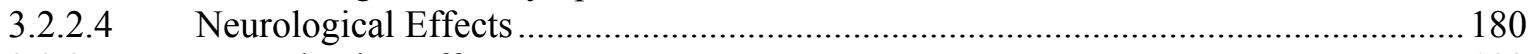

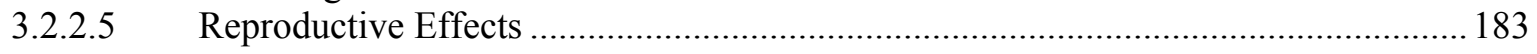




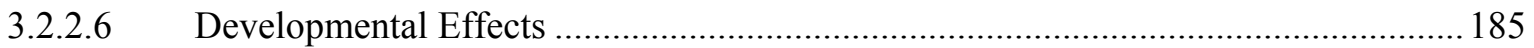

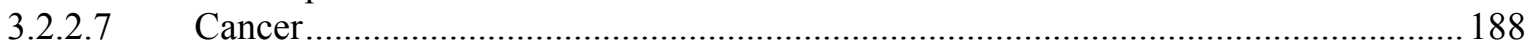

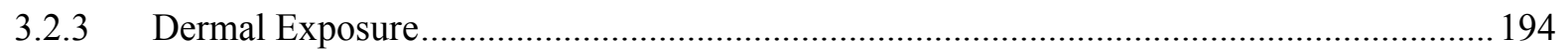

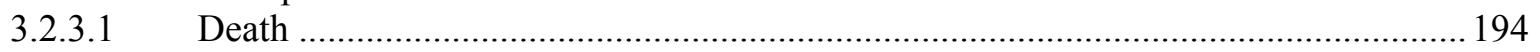

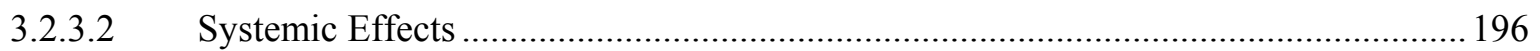

3.2.3.3 Immunological and Lymphoreticular Effects ........................................................... 198

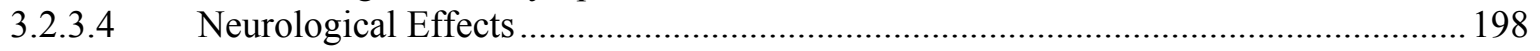

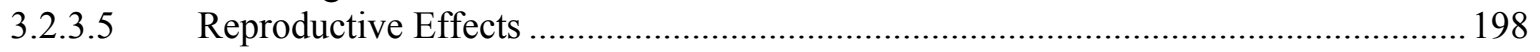

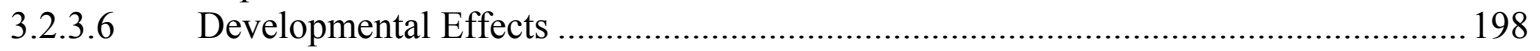

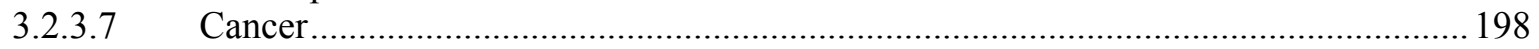

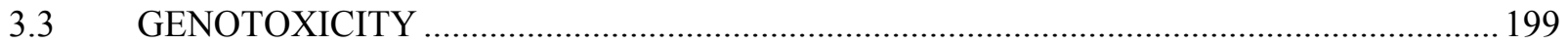

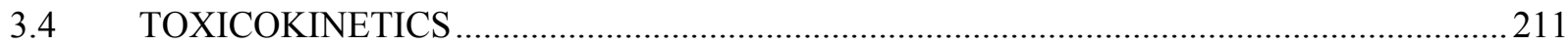

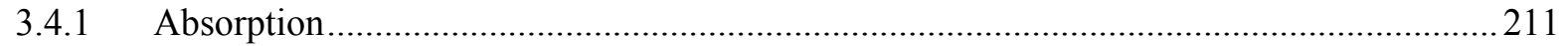

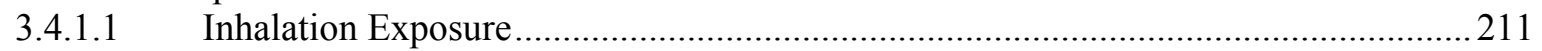

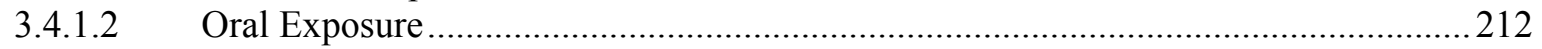

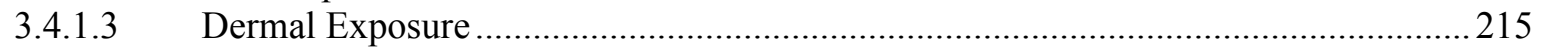

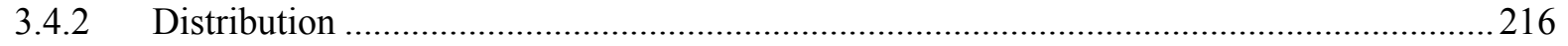

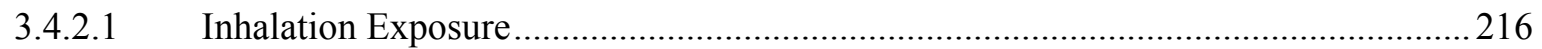

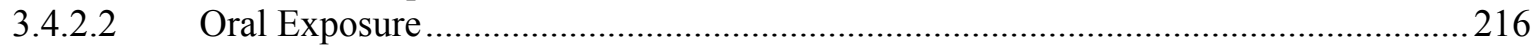

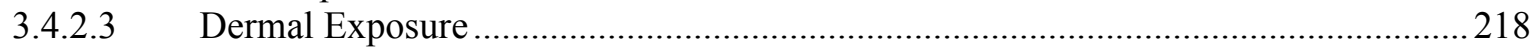

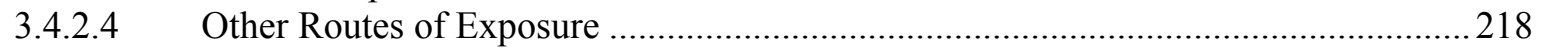

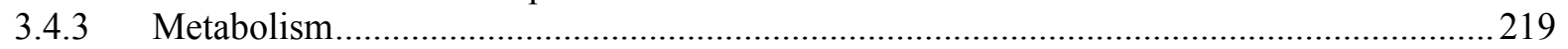

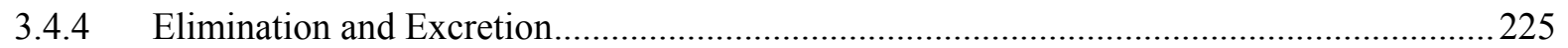

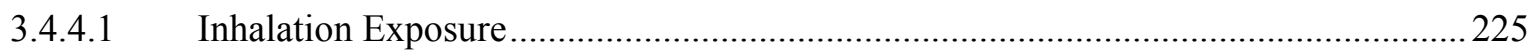

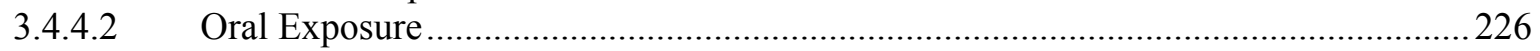

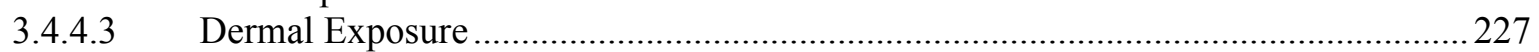

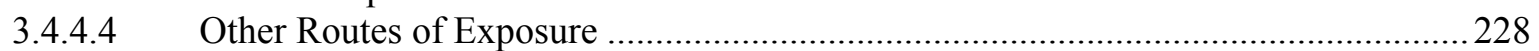

3.4.5 Physiologically Based Pharmacokinetic (PBPK)/Pharmacodynamic (PD) Models ............228

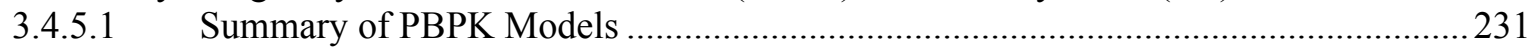

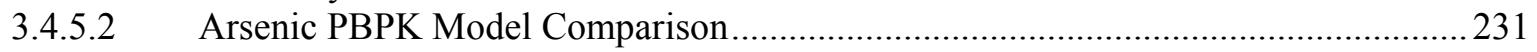

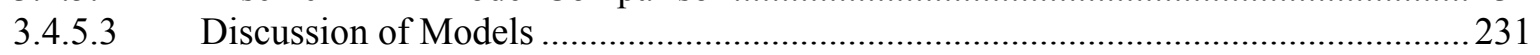

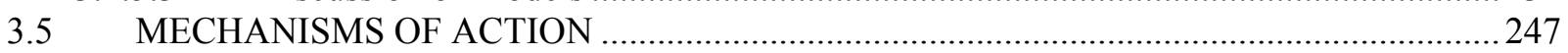

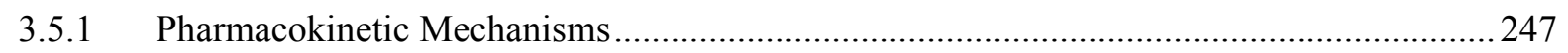

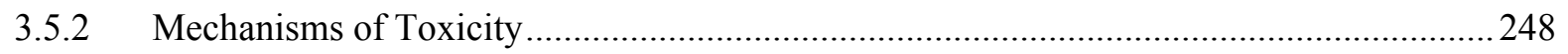

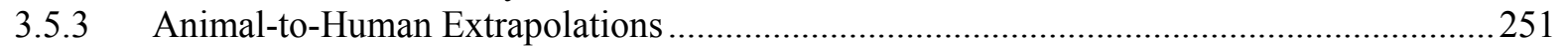

3.6 TOXICITIES MEDIATED THROUGH THE NEUROENDOCRINE AXIS .......................2252

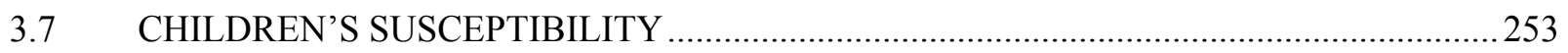

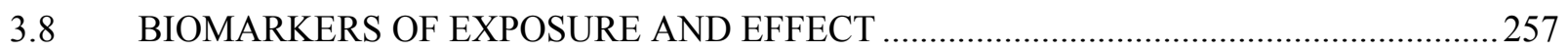

3.8.1 Biomarkers Used to Identify or Quantify Exposure to Arsenic ......................................258

3.8.2 Biomarkers Used to Characterize Effects Caused by Arsenic ........................................261

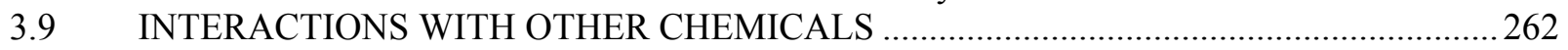

3.10 POPULATIONS THAT ARE UNUSUALLY SUSCEPTIBLE .......................................264

3.11 METHODS FOR REDUCING TOXIC EFFECTS .....................................................26

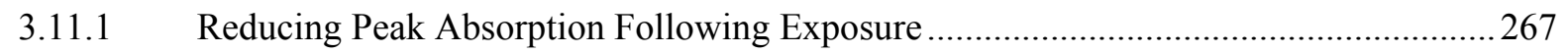

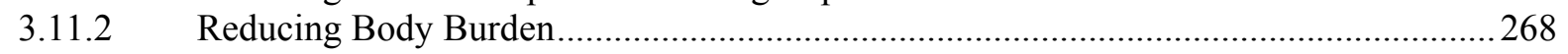

3.11.3 Interfering with the Mechanism of Action for Toxic Effects ......................................269

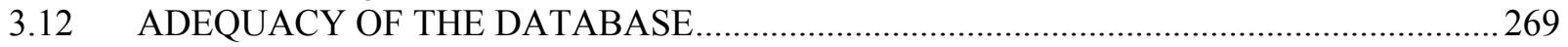

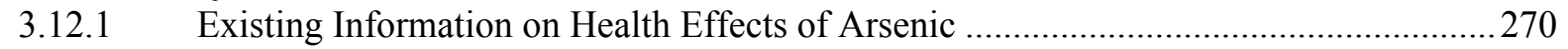

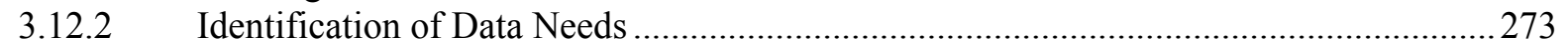

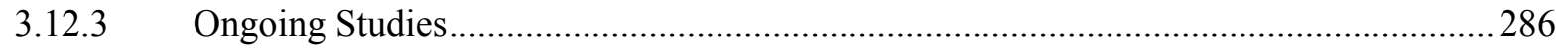




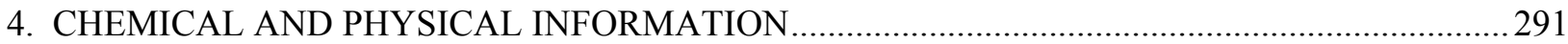

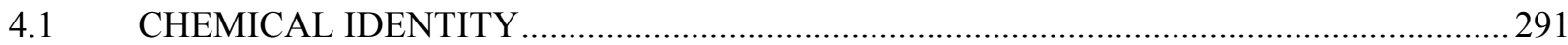

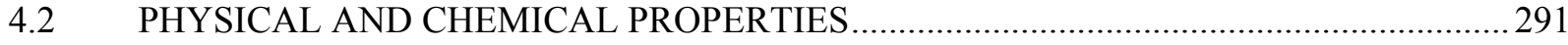

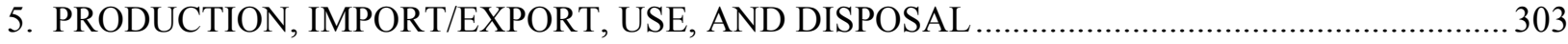

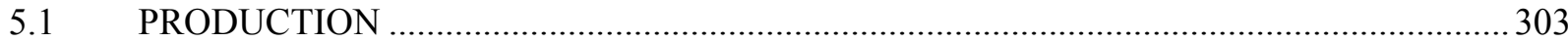

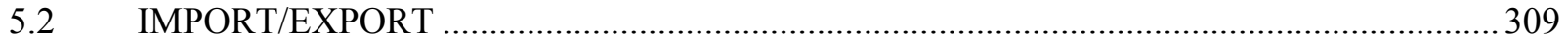

5.3 USE

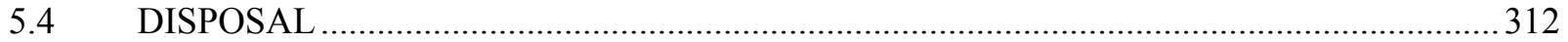

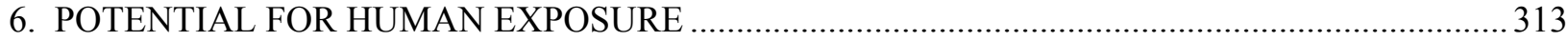

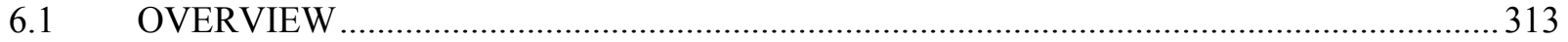

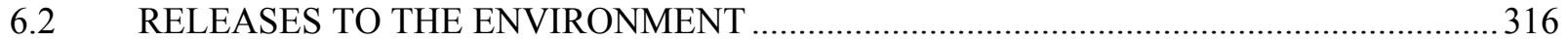

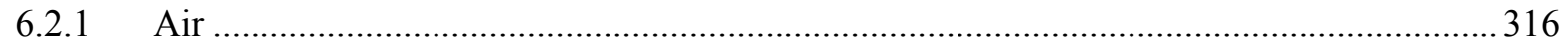

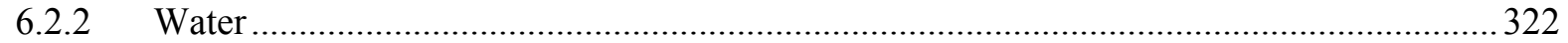

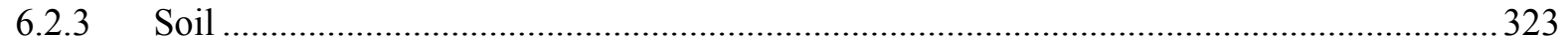

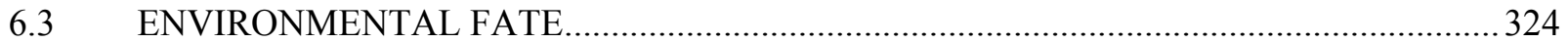

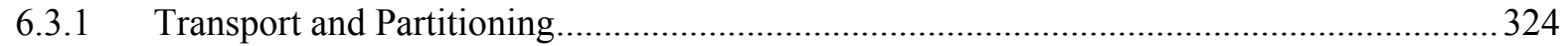

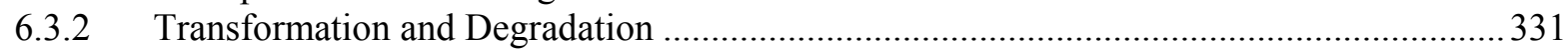

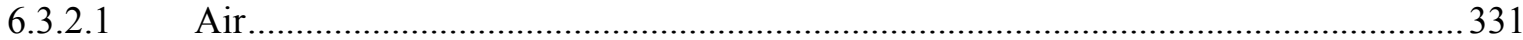

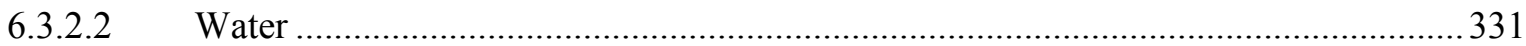

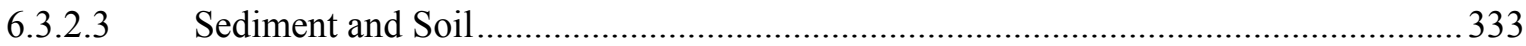

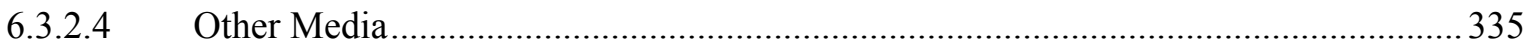

6.4 LEVELS MONITORED OR ESTIMATED IN THE ENVIRONMENT …............................336

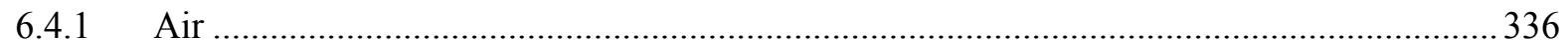

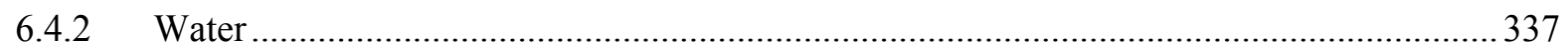

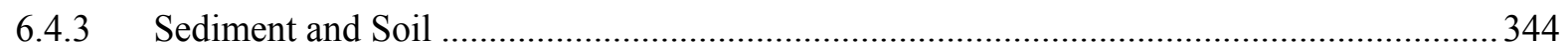

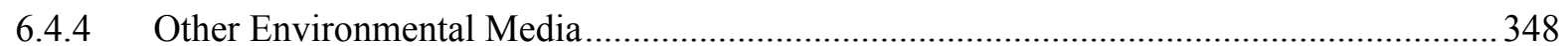

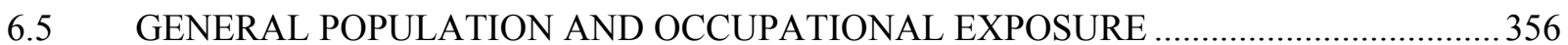

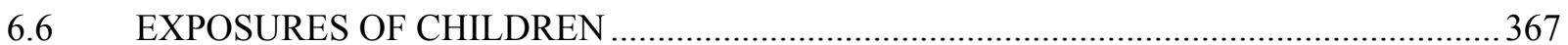

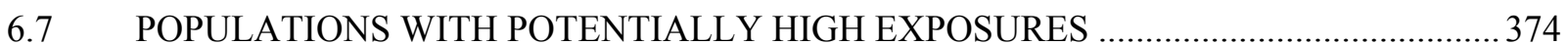

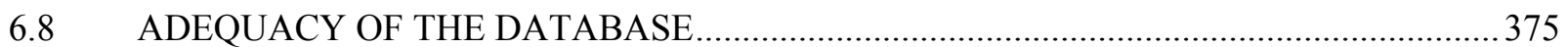

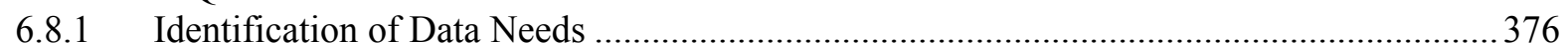

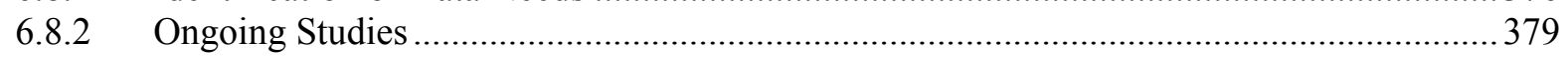

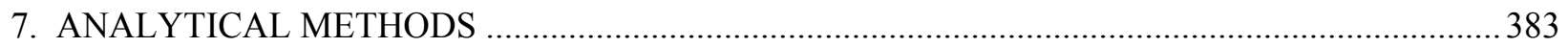

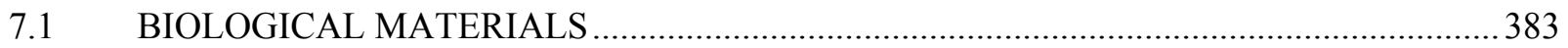

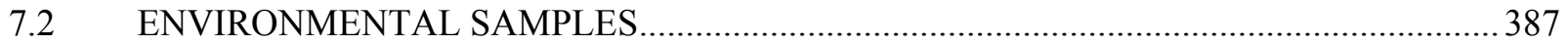

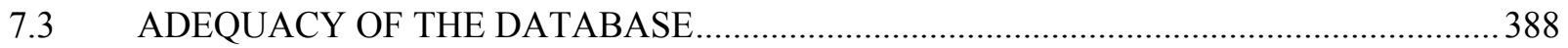

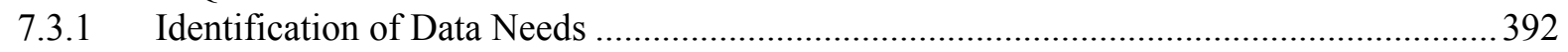

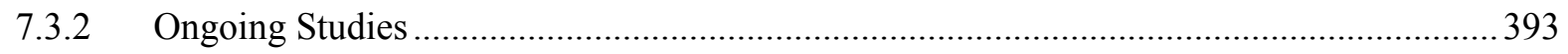

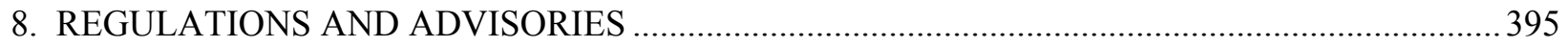

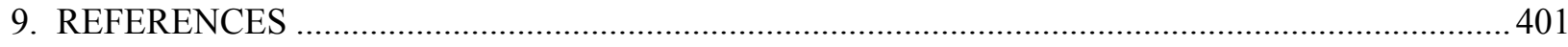

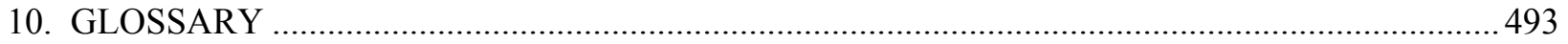


APPENDICES

A. ATSDR MINIMAL RISK LEVELS AND WORKSHEETS …...................................................

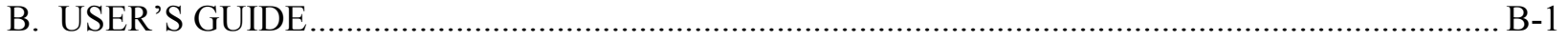

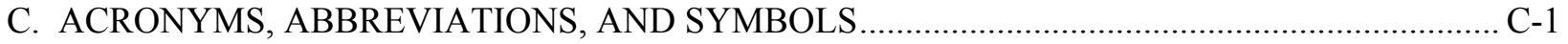

D. INDEX 


\section{LIST OF FIGURES}

3-1. Levels of Significant Exposure to Inorganic Arsenic - Inhalation............................................... 49

3-2. Levels of Significant Exposure to Dimethylarsinic Acid - Inhalation ........................................... 54

3-3. Levels of Significant Exposure to Inorganic Arsenic - Oral ..................................................... 117

3-4. Levels of Significant Exposure to Monomethylarsonic Acid - Oral............................................. 133

3-5. Levels of Significant Exposure to Dimethylarsinic Acid - Oral .................................................. 149

3-6. Levels of Significant Exposure to Roxarsone - Oral ............................................................. 159

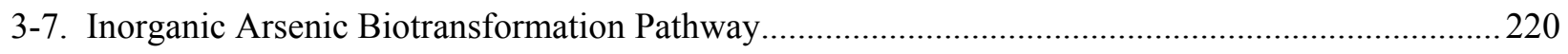

3-8. Alternative Inorganic Arsenic Biotransformation Pathway.......................................................223

3-9. Conceptual Representation of a Physiologically Based Pharmacokinetic (PBPK) Model for a

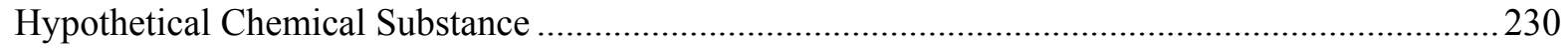

3-10. Parameters Used in the Mann PBPK Model for Animals .........................................................233

3-11. Parameters Used in the Mann PBPK Model for Humans............................................................2238

3-12. Parameters Used in the Yu PBPK Model for Animals ............................................................ 244

3-13. Existing Information on Health Effects of Inorganic Arsenic ................................................22

3-14. Existing Information on Health Effects of Organic Arsenic …................................................22

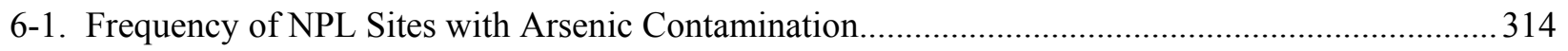

6-2. Counties in Which at Least 25\% of Wells Exceed Different Arsenic Levels.................................. 339 
This page is intentionally blank. 


\section{LIST OF TABLES}

3-1. Levels of Significant Exposure to Inorganic Arsenic - Inhalation................................................ 45

3-2. Levels of Significant Exposure to Dimethylarsinic Acid - Inhalation ........................................... 52

3-3. Levels of Significant Exposure to Inorganic Arsenic - Oral ....................................................... 75

3-4. Levels of Significant Exposure to Monomethylarsonic Acid - Oral........................................... 125

3-5. Levels of Significant Exposure to Dimethylarsinic Acid - Oral ................................................. 137

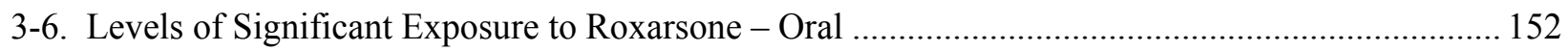

3-7. Levels of Significant Exposure to Inorganic Arsenic - Dermal ................................................. 195

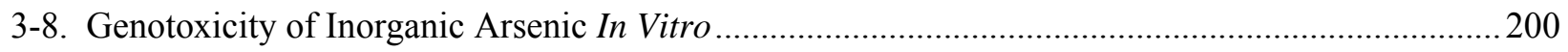

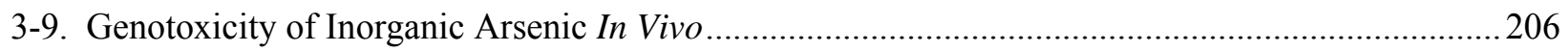

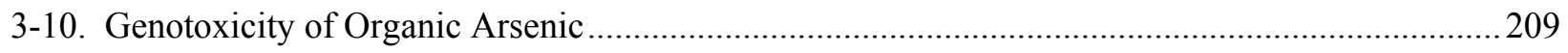

3-11. Parameters Used in the Mann PBPK Model for Animals ............................................................ 234

3-12. Tissue Affinity Constants $\left(\mathrm{K}_{\mathrm{ij}}\right)$ Obtained for the Mann PBPK Model for Animals by Fitting

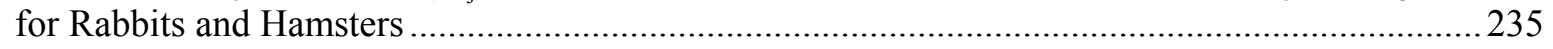

3-13. Metabolic Rate Constants for the Mann PBPK Model for Animals Obtained by Fitting for

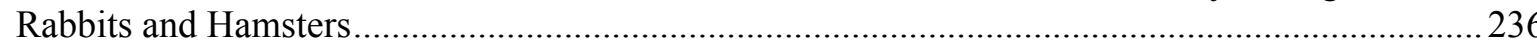

3-14. Fitted Gastrointestinal Tract and Lung Absorption Half-time for the Hamster for the Mann

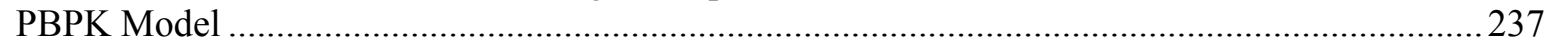

3-15. Physiological Data Used in the Mann PBPK Model for Humans .................................................239

3-16. Tissue Affinity Constants $\left(\mathrm{K}_{\mathrm{ij}}\right)$ Obtained by Fitting the Mann PBPK Animal Model for Use

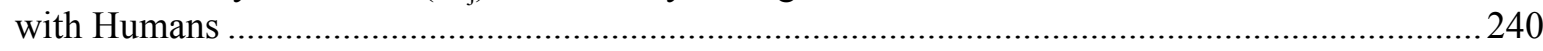

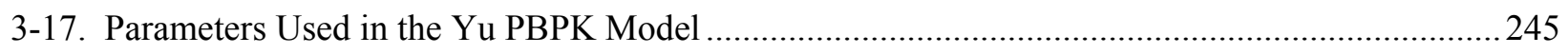

3-18. Ongoing Studies on Health Effects of Arsenic, Federally Funded ............................................287

4-1. Chemical Identity of Arsenic and Selected Inorganic Arsenic Compounds..................................292

4-2. Chemical Identity of Selected Organic Arsenic Compounds .....................................................2 294

4-3. Physical and Chemical Properties of Arsenic and Selected Inorganic Arsenic Compounds............297

4-4. Physical and Chemical Properties of Selected Organic Arsenic Compounds ................................299 
5-1. Facilities that Produce, Process, or Use Arsenic 304

5-2. Facilities that Produce, Process, or Use Arsenic Compounds .306

5-3. Current U.S. Manufacturers of Selected Arsenic Compounds 308

6-1. Releases to the Environment from Facilities that Produce, Process, or Use Arsenic 317

6-2. Releases to the Environment from Facilities that Produce, Process, or Use Arsenic Compounds

6-3. Regional Occurrence of Arsenic in U.S. Water Sources and Finished Drinking Water 342

6-4. Levels of Arsenic in Fish and Shellfish-Recent Studies 349

6-5. Mean Daily Dietary Intake of Arsenic for Selected U.S. Population Groups 357

6-6. Levels of Arsenic in Human Tissue and Urine-Recent Studies 361

6-7. Ongoing Studies on the Environmental Fate and Exposure of Humans to Arsenic 380

7-1. Analytical Methods for Determining Arsenic in Biological Samples 385

7-2. Analytical Methods for Determining Arsenic in Environmental Samples

7-3. Ongoing Studies on Analytical Methods for Arsenic in Environmental and Biological Samples

8-1. Regulations and Guidelines Applicable to Arsenic and Arsenic Compounds. 396 


\section{PUBLIC HEALTH STATEMENT}

This public health statement tells you about arsenic and the effects of exposure to it.

The Environmental Protection Agency (EPA) identifies the most serious hazardous waste sites in the nation. These sites are then placed on the National Priorities List (NPL) and are targeted for long-term federal clean-up activities. Arsenic has been found in at least 1,149 of the 1,684 current or former NPL sites. Although the total number of NPL sites evaluated for this substance is not known, the possibility exists that the number of sites at which arsenic is found may increase in the future as more sites are evaluated. This information is important because these sites may be sources of exposure and exposure to this substance may harm you.

When a substance is released either from a large area, such as an industrial plant, or from a container, such as a drum or bottle, it enters the environment. Such a release does not always lead to exposure. You can be exposed to a substance only when you come in contact with it. You may be exposed by breathing, eating, or drinking the substance, or by skin contact.

If you are exposed to arsenic, many factors will determine whether you will be harmed. These factors include the dose (how much), the duration (how long), and how you come in contact with it. You must also consider any other chemicals you are exposed to and your age, sex, diet, family traits, lifestyle, and state of health.

\subsection{WHAT IS ARSENIC?}

Arsenic is a naturally occurring element that is widely distributed in the Earth's crust. Arsenic is classified chemically as a metalloid, having both properties of a metal and a nonmetal; however, it is frequently referred to as a metal. Elemental arsenic (sometimes referred to as metallic arsenic) is a steel grey solid material. However, arsenic is usually found in the environment combined with other elements such as oxygen, chlorine, and sulfur. Arsenic combined with 
these elements is called inorganic arsenic. Arsenic combined with carbon and hydrogen is referred to as organic arsenic.

Most inorganic and organic arsenic compounds are white or colorless powders that do not evaporate. They have no smell, and most have no special taste. Thus, you usually cannot tell if arsenic is present in your food, water, or air.

Inorganic arsenic occurs naturally in soil and in many kinds of rock, especially in minerals and ores that contain copper or lead. When these ores are heated in smelters, most of the arsenic goes up the stack and enters the air as a fine dust. Smelters may collect this dust and take out the arsenic as a compound called arsenic trioxide $\left(\mathrm{As}_{2} \mathrm{O}_{3}\right)$. However, arsenic is no longer produced in the United States; all of the arsenic used in the United States is imported.

Presently, about $90 \%$ of all arsenic produced is used as a preservative for wood to make it resistant to rotting and decay. The preservative is copper chromated arsenate (CCA) and the treated wood is referred to as "pressure-treated." In 2003, U.S. manufacturers of wood preservatives containing arsenic began a voluntary transition from CCA to other wood preservatives that do not contain arsenic in wood products for certain residential uses, such as play structures, picnic tables, decks, fencing, and boardwalks. This phase out was completed on December 31, 2003; however, wood treated prior to this date could still be used and existing structures made with CCA-treated wood would not be affected. CCA-treated wood products continue to be used in industrial applications. It is not known whether, or to what extent, CCAtreated wood products may contribute to exposure of people to arsenic.

In the past, inorganic arsenic compounds were predominantly used as pesticides, primarily on cotton fields and in orchards. Inorganic arsenic compounds can no longer be used in agriculture. However, organic arsenic compounds, namely cacodylic acid, disodium methylarsenate (DSMA), and monosodium methylarsenate (MSMA), are still used as pesticides, principally on cotton. Some organic arsenic compounds are used as additives in animal feed. Small quantities of elemental arsenic are added to other metals to form metal mixtures or alloys with improved 
properties. The greatest use of arsenic in alloys is in lead-acid batteries for automobiles. Another important use of arsenic compounds is in semiconductors and light-emitting diodes.

To learn more about the properties and uses of arsenic, see Chapters 4 and 5.

\subsection{WHAT HAPPENS TO ARSENIC WHEN IT ENTERS THE ENVIRONMENT?}

Arsenic occurs naturally in soil and minerals and it therefore may enter the air, water, and land from wind-blown dust and may get into water from runoff and leaching. Volcanic eruptions are another source of arsenic. Arsenic is associated with ores containing metals, such as copper and lead. Arsenic may enter the environment during the mining and smelting of these ores. Small amounts of arsenic also may be released into the atmosphere from coal-fired power plants and incinerators because coal and waste products often contain some arsenic.

Arsenic cannot be destroyed in the environment. It can only change its form, or become attached to or separated from particles. It may change its form by reacting with oxygen or other molecules present in air, water, or soil, or by the action of bacteria that live in soil or sediment. Arsenic released from power plants and other combustion processes is usually attached to very small particles. Arsenic contained in wind-borne soil is generally found in larger particles. These particles settle to the ground or are washed out of the air by rain. Arsenic that is attached to very small particles may stay in the air for many days and travel long distances. Many common arsenic compounds can dissolve in water. Thus, arsenic can get into lakes, rivers, or underground water by dissolving in rain or snow or through the discharge of industrial wastes. Some of the arsenic will stick to particles in the water or sediment on the bottom of lakes or rivers, and some will be carried along by the water. Ultimately, most arsenic ends up in the soil or sediment. Although some fish and shellfish take in arsenic, which may build up in tissues, most of this arsenic is in an organic form called arsenobetaine (commonly called "fish arsenic") that is much less harmful.

For more information on how arsenic behaves in the environment, see Chapter 6. 


\subsection{HOW MIGHT I BE EXPOSED TO ARSENIC?}

Since arsenic is found naturally in the environment, you will be exposed to some arsenic by eating food, drinking water, or breathing air. Children may also be exposed to arsenic by eating soil. Analytical methods used by scientists to determine the levels of arsenic in the environment generally do not determine the specific form of arsenic present. Therefore, we do not always know the form of arsenic a person may be exposed to. Similarly, we often do not know what forms of arsenic are present at hazardous waste sites. Some forms of arsenic may be so tightly attached to particles or embedded in minerals that they are not taken up by plants and animals.

The concentration of arsenic in soil varies widely, generally ranging from about 1 to 40 parts of arsenic to a million parts of soil ( $\mathrm{ppm}$ ) with an average level of 3-4 ppm. However, soils in the vicinity of arsenic-rich geological deposits, some mining and smelting sites, or agricultural areas where arsenic pesticides had been applied in the past may contain much higher levels of arsenic. The concentration of arsenic in natural surface and groundwater is generally about 1 part in a billion parts of water ( $1 \mathrm{ppb}$ ), but may exceed 1,000 ppb in contaminated areas or where arsenic levels in soil are high. Groundwater is far more likely to contain high levels of arsenic than surface water. Surveys of U.S. drinking water indicate that about $80 \%$ of water supplies have less than $2 \mathrm{ppb}$ of arsenic, but $2 \%$ of supplies exceed $20 \mathrm{ppb}$ of arsenic. Levels of arsenic in food range from about 20 to $140 \mathrm{ppb}$. However, levels of inorganic arsenic, the form of most concern, are far lower. Levels of arsenic in the air generally range from less than 1 to about 2,000 nanograms (1 nanogram equals a billionth of a gram) of arsenic per cubic meter of air (less than $1-2,000 \mathrm{ng} / \mathrm{m}^{3}$ ), depending on location, weather conditions, and the level of industrial activity in the area. However, urban areas generally have mean arsenic levels in air ranging from 20 to $30 \mathrm{ng} / \mathrm{m}^{3}$.

You normally take in small amounts of arsenic in the air you breathe, the water you drink, and the food you eat. Of these, food is usually the largest source of arsenic. The predominant dietary source of arsenic is seafood, followed by rice/rice cereal, mushrooms, and poultry. While seafood contains the greatest amounts of arsenic, for fish and shellfish, this is mostly in an organic form of arsenic called arsenobetaine that is much less harmful. Some seaweeds may 
contain arsenic in inorganic forms that may be more harmful. Children are likely to eat small amounts of dust or soil each day, so this is another way they may be exposed to arsenic. The total amount of arsenic you take in from these sources is generally about 50 micrograms (1 microgram equals one-millionth of a gram) each day. The level of inorganic arsenic (the form of most concern) you take in from these sources is generally about 3.5 microgram/day. Children may be exposed to small amounts of arsenic from hand-to-mouth activities from playing on play structures or decks constructed out of CCA-treated wood. The potential exposure that children may receive from playing in play structures constructed from CCA-treated wood is generally smaller than that they would receive from food and water. Hand washing can reduce the potential exposure of children to arsenic after playing on play structures constructed with CCAtreated wood, since most of the arsenic on the children's hands was removed with water.

In addition to the normal levels of arsenic in air, water, soil, and food, you could be exposed to higher levels in several ways, such as the following:

- Some areas of the United States contain unusually high natural levels of arsenic in rock, and this can lead to unusually high levels of arsenic in soil or water. If you live in an area like this, you could take in elevated amounts of arsenic in drinking water. Children may be taking in higher amounts of arsenic because of hand-to-mouth contact or eating soil in areas with higher than usual arsenic concentrations.

- Some hazardous waste sites contain large quantities of arsenic. If the material is not properly disposed of, it can get into surrounding water, air, or soil. If you live near such a site, you could be exposed to elevated levels of arsenic from these media.

- If you work in an occupation that involves arsenic production or use (for example, copper or lead smelting, wood treating, or pesticide application), you could be exposed to elevated levels of arsenic during your work.

- If you saw or sand arsenic-treated wood, you could inhale some of the sawdust into your nose or throat. Similarly, if you burn arsenic-treated wood, you could inhale arsenic in the smoke.

- If you live in a former agricultural area where arsenic was used on crops, the soil could contain high levels of arsenic. 
- In the past, several kinds of products used in the home (rat poison, ant poison, weed killer, some types of medicines) had arsenic in them. However, most of these uses of arsenic have ended, so you are not likely to be exposed from home products any longer.

You can find more information on how you may be exposed to arsenic in Chapter 6.

\subsection{HOW CAN ARSENIC ENTER AND LEAVE MY BODY?}

If you swallow arsenic in water, soil, or food, most of the arsenic may quickly enter into your body. The amount that enters your body will depend on how much you swallow and the kind of arsenic that you swallow. This is the most likely way for you to be exposed near a waste site. If you breathe air that contains arsenic dusts, many of the dust particles settle onto the lining of the lungs. Most of the arsenic in these particles is then taken up from the lungs into the body. You might be exposed in this way near waste sites where arsenic-contaminated soils are allowed to blow into the air, or if you work with arsenic-containing soil or products. If you get arseniccontaminated soil or water on your skin, only a small amount will go through your skin into your body, so this is usually not of concern.

Both inorganic and organic forms leave your body in your urine. Most of the inorganic arsenic will be gone within several days, although some will remain in your body for several months or even longer. If you are exposed to organic arsenic, most of it will leave your body within several days.

You can find more information on how arsenic enters and leaves your body in Chapter 3 .

\subsection{HOW CAN ARSENIC AFFECT MY HEALTH?}

Scientists use many tests to protect the public from harmful effects of toxic chemicals and to find ways for treating persons who have been harmed. 
One way to learn whether a chemical will harm people is to determine how the body absorbs, uses, and releases the chemical. For some chemicals, animal testing may be necessary. Animal testing may also help identify health effects such as cancer or birth defects. Without laboratory animals, scientists would lose a basic method for getting information needed to make wise decisions that protect public health. Scientists have the responsibility to treat research animals with care and compassion. Scientists must comply with strict animal care guidelines because laws today protect the welfare of research animals.

Inorganic arsenic has been recognized as a human poison since ancient times, and large oral doses (above $60,000 \mathrm{ppb}$ in water which is 10,000 times higher than $80 \%$ of U.S. drinking water arsenic levels) can result in death. If you swallow lower levels of inorganic arsenic (ranging from about 300 to $30,000 \mathrm{ppb}$ in water; 100-10,000 times higher than most U.S. drinking water levels), you may experience irritation of your stomach and intestines, with symptoms such as stomachache, nausea, vomiting, and diarrhea. Other effects you might experience from swallowing inorganic arsenic include decreased production of red and white blood cells, which may cause fatigue, abnormal heart rhythm, blood-vessel damage resulting in bruising, and impaired nerve function causing a "pins and needles" sensation in your hands and feet.

Perhaps the single-most characteristic effect of long-term oral exposure to inorganic arsenic is a pattern of skin changes. These include patches of darkened skin and the appearance of small "corns" or "warts" on the palms, soles, and torso, and are often associated with changes in the blood vessels of the skin. Skin cancer may also develop. Swallowing arsenic has also been reported to increase the risk of cancer in the liver, bladder, and lungs. The Department of Health and Human Services (DHHS) has determined that inorganic arsenic is known to be a human carcinogen (a chemical that causes cancer). The International Agency for Research on Cancer (IARC) has determined that inorganic arsenic is carcinogenic to humans. EPA also has classified inorganic arsenic as a known human carcinogen.

If you breathe high levels of inorganic arsenic, then you are likely to experience a sore throat and irritated lungs. You may also develop some of the skin effects mentioned above. The exposure level that produces these effects is uncertain, but it is probably above 100 micrograms of arsenic 
per cubic meter $\left(\mu \mathrm{g} / \mathrm{m}^{3}\right)$ for a brief exposure. Longer exposure at lower concentrations can lead to skin effects, and also to circulatory and peripheral nervous disorders. There are some data suggesting that inhalation of inorganic arsenic may also interfere with normal fetal development, although this is not certain. An important concern is the ability of inhaled inorganic arsenic to increase the risk of lung cancer. This has been seen mostly in workers exposed to arsenic at smelters, mines, and chemical factories, but also in residents living near smelters and arsenical chemical factories. People who live near waste sites with arsenic may have an increased risk of lung cancer as well.

If you have direct skin contact with high concentrations of inorganic arsenic compounds, your skin may become irritated, with some redness and swelling. However, it does not appear that skin contact is likely to lead to any serious internal effects.

Almost no information is available on the effects of organic arsenic compounds in humans. Studies in animals show that most simple organic arsenic compounds (such as methyl and dimethyl compounds) are less toxic than the inorganic forms. In animals, ingestion of methyl compounds can result in diarrhea, and lifetime exposure can damage the kidneys. Lifetime exposure to dimethyl compounds can damage the urinary bladder and the kidneys.

You can find more information on the health effects of inorganic and organic arsenic in Chapters 2 and 3.

\subsection{HOW CAN ARSENIC AFFECT CHILDREN?}

This section discusses potential health effects in humans from exposures during the period from conception to maturity at 18 years of age.

Children are exposed to arsenic in many of the same ways that adults are. Since arsenic is found in the soil, water, food, and air, children may take in arsenic in the air they breathe, the water they drink, and the food they eat. Since children tend to eat or drink less of a variety of foods and beverages than do adults, ingestion of contaminated food or juice or infant formula made 
with arsenic-contaminated water may represent a significant source of exposure. In addition, since children often play in the soil and put their hands in their mouths and sometimes intentionally eat soil, ingestion of contaminated soil may be a more important source of arsenic exposure for children than for adults. In areas of the United States where natural levels of arsenic in the soil and water are high, or in areas in and around contaminated waste sites, exposure of children to arsenic through ingestion of soil and water may be significant. In addition, contact with adults who are wearing clothes contaminated with arsenic (e.g., with dust from copper- or lead-smelting factories, from wood-treating or pesticide application, or from arsenic-treated wood) could be a source of exposure. Because of the tendency of children to taste things that they find, accidental poisoning from ingestion of pesticides is also a possibility. Thus, although most of the exposure pathways for children are the same as those for adults, children may be at a higher risk of exposure because of normal hand-to-mouth activity.

Children who are exposed to inorganic arsenic may have many of the same effects as adults, including irritation of the stomach and intestines, blood vessel damage, skin changes, and reduced nerve function. Thus, all health effects observed in adults are of potential concern in children. There is also some evidence that suggests that long-term exposure to inorganic arsenic in children may result in lower IQ scores. We do not know if absorption of inorganic arsenic from the gut in children differs from adults. There is some evidence that exposure to arsenic in early life (including gestation and early childhood) may increase mortality in young adults.

There is some evidence that inhaled or ingested inorganic arsenic can injure pregnant women or their unborn babies, although the studies are not definitive. Studies in animals show that large doses of inorganic arsenic that cause illness in pregnant females can also cause low birth weight, fetal malformations, and even fetal death. Arsenic can cross the placenta and has been found in fetal tissues. Arsenic is found at low levels in breast milk.

In animals, exposure to organic arsenic compounds can cause low birth weight, fetal malformations, and fetal deaths. The dose levels that cause these effects also result in effects in the mothers. 
You can find more information about how arsenic can affect children in Sections 3.7 and 6.6.

\subsection{HOW CAN FAMILIES REDUCE THE RISK OF EXPOSURE TO ARSENIC?}

If your doctor finds that you have been exposed to substantial amounts of arsenic, ask whether your children might also have been exposed. Your doctor might need to ask your state health department to investigate.

Many communities may have high levels of arsenic in their drinking water, particularly from private wells, because of contamination or as a result of the geology of the area. The north central region and the western region of the United States have the highest arsenic levels in surface water and groundwater sources, respectively. Wells used to provide water for drinking and cooking should be tested for arsenic. As of January 2006, EPA's Maximum Contaminant Level (MCL) for arsenic in drinking water is $10 \mathrm{ppb}$. If you have arsenic in your drinking water at levels higher that the EPA's MCL, an alternative source of water should be used for drinking and cooking should be considered.

If you use arsenic-treated wood in home projects, personal protection from exposure to arseniccontaining sawdust may be helpful in limiting exposure of family members. These measures may include dust masks, gloves, and protective clothing. Arsenic-treated wood should never be burned in open fires, or in stoves, residential boilers, or fire places, and should not be composted or used as mulch. EPA's Consumer Awareness Program (CAP) for CCA is a voluntary program established by the manufacturers of CCA products to inform consumers about the proper handling, use, and disposal of CCA-treated wood. You can find more information about this program in Section 6.5. Hand washing can reduce the potential exposure of children to arsenic after playing on play structures constructed with CCA-treated wood, since most of the arsenic on the children's hands was removed with water.

If you live in an area with a high level of arsenic in the water or soil, substituting cleaner sources of water and limiting contact with soil (for example, through use of a dense groundcover or thick lawn) would reduce family exposure to arsenic. By paying careful attention to dust and soil 
control in the home (air filters, frequent cleaning), you can reduce family exposure to contaminated soil. Some children eat a lot of soil. You should prevent your children from eating soil. You should discourage your children from putting objects in their mouths. Make sure they wash their hands frequently and before eating. Discourage your children from putting their hands in their mouths or engaging in other hand-to-mouth activities. Since arsenic may be found in the home as a pesticide, household chemicals containing arsenic should be stored out of reach of young children to prevent accidental poisonings. Always store household chemicals in their original labeled containers; never store household chemicals in containers that children would find attractive to eat or drink from, such as old soda bottles. Keep your Poison Control Center's number by the phone.

It is sometimes possible to carry arsenic from work on your clothing, skin, hair, tools, or other objects removed from the workplace. This is particularly likely if you work in the fertilizer, pesticide, glass, or copper/lead smelting industries. You may contaminate your car, home, or other locations outside work where children might be exposed to arsenic. You should know about this possibility if you work with arsenic.

Your occupational health and safety officer at work can and should tell you whether chemicals you work with are dangerous and likely to be carried home on your clothes, body, or tools and whether you should be showering and changing clothes before you leave work, storing your street clothes in a separate area of the workplace, or laundering your work clothes at home separately from other clothes. Material safety data sheets (MSDS) for many chemicals used should be found at your place of work, as required by the Occupational Safety and Health Administration (OSHA) in the U.S. Department of Labor. MSDS information should include chemical names and hazardous ingredients, and important properties, such as fire and explosion data, potential health effects, how you get the chemical(s) in your body, how to properly handle the materials, and what to do in the case of emergencies. Your employer is legally responsible for providing a safe workplace and should freely answer your questions about hazardous chemicals. Your state OSHA-approved occupational safety and health program or OSHA can answer any further questions and help your employer identify and correct problems with hazardous substances. Your state OSHA-approved occupational safety and health program or 
OSHA will listen to your formal complaints about workplace health hazards and inspect your workplace when necessary. Employees have a right to seek safety and health on the job without fear of punishment.

You can find more information about how arsenic can affect children in Sections 3.7 and 6.6.

\subsection{IS THERE A MEDICAL TEST TO DETERMINE WHETHER I HAVE BEEN EXPOSED TO ARSENIC?}

Several sensitive and specific tests can measure arsenic in your blood, urine, hair, or fingernails, and these tests are often helpful in determining if you have been exposed to above-average levels of arsenic in the past. These tests are not usually performed in a doctor's office. They require sending the sample to a testing laboratory.

Measurement of arsenic in your urine is the most reliable means of detecting arsenic exposures that you experienced within the last several days. Most tests measure the total amount of arsenic present in your urine. This can sometimes be misleading, because the nonharmful forms of arsenic in fish and shellfish can give a high reading even if you have not been exposed to a toxic form of arsenic. For this reason, laboratories sometimes use a more complicated test to separate "fish arsenic" from other forms. Because most arsenic leaves your body within a few days, analysis of your urine cannot detect if you were exposed to arsenic in the past. Tests of your hair or fingernails can tell if you were exposed to high levels over the past 6-12 months, but these tests are not very useful in detecting low-level exposures. If high levels of arsenic are detected, this shows that you have been exposed, but unless more is known about when you were exposed and for how long, it is usually not possible to predict whether you will have any harmful health effects.

You can find more information on how arsenic can be measured in your hair, urine, nails, and other tissues in Chapters 3 and 7. 


\subsection{WHAT RECOMMENDATIONS HAS THE FEDERAL GOVERNMENT MADE TO PROTECT HUMAN HEALTH?}

The federal government develops regulations and recommendations to protect public health. Regulations can be enforced by law. The EPA, the Occupational Safety and Health Administration (OSHA), and the Food and Drug Administration (FDA) are some federal agencies that develop regulations for toxic substances. Recommendations provide valuable guidelines to protect public health, but cannot be enforced by law. The Agency for Toxic Substances and Disease Registry (ATSDR) and the National Institute for Occupational Safety and Health (NIOSH) are two federal organizations that develop recommendations for toxic substances.

Regulations and recommendations can be expressed as "not-to-exceed" levels, that is, levels of a toxic substance in air, water, soil, or food that do not exceed a critical value that is usually based on levels that affect animals; they are then adjusted to levels that will help protect humans. Sometimes these not-to-exceed levels differ among federal organizations because they used different exposure times (an 8-hour workday or a 24-hour day), different animal studies, or other factors.

Recommendations and regulations are also updated periodically as more information becomes available. For the most current information, check with the federal agency or organization that provides it. Some regulations and recommendations for ARSENIC include the following:

The federal government has taken several steps to protect humans from arsenic. First, EPA has set limits on the amount of arsenic that industrial sources can release into the environment. Second, EPA has restricted or canceled many of the uses of arsenic in pesticides and is considering further restrictions. Third, in January 2001, the EPA lowered the limit for arsenic in drinking water from 50 to $10 \mathrm{ppb}$. Finally, OSHA has established a permissible exposure limit (PEL), 8-hour time-weighted average, of $10 \mu \mathrm{g} / \mathrm{m}^{3}$ for airborne arsenic in various workplaces that use inorganic arsenic.

You can find more information on regulations and guidelines that apply to arsenic in Chapter 8. 


\title{
1.10 WHERE CAN I GET MORE INFORMATION?
}

If you have any more questions or concerns, please contact your community or state health or environmental quality department, or contact ATSDR at the address and phone number below.

ATSDR can also tell you the location of occupational and environmental health clinics. These clinics specialize in recognizing, evaluating, and treating illnesses that result from exposure to hazardous substances.

Toxicological profiles are also available on-line at www.atsdr.cdc.gov and on CD-ROM. You may request a copy of the ATSDR ToxProfiles ${ }^{\mathrm{TM}} \mathrm{CD}-\mathrm{ROM}$ by calling the toll-free information and technical assistance number at 1-800-CDCINFO (1-800-232-4636), by e-mail at cdcinfo@cdc.gov, or by writing to:

\author{
Agency for Toxic Substances and Disease Registry \\ Division of Toxicology and Environmental Medicine \\ 1600 Clifton Road NE \\ Mailstop F-32 \\ Atlanta, GA 30333 \\ Fax: $1-770-488-4178$
}

Organizations for-profit may request copies of final Toxicological Profiles from the following:

National Technical Information Service (NTIS)

5285 Port Royal Road

Springfield, VA 22161

Phone: $1-800-553-6847$ or $1-703-605-6000$

Web site: http://www.ntis.gov/ 


\section{RELEVANCE TO PUBLIC HEALTH}

\subsection{BACKGROUND AND ENVIRONMENTAL EXPOSURES TO ARSENIC IN THE UNITED STATES}

Arsenic is widely distributed in the Earth's crust, which contains $\sim 3.4 \mathrm{ppm}$ arsenic. In nature, arsenic is mostly found in minerals and only to a small extent in its elemental form. Arsenic is mainly obtained as a byproduct of the smelting of copper, lead, cobalt, and gold ores. Arsenic trioxide is the primary form in which arsenic is marketed and consumed. There has been no domestic production of arsenic since 1985. In 2003, the world's largest producer of arsenic compounds was China, followed by Chile and Peru.

In 2003, the United States was the world's largest consumer of arsenic. Production of wood preservatives, primarily copper chromated arsenate (CCA), $\mathrm{CrO}_{3} \cdot \mathrm{CuO} \bullet \mathrm{As}_{2} \mathrm{O}_{5}$, accounted for $>90 \%$ of domestic consumption of arsenic trioxide. In response to consumer concerns, U.S. manufacturers of arsenical wood preservative began a voluntary transition from CCA to other wood preservatives for certain residential wood products. This phase-out was completed on December 31, 2003; wood treated prior to this date could still be used and CCA-treated wood products continue to be used in industrial applications.

Other uses for arsenic compounds include the production of agricultural chemicals, as an alloying element in ammunition and solders, as an anti-friction additive to metals used for bearings, and to strengthen leadacid storage battery grids. High-purity arsenic (99.9999\%) is used by the electronics industry for galliumarsenide semiconductors for telecommunications, solar cells, and space research. Various organic arsenicals are still used in the United States as herbicides and as antimicrobial additives for animal and poultry feed. However, the use of inorganic arsenic compounds in agriculture has virtually disappeared beginning around the 1960s. Arsenic trioxide and arsenic acid were used as a decolorizer and fining agent in the production of bottle glass and other glassware. Arsenic compounds also have a long history of use in medicine, and have shown a re-emergence of late with the recent introduction of arsenic trioxide treatment for acute promyelocytic leukemia.

The principal route of exposure to arsenic for the general population is likely to be the oral route, primarily in the food and in the drinking water. Dietary exposures to total arsenic were highly variable, with a mean of $50.6 \mu \mathrm{g} /$ day (range of $1.01-1,081 \mu \mathrm{g} /$ day) for females and $58.5 \mu \mathrm{g} /$ day (range of $0.21-$ $1,276 \mu \mathrm{g} /$ day) for males. U.S. dietary intake of inorganic arsenic has been estimated to range from 1 to $20 \mu \mathrm{g} /$ day, with grains and produce expected to be significant contributors to dietary inorganic arsenic 
intake. Drinking water generally contains an average of $2 \mu \mathrm{g} / \mathrm{L}$ of arsenic, although $12 \%$ of water supplies from surface water sources in the North Central region of the country and $12 \%$ of supplies from groundwater sources in the Western region have levels exceeding $20 \mu \mathrm{g} / \mathrm{L}$. Arsenic is also widely distributed in surface water, groundwater, and finished drinking water in the United States. Surveys of arsenic concentrations in rivers and lakes indicate that most values are below $10 \mu \mathrm{g} / \mathrm{L}$, although individual samples may range up to $3,400 \mu \mathrm{g} / \mathrm{L}$. Arsenic released to the land at hazardous waste sites is likely to be relatively immobile due to a high capacity for soil binding, particularly to iron and manganese oxides. Exposure to arsenic from other pathways is generally small, but may be significant for areas with high levels of arsenic contamination or in occupational settings. For a more complete discussion of possible exposures to arsenic, see Chapter 6 of the profile.

\subsection{SUMMARY OF HEALTH EFFECTS}

Arsenic is a potent toxicant that may exist in several oxidation states and in a number of inorganic and organic forms. Most cases of arsenic-induced toxicity in humans are due to exposure to inorganic arsenic, and there is an extensive database on the human health effects of the common arsenic oxides and oxyacids. Although there may be some differences in the potency of different chemical forms (e.g., arsenites tend to be somewhat more toxic than arsenates), these differences are usually minor. An exception would be arsine, which is highly toxic. However, because arsine and its methyl derivatives are gases or volatile liquids and are unlikely to be present at levels of concern at hazardous waste sites, health effect data for these compounds are not discussed in this document. Humans may be exposed to organic arsenicals (mainly methyl and phenyl derivatives of arsenic acid) that are used in agriculture and to organic arsenicals found in fish and shellfish (arsenobetaine and arsenocholine). Although the toxicity of organic arsenicals has not been as extensively investigated as inorganic arsenicals, there are sufficient animal data to evaluate the toxicity of methyl arsenates (e.g., monomethylarsonic acid [MMA] and dimethylarsinic acid [DMA]) and roxarsone. The so-called "fish arsenic" compounds (e.g., arsenobetaine) are not thought to be toxic and health effects data are not discussed in this document.

It is generally accepted that the arsenic-carbon bond is quite strong and most mammalian species do not have the capacity to break this bond; thus, inorganic arsenic is not formed during the metabolism of organic arsenicals. In most species, including humans, ingested (or exogenous) MMA(V) and DMA(V) undergo limited metabolism, do not readily enter the cell, and are primarily excreted unchanged in the urine. This is in contrast to inorganic arsenic, which undergoes sequential reduction and methylation reactions leading to the formation of MMA and DMA. Inorganic $\mathrm{As}(\mathrm{V})$ is readily reduced to inorganic 
As(III), which is taken up by the cell. Within the cell (primarily in the liver), As(III) is methylated to form MMA(V), which is reduced to MMA(III); MMA(III) subsequently undergoes oxidative methylations to form $\mathrm{DMA}(\mathrm{V})$. $\mathrm{DMA}(\mathrm{V})$ is the primary excretion product in humans. Because inorganic and organic arsenicals exhibit distinct toxicokinetic characteristics, the health effects and MRLs are considered separately.

Inorganic Arsenicals. Exposures of humans near hazardous waste sites could involve inhalation of arsenic dusts in air, ingestion of arsenic in water, food, or soil, or dermal contact with contaminated soil or water. Increased risk of lung cancer, respiratory irritation, nausea, skin effects, and neurological effects have been reported following inhalation exposure. There are only a few quantitative data on noncancer effects in humans exposed to inorganic arsenic by the inhalation route. Animal data similarly identify effects on the respiratory system as the primary noncancer effect of inhaled inorganic arsenic compounds, although only a few studies are available. Only limited data on the effects of inhaled organic arsenic compounds in humans or animals are available; these studies are generally limited to high-dose, short-term exposures, which result in frank effects.

Relatively little information is available on effects due to direct dermal contact with inorganic arsenicals, but several studies indicate that the chief effect is local irritation and dermatitis, with little risk of other adverse effects.

The database for the oral toxicity of inorganic arsenic is extensive, containing a large number of studies of orally-exposed human populations. These studies have identified effects on virtually every organ or tissue evaluated, although some end points appear to be more sensitive than others. The available data from humans identify the skin as the most sensitive noncancer target following long-term oral arsenic exposure. Typical dermal effects include hyperkeratinization of the skin (especially on the palms and soles), formation of multiple hyperkeratinized corns or warts, and hyperpigmentation of the skin with interspersed spots of hypopigmentation. Oral exposure data from studies in humans indicate that these lesions typically begin to manifest at exposure levels of about $0.002-0.02 \mathrm{mg} \mathrm{As} / \mathrm{kg} / \mathrm{day}$, but one study suggests that lesions may appear at even lower levels. At these exposure levels, peripheral vascular effects are also commonly noted, including cyanosis, gangrene, and, in Taiwanese populations, the condition known as "Blackfoot Disease." Other reported cardiovascular effects of oral exposure to inorganic arsenic include increased incidences of high blood pressure and circulatory problems. The use of intravenous arsenic trioxide as therapy for acute promyelocytic leukemia has raised further concerns about the cardiovascular effects of arsenic, including alterations in cardiac QT interval and the 
development of torsades de pointes. Decrements in lung function, assessed by spirometry, have been reported in subjects exposed to approximately $0.008-0.04 \mathrm{mg} \mathrm{As} / \mathrm{kg} /$ day in the drinking water who exhibited skin lesions.

In addition to dermal, cardiovascular, and respiratory effects, oral exposure to inorganic arsenic may result in effects on other organ systems. Nausea, vomiting, and diarrhea are very common symptoms in humans following oral exposure to inorganic arsenicals, both after acute high-dose exposure and after repeated exposure to lower doses; these effects are likely due to a direct irritation of the gastrointestinal mucosa. Acute, high-dose exposure can lead to encephalopathy, with clinical signs such as confusion, hallucinations, impaired memory, and emotional lability, while long-term exposure to lower levels can lead to the development of peripheral neuropathy characterized by a numbness in the hands and feet that may progress to a painful "pins and needles" sensation. Recent studies also have reported neurobehavioral alterations in arsenic-exposed children.

Chronic exposure of humans to inorganic arsenic in the drinking water has been associated with excess incidence of miscarriages, stillbirths, preterm births, and infants with low birth weights. Animal data suggest that arsenic may cause changes to reproductive organs of both sexes, including decreased organ weight and increased inflammation of reproductive tissues, although these changes may be secondary effects. However, these changes do not result in a significant impact on reproductive ability. Animal studies of oral inorganic arsenic exposure have reported developmental effects, but generally only at concentrations that also resulted in maternal toxicity.

Arsenic is a known human carcinogen by both the inhalation and oral exposure routes. By the inhalation route, the primary tumor types are respiratory system cancers, although a few reports have noted increased incidence of tumors at other sites, including the liver, skin, and digestive tract. In humans exposed chronically by the oral route, skin tumors are the most common type of cancer. In addition to skin cancer, there are a number of case reports and epidemiological studies that indicate that ingestion of arsenic also increases the risk of internal tumors (mainly of bladder and lung, and to a lesser extent, liver, kidney, and prostate).

The Department of Health and Human Services (DHHS) has concluded that inorganic arsenic is known to be a human carcinogen. The International Agency for Research on Cancer (IARC) cites sufficient evidence of a relationship between exposure to arsenic and human cancer. The IARC classification of arsenic is Group 1. The EPA has determined that inorganic arsenic is a human carcinogen by the 
inhalation and oral routes, and has assigned it the cancer classification, Group A. EPA has calculated an oral cancer slope factor of $1.5(\mathrm{mg} / \mathrm{kg} / \mathrm{day})^{-1}$ and a drinking water unit risk of $5 \times 10^{-5}(\mu \mathrm{g} / \mathrm{L})^{-1}$ for inorganic arsenic based on human dose-response data. The inhalation unit risk for cancer is calculated to be $0.0043\left(\mu \mathrm{g} / \mathrm{m}^{3}\right)^{-1}$. The unit risk is the upper-bound excess lifetime cancer risk estimated to result from continuous exposure to an agent at a concentration of $1 \mu \mathrm{g} / \mathrm{L}$ in water or $1 \mu \mathrm{g} / \mathrm{m}^{3}$ in air. EPA is currently revising the assessment for inorganic arsenic; a more detailed discussion of the uncertainties associated with human cancer risk levels for arsenic is presented in Section 3.2.2.7.

The following sections discuss significant effects resulting from exposure to inorganic arsenic in greater detail: dermal, cardiovascular, respiratory, gastrointestinal, neurological, and cancer. Additional information on these effects and on other effects is discussed in Section 3.2.

Dermal Effects. The most characteristic effect of long-term oral exposure to inorganic arsenic compounds is the development of skin lesions; these lesions are often used as diagnostic criteria for arsenicosis. The three lesions most often associated with chronic arsenicosis are hyperkeratinization of the skin (especially on the palms and soles), formation of multiple hyperkeratinized corns or warts, and hyperpigmentation of the skin with interspersed spots of hypopigmentation. Numerous studies of longterm, low-level exposure to inorganic arsenic in humans have reported the presence of these lesions. In general, they begin to manifest at chronic exposure levels $>0.02 \mathrm{mg} \mathrm{As} / \mathrm{kg} / \mathrm{day}$. Chronic oral studies of lower exposure levels, ranging from 0.0004 to $0.01 \mathrm{mg} \mathrm{As} / \mathrm{kg} /$ day, have generally not reported dermal effects. However, in a study with detailed exposure assessment, all confirmed cases of skin lesions ingested water containing $>100 \mu \mathrm{g} / \mathrm{L}$ arsenic (approximately $0.0037 \mathrm{mg} \mathrm{As} / \mathrm{kg} /$ day) and the lowest known peak arsenic concentration ingested by a case was $0.115 \mu \mathrm{g} / \mathrm{L}$ (approximately $0.0043 \mathrm{mg} \mathrm{As} / \mathrm{kg} /$ day). Another large study reported increased incidence of skin lesions associated with estimated doses of $0.0012 \mathrm{mg} \mathrm{As} / \mathrm{kg} /$ day (0.023 mg As/L drinking water). The mechanism(s) by which inorganic arsenic causes dermal effects is not well-understood. Elucidating the mechanism of dermal effects has been particularly difficult because the dermal effects common in humans have not been seen in studies in animals.

Dermal effects have also been reported following inhalation exposures to inorganic arsenic, although they are not as diagnostic as for oral exposure. Several studies of arsenic-exposed workers have reported the development of dermatitis; exposure levels required to produce this condition are not well-established. Altered dermal pigmentation and hyperkeratosis have also been reported in studies of humans exposed to inorganic arsenic by inhalation, although exposure levels have varied considerably. Direct dermal contact 
with inorganic arsenicals may cause irritation and contact dermatitis. Usually, the effects are mild (erythema and swelling), but may progress to papules, vesicles, or necrotic lesions in extreme cases; these conditions tend to heal without treatment if exposure ceases.

Cardiovascular Effects. A large number of studies in humans have reported cardiovascular effects following oral exposure to inorganic arsenic compounds. The cardiac effects of arsenic exposure are numerous, and include altered myocardial depolarization (prolonged QT interval, nonspecific ST segment changes), cardiac arrhythmias, and ischemic heart disease. These effects have been seen after acute and long-term exposure to inorganic arsenic in the environment, as well as side effects from intravenous therapy with arsenic trioxide for acute promyelocytic leukemia. Exposure levels for environmental exposures have not been well characterized, but intravenous doses for arsenic trioxide therapy are generally on the order of $0.15 \mathrm{mg} \mathrm{As} / \mathrm{kg} / \mathrm{day}$.

Chronic exposure to inorganic arsenic has also been shown to lead to effects on the vascular system. The most dramatic of these effects is "Blackfoot Disease," a disease characterized by a progressive loss of circulation in the hands and feet, leading ultimately to necrosis and gangrene. Blackfoot Disease is endemic in an area of Taiwan where average drinking water levels of arsenic range from 0.17 to $0.80 \mathrm{ppm}$, corresponding to doses of about $0.014-0.065 \mathrm{mg} \mathrm{As} / \mathrm{kg} /$ day. The results of a another study suggested that individuals with a lower capacity to methylate inorganic arsenic to DMA have a higher risk of developing peripheral vascular disease in the Blackfoot Disease-hyperendemic area in Taiwan. Arsenic exposure in Taiwan has also been associated with an increased incidence of cerebrovascular and microvascular diseases and ischemic heart disease. While Blackfoot Disease itself has not been reported outside of Taiwan, other vascular effects are common in areas with high arsenic exposures, and include such severe effects as increases in the incidences of Raynaud's disease and of cyanosis of fingers and toes as well as hypertension, thickening and vascular occlusion of blood vessels, and other unspecified cardiovascular conditions. However, while the majority of human studies have reported cardiovascular effects following exposure to inorganic arsenic, some have found no such effects.

Changes in cardiac rhythm and in some vascular end points have also been reported in animal studies of inorganic arsenicals, but generally only at higher exposure levels and not to the degree of severity seen in humans.

Respiratory Effects. While case reports and small cohort studies have routinely reported an increase in respiratory symptoms of humans exposed occupationally to inorganic arsenic, dose-response data for 
these symptoms are generally lacking. The only study that evaluated respiratory effects (changes in chest $\mathrm{x}$-ray or respiratory performance) and reported an exposure estimate did not report significant changes at an exposure level of $0.613 \mathrm{mg} \mathrm{As} / \mathrm{m}^{3}$. Exposed workers often report irritation of the mucous membranes of the nose and throat, which may lead to laryngitis, bronchitis, or rhinitis. Increased mortality due to respiratory disease has been reported in some cohort mortality studies of arsenic-exposed workers, but no conclusive evidence of an association of these diseases with arsenic exposure has been presented. It is not known whether respiratory effects following inhaled inorganic arsenic compounds are due to a direct effect of arsenic on respiratory tissues, general effects of foreign material in the lungs, or an effect of arsenic on the pulmonary vasculature. Similar responses, including rales, labored breathing, and respiratory hyperplasia, have been noted in animal studies of inhaled or instilled inorganic arsenic compounds.

Respiratory effects have also been reported following oral exposure of humans to inorganic arsenic. Acute oral exposure to $\geq 8 \mathrm{mg} \mathrm{As} / \mathrm{kg}$ may result in serious respiratory effects, including respiratory distress, hemorrhagic bronchitis, and pulmonary edema; however, it is not clear whether these are primary effects or are the result of damage to the pulmonary vascular system. In general, respiratory effects have not been widely associated with long-term oral exposure to low arsenic doses. However, some studies have reported minor respiratory symptoms, such as cough, sputum, rhinorrhea, and sore throat, in people with repeated oral exposure to $0.03-0.05 \mathrm{mg} \mathrm{As} / \mathrm{kg} /$ day. More serious respiratory effects, such as bronchitis and sequelae (bronchiectasis, bronchopneumonia) have been observed in patients chronically exposed to arsenic and at autopsy in some chronic poisoning cases. There are few animal data reporting respiratory effects of oral exposure to inorganic arsenic, and those studies generally found effects only at very high dose levels.

Gastrointestinal Effects. Both short-term and chronic oral exposures to inorganic arsenicals have been reported to result in irritant effects on gastrointestinal tissues. Numerous studies of acute, high-dose exposure to inorganic arsenicals have reported nausea, vomiting, diarrhea, and abdominal pain, although specific dose levels associated with the onset of these symptoms have not been identified. Chronic oral exposure to $0.01 \mathrm{mg} \mathrm{As} / \mathrm{kg} /$ day generally results in similar reported symptoms. For both acute and chronic exposures, the gastrointestinal effects generally diminish or resolve with cessation of exposure. Similar gastrointestinal effects have been reported after occupational exposures to inorganic arsenicals, although it is not known if these effects were due to absorption of arsenic from the respiratory tract or from mucociliary clearance resulting in eventual oral exposure. 
Neurological Effects. A common effect following both oral and inhalation exposure to inorganic is the development of peripheral neuropathy. Following occupational exposure to inorganic arsenic in pesticide plants or smelters, exposed workers have shown increased incidence of neurological changes, including altered nerve conduction velocities. One study reported that these effects were seen after 28 years of exposure to $0.31 \mathrm{mg} \mathrm{As} / \mathrm{m}^{3}$. In another study, signs and symptoms of sensory and motor polyneuropathy on both upper and lower extremities were reported in workers at a power station in Slovakia. The average length of exposure was 22.3 years (standard deviation [SD] \pm 8.4 years) and the average arsenic exposure in inhaled air ranged from 4.6 to $142.7 \mu \mathrm{g} / \mathrm{m}^{3}$.

Following high-dose ( $>2 \mathrm{mg} \mathrm{As} / \mathrm{kg} /$ day) acute oral exposures to inorganic arsenicals in humans, reported effects include headache, lethargy, mental confusion, hallucination, seizures, and coma. Following longer-term exposure to $0.03-0.1 \mathrm{mg} \mathrm{As} / \mathrm{kg} / \mathrm{day}$, peripheral neuropathy, characterized initially by numbness of the hands and feet and a "pins and needles" sensation and progressing to muscle weakness, wrist-drop and/or ankle-drop, diminished sensitivity, and altered reflex action. Histological features of the neuropathy include a dying-back axonopathy and demyelination. Following removal from exposure, the neuropathy is only partially reversible and what recovery does occur is generally slow. Reports of neurological effects at lower arsenic levels (0.004-0.006 mg As/kg/day) have been inconsistent, with some human studies reporting fatigue, headache, depression, dizziness, insomnia, nightmare, and numbness while others reported no neurological effects. Some studies also have reported that exposure to arsenic may be associated with intellectual deficits in children. Neurological effects have also been reported in oral studies of arsenic toxicity in animals, although these were generally performed at higher doses (0.4-26.6 mg As/kg/day) than has been reported in exposed human populations. The mechanism(s) of arsenic-induced neurological changes has not been determined.

Cancer. There is clear evidence from studies in humans that exposure to inorganic arsenic by either the inhalation or oral routes increases the risk of cancer. Numerous studies of copper smelters or miners exposed to arsenic trioxide have reported an increased risk of lung cancer. Increased incidence of lung cancer has also been observed at chemical plants where exposure was primarily to arsenate. Other studies suggest that residents living near smelters or arsenical chemical plants may have increased risk of lung cancer, although the reported increases are small and are not clearly detectable in all cases. In general, studies reporting long-term exposure to $0.07 \mathrm{mg} \mathrm{As} / \mathrm{m}^{3}$ or greater have shown an increased incidence of lung cancer, while at lower exposure levels, the association has been less clear or not present. 
There is convincing evidence from a large number of epidemiological studies and case reports that ingestion of inorganic arsenic increases the risk of developing skin cancer. The most common tumors seen are squamous cell carcinomas, which may develop from the hyperkeratotic warts or corns commonly seen as a dermal effect of oral inorganic arsenic exposure. Early studies of populations within the United States did not suggest an increased risk of cancer from oral inorganic arsenic exposure. Later studies have found suggestive evidence that the possibility of arsenic-induced skin cancers cannot be discounted based on an association between toenail arsenic levels and incidence of skin cancer.

There is increasing evidence that long-term exposure to arsenic can result in the development of bladder cancer, with transitional cell cancers being the most prevalent. While studies have noted statistical doseresponse trends in arsenic-induced bladder cancers, reliable quantitative assessments of dose-response relationships have not been presented. Several studies have also shown that chronic oral exposure to arsenic results in the development of respiratory tumors, making lung cancer an established cause of death from exposure to arsenic in drinking water. Exposure levels in studies evaluating respiratory and bladder cancers have been comparable to those in studies evaluating skin tumors. Studies of U.S. populations have not identified an increased risk of bladder or respiratory tumors following oral exposure to inorganic arsenic.

Animal studies of both inhalation and oral exposure to inorganic arsenicals have not resulted in increased incidence of cancer formation in adult animals. However, a series of studies have shown that inorganic arsenic can induce cancer in the offspring from mice exposed to arsenic during gestation (transplacental carcinogen) and acts as a co-carcinogen with UV light and polycyclic aromatic hydrocarbons (PAHs).

Organic Arsenicals. Humans may be exposed to organic arsenicals via inhalation of dusts, ingestion of organic arsenic in water, food, soil, or dermal contact with contaminated soil, water or plants following pesticide application. There are limited data on the toxicity of organic arsenicals following inhalation exposure in humans and animals and these data do not allow for identification of critical effects. Keratosis was observed in workers exposed to $0.065 \mathrm{mg} / \mathrm{m}^{3}$ arsanilic acid (i.e., 4-aminophenyl arsenic acid); no alterations in gastrointestinal symptoms or hematological alterations were observed. In animals, very high concentrations $\left(>3,000 \mathrm{mg} / \mathrm{m}^{3}\right)$ of DMA results in respiratory distress, diarrhea, and erythematous lesions on the feet and ears. No adverse effects were observed in rats exposed to DMA concentrations as high as $100 \mathrm{mg} \mathrm{DMA} / \mathrm{m}^{3}$ for 95 days. 
Similarly, the available dermal toxicity data do not allow for identification of critical effects. Contact dermatitis was observed in workers applying DMA (and its sodium salt) and mild dermal irritation was observed in a Draize test in rabbits (adverse effect level not reported). Intermediate duration (21 days) exposure studies in rabbits did not result in systemic toxicity or skin irritation following 5 day/week exposure to $1,000 \mathrm{mg} / \mathrm{kg} /$ day MMA or DMA.

The preponderance of toxicity data for organic arsenicals involves oral exposure. Human data are limited to three case reports of individuals intentionally ingesting pesticides containing organic arsenicals. Gastrointestinal irritation (vomiting, nausea, and diarrhea) were consistently reported in these cases. Animal data has primarily focused on the toxicity of MMA, DMA, and roxarsone; these data suggest that the targets of toxicity may differ between the compounds.

$\boldsymbol{M M A}$. The gastrointestinal tract appears to be the most sensitive target of toxicity for MMA. Diarrhea and tissue damage in the large intestine have been reported in several animal species following dietary, gavage, and capsule exposure. For diarrhea, both the time of onset and incidence appear to be doserelated. In rats, diarrhea was observed in $100 \%$ of females exposed to $98.5 \mathrm{mg} \mathrm{MMA} / \mathrm{kg} / \mathrm{day}, 55 \%$ of females exposed to $33.9 \mathrm{mg} \mathrm{MMA} / \mathrm{kg} /$ day, and $5.1 \%$ of females exposed to $3.9 \mathrm{mg} \mathrm{MMA} / \mathrm{kg} / \mathrm{day}$. The increased incidence of diarrhea was observed after 3 weeks of exposure to $98.5 \mathrm{mg} \mathrm{MMA} / \mathrm{kg} / \mathrm{day}$, 4 weeks at $33.9 \mathrm{mg} \mathrm{MMA} / \mathrm{kg} /$ day, and 18 months at $3.9 \mathrm{mg} \mathrm{MMA} / \mathrm{kg} /$ day. Histological damage consisting of squamous metaplasia of the epithelial columnar absorptive cells in the cecum, colon, and rectum was observed in rats and mice chronically exposed to 72.4 or $67.1 \mathrm{mg} \mathrm{MMA} / \mathrm{kg} /$ day, respectively. Hemorrhagic, necrotic, ulcerated, or perforated mucosa were also observed in the large intestine of rats exposed to $67.1 \mathrm{mg} \mathrm{MMA} / \mathrm{kg} /$ day for 2 years. In rats, the damage to the large intestine resulted in intestinal contents leaking into the abdominal cavity and the development of peritonitis. The available data provide suggestive evidence that there may be some species differences in the sensitivity to gastrointestinal damage; however, some of these differences may be due to the route of administration. The lowest adverse effect levels, regardless of duration of exposure, for gastrointestinal effects in rats, mice, rabbits, and dogs are 25.7, 67.1,12, and $2 \mathrm{mg} \mathrm{MMA} / \mathrm{kg} /$ day, respectively; the no adverse effect levels in rats and mice (NOAELs were not identified in rabbits and dogs) were 3.0 and $24.9 \mathrm{mg}$ MMA/kg/day. However, the rabbit and dog studies involved bolus administration (gavage and capsule administration), which may have increased sensitivity; the rat and mouse studies involved dietary exposure. 
The kidney also appears to be a sensitive target in rats and mice chronically exposed to MMA. An increase in the severity of progressive glomerulonephropathy was observed in female rats exposed to $33.9 \mathrm{mg} \mathrm{MMA} / \mathrm{kg} /$ day for 2 years and an increase in the incidence of progressive glomerulonephropathy was observed in male mice exposed to $6.0 \mathrm{mg} \mathrm{MMA} / \mathrm{kg} /$ day for 2 years. Other adverse effects that have been observed in animals exposed to MMA include hypertrophy of the thyroid follicular cells in rats exposed to $33.9 \mathrm{mg} \mathrm{MMA} / \mathrm{kg} /$ day in the diet for 2 years, reproductive toxicity, and developmental toxicity. Decreases in pregnancy rate and male fertility index were observed in $\mathrm{F}_{0}$ and $\mathrm{F}_{1}$ rats exposed to $76 \mathrm{mg} \mathrm{MMA} / \mathrm{kg} /$ day for 14 weeks prior to mating and during the mating period; the findings were not significantly different than control values but were considered treatment-related because they were outside the range found in historical controls. This study also reported a decrease in pup survival in the $\mathrm{F}_{1}$ and $\mathrm{F}_{2}$ offspring of rats exposed to $76 \mathrm{mg} \mathrm{MMA} / \mathrm{kg} /$ day; as with the reproductive effects, the incidence was not statistically different from controls but was considered biologically significant because survival in the MMA pups was outside the range found in historical controls. Another study reported impaired fetal growth (decreases in fetal weights and incomplete ossification) and minor skeletal defects (an increase in the number of fetuses with supernumerary thoracic ribs and eight lumbar vertebrae) in rat and rabbit fetuses exposed to 500 or $12 \mathrm{mg} \mathrm{MMA} / \mathrm{kg} /$ day, respectively; maternal toxicity was also observed at these dose levels and the effects may be secondary to maternal stress rather than a direct effect on the developing organisms. A 2-year bioassay did not result in significant increases in the incidence of neoplastic lesions in rats and mice exposed to doses as high as 72.4 and $67.1 \mathrm{mg} \mathrm{MMA} / \mathrm{kg} / \mathrm{day}$, respectively.

$\boldsymbol{D M A}$. The most sensitive targets of DMA toxicity in rats are the urinary bladder and kidneys. In the bladder, the effects progress from cytotoxicity to cellular necrosis to regenerative proliferation and hyperplasia. At dietary doses of $11 \mathrm{mg} \mathrm{DMA} / \mathrm{kg} /$ day, cytotoxicity is observed as early as 6 hours after exposure initiation and cellular proliferation (as evident by increased BrdU labeling) was observed after 2 weeks of exposure. After 10 weeks of exposure, necrosis and hyperplasia were also observed. The lowest adverse effect levels for urinary bladder effects following intermediate or chronic duration exposure were $5 \mathrm{mg}$ DMA/kg/day for evidence of regenerative proliferation and $3.1 \mathrm{mg} \mathrm{DMA} / \mathrm{kg} / \mathrm{day}$ for vacuolar degeneration of urothelium and hyperplasia. Vacuolization of the superficial cells of the urothelium was observed in mice exposed to $7.8 \mathrm{mg} \mathrm{DMA} / \mathrm{kg} /$ day and higher for 2 years. However, unlike the vacuolar degeneration observed in rats, the vacuolization observed in mice was not associated with cytotoxicity, necrosis, inflammation, or hyperplasia. 
Kidney damage characterized by increased urinary calcium levels, calcification, nephrocalcinosis, and necrosis of the renal papillae have been observed in rats following intermediate- or chronic-duration exposure. Increases in urine calcium levels and corticomedullary junction calcification were observed in rats exposed to 5 or $10 \mathrm{mg} \mathrm{DMA} / \mathrm{kg} /$ day for 10 weeks and cortical degeneration and necrosis were observed in rats exposed to $57 \mathrm{mg}$ DMA/kg/day for 4 weeks. Chronic-duration exposure to $3.1 \mathrm{mg}$ DMA $/ \mathrm{kg} /$ day resulted in an increased incidence of nephrocalcinosis and necrosis of the renal papillae in rats; these lesions are typical in aged rats, although DMA exposure appeared to exacerbate them. An exacerbation of age-related kidney lesion (progressive glomerulonephropathy and nephrocalcinosis) has also been observed in male mice exposed to 37 or $94 \mathrm{mg} \mathrm{DMA} / \mathrm{kg} /$ day, respectively, for 2 years. A consistent finding in intermediate and chronic rat studies is an increase in urine volume, which corresponds to an increase in water consumption; the toxicological significance of this finding is not known. The observed decreases in electrolyte levels and specific gravity are likely due to the higher urine volume.

Although gastrointestinal effects have been observed in animals exposed to DMA, it does not appear to be as sensitive a target compared to MMA. Diarrhea has been observed in rats exposed to a lethal dose of $190 \mathrm{mg}$ DMA/kg/day for 4 weeks and in dogs administered via 16 mg DMA/kg/day. No gastrointestinal effects were observed in rats or mice chronically exposed to 7.8 or $94 \mathrm{mg} \mathrm{DMA} / \mathrm{kg} /$ day.

Other adverse effects that have been observed in animals exposed to organic arsenicals include hypertrophy of thyroid follicular cells in rats exposed to $4.0 \mathrm{mg} \mathrm{DMA} / \mathrm{kg} / \mathrm{day}$ in the diet for 13 weeks and $7.8 \mathrm{mg}$ DMA $/ \mathrm{kg} /$ day in the diet for 2 years and developmental effects in rats and mice. Decreases in fetal growth and delays in ossification have been observed in rat fetuses exposed to $\geq 36 \mathrm{mg} \mathrm{DMA} / \mathrm{kg} /$ day; these alterations typically occur at doses associated with decreases in maternal weight gain. Other developmental effects that have been reported include an increase in the incidences of irregular palatine rugae in rats exposed to $30 \mathrm{mg} \mathrm{DMA} / \mathrm{kg} /$ day, diaphragmatic hernia in rats exposed to $36 \mathrm{mg}$ $\mathrm{DMA} / \mathrm{kg} /$ day, and cleft palate in mice exposed to $400 \mathrm{mg} \mathrm{DMA} / \mathrm{kg} / \mathrm{day}$. No developmental effects were observed in rabbits exposed to $12 \mathrm{mg} \mathrm{DMA} / \mathrm{kg} /$ day.

The available data provide strong evidence that DMA is carcinogenic in rats. A 2-year exposure to DMA resulted in significant increases in the incidence of neoplastic urinary bladder tumors in rats exposed to $7.8 \mathrm{mg} \mathrm{DMA} / \mathrm{kg} /$ day in the diet or $3.4 \mathrm{mg} \mathrm{DMA} / \mathrm{kg} /$ day in drinking water. No increases in neoplastic tumors were observed in mice exposed to doses as high as $94 \mathrm{mg} \mathrm{DMA} / \mathrm{kg} /$ day for 2 years; however, a 
50-week exposure to $10.4 \mathrm{mg} \mathrm{DMA} / \mathrm{kg} /$ day did result in an increased incidence of lung tumors in $\mathrm{A} / \mathrm{J}$ mice.

The available data for DMA suggest that there are species differences in terms of the critical effects and sensitivity. In rats, the urinary bladder and kidneys are the most sensitive targets with effects occurring at $5 \mathrm{mg} \mathrm{DMA} / \mathrm{kg} /$ day following intermediate-duration exposure and $3.1 \mathrm{mg} \mathrm{DMA} / \mathrm{kg} /$ day following chronic-duration exposure. Although the urinary bladder and kidneys are also sensitive targets in mice with LOAELs of 7.8 and $37 \mathrm{mg}$ DMA/kg/day, respectively, following chronic exposure, the effects are not associated with cytotoxicity or elevated urine calcium levels. In dogs, the most sensitive effect is gastrointestinal tract irritation (diarrhea), which occurs at $16 \mathrm{mg} \mathrm{DMA} / \mathrm{kg} / \mathrm{day}$.

There is concern that the rat may not be a good model to predict the human risk associated with organic arsenic exposure due to the unique toxicokinetic properties of DMA in rats. In humans and most animal species, DMA is rapidly eliminated from the body; $>90 \%$ of the dose is excreted $2-3$ days after dosing. In contrast, DMA is slowly eliminated in rats. One study estimated that $45 \%$ of an initial oral DMA dose was eliminated with a half-time of 13 hours; the remaining 55\% of the dose DMA dose had an elimination half-time of 50 days. In rats, DMA has a strong affinity for hemoglobin resulting in an accumulation of DMA in erythrocytes. Species differences in DMA metabolism have also been found. In particular, DMA undergoes further methylation to trimethylarsine oxide (TMAO) in rats. In most animal species, almost the entire oral DMA dose is excreted in the urine unchanged; however, in rats, about half of the dose is excreted in the urine as DMA and the other half as TMAO. During the metabolism of DMA to TMAO, DMA(III) is formed as a metabolic intermediate. The formation of this highly reactive intermediate and the excretion of small amounts of DMA(III) in urine may damage the urinary bladder.

There are limited data on the mode of action of DMA for most end points. Recently, there has been considerable research on the mode of action for the development of neoplastic urinary bladder tumors in rats. Although the mechanisms have not been fully elucidated, it has been proposed that the mode of action involves cytotoxicity leading to necrosis and subsequent regeneration of the urinary bladder urothelium. There is strong evidence to suggest that DMA(III) is the causative agent for the urothelial cytotoxicity. The strongest evidence comes from the finding that urinary concentrations of DMA(III) measured in rats exhibiting urothelial cytotoxicity are equivalent to DMA(III) concentrations that are cytotoxic to urothelial cells in vitro. Urothelial cytotoxicity, regenerative urothelial proliferation, and urothelial tumors have not been detected in other animal species. Other animal species, including 
humans, only metabolize a small percentage of ingested DMA to TMAO; thus, much lower levels of DMA(III) are produced, suggesting that rats may be very sensitive to toxicity of DMA and therefore are not an appropriate model for human risk assessment.

Roxarsone. The available data on the toxicity of roxarsone suggest that following bolus administration, the gastrointestinal tract, kidney, and nervous system are sensitive end points of roxarsone toxicity. Vomiting and gastrointestinal hemorrhage were observed in dogs receiving a single capsulized dose of $50 \mathrm{mg} / \mathrm{kg}$ roxarone; no gastrointestinal effects were observed in rats or mice administered 4 or $42 \mathrm{mg} / \mathrm{kg}$ /day roxarsone for 2 years. Kidney effects included increases in kidney weight, minimal tubular epithelial cell degeneration, and focal mineralization in rats exposed to $32 \mathrm{mg}$ roxarsone $/ \mathrm{kg} / \mathrm{day}$ for 13 weeks; no kidney effects were observed at $16 \mathrm{mg} / \mathrm{kg} /$ day or in mice exposed to doses as high as $136 \mathrm{mg} / \mathrm{kg} / \mathrm{day}$ for 13 weeks or $43 \mathrm{mg} / \mathrm{kg} / \mathrm{day}$ for 2 years. Hyperexcitability, ataxia, and/or trembling were observed in rats exposed to $20 \mathrm{mg} / \mathrm{kg} /$ day for 13 weeks or $64 \mathrm{mg} / \mathrm{kg} / \mathrm{day}$ for 13 weeks. A 14-day study in rats reported slight inactivity in rats exposed to $32 \mathrm{mg} / \mathrm{kg} /$ day, but this was not observed in longer-term studies. Neurological effects were observed in mice exposed to doses as high as $136 \mathrm{mg} / \mathrm{kg} / \mathrm{day}$ for 13 weeks or $43 \mathrm{mg} / \mathrm{kg} /$ day for 2 years, although a slight decrease in activity at $42 \mathrm{mg} / \mathrm{kg} /$ day was reported in a 14 day study. Pigs appear to be especially sensitive to the neurotoxicity of roxarsone. Muscle tremors have been observed at doses of $\geq 6.3 \mathrm{mg}$ roxarsone $/ \mathrm{kg} /$ day and myelin degeneration in the spinal cord was noted at $6.3 \mathrm{mg} / \mathrm{kg} / \mathrm{day}$. Both the clinical signs of neuropathy and the myelin degeneration followed a time-related pattern. Mild lethargy and ataxia were observed 7 days after exposure initiation, exercise-induced muscle tremors and clonic seizures were observed at day 11, paraparesis was observed at day 22, and paraplegia was observed at day 33. At day 11, equivocal lesions were observed in the cervical spinal cord, and the severity of these lesions increased with time; myelin degeneration was observed in the peripheral nerves and optic nerve starting at day 32 (2 days after exposure termination). Equivocal evidence of carcinogenicity (a slight increase in the incidence of pancreatic tumors) was found in male rats chronically exposed to roxarsone; no increases in neoplastic tumors were observed in female rats or male and female mice.

\subsection{MINIMAL RISK LEVELS (MRLS)}

Estimates of exposure levels posing minimal risk to humans (MRLs) have been made for arsenic. An MRL is defined as an estimate of daily human exposure to a substance that is likely to be without an appreciable risk of adverse effects (noncarcinogenic) over a specified duration of exposure. MRLs are derived when reliable and sufficient data exist to identify the target organ(s) of effect or the most sensitive 
health effect(s) for a specific duration within a given route of exposure. MRLs are based on noncancerous health effects only and do not consider carcinogenic effects. MRLs can be derived for acute, intermediate, and chronic duration exposures for inhalation and oral routes. Appropriate methodology does not exist to develop MRLs for dermal exposure.

Although methods have been established to derive these levels (Barnes and Dourson 1988; EPA 1990i), uncertainties are associated with these techniques. Furthermore, ATSDR acknowledges additional uncertainties inherent in the application of the procedures to derive less than lifetime MRLs. As an example, acute inhalation MRLs may not be protective for health effects that are delayed in development or are acquired following repeated acute insults, such as hypersensitivity reactions, asthma, or chronic bronchitis. As these kinds of health effects data become available and methods to assess levels of significant human exposure improve, these MRLs will be revised.

\section{Inorganic Arsenicals}

Inhalation MRLs. No inhalation MRLs were derived for inorganic arsenic. Human data suggest that dermal or respiratory effects may be the most prevalent (Lagerkvist et al. 1986; Mohamed 1998; Perry et al. 1948); respiratory or immunological effects appeared to be the most common following inhalation exposure to inorganic arsenic in animals (Aranyi et al. 1985; Holson et al. 1999). Adequate human studies evaluating dose-response relationships for noncancer end points were not located for inorganic arsenic, and animal data on the health effects of inorganic arsenic following inhalation exposure are limited to studies that did not evaluate a suitable range of health effects. Lacking suitable studies upon which to base the MRLs, no inhalation MRLs were derived for inorganic arsenic.

\section{Oral MRLs}

- An MRL of $0.005 \mathrm{mg} \mathrm{As} / \mathrm{kg} /$ day has been derived for acute-duration (14 days or less) oral exposure to inorganic arsenic.

Mizuta et al. (1956) summarized findings from 220 poisoning cases associated with an episode of arsenic contamination of soy sauce in Japan. The soy sauce was contaminated with approximately $0.1 \mathrm{mg}$ $\mathrm{As} / \mathrm{mL}$, probably as calcium arsenate. Arsenic intake in the cases was estimated by the researchers to be $3 \mathrm{mg} /$ day $(0.05 \mathrm{mg} / \mathrm{kg} /$ day, assuming $55 \mathrm{~kg}$ average body weight for this Asian population). The duration of exposure was 2-3 weeks in most cases. The primary symptoms were edema of the face, and gastrointestinal and upper respiratory symptoms initially, followed by skin lesions and neuropathy in 
some patients. Other effects included mild anemia and leukopenia, mild degenerative liver lesions and hepatic dysfunction, abnormal electrocardiogram, and ocular lesions. For derivation of the acute oral MRL, facial edema and gastrointestinal symptoms (nausea, vomiting, diarrhea), which were characteristic of the initial poisoning and then subsided, were considered to be the critical effects. The MRL of $0.005 \mathrm{mg} \mathrm{As} / \mathrm{kg} /$ day was calculated by applying an uncertainty factor of 10 (10 for use of a lowestobserved-adverse-effect level (LOAEL) and 1 for human variability) to the LOAEL of $0.05 \mathrm{mg} \mathrm{As} / \mathrm{kg} / \mathrm{day}$ (see Appendix A for MRL worksheets).

An intermediate-duration oral MRL for inorganic arsenic was not derived due to inadequacy of the database. The lowest LOAEL identified in a limited number of intermediate-duration human studies available was $0.05 \mathrm{mg} \mathrm{As} / \mathrm{kg} /$ day in a study by Mizuta et al. (1956) (summarized above). While this study was considered appropriate to derive an acute-duration oral MRL for inorganic arsenic, there is considerable uncertainty regarding what the effects and severity might be beyond the relatively short 23 weeks of exposure that most subjects experienced. There are numerous studies in animals dosed for intermediate durations, but as indicated in Section 3.5.3, animals are not appropriate models for effects of inorganic arsenic in humans.

- An MRL of $0.0003 \mathrm{mg} \mathrm{As} / \mathrm{kg} /$ day has been derived for chronic-duration (365 days or more) oral exposure to inorganic arsenic.

Tseng et al. (1968) and Tseng (1977) investigated the incidence of Blackfoot Disease and dermal lesions (hyperkeratosis and hyperpigmentation) in a large number of poor farmers (both male and female) exposed to high levels of arsenic in well water in Taiwan. A control group consisting of 17,000 people, including one group in which arsenic exposure was "undetermined" and which included those villages where arsenic-contaminated wells were no longer used or the level could not be classified, and a control population of 7,500 people who consumed water from wells almost free of arsenic (0.001-0.017 ppm) was also examined. The authors stated that the incidence of dermal lesions increased with dose, but individual doses were not provided. However, incidence data were provided based on stratification of the exposed population into low $(<300 \mu \mathrm{g} / \mathrm{L})$, medium $(300-600 \mu \mathrm{g} / \mathrm{L})$, or high $(>600 \mu \mathrm{g} / \mathrm{L})$ exposure levels. Doses were calculated from group mean arsenic concentrations in well water, assuming the intake parameters described by IRIS (IRIS 2007). Accordingly, the control, low-, medium-, and high-exposure levels correspond to doses of $0.0008,0.014,0.038$, and $0.065 \mathrm{mg} \mathrm{As} / \mathrm{kg} /$ day, respectively. The noobserved-adverse-effect level (NOAEL) identified by Tseng (1977) (0.0008 mg As/kg/day) was limited by the fact that the majority of the population was $<20$ years of age and the incidence of skin lesions increased as a function of age, and because the estimates of water intake and dietary arsenic intake are 
highly uncertain. Schoof et al. (1998) estimated that dietary intakes of arsenic from rice and yams may have been $15-211 \mu \mathrm{g} / \mathrm{day}$ (mean=61 $\mu \mathrm{g} /$ day), based on arsenic analyses of foods collected in Taiwan in 1993-1995. Use of the $50 \mu \mathrm{g} /$ day estimate would result in an approximate doubling of the NOAEL $(0.0016 \mathrm{mg} / \mathrm{kg} /$ day) (see Appendix A for MRL worksheets). The MRL was derived by applying an uncertainty factor of 3 (for human variability) to the NOAEL of $0.0008 \mathrm{mg} / \mathrm{kg} / \mathrm{day}$.

The MRL is supported by a large number of well-conducted epidemiological studies that identify reliable NOAELs and LOAELs for dermal effects. EPA (1981b) identified a NOAEL of 0.006-0.007 mg $\mathrm{As} / \mathrm{kg} /$ day for dermal lesions in several small populations in Utah. Harrington et al. (1978) identified a NOAEL of $0.003 \mathrm{mg} \mathrm{As} / \mathrm{kg} /$ day for dermal effects in a small population in Alaska. Guha Mazumder et al. (1988) identified a NOAEL of $0.009 \mathrm{mg}$ As/ $/ \mathrm{kg} / \mathrm{day}$ and a LOAEL of $0.006 \mathrm{mg} \mathrm{As} / \mathrm{kg} / \mathrm{day}$ for pigmentation changes and hyperkeratosis in a small population in India. Haque et al. (2003) identified a LOAEL of $0.002 \mathrm{mg} \mathrm{As} / \mathrm{kg} /$ day for hyperpigmentation and hyperkeratosis in a case-control study in India. Cebrián et al. (1983) identified a NOAEL of $0.0004 \mathrm{mg} \mathrm{As} / \mathrm{kg} /$ day and a LOAEL of $0.022 \mathrm{mg}$ As/kg/day in two regions in Mexico. Borgoño and Greiber (1972) and Zaldívar (1974) identified a LOAEL of $0.02 \mathrm{mg} \mathrm{As} / \mathrm{kg} /$ day for abnormal skin pigmentation in patients in Chile, and Borgoño et al. (1980) identified a LOAEL of $0.01 \mathrm{mg} \mathrm{As} / \mathrm{kg} /$ day for the same effect in school children in Chile. Valentine et al. (1985) reported a NOAEL of $0.02 \mathrm{mg} \mathrm{As} / \mathrm{kg} /$ day for dermal effects in several small populations in California. Collectively, these studies indicate that the threshold dose for hyperpigmentation and hyperkeratosis is approximately $0.002 \mathrm{mg} \mathrm{As} / \mathrm{kg} /$ day. While many of these studies also identified effects on other end points at these exposure levels, including effects on gastrointestinal (Borgoño and Greiber 1972; Cebrián et al. 1983; Guha Mazumder et al. 1988; Zaldívar 1974), cardiovascular (Tseng et al. 1995, 1996), hepatic (Hernández-Zavala et al. 1998), and neurological end points (Guha Mazumder et al. 1988; Lianfang and Jianzhong 1994; Tsai et al. 2003), the overall database for dermal effects is considerably stronger than for effects on other end points.

\section{Organic Arsenicals}

Inhalation MRLs. No inhalation MRLs were derived for organic arsenic. Human data are limited to an occupational exposure study of workers exposed to $0.065 \mathrm{mg} / \mathrm{m}^{3}$ ansanilic acid (Watrous and McCaughey 1945). The exposed workers more frequently complained of keratosis than nonexposed workers. A limited number of animal studies have examined the toxicity of organic arsenicals following inhalation exposure. Respiratory distress and diarrhea were observed in rats and mice exposed to high concentrations of MMA and DMA (Stevens et al. 1979); at lower concentrations (1,540-3,150 mg $\mathrm{DMA} / \mathrm{m}^{3}$ ), respiratory irritation, as evidenced by a decrease in respiration rate, was observed in animals 
exposed to MMA or DMA (Stevens et al. 1979). The acute-duration studies do not clearly identify the most sensitive targets of inorganic arsenical toxicity; the available studies are of limited scope and none included a comprehensive histological examination.

One study examined the toxicity of DMA in rats following intermediate-duration exposure. This study (Whitman 1994) found an increase in intracytoplasmic eosinophilic globules in the nasal turbinates of rats exposed to 34 or $100 \mathrm{mg} / \mathrm{m}^{3}$ DMA 6 hours/day, 5 days/week for 67-68 exposures; no other adverse effects were observed in this comprehensive study. As discussed in greater detail in the oral MRL section, the toxicokinetic properties of DMA in rats differ from other species and rats do not appear to be a good model for human exposure. The half-time of DMA in the body is much longer in rats compared to other species, including humans, and DMA is more extensively methylated in rats. In the absence of data to determine whether the observed effect is due to a direct interaction of DMA, derivation of an intermediate-duration MRL using rat data is not recommended at this time.

No studies examined the chronic toxicity of organic arsenicals precluding the derivation of a chronicduration inhalation MRL.

\section{Oral MRLs}

MMA. A limited number of animal studies have examined the acute oral toxicity of MMA. These studies consisted of $\mathrm{LD}_{50}$ studies in rats (Gur and Nyska 1990), mice (Kaise et al. 1989), and rabbits (Jaghabir et al. 1988) and developmental toxicity studies in rats (Irvine et al. 2006) and rabbits (Irvine et al. 2006); all studies administered MMA via gavage. Adverse effects reported in the $\mathrm{LD}_{50}$ studies included diarrhea in rats at 2,030 mg monosodium methane arsonate (MSMA)/kg (Gur and Nyska 1990), mice at 2,200 mg MMA/kg (Kaise et al. 1989) and rabbits at $60 \mathrm{mg}$ MSMA/kg (Jaghabir et al. 1988) and respiratory arrest in mice at $1,800 \mathrm{mg} \mathrm{MMA} / \mathrm{kg} /$ day (Kaise et al. 1989). These doses were at or near the $\mathrm{LD}_{50}$ levels of 2,449 mg MSMA/kg, 1,800 mg MMA $/ \mathrm{kg}, 100 \mathrm{mg} \mathrm{MSMA} / \mathrm{kg}$ for the rats, mice, and rabbits, respectively. In the developmental toxicity studies (Irvine et al. 2006), maternal effects included decreases in maternal body weight gain in rats (17\% less than controls) and rabbits $(70 \%$ less than controls) receiving gavage doses of 100 and $12 \mathrm{mg} \mathrm{MMA} / \mathrm{kg} /$ day, respectively, and loose feces/diarrhea in rabbit does administered $12 \mathrm{mg} \mathrm{MMA} / \mathrm{kg} / \mathrm{day}$. The NOAELs for maternal effects were 10 and $7 \mathrm{mg}$ MMA/kg/day in the rats and rabbits, respectively. Minor developmental effects (decreased fetal weight, incomplete ossification, and supernumerary ribs) were also observed at the maternally toxic doses in the rats and rabbits (Irvine et al. 2006); these effects were probably secondary to the maternal stress. These 
data, coupled with the results of longer-term studies (Arnold et al. 2003; Waner and Nyska 1988), suggest that the gastrointestinal tract is a sensitive target of MMA toxicity. The rabbit developmental toxicity study (Irvine et al. 2006) identified the lowest LOAEL (12 mg MMA/kg/day) for gastrointestinal irritation. However, this study is not suitable for the derivation of an acute-duration oral MRL for MMA because the MMA was administered via bolus doses. It is likely that the observed gastrointestinal effect is a concentration-dependent effect; thus, at a given dose level, effects are more likely to occur following bolus administration. A marked decrease in body weight gain was also observed at this dose level.

- An MRL of $0.1 \mathrm{mg}$ MMA/kg/day has been derived for intermediate-duration (15-364 days) oral exposure to MMA.

Three studies have examined the intermediate-duration toxicity of MMA; two of these are chronicduration studies reporting diarrhea and decreases in body weight gain after MMA exposure for $<1$ year. Diarrhea was observed in rats exposed to $30.2 \mathrm{mg} \mathrm{MMA} / \mathrm{kg} /$ day in the diet (Arnold et al. 2003) and in dogs exposed via a capsule to $2 \mathrm{mg}$ MMA/kg/day (Waner and Nyska 1988). Decreases in body weight were observed at the next highest doses, $106.9 \mathrm{mg} \mathrm{MMA} / \mathrm{kg} /$ day in rats and $8 \mathrm{mg} \mathrm{MMA} / \mathrm{kg} / \mathrm{day}$ in dogs. In the rat study (Arnold et al. 2003), diarrhea was observed in 16.7 and $40 \%$ of the males and females, respectively, exposed to 30.2/35.9 mg MMA/kg/day during the first 52 weeks of the study; diarrhea first occurred after 4 weeks of exposure. At the highest dose level (106.9 mg MMA/kg/day), diarrhea was observed in all exposed male and female rats. In dogs, the increased incidence of diarrhea first occurred during weeks 25-28; at the highest dose tested in the study (35 mg MMA/kg/day), vomiting was also observed. A NOAEL of $3.5 \mathrm{mg} \mathrm{MMA} / \mathrm{kg} /$ day was identified in the rat study; a NOAEL was not identified in the dog study. The remaining study in the intermediate-duration database is a 2-generation study that reported reproductive (decreased pregnancy rate and male fertility index in $\mathrm{F}_{0}$ and $\mathrm{F}_{1}$ generations) and developmental (decreased pup survival in $\mathrm{F}_{1}$ and $\mathrm{F}_{2}$ generation) effects in rats exposed to $76 \mathrm{mg}$ MMA/kg/day in the diet (Schroeder 1994). The lowest LOAEL identified in the intermediateduration database is $2 \mathrm{mg} \mathrm{MMA} / \mathrm{kg} /$ day for diarrhea in dogs (Waner and Nyska 1988). Although dogs appear to be more sensitive to the gastrointestinal effects of MMA, a direct comparison of the two studies is not possible due to the difference in the routes of exposure. It is possible that the bolus administration of MMA, in the form of a capsule, resulted in increased sensitivity of the dogs. Because the most likely route of exposure for humans would be ingestion and the critical effect appears to be irritation of the gastrointestinal tract, studies involving bolus administration (gavage or capsule) were not considered for derivation of oral MRLs. The Arnold et al. (2003) and Schroeder (1994) studies were considered as the basis for an intermediate-duration MRL. Of these two studies, Arnold et al. (2003) identified the lowest 
LOAEL, $30.2 \mathrm{mg} \mathrm{MMA} / \mathrm{kg} / \mathrm{day}$, for gastrointestinal effects and was selected as the principal study for the intermediate-duration oral MRL.

Arnold et al. (2003) exposed groups of 60 male and 60 female Fischer 344 rats to 0, 50, 400, or 1,300 ppm MMA in the diet for 104 weeks. Using the average doses for weeks 1-50 reported in an unpublished version of this study (Crown et al. 1990), doses of 0, 3.5, 30.2, and 106.9 mg MMA/kg/day and $0,4.2,35.9$, and $123.3 \mathrm{mg} \mathrm{MMA} / \mathrm{kg} / \mathrm{day}$ were calculated for males and females, respectively. Body weights, food consumption, and water intake were monitored regularly. Blood was taken at 3, 6, and 12 months for clinical chemistry measurements, and urine samples were collected at the same interval. Mortality was increased in high-dose males and females during the first 52 weeks of the study. Body weights were decreased in the mid- and high-dose groups of both sexes; however, at 51 weeks, only the body weight for the high-dose males was $<10 \%$ of the control weight (14.5\%). Food and water consumption was increased in the mid- and high-dose groups. Diarrhea was observed in $100 \%$ of the high-dose males and females and in 16.7 and $40 \%$ of the mid-dose males and females during the first 52 weeks of exposure. Diarrhea first occurred after 3 weeks of exposure to the high dose and 4 weeks of exposure to the mid-dose group; the severity of the diarrhea was dose-related. The gastrointestinal system was the primary target in animals dying early; numerous macroscopic and histological alterations were observed.

A benchmark dose (BMD) analysis of the incidence data for diarrhea was conducted; details of this analysis are presented in Appendix A. Using the female incidence data, a BMD (BMD $\left.{ }_{10}\right)$ of $16.17 \mathrm{mg}$ MMA $/ \mathrm{kg} /$ day, which corresponds to a $10 \%$ increase in the incidence of diarrhea, was calculated; the $95 \%$ lower confidence limit on the $\mathrm{BMD}\left(\mathrm{BMDL}_{10}\right)$ was $12.38 \mathrm{mg} \mathrm{MMA} / \mathrm{kg} /$ day. The female incidence data were selected over the male data because the females may be more sensitive than the males. Thus, the intermediate-duration oral MRL of $0.1 \mathrm{mg} \mathrm{MMA} / \mathrm{kg} /$ day is based on the $\mathrm{BMDL}_{10}$ of $12.38 \mathrm{mg}$ $\mathrm{MMA} / \mathrm{kg} / \mathrm{day}$ in female rats and an uncertainty factor of 100 (10 to account for animal to human extrapolation and 10 for human variability).

- An MRL of $0.01 \mathrm{mg} \mathrm{MMA} / \mathrm{kg} /$ day has been derived for chronic-duration (365 days or longer) oral exposure to MMA.

The available data on the chronic toxicity of MMA in animals (no human data are available) suggest that the gastrointestinal tract and the kidney are the most sensitive targets. Diarrhea has been observed in rats and mice exposed to MMA in the diet for 2 years (Arnold et al. 2003). The NOAEL and LOAEL values for diarrhea are 3.0 and $25.7 \mathrm{mg} \mathrm{MMA} / \mathrm{kg} /$ day in rats, respectively, and 24.9 and $67.1 \mathrm{mg} \mathrm{MMA} / \mathrm{kg} / \mathrm{day}$ 
in mice, respectively. At $72.4 \mathrm{mg} \mathrm{MMA} / \mathrm{kg} /$ day, necrotic, ulcerated, or perforated mucosa and metaplasia were observed in the cecum, colon, and rectum of rats. Squamous metaplasia was also observed in the cecum, colon, and rectum of mice exposed to $67.1 \mathrm{mg} \mathrm{MMA} / \mathrm{kg} /$ day. Diarrhea was observed in dogs exposed via capsule to $2 \mathrm{mg} \mathrm{MMA} / \mathrm{kg}$ /day for 52 weeks (Waner and Nyska 1988). The bolus administration used in the dog study probably increased the dog's sensitivity to MMA. In both the rats and mice, chronic administration of MMA resulted in an exacerbation of chronic progressive nephropathy. In female rats, significant increases in the severity of chronic progressive nephropathy were observed at 33.9 and $98.5 \mathrm{mg}$ MMA/kg/day; the NOAEL was $3.9 \mathrm{mg} \mathrm{MMA} / \mathrm{kg} /$ day (Arnold et al. 2003). In male mice, there was an increased incidence of slight progressive nephropathy at doses $\geq 6.0 \mathrm{mg}$ MMA/kg/day; the NOAEL was $1.2 \mathrm{mg} \mathrm{MMA} / \mathrm{kg} /$ day (Arnold et al. 2003; incidence data reported in Gur et al. 1991). Nephrocalcinosis was also observed in male mice exposed to $\geq 24.9 \mathrm{mg} \mathrm{MMA} / \mathrm{kg} / \mathrm{day}$ (Arnold et al. 2003). Other effects that have been observed following chronic exposure MMA include decreased weight gain in male and female rats exposed to 25.7/33.9 mg MMA/kg/day and higher (Arnold et al. 2003) and hypertrophy of the thyroid follicular epithelium in female rats exposed to $\geq 33.9 \mathrm{mg}$ MMA $/ \mathrm{kg} /$ day (Arnold et al. 2003). A variety of other lesions including peritonitis, pancreatitis, inflammation of the ureter, uterus, prostate, testes, epididymis, and seminal vesicles, hydronephrosis, pyelonephritis, and cortical tubular cystic dilation were also observed in rats; however, these alterations were probably secondary to the ulceration and perforation of the large intestine, which resulted in leaking of gastrointestinal contents into the abdominal cavity. Hyperplasia of the urinary bladder was also observed in rats exposed to $2.1 \mathrm{mg}$ MMA/kg/day as MMA in drinking water for 2 years (Shen et al. 2003). Although hyperplasia of the urinary bladder is commonly observed in rats exposed to DMA, it was not observed in the Arnold et al. (2003) study at doses as high as $72.4 \mathrm{mg}$ MMA/kg/day; thus, the significance of the results of the Shen et al. (2003) study is not known.

The lowest reliable LOAEL identified in the chronic oral MMA database was $6.0 \mathrm{mg} \mathrm{MMA} / \mathrm{kg} /$ day for an increased incidence of progressive glomerulonephropathy in mice (Arnold et al. 2003). Although the investigators noted that the kidney lesions were consistent with the normal spectrum of spontaneous renal lesions and that there was no difference in character or severity of lesions between groups, ATSDR considers the dose-related increase in glomerulonephropathy to be treatment-related.

In the Arnold et al. (2003) study (incidence data reported in Gur et al. 1991), groups of 52 male and 52 female $\mathrm{B}_{6 \mathrm{C} 3 \mathrm{~F}_{1}}$ mice were exposed to $0,10,50,200$, or $400 \mathrm{ppm}$ of MMA in the diet for 104 weeks. The average doses reported in Gur et al. (1991) were 0, 1.2, 6.0, 24.9, and 67.1 mg MMA/kg/day for males and $0,1.4,7.0,31.2$, and $101 \mathrm{mg} \mathrm{MMA} / \mathrm{kg} /$ day for females. Body weights, food consumption, and 
water intake were monitored regularly. Blood was taken at 3, 6, 12, 18, and 24 months for white cell counts. At sacrifice, complete necropsies were performed, including histological examination of at least 13 organs. No treatment-related increases in mortality were observed. Significant decreases in body weights were observed in males and females exposed to 67.1 or $101 \mathrm{mg} \mathrm{MMA} / \mathrm{kg} /$ day, respectively; at week 104, the males and females weighed 17 and 23\%, respectively, less than controls. Food consumption was increased in females exposed to $101 \mathrm{mg} \mathrm{MMA} / \mathrm{kg} / \mathrm{day}$, and water consumption was increased in $67.1 \mathrm{mg} \mathrm{MMA} / \mathrm{kg} /$ day males and 31.2 and $101 \mathrm{mg} \mathrm{MMA} / \mathrm{kg} /$ day females. Loose and mucoid feces were noted in mice exposed to 67.1/101 mg MMA/kg/day. No changes were seen in white cell counts of either sex. Small decreases in the weights of heart, spleen, kidney, and liver weights were observed in some animals, but the decreases were not statistically significant. Squamous metaplasia of the cecum, colon, and rectum was observed at $67.1 / 101 \mathrm{mg} \mathrm{MMA} / \mathrm{kg} /$ day. The incidence of metaplasia in the cecum, colon, and rectum were $29 / 49,14 / 49$, and 39/49 in males and 38/52, 17/52, and 42/52 in females; metaplasia was not observed in other groups of male or female mice. An increased incidence of progressive glomerulonephropathy (incidence of 25/52, 27/52, 38/52, 39/52, and 46/52 in the 0, 1.2, 6.0, 24.9, and $67.1 \mathrm{mg} \mathrm{MMA} / \mathrm{kg} /$ day males, respectively) was observed in males; the incidence was significantly higher (Fisher Exact Test) than controls at $\geq 6.0 \mathrm{mg} \mathrm{MMA} / \mathrm{kg} /$ day. Significant increases in the incidence of nephrocalcinosis was observed in the males at 24.9 and $67.1 \mathrm{mg} \mathrm{MMA} / \mathrm{kg} / \mathrm{day}$ (Fisher Exact Test) (incidences of 25/52, 30/52, 30/52, 45/52, and 45/51 in males and 0/52, 1/52, 1/52, 2/52, and $5 / 52$ in females). A reduction in the incidence of cortical focal hyperplasia in the adrenal gland of male mice exposed to $67.1 \mathrm{mg} \mathrm{MMA} / \mathrm{kg} /$ day was possibly related to MMA exposure; the toxicological significance of this effect is not known. Thus, this study identifies a NOAEL of $1.2 \mathrm{mg} \mathrm{MMA} / \mathrm{kg} / \mathrm{day}$ and a LOAEL of $6.0 \mathrm{mg} \mathrm{MMA} / \mathrm{kg} /$ day for progressive glomerulonephropathy in male mice.

As described in greater detail in Appendix A, BMD was applied to the incidence data for progressive glomerulonephropathy in male mice using all available dichotomous models in EPA's Benchmark Dose Software (version 1.4.1) to calculate predicted doses associated with a $10 \%$ extra risk. As assessed by the Akaike's Information Criteria (AIC), the log-logistic model provided the best fit to the data. The predicted $\mathrm{BMD}_{10}$ and $\mathrm{BMDL}_{10}$ are 2.09 and $1.09 \mathrm{mg} \mathrm{MMA} / \mathrm{kg} / \mathrm{day}$. The $\mathrm{BMDL}_{10}$ was selected as the point of departure and divided by an uncertainty factor of 100 (10 for extrapolation from animals to humans and 10 for human variability) to derive a chronic-duration oral MRL of $0.01 \mathrm{mg} \mathrm{MMA} / \mathrm{kg} / \mathrm{day}$.

DMA. As discussed in greater detail in Section 2.2, urinary bladder effects characterized by cytotoxicity and regenerative proliferation and hyperplase have been observed in rats, but not in other species. The LOAELs for these effects are lower than the LOAELs for sensitive effects in other species. Additionally, 
rats have a much greater capacity than other species to metabolize ingested DMA to form DMA(III) (a reactive intermediate) and TMAO (Cohen et al. 2006; Marafante et al. 1987b; Yoshida et al. 1998). It is likely that DMA(III) is the causative agent for the urothelial cytoxicity observed in rats (Cohen et al. 2006). Thus, rats were not considered a suitable model for humans and these data were not considered for derivation of MRLs for DMA.

There are limited data to assess the acute toxicity of DMA in species other than rats. Diarrhea, increased startle reflex, and ataxia were observed in mice exposed to a lethal gavage dose of 1,757 $\mathrm{mg}$ DMA/kg (Kaise et al. 1989); vomiting and diarrhea were also observed during the second week of a 52-week study in dogs exposed via capsule to $16 \mathrm{mg}$ DMA/kg/day (Zomber et al. 1989). The remaining studies in the acute database are developmental toxicity studies in mice and rabbits. Rabbits appear to be more sensitive than mice to maternal and developmental effects. Gavage exposure to $48 \mathrm{mg}$ DMA/kg/day on gestational days 7-19 resulted in maternal weight loss and abortion in approximately $75 \%$ of the does; no adverse effects were observed at $12 \mathrm{mg} \mathrm{DMA} / \mathrm{kg} /$ day (Irvine et al. 2006). In mice, decreases in maternal body weight gain were observed at gavage doses of $200 \mathrm{mg}$ DMA/kg/day on gestational days 716 (Rogers et al. 1981), decreases in fetal body weight, delays in ossification, and increased incidence of cleft palate were observed at $400 \mathrm{mg}$ DMA/kg/day on gestational days 7-16 (Rogers et al. 1981) and fetal deaths, decreases in growth, and increased incidence of malformations were observed in mice administered 1,600 mg DMA/kg on gestational day 8 (Kavlock et al. 1985). The acute-duration database for DMA was not considered adequate for derivation of an oral MRL. The database is lacking a comprehensive toxicity study, which would be useful in establishing the critical target of toxicity. In a chronic-duration study in mice (Arnold et al. 2006), vacuolization was observed in the urinary bladder at $\geq 7.8 \mathrm{mg} \mathrm{DMA} / \mathrm{kg} / \mathrm{day}$; it is not known if these effects would also be observed after acute-duration exposure. Thus, it is not known if systemic effects would occur at lower doses than the maternal developmental effects observed in rabbits exposed to $48 \mathrm{mg} \mathrm{DMA} / \mathrm{kg} /$ day (Irvine et al. 2006); an acuteduration oral MRL for DMA is not recommended at this time.

Excluding rat studies, the database on the toxicity of DMA following intermediate-duration oral exposure is limited to a chronic study of dogs exposed to DMA via capsule 6 days/week for 52 weeks (Zomber et al. 1989). Diarrhea and vomiting were observed at 16 and $40 \mathrm{mg} \mathrm{DMA} / \mathrm{kg} /$ day starting after the first week of exposure. A slight decrease in erythrocyte levels and increase in total leukocyte levels were observed in males exposed to $40 \mathrm{mg}$ DMA/kg/day for 51 weeks. This dog study was not selected as the basis of an MRL because it is likely that bolus administration of DMA would increase sensitivity to the gastrointestinal effects. 
- An MRL of $0.02 \mathrm{mg} \mathrm{DMA} / \mathrm{kg} /$ day has been derived for chronic-duration (365 days or longer) oral exposure to DMA.

Two studies investigated the chronic-duration toxicity of DMA in a species other than rats. In dogs, diarrhea and vomiting were observed after 52 weeks of exposure to 16 or $40 \mathrm{mg} \mathrm{As} / \mathrm{kg} /$ day (Zomber et al. 1989); no histological alterations were observed. In mice exposed to DMA in the diet for 2 years, vacuolization of the urothelium in the urinary bladder was observed at $\geq 7.8 \mathrm{mg} \mathrm{DMA} / \mathrm{kg} / \mathrm{day}$ and progressive glomerulonephropathy was observed at $\geq 37 \mathrm{mg} \mathrm{DMA} / \mathrm{kg} /$ day (Arnold et al. 2006). As noted in Section 2.2, the vacuolization was not associated with cytotoxicity or proliferation. Because the bladder effects in mice occurred at the lowest adverse effect level for the database, it was selected as the critical effect and Arnold et al. (2006) was selected as the principal study.

In the Arnold et al. (2006) study, groups of 56 male and 56 female $\mathrm{B}_{6} \mathrm{C} 3 \mathrm{~F}_{1}$ mice were exposed to 0, 8, 40, 200 , or 500 ppm DMA in the diet for 2 years; the results of this study were also reported in an unpublished paper (Gur et al. 1989b) submitted to EPA under Federal Insecticide, Fungicide, and Rodenticide Act (FIFRA). The investigators reported dietary doses of approximately 0, 1.3, 7.8, 37, and $94 \mathrm{mg}$ DMA/kg/day. The following parameters were used to assess toxicity: clinical observations, body weight, food consumption, water consumption, differential leukocyte levels measured at 12, 18, and 24 months in mice in the control and $94 \mathrm{mg} \mathrm{DMA} / \mathrm{kg} /$ day groups, organ weights (brain, kidneys, liver, and testes), and histopathological examination of major tissues and organs. No deaths or treatmentrelated clinical signs were observed. Decreases in body weight gain were observed in the male mice exposed to $94 \mathrm{mg} \mathrm{DMA} / \mathrm{kg} /$ day; the difference was $<10 \%$ and not considered adverse. An increase in water consumption was observed in males exposed to $94 \mathrm{mg} \mathrm{DMA} / \mathrm{kg} / \mathrm{day}$ during weeks 60-96. In the female mice exposed to $51 \mathrm{mg}$ As/ $/ \mathrm{kg} /$ day, a statistically significant decrease in lymphocytes and an increase in monocytes were observed at 24 months. Treatment related nonneoplastic alterations were observed in the urinary bladder and kidneys. In the urinary bladder, increases in the vacuolization of the superficial cells of the urothelium were observed in males exposed to 37 or $94 \mathrm{mg}$ DMA/kg/day (0/44, $1 / 50,0 / 50,36 / 45,48 / 48)$ and in females exposed to $7.8,37$, and $94 \mathrm{mg}$ DMA/kg/day $(1 / 45,1 / 48,26 / 43$, 47/47, 43/43); incidence data reported in Gur et al. (1989b). An increased incidence of progressive glomerulonephropathy was observed in males at $37 \mathrm{mg}$ DMA/kg/day $(16 / 44,22 / 50,17 / 50,34 / 45,30 / 50)$ and an increased incidence of nephrocalcinosis was also observed in male mice at $94 \mathrm{mg} \mathrm{DMA} / \mathrm{kg} / \mathrm{day}$ $(30 / 44,25 / 50,27 / 50,29 / 50,45 / 50)$. Neoplastic alterations were limited to an increased incidence of fibrosarcoma of the skin in females exposed to $94 \mathrm{mg}$ DMA/kg/day (the incidence of 3/56, 0/55, 1/56, 
$1 / 56$, and $6 / 56$ in the $0,1.3,7.8,37$, and $94 \mathrm{mg} \mathrm{DMA} / \mathrm{kg} /$ day groups, respectively); however, it was concluded that this lesion was not related to DMA exposure.

As described in detail in Appendix A, BMD analysis was applied to the incidence data for vacuolization of the urothelium in the urinary bladder of female mice using all available dichotomous models in EPA's Benchmark Dose Software (version 1.4.1) to calculate predicted doses associated with a $10 \%$ extra risk. As assessed by the AIC, the multi-stage model provided the best fit to the data. The predicted $\mathrm{BMD}_{10}$ and $\mathrm{BMDL}_{10}$ are 2.68 and $1.80 \mathrm{mg} \mathrm{DMA} / \mathrm{kg} /$ day. The $\mathrm{BMDL}_{10}$ was selected as the point of departure and divided by an uncertainty factor of 100 (10 for extrapolation from animals to humans and 10 for human variability) to derive a chronic-duration oral MRL of $0.02 \mathrm{mg} \mathrm{DMA} / \mathrm{kg} /$ day.

Roxarsone. A series of three National Toxicology Program (NTP) studies in rats and mice (NTP 1989b) and a study in dogs (Kerr et al. 1963) have examined the acute toxicity of roxarsone; adverse effects have also been reported within the first 2 weeks of a longer-term study in pigs (Rice et al. 1985; Kennedy et al. 1986). A single exposure study reported diarrhea and ataxia in rats and mice exposed to doses that exceeded the $\mathrm{LD}_{50}$ (NTP 1989b). In another study, no alterations in hematological parameters (only end point assessed) were found after 10 or 9 days of dietary exposure in rats and mice, respectively (NTP 1989b). In a 14-day study (NTP 1989b), a decrease in body weight gain and slight inactivity were observed in rats exposed to $32 \mathrm{mg}$ roxarsone $/ \mathrm{kg} /$ day and slight inactivity was observed in mice exposed to $42 \mathrm{mg}$ roxarsone/ $\mathrm{kg} /$ day; a decrease in body weight gain was also observed in mice exposed to $168 \mathrm{mg}$ roxarsone $/ \mathrm{kg} / \mathrm{day}$. The dog study was considered inadequate because a small number $(\mathrm{n}=3)$ of animals were tested and no control group was used. In a 30-day dietary exposure study in pigs (Rice et al. 1985; Kennedy et al. 1986), mild lethargy and ataxia were observed from day 7 forward and exercise-induced muscle tremors and clonic seizures were observed from day 11 forward in pigs exposed to $6.3 \mathrm{mg}$ roxarsone/kg/day; equivocal evidence of myelin degeneration was also observed in pigs sacrificed after 11 days of exposure. These data clearly identify pigs as the most sensitive species following acuteduration oral exposure; in the absence of data to the contrary, it is assumed that pigs are a good model to predict the toxic potential of roxarsone in humans. Because the lowest dose tested in pigs was a serious LOAEL for neurotoxicity and a NOAEL for this effect was not identified, an acute-duration oral MRL cannot be derived for roxarsone.

As with the acute-duration database, pigs appear to be the most sensitive species; neurotoxicity has been observed at $\geq 6.3 \mathrm{mg}$ roxarsone/ $\mathrm{kg} /$ day. In a study reported by Rice et al. (1985) and Kennedy et al. (1986), exercise-induced muscle tremors and clonic convulsions were observed in pigs during the early 
part of the study; when the pigs returned to a recumbent position, the seizures and tremors stopped. Paraparesis, evidenced by reluctance to rise and the pigs dragging their hindquarters on the ground, was observed at day 22; paraplegia was observed 2 days after exposure termination. In addition to these clinical signs of neuropathy, histological alterations consisting of myelin degeneration was observed in the spinal cord, peripheral nerves, and optic nerve. The lesions were first detected in the spinal cord on day 15 and in the peripheral nerves and optic nerve 2 days after exposure termination. The Rice et al. (1985) and Kennedy et al. (1986) studies did not identify a NOAEL. Muscle tremors were also observed in pigs exposed to $10 \mathrm{mg}$ roxarsone $/ \mathrm{kg} /$ day for 28 days (Edmonds and Baker 1986). This study was not designed to assess neurotoxicity and did not include histological examination of the spinal cord or nerves. Trembling, ataxia, and hyperexcitability were also observed in rats exposed to $64 \mathrm{mg}$ roxarsone $/ \mathrm{kg} / \mathrm{day}$ for 13 weeks (NTP 1989b). Other effects that have been observed include tubular degeneration and focal regenerative hyperplasia in the kidney and decreased body weight in rats exposed to $32 \mathrm{mg}$ roxarsone/ $\mathrm{kg} /$ day for 13 weeks (NTP 1989b) and decreased body weight in mice at $136 \mathrm{mg}$ roxarsone $/ \mathrm{kg} /$ day for 13 weeks (NTP 1989b). The lowest identified adverse effect level is $6.3 \mathrm{mg}$ roxarsone $/ \mathrm{kg} / \mathrm{day}$ for serious neurological effects in pigs (Kennedy et al. 1986; Rice et al. 1985) and is not suitable for the derivation of an intermediate-duration oral MRL.

The chronic toxicity of roxarsone has been examined in rats (NTP 1989b; Prier et al. 1963), mice (NTP 1989b; Prier et al. 1963), and dogs (Prier et al. 1963) in 2-year dietary exposure studies. None of these studies reported adverse effects at the highest doses tested; the highest NOAELs for each species are 10, 43, and $5 \mathrm{mg}$ roxarsone $/ \mathrm{kg} /$ day for rats, mice, and dogs, respectively. The results from shorter duration studies suggest that pigs are more sensitive to the neurotoxic effects of roxarsone than rats, mice, or dogs. Because no chronic duration pig studies were identified and deriving an MRL using a potentially less sensitive species may not be protective of human health, a chronic-duration oral MRL is not recommended at this time. 


\section{HEALTH EFFECTS}

\subsection{INTRODUCTION}

The primary purpose of this chapter is to provide public health officials, physicians, toxicologists, and other interested individuals and groups with an overall perspective on the toxicology of arsenic. It contains descriptions and evaluations of toxicological studies and epidemiological investigations and provides conclusions, where possible, on the relevance of toxicity and toxicokinetic data to public health.

A glossary and list of acronyms, abbreviations, and symbols can be found at the end of this profile.

\subsection{DISCUSSION OF HEALTH EFFECTS BY ROUTE OF EXPOSURE}

To help public health professionals and others address the needs of persons living or working near hazardous waste sites, the information in this section is organized first by route of exposure (inhalation, oral, and dermal) and then by health effect (death, systemic, immunological, neurological, reproductive, developmental, genotoxic, and carcinogenic effects). These data are discussed in terms of three exposure periods: acute (14 days or less), intermediate (15-364 days), and chronic (365 days or more).

Levels of significant exposure for each route and duration are presented in tables and illustrated in figures. The points in the figures showing no-observed-adverse-effect levels (NOAELs) or lowestobserved-adverse-effect levels (LOAELs) reflect the actual doses (levels of exposure) used in the studies. LOAELs have been classified into "less serious" or "serious" effects. "Serious" effects are those that evoke failure in a biological system and can lead to morbidity or mortality (e.g., acute respiratory distress or death). "Less serious" effects are those that are not expected to cause significant dysfunction or death, or those whose significance to the organism is not entirely clear. ATSDR acknowledges that a considerable amount of judgment may be required in establishing whether an end point should be classified as a NOAEL, "less serious" LOAEL, or "serious" LOAEL, and that in some cases, there will be insufficient data to decide whether the effect is indicative of significant dysfunction. However, the Agency has established guidelines and policies that are used to classify these end points. ATSDR believes that there is sufficient merit in this approach to warrant an attempt at distinguishing between "less serious" and "serious" effects. The distinction between "less serious" effects and "serious" effects is considered to be important because it helps the users of the profiles to identify levels of exposure at which major health effects start to appear. LOAELs or NOAELs should also help in determining whether or not 
the effects vary with dose and/or duration, and place into perspective the possible significance of these effects to human health.

The significance of the exposure levels shown in the Levels of Significant Exposure (LSE) tables and figures may differ depending on the user's perspective. Public health officials and others concerned with appropriate actions to take at hazardous waste sites may want information on levels of exposure associated with more subtle effects in humans or animals (LOAELs) or exposure levels below which no adverse effects (NOAELs) have been observed. Estimates of levels posing minimal risk to humans (Minimal Risk Levels or MRLs) may be of interest to health professionals and citizens alike.

Levels of exposure associated with carcinogenic effects (Cancer Effect Levels, CELs) of arsenic are indicated in Tables 3-1 and 3-3 and Figures 3-1 and 3-3. Because cancer effects could occur at lower exposure levels, Figures 3-1 and 3-3 also show a range for the upper bound of estimated excess risks, ranging from a risk of 1 in 10,000 to 1 in $10,000,000\left(10^{-4}\right.$ to $\left.10^{-7}\right)$, as developed by EPA.

A User's Guide has been provided at the end of this profile (see Appendix B). This guide should aid in the interpretation of the tables and figures for Levels of Significant Exposure and the MRLs.

Chemical Forms of Concern. Analysis of the toxic effects of arsenic is complicated by the fact that arsenic can exist in several different oxidation states and many different inorganic and organic compounds. Most cases of human toxicity from arsenic have been associated with exposure to inorganic arsenic, so these compounds are the main focus of this profile.

The most common inorganic arsenical in air is arsenic trioxide $\left(\mathrm{As}_{2} \mathrm{O}_{3}\right)$, while a variety of inorganic arsenates $\left(\mathrm{AsO}_{4}{ }^{-3}\right)$ or arsenites $\left(\mathrm{AsO}_{2}{ }^{-}\right)$occur in water, soil, or food. A number of studies have noted differences in the relative toxicity of these compounds, with trivalent arsenites tending to be somewhat more toxic than pentavalent arsenates (Byron et al. 1967; Gaines 1960; Maitani et al. 1987a; Sardana et al. 1981; Willhite 1981). However, these distinctions have not been emphasized in this profile, for several reasons: (1) in most cases, the differences in the relative potency are reasonably small (about $2-$ 3 -fold), often within the bounds of uncertainty regarding NOAEL or LOAEL levels; (2) different forms of arsenic may be interconverted, both in the environment (see Section 6.3) and the body (see Section 3.4); and (3) in many cases of human exposure (especially those involving intake from water or soil, which are of greatest concern to residents near wastes sites), the precise chemical speciation is not known. 
Gallium arsenide (GaAs) is another inorganic arsenic compound of potential human health concern, due to its widespread use in the microelectronics industry. Available toxicokinetic data suggest that although gallium arsenide is poorly soluble, it undergoes slow dissolution and oxidation to form gallium trioxide and arsenite (Webb et al. 1984, 1986). Therefore, the toxic effects of this compound are expected to be attributable to the arsenite that is liberated, plus the additional effects of the gallium species.

It is beyond the scope of this profile to provide detailed toxicity data on other less common inorganic arsenic compounds (e.g., $\mathrm{As}_{2} \mathrm{~S}_{3}$ ), but these are expected to be of approximately equal or lesser toxicity than the oxycompounds, depending mainly on solubility (see Section 3.4).

Although organic arsenicals are usually viewed as being less toxic than the inorganics, several methyl and phenyl derivatives of arsenic that are widely used in agriculture are of possible human health concerns based on their toxicity in animal species (Arnold et al. 2003, 2006; NTP 1989b). Chief among these are monomethylarsonic acid (MMA) and its salts (monosodium methane arsonate [MSMA] and disodium methane arsonate [DSMA]), dimethylarsinic acid (DMA, also known as cacodylic acid) and its sodium salt (sodium dimethyl arsinite, or sodium cacodylate), and roxarsone (3-nitro-4-hydroxyphenylarsonic acid). However, it should be noted that food is the largest contributor to background intakes of organic arsenicals. Estimates on the concentration of organic arsenicals in the diet were not located; Cohen et al. (2006) estimated that the intake of DMA from food and water is $<1 \mathrm{ng} / \mathrm{kg} / \mathrm{day}$. As with the inorganic compounds, there are toxicological differences between these various organic derivatives; because of these differences, the discussion of the health effects of MMA, DMA, and roxarsone are discussed separately. As discussed below, animals do not appear to be good quantitative models for inorganic arsenic toxicity in humans, but it is not known if this also applies to toxicity of organic arsenicals.

Several organic arsenicals are found to accumulate in fish and shellfish. These derivatives (mainly arsenobetaine and arsenocholine, also referred to as "fish arsenic") have been studied by several researchers and have been found to be essentially nontoxic (Brown et al. 1990; Cannon et al. 1983; Charbonneau et al. 1978; Kaise et al. 1985; Luten et al. 1982; Siewicki 1981; Tam et al. 1982; Yamauchi et al. 1986). Thus, these compounds are not considered further here.

Arsine $\left(\mathrm{AsH}_{3}\right)$ and its methyl derivatives, although highly toxic, are also not considered in this profile, since these compounds are either gases or volatile liquids that are unlikely to be present at levels of concern at hazardous waste sites. 
Use of Animal Data. An additional complexity to the analysis of arsenic toxicity is that most laboratory animals appear to be substantially less susceptible to inorganic arsenic than humans. For example, chronic oral exposure of humans to inorganic arsenic at doses of $0.05-0.1 \mathrm{mg} / \mathrm{kg} / \mathrm{day}$ is frequently associated with neurological (Barton et al. 1992; Goddard et al. 1992; Guha Mazumder et al. 1988; Haupert et al. 1996; Hindmarsh et al. 1977; Huang et al. 1985; Sass et al. 1993; Silver and Wainman 1952; Szuler et al. 1979; Tay and Seah 1975; Valentine et al. 1981) or hematological signs of arsenic toxicity (Glazener et al. 1968; Guha Mazumder et al. 1988; Prasad and Rossi 1995; Sass et al. 1993; Tay and Seah 1975), but no characteristic neurological or hematological signs of arsenism were detected in monkeys, dogs, or rats chronically exposed to arsenate or arsenite at doses of $0.7-2.8 \mathrm{mg} \mathrm{As} / \mathrm{kg} / \mathrm{day}$ (Byron et al. 1967; EPA 1980f; Heywood and Sortwell 1979). This may be because the studies were not conducted for a sufficient length of time, or because too few animals were used. Moreover, while there is good evidence that inorganic arsenic is carcinogenic in humans by both oral and inhalation routes, evidence of inorganic arsenic-induced carcinogenicity in animals is mostly negative, with the exception of studies in mice demonstrating transplacental carcinogenesis. For these reasons, quantitative doseresponse data from animals are not judged to be reliable for determining levels of significant human exposure, and will be considered only briefly except when human data are lacking.

\subsubsection{Inhalation Exposure}

Most information on human inhalation exposure to arsenic derives from occupational settings such as smelters and chemical plants, where the predominant form of airborne arsenic is arsenic trioxide dust. One limitation to this type of study is that exposure data are usually difficult to obtain, especially from earlier time periods when exposure levels were higher than in recent years. This is further complicated by the fact that significant oral and dermal exposures are also likely to occur under these conditions and co exposure to other metals and chemicals is also common. Thus, studies of this type are, like virtually all epidemiological studies, subject to some limitations and uncertainties. Table 3-1 and Figure 3-1 summarize studies that provide the most reliable quantitative data on health effects in humans, along with several studies in animals exposed to arsenic trioxide and other inorganic arsenic compounds by the inhalation route. Data for DMA are shown in Table 3-2 and Figure 3-2. All exposure data are expressed as milligrams of arsenic (as the element) per cubic meter of air $\left(\mathrm{mg} \mathrm{As} / \mathrm{m}^{3}\right)$. These studies and others that provide useful qualitative information on health effects of inorganic and organic arsenicals are discussed below. 
Table 3-1 Levels of Significant Exposure to Inorganic Arsenic - Inhalation

\begin{tabular}{|c|c|c|c|c|c|c|c|c|}
\hline \multirow[b]{2}{*}{$\begin{array}{l}\text { Key to } \\
\text { Figure }\end{array}$} & \multirow[b]{2}{*}{$\begin{array}{l}\text { Species } \\
\text { (Strain) }\end{array}$} & \multirow{2}{*}{$\begin{array}{l}\text { Exposurel } \\
\text { Duration/ } \\
\text { Frequency } \\
\text { (Route) }\end{array}$} & \multirow[b]{2}{*}{ System } & \multirow[b]{2}{*}{$\begin{array}{l}\text { NOAEL } \\
\left(\mathrm{mg} / \mathrm{m}^{3}\right)\end{array}$} & \multicolumn{3}{|c|}{ LOAEL } & \multirow[b]{2}{*}{ Comments } \\
\hline & & & & & $\begin{array}{l}\text { Less Serious } \\
\qquad\left(\mathrm{mg} / \mathrm{m}^{3}\right)\end{array}$ & $\begin{array}{l}\text { Serious } \\
\left(\mathrm{mg} / \mathrm{m}^{3}\right)\end{array}$ & $\begin{array}{l}\text { Reference } \\
\text { Chemical Form }\end{array}$ & \\
\hline
\end{tabular}

ACUTE EXPOSURE

Immunol Lymphoret

$1 \quad$ Mouse $3 \mathrm{hr}$

(CD-1)

2 Mouse $5 d$

(CD-1) $\quad 3 \mathrm{hr} / \mathrm{d}$

Developmental

3 Mouse

(CFLP)

Gd 9-12

$4 \mathrm{hr} / \mathrm{d}$

0.2

$2.2 \quad(10 \%$ decreased average fetal body weight)

21.6

(increased fetal deaths, skeletal malformations,

and retarded growth)

\section{Nagymajtenyi et al. 1985}

As $(+3)$

Aranyi et al. 1985

As $(+3)$

bactericidal activity and

ncreased susceptibility

\section{INTERMEDIATE EXPOSURE}

Death

$\begin{array}{lll}4 & \text { Rat } & 14 \text { pmd- Gd } 19 \\ & \text { (CD) } & 7 \mathrm{~d} / \mathrm{wk}\end{array}$

Systemic

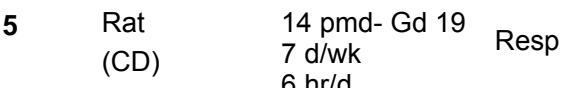

Bd Wt
$2 \mathrm{~F}$

$2 \mathrm{~F}$
$8 \mathrm{~F}$ (rales, dried red material around nose)

$8 \mathrm{~F}$ (decreased body weight gain during gestation)

$$
\text { As(+3) }
$$

Holson et al. 1999

As(+3)
$20 \mathrm{~F}$ (5/10 dams died)
Aranyi et al. 1985

As(+3) 
Table 3-1 Levels of Significant Exposure to Inorganic Arsenic - Inhalation

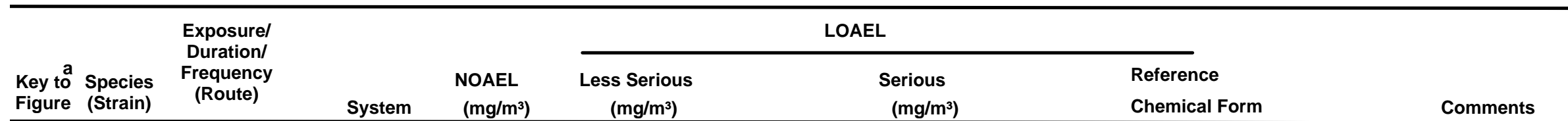

$\begin{array}{lllll}\text { Rat } & 14 \mathrm{pmd}-\mathrm{Gd} 19 & & & \\ \text { (CD) } & 7 \mathrm{~d} / \mathrm{wk} & \text { Resp } & 0.9 \mathrm{~F} & 8 \mathrm{~F} \text { (rales) } \\ & 6 \mathrm{hr} / \mathrm{d} & & & \end{array}$

Gastro $8 \mathrm{~F}$

Bd Wt

Holson et al. 1999 As(+3)

Holson et al. 1999 As(+3)

Holson et al. 1999 As(+3)

\section{Developmental}

\section{Rat}

(CD)
14 pmd- Gd 19

$$
6 \mathrm{hr} / \mathrm{d}
$$

14 pmd- Gd 19

$$
7 \mathrm{~d} / \mathrm{wk}
$$


Table 3-1 Levels of Significant Exposure to Inorganic Arsenic - Inhalation

\begin{tabular}{|c|c|c|c|c|c|c|c|c|}
\hline \multirow[b]{2}{*}{$\begin{array}{l}\text { Key to } \\
\text { Figure }\end{array}$} & \multirow[b]{2}{*}{$\begin{array}{l}\text { Species } \\
\text { (Strain) }\end{array}$} & \multirow{2}{*}{$\begin{array}{l}\text { Exposurel } \\
\text { Durationl } \\
\text { Frequency } \\
\text { (Route) }\end{array}$} & \multirow[b]{2}{*}{ System } & \multirow[b]{2}{*}{$\begin{array}{l}\text { NOAEL } \\
\left(\mathrm{mg} / \mathrm{m}^{3}\right)\end{array}$} & \multicolumn{3}{|c|}{ LOAEL } & \multirow[b]{2}{*}{$\begin{array}{l}\text { Reference } \\
\text { Chemical Form }\end{array}$} \\
\hline & & & & & $\begin{array}{l}\text { Less Serious } \\
\left(\mathrm{mg} / \mathrm{m}^{3}\right)\end{array}$ & $\begin{array}{r}\mathrm{Se} \\
(\mathrm{r}\end{array}$ & $\begin{array}{l}\text { ious } \\
\left.\mathrm{ng} / \mathrm{m}^{3}\right)\end{array}$ & \\
\hline 11 & $\begin{array}{l}\text { Rat } \\
\text { (CD) }\end{array}$ & $\begin{array}{l}14 \mathrm{pmd}-\mathrm{Gd} 19 \\
7 \mathrm{~d} / \mathrm{wk} \\
6 \mathrm{hr} / \mathrm{d}\end{array}$ & & 8 & & 20 & $\begin{array}{l}\text { (marked increase in post- } \\
\text { implantation loss and } \\
\text { marked decrease in } \\
\text { viable fetuses) }\end{array}$ & $\begin{array}{l}\text { Holson et al. } 1999 \\
\text { As(+3) }\end{array}$ \\
\hline
\end{tabular}

\section{CHRONIC EXPOSURE}

Systemic
12 Human
$23 \mathrm{yr}$ (avg)
Cardio
(occup)

viable fetuses)

(a)

13 Human

$0.5-50 \mathrm{yr} \quad$ Resp
(occup)

0.613

Dermal

0.078 (mild pigmentation
keratosis of skin)

$0.36 \mathrm{M}$ (increased incidence of vasospasticity and clinical Raynaud's

Lagerkvist et al. 1986 phenomenon)

As(+3)

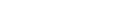

Perry et al. 1948
As $(+3)$

\section{Neurological}

14 Human $\begin{aligned} & 28 \mathrm{yr} \text { (avg) } \\ & \text { (occup) }\end{aligned}$

$0.31 \mathrm{M}$ (decreased nerve

conduction velocity)

Lagerkvist and Zetterlund 1994

As(+3)

\section{Cancer}

\begin{tabular}{|c|c|c|}
\hline 15 & Human & $\begin{array}{l}1->30 \mathrm{yr} \\
\text { (occup) }\end{array}$ \\
\hline 16 & Human & $\begin{array}{l}19.5 \mathrm{yr} \\
\text { (avg) } \\
\text { (occup) }\end{array}$ \\
\hline
\end{tabular}

$\begin{array}{ll}0.213 \text { M (CEL: lung cancer) } & \text { Enterline et al. 1987a } \\ & \text { As }(+3) \\ 0.069 \text { M (CEL: lung cancer) } & \begin{array}{l}\text { Enterline et al. 1987b } \\ \text { As }(+3)\end{array}\end{array}$


Table 3-1 Levels of Significant Exposure to Inorganic Arsenic - Inhalation

(continued)

\begin{tabular}{|c|c|c|c|c|c|c|c|c|}
\hline \multirow[b]{2}{*}{$\begin{array}{l}\text { Key to } \\
\text { Figure }\end{array}$} & \multirow[b]{2}{*}{$\begin{array}{c}\text { Species } \\
\text { (Strain) }\end{array}$} & \multirow{2}{*}{$\begin{array}{l}\text { Exposurel } \\
\text { Duration/ } \\
\text { Frequency } \\
\text { (Route) }\end{array}$} & \multirow[b]{2}{*}{ System } & \multirow[b]{2}{*}{$\begin{array}{l}\text { NOAEL } \\
\left(\mathrm{mg} / \mathrm{m}^{3}\right)\end{array}$} & \multicolumn{2}{|c|}{ LOAEL } & \multirow[b]{2}{*}{$\begin{array}{l}\text { Reference } \\
\text { Chemical Form }\end{array}$} & \multirow[b]{2}{*}{ Comments } \\
\hline & & & & & $\begin{array}{l}\text { Less Serious } \\
\left(\mathrm{mg} / \mathrm{m}^{3}\right)\end{array}$ & $\begin{array}{l}\text { Serious } \\
\left(\mathrm{mg} / \mathrm{m}^{3}\right)\end{array}$ & & \\
\hline
\end{tabular}

17 Human 3 mo- $>30 \mathrm{yr}$

$$
\text { (occup) }
$$

18 Human 3 mo- $>30 \mathrm{yr}$

$$
\text { (occup) }
$$

$$
\begin{array}{ll}
\text { Human } & 1->30 \mathrm{yr} \\
& \text { (occup) }
\end{array}
$$

20

$$
\begin{array}{ll}
\text { Human } & >25 \mathrm{yr} \\
& \text { (occup) }
\end{array}
$$

21

$$
\begin{array}{ll}
\text { Human } & \begin{array}{l}
14.8 \mathrm{yr} \text { (avg) } \\
\text { (occup) }
\end{array}
\end{array}
$$

$0.2 \mathrm{M}$ (CEL: lung cancer)

0.05 M (CEL: lung cancer)

$0.38 \mathrm{M}$ (CEL: lung cancer)

$0.29 \mathrm{M}$ (CEL: lung cancer)

$0.3 \mathrm{M}$ (CEL: lung cancer)
Chemical Form

Jarup and Pershagen 1991

As(+3)

Jarup et al. 1989

As $(+3)$

Lee-Feldstein 1986

As $(+3)$

Lubin et al. 2000

As(+3)

Welch et al. 1982

As(+3)

a The number corresponds to entries in Figure 3-1.

avg = average; $\mathrm{Bd} \mathrm{Wt}=$ body weight; Cardio = cardiovascular; $\mathrm{CEL}=$ cancer effect level; $\mathrm{d}=$ day $(\mathrm{s}) ; \mathrm{F}=$ female; Gastro = gastrointestinal; $\mathrm{Gd}=\mathrm{gestation}$ day; $\mathrm{hr}=\mathrm{hour}(\mathrm{s})$;

Immuno/Lymphoret = immunological/lymphoreticular; LOAEL = lowest-observable-adverse-effect level; $\mathrm{M}=$ male; $\mathrm{mo}=$ month(s); NOAEL = no-observable-adverse-effect level; NS = not specified; occup = occupational; pmd = pre-mating day; Resp = respiratory; $w \mathrm{w}=$ week(s); yr = year(s) 
Figure 3-1 Levels of Significant Exposure to Inorganic Arsenic - Inhalation

Acute $(\leq 14$ days $)$

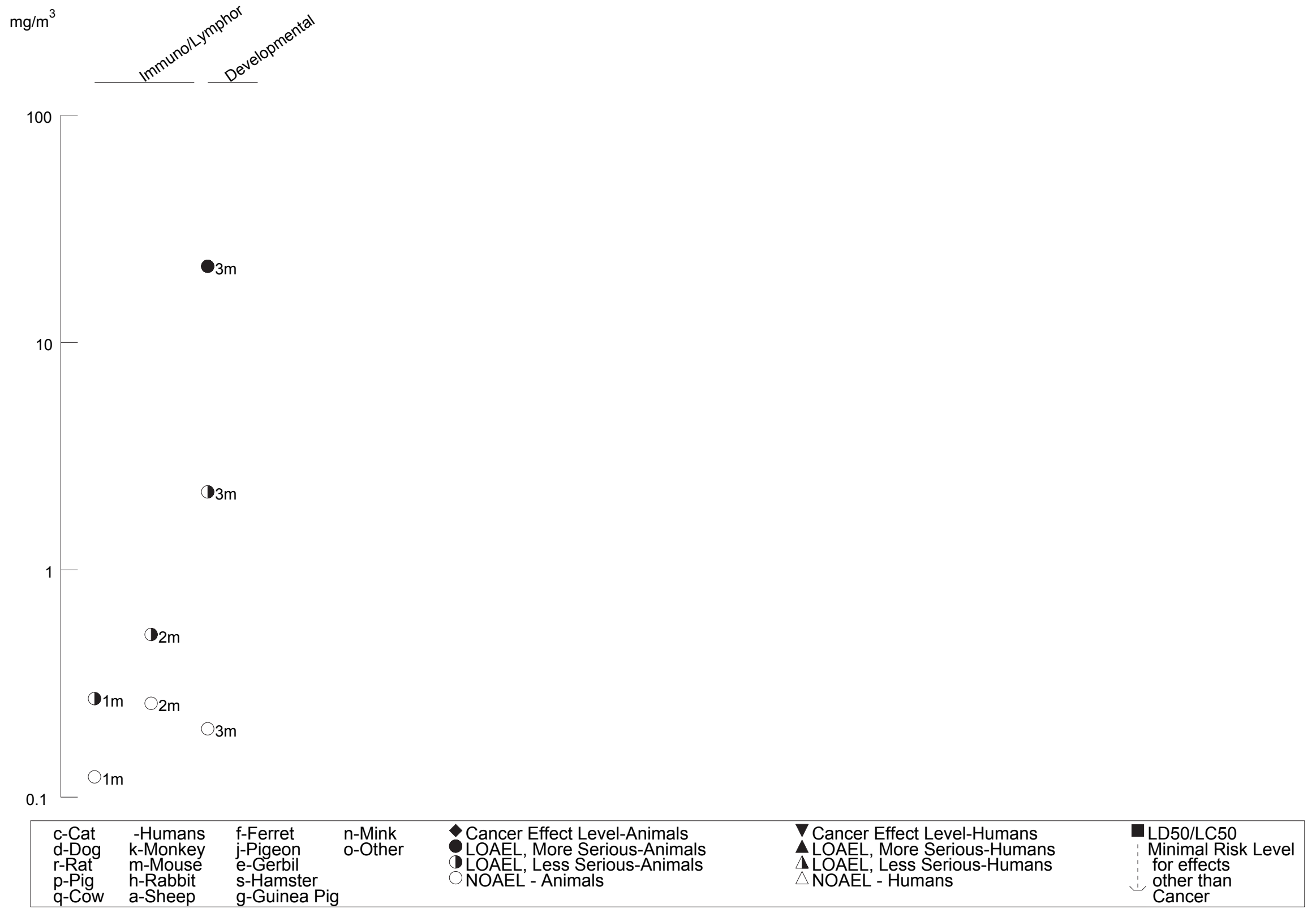


Figure 3-1 Levels of Significant Exposure to Inorganic Arsenic - Inhalation (Continued)

Intermediate (15-364 days)

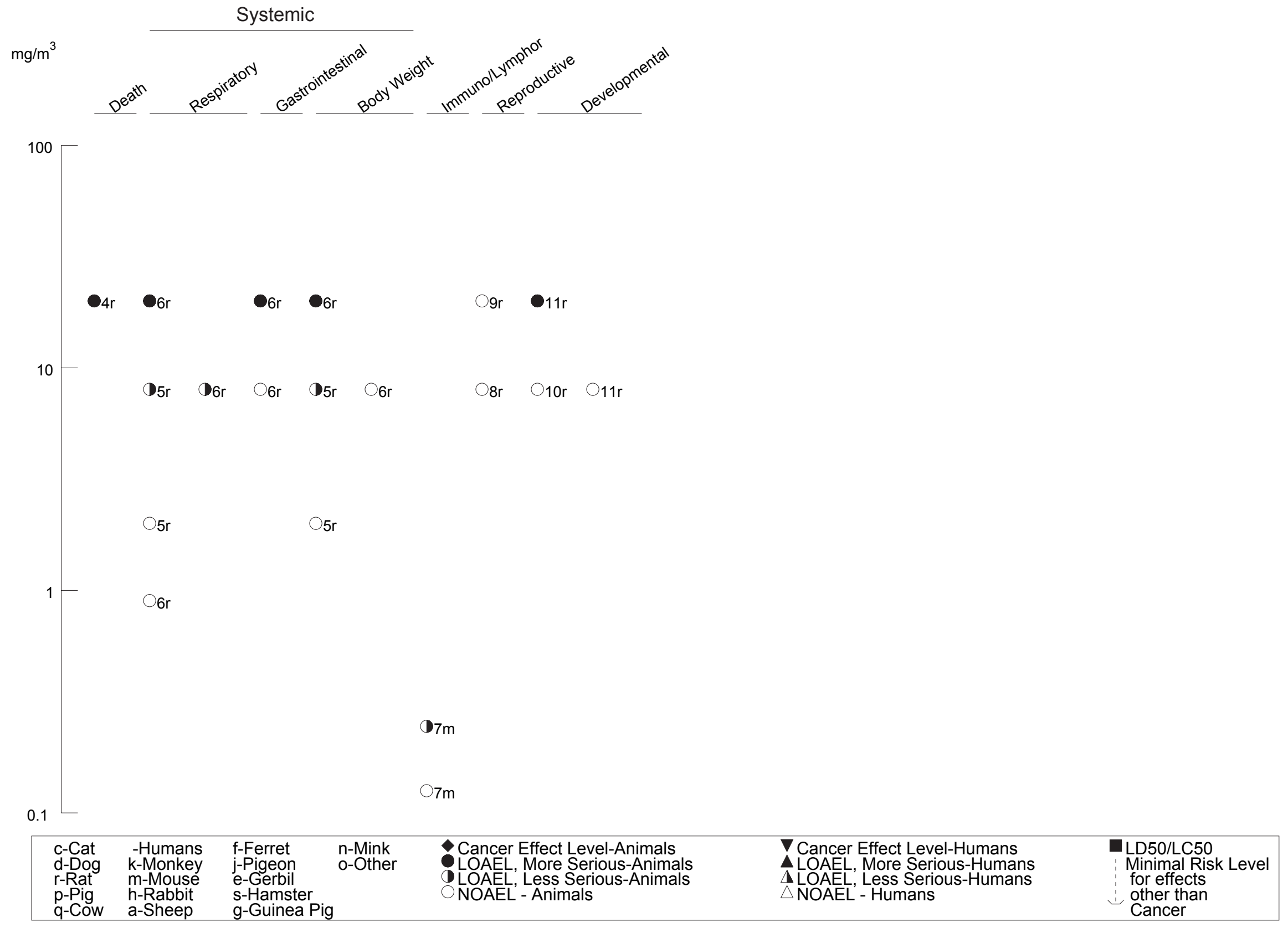


Figure 3-1 Levels of Significant Exposure to Inorganic Arsenic - Inhalation (Continued)

Chronic ( $\geq 365$ days)

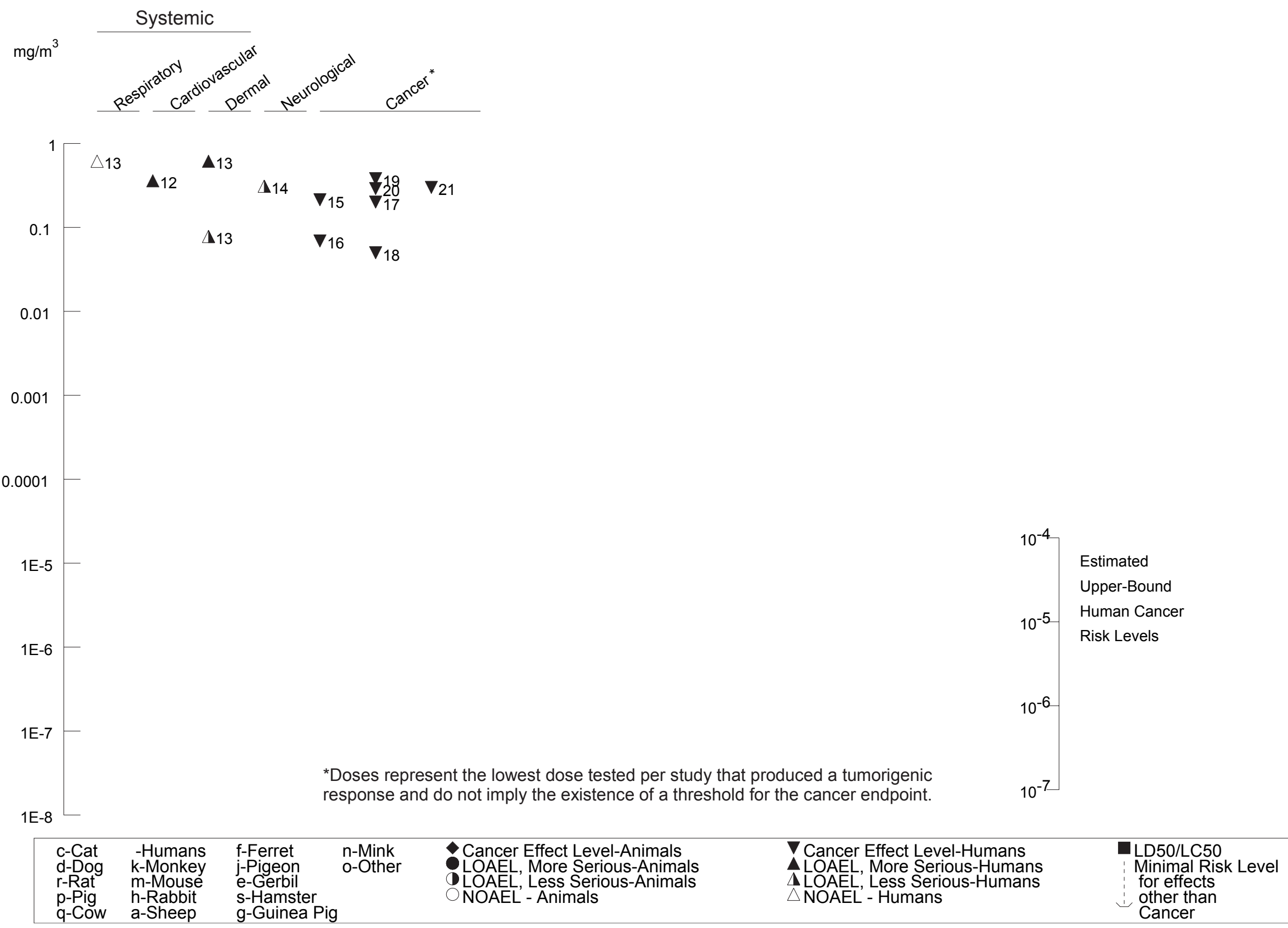


Table 3-2 Levels of Significant Exposure to Dimethylarsinic Acid - Inhalation

\begin{tabular}{llr}
\hline & & $\begin{array}{r}\text { Exposu } \\
\text { Duration }\end{array}$ \\
Key to & Species & $\begin{array}{r}\text { Freque } \\
\text { (Rout }\end{array}$ \\
Figure & (Strain) & \\
\hline ACUTE EXPOSURE \\
Death \\
1 & Rat & \\
& (Sherman) & \\
\end{tabular}

Systemic

2 Rat (Sherman)

\section{el}

urationl

(Route)

System

NOAEL

LOAEL

System

$\left(\mathrm{mg} / \mathrm{m}^{3}\right)$

Less Serious
\[ \left(\mathrm{mg} / \mathrm{m}^{3}\right) \]

Serious
$\left(\mathrm{mg} / \mathrm{m}^{3}\right)$

3900 F (LC50)

4000

(respiratory distress)

\begin{tabular}{|c|c|c|c|}
\hline Resp & & & \\
\hline Gastro & & 4000 & (diarrhea) \\
\hline Dermal & 4100 & $6900 \mathrm{~F}$ & $\begin{array}{l}\text { (erythematous lesions of } \\
\text { ears and feet) }\end{array}$ \\
\hline Ocular & & 4000 & (eye encrustation) \\
\hline $\mathrm{Bd} W \mathrm{t}$ & & 4000 & $\begin{array}{l}\text { (unspecified decrease in } \\
\text { body weight) }\end{array}$ \\
\hline
\end{tabular}

3 Mouse $5 \mathrm{~min}$

Mouse $\quad 5$ min $\quad$ Resp

Webster)

\section{INTERMEDIATE EXPOSURE}

Systemic

4 Rat

$\begin{array}{ll}\text { Rat } & 6 \mathrm{hr} / \mathrm{d} \\ \text { (Sprague- } & 5 \mathrm{~d} / \mathrm{wk} \\ \text { Dawley) } & 67-68\end{array}$

exposures

4000 (diarrhea)

Resp

3150 M (RD50)

10

Cardio

100

Gastro 100

Hemato $\quad 100$

Hepatic $\quad 100$

Renal $\quad 100$

Endocr $\quad 100$

Dermal 100
Reference

Chemical Form

Comments

Stevens et al. 1979

DMA

Stevens et al. 1979

DMA
DMA

Whitman 1994

DMA 
Table 3-2 Levels of Significant Exposure to Dimethylarsinic Acid - Inhalation

(continued)

\begin{tabular}{|c|c|c|c|c|c|c|c|c|}
\hline \multirow[b]{2}{*}{$\begin{array}{l}\text { Key to } \\
\text { Figure }\end{array}$} & \multirow[b]{2}{*}{$\begin{array}{c}\text { Species } \\
\text { (Strain) }\end{array}$} & \multirow{2}{*}{$\begin{array}{l}\text { Exposurel } \\
\text { Durationl } \\
\text { Frequency } \\
\text { (Route) }\end{array}$} & \multirow[b]{2}{*}{ System } & \multirow[b]{2}{*}{$\begin{array}{l}\text { NOAEL } \\
\left(\mathrm{mg} / \mathrm{m}^{3}\right)\end{array}$} & \multicolumn{3}{|c|}{ LOAEL } & \multirow[b]{2}{*}{ Comments } \\
\hline & & & & & $\begin{array}{l}\text { Less Serious } \\
\qquad\left(\mathrm{mg} / \mathrm{m}^{3}\right)\end{array}$ & $\begin{array}{l}\text { Serious } \\
\left(\mathrm{mg} / \mathrm{m}^{3}\right)\end{array}$ & $\begin{array}{l}\text { Reference } \\
\text { Chemical Form }\end{array}$ & \\
\hline
\end{tabular}

Bd Wt $\quad 100$

a The number corresponds to entries in Figure 3-2.

$\mathrm{Bd} \mathrm{Wt}=$ body weight; Cardio = cardiovascular; $\mathrm{d}=$ day(s); $\mathrm{DMA}=$ dimethylarsinic acid; $\mathrm{F}=$ female; Gastro = gastrointestinal; Hemato = hematological; $\mathrm{hr}=$ hour(s); LC50 = lethal concentration, $50 \%$ kill; LOAEL = lowest-observable-adverse-effect level; $\mathrm{M}=$ male; $\mathrm{min}=$ minute(s); NOAEL = no-observable-adverse-effect level; RD50 = 50\% decrease in respiration rate; Resp = respiratory; wk = week(s); yr = year(s) 
Figure 3-2 Levels of Significant Exposure to Dimethylarsinic Acid - Inhalation Acute $(\leq 14$ days $)$

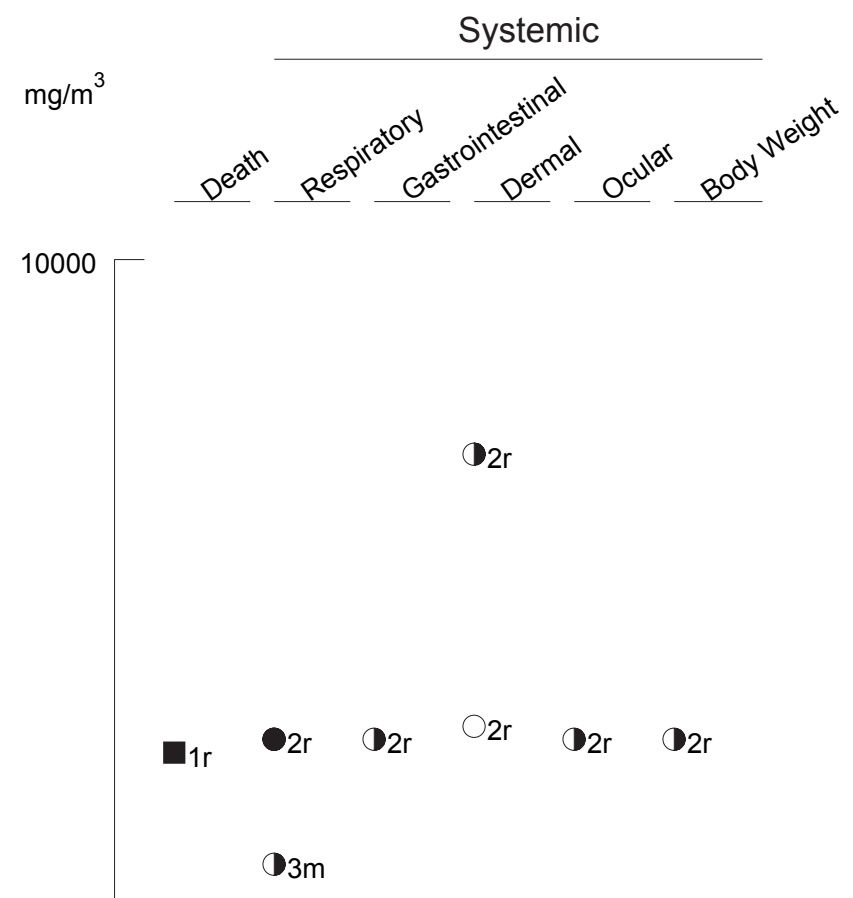

$1000 \leftarrow$

\begin{tabular}{|c|c|c|c|c|c|c|}
\hline $\begin{array}{l}\text { c-Cat } \\
\text { d-Dog } \\
\text { r-Rat } \\
\text { p-Pig } \\
\text { q-Cow }\end{array}$ & $\begin{array}{l}\text {-Humans } \\
\text { k-Monkey } \\
\text { m-Mouse } \\
\text { h-Rabbit } \\
\text { a-Sheep }\end{array}$ & $\begin{array}{l}\text { f-Ferret } \\
\text { j-Pigeon } \\
\text { e-Gerbil } \\
\text { s-Hamster } \\
\text { g-Guinea Pig }\end{array}$ & $\begin{array}{l}\text { n-Mink } \\
\text { o-Other }\end{array}$ & $\begin{array}{l}\text { Cancer Effect Level-Animals } \\
\text { LOAEL, More Serious-Animals } \\
\text { LOAEL, Less Serious-Animals } \\
\text { NOAEL - Animals }\end{array}$ & $\begin{array}{l}\nabla \text { Cancer Effect Level-Humans } \\
\Delta \text { LOAEL, More Serious-Humans } \\
\Delta \text { LOAEL, Less Serious-Humans } \\
\triangle \text { NOAEL - Humans }\end{array}$ & $\begin{array}{l}\text { LD50/LC50 } \\
\text { Minimal Risk Level } \\
\text { for effects } \\
\text { other than } \\
\text { Cancer }\end{array}$ \\
\hline
\end{tabular}


Figure 3-2 Levels of Significant Exposure to Dimethylarsinic Acid - Inhalation (Continued)

Intermediate (15-364 days)

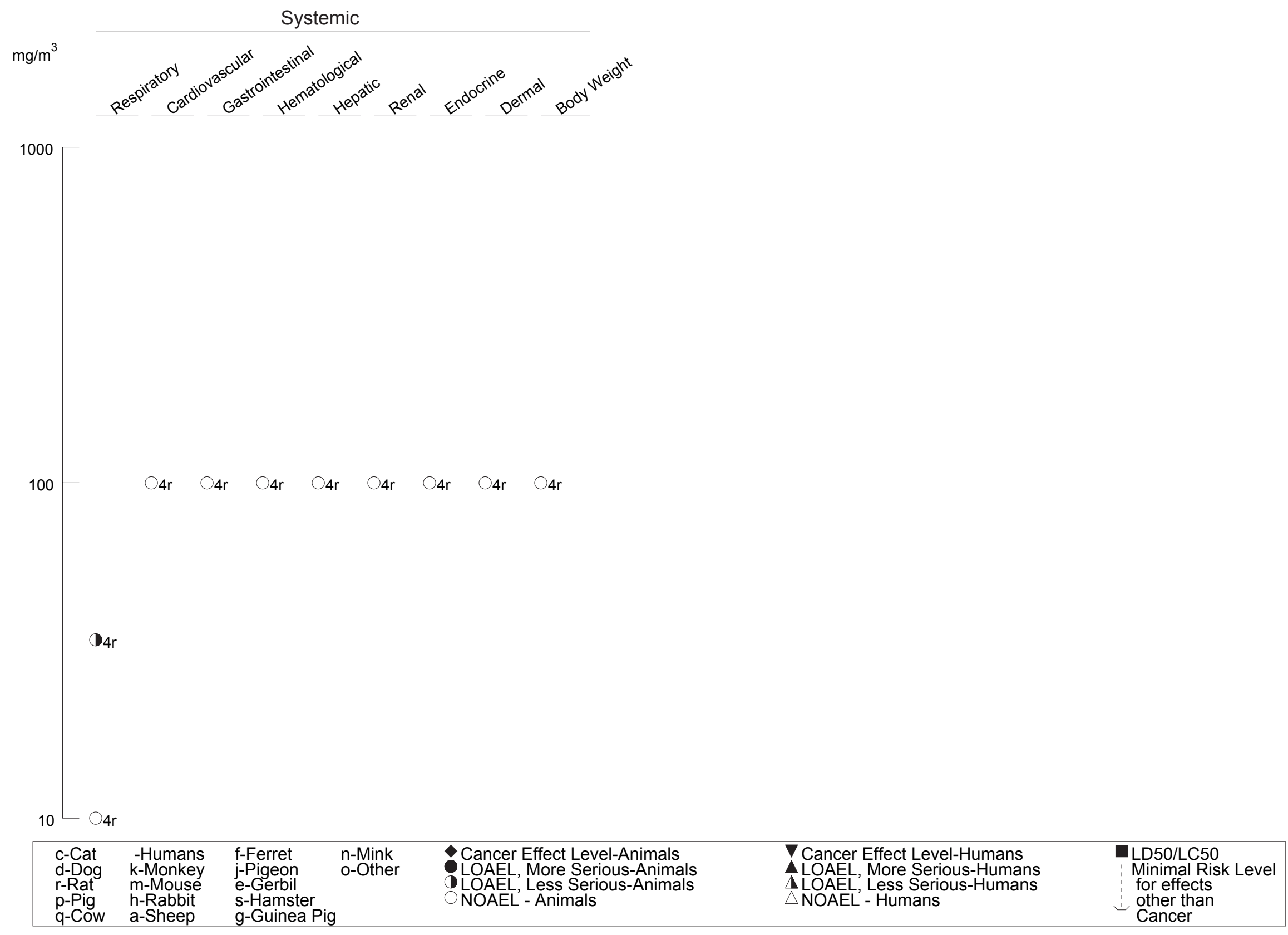

$10-\mathrm{O}_{4}$ 


\subsubsection{Death}

Inorganic Arsenicals. Although there are many studies of humans exposed to arsenic in air, no cases of lethality from short-term exposure were located. This suggests that death is not likely to be of concern following acute exposure, even at the very high exposure levels (1-100 $\mathrm{mg} \mathrm{As} / \mathrm{m}^{3}$ ) found previously in the workplace (e.g., Enterline and Marsh 1982; Järup et al. 1989; Lee-Feldstein 1986). Delayed lethality from chronic exposure attributable to increased risk of cardiovascular disease or lung cancer is discussed below in Sections 3.2.1.2 and 3.2.1.7, respectively. The only report of a lethal effect of inhaled inorganic arsenic in animals was a developmental toxicology study in which four of nine pregnant rats died, and one rat was euthanized in extremis, between days 12 and 19 of gestation after 30-35 days of exposure to an aerosol of arsenic trioxide at an exposure concentration of $20 \mathrm{mg} \mathrm{As} / \mathrm{m}^{3}$ (Holson et al. 1999). These animals exhibited severe hyperemia and plasma discharge into the intestinal lumen at autopsy. In this same study, there was $100 \%$ mortality in groups of 10 pregnant rats after 1 day of exposure to concentrations $\geq 100 \mathrm{mg} / \mathrm{m}^{3}$ (76 $\left.\mathrm{mg} \mathrm{As} / \mathrm{m}^{3}\right)$.

Organic Arsenicals. No studies were located regarding death in humans after inhalation exposure to organic arsenicals. A 2-hour $\mathrm{LC}_{50}$ of 3,900 $\mathrm{mg} \mathrm{DMA} / \mathrm{m}^{3}$ was calculated for DMA in female rats (Stevens et al. 1979). This $\mathrm{LC}_{50}$ is shown in Table 3-2 and Figure 3-2. Male rats and mice of both sexes were less susceptible, with only a few deaths after 2-hour exposures as high as $6,900 \mathrm{mg} \mathrm{DMA} / \mathrm{m}^{3}$ in rats and $6,400 \mathrm{mg} \mathrm{DMA} / \mathrm{m}^{3}$ in mice (Stevens et al. 1979). The cause of death was not specified, but was probably due to lung injury (see Section 3.2.1.2). No deaths were observed among rats and mice exposed to DSMA (the disodium salt of MMA) at concentrations up to $6,100 \mathrm{mg} \mathrm{DSMA} / \mathrm{m}^{3}$ in rats and $6,900 \mathrm{mg}$ DSMA $/ \mathrm{m}^{3}$ in mice (Stevens et al. 1979). Chamber atmospheres at these high concentrations were so dense that it was difficult to see the animals clearly. These data indicate that there is no significant risk of acute lethality from concentrations of DMA or MMA that might be encountered in the environment or the workplace.

\subsubsection{Systemic Effects}

The highest NOAEL values and all reliable LOAEL values for systemic effects from inhalation exposure to inorganic arsenicals in each species and duration category are recorded in Table 3-1 and plotted in Figure 3-1, while the corresponding data for DMA are shown in Table 3-2 and Figure 3-2. 


\section{Respiratory Effects.}

Inorganic Arsenicals. Workers exposed to arsenic dusts in air often experience irritation to the mucous membranes of the nose and throat. This may lead to laryngitis, bronchitis, or rhinitis (Dunlap 1921; Morton and Caron 1989; Pinto and McGill 1953), and very high exposures (characteristic of workplace exposures in the past) can cause perforation of the nasal septum (Dunlap 1921; Pinto and McGill 1953; Sandstrom et al. 1989). Despite the known respiratory irritant effects of arsenic, there have been few systematic investigations of respiratory effects in humans exposed to arsenic. Perry et al. (1948) found no difference in chest x-rays or respiratory performance (vital capacity and exercise-tolerance tests) between unexposed and exposed workers in a cross-sectional study at a factory where sodium arsenite was prepared. The NOAEL of $0.613 \mathrm{mg} \mathrm{As} / \mathrm{m}^{3}$ for respiratory effects in this study is shown in Table 3-1 and plotted in Figure 3-1.

Increased mortality due to respiratory disease has been reported in some cohort mortality studies of arsenic-exposed workers, but no conclusive evidence of an association with arsenic has been produced. In studies of workers exposed to arsenic trioxide at the Anaconda copper smelter in Montana, mortality due to noncancer respiratory disease (e.g., emphysema) was significantly increased compared to the general population (Lee-Feldstein 1983; Lubin et al. 2000; Welch et al. 1982). However, the data were not adjusted for smoking (a well-known confounder for respiratory disease), and analysis of the data with respect to arsenic exposure level did not show a clear dose-response. Similarly, Enterline et al. (1995) found a significant excess of nonmalignant respiratory disease mortality in workers at the ASARCO copper smelter in Tacoma, Washington, but only a slight negative relation to cumulative arsenic exposure. Xuan et al. (1993) found an increase in the relative risk of mortality from pneumoconiosis associated with arsenic exposure in a cohort of tin miners in China. However, this finding was based on a small number of observations ( $\mathrm{n}=32$ ), a clear exposure-response relationship with arsenic was not established, and the miners experienced confounding exposures to dust (a known risk factor for pneumoconiosis) and to radon. These studies were all considered to be inconclusive as to the relationship between inhaled inorganic arsenic and respiratory disease.

Respiratory symptoms were observed in a study of developmental effects in rats. Pregnant female rats exposed to arsenic trioxide dust starting 14 days prior to mating and continuing through mating and gestation exhibited rales at $8 \mathrm{mg} \mathrm{As} / \mathrm{m}^{3}$ and labored breathing and gasping at $20 \mathrm{mg} \mathrm{As} / \mathrm{m}^{3}$, with no symptoms at $2 \mathrm{mg} \mathrm{As} / \mathrm{m}^{3}$ (Holson et al. 1999). The lungs were examined by gross necropsy and no lesions were found. Intratracheal instillation of arsenic trioxide (13 mg As $/ \mathrm{kg}$ ) or gallium arsenide (1.5- 
$52 \mathrm{mg} \mathrm{As} / \mathrm{kg}$ ) can cause marked irritation and hyperplasia in the lungs of rats and hamsters (Goering et al. 1988; Ohyama et al. 1988; Webb et al. 1986, 1987). Since this sort of response is produced by a number of respirable particulate materials, it is likely that the inflammatory response is not specifically due to the arsenic.

Organic Arsenicals. No studies were located regarding respiratory effects in humans exposed to organic arsenicals. Short-term exposure of rats and mice to high concentrations $\left(\geq 4,000 \mathrm{mg} / \mathrm{m}^{3}\right)$ of DMA caused respiratory distress, and necropsy of animals that died revealed bright red lungs with dark spots (Stevens et al. 1979). Respiratory distress was also observed in rats and mice exposed to high levels $\left(\geq 6,100 \mathrm{mg} / \mathrm{m}^{3}\right.$ ) of the disodium salt of MMA (Stevens et al. 1979), although none of the MMA-exposed animals died. Respiratory distress appears to be associated with inhalation of very high concentrations of organic arsenicals. In 5-minute whole-body plethysmography trials, DMA and the disodium salt of MMA had $\mathrm{RD}_{50}$ (concentration calculated to produce a $50 \%$ decrease in respiration rate) values of 3,150 and $1,540 \mathrm{mg} / \mathrm{m}^{3}$, respectively (Stevens et al. 1979). Based on these $\mathrm{RD}_{50}$ values, neither DMA nor MMA is considered to be a potent respiratory irritant. At low concentrations of DMA (34 or $100 \mathrm{mg}$ $\mathrm{DMA} / \mathrm{m}^{3}$ ), an increase in intracytoplasmic eosinophilic globules were found in the nasal turbinates of rats exposed to DMA 6 hours/day, 5 days/week for 67-68 exposures (Whitman 1994).

\section{Cardiovascular Effects.}

Inorganic Arsenicals. There is some evidence from epidemiological studies that inhaled inorganic arsenic can produce effects on the cardiovascular system. Cardiovascular effects following oral exposure to arsenic are well known (see Section 3.2.2.2). A cross-sectional study of workers exposed to an estimated time-weighted average of $0.36 \mathrm{mg} \mathrm{As} / \mathrm{m}^{3}$ (as arsenic trioxide) at the Ronnskar copper smelter in Sweden for an average of 23 years showed that smelter workers had significantly increased incidences of Raynaud's phenomenon (a peripheral vascular disease characterized by spasm of the digital arteries and numbness of the fingers) and showed increased vasospasticity (constriction of blood vessels) in response to cold when tested in the fingers (Lagerkvist et al. 1986). A follow-up study conducted 2-3 years later found that vasospasticity measurements in exposed workers had improved concurrent with a reduction in arsenic exposure levels, although symptoms of peripheral vascular effects (cold hands or feet, white fingers, numbness in fingers or feet) were still common (Lagerkvist et al. 1988). A cross-sectional study including 46 workers in Denmark with varying, unquantified occupational exposure to arsenic in different occupations found that systolic blood pressure was significantly increased in the arsenic workers (median=125 mmHg) compared with controls (median=117 mmHg) (Jensen and Hansen 1998). Diastolic 
pressure was also increased in this study ( 77.9 vs. $74.7 \mathrm{mmHg})$, although the difference from controls was not statistically significant.

Cohort mortality studies of arsenic-exposed workers at the ASARCO copper smelter in Tacoma, Washington (Enterline et al. 1995), Anaconda copper smelter in Montana (Lee-Feldstein 1983; Welch et al. 1982), Ronnskar copper smelter in Sweden (Wall 1980), orchard workers in Washington state (Tollestrup et al. 1995), and tin miners in China (Qiao et al. 1997; Xuan et al. 1993) have all reported increased risk of mortality from cardiovascular disease, specifically ischemic heart disease and cerebrovascular disease, in the cohorts studied. However, none of these studies provided conclusive evidence that the observed increase in risk was due to arsenic exposure. The studies in the ASARCO and Anaconda copper smelter workers failed to find a clear dose-response relationship with arsenic (Enterline et al. 1995; Welch et al. 1982), while a follow-up study of the Ronnskar smelter workers not only found lack of a dose-response, but also that the risk of cardiovascular disease was no longer elevated in the cohort (Järup et al. 1989). The studies in orchard workers and tin miners were limited by confounding exposures to copper, lead, and radon, respectively (Qiao et al. 1997; Tollestrup et al. 1995). The risk of cardiovascular disease mortality in the tin miners not only showed no dose-response relationship with arsenic exposure, but was positively associated with radon exposure, suggesting that radon may have been responsible for the increased cardiovascular risk in this cohort (Xuan et al. 1993).

The LOAEL for Raynaud's phenomenon and vasospasticity identified by Lagerkvist et al. (1986) is shown in Table 3-1 and Figure 3-1. No studies were located regarding cardiovascular effects in animals after inhalation exposure to inorganic arsenic.

Organic Arsenicals. No studies were located regarding cardiovascular effects in humans after inhalation exposure to organic arsenicals. No histological alterations were observed in the hearts of rats exposed to $100 \mathrm{mg}$ DMA $/ \mathrm{m}^{3}$ for 67-68 exposures (Whitman 1994).

\section{Gastrointestinal Effects.}

Inorganic Arsenicals. Several case studies have reported nausea, vomiting, and diarrhea in workers with acute arsenic poisoning following occupational inhalation exposure (Beckett et al. 1986; Bolla-Wilson and Bleecker 1987; Ide and Bullough 1988; Morton and Caron 1989; Pinto and McGill 1953). Although gastrointestinal effects are not typically associated with arsenic poisoning by inhalation (Pinto and McGill 1953), such effects are a common feature of oral ingestion of high doses of arsenic (see Section 3.2.2.2), 
and it is possible that mucociliary transport of arsenic dust from the lungs to the gut could be responsible for the effects in these cases. Exposure levels were not reliably estimated for any of these cases.

The only report of gastrointestinal effects of inhaled inorganic arsenic in animals was a developmental toxicology study in which four of nine pregnant rats died, and one rat was euthanized in extremis, between days 12 and 19 of gestation after 30-35 days of exposure to an aerosol of arsenic trioxide at an exposure concentration of $20 \mathrm{mg} \mathrm{As} / \mathrm{m}^{3}$ (Holson et al. 1999). These animals exhibited severe hyperemia and plasma discharge into the intestinal lumen at autopsy. Exposure to $8 \mathrm{mg} \mathrm{As} / \mathrm{m}^{3}$ did not produce gross gastrointestinal lesions.

Organic Arsenicals. Data regarding gastrointestinal effects in people exposed to organic arsenic in the air are limited. The frequency of gastrointestinal complaints was no higher than controls in workers exposed to arsanilic acid (i.e., 4-aminophenyl arsonic acid) at mean concentrations up to $0.17 \mathrm{mg} / \mathrm{m}^{3}$ in a chemical factory (Watrous and McCaughey 1945). However, this sort of data might easily be biased by workers who chose not to complain about minor symptoms, so no conclusion can be reached. Rats and mice exposed to very high levels (above $3,000 \mathrm{mg} / \mathrm{m}^{3}$ ) of MMA (disodium salt) or DMA experienced diarrhea (Stevens et al. 1979). The diarrhea could be due to transport of inhaled particulate material from the lungs to the gastrointestinal system or to direct ingestion of the compound (e.g., from grooming of the fur). No gastrointestinal effects were observed in rats repeatedly exposed to $100 \mathrm{mg} \mathrm{DMA} / \mathrm{m}^{3}$ 6 hours/day, 5 days/week for 67-68 exposures (Whitman 1994).

\section{Hematological Effects.}

Inorganic Arsenicals. Although anemia is a common feature of arsenic poisoning following oral exposure in humans (see Section 3.2.2.2), case studies of workers with arsenic poisoning from occupational inhalation exposure reported no effects on red blood cell count (Beckett et al. 1986; BollaWilson and Bleecker 1987; Ide and Bullough 1988; Morton and Caron 1989). The reason for this apparent route specificity is not clear, but might simply be related to dose. No studies were located regarding hematological effects in animals after inhalation exposure to inorganic arsenicals.

Organic Arsenicals. No effect on levels of hemoglobin, red cells, or white cells was detected in the blood of manufacturing workers (323 counts in 35 workers) exposed to airborne arsanilic acid dusts at a mean concentration of $0.17 \mathrm{mg} / \mathrm{m}^{3}$ in the workplace (Watrous and McCaughey 1945). Controls were an unspecified number of unexposed manufacturing workers with 221 complete blood counts. No 
hematological alterations were observed in rats exposed to $100 \mathrm{mg} \mathrm{DMA} / \mathrm{m}^{3}$ for an intermediate duration (Whitman 1994).

\section{Musculoskeletal Effects.}

Inorganic Arsenicals. Few data were located regarding musculoskeletal effects associated with inhalation exposure to inorganic arsenic, and none to suggest the existence of any such effects. Electromyographic examination of the calves and feet showed no differences between control and arsenic-exposed workers in a cross-sectional study of workers at the Ronnskar copper smelter in Sweden (Blom et al. 1985). No studies were located regarding musculoskeletal effects in animals after inhalation exposure to inorganic arsenicals.

Organic Arsenicals. No studies were located regarding musculoskeletal effects in humans or animals after inhalation exposure to organic arsenicals.

\section{Hepatic Effects.}

Inorganic Arsenicals. There is no evidence that inhaled inorganic arsenic produces effects on the liver, although few data are available. Case studies of workers with inhalation arsenic poisoning that included liver function tests did not find any evidence of hepatic dysfunction (Bolla-Wilson and Bleecker 1987; Ide and Bullough 1988). No studies were located regarding hepatic effects in animals after inhalation exposure to inorganic arsenicals.

Organic Arsenicals. No studies were located regarding hepatic effects in humans after inhalation exposure to organic arsenicals. No histological alterations were observed in the livers of rats exposed to $100 \mathrm{mg}$ DMA $/ \mathrm{m}^{3}$ for 67-68 exposures (Whitman 1994).

\section{Renal Effects.}

Inorganic Arsenicals. The limited data available do not suggest any relationship between inhalation of inorganic arsenic and kidney effects. A cross-sectional study of renal function parameters in glass factory workers exposed to arsenic (concentrations unknown) found no meaningful differences from controls in urinary levels of several proteins (albumin, retinol binding protein, $\beta_{2}$-microglobulin, brush-border antigen) used as markers of glomerular damage or tubular cell exfoliation (Foà et al. 1987). Routine 
clinical urinalysis was normal when included in case studies of workers with inhalation arsenic poisoning (Ide and Bullough 1988; Morton and Caron 1989). No studies were located regarding renal effects in animals after inhalation exposure to inorganic arsenicals.

Organic Arsenicals. No studies were located regarding renal effects in humans after inhalation exposure to organic arsenicals. No renal effects were reported in rats exposed to $100 \mathrm{mg} \mathrm{DMA} / \mathrm{m}^{3} 6 \mathrm{hours} / \mathrm{day}$, 5 days/week for 67-68 exposures (Whitman 1994).

\section{Dermal Effects.}

Inorganic Arsenicals. Dermatitis has frequently been observed in industrial workers exposed to inorganic arsenic in the air, with the highest rates occurring in the workers with the greatest arsenic exposure (Cöl et al. 1999; Dunlap 1921; Holmqvist 1951; Lagerkvist et al. 1986; Pinto and McGill 1953). Limited quantitative information is available regarding the exposure levels that produce dermatitis, and the high likelihood of co-exposure by the dermal route makes dose-response analysis difficult. A crosssectional study of workers at a factory where sodium arsenite was prepared found that workers with the highest arsenic exposure (mean air levels ranging from 0.384 to $1.034 \mathrm{mg} \mathrm{As} / \mathrm{m}^{3}$ and estimated to average $0.613 \mathrm{mg} \mathrm{As} / \mathrm{m}^{3}$ ) tended to be grossly pigmented with hyperkeratinization of exposed skin and to have multiple warts (Perry et al. 1948). In the same study, workers with lower arsenic exposure (estimated to average $0.078 \mathrm{mg} \mathrm{As} / \mathrm{m}^{3}$ ) were much less affected, but still had a higher incidence of pigmentation keratosis than controls. LOAEL values identified by Perry et al. (1948) and Mohamed (1998) are shown in Table 3-1 and Figure 3-1. NOAEL values for dermal irritation have not been identified. Dermal effects (hyperkeratoses, hyperpigmentation) are also very common in people exposed to inorganic arsenic by the oral route (see Section 3.2.2.2). No studies were located on dermal effects in animals after inhalation exposure to inorganic arsenicals.

Organic Arsenicals. Data regarding dermal effects in people exposed to organic arsenic in the air are limited. Complaints of keratosis were roughly 2-fold higher than unexposed controls in female packaging workers exposed to arsanilic acid at an average concentration of $0.065 \mathrm{mg} / \mathrm{m}^{3}$ and in male manufacturing workers exposed to an average concentration of $0.17 \mathrm{mg} / \mathrm{m}^{3}$ in a chemical factory (Watrous and McCaughey 1945). Limitations in study methodology (e.g., alternate sources of effects were not investigated, workers might choose not to report minor complaints to company officials) make the reliability of this observation uncertain. Female rats exposed to DMA at $6,900 \mathrm{mg} / \mathrm{m}^{3}$ developed erythematous lesions on the feet and ears (Stevens et al. 1979); these lesions did not develop in females 
exposed at lower concentrations $\left(4,100 \mathrm{mg} / \mathrm{m}^{3}\right)$ or males. It seems likely that these effects were due to direct irritation from dermal contact with the dust. No dermal effects were observed in rats repeatedly exposed to lower levels of DMA (100 mg/m³) (Whitman 1994).

\section{Ocular Effects.}

Inorganic Arsenicals. Chemical conjunctivitis, characterized by redness, swelling, and pain, has been observed in workers exposed to arsenic dusts in air, usually accompanied by facial dermatitis (Dunlap 1921; Pinto and McGill 1953). No information was located regarding air levels of arsenic that produce this effect. No studies were located on ocular effects in animals after inhalation exposure to inorganic arsenicals.

Organic Arsenicals. No studies were located on ocular effects in humans after inhalation exposure to organic arsenicals. Rats and mice exposed to high concentrations of DMA $\left(\geq 4,000 \mathrm{mg} / \mathrm{m}^{3}\right)$ developed an encrustation around the eyes (Stevens et al. 1979). It seems likely that these effects were due to direct irritation from ocular contact with the dust.

\section{Body Weight Effects.}

Inorganic Arsenicals. No studies were located on body weight effects in humans after inhalation exposure to inorganic arsenicals. Female rats exposed to arsenic trioxide dust starting 14 days before mating and continuing through mating and gestation showed a marked decrease in body weight and food consumption at $20 \mathrm{mg} \mathrm{As} / \mathrm{m}^{3}$ (preliminary study) and a smaller decrease at $8 \mathrm{mg} \mathrm{As} / \mathrm{m}^{3}$ (definitive study), with no effect at $2 \mathrm{mg} \mathrm{As} / \mathrm{m}^{3}$ (Holson et al. 1999).

Organic Arsenicals. No studies were located on body weight effects in humans after inhalation exposure to organic arsenicals. Rats and mice exposed to high concentrations of DMA $\left(\geq 4,000 \mathrm{mg} / \mathrm{m}^{3}\right)$ for 2 hours had an unspecified decrease in body weight gain during the subsequent 14 days (Stevens et al. 1979). No alterations in body weight gain were observed in rats exposed to $100 \mathrm{mg} \mathrm{DMA} / \mathrm{m}^{3}$ for $67-68$ exposures (Whitman 1994). 


\subsubsection{Immunological and Lymphoreticular Effects}

Inorganic Arsenicals. A single study was located regarding the immunological and lymphoreticular effects of inhaled inorganic arsenic in humans. Bencko et al. (1988) detected no abnormalities in serum levels of immunoglobins in 47 workers exposed to arsenic (exposure levels not measured) in a coalburning power plant. However, serum levels of other proteins such as transferrin, orosomucoid, and ceruloplasmin were significantly elevated compared to levels in a group of 27 workers from a different plant in which the arsenic content in the coal was 10 times lower. The investigators suggested that the increased levels of ceruloplasmin might be related to higher cancer mortality rates found among these workers.

The immune effects of inhaled arsenic in animals were studied by Aranyi et al. (1985). Female mice exposed to arsenic trioxide aerosol for 3 hours showed a concentration-related decrease in pulmonary bactericidal activity (presumably as a result of injury to alveolar macrophages) and a corresponding concentration-related increase in susceptibility to introduced respiratory bacterial pathogens. Similar results were found when the exposure was repeated over 1- and 4-week periods. The NOAEL and LOAEL values for this study are shown in Table 3-1 and Figure 3-1.

Intratracheal studies in animals offer some support for an immune effect of inhaled inorganic arsenic. Decreases in humoral response to antigens and in several complement proteins were noted in mice given an intratracheal dose of $5.7 \mathrm{mg} \mathrm{As} / \mathrm{kg}$ as sodium arsenite (Sikorski et al. 1989), although these changes were not accompanied by any decrease in resistance to bacterial or tumor cell challenges. Animals given an intratracheal dose of GaAs (25 mg As/kg or higher) also displayed a variety of changes in numerous immunological end points (some increased, some decreased) (Burns and Munson 1993; Sikorski et al. 1989). Whether these effects were due to a direct effect on the immune system or were secondary to the inflammatory effect of GaAs on the lung (see Section 3.2.1.2, above) is uncertain.

Organic Arsenicals. No studies were located regarding immunological and lymphoreticular effects in humans or animals after inhalation exposure to organic arsenicals.

\subsubsection{Neurological Effects}

Inorganic Arsenicals. There is evidence from epidemiological studies that inhaled inorganic arsenic can produce neurological effects. A study by Gerr et al. (2000) reported an elevated incidence of peripheral neuropathy in subjects who lived near an arsenic-using pesticide plant $(13 / 85=15.3 \%$; odds ratio 
$[\mathrm{OR}]=5.1, \mathrm{p}=0.004)$, relative to subjects who lived farther from the plant $(4 / 118=3.4 \%)$. Concentrations of arsenic in soil and house dust were elevated $(\sim 30-300 \mu \mathrm{g} \mathrm{As} / \mathrm{g})$ for residences near the plant, according to 1993-1995 monitoring data. Studies of copper smelter workers at the ASARCO smelter in Tacoma, Washington (Feldman et al. 1979), a power station in Slovakia (Buchancová et al. 1998), and the Ronnskar smelter in Sweden (Blom et al. 1985; Lagerkvist and Zetterlund 1994) have demonstrated peripheral neurological effects in workers associated with arsenic trioxide exposure. At the ASARCO smelter, the prevalence of clinically diagnosed peripheral neuropathy was markedly higher in arsenicexposed workers $(26 / 61=43 \%)$ than controls $(4 / 33=12 \%)$, and although the difference in mean nerve conduction velocities (NCV) was not statistically significant, mean peroneal motor NCV was lower in arsenic-exposed workers than controls and all 12 cases of abnormally low NCV occurred in the arsenic group (Feldman et al. 1979). In the study of 70 workers in Slovakia, the investigators described 16 cases of arsenic intoxication. Among these, 13 had signs and symptoms of sensory and motor polyneuropathy on both upper and lower extremities, 10 were diagnosed with pseudoneurasthenic syndrome, and 6 suffered from toxic encephalopathy (Buchancová et al. 1998). The average length of exposure was 22.3 years ( $\mathrm{SD} \pm 8.4$ years) and the average arsenic exposure in inhaled air ranged from 4.6 to $142.7 \mu \mathrm{g} / \mathrm{m}^{3}$. Similar results were observed at the Ronnskar smelter, where Blom et al. (1985) reported significantly increased prevalence of workers with abnormally low NCV in the exposed group, and lower, but not statistically significant, mean NCV in five peripheral nerves. A follow-up study on the Ronnskar workers 5 years later found that the prevalence of abnormally low NCV remained significantly increased in the exposed workers, but that the decrease in mean NCV was now also statistically significant in the tibial (motor) and sural (sensory) nerves (Lagerkvist and Zetterlund 1994). Blood lead was monitored in this study as a potential confounder, but levels were low and not considered likely by the researchers to have had any influence on the results. The follow-up Ronnskar study provided enough information to estimate that mean arsenic exposure was $0.31 \mathrm{mg} \mathrm{As} / \mathrm{m}^{3}$ and lasted an average of 28 years in the exposed group, and this LOAEL is shown in Table 3-1 and Figure 3-1.

The literature also contains several case studies of workers with inhalation arsenic poisoning who developed neurological symptoms. Although these studies do not provide reliable information on exposure levels or conclusive evidence that the observed effects were related to arsenic, the findings are suggestive. Symptoms in these cases included not only indicators of peripheral neuropathy (numbness, loss of reflexes, muscle weakness, tremors) (Ide and Bullough 1988; Morton and Caron 1989), but also frank encephalopathy (hallucinations, agitation, emotional lability, memory loss) (Beckett et al. 1986; Bolla-Wilson and Bleecker 1987; Morton and Caron 1989). Both peripheral neuropathy and encephalopathy are associated with oral exposure to inorganic arsenic (see Section 3.2.2.4). 
The possible association between arsenic in air and neurological effects in children has also been examined. A study by Bencko et al. (1977) reported that children of approximately 10 years of age $(n=56)$ living near a power plant burning coal of high arsenic content showed significant hearing losses (increased threshold) compared to a control group of children $(n=51)$ living outside the polluted area (Bencko et al. 1977). The effect was most marked at low frequencies. The precise site affected within the auditory pathway was not determined and could have been in the periphery, centrally-located, or both. A small study of children in Mexico reported a significant negative correlation between tests of verbal IQ and urinary arsenic in children $(n=41)$ living in an urban area near a smelter complex (Calderón et al. 2001). Exposure concentrations were not available in either study.

No studies were located regarding neurological effects in animals after inhalation exposure to inorganic arsenicals. Mice given a single intratracheal dose of $200 \mathrm{mg} / \mathrm{kg}$ of GaAs displayed a decrease in overall activity 6-8 hours later, but no additional neurological evaluations were conducted on these animals (Burns and Munson 1993).

Organic Arsenicals. Data regarding neurological effects in people exposed to organic arsenic in the air are limited to a single study. The frequency of central nervous system complaints was no higher than controls in workers at a chemical factory exposed to arsanilic acid at mean concentrations up to $0.17 \mathrm{mg} / \mathrm{m}^{3}$ (Watrous and McCaughey 1945). Although peripheral nerve complaints were higher in arsenic packaging workers (mean exposure $=0.065 \mathrm{mg} / \mathrm{m}^{3}$ ) than in unexposed controls, this was not the case in manufacturing workers with higher arsenic exposure (mean $=0.17 \mathrm{mg} / \mathrm{m}^{3}$ ). This suggests that the effects on the peripheral nerves in the exposed packaging workers were not due to arsenic. The reliability of these data is limited by shortcomings in the study methodology (e.g., the data might easily be biased by workers who chose not to complain about minor symptoms). No studies were located regarding neurological effects in animals after inhalation exposure to organic arsenicals.

\subsubsection{Reproductive Effects}

Inorganic Arsenicals. No studies were located regarding reproductive effects in humans after inhalation exposure to inorganic arsenicals. Reproductive performance was evaluated in female rats exposed to $0.08-20 \mathrm{mg} \mathrm{As} / \mathrm{m}^{3}$ (preliminary study) or $0.2-8 \mathrm{mg} \mathrm{As} / \mathrm{m}^{3}$ (definitive study) as $\mathrm{As}_{2} \mathrm{O}_{3} 6$ hours daily from 14 days prior to mating through gestation day 19 (Holson et al. 1999). No changes occurred in the precoital interval (time to mating), mating index (percentage of rats mated), or fertility index (percentage 
of matings resulting in pregnancy). The NOAEL values for this study are shown in Table 3-1 and Figure 3-1.

Organic Arsenicals. No studies were located regarding reproductive effects in humans or animals after inhalation exposure to organic arsenicals.

\subsubsection{Developmental Effects}

Inorganic Arsenicals. Developmental effects associated with occupational and environmental exposure to airborne arsenic have been investigated in a series of studies at the Ronnskar copper smelter in northern Sweden (Nordström et al. 1978a, 1978b, 1979a, 1979b). In comparison to a northern Swedish reference population, female employees of the smelter had a significantly increased incidence of spontaneous abortion (Nordström et al. 1979a), and their children had a significantly increased incidence of congenital malformations (Nordström et al. 1979b) and significantly decreased average birth weight (Nordström et al. 1978a). Increased incidence of spontaneous abortion and decreased average birth weight of children were also found in populations living in close proximity to the smelter (Nordström et al. 1978a, 1978b, 1979b). While these data are suggestive of developmental effects associated with occupational and environmental exposure from the smelter, the reported effects are not large, the analyses include only limited consideration of potential confounders (e.g., smoking), and there are no data relating the apparent effects specifically to arsenic exposure.

Ihrig et al. (1998) conducted a case-control study of stillbirths in the vicinity of a Texas arsenic pesticide factory that included estimation of environmental arsenic exposures using atmospheric dispersion modeling and multiple regression analysis considering arsenic exposure, race/ethnicity, maternal age, median income, and parity as explanatory variables. There was a statistically significant increase in the risk of stillbirth in the highest exposure category $\left(>100 \mathrm{ng} \mathrm{As} / \mathrm{m}^{3}\right.$, midpoint $\left.=682 \mathrm{ng} / \mathrm{m}^{3}\right)$. Further analysis showed that this increase in risk was limited to people of Hispanic descent, who the researchers speculated may be an especially sensitive population due to a genetic impairment in folate metabolism. Interpretation of this study is limited by small numbers of cases and controls in the high exposure group, lack of data on smoking, potential confounding exposures to other chemicals from the factory, and failure to take into account previous years of deposition in the exposure estimates.

Arsenic has been shown to produce developmental effects by inhalation exposure in laboratory animals, although it is unclear whether or not the effects occur only at maternally toxic doses. Mice exposed to 
$22 \mathrm{mg} \mathrm{As} / \mathrm{m}^{3}$ (as $\mathrm{As}_{2} \mathrm{O}_{3}$ ) for 4 hours on days 9-12 of gestation had serious developmental effects (significant increases in the percentage of dead fetuses, skeletal malformations, and the number of fetuses with retarded growth), while those exposed to $2.2 \mathrm{mg} \mathrm{As} / \mathrm{m}^{3}$ had only a $10 \%$ decrease in average fetal body weight, and those exposed to $0.20 \mathrm{mg} \mathrm{As} / \mathrm{m}^{3}$ had no effects (Nagymajtényi et al. 1985). The study was limited by failure to quantify malformations on a litter basis, discuss the nature and severity of the observed malformations, or report on the occurrence of maternal effects. No increases in fetal resorptions, fetal mortality, or malformations, and no decreases in fetal body weight occurred when rats were exposed to $0.2-8 \mathrm{mg} \mathrm{As} / \mathrm{m}^{3}$ (as $\mathrm{As}_{2} \mathrm{O}_{3}$ ), 6 hours daily from 14 days prior to mating through gestation day 19 (Holson et al. 1999). At the $8 \mathrm{mg} / \mathrm{m}^{3}$ exposure level, toxicity was observed in the dams, including rales, a dried red exudate at the nose, and lower gains in net body weight than controls. In a preliminary dose-range study, there was a marked significant increase in postimplantation loss (primarily early resorptions) and consequent marked significant decrease in viable fetuses per litter at $20 \mathrm{mg} \mathrm{As} / \mathrm{m}^{3}$, a concentration that also produced severe maternal effects including mortality (Holson et al. 1999).

The NOAEL and LOAEL values for increased risk of stillbirth in humans identified by Ihrig et al. (1998) and those for developmental effects in rodents found by Nagymajtényi et al. (1985) and Holson et al. (1999) are shown in Table 3-1 and Figure 3-1.

Organic Arsenicals. No studies were located regarding developmental effects in humans or animals after inhalation exposure to organic arsenicals.

\subsubsection{Cancer}

Inorganic Arsenicals. There is convincing evidence from a large number of epidemiological studies that inhalation exposure to inorganic arsenic increases the risk of lung cancer. Most studies involved workers exposed primarily to arsenic trioxide dust in air at copper smelters (Axelson et al. 1978; Brown and Chu 1982, 1983a, 1983b; Enterline and Marsh 1982; Enterline et al. 1987a, 1987b, 1995; Ferreccio et al. 1996; Järup and Pershagen 1991; Järup et al. 1989; Lee and Fraumeni 1969; Lee-Feldstein 1983, 1986; Lubin et al. 2000; Mazumdar et al. 1989; Pinto et al. 1977, 1978; Sandstrom et al. 1989; Viren and Silvers 1999; Wall 1980; Welch et al. 1982) and mines (Liu and Chen 1996; Qiao et al. 1997; Taylor et al. 1989; Xuan et al. 1993), but increased incidence of lung cancer has also been observed at chemical plants where exposure was primarily to arsenate (Bulbulyan et al. 1996; Mabuchi et al. 1979; Ott et al. 1974; Sobel et al. 1988). In addition, several studies suggest that residents living near smelters or arsenical chemical plants may also have increased risk of lung cancer (Brown et al. 1984; Cordier et al. 1983; Matanoski et 
al. 1981; Pershagen 1985), although the increases are small and are not clearly detectable in all cases (e.g., Frost et al. 1987). The strongest evidence that arsenic is responsible for the observed lung cancer comes from quantitative dose-response data relating specific arsenic exposure levels to lung cancer risk. These data are available for arsenic-exposed workers at the ASARCO copper smelter in Tacoma, Washington (Enterline and Marsh 1982; Enterline et al. 1987a, 1995; Mazumdar et al. 1989), the Anaconda copper smelter in Montana (Lee-Feldstein 1986; Welch et al. 1982), eight other U.S. copper smelters (Enterline et al. 1987b), and the Ronnskar copper smelter in Sweden (Järup and Pershagen 1991; Järup et al. 1989). A common limitation of these studies is confounding exposure to other chemicals, such as sulfur dioxide, and cigarette smoking.

Enterline and Marsh (1982) reported a significant increase in respiratory cancer mortality (standard mortality ratio $[\mathrm{SMR}]=189.4$ ) based on 104 observed respiratory cancer deaths and only 54.9 expected over the years 1941-1976 in a cohort of 2,802 male workers employed for $\geq 1$ year between 1940 and 1964 at the ASARCO smelter. When the cohort was separated into low and high arsenic exposure groups, with mean estimated time-weighted average arsenic exposures of 0.054 and $0.157 \mathrm{mg} \mathrm{As} / \mathrm{m}^{3}$, respectively (based on work history, historical urinary arsenic measurements, and an experimentally derived relationship between urinary and inhaled arsenic), respiratory cancer mortality was significantly increased in both groups in a concentration-related fashion (SMR=227.7 and 291.4 in the low and high groups, respectively). Enterline et al. (1987a) re-analyzed these data using improved exposure estimates that incorporated historical measurements of arsenic in the ambient air and personal breathing zone of workers. Respiratory cancer mortality was significantly increased in a concentration-related fashion in the low $(\mathrm{SMR}=213.0)$, medium $(\mathrm{SMR}=312.1)$, and high $(\mathrm{SMR}=340.9)$ arsenic exposure groups, which had mean estimated time-weighted average arsenic exposures of $0.213,0.564$, and $1.487 \mathrm{mg} \mathrm{As} / \mathrm{m}^{3}$, respectively. An alternative analysis of these data by Mazumdar et al. (1989) produced similar results. Enterline et al. (1995) extended the mortality follow-up from 1976 to 1986, but reported findings similar to the earlier study in a less thorough analysis. The CEL from Enterline et al. (1987a), the most complete analysis of the ASARCO cohort with the best exposure estimates, is presented in Table 3-1 and Figure 3-1.

Respiratory cancer mortality was significantly increased $(\mathrm{SMR}=285)$ based on 302 observed respiratory deaths between 1938 and 1977 in a cohort of 8,045 white male workers employed for at least 1 year between 1938 and 1956 at the Anaconda smelter (Lee-Feldstein 1986). When workers were categorized according to cumulative arsenic exposure and date of hire, lung cancer mortality was significantly increased in all groups hired between 1925 and 1947. Workers in the lowest cumulative exposure group 
$\left(<10 \mathrm{mg}-\mathrm{mo} / \mathrm{m}^{3}\right)$ were reported to have had $<2$ years of exposure at an average arsenic concentration of $0.38 \mathrm{mg} / \mathrm{m}^{3}$. An alternative analysis of a subset of the Anaconda cohort $(\mathrm{n}=1,800$, including all 277 employees with heavy arsenic exposure and $20 \%$ of the others) that included information on smoking and other occupational exposures was performed by Welch et al. (1982). This analysis showed that lung cancer mortality increased with increasing time-weighted average arsenic exposure, with a small nonsignificant increase in the low group $(\mathrm{SMR}=138)$ exposed to $0.05 \mathrm{mg} / \mathrm{m}^{3}$ and significant increases in the medium $(\mathrm{SMR}=303)$, high $(\mathrm{SMR}=375)$, and very high $(\mathrm{SMR}=704)$ groups exposed to $0.3,2.75$, and $5.0 \mathrm{mg} / \mathrm{m}^{3}$, respectively. Cohort members were more likely to be smokers than U.S. white males, but smoking did not differ among the arsenic exposure groups. Exposure-response analysis of smokers was similar to the analysis based on the full subcohort, while analysis of nonsmokers (limited by small group sizes) also showed a similar pattern, but with lower SMRs. In a followup analysis of the same cohort, Lubin et al. (2000) re-weighted the exposure concentrations based on duration and time of exposure and re-evaluated the effects of exposure. Relative risks for respiratory cancer increased with increasing duration in each arsenic exposure area (light, medium, and heavy) after adjustment for duration in the other two exposure areas. SMRs were significantly elevated following exposure to $0.58 \mathrm{mg} / \mathrm{m}^{3}$ (medium; $\mathrm{SMR}=3.01,95 \% \mathrm{CI}=2.0-4.6$ ) or $11.3 \mathrm{mg} / \mathrm{m}^{3}$ (high; $\mathrm{SMR}=3.68,95 \% \mathrm{CI}=2.1-6.4$ ) for 10 or more years, and following exposure to $0.29 \mathrm{mg} / \mathrm{m}^{3}$ (low; $\mathrm{SMR}=1.86,95 \% \mathrm{CI}=1.2-2.9$ ) for 25 or more years. The CELs from the analyses of the Anaconda cohort are presented in Table 3-1 and Figure 3-1.

Enterline et al. (1987b) studied the mortality experience from 1949 to 1980 of a cohort of 6,078 white males who had worked for 3 years or more between 1946 and 1976 at one of eight U.S. copper smelters in Arizona, Utah, Tennessee, and Nevada. Lung cancer mortality was significantly increased only in the Utah smelter $(\mathrm{SMR}=226.7)$, which had the highest average arsenic exposure concentration $\left(0.069 \mathrm{mg} / \mathrm{m}^{3}\right.$ vs. $0.007-0.013 \mathrm{mg} / \mathrm{m}^{3}$ in the other smelters) and also contributed the largest number of cohort members ( $\mathrm{n}=2,288$ vs. 189-965 from the other smelters). A nested case-control study showed that arsenic exposure and cigarette smoking were significant risk factors for lung cancer in the smelter workers. Smoking was lower in the Utah smelter workers than in the other smelter workers, but still higher than in the referent Utah population, suggesting that the risk attributable to arsenic in this study population is somewhat lower than indicated by the SMR reported above. The CEL from this study is presented in Table 3-1 and Figure 3-1.

Järup et al. (1989) reported significantly increased lung cancer mortality (SMR=372, 95\% confidence interval $[\mathrm{CI}]=304-450$ ) based on 106 lung cancer deaths in a cohort of 3,916 male workers employed for $\geq 3$ months between 1928 and 1967 at the Ronnskar smelter and followed for mortality through 1981 . 
Workers were separated into low, medium, and high arsenic exposure groups with mean time-weighted average exposure estimates of $0.05,0.2$, and $0.4 \mathrm{mg} / \mathrm{m}^{3}$, respectively. Lung cancer mortality was significantly increased in all three exposure groups in a concentration-related fashion (SMR=201, 353, and 480, respectively). A nested case-control analysis of 102 lung cancer cases and 190 controls from the cohort showed that lung cancer risk increased with increasing arsenic exposure in nonsmokers, light smokers, and heavy smokers (Järup and Pershagen 1991). The results demonstrated that arsenic is a risk factor for lung cancer in the smelter workers, but also suggested a greater-than-additive interaction between smoking and arsenic exposure. In this analysis, in contrast to the cohort study, lung cancer risk due to arsenic was increased only in the higher arsenic-exposure groups. Potential explanations for this difference between the cohort and case-control analyses include a higher proportion of smokers in the smelter workers than in the regional referent population in the cohort study, and limited power to detect increased risk in the case-control study due to small group sizes in the dose-response analysis. The CELs from both the cohort and case-control studies are presented in Table 3-1 and Figure 3-1.

Several researchers have examined the histological cell types of lung cancer (epidermoid carcinoma, small cell carcinoma, adenocarcinoma) in arsenic-exposed workers (e.g., Axelson et al. 1978; Newman et al. 1976; Pershagen et al. 1987; Qiao et al. 1997; Wicks et al. 1981). Although the incidence of the various cell types varied from population to population, all studies found an increase in several tumor types. This indicates that arsenic does not specifically increase the incidence of one particular type of lung cancer.

The studies of the ASARCO cohort (Enterline and Marsh 1982; Enterline et al. 1987a, 1995) noted a supralinear exposure-response relationship (i.e., steeper at lower doses) between arsenic exposure and lung cancer mortality. Hertz-Picciotto and Smith (1993) extended this observation to several other occupationally exposed cohorts with quantitative exposure information. The authors suggest that neither toxicokinetic mechanisms nor confounding from age, smoking, or other workplace carcinogens that differ by exposure level are likely explanations for the curvilinearity. Plausible explanations offered include: (1) synergism (with smoking), which varies in magnitude according to the level of arsenic exposure, (2) long-term survivorship at higher exposures among the healthier, less susceptible individuals, and (3) exposure estimate errors that were more prominent at higher-exposure levels as a result of past industrial hygiene sampling or worker protection practices.

Quantitative risk estimates for inhaled inorganic arsenic have been derived using the exposure-response data. EPA derived a unit risk estimate (the excess risk of lung cancer associated with lifetime exposure to 
$\left.1 \mu \mathrm{g} / \mathrm{m}^{3}\right)$ of $4.3 \times 10^{-3} \mathrm{per}\left(\mu \mathrm{g} / \mathrm{m}^{3}\right)$ based on the dose-response relationships between arsenic exposure and excess lung cancer mortality in workers at the Anaconda smelter in Montana (Brown and Chu 1982, 1983a, 1983b; Lee-Feldstein 1983; and an unpublished paper by Higgins and associates) and the ASARCO smelter in Tacoma, Washington (Enterline and Marsh 1982; EPA 1984a; IRIS 2007). In some cases, calculations of exposure, as well as the procedures for generating quantitative risk estimates, are quite complex and the interested reader is referred to the EPA documents (EPA 1981c, 1984a, 1987e, 1996b; IRIS 2007) for a detailed description. Viren and Silvers (1994) re-evaluated the unit risk estimate using the same methods as EPA, but incorporating updated results from the ASARCO smelter (Enterline et al. 1987a; Mazumdar et al. 1989) and the findings from the Swedish smelter (Järup et al. 1989). Their analysis yielded a revised unit risk of $1.28 \times 10^{-3} \mathrm{per}\left(\mu \mathrm{g} / \mathrm{m}^{3}\right)$ that, when pooled with the earlier estimate from the Montana smelter cohort, yielded a composite unit risk of $1.43 \times 10^{-3} \mathrm{per}\left(\mu \mathrm{g} / \mathrm{m}^{3}\right)$. This unit risk estimate is a factor of 3 smaller than the EPA's current estimate of $4.3 \times 10^{-3}$ per $\left(\mu \mathrm{g} / \mathrm{m}^{3}\right)$. Figure $3-1$ shows the air concentrations that correspond to excess lifetime cancer risks of $10^{-4}-10^{-7}$ based on the EPA unit risk estimate.

There have been occasional reports of other types of cancer (i.e., nonrespiratory cancer) potentially associated with inhalation exposure to inorganic arsenic, but there is no strong evidence for any of them. For example, Enterline et al. (1995) found significantly increased mortality due to cancer of the large intestine and bone cancer in the ASARCO cohort. However, neither cancer showed any relation to cumulative arsenic exposure, and the purported increase in bone cancer risk was based on a very small number of observations. Pesch et al. (2002) reported an increase in nonmelanoma skin cancers resulting from exposure from a Slovakian coal-burning power plant, but exposure levels associated with the lesions were not presented. Bencko et al. (2005) also reported an increase in the incidence of nonmelanoma skin cancer among workers of a power plant burning coal of a high arsenic content and in the population living in the vicinity of the power plant. Bulbulyan et al. (1996) reported an increase in risk of stomach cancer among workers exposed to the highest average arsenic concentrations at a Russian fertilizer plant, but this finding, which was based on a small number of observations and was only marginally statistically significant, was confounded by exposure to nitrogen oxides, which were more convincingly associated with stomach cancer in this study. Wingren and Axelson (1993) reported an association between arsenic exposure and stomach and colon cancer in Swedish glass workers, but this result was confounded by concomitant exposure to other metals. Lee-Feldstein (1983) observed a small, marginally significant increase in digestive tract cancer $(\mathrm{SMR}=125)$ in one study of the Anaconda cohort, but this was not found in other studies of this cohort (Lee and Fraumeni 1969; Lee-Feldstein 1986; Welch et al. 1982). Wulff et al. (1996) observed an apparent increase in the risk of childhood cancer (all types combined) in the 
population living within $20 \mathrm{~km}$ of the Ronnskar smelter, but the apparent increase was based on a small number of cases (13 observed vs. 6.7 expected) and was not statistically significant, and exposure to arsenic was confounded by exposure to lead, copper, cadmium, sulfur dioxide, and possibly other emissions such as nickel and selenium. A retrospective study of deaths due to unspecified types of malignancies among workers of power plants found no significant differences in death rate between two groups whose exposure levels to arsenic had a difference of one order of magnitude (Bencko et al. 1980). However, the mean age of those deceased due to cancer in the high-exposure group was 55.9 years compared to 61.2 years in the low-exposure group, and this difference was statistically significant $(p<0.05)$. Also, when the workers were stratified by exposure-duration, there was a significantly higher frequency of tumors in the high-exposure group after shorter employment periods ( $<5$ or $6-10$ years) than after a longer employment period ( $\geq 11$ years). No information was provided regarding specific types of cancer. Various case reports have implicated occupational arsenic exposure as a potential contributing factor in workers who developed sinonasal cancer (Battista et al. 1996), hepatic angiosarcoma (Tsai et al. 1998a), and skin cancer (Cöl et al. 1999; Tsuruta et al. 1998), but provide no proof that inhaled arsenic was involved in the etiology of the observed tumors. Wong et al. (1992) found no evidence that environmental exposure to airborne arsenic produced skin cancer in residents living near the Anaconda smelter or an open pit copper mine.

No studies were located regarding cancer in animals after inhalation exposure to inorganic arsenicals, although several intratracheal instillation studies in hamsters have provided evidence that both arsenite and arsenate can increase the incidence of lung adenomas and/or carcinomas (Ishinishi et al. 1983; Pershagen and Björklund 1985; Pershagen et al. 1984; Yamamoto et al. 1987). These data support the conclusion that inhalation of arsenic may lead to lung cancer in humans.

Organic Arsenicals. No studies were located regarding cancer effects in humans or animals after inhalation exposure to organic arsenicals.

\subsubsection{Oral Exposure}

There are a large number of studies in humans and animals on the toxic effects of ingested arsenic. In humans, most cases of toxicity have resulted from accidental, suicidal, homicidal, or medicinal ingestion of arsenic-containing powders or solutions or by consumption of contaminated food or drinking water. In some cases, the chemical form is known (e.g., the most common arsenic medicinal was Fowler's solution, which contained $1 \%$ potassium arsenite or arsenic trioxide), but in many cases (e.g., exposures through 
drinking water), the chemical form is not known. In these cases, it is presumed that the most likely forms are either inorganic arsenate $[\mathrm{As}(+5)]$, inorganic arsenite $[\mathrm{As}(+3)]$, or a mixture. Table 3-3 and Figure 3-3 summarize a number of studies that provide reliable quantitative data on health effects in humans and animals exposed to inorganic arsenicals by the oral route. Similar data for MMA, DMA, and roxarsone are listed in Tables 3-4, 3-5, and 3-6, and shown in Figures 3-4, 3-5, and 3-6, respectively. All exposure data are expressed as milligrams of arsenic (as the element) per kilogram body weight per day (mg As/kg/day). These studies and others that provide useful qualitative information are summarized below.

\subsubsection{Death}

Inorganic Arsenicals. There are many case reports of death in humans due to ingestion of high doses of arsenic. In nearly all cases, the most immediate effects are vomiting, diarrhea, and gastrointestinal hemorrhage, and death may ensue from fluid loss and circulatory collapse (Levin-Scherz et al. 1987; Saady et al. 1989; Uede and Furukawa 2003). In other cases, death may be delayed and result from the multiple tissue injuries produced by arsenic (Campbell and Alvarez 1989). Some accounts of fatal arsenic poisoning describe both gastrointestinal effects soon after ingestion and extensive damage to multiple organ systems prior to death (Quatrehomme et al. 1992). A precise estimate of the ingested dose is usually not available in acute poisonings, so quantitative information on lethal dose in humans is sparse. The lethal doses ranged from 22 to $121 \mathrm{mg} \mathrm{As} / \mathrm{kg}$ in four cases where known amounts were ingested as a single bolus (Civantos et al. 1995; Hantson et al. 1996; Levin-Scherz et al. 1987; Quatrehomme et al. 1992). Two people in a family of eight died from ingestion of water containing about $110 \mathrm{ppm}$ of arsenic for a week (Armstrong et al. 1984). This corresponded to a dose of about $2 \mathrm{mg} \mathrm{As} / \mathrm{kg} / \mathrm{day}$. Based on a review of clinical reports in the older literature, Holland (1904) estimated the minimum lethal dose to be about $130 \mathrm{mg}$ (also about $2 \mathrm{mg} / \mathrm{kg}$ ). A similar estimate of 70-180 mg (about 1-3 mg/kg) was provided by Vallee et al. (1960). Death due to chronic arsenic exposure has been reported at lower concentrations. Five children between the ages of 2 and 7 years died from late sequelae of chronic arsenic poisoning after drinking contaminated water throughout their lives at estimated average doses of $0.05-0.1 \mathrm{mg} \mathrm{As} / \mathrm{kg} / \mathrm{day}$ (Zaldívar and Guillier 1977). A 22-year-old man with chronic arsenical dermatosis died from arsenicrelated effects after lifetime exposure to an estimated average dose of $0.014 \mathrm{mg} \mathrm{As} / \mathrm{kg} /$ day in the drinking water (Zaldívar et al. 1981). Systematic studies of lethality from chronic exposure attributable to increased risk of cardiovascular disease or cancer are discussed below in Sections 3.2.2.2 and 3.2.2.7, respectively. 
Table 3-3 Levels of Significant Exposure to Inorganic Arsenic - Oral

\begin{tabular}{|c|c|c|c|c|c|c|c|}
\hline \multirow[b]{2}{*}{$\begin{array}{l}\text { Key to } \\
\text { Figure }\end{array}$} & \multirow[b]{2}{*}{$\begin{array}{l}\text { Species } \\
\text { (Strain) }\end{array}$} & \multirow{2}{*}{$\begin{array}{l}\text { Exposurel } \\
\text { Durationl } \\
\text { Frequency } \\
\text { (Route) }\end{array}$} & \multirow[b]{2}{*}{ System } & \multirow[b]{2}{*}{$\begin{array}{l}\text { NOAEL } \\
\text { (mg/kg/day) }\end{array}$} & \multicolumn{2}{|c|}{ LOAEL } & \multirow[b]{2}{*}{$\begin{array}{l}\text { Reference } \\
\text { Chemical Form }\end{array}$} \\
\hline & & & & & $\begin{array}{r}\text { Less Serious } \\
(\mathrm{mg} / \mathrm{kg} / \mathrm{day}) \\
\end{array}$ & $\begin{array}{l}\text { Serious } \\
\text { (mg/kg/day) }\end{array}$ & \\
\hline \multicolumn{8}{|c|}{$\begin{array}{l}\text { ACUTE EXPOSURE } \\
\text { Death }\end{array}$} \\
\hline 1 & Human & $\begin{array}{l}1 \mathrm{wk} \\
(\mathrm{W})\end{array}$ & & & & 2 (death) & $\begin{array}{l}\text { Armstrong et al. } 1984 \\
\text { NS }\end{array}$ \\
\hline 2 & Human & $\begin{array}{l}\text { once } \\
\text { (IN) }\end{array}$ & & & & $121 \mathrm{M}$ (death) & $\begin{array}{l}\text { Civantos et al. } 1995 \\
\text { As }(+5)\end{array}$ \\
\hline 3 & Human & $\begin{array}{l}\text { once } \\
\text { (IN) }\end{array}$ & & & & $108 \mathrm{M}$ (death) & $\begin{array}{l}\text { Hantson et al. } 1996 \\
\text { As }(+3)\end{array}$ \\
\hline 4 & Human & $\begin{array}{l}\text { once } \\
(\mathrm{IN})\end{array}$ & & & & $22 \mathrm{M}$ (death) & $\begin{array}{l}\text { Levin-Scherz et al. } 1987 \\
\text { As }(+3)\end{array}$ \\
\hline 5 & Human & $\begin{array}{l}\text { once } \\
\text { (IN) }\end{array}$ & & & & $93 \mathrm{M}$ (death) & $\begin{array}{l}\text { Quatrehomme et al. } 1992 \\
\text { As }(+3)\end{array}$ \\
\hline 6 & $\begin{array}{l}\text { Rat } \\
\text { (wild Norway) }\end{array}$ & $\begin{array}{l}\text { once } \\
\text { y) }(G)\end{array}$ & & & & 104 (LD50) & $\begin{array}{l}\text { Dieke and Richter } 1946 \\
\text { As }(+3)\end{array}$ \\
\hline 7 & $\begin{array}{l}\text { Rat } \\
\text { (Sherman) }\end{array}$ & $\begin{array}{l}\text { once } \\
(G)\end{array}$ & & & & 112 F (LD50) & $\begin{array}{l}\text { Gaines } 1960 \\
\text { As }(+5) \text { calcium arsenate }\end{array}$ \\
\hline 8 & $\begin{array}{l}\text { Rat } \\
\text { (Sherman) }\end{array}$ & $\begin{array}{l}\text { once } \\
\text { (G) }\end{array}$ & & & & $44 \mathrm{~F}$ (LD50) & $\begin{array}{l}\text { Gaines } 1960 \\
\text { As(+3) }\end{array}$ \\
\hline 9 & $\begin{array}{l}\text { Rat } \\
\text { (Sherman) }\end{array}$ & $\begin{array}{l}\text { once } \\
(G)\end{array}$ & & & & $175 \mathrm{~F}$ (LD50) & $\begin{array}{l}\text { Gaines } 1960 \\
\text { As }(+5) \text { lead arsenate }\end{array}$ \\
\hline 10 & $\begin{array}{l}\text { Rat } \\
\text { (Sprague- } \\
\text { Dawley) }\end{array}$ & $\begin{array}{l}\text { once } \\
(\mathrm{GW})\end{array}$ & & & & $15 \mathrm{M}$ (LD50) & $\begin{array}{l}\text { Harrisson et al. } 1958 \\
\text { As }(+3)\end{array}$ \\
\hline
\end{tabular}

Comments 
Table 3-3 Levels of Significant Exposure to Inorganic Arsenic - Oral

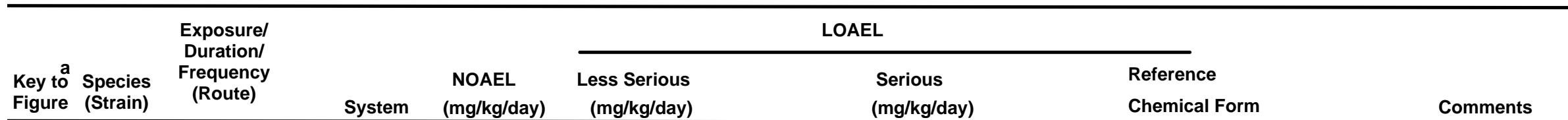

11 Rat once

(Sprague- (F)

Dawley)

12 Rat

$(\mathrm{GW})$

13 Mouse once

(Swiss- (GW)

Webster)

$14 \quad$ Mouse

15 Mouse once

(Dba2) (GW)

16 Mouse once

$(\mathrm{C} 3 \mathrm{H}) \quad(\mathrm{GW})$

17 Mouse once

$(\mathrm{ddY}) \quad(\mathrm{GW})$

$18 \quad$ Rabbit Gd 6-18

(New $\quad 1 \mathrm{x} / \mathrm{d}$

Zealand) (GW)

145 M (LD50) $\quad \begin{aligned} & \text { Harrisson et al. } 1958 \\ & \text { As }(+3)\end{aligned}$

$23 \mathrm{~F}(7 / 25$ dams died $)$

Stump et al. 1999

As(+3)

$39 \mathrm{M}(\mathrm{LD} 50)$

26 M (LD50)

32 M (LD50)

26 M (LD50)

26 M (LD50)

$1.49 \mathrm{~F}$ (7/20 dams died $)$
Harrisson et al. 1958

As(+3)

Harrisson et al. 1958

As(+3)

Harrisson et al. 1958

As(+3)

Harrisson et al. 1958

As(+3)

Kaise et al. 1985

As(+3)

Nemec et al. 1998

As(+5) 
Table 3-3 Levels of Significant Exposure to Inorganic Arsenic - Oral

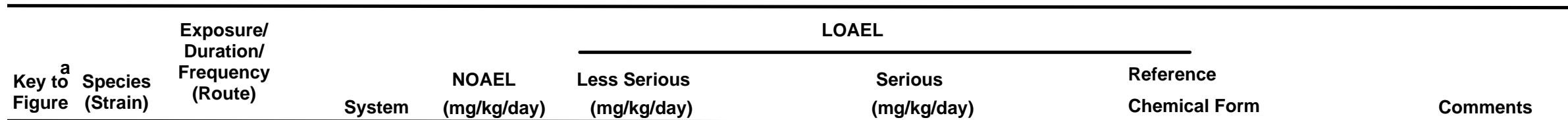

\section{Systemic}

19 Human

$1 \mathrm{wk}$

(W)

Gastro
Hemato
Hepatic
Renal
Ocular

Resp

once

(IN)

Cardio

Gastro
0.2 (vomiting, diarrhea, abdominal pain)

0.2 (pancytopenia leukopenia)

0.4 (hepatitis)

0.2 (nephropathy)

$0.2 \quad$ (periorbital swelling) the GI tract)
$2 \mathrm{M}$ (diffuse inflammation of Armstrong et al. 1984

NS

$121 \mathrm{M}$ (respiratory distress, lung Civantos et al. 1995 hemmorhage and As(+5) edema)

$121 \mathrm{M}$ (hypotension, ventricular fibrillation, cardiac arrest)

$121 \mathrm{M}$ (ulceration of upper gastrointestinal tract) 
Table 3-3 Levels of Significant Exposure to Inorganic Arsenic - Oral

\begin{tabular}{|c|c|c|c|c|}
\hline $\begin{array}{l}\text { Key to } \\
\text { Figure }\end{array}$ & $\begin{array}{l}\text { Species } \\
\text { (Strain) }\end{array}$ & $\begin{array}{l}\text { Exposurel } \\
\text { Duration/ } \\
\text { Frequency } \\
\text { (Route) }\end{array}$ & System & $\begin{array}{l}\text { NOAEL } \\
\text { (mg/kg/day }\end{array}$ \\
\hline \multirow[t]{5}{*}{21} & Human & $\begin{array}{l}\text { once } \\
\text { (IN) }\end{array}$ & Cardio & \\
\hline & & & Gastro & \\
\hline & & & Hemato & $19 \mathrm{~F}$ \\
\hline & & & Hepatic & $19 \mathrm{~F}$ \\
\hline & & & Renal & $19 \mathrm{~F}$ \\
\hline
\end{tabular}

Human once Resp

(NS)

Resp

Cardio

$8 \mathrm{M}$ (hemorrhagic bronchitis, pulmonary edema)

Fincher and Koerker 1987

As $(+3)$

$8 \mathrm{M}$ (hypotension,

tachycardia, massive

cardiomegaly)

Reference

Chemical Form

Comments

(a)

Gastro

Hemato

Musc/skel

Renal

Dermal

Gastro

1 or $2 \times$

(W)

0.05

(occasional nausea,

diarrhea, and abdominal cramps)
$8 \mathrm{M}$ (gastrointestinal bleeding)

$8 \mathrm{M}$ (hemolysis)

$8 \mathrm{M}$ (marked atrophy of distal muscle groups)

$8 \mathrm{M}$ (acute renal failure)

$8 \mathrm{M}$ (truncal macular rash)
Franzblau and Lilis 1989

As $(+3)$ As (+5) 
Table 3-3 Levels of Significant Exposure to Inorganic Arsenic - Oral

\begin{tabular}{|c|c|c|c|c|c|c|c|}
\hline \multirow[b]{2}{*}{$\begin{array}{l}\text { Key to } \\
\text { Figure }\end{array}$} & \multirow[b]{2}{*}{$\begin{array}{l}\text { Species } \\
\text { (Strain) }\end{array}$} & \multirow{2}{*}{$\begin{array}{c}\text { Exposurel } \\
\text { Duration/ } \\
\text { Frequency } \\
\text { (Route) }\end{array}$} & \multirow[b]{2}{*}{ System } & \multirow[b]{2}{*}{$\begin{array}{c}\text { NOAEL } \\
\text { (mg/kg/day) }\end{array}$} & \multicolumn{2}{|c|}{ LOAEL } & \multirow[b]{2}{*}{$\begin{array}{l}\text { Reference } \\
\text { Chemical Form }\end{array}$} \\
\hline & & & & & $\begin{array}{r}\text { Less Serious } \\
\text { (mg/kg/day) }\end{array}$ & $\begin{array}{l}\text { Serious } \\
\text { (mg/kg/day) }\end{array}$ & \\
\hline \multirow[t]{3}{*}{24} & Human & $\begin{array}{l}\text { once } \\
\text { (W) }\end{array}$ & Gastro & & & $120 \mathrm{M}$ (vomiting and diarrhea) & $\begin{array}{l}\text { Goebel et al. } 1990 \\
\text { NS }\end{array}$ \\
\hline & & & Renal & & & $120 \mathrm{M}$ (anuria) & \\
\hline & & & Dermal & & 120 M (hyperkeratosis) & & \\
\hline \multirow[t]{3}{*}{25} & Human & $\begin{array}{l}\text { once } \\
(\mathrm{IN})\end{array}$ & Gastro & & $2 \mathrm{~F}$ (vomiting) & & $\begin{array}{l}\text { Hantson et al. } 1996 \\
\text { As }(+3)\end{array}$ \\
\hline & & & Hepatic & & $\begin{array}{l}2 \mathrm{~F} \text { (slight increase in serum } \\
\text { bilirubin) }\end{array}$ & & \\
\hline & & & Renal & & $\begin{array}{l}2 \mathrm{~F} \text { (altered renal function } \\
\text { tests) }\end{array}$ & & \\
\hline \multirow[t]{4}{*}{26} & Human & $\begin{array}{l}\text { once } \\
(\mathrm{IN})\end{array}$ & Gastro & & & $\begin{array}{l}13 \mathrm{M} \text { (frequent vomiting, } \\
\text { diarrhea) }\end{array}$ & $\begin{array}{l}\text { Kamijo et al. } 1998 \\
\text { As }(+3)\end{array}$ \\
\hline & & & Hepatic & & & $\begin{array}{l}13 \mathrm{M} \text { (large increase in serum } \\
\text { bilirubin, ALT, AST, LDH) }\end{array}$ & \\
\hline & & & Dermal & & $13 \mathrm{M}$ (erythematous eruption) & & \\
\hline & & & Ocular & & $13 \mathrm{M}$ (constricted vision) & & \\
\hline
\end{tabular}


Table 3-3 Levels of Significant Exposure to Inorganic Arsenic - Oral

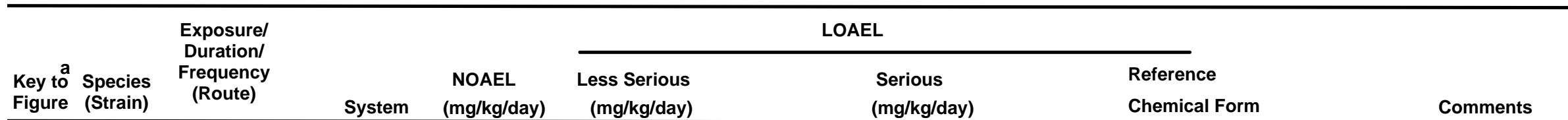
Human once

Resp

(IN)

Resp

Cardio

Gastro

Hepatic

Renal

once

pregnancy wk Cardio

30

(IN)

Gastro

Hemato

$6 \mathrm{~F}$ (high leukocyte count, low hematocrit)

Renal
$22 \mathrm{M}$ (tachypnea, respiratory failure)

Levin-Scherz et al. 1987

As(+3)

$22 \mathrm{M}$ (cyanosis, hypotension, tachycardia, ventricular fibrillation)

$22 \mathrm{M}$ (abdominal pain, nausea, diarrhea, massive

vomiting, dysphagia,

hemorrhage)

$22 \mathrm{M}$ (large increase in serum creatinine and BUN

indicating acute renal

failure)

$6 \mathrm{~F}$ (hypotension, rapid

Lugo et al. 1969 pulse)

As(+3)

$6 \mathrm{~F}$ (abdominal pain vomiting)

$6 \mathrm{~F}$ (acute renal failure) 
Table 3-3 Levels of Significant Exposure to Inorganic Arsenic - Oral

\begin{tabular}{|c|c|c|c|c|c|c|c|c|}
\hline \multirow[b]{2}{*}{$\begin{array}{l}\text { Key to } \\
\text { Figure }\end{array}$} & \multirow[b]{2}{*}{$\begin{array}{l}\text { Species } \\
\text { (Strain) }\end{array}$} & \multirow{2}{*}{$\begin{array}{l}\text { Exposurel } \\
\text { Durationl } \\
\text { Frequency } \\
\text { (Route) }\end{array}$} & \multicolumn{5}{|c|}{ LOAEL } & \\
\hline & & & System & $\begin{array}{l}\text { NOAEL } \\
\text { (mg/kg/day) }\end{array}$ & $\begin{array}{r}\text { Less Serious } \\
\text { (mg/kg/day) }\end{array}$ & $\begin{array}{l}\text { Serious } \\
\text { (mg/kg/day) }\end{array}$ & $\begin{array}{l}\text { Reference } \\
\text { Chemical Form }\end{array}$ & Comments \\
\hline
\end{tabular}

29 Human 2-3 wk

(F)

Resp

Cardio

Gastro

Hemato

Musc/skel

Hepatic

Renal 0.05

Dermal

Ocular
0.05 (sore throat, rhinorrhea,

cough, sputum)
Mizuta et al. 1956

As(+5)
0.05 (abnormal

electrocardiogram)

$0.05^{\mathrm{b}}$ (nausea, vomiting,

diarrhea, occult blood in

feces and gastric and

duodenal juice) 
Table 3-3 Levels of Significant Exposure to Inorganic Arsenic - Oral

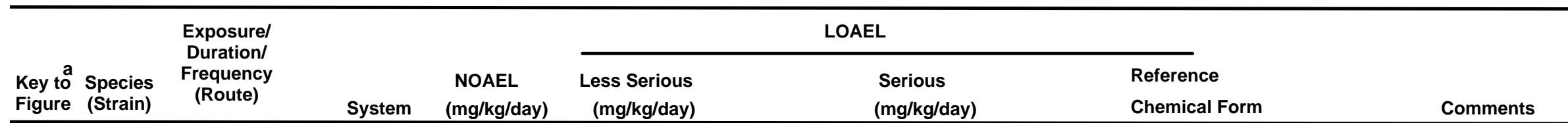

30 Human once

(IN)

\begin{tabular}{|c|c|c|}
\hline Resp & $11 \mathrm{M}$ & \\
\hline Cardio & $11 \mathrm{M}$ & \\
\hline Gastro & & \\
\hline Hemato & $43 \mathrm{M}$ & \\
\hline Renal & & $\begin{array}{l}11 \mathrm{M} \text { (increased serum } \\
\text { creatinine) }\end{array}$ \\
\hline
\end{tabular}

31

$\begin{array}{ll}\text { Human } & \begin{array}{l}\text { once } \\ \text { (IN) }\end{array} \\ & \text { Resp } \\ \text { Hepatic } \\ \\ \text { Renal } \\ \text { Dermal }\end{array}$

Dermal
$43 \mathrm{M}$ (shortness of breath, decreased oxygen saturation)

Moore et al. 1994a

As(+3)

$43 \mathrm{M}$ (hypotension, asystolic cardiac arrest)

$11 \mathrm{M}$ (profuse diarrhea and vomiting, severe abdominal pain)

$43 \mathrm{M}$ (acute renal failure)

$93 \mathrm{M}$ (pulmonary edema)

$93 \mathrm{M}$ (ulcero-necrotic hemorrhagic gastritis)

$93 \mathrm{M}$ (hepatomegaly, diffuse fatty degeneration)

$93 \mathrm{M}$ (glomerular congestion)

$93 \mathrm{M}$ (dermoepidermic separation)

\section{Quatrehomme et al. 1992} As(+3) 
Table 3-3 Levels of Significant Exposure to Inorganic Arsenic - Oral

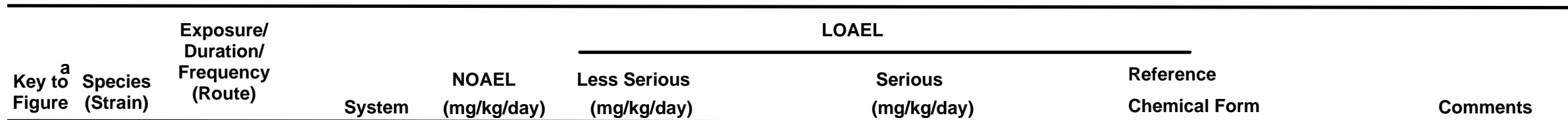

$32 \quad$ Monkey $13 d$

(Rhesus) $1 \mathrm{x} / \mathrm{d}$

(IN)

Gastro 3

$\begin{array}{llll}\text { Hepatic } & 3 & 6 & \begin{array}{l}\text { (decreased liver } \\ \text { glycogen, vacuolation of } \\ \text { hepatocytes) }\end{array} \\ \text { Renal } & 3 & 6 & \begin{array}{l}\text { (dilation of proximal } \\ \text { tubules) }\end{array}\end{array}$

33 Rat

4-14 d

(Wistar- $\quad 5 \mathrm{~d} / \mathrm{wk}$

Barby) $\quad 1 \mathrm{x} / \mathrm{G}$

\section{Cardio}

$2 \mathrm{~F}$

$11 \mathrm{~F}$ (decreased

asoreactivity

Gastro

$2 \mathrm{~F}$

$34 \quad$ Rat

$\begin{array}{lll}\text { Rat } & 2 \mathrm{x} & \text { Resp } \\ \text { (Sprague- } & (\mathrm{GW}) & \end{array}$

Hepatic
ornithine decarboxylase
and heme oxygenase
activity in liver)

$0.9 \mathrm{~F}$ (slight increased

Dermal

$14 \mathrm{~F}$

35 Rat

$\begin{array}{lll}\text { Rat } & 2 \mathrm{x} & \text { Hepatic } \\ \begin{array}{l}\text { (Sprague- } \\ \text { Dawley) }\end{array} & (\mathrm{GW}) & \end{array}$
$\begin{array}{lll}\text { (vomiting, unformed } & \text { Heywood and Sortwell } 1979 \\ \text { stool, "loss of condition") } & \text { As }(+5)\end{array}$
Brown and Kitchin 1996

As(+3)
Bekemeier and Hirschelmann

1989

As $(+3)$

$11 \mathrm{~F}$ (diarrhea, bloody stools)

Brown et al. 1997c

As(+5) 
Table 3-3 Levels of Significant Exposure to Inorganic Arsenic - Oral

\begin{tabular}{|c|c|c|c|c|c|c|c|c|}
\hline \multirow[b]{2}{*}{$\begin{array}{l}\text { Key to } \\
\text { Figure }\end{array}$} & \multirow[b]{2}{*}{$\begin{array}{l}\text { Species } \\
\text { (Strain) }\end{array}$} & \multirow{2}{*}{$\begin{array}{l}\text { Exposurel } \\
\text { Duration/ } \\
\text { Frequency } \\
\text { (Route) }\end{array}$} & \multicolumn{5}{|c|}{ LOAEL } & \multirow[b]{2}{*}{ Comments } \\
\hline & & & System & $\begin{array}{l}\text { NOAEL } \\
\text { (mg/kg/day) }\end{array}$ & $\begin{array}{r}\text { Less Serious } \\
\text { (mg/kg/day) }\end{array}$ & $\begin{array}{l}\text { Serious } \\
\text { (mg/kg/day) }\end{array}$ & $\begin{array}{l}\text { Reference } \\
\text { Chemical Form }\end{array}$ & \\
\hline
\end{tabular}

36 Rat

$\begin{array}{ll}\text { Rat } & 1 \mathrm{x} / \mathrm{d} \\ \text { (Sprague- } & 15 \mathrm{~d} \\ \text { Dawley) } & (\mathrm{G})\end{array}$

$\begin{array}{ll}\text { Rat } & \text { once } \\ \text { (CD) } & \text { Gd } 9\end{array}$

(GW)

38

Mouse $\quad$ Gd 6-15

(CD-1)

Gd 6-15
$1 \times / d$
$(G W)$

39

$\begin{array}{ll}\text { Mouse } & 1 \text { or } 4 \mathrm{~d} \\ \text { (B6C3F1) } & 1 \mathrm{x} / \mathrm{d} \\ & (\mathrm{GW})\end{array}$

(GW)

$40 \quad$ Gn Pig

$1 \mathrm{x} / \mathrm{d}$

$8 d$
$(G)$

41

$\begin{array}{ll}\text { Rabbit } & \text { Gd 6-18 } \\ \text { (New } & 1 \times / d \\ \text { Zealand) } & (G W)\end{array}$

Neurological

42 Human 1 wk

(W)
Bd Wt

$10 \mathrm{M}$

$20 \mathrm{M}(20-25 \%$ decreased body

weight)

$\mathrm{Bd} W \mathrm{t}$

$15 \mathrm{~F}$

$23 \mathrm{~F}$ (decreased body weight

gain)

$12 \mathrm{~F} \quad 24 \mathrm{~F}$ (decreased body weight gain during gestation)

Hemato

$3 \mathrm{M}$

$6 \mathrm{M}$ (decreased

polychromatic
erythrocytes in bone

marrow)

3.8 M (prolongation of QT

interval)

$0.37 \mathrm{~F}$

$1.49 \mathrm{~F}$ (loss of body weight

during treatment during

gestation)
Rodriguez et al. 2001

As(+3)

Stump et al. 1999

As(+3)

Nemec et al. 1998

As(+5)

Tice et al. 1997

As(+3)

Chiang et al. 2002

As2O3

Nemec et al. 1998

As(+5)

2 (encephalopathy, peripheral neuropathy)
Armstrong et al. 1984

NS 
Table 3-3 Levels of Significant Exposure to Inorganic Arsenic - Oral

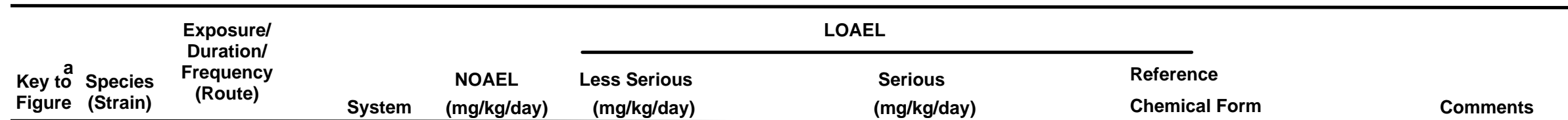

43 Human once

(IN)

44 Human once

(IN)

45 Human once

(NS)

46 Human once

(W)

47 Human once

(IN)

48 Human once

(IN)

49 Human

once

$(\mathrm{IN})$

50 Human 2-3 wk

(F)

51 Human once

(IN)
121 M (confusion, brain edema) Civantos et al. 1995

As(+5)

$19 \mathrm{~F}$ (lethargy)

Cullen et al. 1995

As (+5)

$8 \mathrm{M}$ (severe, persistent encephalopathy and

Fincher and Koerker 1987

As(+3)

peripheral neuropathy)

120 M (severe polyneuropathy) Goebel et al. 1990 NS

216 M (peripheral neuropathy) Hantson et al. 1996 As(+3)

$13 \mathrm{M}$ (peripheral neuropathy) Kamijo et al. 1998 As(+3)

22 M (agitation, disorientation, Levin-Scherz et al. 1987 paranoia, violent $\quad \mathrm{As}(+3)$
reactions)

$$
\text { reactions) }
$$

0.05 (hypesthesia in legs,

Mizuta et al. 1956 abnormal patellar reflex) As(+5) 
Table 3-3 Levels of Significant Exposure to Inorganic Arsenic - Oral

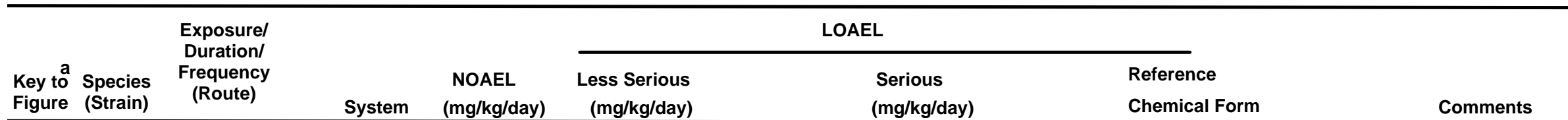

52 Human once

(IN)

$53 \quad$ Monkey $13 \mathrm{~d}$ (Rhesus) $1 \mathrm{x} / \mathrm{d}$

(IN)

$54 \quad$ Rat $1 \times / d$

(Sprague- $\quad 15 \mathrm{~d}$

Dawley) (G)

55 Rabbit Gd 6-18

(New $\quad 1 \mathrm{x} / \mathrm{d}$

Zealand $\quad(\mathrm{GW})$

Developmental

56 Human

once

pregnancy wk

30

(IN)

$57 \quad$ Rat

(CD) Gd 9

(GW)
3 locomotor activity)

$0.37 \mathrm{~F}$

hemorrhage that may (severe pulmonary

have contributed to death

in premature neonate)

$\begin{array}{cl}93 \text { M (encephalopathy) } & \begin{array}{l}\text { Quatrehomme et al. } 1992 \\ \text { As }(+3)\end{array} \\ 6 \quad \begin{array}{l}\text { (marked salivation, } \\ \text { uncontrolled head } \\ \text { shaking) }\end{array} & \text { Heywood and Sortwell } 1979\end{array}$

Rodriguez et al. 2001

As(+3)

Nemec et al. 1998

As(+5)

Lugo et al. 1969

As(+3)

tump et al. 1999

post-implantation loss
and decreased viable

fetuses)

$\mathrm{As}(+3)$ 
Table 3-3 Levels of Significant Exposure to Inorganic Arsenic - Oral

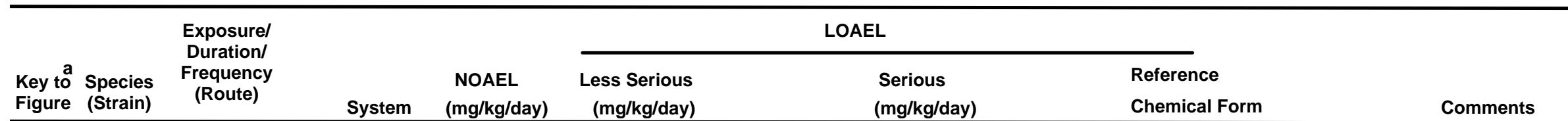

\section{$58 \quad$ Mouse once}

(CD-1) Gd 8-15

$(\mathrm{GW})$

59 Mouse once

(CD-1) Gd 7-15

(GW)

60

$\begin{array}{ll}\text { Mouse } & \text { Gd 6-15 } \\ \text { (CD-1) } & 1 \times / d\end{array}$

(GW)

61 Hamster once

(Lak:LVG Gd 8-12

[SYR]) (GW)

62 Rabbit Gd 6-18

(New $1 \mathrm{x} / \mathrm{d}$

Zealand) (GW)
11
23 (increased fetal mortality, Baxley et al. 1981 exencephaly) As(+3)

48 (increased fetal death, Hood et al. 1978 decreased fetal weight, As $(+5)$ gross and skeletal

malformations)

24 (increased resorptions Nemec et al. 1998 per litter, decreased live $\mathrm{As}(+5)$

fetuses per litter,

decreased mean fetal

weight)

14 (increased fetal mortality, Hood and Harrison 1982 decreased fetal weight) $\mathrm{As}(+3)$

1.49 (increased resorptions Nemec et al. 1998 per litter, decreased live $\mathrm{As}(+5)$ fetuses per litter) 
Table 3-3 Levels of Significant Exposure to Inorganic Arsenic - Oral

(continued)

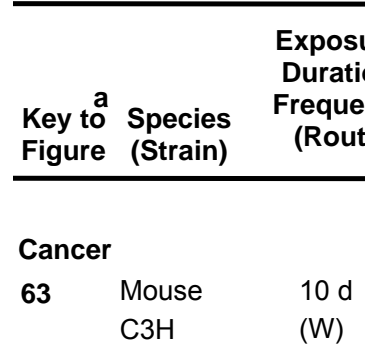

LOAEL

Duration/

(Route)

NOAEL

Less Serious

System

(mg/kg/day)

(mg/kg/day)

(mg/kg/day)

Reference

Chemical Form

Comments

\section{INTERMEDIATE EXPOSURE}

Systemic

64 Human 3 mo

(W)

Gastro

Hemato

Hepatic

Dermal

Ocular

65 Human

$0.5-14 \mathrm{yr}$

(W)

Dermal

65 (W) Dermal

9.55 M (CEL: liver and adrenal tumors)

Waalkes et al. 2003

As(+3)

19.13 F (CEL: ovarian and lung tumors)

0.1 (severe nausea diarrhea, pain, cramps, vomiting, traces of blood

Franzblau and Lilis 1989 in stool)

0.1 (anemia, leukopenia)

$0.1 \quad$ (large increased AST and $\mathrm{ALT}$ ) $\mathrm{As}(+3) \mathrm{As}(+5)$
0.1 (diffuse erythematous and scaly rash)

0.1 (swelling and irritation of the eyes, impaired
peripheral vision)
0.05

(hyperpigmentation with keratosis, possibly pre-cancerous)
Huang et al. 1985 NS 
Table 3-3 Levels of Significant Exposure to Inorganic Arsenic - Oral

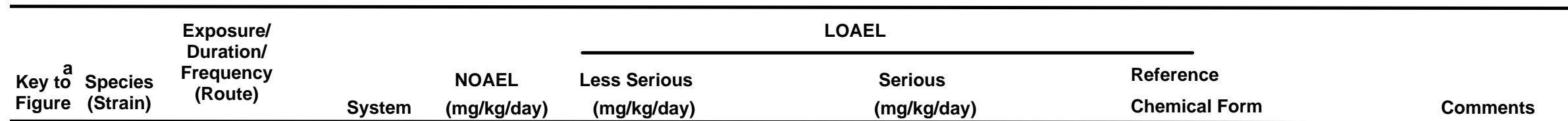

Human 4 mo Gastro

(W)

Gastro

Wagner et al. 1979

diarrhea)

NS

Hemato

$0.06 \mathrm{~F}$ (anemia, leukopenia,

erythroid hyperplasia of

bone marrow)

Dermal

$\mathrm{Bd} \mathrm{Wt}$

$67 \quad$ Rat

$\begin{array}{ll}\text { Rat } & 4 \mathrm{wk} \\ \text { Wistar- } & 5 \mathrm{~d} / \mathrm{wk}\end{array}$

Barby) $\quad 1 \mathrm{x} / \mathrm{d}$

(GW)

$68 \quad$ Rat

$\begin{array}{lll}\text { Rat } & 6 w k & \text { Renal } \\ \text { (Sprague- } & (\mathrm{W}) & \end{array}$

Dawley)

$7 \mathrm{M}$ (increased relative kidney weight, impaired renal

mitochondrial respiration,

ultrastructural changes in

proximal tubule)

$0.06 \mathrm{~F}$ (persistent extensive hyperkeratosis of palms and soles)

$0.06 \mathrm{~F}$ (40 lb weight loss)
Bekemeier and Hirschelmann

As(+3)

Brown et al. 1976

As(+5)

$\mathrm{Bd} \mathrm{Wt}$ 
Table 3-3 Levels of Significant Exposure to Inorganic Arsenic - Oral

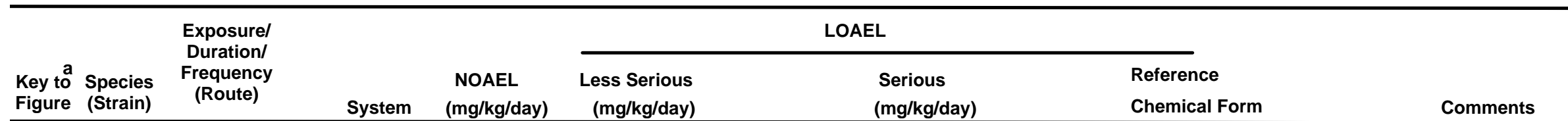

$69 \quad$ Rat $\quad 1 \times / d$ (Wistar) 28

(G)

$\mathrm{Bd} \mathrm{Wt}$

$0.14 \mathrm{~F}$

wk

$\begin{array}{ll}\text { Rat } & 6 w k \\ (C D) & (W)\end{array}$

(W) Hepatic

Hepatic

$3 \mathrm{M}$

M (ultrastructural changes

in hepatocytes, impaired

liver mitochondrial

respiration)

$\mathrm{Bd} \mathrm{Wt} \quad 6 \mathrm{M}$

71

$\begin{array}{ll}\text { Rat } & 14 \mathrm{pmd}-\mathrm{Gd} 19 \text { Gastro } \\ \text { (CD) } & 7 \mathrm{~d} / \mathrm{wk} \\ & 6 \mathrm{hr} / \mathrm{d} \\ & (\mathrm{GW})\end{array}$

$\begin{array}{lll}\text { Hepatic } & 2 \mathrm{~F} & 4 \mathrm{~F} \text { (increased liver weight) } \\ \text { Renal } & 4 \mathrm{~F} & 8 \mathrm{~F} \text { (increased kidney weight) } \\ \text { Bd Wt } & 4 \mathrm{~F} & 8 \mathrm{~F} \text { (decreased body weight } \\ & & \text { gain) }\end{array}$

$\begin{array}{lll}\text { Rat } & 16 w k & \text { Hemato } \\ \text { (NS) } & \text { (W) }\end{array}$

Hepatic
$6 \mathrm{M}$

$4 \mathrm{~F}$

gain)

$0.92 \mathrm{M}$ (decreased erythrocyte and leukocyte numbers)
Chattopadhyay et al. 2001

As(+3)

Fowler et al. 1977

As(+5)

$12 \mathrm{M}$ (final body weight $28 \%$ lower than controls)
$8 \mathrm{~F}$ (stomach adhesions, Holson et al. 2000
eroded luminal
epithelium in the
As $(+3)$

stomach) 
Table 3-3 Levels of Significant Exposure to Inorganic Arsenic - Oral

\begin{tabular}{|c|c|c|c|c|c|c|c|c|}
\hline \multirow[b]{2}{*}{$\begin{array}{l}\text { Key to } \\
\text { Figure }\end{array}$} & \multirow[b]{2}{*}{$\begin{array}{l}\text { Species } \\
\text { (Strain) }\end{array}$} & \multirow{2}{*}{$\begin{array}{l}\text { Exposurel } \\
\text { Durationl } \\
\text { Frequency } \\
\text { (Route) }\end{array}$} & \multicolumn{5}{|c|}{ LOAEL } & \multirow[b]{2}{*}{ Comments } \\
\hline & & & System & $\begin{array}{c}\text { NOAEL } \\
\text { (mg/kg/day) }\end{array}$ & $\begin{array}{r}\text { Less Serious } \\
(\mathrm{mg} / \mathrm{kg} / \text { day })\end{array}$ & $\begin{array}{l}\text { Serious } \\
\text { (mg/kg/day) }\end{array}$ & $\begin{array}{l}\text { Reference } \\
\text { Chemical Form }\end{array}$ & \\
\hline
\end{tabular}

73 Rat

$\begin{array}{ll}\text { Rat } & 4 \mathrm{wk} \\ \text { (Sprague- } & \text { (W) } \\ \text { Dawley) } & \end{array}$

74

$\begin{array}{ll}\text { Rat } & 1 \mathrm{x} / \mathrm{d} \\ \text { (NS) } & 30 \mathrm{~d}\end{array}$

(G)

75

$\begin{array}{ll}\text { Rat } & 1 \mathrm{x} / \mathrm{d} \\ \text { (Wistar) } & 5 \mathrm{~d} / \mathrm{wk} \\ & 12 \mathrm{wk}\end{array}$

(G)

76

Mouse $\quad 6 \mathrm{wk}$

(C57BL)

(W)

Hepatic

$\mathrm{Bd} \mathrm{Wt}$
$5 \mathrm{M}$
$10 \mathrm{M}$ (ultrastructural changes in hepatocytes, impaired liver mitochondrial
respiration)

0.3 (increased platelet

aggregation)
2.3 M (decreased islet cells in
pancreas, increased

catalase)

9.5 M $19 \mathrm{M}(\sim 17 \%$ decreased body weight gain)

$\mathrm{M}$ (decreased body weight gain)
Lee et al. 2002

As $(+3)$

Mukherjee et al. 2003

As2O3

Schulz et al. 2002

As(+3) 
Table 3-3 Levels of Significant Exposure to Inorganic Arsenic - Oral

\begin{tabular}{|c|c|c|c|c|c|c|c|c|}
\hline \multirow[b]{2}{*}{$\begin{array}{l}\text { Key to } \\
\text { Figure }\end{array}$} & \multirow[b]{2}{*}{$\begin{array}{l}\text { Species } \\
\text { (Strain) }\end{array}$} & \multirow{2}{*}{$\begin{array}{l}\text { Exposurel } \\
\text { Duration/ } \\
\text { Frequency } \\
\text { (Route) }\end{array}$} & \multicolumn{5}{|c|}{ LOAEL } & \multirow[b]{2}{*}{ Comments } \\
\hline & & & System & $\begin{array}{l}\text { NOAEL } \\
\text { (mg/kg/day) }\end{array}$ & $\begin{array}{r}\text { Less Serious } \\
\text { (mg/kg/day) }\end{array}$ & $\begin{array}{l}\text { Serious } \\
\text { (mg/kg/day) }\end{array}$ & $\begin{array}{l}\text { Reference } \\
\text { Chemical Form }\end{array}$ & \\
\hline
\end{tabular}

$77 \quad$ Mouse $14 \mathrm{wk}$

(C57BL/6 B6) (W)

78
(NS)
16 wk
(W)

Hemato

$\begin{array}{ll}\text { Hepatic } & 25 \mathrm{M} \\ \text { Renal } & 25 \mathrm{M}\end{array}$

Hepatic

$0.69 \mathrm{M}$ (increased ALAS activity)

79

$\begin{array}{llll}\begin{array}{l}\text { Dog } \\ \text { (Beagle) }\end{array} & \begin{array}{l}26 \mathrm{wk} \\ \text { ad lib } \\ (\mathrm{F})\end{array} & \text { Hemato } & 1.9 \mathrm{~F} \\ & \text { Hepatic } & \\ & & \\ & \text { Renal } & 1.9 \mathrm{~F} \\ & \text { Bd Wt } & 0.8 \mathrm{~F}\end{array}$

mmunol Lymphoret

80 Mouse $14 \mathrm{wk}$

(C57BL/6 B6) (W)

Neurological

81 Human 3 mo

(W)
$25 \mathrm{M}$ number and leukocyte

number, decreased

ALAD levels)

Kerkvliet et al. 1980

As(+5)

Kannan et al. 2001

As(+3)

Neiger and Osweiler 1989

As(+3)

0.8 F (mild increased serum ALT/AST)

$1.9 \mathrm{~F}(25 \%$ decrease in body weight) gain)

Kerkvliet et al. 1980

As(+5)

0.1 (paresthesia of hands and feet; confusion,

disorientation and mental

Franzblau and Lilis 1989

sluggishness 
Table 3-3 Levels of Significant Exposure to Inorganic Arsenic - Oral

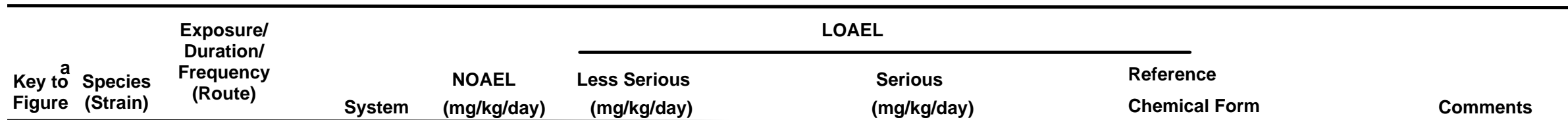

82 Human 4 mo

(W)

83 Rat 16 wk

(NS) (W)

$84 \quad$ Rat

$\begin{array}{ll}\text { (Wistar) } & 5 \mathrm{~d} / \mathrm{wk} \\ & 12 \mathrm{wk}\end{array}$

(G)

85

Gn Pig $\quad 16$ wk

(NS) (W)

Reproductive

86 Rat

(Wistar) $28 \mathrm{~d}$

(G)

87

$\begin{array}{ll}\text { Rat } & 14 \mathrm{pmd}-\mathrm{Gd} 19 \\ \text { (CD) } & 7 \mathrm{~d} / \mathrm{wk} \\ & 6 \mathrm{hr} / \mathrm{d} \\ & (\mathrm{GW})\end{array}$

88

Mouse 3 gen

(CD) (W)
$0.92 \mathrm{M}$

neurotransmitter levels

( paresthesia)

NS

Kannan et al. 2001

As(+3)

Schulz et al. 2002

As $(+3)$

(changes in brain
neurotransmitter levels)

$0.14 \mathrm{~F}$ (changes in uterine and

ovarian weights

decreased estradiol)

$8 \mathrm{~F}$

1 (decreased litter size)
Kannan et al. 2001

As(+3)

Chattopadhyay et al. 2001

As(+3)

Holson et al. 2000

As(+3)

Schroeder and Mitchener 1971

As(+3) 
Table 3-3 Levels of Significant Exposure to Inorganic Arsenic - Oral

\begin{tabular}{|c|c|c|}
\hline $\begin{array}{l}\text { Key to } \\
\text { Figure }\end{array}$ & $\begin{array}{l}\text { Species } \\
\text { (Strain) }\end{array}$ & $\begin{array}{l}\text { Exposurel } \\
\text { Durationl } \\
\text { Frequency } \\
\text { (Route) }\end{array}$ \\
\hline \multicolumn{3}{|c|}{ Developmental } \\
\hline 89 & $\begin{array}{l}\text { Rat } \\
\text { (CD) }\end{array}$ & $\begin{array}{l}14 \mathrm{pmd}-\mathrm{Gd} 19 \\
7 \mathrm{~d} / \mathrm{wk} \\
6 \mathrm{hr} / \mathrm{d} \\
(\mathrm{GW})\end{array}$ \\
\hline
\end{tabular}

LOAEL

$\begin{array}{lll}90 & \begin{array}{l}\text { Rat } \\ \text { (Sprague- }\end{array} & \begin{array}{l}\text { Gd 15 or pnd 1- } \\ \text { Dawley) }\end{array} \\ & \text { (W) }\end{array}$

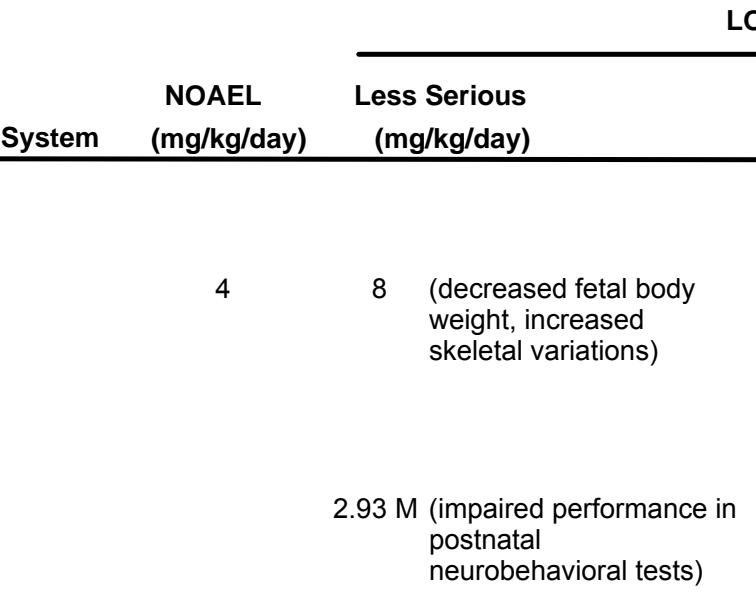

91 Mouse 3 gen
(CD)
(W)

\section{CHRONIC EXPOSURE}

Death

92 Human $\begin{aligned} & 2-7 \mathrm{yr} \\ & \text { children }\end{aligned}$

(W)

93 Human $22 \mathrm{yr}$

(W)

$94 \quad$ Monkey $1 \mathrm{yr}$

(Rhesus) (IN)

95 Rat $27 \mathrm{mo}$

(Wistar) (F)

$96 \quad$ Mouse $2 \mathrm{yr}$

(CD) (W)

Reference

Chemical Form

Comments
Holson et al. 2000

As(+3)

Rodriguez et al. 2002

As(+3)

1 (decreased litter size)

Schroeder and Mitchener 1971 As(+3)

0.05 (death)

Zaldivar and Guillier 1977

NS

Zaldivar et al. 1981

NS

Cause of death was liver tumor.
$0.014 \mathrm{M}$ (death)

3 (2/7 died)

30 (increased mortality) 1 (increased mortality,
Heywood and Sortwell 1979

As(+5)

Kroes et al. 1974

As $(+5)$ lead arsenate

Schroeder and Balassa 1967

As $(+3)$ 
Table 3-3 Levels of Significant Exposure to Inorganic Arsenic - Oral

\begin{tabular}{|c|c|c|}
\hline $\begin{array}{l}\text { Key to } \\
\text { Figure }\end{array}$ & $\begin{array}{c}\text { Species } \\
\text { (Strain) }\end{array}$ & $\begin{array}{r}\text { Expos } \\
\text { Dura } \\
\text { Frequ } \\
\text { (Rou }\end{array}$ \\
\hline 97 & $\begin{array}{l}\text { Dog } \\
\text { (Beagle) }\end{array}$ & $\begin{array}{l}2 \mathrm{yr} \\
(\mathrm{F})\end{array}$ \\
\hline 98 & $\begin{array}{l}\text { Dog } \\
\text { (Beagle) }\end{array}$ & $\begin{array}{l}2 \mathrm{yr} \\
(\mathrm{F})\end{array}$ \\
\hline
\end{tabular}

Systemic

99 Human NS

(W)

Resp $0.032 \quad$ (cough)

Ocular

100

$\begin{array}{ll}\text { Human } & >8 \\ & (\mathrm{~W})\end{array}$

(W)

Dermal

NOAEL

(mg/kg/day)

System (mg/kg/day) (mg/kg/day)

LOAEL

(mg/kg/day)

Reference

Chemical Form

Comments

$2.4 \quad(6 / 6$ died $)$

Byron et al. 1967

As(+3)

$2.4 \quad(1 / 6$ died $)$

Byron et al. 1967

As(+5)

Ahmad et al. 1997

NS

0.032 (melanosis, keratosis

hyperkeratosis, and

depigmentation)

0.032 (chronic conjunctivitis)

Ahsan et al. 2006

(NS)

premalignant skin lesions)

$0.1 \mathrm{~F}$ (de-pigmentation with hyperkeratosis, possibly pre-cancerous)

Bickley and Papa 1989

As(+3)

(IN)

Dermal

102

Human

NS
(W)

Cardio

0.014 (gangrene of feet)

Biswas et al. 1998

NS

0.014 (melanosis and keratosis of hand palms and foot soles)

Dermal 
Table 3-3 Levels of Significant Exposure to Inorganic Arsenic - Oral

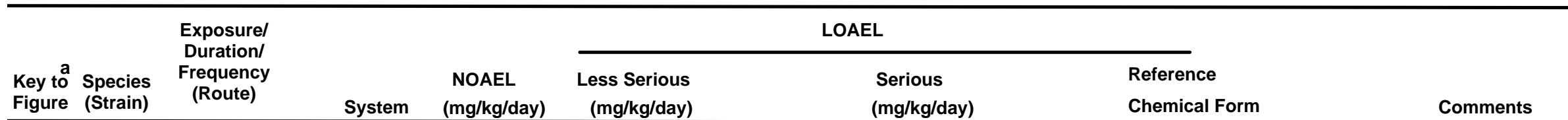

103 Human $12 \mathrm{yr}$

$\begin{array}{ll}12 \mathrm{yr} & \text { Cardio } \\ \text { (W) }\end{array}$

Gastro

Dermal

104 Human

\section{$11-15 \mathrm{yr}$}

(W)

Dermal

0.01 (hypo- and

hyperpigmentation

105 Human NS

(W)

Gastro

0.0004

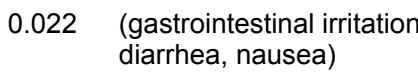

Dermal $\quad 0.0004$

106 Human
$1-11 \mathrm{yr}$

(W)

Hepatic

Dermal
0.02 (Raynaud's disease, gangrene of toes)

Borgono and Greiber 1972

NS

Borgono et al. 1980

NS

Cebrian et al. 1983

As $(+5)$

0.022 (pigmentation changes

with hyperkeratosis,

possibly pre-cancerous)

Chakraborty and Saha 1987

NS

0.046 (pigmentation changes with keratosis, possibly pre-cancerous) 
Table 3-3 Levels of Significant Exposure to Inorganic Arsenic - Oral

\begin{tabular}{|c|c|c|c|c|c|c|c|c|}
\hline \multirow[b]{2}{*}{$\begin{array}{l}\text { Key to } \\
\text { Figure }\end{array}$} & \multirow[b]{2}{*}{$\begin{array}{l}\text { Species } \\
\text { (Strain) }\end{array}$} & \multirow{2}{*}{$\begin{array}{l}\text { Exposurel } \\
\text { Duration/ } \\
\text { Frequency } \\
\text { (Route) }\end{array}$} & \multirow[b]{2}{*}{ System } & \multirow[b]{2}{*}{$\begin{array}{l}\text { NOAEL } \\
\text { (mg/kg/day) }\end{array}$} & \multicolumn{3}{|c|}{ LOAEL } & \multirow[b]{2}{*}{$\begin{array}{l}\text { Reference } \\
\text { Chemical Form }\end{array}$} \\
\hline & & & & & $\begin{array}{r}\text { Less Serious } \\
\text { (mg/kg/day) }\end{array}$ & $\begin{array}{l}\mathrm{Se} \\
(\mathrm{m}\end{array}$ & $\begin{array}{l}\text { lous } \\
\text { kg/day) }\end{array}$ & \\
\hline 107 & Human & $\begin{array}{l}\text { NS } \\
(\mathrm{W})\end{array}$ & Cardio & & & 0.064 & (Blackfoot disease) & $\begin{array}{l}\text { Chen et al. } 1988 b \\
\text { NS }\end{array}$ \\
\hline 108 & Human & $\begin{array}{l}>10 \mathrm{yr} \\
(\mathrm{W})\end{array}$ & Cardio & 0.0008 & & 0.022 & $\begin{array}{l}\text { (increased risk of } \\
\text { ischemic heart disease } \\
\text { mortality) }\end{array}$ & $\begin{array}{l}\text { Chen et al. } 1996 \\
\text { NS }\end{array}$ \\
\hline 109 & Human & $\begin{array}{l}\text { NS } \\
(\mathrm{W})\end{array}$ & Cardio & & & 0.002 & $\begin{array}{l}\text { (increased prevalence of } \\
\text { cerebrovascular disease } \\
\text { and cerebral infarction) }\end{array}$ & $\begin{array}{l}\text { Chiou et al. } 1997 \\
\text { NS }\end{array}$ \\
\hline \multirow[t]{2}{*}{110} & Human & $\begin{array}{l}>5 \mathrm{yr} \\
(\mathrm{W})\end{array}$ & Hemato & $\begin{array}{l}0.006^{\mathrm{C}} \mathrm{M} \\
0.007 \mathrm{~F}\end{array}$ & & & & $\begin{array}{l}\text { EPA 1981b } \\
\text { NS }\end{array}$ \\
\hline & & & Dermal & $\begin{array}{c}0.0009^{\mathrm{C}} \mathrm{M} \\
0.001 \mathrm{~F}\end{array}$ & & & & \\
\hline \multirow[t]{2}{*}{111} & Human & $\begin{array}{l}3-7 \mathrm{yr} \\
(\mathrm{W})\end{array}$ & Cardio & & & 0.05 & (Blackfoot disease) & $\begin{array}{l}\text { Foy et al. } 1992 \\
\text { NS }\end{array}$ \\
\hline & & & Dermal & & & 0.05 & $\begin{array}{l}\text { (melanosis with } \\
\text { hyperkeratosis, possibly } \\
\text { pre-cancerous) }\end{array}$ & \\
\hline
\end{tabular}


Table 3-3 Levels of Significant Exposure to Inorganic Arsenic - Oral

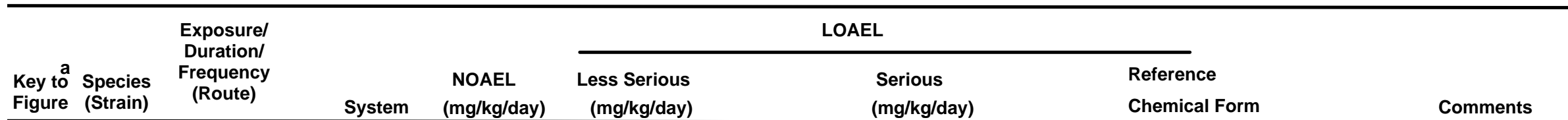

112 Human 2-6 yr

$\begin{array}{ll}2-6 \mathrm{yr} & \text { Hepatic } \\ (\mathrm{IN}) & \text { Dermal }\end{array}$

$0.08 \mathrm{M}$ (cirrhosis, ascites)

Franklin et al. 1950

As(+3)

$0.08 \mathrm{M}$ (pigmentation with hyperkeratosis, possibly pre-cancerous)

113 Human 1-15 yr

(W)

Hepatic 0.16 (portal fibrosis of the
liver)

$\begin{array}{ll}\text { Hepatic } & 0.004 \\ \text { Dermal } & 0.004\end{array}$

115

Human

$\begin{array}{ll}\text { 1-20 yr } & \text { Gastro } \\ & \\ & \text { Hemato } \\ & \text { Hepatic } \\ & \text { Dermal }\end{array}$

116 Human

Dermal

0.0016

$0.06 \quad$ (abdominal pain)
0.06 (anemia)

0.06 (hepatomegaly, fibrosis)

0.06 (hyperpigmentation with hyperkeratosis, possibly pre-cancerous)

0.009 (hyperpigmentation with keratosis, possibly pre-cancerous)

Guha Mazumder et al. 1998a NS

\section{Guha Mazumder 2005}

(NS)

Guha Mazumder et al. 1988

pigmentation changes

with hyperkaratosis,

possibly pre-cancerous)

Guha Mazumder et al. 1988

NS 
Table 3-3 Levels of Significant Exposure to Inorganic Arsenic - Oral

\begin{tabular}{|c|c|c|c|c|c|c|c|c|}
\hline \multirow[b]{2}{*}{$\begin{array}{l}\text { Key to } \\
\text { Figure }\end{array}$} & \multirow[b]{2}{*}{$\begin{array}{l}\text { Species } \\
\text { (Strain) }\end{array}$} & \multirow{2}{*}{$\begin{array}{l}\text { Exposurel } \\
\text { Durationl } \\
\text { Frequency } \\
\text { (Route) }\end{array}$} & \multirow[b]{2}{*}{ System } & \multirow[b]{2}{*}{$\begin{array}{l}\text { NOAEL } \\
\text { (mg/kg/day) }\end{array}$} & \multicolumn{2}{|c|}{ LOAEL } & \multirow[b]{2}{*}{$\begin{array}{l}\text { Reference } \\
\text { Chemical Form }\end{array}$} & \multirow[b]{2}{*}{ Comments } \\
\hline & & & & & $\begin{array}{r}\text { Less Serious } \\
(\mathrm{mg} / \mathrm{kg} / \mathrm{day})\end{array}$ & $\begin{array}{l}\text { Serious } \\
\text { (mg/kg/day) }\end{array}$ & & \\
\hline
\end{tabular}

117 Human (W)

Dermal

0.0014

(arsenical dermatosis)

0.0043 (hyperkeratosis,

(W)

Dermal

hyperpigmentation)

118 Human

\section{$10 \mathrm{yr}$}

(W)

$\begin{array}{ll}\text { Gastro } & 0.0046 \\ \text { Hemato } & 0.0046 \\ \text { Dermal } & 0.0046\end{array}$

120

Human

NS

(W)

0.0008

lifetime

(W)

Hemato

$0.002 \mathrm{~F}$ (anemia during pregnancy) bilirubin)
Guo et al. 2001a

(NS)

Haque et al. 2003

(NS)

Harrington et al. 1978

NS

Hernandez-Zavala et al. 1998 NS

alkaline phosphatase an

Hopenhayn et al. 2006

(NS)

0.067 (ischemic heart disease) Hsueh et al. 1998b dose listed is that

associated with lowest

known peak As

concentration ingested

by a case with

complete water history 
Table 3-3 Levels of Significant Exposure to Inorganic Arsenic - Oral

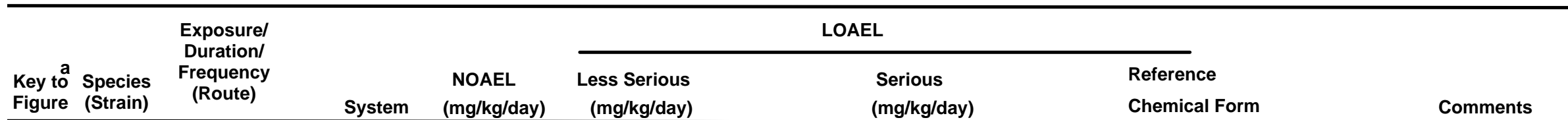

123 Human 0.5-14 yr Dermal

(W)

124 Human $15 \mathrm{yr}$
Gastro

(IN)

Dermal

Cardio $\quad 0.004$

(W)

Dermal $\quad 0.004$

126 Human 3-22 yr

(IN)

Gastro

Hepatic

Dermal
0.05 (hyperpigmentation with Huang et al. 1985

keratosis, possibly NS

pre-cancerous)

$\begin{array}{ll}0.03 \mathrm{M} \text { (hematemesis, } & \text { Lander et al. } 1975 \\ \text { hemoperitoneum, } & \mathrm{As}(+3)\end{array}$

melena)

$0.03 \mathrm{M}$ (hyperkeratosis - possibly

pre-cancerous)

0.005 (cyanosis of extremities, Lianfang and Jianzhong 1994

palpitations/chest NS

discomfort)

0.005 (keratosis

hyperpigmentation,

depigmentation)

$0.05 \mathrm{M}$ (gastrointestinal hemorrhages)

Morris et al. 1974

As(+3)

$0.05 \mathrm{M}$ (vascular fibrosis, portal hypertension)

$0.05 \mathrm{M}$ (hyperpigmentation with keratoses, possibly pre-cancerous) 
Table 3-3 Levels of Significant Exposure to Inorganic Arsenic - Oral

(continued)

\begin{tabular}{llll}
\hline Key to & & $\begin{array}{c}\text { Exposurel } \\
\text { Durationl }\end{array}$ & \\
Figure & (Strain) & $\begin{array}{c}\text { Frequency } \\
\text { (Route) }\end{array}$ & System \\
\hline & & & \\
127 & Human & $\begin{array}{l}15 \mathrm{yr} \\
\text { (IN) }\end{array}$ & Hepatic \\
& & & Dermal
\end{tabular}

128

Human

NS

(W)

Endocr

129 Human

0.018

0.055 (hypertension)

LOAEL

NOAEL Less Serious $\quad$ Serious $\quad$ Reference

(mg/kg/day)

Chemical Form

Comments
$0.05 \mathrm{~F}$ (central fibrosis) Piontek et al. 1989

$0.05 \mathrm{~F}$ (hyperkeratosis, possibly pre-cancerous)

0.11 (diabetes mellitus)

Rahman et al. 1998

NS

Rahman et al. 1999

NS 
Table 3-3 Levels of Significant Exposure to Inorganic Arsenic - Oral

\begin{tabular}{|c|c|c|c|c|c|c|c|}
\hline \multirow[b]{2}{*}{$\begin{array}{l}\text { Key to } \\
\text { Figure }\end{array}$} & \multirow[b]{2}{*}{$\begin{array}{l}\text { Species } \\
\text { (Strain) }\end{array}$} & \multirow{2}{*}{$\begin{array}{l}\text { Exposurel } \\
\text { Durationl } \\
\text { Frequency } \\
\text { (Route) }\end{array}$} & \multirow[b]{2}{*}{ System } & \multirow[b]{2}{*}{$\begin{array}{l}\text { NOAEL } \\
\text { (mg/kg/day) }\end{array}$} & \multicolumn{2}{|c|}{ LOAEL } & \multirow[b]{2}{*}{$\begin{array}{l}\text { Reference } \\
\text { Chemical Form }\end{array}$} \\
\hline & & & & & $\begin{array}{r}\text { Less Serious } \\
\text { (mg/kg/day) }\end{array}$ & $\begin{array}{l}\text { Serious } \\
\text { (mg/kg/day) }\end{array}$ & \\
\hline \multirow[t]{7}{*}{130} & Human & $\begin{array}{l}28 \mathrm{mo} \\
(\mathrm{IN})\end{array}$ & Cardio & $0.06 \mathrm{~F}$ & & & $\begin{array}{l}\text { Silver and Wainman } 1952 \\
\text { As }(+3)\end{array}$ \\
\hline & & & Gastro & & $\begin{aligned} & 0.06 \mathrm{~F} \text { (intermittent, } \\
& \text { progressively severe } \\
& \text { nausea, cramps, and } \\
& \text { diarrhea) }\end{aligned}$ & & \\
\hline & & & Hemato & $0.06 \mathrm{~F}$ & & & \\
\hline & & & Hepatic & & $\begin{array}{c}0.06 \mathrm{~F} \text { (hepatomegaly, fatty } \\
\text { liver) }\end{array}$ & & \\
\hline & & & Renal & $0.06 \mathrm{~F}$ & & & \\
\hline & & & Dermal & & & $\begin{aligned} & 0.06 \mathrm{~F} \text { (melanosis with } \\
& \text { hyperkeratosis, possibly } \\
& \text { pre-cancerous) }\end{aligned}$ & \\
\hline & & & Ocular & & $\begin{array}{c}0.06 \mathrm{~F} \text { (conjunctival injection, } \\
\text { periocular edema) }\end{array}$ & & \\
\hline
\end{tabular}

131 Human $55 \mathrm{yr}$

(IN)

Hepatic

Dermal

132 Human

Cardio

$45 \mathrm{yr}$

(W)
$0.03 \mathrm{M}$ (portal fibrosis and

hypertension, bleeding

Szuler et al. 1979

from esophageal varices)

$0.03 \mathrm{M}$ (hyperpigmentation with hyperkeratosis, possibly pre-cancerous)

0.014 (Blackfoot disease)

Tseng 1977 
Table 3-3 Levels of Significant Exposure to Inorganic Arsenic - Oral

\begin{tabular}{|c|c|c|c|c|c|c|c|c|}
\hline \multirow[b]{2}{*}{$\begin{array}{l}\text { Key to } \\
\text { Figure }\end{array}$} & \multirow[b]{2}{*}{$\begin{array}{l}\text { Species } \\
\text { (Strain) }\end{array}$} & \multirow{2}{*}{$\begin{array}{l}\text { Exposurel } \\
\text { Durationl } \\
\text { Frequency } \\
\text { (Route) }\end{array}$} & \multirow[b]{2}{*}{ System } & \multicolumn{4}{|c|}{ LOAEL } & \multirow[b]{2}{*}{$\begin{array}{l}\text { Reference } \\
\text { Chemical Form }\end{array}$} \\
\hline & & & & $\begin{array}{l}\text { NOAEL } \\
\text { (mg/kg/day) }\end{array}$ & $\begin{array}{r}\text { Less Serious } \\
\text { (mg/kg/day) }\end{array}$ & $\begin{array}{l}\mathrm{Se} \\
(\mathrm{mg}\end{array}$ & $\begin{array}{l}\text { lous } \\
\text { kg/day) }\end{array}$ & \\
\hline 133 & Human & $\begin{array}{l}\text { NS } \\
(W)\end{array}$ & Cardio & & & 0.014 & (Blackfoot disease) & $\begin{array}{l}\text { Tseng } 1989 \\
\text { NS }\end{array}$ \\
\hline 134 & Human & $\begin{array}{l}>45 \mathrm{yr} \\
(\mathrm{W})\end{array}$ & Dermal & $0.0008^{d} \mathrm{M}$ & $\begin{array}{c}0.014 \mathrm{M} \text { (hyperkeratosis and } \\
\text { hyperpigmentation) }\end{array}$ & & & $\begin{array}{l}\text { Tseng et al. } 1968 \\
\text { NS }\end{array}$ \\
\hline 135 & Human & $\begin{array}{l}>30 \mathrm{yr} \\
(\mathrm{W})\end{array}$ & Cardio & & $\begin{array}{l}0.064 \mathrm{M} \text { (deficits in cutaneous } \\
\text { microcirculation of the } \\
\text { toes) }\end{array}$ & & & $\begin{array}{l}\text { Tseng et al. } 1995 \\
\text { As(+3) }\end{array}$ \\
\hline 136 & Human & $\begin{array}{l}52.6 \mathrm{yr} \\
\text { (avg) } \\
\text { (W) }\end{array}$ & Cardio & 0.016 & & 0.031 & $\begin{array}{l}\text { (peripheral vascular } \\
\text { disease) }\end{array}$ & $\begin{array}{l}\text { Tseng et al. } 1996 \\
\text { NS }\end{array}$ \\
\hline \multirow[t]{5}{*}{137} & Human & $\begin{array}{l}16 \mathrm{mo} \\
(\mathrm{IN})\end{array}$ & Resp & $0.1 \mathrm{M}$ & & & & $\begin{array}{l}\text { Wade and Frazer } 1953 \\
\mathrm{As}(+3)\end{array}$ \\
\hline & & & Cardio & $0.1 \mathrm{M}$ & & & & \\
\hline & & & Hemato & $0.1 \mathrm{M}$ & & & & \\
\hline & & & Hepatic & & $0.1 \mathrm{M}$ (liver enlargment) & & & \\
\hline & & & Dermal & & & 0.1 & $\begin{array}{l}\text { (hyperkeratosis, } \\
\text { hyperpigmentation with } \\
\text { hyperkeratosis, possibly } \\
\text { pre-cancerous) }\end{array}$ & \\
\hline
\end{tabular}


Table 3-3 Levels of Significant Exposure to Inorganic Arsenic - Oral

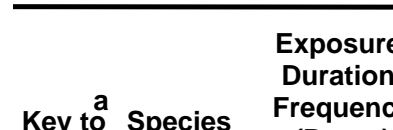

Durationl

Frequency
(Route)

Figure (Strain)

System

NOAEL

(mg/kg/day)

138

Human

30-33 yr Dermal
(W)

(W)

Dermal

139 Human $12 \mathrm{yr}$

(W)

Resp

$0.015^{\mathrm{C}} \mathrm{M}$ (bronchitis, bronchiectasis)

Zaldivar 1974

NS

Reference

Chemical Form

$0.018 \mathrm{~F}$ (bronchitis,

bronchiectasis)

Cardio

$0.015^{\mathrm{C}} \mathrm{M}$ (Raynaud's disease, thrombosis)

Comments

$0.018 \mathrm{~F}$

Gastro

$0.015^{\mathrm{C}} \mathrm{M}$ (diarrhea)

$0.018 \mathrm{~F}$ (diarrhea)

Dermal

$0.015^{\mathrm{C}} \mathrm{M}$ (scaling of skin,

hyperkeratosis,
leukoderma,

melanoderma)

$0.018 \mathrm{~F}$

$\mathrm{Bd} \mathrm{Wt}$

$0.015^{\mathrm{C}} \mathrm{M}$ (unspecified decreased

body weight)

$0.018 \mathrm{~F}$ (unspecified decreased

body weight) 
Table 3-3 Levels of Significant Exposure to Inorganic Arsenic - Oral

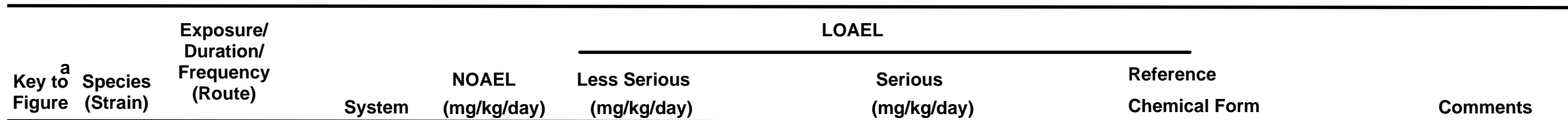

(W) Dermal

$\begin{array}{ll}2-7 \text { yr } & \text { Resp } \\ \text { children } & \end{array}$

children

(W)

Cardio

Gastro

Hemato

Hepatic

Renal

Dermal

142

Human

$1-39$ y

(W)

Cardio
(hyperpigmentation with
keratoses, possibly
pre-cancerous)

\section{Zaldivar 1977}

NS

0.08 (inflammation of bronchi Zaldivar and Guillier 1977 and larynx,

bronchopneumonia)

NS

0.05 (vascular spasms,

thrombosis, ischemia,

hypotension, cardiac

failure)

0.05 (nause, vomiting,

diarrhea, intestina

hemorrhage)

0.05 (anemia)

0.08 (cirrhosis)

0.05 (hyperkeratosis of palms and soles, melanoderma, leukoderma)

0.06 (arterial thickening Raynaud's disease)
Zaldivar and Guillier 1977

NS 
Table 3-3 Levels of Significant Exposure to Inorganic Arsenic - Oral

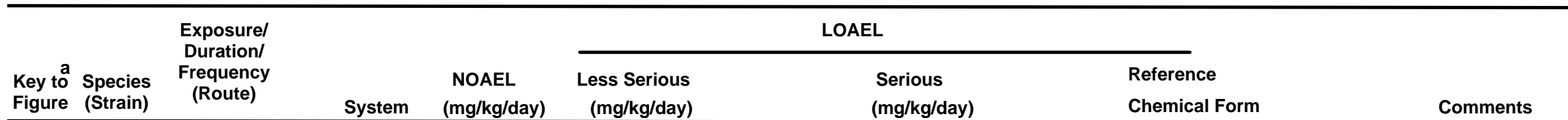

$\begin{array}{lll}\begin{array}{l}\text { Rat } \\ \text { (Osborne- } \\ \text { Mendel) }\end{array} & \begin{array}{l}2 \mathrm{yr} \\ (\mathrm{F})\end{array} & \text { Resp } \\ & & \\ & \text { Cardio } \\ & \text { Gastro } \\ & \text { Hemato }\end{array}$

20

20

Hepatic 4

$\begin{array}{ll}\text { Renal } & 9 \\ \text { Bd Wt } & 2\end{array}$

Resp

30

(Osborne-

Mendel)

(slight transient decrease in $\mathrm{Hb}$ and $\mathrm{Hct}$ values)

9 (enlarged bile duct, bile duct proliferation)

Byron et al. 1967

As(+3)

$\begin{array}{lrrl}\text { Cardio } & 30 & & \\ \text { Gastro } & 30 & & \\ \text { Hemato } & 30 & & \\ \text { Hepatic } & 9 & 20 & \text { (enlarged bile duct) } \\ \text { Renal } & 9 & 20 & \text { (pigmentation, cysts) } \\ \text { Bd Wt } & & 2 & \begin{array}{l}\text { (decreased body weight } \\ \text { gain in females) }\end{array}\end{array}$

Byron et al. 1967

As $(+5)$ 
Table 3-3 Levels of Significant Exposure to Inorganic Arsenic - Oral

\begin{tabular}{|c|c|c|c|c|}
\hline $\begin{array}{l}\text { Key to } \\
\text { Figure }\end{array}$ & $\begin{array}{l}\text { Species } \\
\text { (Strain) }\end{array}$ & $\begin{array}{l}\text { Exposurel } \\
\text { Durationl } \\
\text { Frequency } \\
\text { (Route) }\end{array}$ & System & $\begin{array}{r}\text { NOAE } \\
(\mathrm{mg} / \mathrm{kg}\end{array}$ \\
\hline \multirow[t]{8}{*}{145} & $\begin{array}{l}\text { Rat } \\
\text { (Wistar) }\end{array}$ & $\begin{array}{l}27 \mathrm{mo} \\
(\mathrm{F})\end{array}$ & Resp & 7 \\
\hline & & & Cardio & 7 \\
\hline & & & Gastro & 7 \\
\hline & & & Hemato & 7 \\
\hline & & & Musc/skel & 7 \\
\hline & & & Hepatic & 7 \\
\hline & & & Renal & 7 \\
\hline & & & Endocr & 7 \\
\hline
\end{tabular}

LOAEL

Reference

Serious

Chemical Form

$\mathrm{Bd} \mathrm{Wt}$

7 (decreased body weight

gain)

Kroes et al. 1974

As(+5) 
Table 3-3 Levels of Significant Exposure to Inorganic Arsenic - Oral

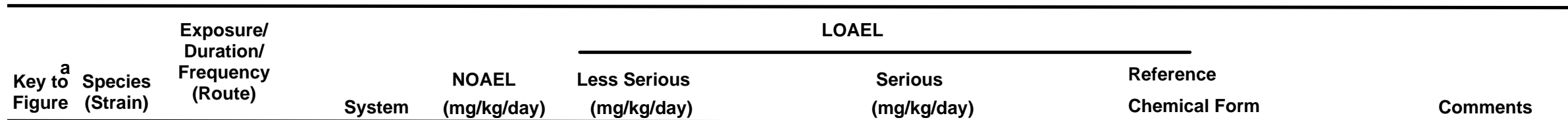

146 Rat

$27 \mathrm{mo}$

(F)

$\begin{array}{lr}\text { Resp } & 30 \\ \text { Cardio } & 30 \\ \text { Gastro } & 30 \\ \text { Hemato } & 7 \\ \text { Musc/skel } & 30 \\ \text { Hepatic } & 7\end{array}$

(slight anemia)

30 (enlarged bile duct with

extensive dilation and

inflammation)

Kroes et al. 1974

As $(+5)$ lead arsenate

Renal $\quad 30$

Endocr $\quad 30$

Bd Wt $\quad 7$

30 (decreased body weight

gain)

Resp

Dermal $\quad 0.6$

Bd Wt $\quad 0.6$

Schroeder et al. 1968 As(+3) 
Table 3-3 Levels of Significant Exposure to Inorganic Arsenic - Oral

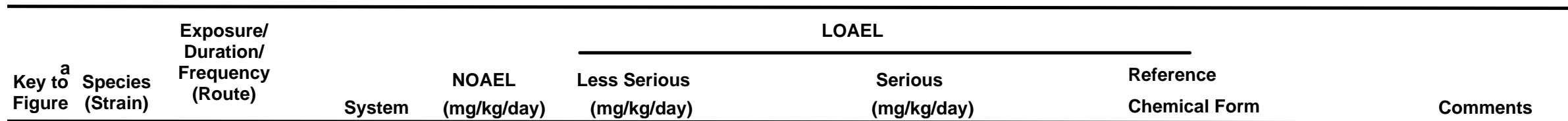

$\begin{array}{lll}\text { Mouse } & 48 w k & \text { Hepatic } \\ \text { (NS) } & \text { (W) } & \\ & & \end{array}$

11.1

5.6 (histological alterations of the kidney)

Bd Wt $\quad 11.1$

149

\begin{tabular}{|c|c|c|}
\hline $\begin{array}{l}\text { Mouse } \\
\text { (NS) }\end{array}$ & $\begin{array}{l}48 w k \\
\text { (W) }\end{array}$ & Hepatic \\
\hline & & Renal \\
\hline
\end{tabular}

$\begin{array}{lll}\text { Mouse } & 15 \text { mo } & \text { Hepatic } \\ \text { (BALB/c) } & \text { (W) } & \end{array}$

$\mathrm{Bd} \mathrm{Wt}$ $0.7 \mathrm{M}(13-17 \%$ decreased body
weight)

151 Mouse
(CD)
(W)
Bd Wt

$2 \mathrm{yr}$
1 (decreased body weight gain after the first 6 months of the study)

$0.7 \mathrm{M}$ (increased liver weight, altered liver

histopathology,

decreased hepatic

enzymes in serum)

(mg/kg/day)

Chemical Form

Comments

Liu et al. 2000

As(+3)

Liu et al. 2000

As (+5)

Santra et al. 2000

(NS) 
Table 3-3 Levels of Significant Exposure to Inorganic Arsenic - Oral

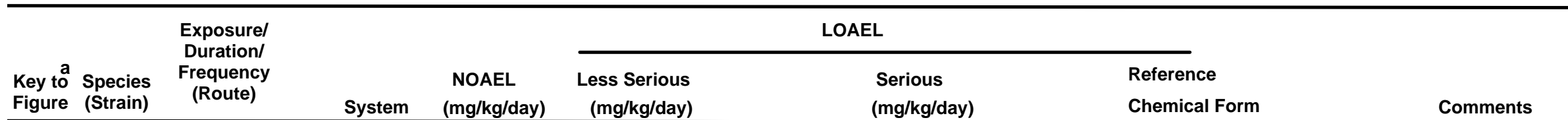

Dog 2 yr

(Beagle) (F)

$\begin{array}{llr}2 \mathrm{yr} & \text { Resp } & 2.4 \\ & & \\ & \text { Cardio } & 2.4 \\ & \text { Gastro } & 1 \\ & \text { Hemato } & 1 \\ & \\ & \text { Hepatic } & 1 \\ & \\ & \text { Renal } & 2.4 \\ \text { Bd Wt } & 1\end{array}$

1

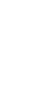

$\begin{array}{ll}2.4 & \text { (slight to moderate } \\ \text { anemia) }\end{array}$
$\begin{array}{ll}2.4 & \text { (hemosiderin deposits in } \\ \text { hepatic macrophages) }\end{array}$

153 Dog (Beagle)

\begin{abstract}
$2 \mathrm{yr}$
\end{abstract}

$\begin{array}{lr}\text { Resp } & 2.4 \\ \text { Cardio } & 2.4 \\ \text { Gastro } & 2.4 \\ \text { Hemato } & 1 \\ \text { Hepatic } & 1 \\ & \\ \text { Renal } & 2.4 \\ \text { Bd Wt } & 1\end{array}$

$\mathrm{Bd} \mathrm{Wt}$
2.4 (bleeding in the gut)

Byron et al. 1967

As(+3)

2.4 (44-61\% weight loss)

Byron et al. 1967

As(+5)

2.4 (marked decreased weight gain) 
Table 3-3 Levels of Significant Exposure to Inorganic Arsenic - Oral

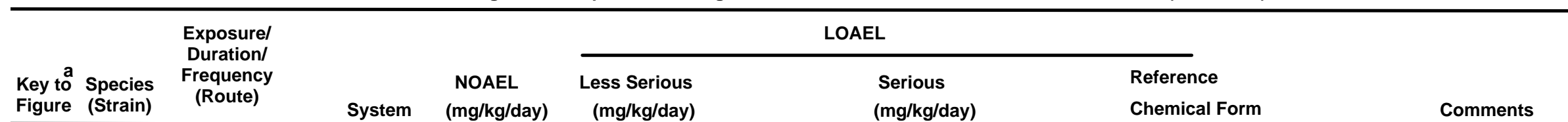

Neurological

154 Human

$>5 \mathrm{yr}$

(W)

$0.006^{\mathrm{C}} \mathrm{M}$

$0.007 \mathrm{~F}$

155 Human 3-7 yr

(W)

156 Human 1-20 yr

(W)

157 Human $10 \mathrm{yr}$

(W)

158 Human

NS

(W)

159 Huma

NS

(W)

$28 \mathrm{mo}$

(IN)

161 Human

$55 \mathrm{yr}$

(IN)

0.0046

0.0014

0.004
0.06 (tingling of hands and feet)

0.005 (fatigue, headache, dizziness, insomnia,
nighmare, numbness

0.04 (functional denervation)

Harrington et al. 1978

NS

Hindmarsh et al. 1977

NS

Lianfang and Jianzhong 1994

NS

Silver and Wainman 1952

As(+3)

Szuler et al. 1979

As(+3)
$0.03 \mathrm{M}$ (absent ankle jerk reflex and vibration sense in legs)
$0.06 \mathrm{~F}$ (paresthesia) 
Table 3-3 Levels of Significant Exposure to Inorganic Arsenic - Oral

\begin{tabular}{|c|c|c|c|c|c|c|c|c|}
\hline \multirow[b]{2}{*}{$\begin{array}{l}\text { Key to } \\
\text { Figure }\end{array}$} & \multirow[b]{2}{*}{$\begin{array}{c}\text { Species } \\
\text { (Strain) }\end{array}$} & \multirow{2}{*}{$\begin{array}{l}\text { Exposurel } \\
\text { Duration/ } \\
\text { Frequency } \\
\text { (Route) }\end{array}$} & \multirow[b]{2}{*}{ System } & \multirow[b]{2}{*}{$\begin{array}{l}\text { NOAEL } \\
\text { (mg/kg/day) }\end{array}$} & \multicolumn{2}{|c|}{ LOAEL } & \multirow[b]{2}{*}{$\begin{array}{l}\text { Reference } \\
\text { Chemical Form }\end{array}$} & \multirow[b]{2}{*}{ Comments } \\
\hline & & & & & $\begin{array}{r}\text { Less Serious } \\
(\mathrm{mg} / \mathrm{kg} / \mathrm{day})\end{array}$ & $\begin{array}{l}\text { Serious } \\
\text { (mg/kg/day) }\end{array}$ & & \\
\hline
\end{tabular}

162 Human NS

(W)

163 Human

lifetime

continuous

(W)

164 Human

lifetime

0.0008

0.003 (decreased score in

Performance domain of

an intelligence scale)
Tsai et al. 2003

(NS)

Wasserman et al. 2004

(NS)

Wasserman et al. 2007

(NS)

$0.008 \mathrm{~F}$ (increased frequencies for spontaneous abortion, (NS)

Ahmad et al. 2001 birth rates)

$0.006 \mathrm{~F}$ (increased incidence of spontaneous abortion)

ilton et al. 2005

(NS)

$0.02 \mathrm{~F}$ (increased risk of stilbirth) von Ehrenstein et al. 2006

(NS)

$98 \%$ of the exposed

group drank water

containing $0.1 \mathrm{mg} \mathrm{As} / \mathrm{L}$

or more. 
Table 3-3 Levels of Significant Exposure to Inorganic Arsenic - Oral

(continued)

\begin{tabular}{|c|c|c|c|c|c|c|c|c|}
\hline \multirow[b]{2}{*}{$\begin{array}{l}\text { Key to } \\
\text { Figure }\end{array}$} & \multirow[b]{2}{*}{$\begin{array}{c}\text { Species } \\
\text { (Strain) }\end{array}$} & \multirow{2}{*}{$\begin{array}{l}\text { Exposurel } \\
\text { Duration/ } \\
\text { Frequency } \\
\text { (Route) }\end{array}$} & \multirow[b]{2}{*}{ System } & \multirow[b]{2}{*}{$\begin{array}{l}\text { NOAEL } \\
\text { (mg/kg/day) }\end{array}$} & \multicolumn{2}{|c|}{ LOAEL } & \multirow[b]{2}{*}{$\begin{array}{l}\text { Reference } \\
\text { Chemical Form }\end{array}$} & \multirow[b]{2}{*}{ Comments } \\
\hline & & & & & $\begin{array}{r}\text { Less Serious } \\
\text { (mg/kg/day) }\end{array}$ & $\begin{array}{l}\text { Serious } \\
\text { (mg/kg/day) }\end{array}$ & & \\
\hline
\end{tabular}

\section{Developmental}

168 Human

continuous

(W)

0.002 (reduced birth weight)

Hopenhayn et al. 2003a

(NS)

169 Human $\quad \begin{aligned} & >1 \mathrm{yr} \\ & 1 \mathrm{x} / \mathrm{d}\end{aligned}$

$1 \mathrm{x} / \mathrm{d}$

0.03 (increased SMR for

malignant and

non-malignant lung

Smith et al. 2006

disease)$$
\text { (1) }
$$

170 Human lifetime

$\begin{array}{ll}\text { lifetime } & 0.008 \\ \text { (W) } & \end{array}$

von Ehrenstein et al. 2006

(NS)

NOAEL is for no

increase in risk of

neonatal mortality or
overall infant mortality.

(CEL: skin cancer)

Cebrian et al. 1983

As(+5)

0.064 (CEL: bladder, lung and liver cancers)

Chen et al. 1986

NS

173 Human NS

(W)

0.064 (CEL: malignant neoplasms of the bladder, skin, lung and

liver)

\section{Chen et al. 1988b} NS

174 Human NS

(W)

Chiou et al. 2001

(NS) 
Table 3-3 Levels of Significant Exposure to Inorganic Arsenic - Oral

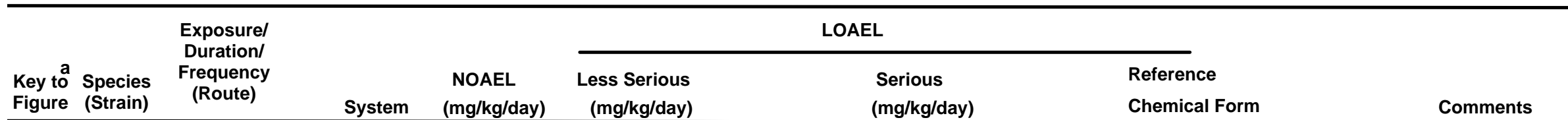

175 Human 2 wk- $12 \mathrm{yr}$

(IN)

176 Human NS

(W)

177 Human NS

(W)

178 Human

NS

(W)

179 Human

NS

(W)

180 Human

NS

(W)

181 Human NS

(W)
3.67 (CEL: bladder cancer

risk)

0.0011 (CEL: lung cancer)

0.0017 (CEL: lung cancers)

0.018 (CEL: lung cancer mortality)

0.018 (CEL: bladder cancer)

incidence of transitiona cell carcinomas of the

bladder, kidney, ureters and all urethral cancer)

$0.0049^{\mathrm{C}} \mathrm{M}$ (CEL: squamous cell carcinoma of the skin)

(NS)
Guo et al. 2001b

Cuzick et al.1992

As(+3)

Ferreccio et al. 1998

NS

Ferreccio et al. 2000

(NS)

Guo 2004

(NS)

Guo and Tseng 2000

(NS)

Guo et al. 1997

NS 
Table 3-3 Levels of Significant Exposure to Inorganic Arsenic - Oral

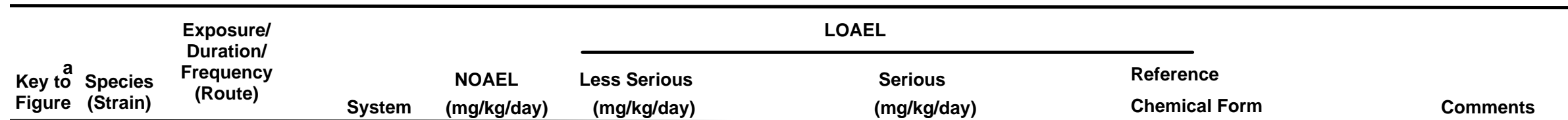

182 Human $>1 \mathrm{yr}$

(W)

183 Human

16 yr (avg)

(IN)

184 Human $60 \mathrm{yr}$

(W)

185

Human $\quad>45 \mathrm{yr}$

(W)

186

Human

$\sim 5 \mathrm{yr}$

(W)
0.0075 (CEL: basal or squamous Haupert et al. 1996 skin carcinoma)

NS

$0.04 \mathrm{M}$ (CEL: basal cell and squamous cell carcinomas of the skin, small cell and squamous

cell carcinoma of the

lung)

0.038 (CEL: intraepidermal carcinoma)

Tseng 1977

NS

0.014 (CEL: squamous cell carcinoma of the skin)

Tseng et al. 1968 NS

0.033 (CEL: lung, urinary tract cancer)
Luchtrath 1983 As(+5) 
Table 3-3 Levels of Significant Exposure to Inorganic Arsenic - Oral

\begin{tabular}{|c|c|c|c|c|c|c|c|c|}
\hline \multirow[b]{2}{*}{$\begin{array}{l}\text { Key to } \\
\text { Figure }\end{array}$} & \multirow[b]{2}{*}{$\begin{array}{l}\text { Species } \\
\text { (Strain) }\end{array}$} & \multirow{2}{*}{$\begin{array}{l}\text { Exposurel } \\
\text { Duration/ } \\
\text { Frequency } \\
\text { (Route) }\end{array}$} & \multicolumn{5}{|c|}{ LOAEL } & \multirow[b]{2}{*}{ Comments } \\
\hline & & & System & $\begin{array}{l}\text { NOAEL } \\
\text { (mg/kg/day) }\end{array}$ & $\begin{array}{l}\text { Less Serious } \\
\text { (mg/kg/day) }\end{array}$ & $\begin{array}{l}\text { Serious } \\
\text { (mg/kg/day) }\end{array}$ & $\begin{array}{l}\text { Reference } \\
\text { Chemical Form }\end{array}$ & \\
\hline
\end{tabular}

187 Human $12 \mathrm{yr}$

The number corresponds to entries in Figure 3-3.
$0.015^{\mathrm{C}} \mathrm{M}$ (CEL: squamous cell carcinoma of the skin)

\section{Zaldivar 1974}

NS

$0.018 \mathrm{~F}$ (CEL: squamous cell carcinoma of the skin)

0.014 M (CEL: basal cell and squamous cell

carcinomas of the skin

hemangioendothelioma

of the liver)
Zaldivar et al. 1981

NS

c Differences in levels of health effects and cancer effects between male and females are not indicated in Figure 3-3. Where such differences exist, only the levels of effect for the most sensitive gender are presented.

d Used to derive chronic oral minimal risk level (MRL) of $0.0003 \mathrm{mg} / \mathrm{kg} / \mathrm{day}$; dose divided by an uncertainty factor of 3 (for human variability)

avg = average; $\mathrm{ALAD}=$ delta-aminolevulinic acid dehydratase $; \mathrm{ALAS}=$ delta-aminolevulinic acid synthetase; $\mathrm{ALT}=$ alanine aminotransferase $; \mathrm{AST}=$ aspartate aminotransferase; $\mathrm{Bd}$ $\mathrm{Wt}$ = body weight; $\mathrm{BUN}=$ blood urea nitrogen; Cardio = cardiovascular; $\mathrm{CEL}=$ cancer effect level; $\mathrm{d}=$ day $(\mathrm{s}) ;$ Endocr = endocrine; $(\mathrm{F})=$ feed; $\mathrm{F}=$ female; $(\mathrm{G})=$ gavage; Gastro = gastrointestinal; $\mathrm{Gd}=$ gestational day; $\mathrm{Gl}=$ gastrointestinal; $(\mathrm{GW})$ = gavage in water; gen = generation; $\mathrm{Gd}=$ gestation day; $\mathrm{Gn}$ pig = guinea pig; Hemato = hematological; $\mathrm{Hb}=$ hemoglobin; Hct = hematocrit; Hemato = hematological; hr = hour(s); (IN) = ingestion; LD50 = lethal dose, 50\% kill; LDH = lactate dehydrogenase; LOAEL =

hemoglobin; Hct = hematocrit; Hemato = hematological; $\mathrm{hr}=$ hour(s); (IN) = ingestion; $\mathrm{LD} 50=$ lethal dose, $50 \%$ kill; $\mathrm{LDH}=\mathrm{lactate}$ dehydrogenase; $\mathrm{LOAEL}=$
lowest-observable-adverse-effect level; $\mathrm{M}=$ male; Metab = metabolic; $\mathrm{mo}=$ month(s); Musc/skel = musculoskeletal; NOAEL = no-observable-adverse-effect level; NS = not specified; lowest-observable-adverse-effect level; $M=$ male; Metab = metabolic; mo = month(s); Musc/skel = musculoskeletal; NOAEL = no-observable-adverse-effect level;
pmd = pre-mating day; pnd = post-natal day; Resp = respiratory; SMR =standardized mortality ratio; $(\mathrm{W})=$ drinking water; wk = week(s); $\mathrm{x}=$ time(s); yr = year(s) 
Figure 3-3 Levels of Significant Exposure to Inorganic Arsenic - Oral

Acute ( $\leq 14$ days)

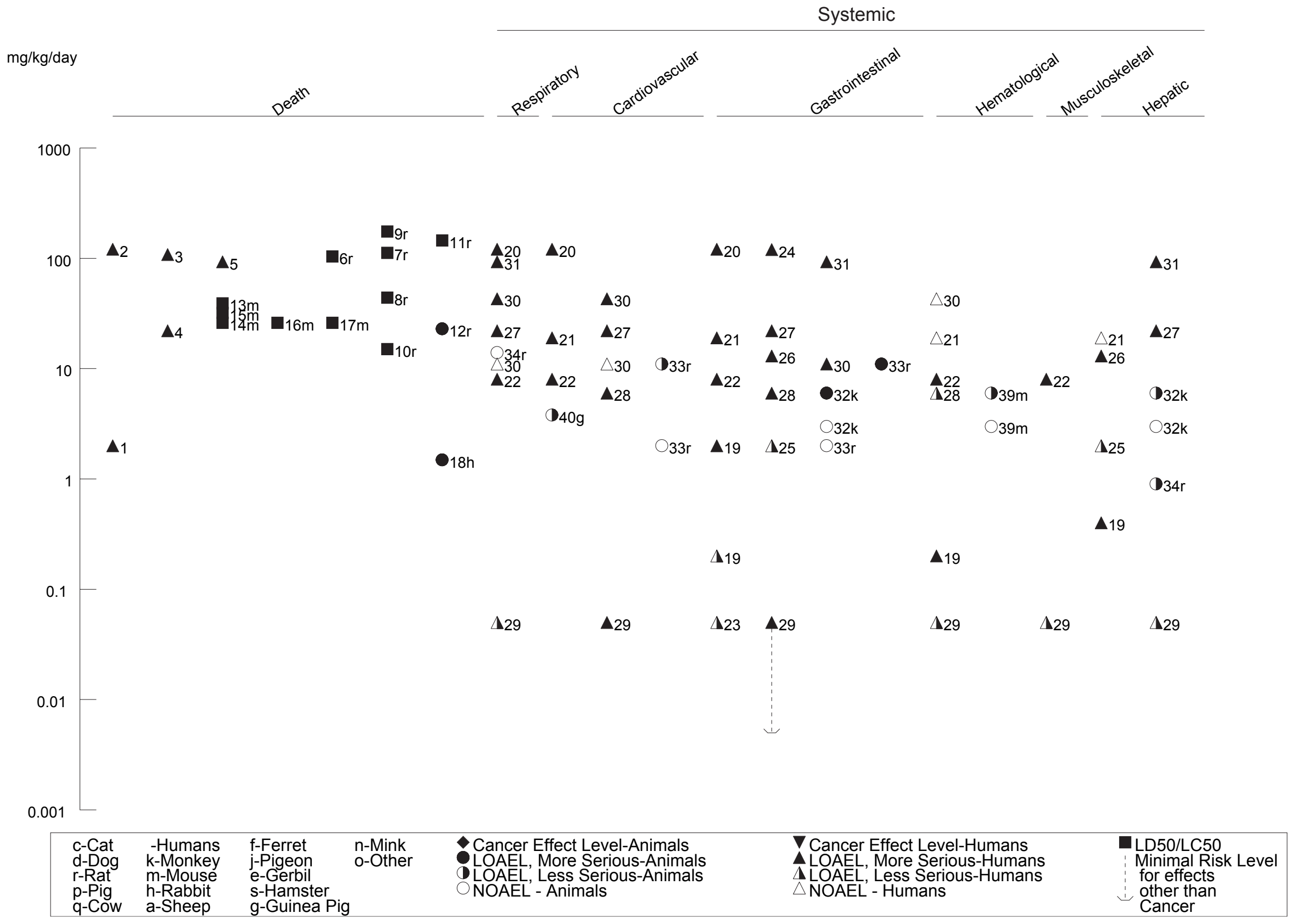


Figure 3-3 Levels of Significant Exposure to Inorganic Arsenic - Oral (Continued)

Acute ( $\leq 14$ days)

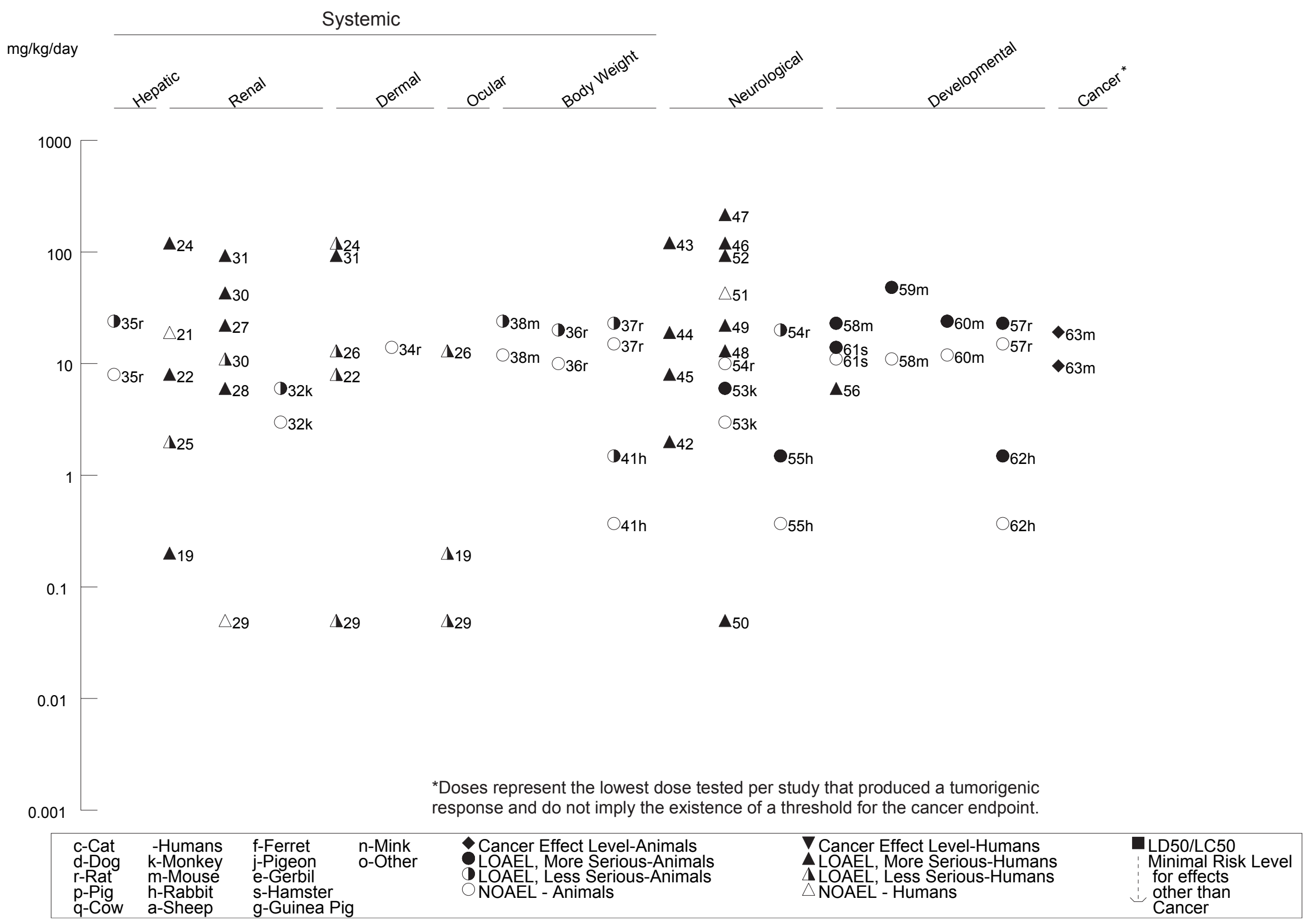

*Doses represent the lowest dose tested per study that produced a tumorigenic sponse and do not imply the existence of a threshold for the cancer endpoint.

\begin{tabular}{|c|c|c|c|c|c|c|}
\hline $\begin{array}{l}\text { c-Cat } \\
\text { d-Dog } \\
\text { r-Rat } \\
\text { p-Pig } \\
\text { q-Cow }\end{array}$ & $\begin{array}{l}\text {-Humans } \\
\text { k-Monkey } \\
\text { m-Mouse } \\
\text { h-Rabbit } \\
\text { a-Sheep }\end{array}$ & $\begin{array}{l}\text { f-Ferret } \\
\text { j-Pigeon } \\
\text { e-Gerbil } \\
\text { s-Hamster } \\
\text { g-Guinea Pic }\end{array}$ & $\begin{array}{l}\text { n-Mink } \\
\text { o-Other }\end{array}$ & $\begin{array}{l}\text { Cancer Effect Level-Animals } \\
\text { LOAEL, More Serious-Animals } \\
\text { LOAEL, Less Serious-Animals } \\
\text { NOAEL - Animals }\end{array}$ & $\begin{array}{l}\nabla \text { Cancer Effect Level-Humans } \\
\Delta \text { LOAEL, More Serious-Humans } \\
\Lambda \text { LOAEL, Less Serious-Humans } \\
\triangle \text { NOAEL - Humans }\end{array}$ & $\begin{array}{l}\text { LD50/LC50 } \\
\text { Minimal Risk Level } \\
\text { for effects } \\
\text { other than } \\
\text { Cancer }\end{array}$ \\
\hline
\end{tabular}


Figure 3-3 Levels of Significant Exposure to Inorganic Arsenic - Oral (Continued)

Intermediate (15-364 days)

Systemic

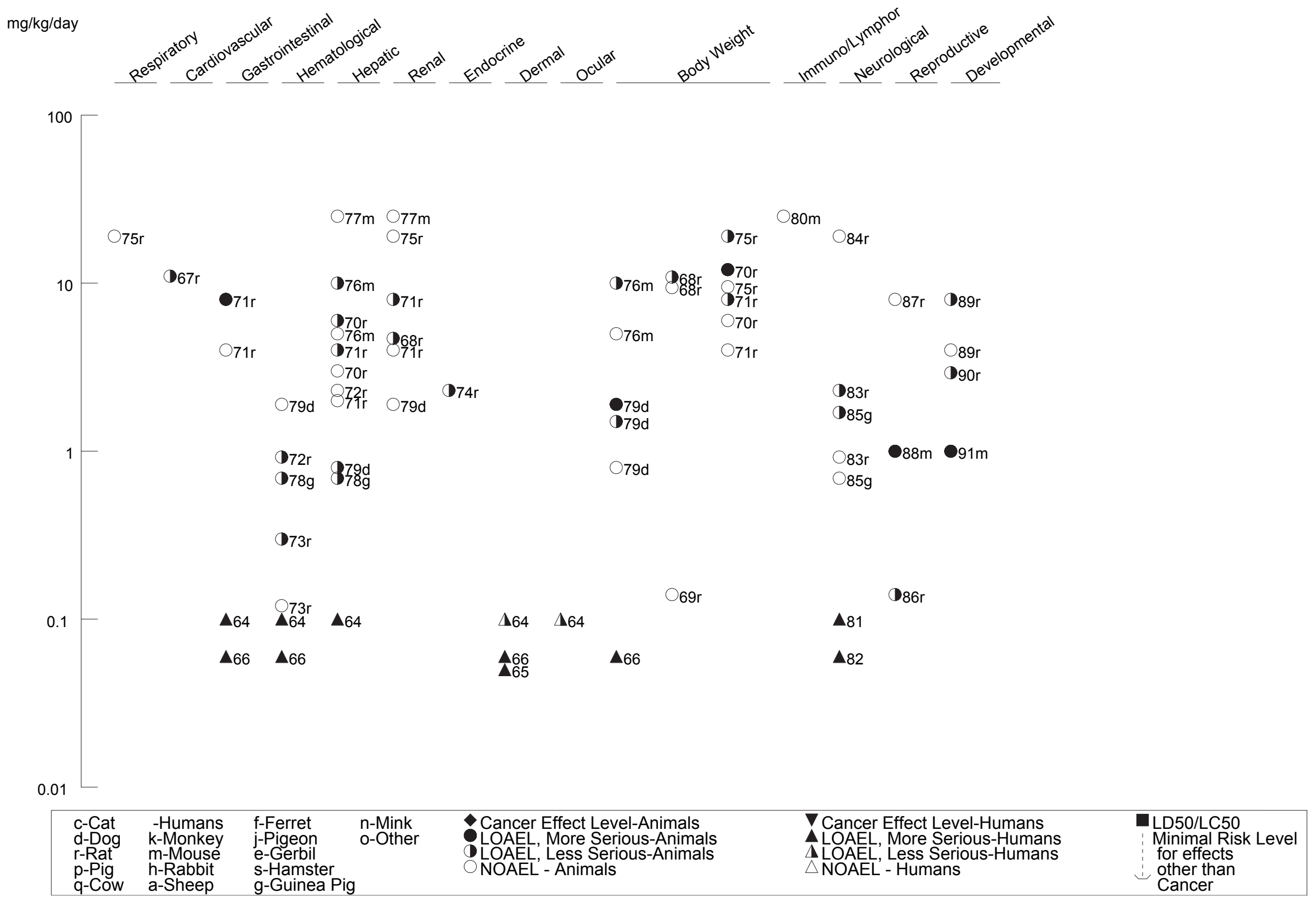


Figure 3-3 Levels of Significant Exposure to Inorganic Arsenic - Oral (Continued)

Chronic ( $\geq 365$ days)

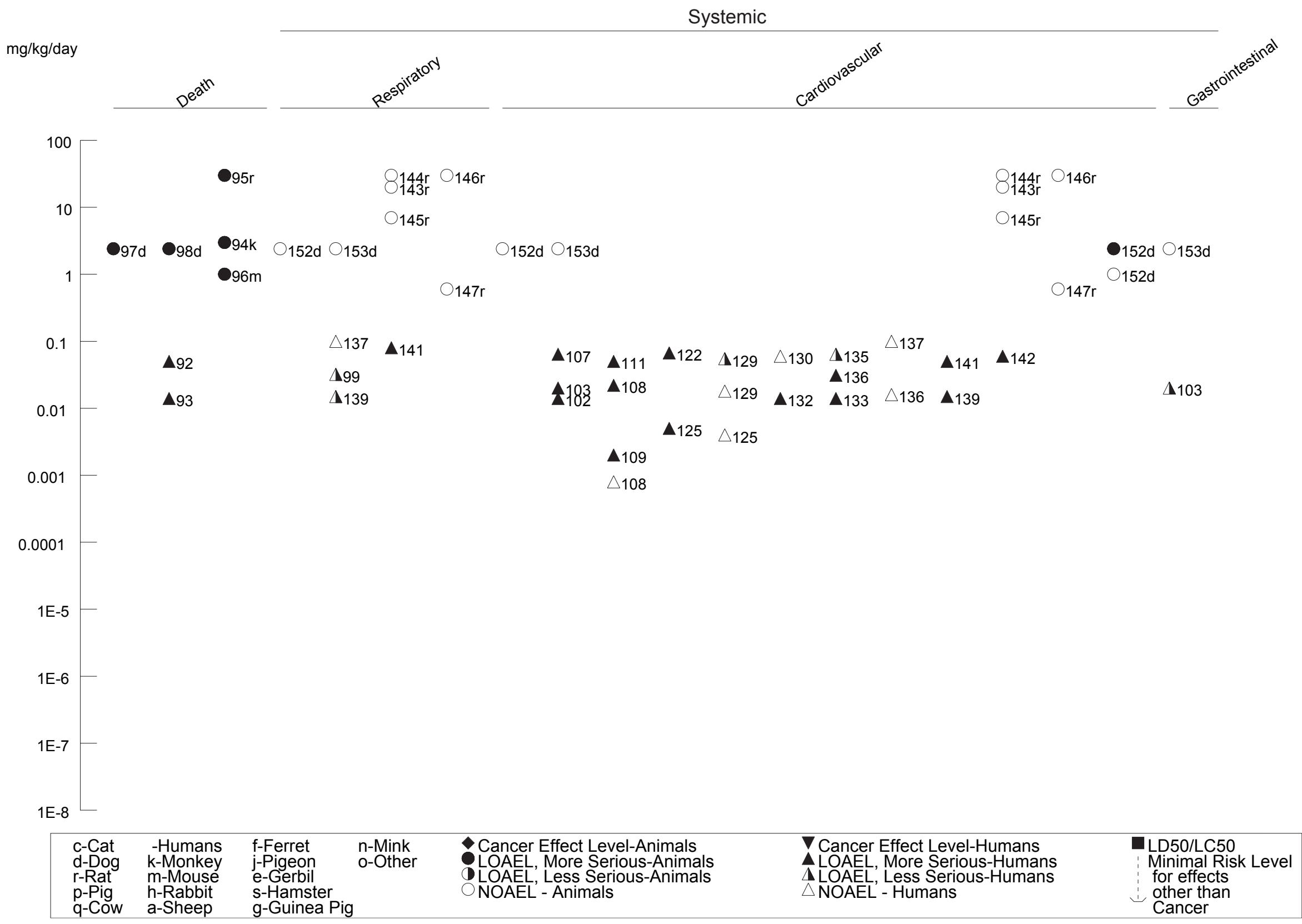


Figure 3-3 Levels of Significant Exposure to Inorganic Arsenic - Oral (Continued)

Chronic ( $\geq 365$ days)

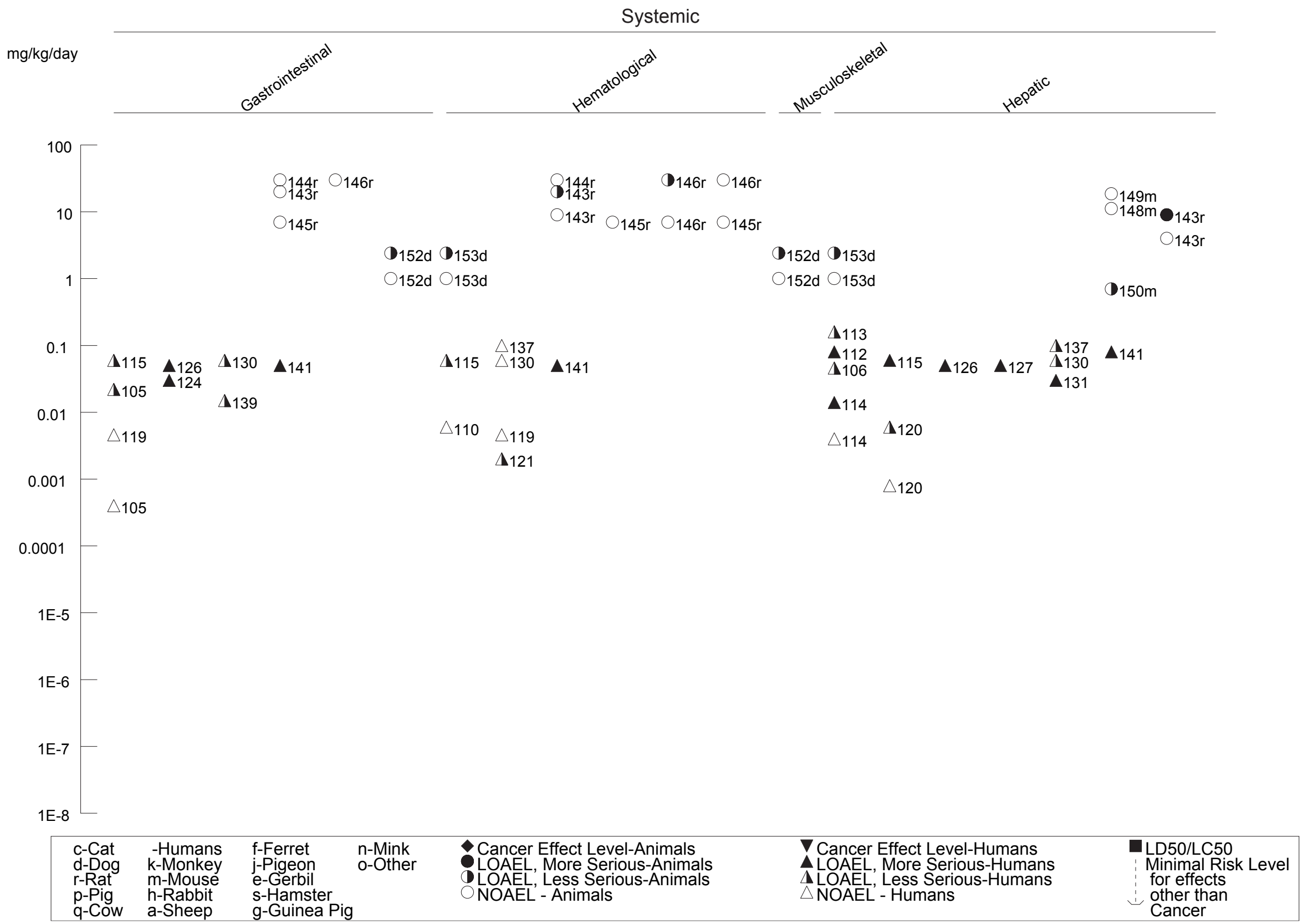


Figure 3-3 Levels of Significant Exposure to Inorganic Arsenic - Oral (Continued)

Chronic ( $\geq 365$ days)

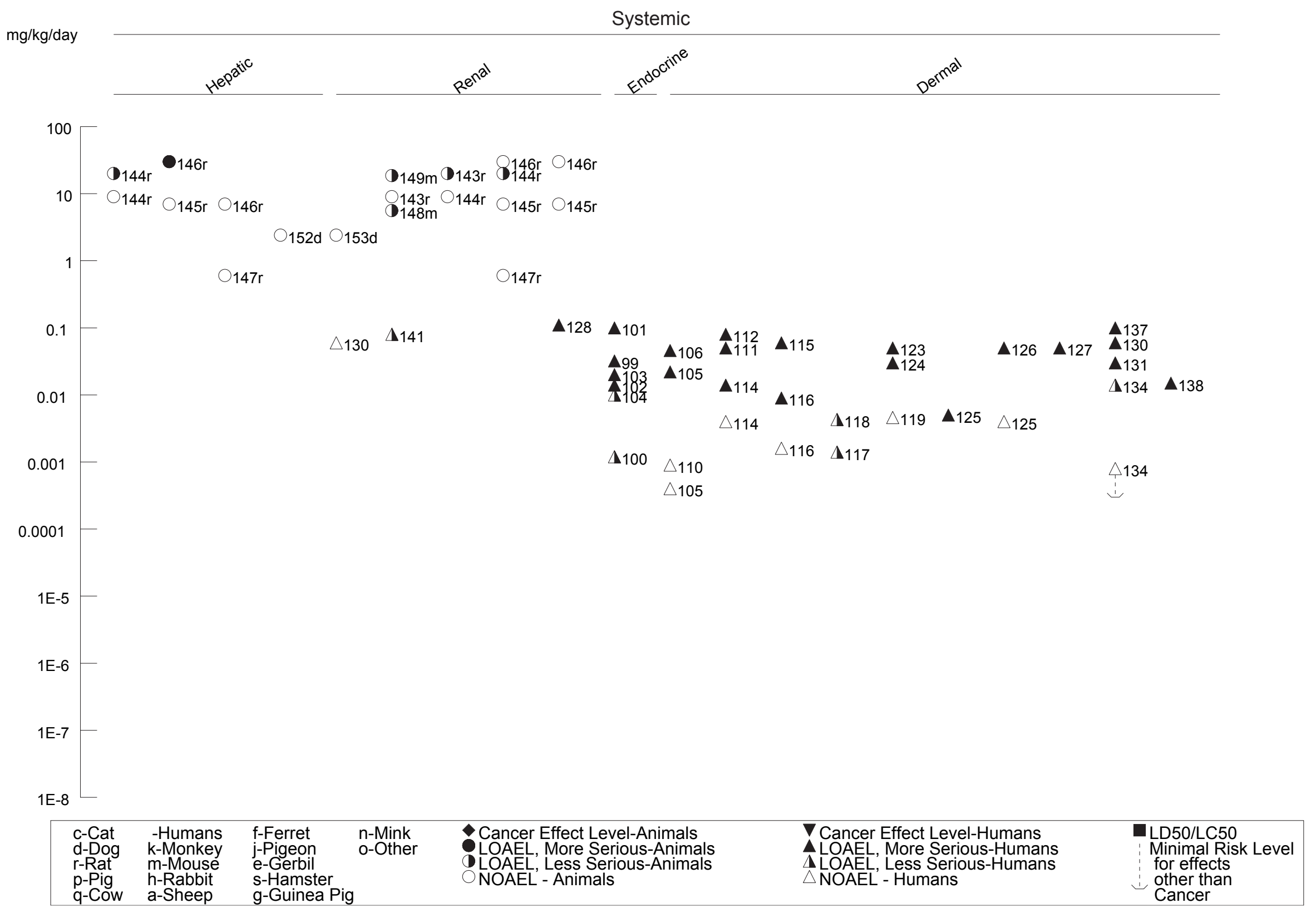


Figure 3-3 Levels of Significant Exposure to Inorganic Arsenic - Oral (Continued)

Chronic ( $\geq 365$ days)

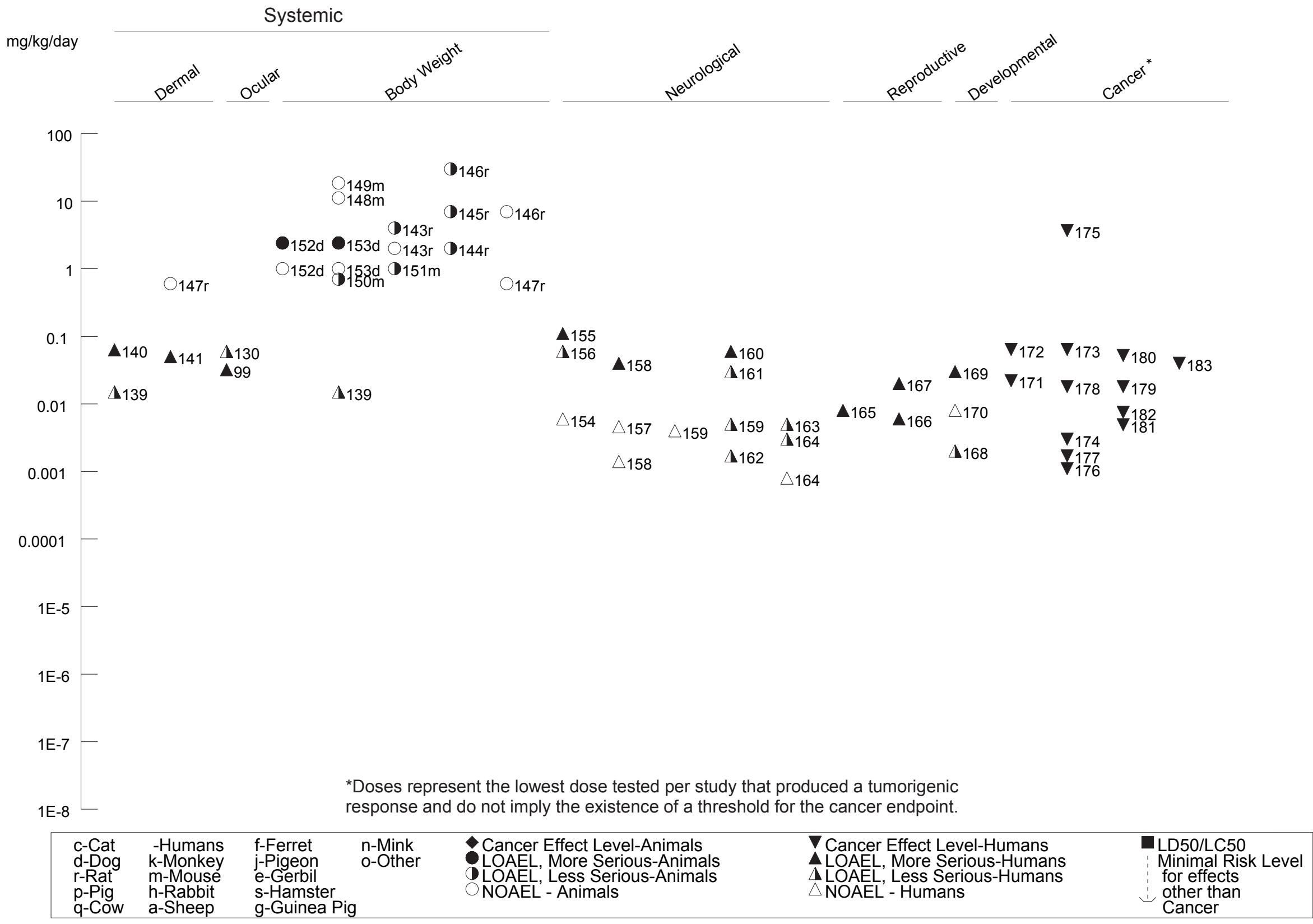

${ }^{*}$ Doses represent the lowest dose tested per study that produced a tumorigenic esponse and do not imply the existence of a threshold for the cancer endpoint.

\begin{tabular}{|c|c|c|c|c|c|c|}
\hline $\begin{array}{l}\text { c-Cat } \\
\text { d-Dog } \\
\text { r-Rat } \\
\text { p-Pig } \\
\text { q-Cow }\end{array}$ & $\begin{array}{l}\text {-Humans } \\
\text { k-Monkey } \\
\text { m-Mouse } \\
\text { h-Rabbit } \\
\text { a-Sheep }\end{array}$ & $\begin{array}{l}\text { f-Ferret } \\
\text { j-Pigeon } \\
\text { e-Gerbil } \\
\text { s-Hamster } \\
\text { g-Guinea Pig }\end{array}$ & $\begin{array}{l}\text { n-Mink } \\
\text { o-Other }\end{array}$ & $\begin{array}{l}\text { Cancer Effect Level-Animals } \\
\text { LOAEL, More Serious-Animals } \\
\text { LOAEL, Less Serious-Animals } \\
\text { NOAEL - Animals }\end{array}$ & $\begin{array}{l}\nabla \text { Cancer Effect Level-Humans } \\
\Delta \text { LOAEL, More Serious-Humans } \\
\Delta \text { LOAEL, Less Serious-Humans } \\
\triangle \text { NOAEL - Humans }\end{array}$ & $\begin{array}{l}\text { LD50/LC50 } \\
\text { Minimal Risk Level } \\
\text { for effects } \\
\text { other than } \\
\text { Cancer }\end{array}$ \\
\hline
\end{tabular}


Figure 3-3 Levels of Significant Exposure to Inorganic Arsenic - Oral (Continued)

Chronic ( $\geq 365$ days)

$\mathrm{mg} / \mathrm{kg} /$ day

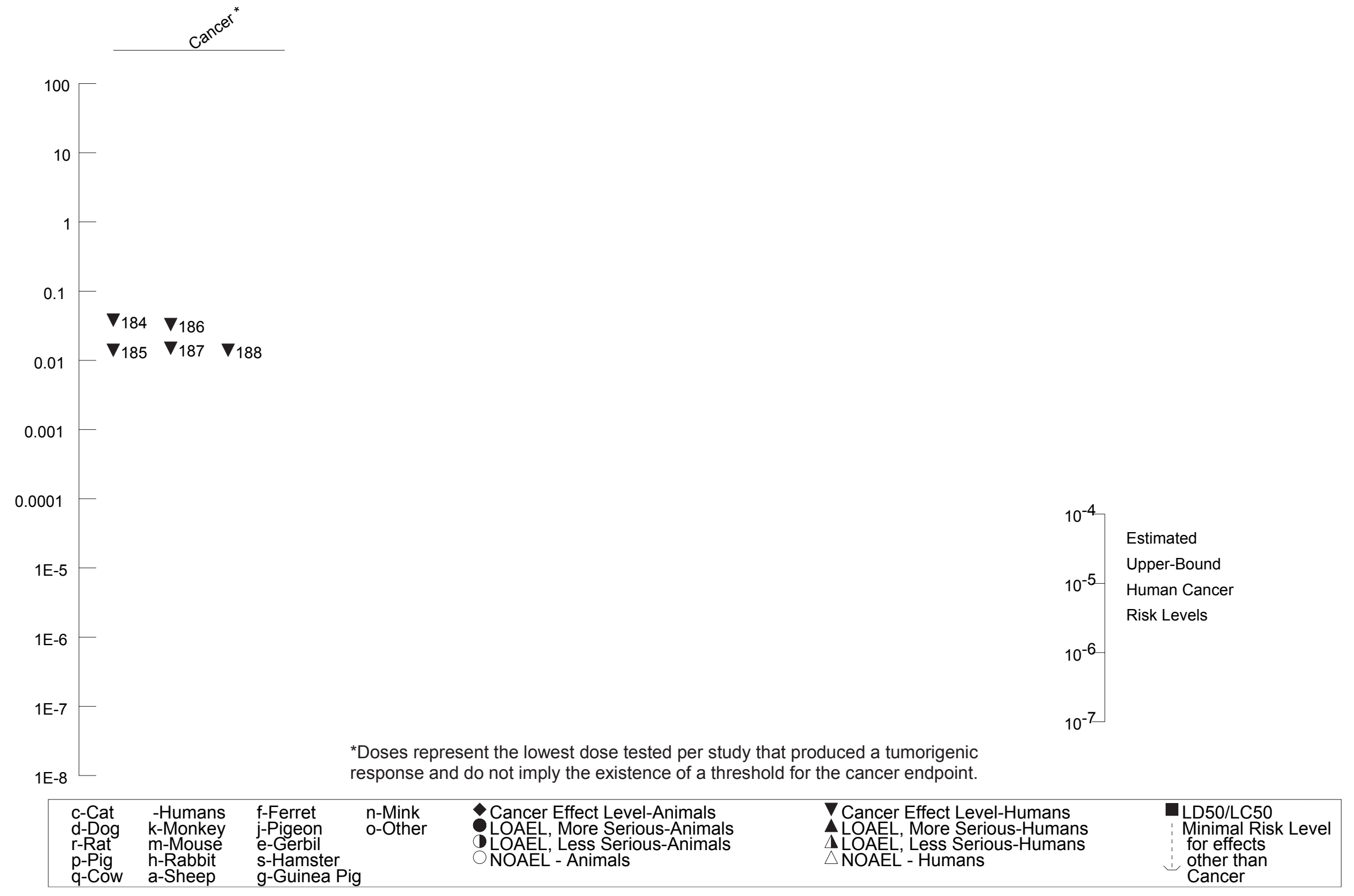

${ }^{*}$ Doses represent the lowest dose tested per study that produced a tumorigenic esponse and do not imply the existence of a threshold for the cancer endpoint. 
Table 3-4 Levels of Significant Exposure to Monomethylarsonic Acid - Oral

\begin{tabular}{|c|c|c|}
\hline $\begin{array}{l}\text { Key to } \\
\text { Figure }\end{array}$ & $\begin{array}{l}\text { Species } \\
\text { (Strain) }\end{array}$ & $\begin{array}{r}\text { Expo } \\
\text { Dura } \\
\text { Frequ } \\
\text { (Ro }\end{array}$ \\
\hline \multicolumn{3}{|c|}{$\begin{array}{l}\text { ACUTE EXPOSURE } \\
\text { Death }\end{array}$} \\
\hline 1 & $\begin{array}{l}\text { Rat } \\
\text { (Sprague- } \\
\text { Dawley) }\end{array}$ & $\begin{array}{l}\text { onc } \\
\text { (GV }\end{array}$ \\
\hline 2 & $\begin{array}{l}\text { Mouse } \\
(\mathrm{dd} Y)\end{array}$ & $\begin{array}{l}\text { onc } \\
\text { (GV }\end{array}$ \\
\hline 3 & $\begin{array}{l}\text { Rabbit } \\
\text { (New } \\
\text { Zealand) }\end{array}$ & $\begin{array}{l}\text { onc } \\
\text { (GV }\end{array}$ \\
\hline
\end{tabular}

OAEL$$
\text { Duration/ }
$$

(Route)

System

NOAEL

Less Serious

(mg/kg/day)

(mglkglday)

Reference

Chemical Form

Comments

Systemic

4 Rat once

(Sprague- (GW)

Dawley)

Gastro

(mucoid feces and
diarrhea)

Bd Wt 2030

5 Rat Gd 6-15

(Sprague- (GW)

$\mathrm{Bd} \mathrm{Wt}$

Dawley)

Bd

$100 \mathrm{~F}$ ( $17 \%$ decrease in

maternal body weight

gain)

$\begin{array}{ll}3184 \mathrm{M} \mathrm{(LD50)} & \text { Gur and Nyska } 1990 \\ \text { b } & \text { MSMA }\end{array}$

$2449 \mathrm{~F}$ (LD50)

1800 M (LD50)

102 M (LD50)

MMA

Jaghabir et al. 1988

MSMA

Gur and Nyska 1990

MSMA

$500 \mathrm{~F}(40 \%$ decrease in maternal body weigh gain)

Irvine et al. 2006

MMA

$1800 \mathrm{M}$ (respiratory arrest)

Kaise et al. 1989

MMA

$\begin{array}{lcc}\begin{array}{l}\text { Mouse } \\ (d d Y)\end{array} & \begin{array}{l}\text { once } \\ (G W)\end{array} & \text { Resp } \\ & \text { Gastro } & 2200 \mathrm{M} \text { (diarrhea, slight } \\ & & \begin{array}{c}\text { congestion of the small } \\ \text { intestine) }\end{array}\end{array}$

congestion of the small 
Table 3-4 Levels of Significant Exposure to Monomethylarsonic Acid - Oral

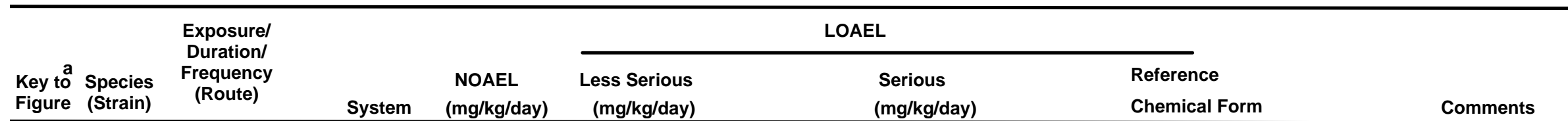

$\begin{array}{llll} & \text { Rabbit } & \text { Gd 7-19 } & \text { Gastro } \\ & \text { (New } & (\mathrm{GW}) & \end{array}$

(New

$\mathrm{Bd} W \mathrm{t}$

$7 \mathrm{~F}$

$\begin{array}{lll}\text { Rabbit } & \text { once } & \text { Gastro } \\ \text { (New } & (\mathrm{GW}) & \\ \text { Zealand) } & & \end{array}$

Developmental

9 Rat

(Sprague-

Gd 6-15

Dawley)

$(\mathrm{GW})$

100

Zealand)

7

12 (supernumerary thoracic ribs and eight lumbar vertebrae)

$60 \mathrm{M}$ (diarrhea)

500 (decreased fetal weight and increased fetal

incidence of imcomplete

ossification of thoracic

vertebrae)

(mg/kg/day)

Chemical Form

Comments

Irvine et al. 2006

MMA
$12 \mathrm{~F} \quad(67 \%$ decrease in maternal body weight gain)

Jaghabir et al. 1988 MSMA

Irvine et al. 2006

MMA

\section{INTERMEDIATE EXPOSURE}

Death
11 Rat
52 wk
(Fischer- 344) (F)

106.9 M (increased mortality)

Irvine et al. 2006

MMA

Arnold et al. 2003

MMA 
Table 3-4 Levels of Significant Exposure to Monomethylarsonic Acid - Oral

\begin{tabular}{|c|c|c|c|c|c|}
\hline $\begin{array}{l}\text { Key to } \\
\text { Figure }\end{array}$ & $\begin{array}{c}\text { Species } \\
\text { (Strain) }\end{array}$ & $\begin{array}{c}\text { Exposurel } \\
\text { Duration/ } \\
\text { Frequency } \\
\text { (Route) }\end{array}$ & System & $\begin{array}{l}\text { NOAEL } \\
\text { (mg/kg/day) }\end{array}$ & $\begin{array}{r}\text { Less Serious } \\
(\mathrm{mg} / \mathrm{kg} / \mathrm{day})\end{array}$ \\
\hline \multicolumn{6}{|c|}{ Systemic } \\
\hline \multirow[t]{2}{*}{12} & $\begin{array}{l}\text { Rat } \\
\text { (Fischer- }\end{array}$ & $\begin{array}{l}52 \mathrm{wk} \\
\text { 4) }(F)\end{array}$ & Gastro & $3.5^{\mathrm{C}} \mathrm{M}$ & $30.2 \mathrm{M}$ (diarrhea) \\
\hline & & & $\mathrm{Bd} \mathrm{Wt}$ & $30.2 \mathrm{M}$ & $\begin{array}{c}106.9 \mathrm{M} \text { (14\% decrease in body } \\
\text { weight) }\end{array}$ \\
\hline
\end{tabular}

LOAEL

\begin{tabular}{ll}
\hline Serious & Reference \\
(mg/kg/day) & Chemical Form
\end{tabular}

13

Rat

Dawley)

146-171 d

pre-mating,

mating,

estation, and

lactation

(F)

14 Dog

(Beagle) (C)

(C)

\begin{tabular}{|c|c|c|}
\hline Gastro & & $2 \mathrm{M}$ (diarrhea) \\
\hline $\mathrm{Bd} \mathrm{Wt}$ & $2 \mathrm{~F}$ & $\begin{array}{l}8 \mathrm{~F} \text { (decrease in body } \\
\text { weight) }\end{array}$ \\
\hline
\end{tabular}

Reproductive

15 Rat

Dawley) mating,

gestation, and

lactation

(F)

$\begin{array}{ll}\text { Mouse } & 19 d \\ \text { (Swiss) } & 3 d / w k \\ & (G W)\end{array}$

Bd Wt $\quad 76$
76 (decreased pregnancy
rate and male fertility
index in F0 and F1)

Chemical Form

Arnold et al. 2003

MMA

Schroeder 1994

MMA

Waner and Nyska 1988

MMA

Schroeder 1994

MMA

Prukop and Savage 1986 MSMA 
Table 3-4 Levels of Significant Exposure to Monomethylarsonic Acid - Oral

(continued)

\begin{tabular}{|c|c|c|}
\hline $\begin{array}{l}\text { Key to } \\
\text { Figure }\end{array}$ & $\begin{array}{l}\text { Species } \\
\text { (Strain) }\end{array}$ & $\begin{array}{l}\text { Exposurel } \\
\text { Duration/ } \\
\text { Frequency } \\
\text { (Route) }\end{array}$ \\
\hline \multicolumn{3}{|c|}{ Developmental } \\
\hline 17 & $\begin{array}{l}\text { Rat } \\
\text { (Sprague- } \\
\text { Dawley) }\end{array}$ & $\begin{array}{l}146-171 \mathrm{~d} \\
\text { pre-mating, } \\
\text { mating, } \\
\text { gestation, and } \\
\text { lactation } \\
\text { (F) }\end{array}$ \\
\hline
\end{tabular}

LOAEL

\section{CHRONIC EXPOSURE}

\section{Death}

18 Rat $104 \mathrm{wk}$

(Fischer- 344) (F)

\begin{tabular}{ccc} 
& & \\
\cline { 3 - 3 } System & $\begin{array}{c}\text { NOAEL } \\
(\mathrm{mg} / \mathrm{kg} / \text { day })\end{array}$ & $\begin{array}{c}\text { Less Serious } \\
(\mathrm{mg} / \mathrm{kg} / \text { day })\end{array}$ \\
\hline & & \\
22 & $76 \quad \begin{array}{l}\text { (decreased pup survival } \\
\text { F1 and F2) }\end{array}$ \\
& & \\
& &
\end{tabular}

Serious Reference

(mg/kg/day) Chemical Form

Comments

$\begin{array}{ll}\text { 72.4 M (increased mortality) } & \begin{array}{l}\text { Arnold et al. } 2003 \\ \text { MMA }\end{array}\end{array}$

Schroeder 1994

MMA 
Table 3-4 Levels of Significant Exposure to Monomethylarsonic Acid - Oral

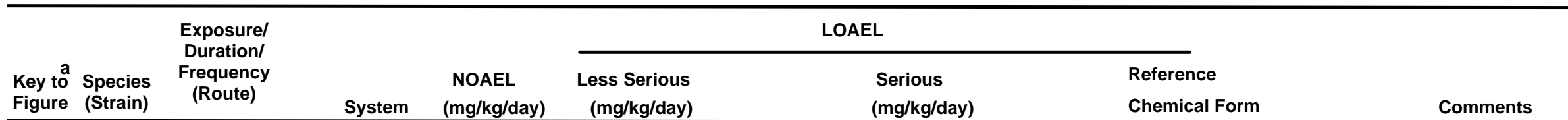

Systemic
19 Rat

Rat $\quad 104$ wk

(Fischer- 344) (F)

$\begin{array}{lr}\text { Gastro } & 3 \mathrm{M} \\ & \\ \text { Hemato } & 72.4 \mathrm{M} \\ \text { Musc/skel } & 72.4 \mathrm{M} \\ \text { Hepatic } & 72.4 \mathrm{M} \\ \text { Renal } & 3.9 \mathrm{~F}\end{array}$

25.7 M (diarrhea)

72.4 M (necrosis, ulceration,

perforation in large

intestine)

Arnold et al. 2003

MMA
33.9 F (increased absolute
kidney weight and
progressive
glomerulonephropathy)
Endocr $\quad 3.9 \mathrm{~F} \quad 33.9 \mathrm{~F}$ (hypertrophy of thyroid
follicular epithelium,
decreased absolute
thyroid weight)

$\begin{array}{lrr}\text { Dermal } & 72.4 \mathrm{M} \\ \text { Ocular } & 72.4 \mathrm{M} \\ \text { Bd Wt } & 3 \mathrm{M} & \begin{array}{c}25.7 \mathrm{M}\left(\begin{array}{l}15 \% \text { decrease in body } \\ \text { weight })\end{array}\right. \\ \end{array}\end{array}$


Table 3-4 Levels of Significant Exposure to Monomethylarsonic Acid - Oral

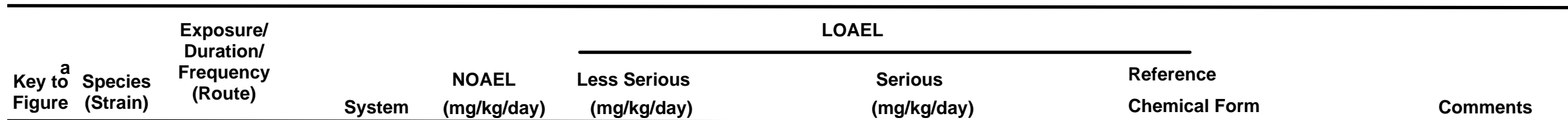

20 Rat (Fischer- 344) (W)

04 wk (Elscher-344) (W)

21 Mouse (B6C3F1)

104 wk

(F)

$\begin{array}{lr}\text { Hemato } & 8.4 \mathrm{M} \\ \text { Hepatic } & 2.1 \mathrm{M}\end{array}$

$$
2.1 \mathrm{M}
$$

Renal

Bd Wt

Cardio

Gastro

Gastro

Musc/skel

Hepatic

Renal

$67.1 \mathrm{M}$

$\begin{array}{ll}\text { Dermal } & 67.1 \mathrm{M} \\ \text { Ocular } & 67.1 \mathrm{M} \\ \text { Bd Wt } & 24.9 \mathrm{M}\end{array}$

$8.4 \mathrm{M}$

$67.1 \mathrm{M}$
$8.4 \mathrm{M}$ (increased

GST-P-positive foci)

2.1 M (hyperplasia of the bladder)

Arnold et al. 2003

MMA

hen et al. 2003

MMA

metaplasia of the cecum

and colon) 
Table 3-4 Levels of Significant Exposure to Monomethylarsonic Acid - Oral

(continued)

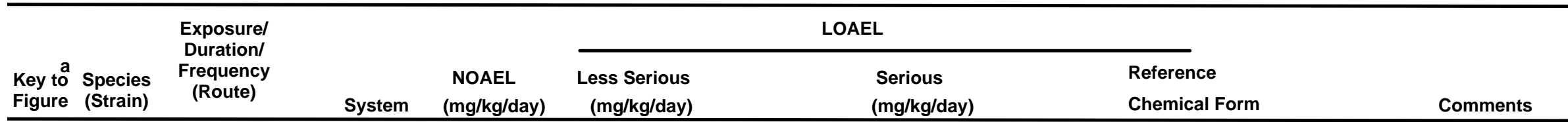

$\begin{array}{ll}\text { Dog } & 52 \\ \text { (Beagle) } & \text { (C) }\end{array}$

\section{$52 \mathrm{wk}$}

(Beagle)

35

Gastro

Hemato

Hepatic

Renal

Ocular

Bd Wt

\section{Neurological}

23 Dog

(Beagle)

52 wk

(C)

Reproductive

24 Dog

(Beagle) (C)

$52 \mathrm{wk}$
8 (increased urine specific gravity; increased kidney weight) $8 \mathrm{~F}$
(42\% decrease in body
weight)

Waner and Nyska 1988

MMA

Waner and Nyska 1988

MMA

$35 \mathrm{M}$
$8 \mathrm{~F}$

Waner and Nyska 1988

MMA 
Table 3-4 Levels of Significant Exposure to Monomethylarsonic Acid - Oral

(continued)

\begin{tabular}{|c|c|c|c|c|c|c|c|}
\hline \multirow[b]{2}{*}{$\begin{array}{l}\text { Key to } \\
\text { Figure }\end{array}$} & \multirow[b]{2}{*}{$\begin{array}{c}\text { Species } \\
\text { (Strain) }\end{array}$} & \multirow{2}{*}{$\begin{array}{l}\text { Exposurel } \\
\text { Durationl } \\
\text { Frequency } \\
\text { (Route) }\end{array}$} & \multirow[b]{2}{*}{ System } & \multirow[b]{2}{*}{$\begin{array}{l}\text { NOAEL } \\
\text { (mg/kg/day) }\end{array}$} & \multicolumn{2}{|c|}{ LOAEL } & \multirow[b]{2}{*}{$\begin{array}{l}\text { Reference } \\
\text { Chemical Form }\end{array}$} \\
\hline & & & & & $\begin{array}{r}\text { Less Serious } \\
(\mathrm{mg} / \mathrm{kg} / \text { day })\end{array}$ & $\begin{array}{l}\text { Serious } \\
\text { (mg/kg/day) }\end{array}$ & \\
\hline \multicolumn{8}{|l|}{ Cancer } \\
\hline 25 & $\begin{array}{l}\text { Rat } \\
\text { (Fischer- }\end{array}$ & $\begin{array}{l}104 \mathrm{wk} \\
4)(W)\end{array}$ & & $8.4 \mathrm{M}$ & & & $\begin{array}{l}\text { Shen et al. } 2003 \\
\text { MMA }\end{array}$ \\
\hline
\end{tabular}

a The number corresponds to entries in Figure 3-4.

b Differences in levels of health effects and cancer effects between male and females are not indicated in Figure 3-4. Where such differences exist, only the levels of effect for the most sensitive gender are presented.

c The intermediate-duration oral MRL of $0.1 \mathrm{mg} \mathrm{MMA} / \mathrm{kg} /$ day was calculated using a benchmark dose analysis. The BMDL10 of $12.38 \mathrm{mg}$ MMA/kg/day was divided by an uncertainty factor of 100 (10 to account for extrapolation from animals to humans and 10 for human variability)

d The chronic-duration oral MRL of $0.01 \mathrm{mg} \mathrm{MMA} / \mathrm{kg} /$ day was calculated using a benchmark dose analysis. The BMDL10 of $1.09 \mathrm{mg}$ MMA $/ \mathrm{kg} / \mathrm{day}$ was divided by an uncertainty factor of 100 (10 to account for extrapolation from animals to humans and 10 for human variability)

Bd Wt = body weight; $(\mathrm{C})$ = capsule; Cardio = cardiovascular; $\mathrm{d}$ = day $(\mathrm{s})$; Endocr = endocrine; $(\mathrm{F})=$ feed; $\mathrm{F}=$ female; Gastro = gastrointestinal; $(\mathrm{GO})=$ gavage in oil; $(\mathrm{GW})=$ gavage in water; Gd = gestation day; GST-P = glutathione S-transferase placental form; Hemato = hematological; IN = ingestion; LD50 = lethal dose, $50 \%$ kill; LOAEL =

lowest-observable-adverse-effect level; $\mathrm{M}=$ male; $\mathrm{MMA}=$ monomethylarsonic acid; $\mathrm{MSMA}=$ monosodium methane arsonate; Musc/skel = musculoskeletal; $\mathrm{NOAEL}=$

no-observable-adverse-effect level; Resp = respiratory; $(W)=$ drinking water; wk = week $(\mathrm{s}) ; \mathrm{x}=$ time $(\mathrm{s})$ 
Figure 3-4 Levels of Significant Exposure to Monomethylarsonic Acid - Oral

Acute ( $\leq 14$ days)

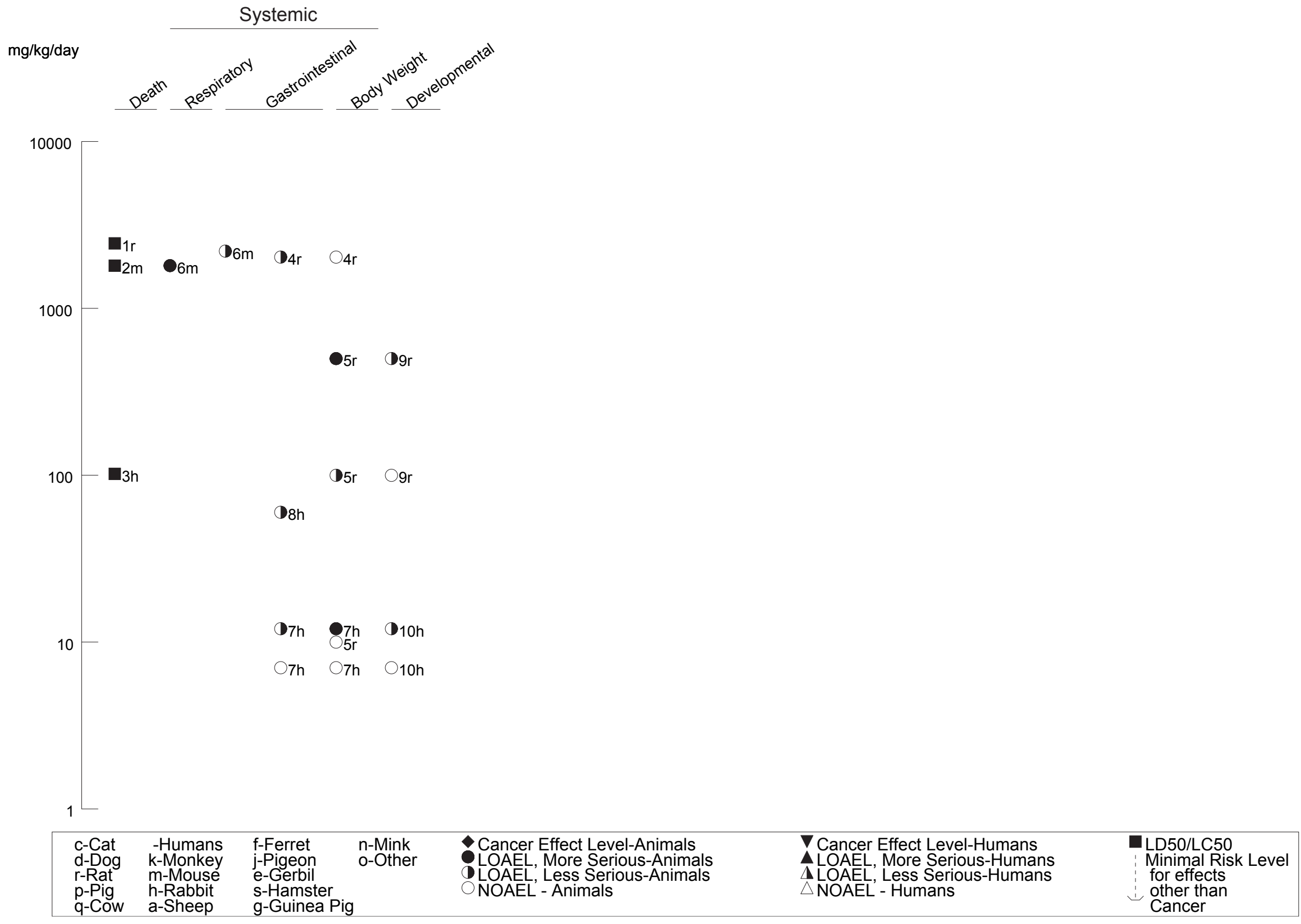


Figure 3-4 Levels of Significant Exposure to Monomethylarsonic Acid - Oral (Continued)

Intermediate (15-364 days)

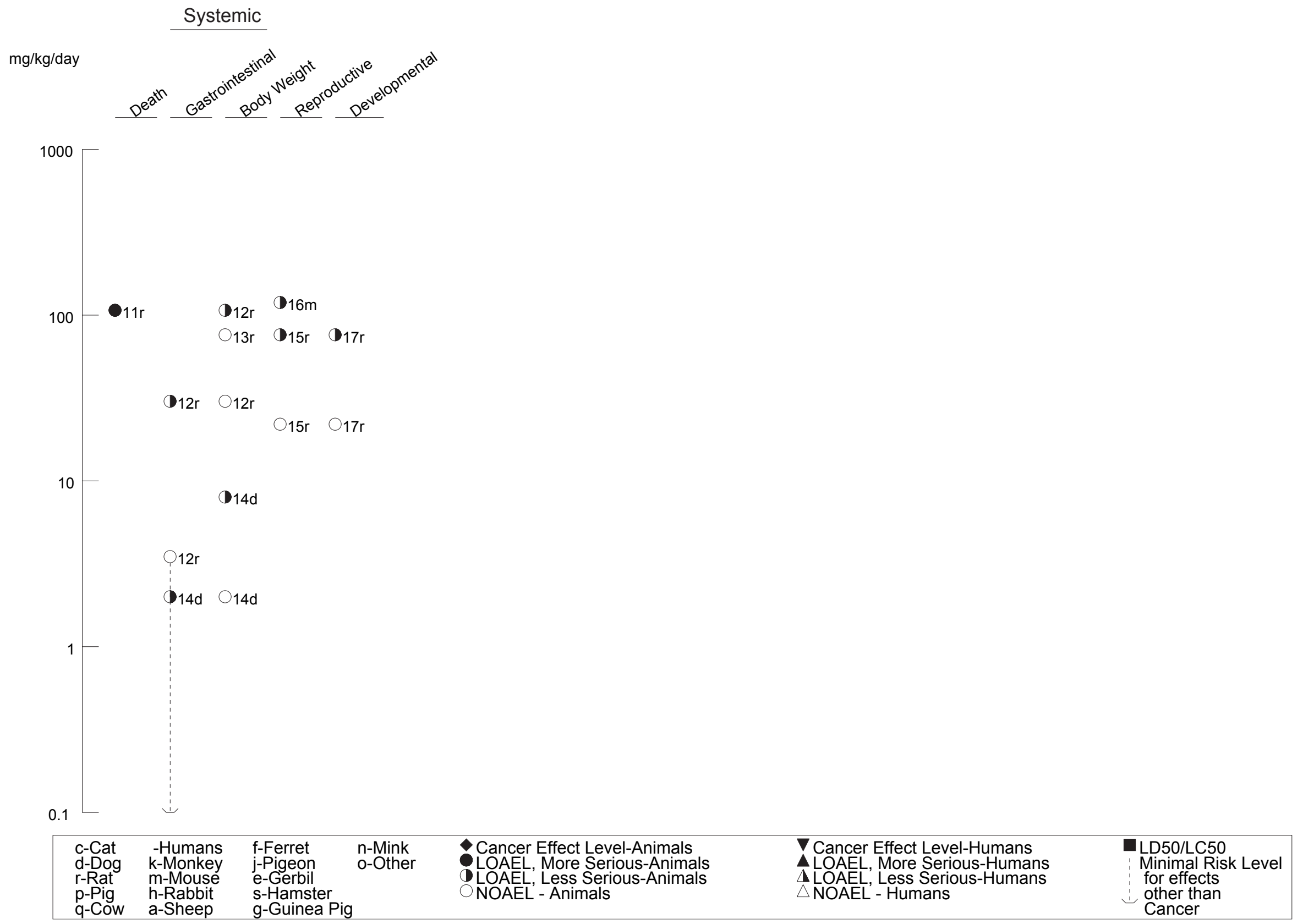


Figure 3-4 Levels of Significant Exposure to Monomethylarsonic Acid - Oral (Continued)

Chronic ( $\geq 365$ days)

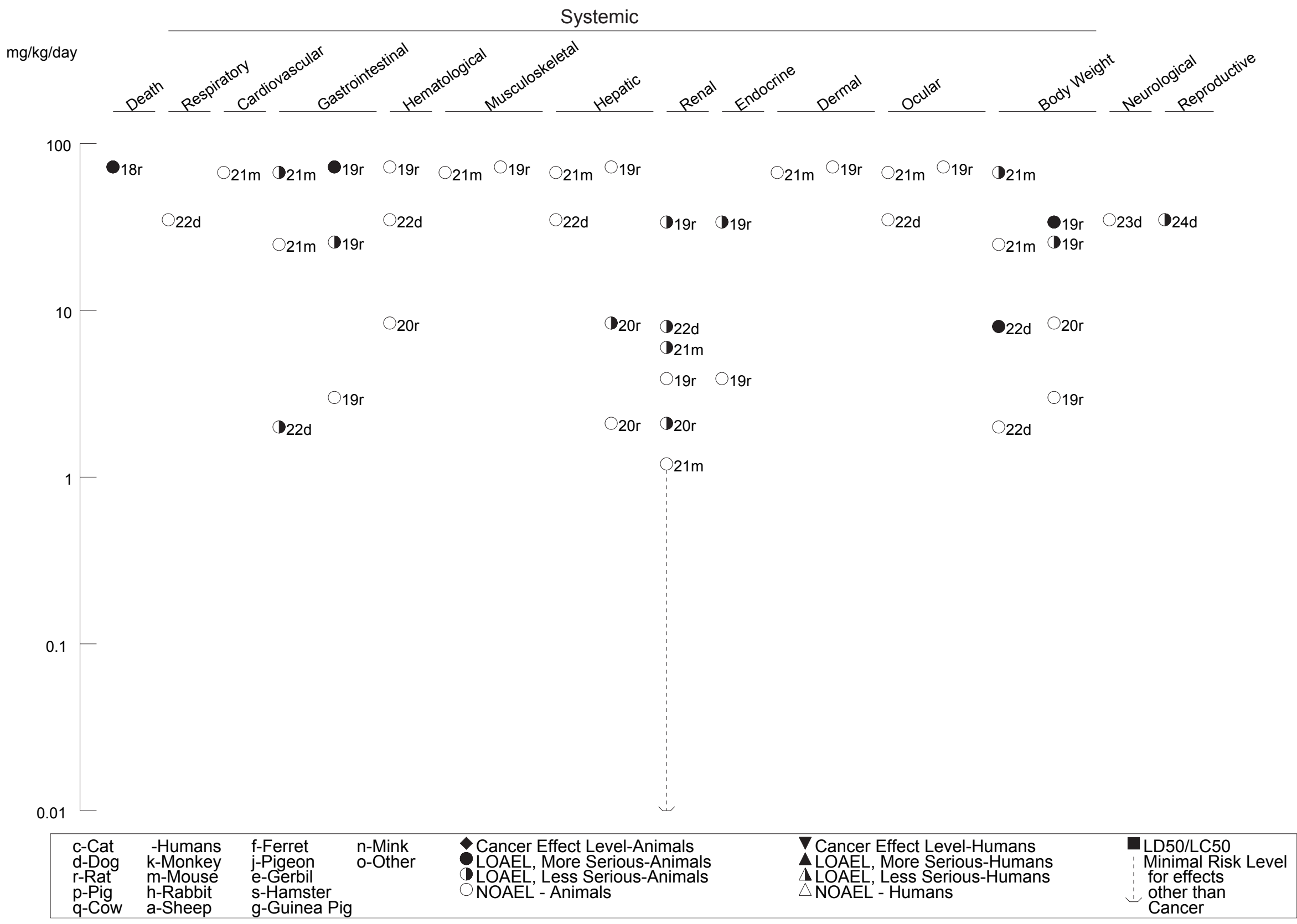


Figure 3-4 Levels of Significant Exposure to Monomethylarsonic Acid - Oral (Continued)

Chronic ( $\geq 365$ days)

$\mathrm{mg} / \mathrm{kg} / \mathrm{day}$

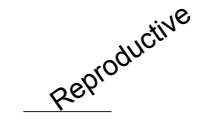

100

$\bigcirc 24 d$

10

$\mathrm{O}_{24 d}$

0.1

1

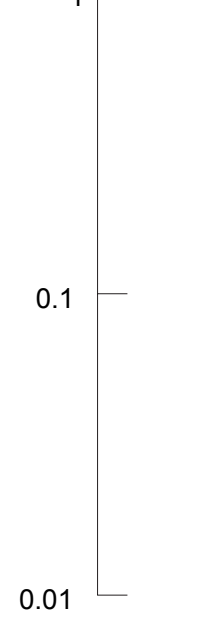

0.01

\begin{tabular}{|c|c|c|c|c|c|c|}
\hline $\begin{array}{l}\text { c-Cat } \\
\text { d-Dog } \\
\text { r-Rat } \\
\text { p-Pig } \\
\text { q-Cow }\end{array}$ & $\begin{array}{l}\text {-Humans } \\
\text { k-Monkey } \\
\text { m-Mouse } \\
\text { h-Rabbit } \\
\text { a-Sheep }\end{array}$ & $\begin{array}{l}\text { f-Ferret } \\
\text { j-Pigeon } \\
\text { e-Gerbil } \\
\text { s-Hamster } \\
\text { g-Guinea Pig }\end{array}$ & $\begin{array}{l}\text { n-Mink } \\
\text { o-Other }\end{array}$ & $\begin{array}{l}\text { Cancer Effect Level-Animals } \\
\text { LOAEL, More Serious-Animals } \\
\text { LOAEL, Less Serious-Animals } \\
\text { NOAEL - Animals }\end{array}$ & $\begin{array}{l}\nabla \text { Cancer Effect Level-Humans } \\
\Delta \text { LOAEL, More Serious-Humans } \\
\Delta \text { LOAEL, Less Serious-Humans } \\
\triangle \text { NOAEL - Humans }\end{array}$ & $\begin{array}{l}\text { LD50/LC50 } \\
\text { Minimal Risk Level } \\
\text { for effects } \\
\text { other than } \\
\text { Cancer }\end{array}$ \\
\hline
\end{tabular}

a-Sheep g-Guinea Pig 
Table 3-5 Levels of Significant Exposure to Dimethylarsinic Acid - Oral

\begin{tabular}{|c|c|c|c|c|c|c|c|c|}
\hline \multirow[b]{2}{*}{$\begin{array}{l}\text { Key to } \\
\text { Figure }\end{array}$} & \multirow[b]{2}{*}{$\begin{array}{l}\text { Species } \\
\text { (Strain) }\end{array}$} & \multirow{2}{*}{$\begin{array}{l}\text { Exposurel } \\
\text { Durationl } \\
\text { Frequency } \\
\text { (Route) }\end{array}$} & \multirow[b]{2}{*}{ System } & \multirow[b]{2}{*}{$\begin{array}{l}\text { NOAEL } \\
\text { (mg/kg/day) }\end{array}$} & \multicolumn{2}{|c|}{ LOAEL } & \multirow[b]{2}{*}{$\begin{array}{l}\text { Reference } \\
\text { Chemical Form }\end{array}$} & \multirow[b]{2}{*}{ Comments } \\
\hline & & & & & $\begin{array}{r}\text { Less Serious } \\
(\mathrm{mg} / \mathrm{kg} / \mathrm{day})\end{array}$ & $\begin{array}{l}\text { Serious } \\
\text { (mg/kg/day) }\end{array}$ & & \\
\hline
\end{tabular}

ACUTE EXPOSURE

Death

1 Rat

(Fischer- 344) (F)

2 Rat
(CD) Gd 7
(GW)

3 Mouse once

(ddY) (GW)

$4 \quad$ Mouse $10 d$

(CD-1) Gd 7-16

$1 \mathrm{X} / \mathrm{d}$
$(\mathrm{GW})$

Systemic

$5 \quad$ Rat 2 wk

(Fischer- 344) (F)

Bd Wt

$11 \mathrm{~F}$

$6 \quad$ Rat $13 w k$

(Fischer- 344) (F)
Gastro
475 M (100\% mortality during first 2 weeks of study)

Crown et al. 1987

DMA

$60 \mathrm{~F}(67 \%$ mortality $)$

Rogers et al. 1981

DMA

1200 M (LD50)

Kaise et al. 1989

DMA

600 F $(59 \%$ mortality $)$

Rogers et al. 1981

DMA

$11 \mathrm{~F}$ (altered bladder cell surface characteristics)

Cohen et al. 2001

DMA

Crown et al. 1987

DMA
$475 \mathrm{M}$ (diarrhea and congestion and hemorrhagic
contents in
gastrointestinal tract in
rats dying during first 2
weeks)


Table 3-5 Levels of Significant Exposure to Dimethylarsinic Acid - Oral

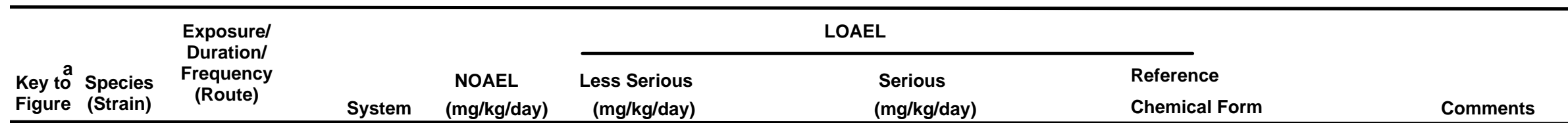

$7 \quad$ Rat

$\begin{array}{llccc}\begin{array}{l}\text { Rat } \\ \text { (Sprague- } \\ \text { Dawley) }\end{array} & \begin{array}{l}\text { Gd 6-15 } \\ (\mathrm{GW})\end{array} & \text { Bd Wt } & 12 \mathrm{~F} & 36 \mathrm{~F} \text { (decreased maternal } \\ \text { body weight gain) }\end{array}$

vine et al. 2006

$8 \quad$ Rat

$\begin{array}{lll}\text { Rat } & 10 d & \text { Bd Wt } \\ \text { (CD) } & \text { Gd 7-16 } & \\ & 1 \times / d & \\ & (G W) & \end{array}$

$9 \quad$ Mouse

$24 \mathrm{hr}$

(B6C3F1) 1 or $2 x$

Resp

$720 \mathrm{~F}$ (decreased lung ODC)

Ahmad et al. 1999a

DMA

Hepatic
$720 \mathrm{~F}$ (decreased liver GSH GSSG, CYP-450 and ODC; increased serum ALT)

$40 \mathrm{~F}(27 \%$ decreased maternal weight gain)

Rogers et al. 1981 DMA

$900 \mathrm{M}$ (respiratory arrest)

Kaise et al. 1989 DMA

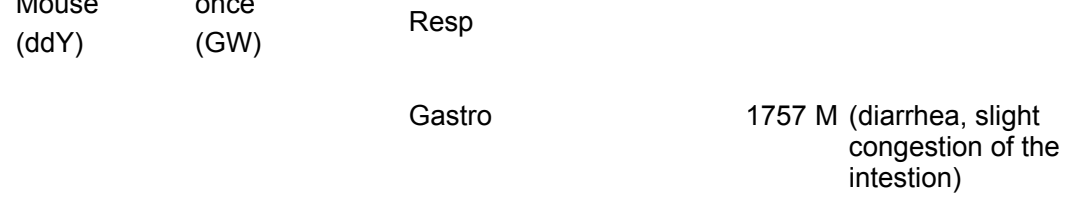

11 Mouse $10 \mathrm{~d}$

$\begin{array}{lll}\text { (CD-1) } & \text { Gd 7-16 } & \text { Bd Wt }\end{array}$

(GW)

52 wk Gastro

Gastro

6.5

16 (vomiting and diarrhea)

$200 \mathrm{~F}$ (26\% decreased maternal weight gain)

DMA

Zomber et al. 1989

DMA 
Table 3-5 Levels of Significant Exposure to Dimethylarsinic Acid - Oral

\begin{tabular}{|c|c|c|c|c|c|c|c|c|}
\hline \multirow[b]{2}{*}{$\begin{array}{l}\text { Key to } \\
\text { Figure }\end{array}$} & \multirow[b]{2}{*}{$\begin{array}{l}\text { Species } \\
\text { (Strain) }\end{array}$} & \multirow{2}{*}{$\begin{array}{l}\text { Exposurel } \\
\text { Durationl } \\
\text { Frequency } \\
\text { (Route) }\end{array}$} & \multicolumn{5}{|c|}{ LOAEL } & \multirow[b]{2}{*}{ Comments } \\
\hline & & & System & $\begin{array}{l}\text { NOAEL } \\
\text { (mg/kg/day) }\end{array}$ & $\begin{array}{l}\text { Less Serious } \\
\text { (mg/kg/day) }\end{array}$ & $\begin{array}{l}\text { Serious } \\
\text { (mg/kg/day) }\end{array}$ & $\begin{array}{l}\text { Reference } \\
\text { Chemical Form }\end{array}$ & \\
\hline
\end{tabular}

13 Rabbit Gd 7-19

$\begin{array}{lll}\text { Rabbit } & \text { Gd 7-19 } & \text { Gastro } \\ \text { (New } & \text { (GW) } & \end{array}$

Zealand)

$48 \mathrm{~F}$ (fluid gastrointestinal

tract contents)

Irvine et al. 2006

DMA

$\mathrm{Bd} \mathrm{Wt} \quad 12 \mathrm{~F}$

$48 \mathrm{~F}$ (maternal weight loss)

\section{Neurological}

14 Mouse once

(ddY) (GW)

Developmental

15 Rat

(Sprague- $\quad(\mathrm{GW})$

Dawley)

16 Rat

(Sprague- (GW)

Dawley)

live fetuses and fetal

weight; increases in

fetuses with

diaphragmatic hernia;

delayed ossification)

1757 M (increased startle reflex; Kaise et al. 1989 ataxia)

DMA

Chernoff et al. 1990

DMA

Irvine et al. 2006

DMA

30 (malformed palates in

$15 \%)$

Rogers et al. 1981

DMA

\%)

$\mathrm{x} / \mathrm{d}$

(GW)

$\begin{array}{ll}\text { Rat } & 10 \mathrm{~d} \\ \text { (CD) } & \text { Gd 7-16 } \\ & 1 \times / d \\ & (G W)\end{array}$

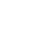


Table 3-5 Levels of Significant Exposure to Dimethylarsinic Acid - Oral

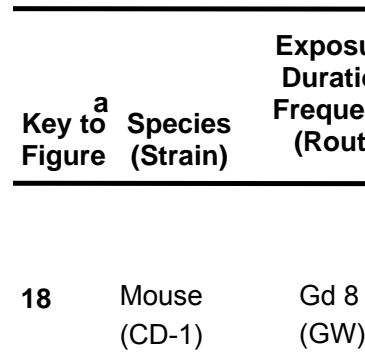

$\begin{array}{ll}\text { Mouse } & 10 \mathrm{~d} \\ \text { (CD-1) } & \text { Gd 7-16 } \\ & 1 \times / d \\ & (G W)\end{array}$

$\begin{array}{ll}\text { Rabbit } & \text { Gd 7-19 } \\ \text { (New } & (\mathrm{GW}) \\ \text { Zealand) } & \end{array}$

\section{INTERMEDIATE EXPOSURE}

Death
21 Rat
(Fischer- 344) (F)
22 Rat
$\begin{array}{ll}\text { Rat } & 4 \mathrm{wk} \\ \text { (Fischer- 344) } & 5 \mathrm{~d} / \mathrm{wk} \\ & 1 \mathrm{x} / \mathrm{d}\end{array}$

(G)
$400 \quad(18 \%$ decrease in fetal

weight, delayed

ossification, cleft palate

in $12 / 28$; irregular

Reference

Chemical Form

Comments

Kavlock et al. 1985

DMA

etal weight, delayed

ossification, skeleta

malformations)

palatine rugae in $4.8 \%$ )

Rogers et al. 1981

DMA
$90 \mathrm{M}(100 \%$ mortality during first 4 weeks of study)

Irvine et al. 2006

DMA

Crown et al. 1987

DMA

57 (50\% survival in males; Murai et al. 1993

$20 \%$ survival in females) DMA 
Table 3-5 Levels of Significant Exposure to Dimethylarsinic Acid - Oral

(continued)

\begin{tabular}{lll}
\hline & & $\begin{array}{r}\text { Exposu } \\
\text { Duration }\end{array}$ \\
$\begin{array}{l}\text { Key to } \\
\text { Figure }\end{array}$ & $\begin{array}{r}\text { Species } \\
\text { (Strain) }\end{array}$ & $\begin{array}{r}\text { Freque } \\
\text { (Route) }\end{array}$ \\
\hline & & \\
23 & $\begin{array}{l}\text { Rat } \\
\text { (Fischer- 344) (W) }\end{array}$
\end{tabular}

LAEL

Reference

uration/

Route)

NOAEL

Less Serious

System (mg/kg/day) (mg/kg/day)

(mg/kg/day)

Chemical Form

Comments

Systemic

24 Rat

10 or $20 \mathrm{wk}$

(Fischer- 344) (F)

Renal

$1 \mathrm{~F}$

$10 \mathrm{M}$ (necrosis in bladder

epithelium)

17 M (10/10 died)

Wanibuchi et al. 1996

DMA

Arnold et al. 1999

DMA

${ }_{5}^{b} \mathrm{~F}$ (increased kidney weight, calcification at

calcification at

increased bladder weight

and increased BrdU

labelling in bladder

epithelium)

$25 \quad$ Rat $10 \mathrm{wk}$

(Fischer- 344) (F)
$11 \mathrm{~F}$ (increased bladder and

kidney weights,

hyperplasia and necrosis

of bladder epithelium)
Cohen et al. 2001

DMA 
Table 3-5 Levels of Significant Exposure to Dimethylarsinic Acid - Oral

\begin{tabular}{|c|c|c|c|c|c|c|c|c|}
\hline \multirow[b]{2}{*}{$\begin{array}{l}\text { Key to } \\
\text { Figure }\end{array}$} & \multirow[b]{2}{*}{$\begin{array}{l}\text { Species } \\
\text { (Strain) }\end{array}$} & \multirow{2}{*}{$\begin{array}{l}\text { Exposurel } \\
\text { Durationl } \\
\text { Frequency } \\
\text { (Route) }\end{array}$} & \multicolumn{5}{|c|}{ LOAEL } & \\
\hline & & & System & $\begin{array}{l}\text { NOAEL } \\
\text { (mg/kg/day) }\end{array}$ & $\begin{array}{r}\text { Less Serious } \\
\text { (mg/kg/day) }\end{array}$ & $\begin{array}{l}\text { Serious } \\
\text { (mg/kg/day) }\end{array}$ & $\begin{array}{l}\text { Reference } \\
\text { Chemical Form }\end{array}$ & Comments \\
\hline
\end{tabular}

26 Rat

(Fischer- 344) (F)

\section{$3 \mathrm{wk}$}

Resp

Hepatic

Renal

Endocr

$\mathrm{Bd} W \mathrm{t}$

Renal

Bd Wt
43.2 M

$43.2 \mathrm{M}$

$43.2 \mathrm{M}$

$90 \mathrm{M}$ (diarrhea and congestion

and hemorrhagic

contents in

gastrointestinal tissues)

0.44 F $\quad$ 4.5 F (decreased hemoglobin

and erythrocyte levels)

$23.5 \mathrm{M}$

$0.4 \mathrm{M}$

$4 \mathrm{M}$ (increased urine volume and decreased specific gravity)

$0.4 \mathrm{M}$

$4 \mathrm{M}$ (hypertrophy of thyroid follicle epithelium)

$43.2 \mathrm{M}$

57 (decreased body weight)

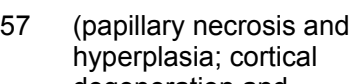

degeneration and

necrosis)
Crown et al. 1987

DMA 
Table 3-5 Levels of Significant Exposure to Dimethylarsinic Acid - Oral

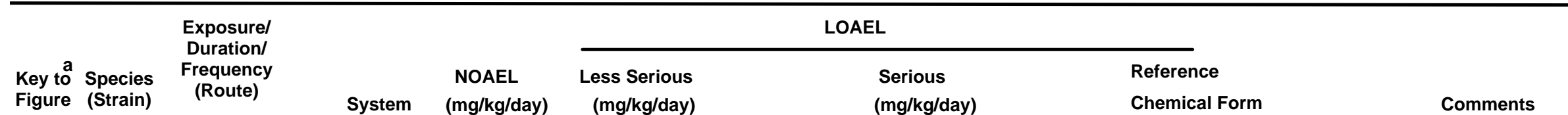

28 Rat

$\begin{array}{lll}\text { Rat } & 10 \text { wk } & \text { Resp } \\ \text { (Sprague- } & \text { pre-mating, } & \text { gestation and } \\ \text { Dawley) } & \text { lactation } \\ & \text { periods } \\ & \text { (F) }\end{array}$

Resp $\quad 16.5$

\begin{tabular}{|c|c|c|}
\hline Cardio & 16.5 & \\
\hline Hemato & $0.34 \mathrm{M}$ & $\begin{array}{l}2.3 \mathrm{M} \text { (decreased mean } \\
\text { corpscular hemoglobin } \\
\text { concentration) }\end{array}$ \\
\hline Hepatic & 16.5 & \\
\hline Renal & 16.5 & \\
\hline Endocr & $2.3 \mathrm{~F}$ & $\begin{array}{c}16.5 \mathrm{~F} \text { (hypertrophy of thyroid } \\
\text { follicle epithelium) }\end{array}$ \\
\hline $\mathrm{Bd} W \mathrm{t}$ & 16.5 & \\
\hline
\end{tabular}

29 Rat

(Sprague-

$42 \mathrm{~d}$

Dawley)
Hemato

$3.7 \mathrm{M}$

Hepatic

Renal

Bd Wt

$3.7 \mathrm{M}$

$3.7 \mathrm{M}$
Rubin et al. 1989

DMA

Siewicki 1981

DMA 
Table 3-5 Levels of Significant Exposure to Dimethylarsinic Acid - Oral

\begin{tabular}{|c|c|c|c|c|c|}
\hline $\begin{array}{l}\text { Key to } \\
\text { Figure }\end{array}$ & $\begin{array}{l}\text { Species } \\
\text { (Strain) }\end{array}$ & $\begin{array}{l}\text { Exposurel } \\
\text { Duration/ } \\
\text { Frequency } \\
\text { (Route) }\end{array}$ & System & $\begin{array}{l}\text { NOAEL } \\
\text { (mg/kg/day) }\end{array}$ & $\begin{array}{r}\text { Less Serious } \\
(\mathrm{mg} / \mathrm{kg} / \mathrm{day}) \\
\end{array}$ \\
\hline \multirow[t]{2}{*}{30} & $\begin{array}{l}\text { Dog } \\
\text { (Beagle) }\end{array}$ & $\begin{array}{l}6 \mathrm{~d} / \mathrm{wk} \\
52 \mathrm{wk} \\
\text { (C) }\end{array}$ & Gastro & 6.5 & (vomiting and diarrhea) \\
\hline & & & Hemato & $16 \mathrm{M}$ & $\begin{array}{l}40 \mathrm{M} \text { (decreased erythrocyte } \\
\text { and increased leukocyte } \\
\text { levels) }\end{array}$ \\
\hline
\end{tabular}

LOAEL

Reference

$\begin{array}{ll}\text { Serious } & \text { Reference } \\ (\mathrm{mg} / \mathrm{kg} / \mathrm{day}) & \text { Chemical Form }\end{array}$

Comments

Reproductive

31 Rat

$\begin{array}{ll}\text { Rat } & 10 \text { wk } \\ \text { (Sprague- } & \text { pre-mating, } \\ \text { Dawley) } & \text { gestation and } \\ & \text { lactation } \\ & \text { periods }\end{array}$

and increased leukocyte

levels)

Zomber et al. 1989

DMA

( $F)$

Cancer

32 Mouse $50 \mathrm{wk}$

A/J ad lib

(W)

10.4 M (CEL: lung tumors)

Hayashi et al. 1998

DMA

Rubin et al. 1989

DMA 
Table 3-5 Levels of Significant Exposure to Dimethylarsinic Acid - Oral

(continued)

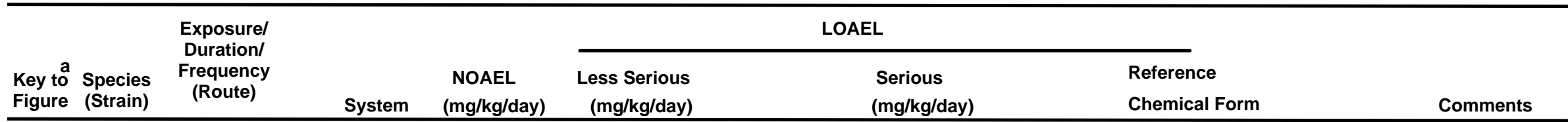

\section{CHRONIC EXPOSURE}

\section{Systemic}

33 Rat

Rat daily (Fischer- 344) 2 yr

Resp

7.8

$(\mathrm{F})$

$(F)$

$\begin{array}{ll}\text { Cardio } & 7.8 \\ \text { Gastro } & 7.8 \\ \text { Hemato } & 7.8 \\ \text { Musc/skel } & 7.8 \\ \text { Hepatic } & 7.8\end{array}$

Renal $\quad 0.77 \mathrm{M}$

3.1 M (nephrocalcinosis)

$0.77 \mathrm{~F}$

3.1 $\mathrm{F}$ (urothelial vacular

degeneration and

hyperplasia of urothelial

cells in urinary bladder)

$\begin{array}{llll}\text { Endocr } & 3.1 & 7.8 & \begin{array}{l}\text { (hypertrophy of thyroid } \\ \text { follicle epithelium) }\end{array}\end{array}$

Dermal $\quad 7.8$

Ocular $\quad 7.8$

$\mathrm{Bd} \mathrm{Wt} \quad 7.8$

34 Rat

104 wk

(Fischer- 344) (W)

Renal

$0.75 \mathrm{M}$

3.4 M (nodular or papillary

hyperplasia in urinary

(mg/kg/day)

Chemical Form

Comments

Arnold et al. 2006

DMA 
Table 3-5 Levels of Significant Exposure to Dimethylarsinic Acid - Oral

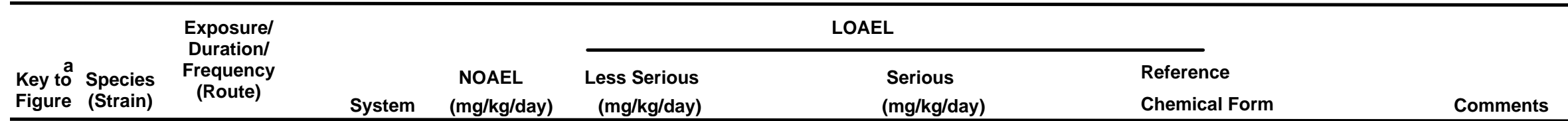

$35 \quad$ Mouse

$\begin{array}{lll}\text { daily } & \text { Resp }\end{array}$

(B6C3F1) $2 \mathrm{yr}$

94

$\begin{array}{ll}\text { Cardio } & 94 \\ \text { Gastro } & 94 \\ \text { Hemato } & 94\end{array}$

$94 \mathrm{~F}$ (decreased lymphocytes and increased monocytes)

Musc/skel $\quad 94$

Hepatic 94

Renal $\quad 1.3^{\mathrm{C}} \mathrm{F}$

\section{$37 \mathrm{M}$ (progressive}

glomerulonephropathy)

7.8 $\mathrm{F}$ (vacuolization of

superficial cells of

urotheliumin urinary

bladder)

Arnold et al. 2006

DMA

$\begin{array}{ll}\text { Dermal } & 94 \\ \text { Ocular } & 94 \\ \text { Bd Wt } & 94\end{array}$


Table 3-5 Levels of Significant Exposure to Dimethylarsinic Acid - Oral

\begin{tabular}{|c|c|c|c|c|c|}
\hline $\begin{array}{l}\text { Key to } \\
\text { Figure }\end{array}$ & $\begin{array}{l}\text { Species } \\
\text { (Strain) }\end{array}$ & $\begin{array}{c}\text { Exposurel } \\
\text { Durationl } \\
\text { Frequency } \\
\text { (Route) }\end{array}$ & System & $\begin{array}{l}\text { NOAEL } \\
(\mathrm{mg} / \mathrm{kg} / \text { day })\end{array}$ & $\begin{array}{r}\text { Less Serious } \\
(\mathrm{mg} / \mathrm{kg} / \text { day })\end{array}$ \\
\hline \multirow[t]{5}{*}{36} & $\begin{array}{l}\text { Dog } \\
\text { (Beagle) }\end{array}$ & $\begin{array}{l}6 \mathrm{~d} / \mathrm{wk} \\
52 \mathrm{wk} \\
\text { (C) }\end{array}$ & Resp & 40 & \\
\hline & & & Cardio & 40 & \\
\hline & & & Gastro & 6.5 & (vomiting and diarrhea) \\
\hline & & & Hepatic & 40 & \\
\hline & & & Renal & 40 & \\
\hline
\end{tabular}

\section{Reproductive}

37 Rat daily

(Fischer- 344) 2 yr

38 Mouse daily

\section{Cancer}

$39 \quad$ Rat

Rat daily
(Fischer- 344) 2 yr

$(\mathrm{F})$

40 Rat

104 wk

(Fischer- 344) (W)

LOAEL

Serious

(mg/kg/day)

Reference

Chemical Form

Comments

Zomber et al. 1989

DMA

Histological

Arnold et al. 2006

DMA

reproductive tissues.

Arnold et al. 2006

DMA

Histological

examination of

reproductive tissues.
7.8 (CEL: urothelial cell papillomas and

carcinomas in urinary bladder)

3.4 M (CEL: urinary bladder tumors)
Arnold et al. 2006

DMA

Wei et al. 1999, 2002

DMA 
Table 3-5 Levels of Significant Exposure to Dimethylarsinic Acid - Oral

(continued)

\begin{tabular}{|c|c|c|c|c|c|c|c|}
\hline \multirow[b]{2}{*}{$\begin{array}{l}\text { Key to } \\
\text { Figure }\end{array}$} & \multirow[b]{2}{*}{$\begin{array}{l}\text { Species } \\
\text { (Strain) }\end{array}$} & \multirow{2}{*}{$\begin{array}{l}\text { Exposurel } \\
\text { Durationl } \\
\text { Frequency } \\
\text { (Route) }\end{array}$} & \multirow[b]{2}{*}{ System } & \multirow[b]{2}{*}{$\begin{array}{l}\text { NOAEL } \\
\text { (mg/kg/day) }\end{array}$} & \multicolumn{2}{|c|}{ LOAEL } & \multirow[b]{2}{*}{$\begin{array}{l}\text { Reference } \\
\text { Chemical Form }\end{array}$} \\
\hline & & & & & $\begin{array}{l}\text { Less Serious } \\
\text { (mg/kg/day) }\end{array}$ & $\begin{array}{l}\text { Serious } \\
\text { (mg/kg/day) }\end{array}$ & \\
\hline 41 & $\begin{array}{l}\text { Mouse } \\
\text { knockout }\end{array}$ & $\begin{array}{l}\text { continuous } \\
18 \text { mo } \\
\text { (W) }\end{array}$ & & & & $11.8 \mathrm{M}(\mathrm{CEL})$ & $\begin{array}{l}\text { Salim et al. } 2003 \\
\text { DMA }\end{array}$ \\
\hline
\end{tabular}

a The number corresponds to entries in Figure 3-5.

b Differences in levels of health effects and cancer effects between male and females are not indicated in Figure 3-5. Where such differences exist, only the levels of effect for the most sensitive gender are presented.

c The chronic-duration oral MRL of $0.02 \mathrm{mg} \mathrm{DMA} / \mathrm{kg} /$ day was calculated using a benchmark dose analysis. The BMDL10 of $1.80 \mathrm{mg}$ DMA/kg/day was divided by an uncertainty factor of 100 (10 to account for extrapolation from animals to humans and 10 for human variability)

ad lib = ad libitum; $\mathrm{ALT}=$ alanine aminotransferase; $\mathrm{Bd} \mathrm{Wt}=$ body weight; $\mathrm{BrdU}=$ bromodeoxyuridine; $(\mathrm{C})=$ capsule; Cardio = cardiovascular; $\mathrm{CEL}=\mathrm{cancer}$ effect level; $\mathrm{CYP}=$ cytochrome p; $d=\operatorname{day}(\mathrm{s}) ; \mathrm{DMA}=$ dimethylarsinic acid; Endocr = endocrine; $(\mathrm{F})=$ feed; $\mathrm{F}=$ female; $(\mathrm{G})$ = gavage; Gastro = gastrointestinal; $\mathrm{Gd}=$ gestation day; $\mathrm{GSH}=$ reduced glutathione; GSSG = oxidized glutathione; $(\mathrm{GW})$ = gavage in water; Hemato = hematological; hr = hour(s); LD50 = lethal dose, 50\% kill; ODC = ornithine decarboxylase; LOAEL = lowest-observable-adverse-effect level; $\mathrm{M}=$ male; $\mathrm{mo}=$ month; Musc/skel = musculoskeletal; NOAEL = no-observable-adverse-effect level; Resp = respiratory; (W) = drinking water; $w k=\operatorname{week}(s) ; x=$ time(s); $y r=\operatorname{year}(s)$ 
Figure 3-5 Levels of Significant Exposure to Dimethylarsinic Acid - Oral

Acute ( $\leq 14$ days)

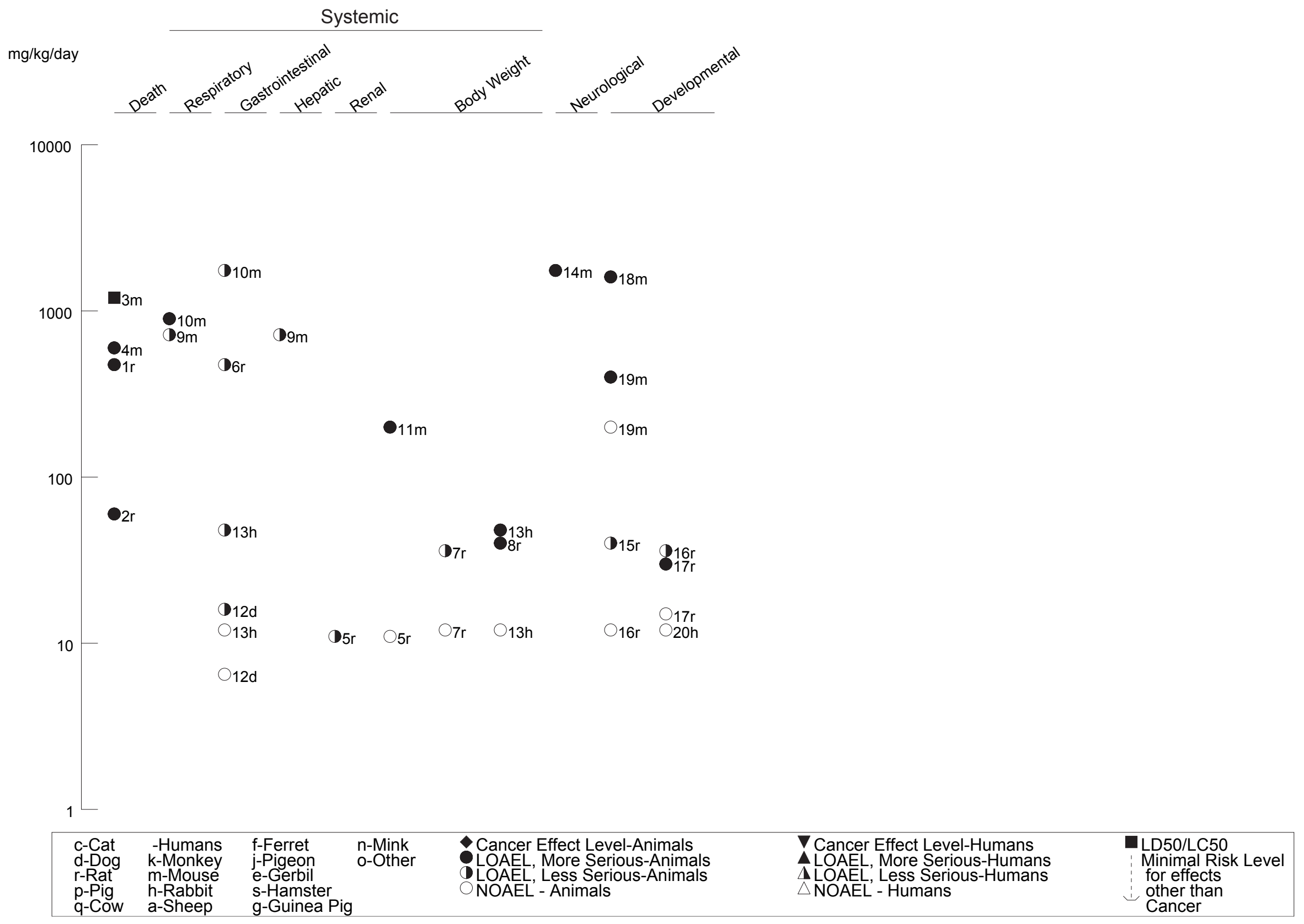


Figure 3-5 Levels of Significant Exposure to Dimethylarsinic Acid - Oral (Continued)

Intermediate (15-364 days)

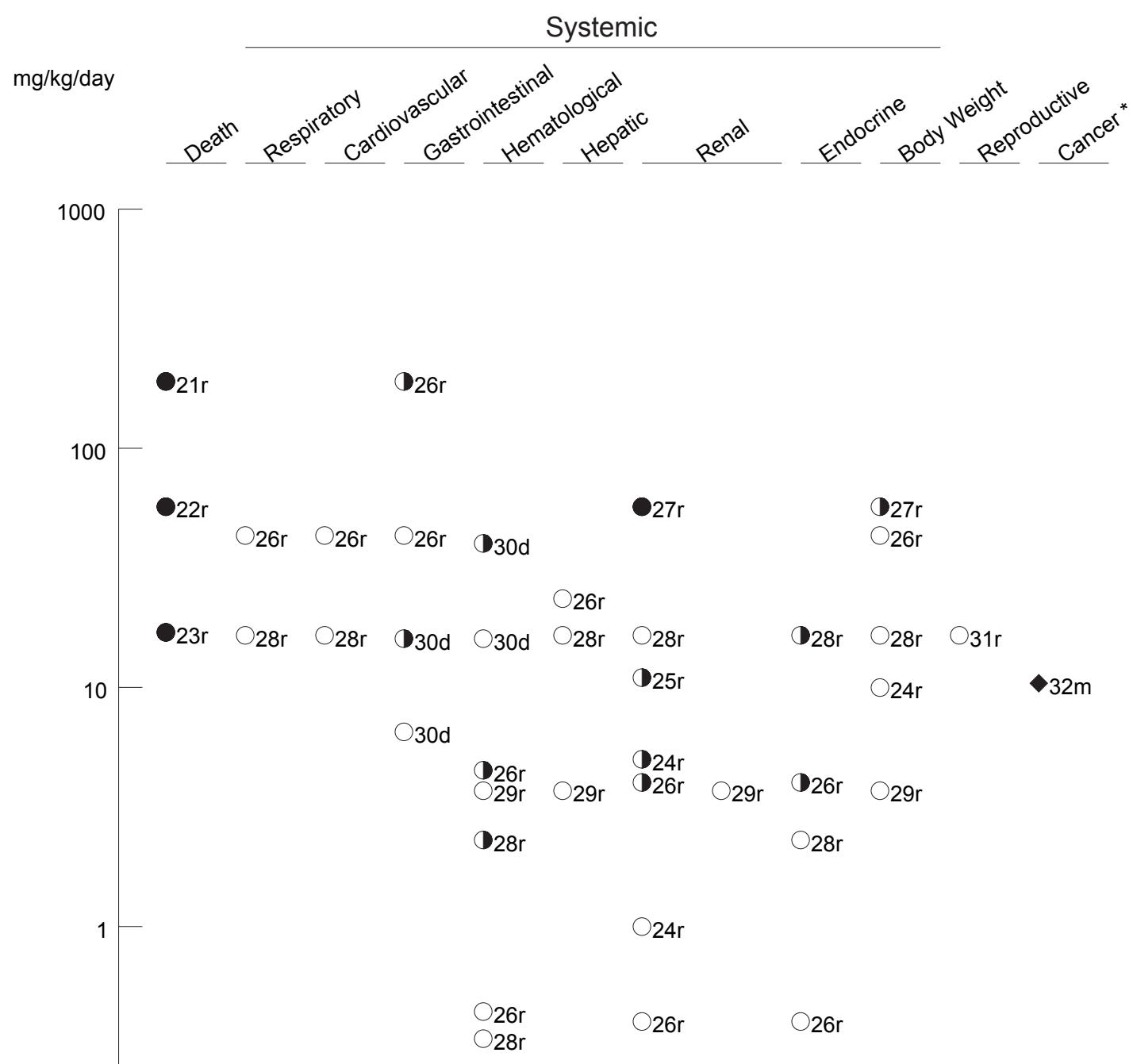

*Doses represent the lowest dose tested per study that produced a tumorigenic response and do not imply the existence of a threshold for the cancer endpoint.

\begin{tabular}{|c|c|c|c|c|c|c|}
\hline $\begin{array}{l}\text { c-Cat } \\
\text { d-Dog } \\
\text { r-Rat } \\
\text { p-Pig } \\
\text { q-Cow }\end{array}$ & $\begin{array}{l}\text {-Humans } \\
\text { k-Monkey } \\
\text { m-Mouse } \\
\text { h-Rabbit } \\
\text { a-Sheep }\end{array}$ & $\begin{array}{l}\text { f-Ferret } \\
\text { j-Pigeon } \\
\text { e-Gerbil } \\
\text { s-Hamster } \\
\text { g-Guinea Pis }\end{array}$ & $\begin{array}{l}\text { n-Mink } \\
\text { o-Other }\end{array}$ & $\begin{array}{l}\text { Cancer Effect Level-Animals } \\
\text { LOAEL, More Serious-Animals } \\
\text { LOAEL, Less Serious-Animals } \\
\text { NOAEL - Animals }\end{array}$ & $\begin{array}{l}\nabla \text { Cancer Effect Level-Humans } \\
\Delta \text { LOAEL, More Serious-Humans } \\
\Delta \text { LOAEL, Less Serious-Humans } \\
\triangle \text { NOAEL - Humans }\end{array}$ & $\begin{array}{l}\text { LD50/LC50 } \\
\text { Minimal Risk Level } \\
\text { for effects } \\
\text { other than } \\
\text { Cancer }\end{array}$ \\
\hline
\end{tabular}


Figure 3-5 Levels of Significant Exposure to Dimethylarsinic Acid - Oral (Continued)

Chronic ( $\geq 365$ days)

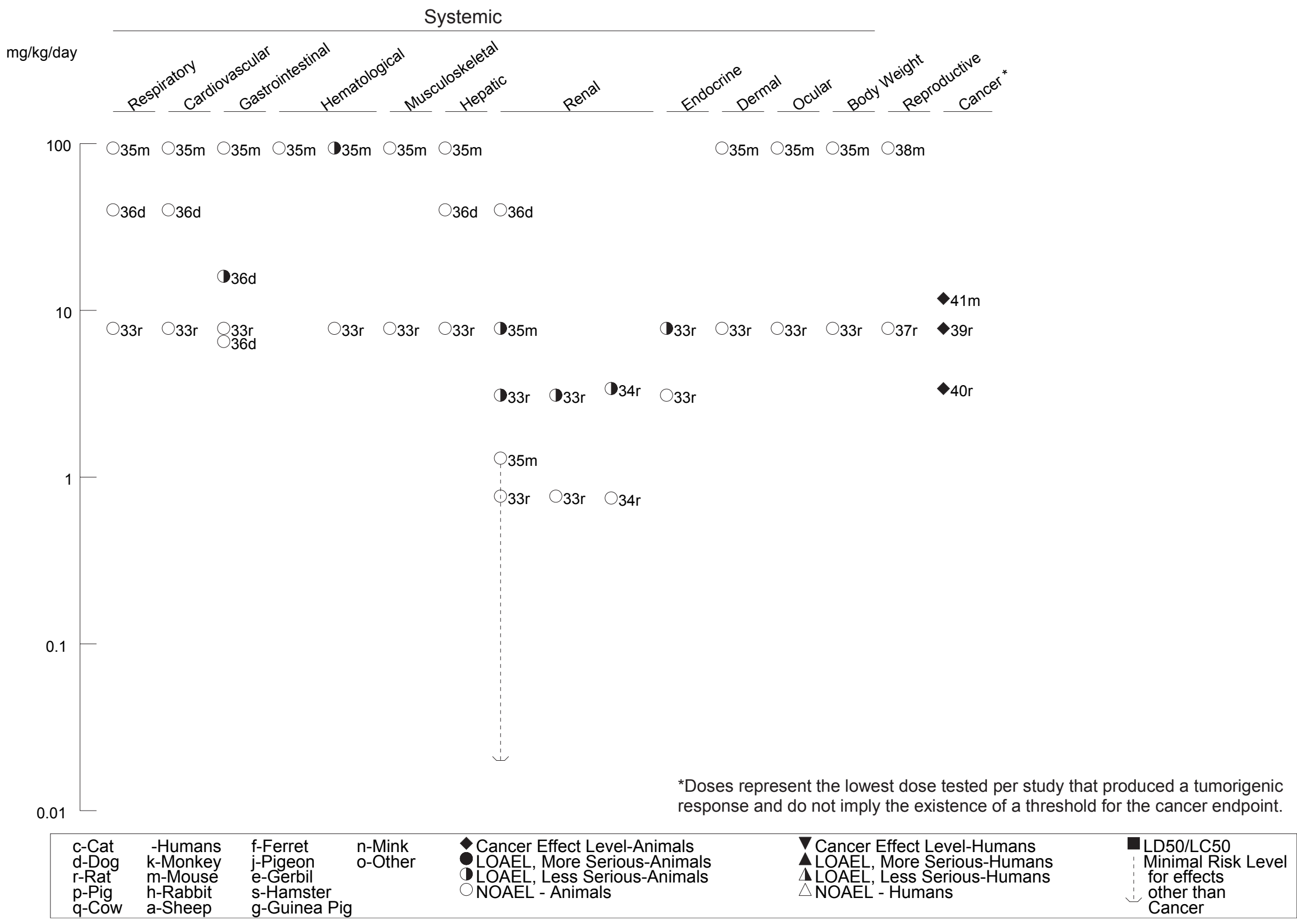

*Doses represent the lowest dose tested per study that produced a tumorigenic response and do not imply the existence of a threshold for the cancer endpoint.

\begin{tabular}{|c|c|c|c|c|c|c|}
\hline $\begin{array}{l}\text { c-Cat } \\
\text { d-Dog } \\
\text { r-Rat } \\
\text { p-Pig } \\
\text { q-Cow }\end{array}$ & $\begin{array}{l}\text {-Humans } \\
\text { k-Monkey } \\
\text { m-Mouse } \\
\text { h-Rabbit } \\
\text { a-Sheep }\end{array}$ & $\begin{array}{l}\text { f-Ferret } \\
\text { j-Pigeon } \\
\text { e-Gerbil } \\
\text { s-Hamster } \\
\text { g-Guinea Pig }\end{array}$ & $\begin{array}{l}\text { n-Mink } \\
\text { o-Other }\end{array}$ & $\begin{array}{l}\text { Cancer Effect Level-Animals } \\
\text { LOAEL, More Serious-Animals } \\
\text { LOAEL, Less Serious-Animals } \\
\text { NOAEL - Animals }\end{array}$ & $\begin{array}{l}\nabla \text { Cancer Effect Level-Humans } \\
\Delta \text { LOAEL, More Serious-Humans } \\
\Delta \text { LOAEL, Less Serious-Humans } \\
\triangle \text { NOAEL - Humans }\end{array}$ & $\begin{array}{l}\text { LD50/LC50 } \\
\text { Minimal Risk Level } \\
\text { for effects } \\
\text { other than } \\
\text { Cancer }\end{array}$ \\
\hline
\end{tabular}


Table 3-6 Levels of Significant Exposure to Roxarsone - Oral

\begin{tabular}{|c|c|c|}
\hline $\begin{array}{l}\text { Key to } \\
\text { Figure }\end{array}$ & $\begin{array}{l}\text { Species } \\
\text { (Strain) }\end{array}$ & $\begin{array}{l}\text { Expos } \\
\text { Durati } \\
\text { Freque } \\
\text { (Rou }\end{array}$ \\
\hline \multicolumn{3}{|c|}{$\begin{array}{l}\text { ACUTE EXPOSURE } \\
\text { Death }\end{array}$} \\
\hline 1 & $\begin{array}{l}\text { Rat } \\
\text { (Holtzman }\end{array}$ & $\begin{array}{l}\text { once } \\
\text { (GW) }\end{array}$ \\
\hline 2 & $\begin{array}{l}\text { Rat } \\
\text { (Fischer-3 }\end{array}$ & $\begin{array}{r}\text { once } \\
\text { 44) (GO) }\end{array}$ \\
\hline 3 & $\begin{array}{l}\text { Rat } \\
\text { (Fischer-3 }\end{array}$ & 44) $\begin{array}{r}14 \mathrm{C} \\
(\mathrm{F})\end{array}$ \\
\hline
\end{tabular}

4 Mouse once

(B6C3F1) (GO)

$5 \quad$ Mouse $14 \mathrm{~d}$

(B6C3F1) (F)

Systemic

6 Rat $14 \mathrm{~d}$

(Fischer- 344) (F)

7 Mouse

(B6C3F1)

$14 \mathrm{~d}$

(F)

$\mathrm{Bd} \mathrm{Wt}$

84

Neurological
8 Rat
$\begin{array}{ll}\text { Rat } & 14 \\ \text { (Fischer- 344) } & (F)\end{array}$

LOAEL

NOAEL

Less Serious

(mg/kg/day)

(mg/kg/day)

Reference

Chemical Form

Comments

(mg/kg/day)

Kerr et al. 1963
ROX

$\begin{array}{ll}150 \mathrm{M}(5 / 5 \text { died }) & \text { NTP 1989b } \\ \mathrm{b} \text { (LD50) } & \text { ROX }\end{array}$

$\begin{array}{ll}128 \mathrm{~b}(3 / 5 \text { died }) & \text { NTP 1989b } \\ & \text { ROX }\end{array}$

$144 \mathrm{~F}(5 / 5$ died $)$

ROX

$\begin{array}{ll}300 \mathrm{M}(5 / 5 \text { died }) & \text { NTP } 1989 \mathrm{~b} \\ \mathrm{~b} & \text { ROX }\end{array}$

168 F (5/5 died)

NTP 1989b

ROX

NTP 1989b

ROX

168 (34\% decrease in body weight)

NTP 1989b

ROX

NTP 1989b

ROX 
Table 3-6 Levels of Significant Exposure to Roxarsone - Oral

(continued)

\begin{tabular}{|c|c|c|}
\hline $\begin{array}{l}\text { Key to } \\
\text { Figure }\end{array}$ & $\begin{array}{l}\text { Species } \\
\text { (Strain) }\end{array}$ & $\begin{array}{l}\text { Expost } \\
\text { Durati } \\
\text { Freque } \\
\text { (Rout }\end{array}$ \\
\hline 9 & $\begin{array}{l}\text { Mouse } \\
\text { (B6C3F1) }\end{array}$ & $\begin{array}{l}14 \mathrm{~d} \\
(\mathrm{~F})\end{array}$ \\
\hline
\end{tabular}

10
Pig
(Landrace)

(F)

\section{INTERMEDIATE EXPOSURE}

\section{Death}

11 Rat 13 wk

(Holtzman) (F)

12 Rat 13 wk

(Fischer- 344) ad lib

(F)

$13 \quad$ Mouse $13 \mathrm{wk}$

(B6C3F1) ad lib

(F)
LOAEL

NOAEL

Less Serious

(mg/kg/day)

(mg/kg/day)

Reference

Chemical Form

Comments

(mg/kg/day)

20

42

fur)

6.3 (muscle tremors and clonic convulsions)

Rice et al. 1985; Kennedy et al

1986

ROX

$20 \quad(10 / 12$ died $)$

Kerr et al. 1963

ROX

$64 \mathrm{M} \mathrm{(3/10} \mathrm{died)}$

NTP 1989b

ROX

$136 \quad(6 / 10$ males and 8/10 females died)
NTP 1989b

ROX 
Table 3-6 Levels of Significant Exposure to Roxarsone - Oral

(continued)

\begin{tabular}{|c|c|c|c|c|c|c|c|c|}
\hline \multirow[b]{2}{*}{$\begin{array}{l}\text { Key to } \\
\text { Figure }\end{array}$} & \multirow[b]{2}{*}{$\begin{array}{c}\text { Species } \\
\text { (Strain) }\end{array}$} & \multirow{2}{*}{$\begin{array}{l}\text { Exposurel } \\
\text { Duration/ } \\
\text { Frequency } \\
\text { (Route) }\end{array}$} & \multirow[b]{2}{*}{ System } & \multirow[b]{2}{*}{$\begin{array}{l}\text { NOAEL } \\
\text { (mg/kg/day) }\end{array}$} & \multicolumn{2}{|c|}{ LOAEL } & \multirow[b]{2}{*}{$\begin{array}{l}\text { Reference } \\
\text { Chemical Form }\end{array}$} & \multirow[b]{2}{*}{ Comments } \\
\hline & & & & & $\begin{array}{l}\text { Less Serious } \\
\text { (mg/kg/day) }\end{array}$ & $\begin{array}{l}\text { Serious } \\
\text { (mg/kg/day) }\end{array}$ & & \\
\hline
\end{tabular}

Systemic

Rat $\quad 31$ or 90

(Fischer- 344) ad lib

Hemato

$32 \mathrm{M}$

(F)

Hepatic $\quad 9 \mathrm{~F} \quad 36 \mathrm{~F}$ (decreased absolute and
relative liver weight)

Renal $8 \mathrm{M} \quad 32 \mathrm{M}$ (increased kidney weight;

minimal tubular

degeneration)

Bd Wt

$8 \mathrm{M}$

$32 \mathrm{M}(27 \%$ decrease in body

weight) 
Table 3-6 Levels of Significant Exposure to Roxarsone - Oral

(continued)

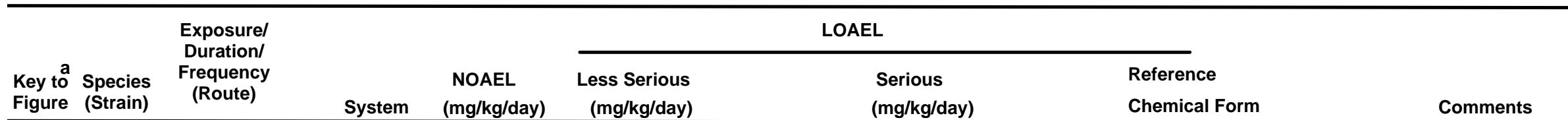

15 Rat 13 wk (Fischer- 344) ad lib

Resp

$64 \mathrm{M}$

$(\mathrm{F})$

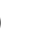

$\begin{array}{lr}\text { Cardio } & 64 \mathrm{M} \\ \text { Gastro } & 64 \mathrm{M} \\ \text { Musc/skel } & 64 \mathrm{M} \\ \text { Hepatic } & 4 \mathrm{M}\end{array}$

$8 \mathrm{M}$ (increased relative liver weight)

Renal

$16 \mathrm{M}$

$32 \mathrm{M}$ (interstitial inflammation,

focal regenerative

hyperplasia of tubular cell

epithelium and

mineralization)

$\begin{array}{lr}\text { Endocr } & 64 \mathrm{M} \\ \text { Dermal } & 64 \mathrm{M} \\ \text { Bd Wt } & 8 \mathrm{M}\end{array}$

16 M (14\% decreased body

weight)

32 M (26\% decreased body

weight) 
Table 3-6 Levels of Significant Exposure to Roxarsone - Oral

(continued)

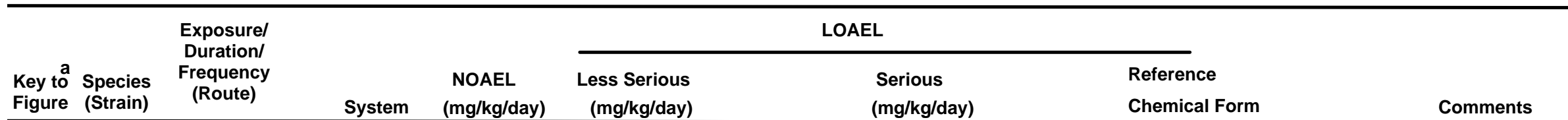

16 Mouse $13 \mathrm{wk}$

$\begin{array}{llll}\text { Mouse } & 13 \mathrm{wk} & \text { Cardio } & 136 \\ \text { (B6C3F1) } & \text { ad lib } & & \end{array}$

(F)

(mg/kg/day)

Chemical Form

Comments

NTP 1989b

ROX

$\begin{array}{ll}\text { Gastro } & 136 \\ \text { Musc/skel } & 136 \\ \text { Hepatic } & 136 \\ \text { Renal } & 136 \\ \text { Endocr } & 136 \\ \text { Dermal } & 136\end{array}$

$\mathrm{Bd} \mathrm{Wt}_{\mathrm{t}}$

$136 \quad(18 \%$ decreased body

weight in males; $11 \%$

decreased body weight in

females)

17 Mouse 29 or 91

Hemato

Neurological

18 Rat 13 wk

(Fischer- 344) ad lib

NTP 1989b

ROX

ROX 
Table 3-6 Levels of Significant Exposure to Roxarsone - Oral

(continued)

\begin{tabular}{|c|c|c|c|c|c|c|c|c|}
\hline \multirow[b]{2}{*}{$\begin{array}{l}\text { Key to } \\
\text { Figure }\end{array}$} & \multirow[b]{2}{*}{$\begin{array}{l}\text { Species } \\
\text { (Strain) }\end{array}$} & \multirow{2}{*}{$\begin{array}{l}\text { Exposurel } \\
\text { Durationl } \\
\text { Frequency } \\
\text { (Route) }\end{array}$} & \multicolumn{6}{|c|}{ LOAEL } \\
\hline & & & System & $\begin{array}{l}\text { NOAEL } \\
\text { (mg/kg/day) }\end{array}$ & $\begin{array}{r}\text { Less Serious } \\
\text { (mg/kg/day) }\end{array}$ & $\begin{array}{l}\mathrm{Se} \\
(\mathrm{mg}\end{array}$ & $\begin{array}{l}\text { ious } \\
\text { lkg/day) }\end{array}$ & $\begin{array}{l}\text { Reference } \\
\text { Chemical Form }\end{array}$ \\
\hline 19 & Pig & $\begin{array}{l}28 d \\
(F)\end{array}$ & & & & 10 & (muscle tremors) & $\begin{array}{l}\text { Edmonds and Baker } 1986 \\
\text { ROX }\end{array}$ \\
\hline 20 & $\begin{array}{l}\text { Pig } \\
\text { (Landrace) }\end{array}$ & $\begin{array}{l}30 \mathrm{~d} \\
\text { ad lib } \\
(\mathrm{F})\end{array}$ & & & & 6.3 & $\begin{array}{l}\text { (paraplegia, myelin } \\
\text { degeneration in spinal } \\
\text { cord, peripheral nerves, } \\
\text { optic nerve) }\end{array}$ & $\begin{array}{l}\text { Rice et al. 1985; Kennedy et al. } \\
1986 \\
\text { ROX }\end{array}$ \\
\hline
\end{tabular}

\section{CHRONIC EXPOSURE}

Systemic

(Fischer- 344) ad lib

Resp

4

NTP 1989b

(F)

ROX

$\begin{array}{ll}\text { Cardio } & 4 \\ \text { Gastro } & 4 \\ \text { Musc/skel } & 4 \\ \text { Hepatic } & 4 \\ \text { Renal } & 4 \\ \text { Endocr } & 4 \\ \text { Dermal } & 4 \\ \text { Ocular } & 4 \\ \text { Bd Wt } & 4\end{array}$

22 Mouse $103 \mathrm{wk}$

(Fischer- 344) ad lib

Resp

$43 \mathrm{M}$

(F)

$\begin{array}{ll}\text { Cardio } & 43 \mathrm{M} \\ \text { Gastro } & 43 \mathrm{M} \\ \text { Musc/skel } & 43 \mathrm{M} \\ \text { Hepatic } & 43 \mathrm{M}\end{array}$

NTP 1989b

ROX 
Table 3-6 Levels of Significant Exposure to Roxarsone - Oral

(continued)

\begin{tabular}{|c|c|c|c|}
\hline $\begin{array}{l}\text { Key to } \\
\text { Figure } \\
\text { Figecies } \\
\text { (Strain) }\end{array}$ & $\begin{array}{c}\text { Exposurel } \\
\text { Durationl } \\
\text { Frequency } \\
\text { (Route) }\end{array}$ & System & $\begin{array}{l}\text { NOAEL } \\
(\mathrm{mg} / \mathrm{kg} / \mathrm{da}\end{array}$ \\
\hline & & Renal & $43 \mathrm{M}$ \\
\hline & & Endocr & $43 \mathrm{M}$ \\
\hline & & Dermal & $43 \mathrm{M}$ \\
\hline & & Ocular & $43 \mathrm{M}$ \\
\hline & & $\mathrm{Bd} \mathrm{Wt}$ & $43 \mathrm{~F}$ \\
\hline
\end{tabular}

a The number corresponds to entries in Figure 3-6.

b Differences in levels of health effects and cancer effects between male and females are not indicated in Figure 3-6. Where such differences exist, only the levels of effect for the most sensitive gender are presented.

ad lib = ad libitum; Bd Wt = body weight; Cardio = cardiovascular; $d=$ day $(\mathrm{s}) ;$ Endocr = endocrine; $(\mathrm{F})=$ feed; $\mathrm{F}=$ Female; Gastro = gastrointestinal; $(\mathrm{GO})=$ gavage in oil; $(\mathrm{GW})=$

gavage in water; Hemato = hematological; LC50 = lethal concentration, $50 \%$ kill, LD50 = lethal dose, 50\% kill; LOAEL = lowest-observed-adverse-effect level; $M=$ male; Musc/skel =

musculoskeletal; NOAEL = no-observed-adverse-effect level; occup = occupational; Resp = respiratory; $w \mathrm{wk}=$ week $(\mathrm{s})$ 
Figure 3-6 Levels of Significant Exposure to Roxarsone - Oral

Acute ( $\leq 14$ days)

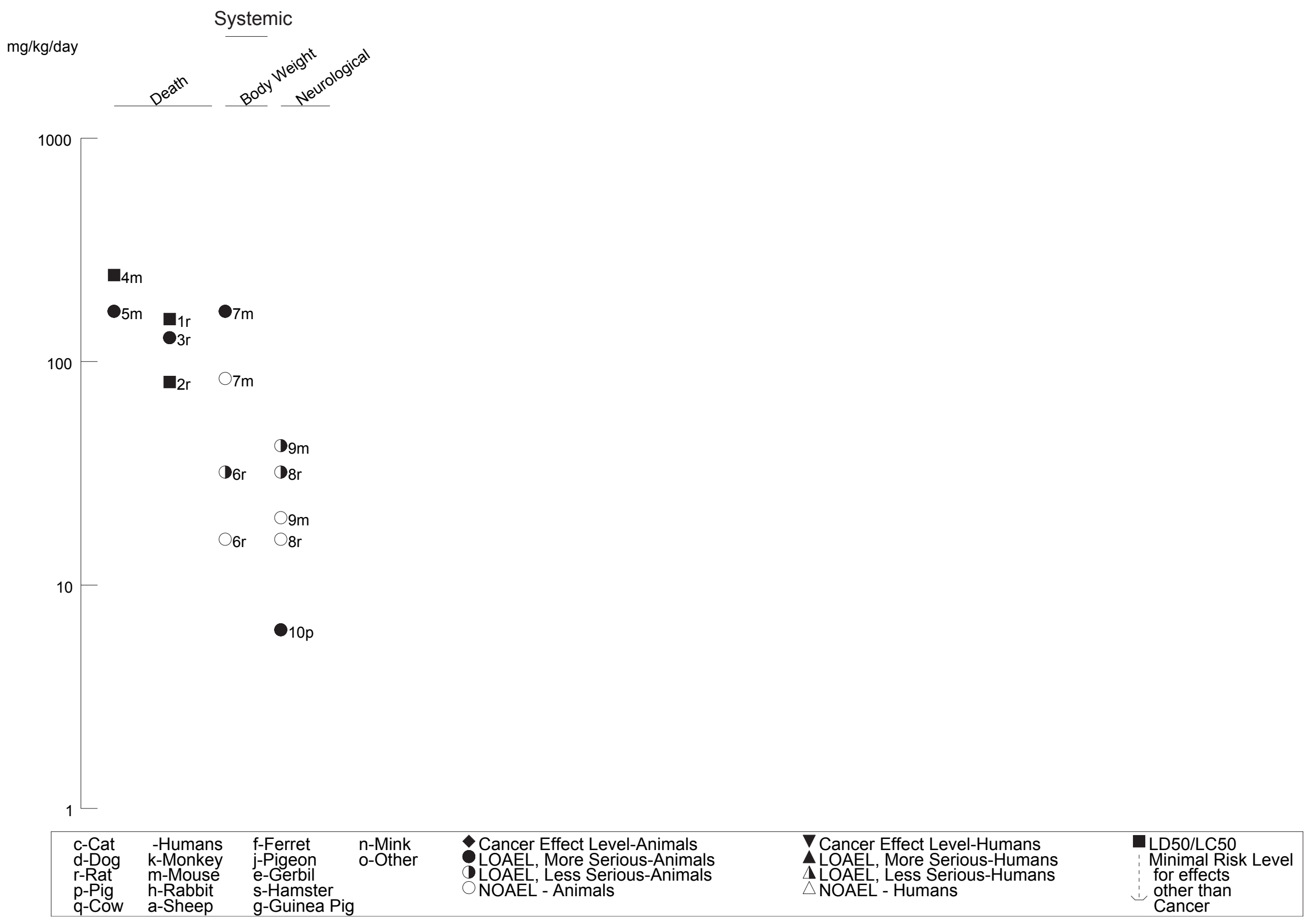


Figure 3-6 Levels of Significant Exposure to Roxarsone - Oral (Continued)

Intermediate (15-364 days)

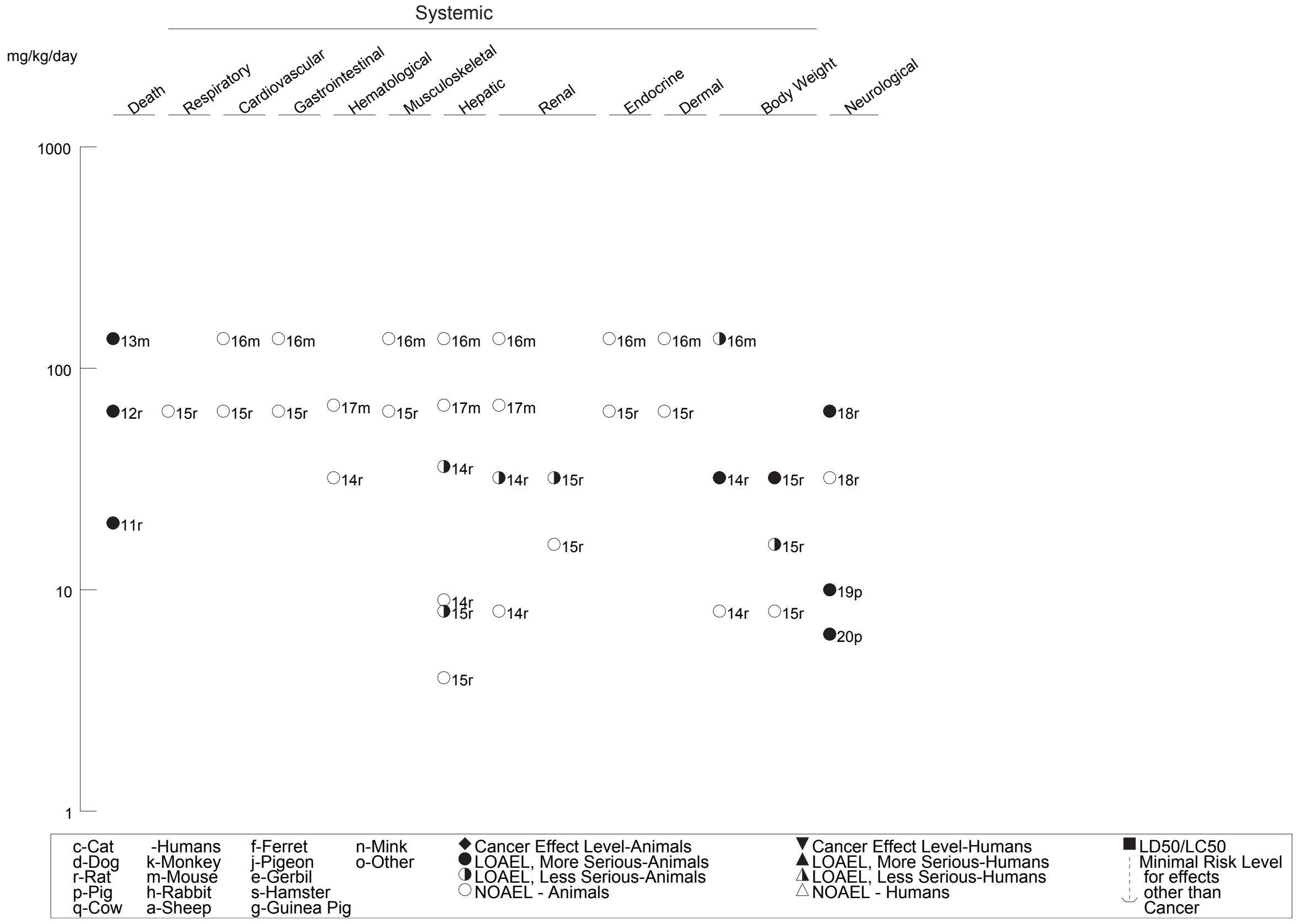


Figure 3-6 Levels of Significant Exposure to Roxarsone - Oral (Continued)

Chronic ( $\geq 365$ days)

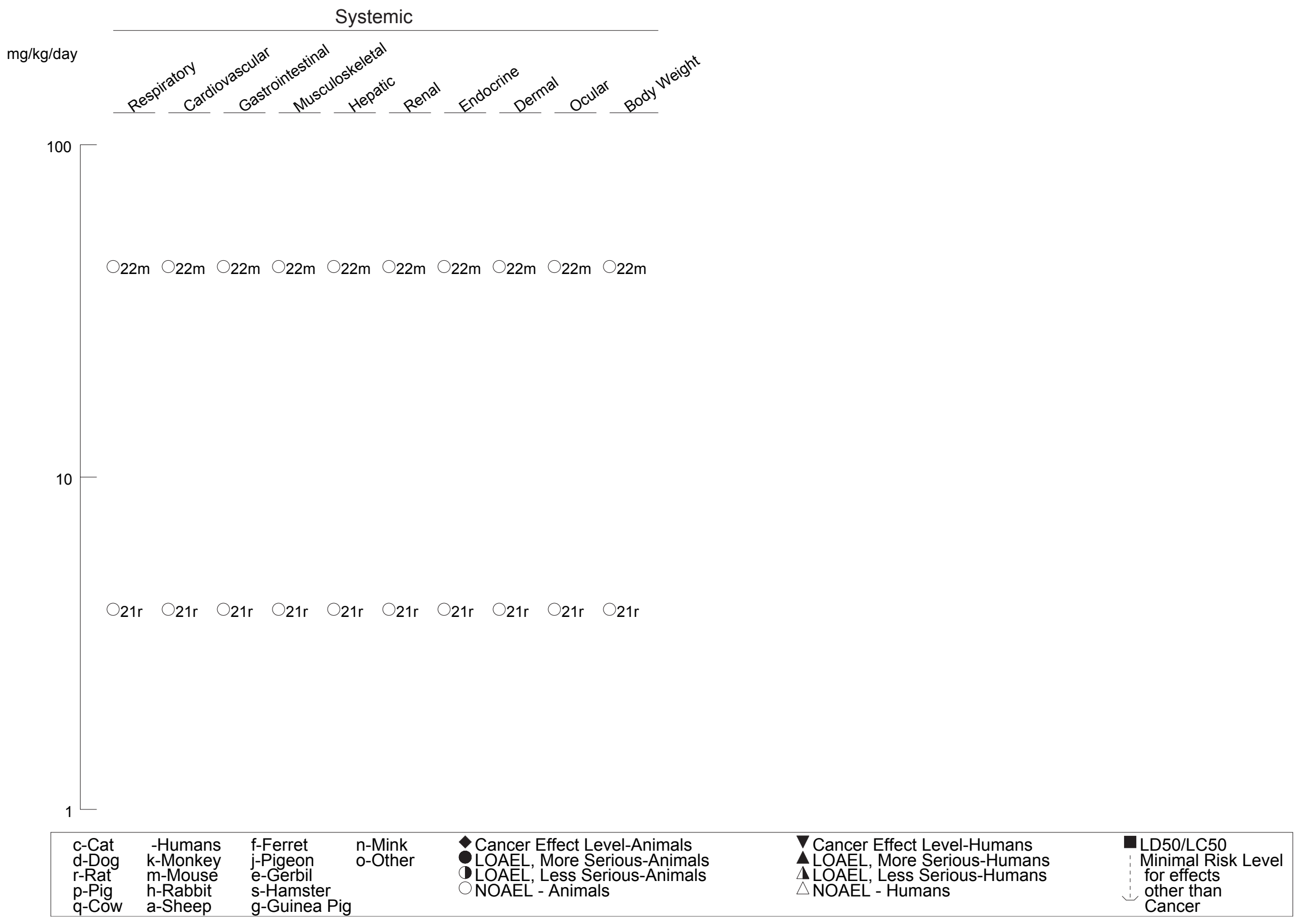


Available $\mathrm{LD}_{50}$ values for arsenate and arsenite in rats and mice range from 15 to $175 \mathrm{mg} \mathrm{As} / \mathrm{kg}$ (Dieke and Richter 1946; Gaines 1960; Harrisson et al. 1958; Kaise et al. 1985). The variability can be attributed to differences based on species, strain, specific route of exposure (feed vs. gavage), specific compound tested, and testing laboratory. Most deaths occurred within 1 day of exposure, but details regarding cause of death were not generally reported. Seven of 25 pregnant rats given a single gavage dose of $23 \mathrm{mg}$ $\mathrm{As} / \mathrm{kg}$ as arsenic trioxide on day 9 of gestation died soon after dosing, while no deaths occurred at doses of 4-15 mg As/kg (Stump et al. 1999). Data on lethality from repeated exposure studies in animals are relatively sparse. Seven of 20 pregnant rabbits died from repeated gavage doses of $1.5 \mathrm{mg} \mathrm{As} / \mathrm{kg} / \mathrm{day}$ as arsenic acid during gestation, while none died at 0.1-0.4 mg As/ $\mathrm{kg} /$ day (Nemec et al. 1998). Chronic studies observed treatment-related mortality in monkeys exposed to $3 \mathrm{mg} \mathrm{As} / \mathrm{kg} / \mathrm{day}$ as arsenate (Heywood and Sortwell 1979), dogs exposed to $2.4 \mathrm{mg}$ As/kg/day as arsenite or arsenate (Byron et al. 1967), mice exposed to $1 \mathrm{mg} \mathrm{As} / \mathrm{kg} /$ day as arsenite (Schroeder and Balassa 1967), and rats exposed to $30 \mathrm{mg} \mathrm{As} / \mathrm{kg} /$ day as lead arsenate (Kroes et al. 1974).

Reliable LOAEL and $\mathrm{LD}_{50}$ values for lethality from oral exposure to inorganic arsenicals in each species and duration category are recorded in Table 3-3 and plotted in Figure 3-3.

Organic Arsenicals. No studies were located regarding death in humans after oral exposure to organic arsenicals, but the acute lethality of MMA, DMA, and roxarsone have been investigated in several animal studies. The $\mathrm{LD}_{50}$ values for MMA (including MSMA), DMA, and roxarsone are $102-3,184 \mathrm{mg} / \mathrm{kg}$ MMA or MSMA (Gur and Nyska 1990; Jaghabir et al. 1988; Kaise et al. 1989), 1,200 mg DMA/kg/day (Kaise et al. 1989), and 14.2-69.5 mg DMA/kg/day (Kerr et al. 1963; NTP 1989b), respectively. The cause of death was not investigated in any of these studies. Intermediate-duration exposure to MMA, DMA, or roxarsone resulted in increased mortality in laboratory animals exposed to $106.9 \mathrm{mg}$ MMA/kg/day (Arnold et al. 2003), 17-190 mg DMA/kg/day (Crown et al. 1987; Murai et al. 1993; Wanibuchi et al. 1996) or 20-64 mg/kg/day roxarsone (Edmonds and Baker 1986; Kerr et al. 1963; NTP $1989 \mathrm{~b})$, respectively. Increased mortality was also observed in rats chronically exposed to $72.4 \mathrm{mg}$ MMA/kg/day (Arnold et al. 2003).

\subsubsection{Systemic Effects}

The highest NOAEL values and all reliable LOAEL values for systemic effects from oral exposure in each species and duration category are recorded in Table 3-3 and plotted in Figure 3-3. Similar data for 
oral exposure to MMA, DMA, and roxarsone are shown in Tables 3-4, 3-5, and 3-6, and shown in Figures 3-4, 3-5, and 3-6, respectively.

\section{Respiratory Effects.}

Inorganic Arsenicals. Serious respiratory effects, including respiratory distress, hemorrhagic bronchitis, and pulmonary edema, have been reported in some cases of acute oral arsenic poisoning at doses of $8 \mathrm{mg}$ As/kg and above (e.g., Civantos et al. 1995; Fincher and Koerker 1987; Levin-Scherz et al. 1987; Moore et al. 1994b; Quatrehomme et al. 1992). These effects may be secondary to injury to the pulmonary vasculature (see Cardiovascular Effects, below). In addition, bronchitis and sequelae (bronchiectasis, bronchopneumonia) have been observed in patients and at autopsy in some chronic poisoning cases (Guha Mazumder et al. 2005; Milton and Rahman 2002; Rosenberg 1974; Tsai et al. 1999; Zaldívar 1974; Zaldívar and Guillier 1977). Bronchopneumonia secondary to arsenic-induced bronchitis was considered to be the cause of death in one young child who died after several years of exposure to an average dose of $0.08 \mathrm{mg} \mathrm{As} / \mathrm{kg} /$ day (Zaldívar and Guillier 1977). Decrements in lung function, measured as decreased $\mathrm{FEV}_{1}, \mathrm{FVC}$, and $\mathrm{FEF}_{25-75}$ have also been reported in subjects exposed to $0.1-0.5 \mathrm{mg} \mathrm{As} / \mathrm{L}$ in the drinking water and exhibiting skin lesions (von Ehrenstein et al. 2005). In general, however, respiratory effects have not been widely associated with repeated oral ingestion of low arsenic doses. Nevertheless, a few studies have reported minor respiratory symptoms, such as cough, sputum, rhinorrhea, and sore throat, in people with repeated oral exposure to 0.03-0.05 mg As/kg/day (Ahmad et al. 1997; Mizuta et al. 1956).

There are few data regarding respiratory effects in animals following acute oral exposure to inorganic arsenic. An infant Rhesus monkey that died after 7 days of oral exposure to a complex arsenate salt at a dose of $3 \mathrm{mg} \mathrm{As} / \mathrm{kg} /$ day exhibited bronchopneumonia with extensive pulmonary hemorrhage, edema, and necrosis (Heywood and Sortwell 1979). Two other monkeys in this treatment group survived a 1-year exposure period and had no gross or microscopic pulmonary lesions at sacrifice. Increased relative lung weights were seen in rats exposed to $6.66 \mathrm{mg} \mathrm{As} / \mathrm{kg} /$ day as sodium arsenite 5 days/week for 12 weeks (Schulz et al. 2002). Chronic oral studies in dogs and rats treated with arsenate or arsenite failed to find respiratory lesions (Byron et al. 1967; Kroes et al. 1974; Schroeder et al. 1968).

One study utilizing gallium arsenide included limited investigation of respiratory function. Respiration rate was significantly decreased in rats following ingestion of a single dose of gallium arsenide at $1,040 \mathrm{mg} \mathrm{As} / \mathrm{kg}$, but was unaffected at a dose of $520 \mathrm{mg} \mathrm{As} / \mathrm{kg}$ (Flora et al. 1997a). Respiration rate was measured 1, 7, and 15 days after dosing, but the decrease was most noticeable after 15 days. 
Organic Arsenicals. No respiratory effects were noted after acute human ingestion of 1,714 mg MSMA/kg (Shum et al. 1995). Mice exhibited respiratory arrest after a single oral dose of 1,800 mg MMA/kg (Kaise et al. 1989) or $900 \mathrm{mg}$ DMA/kg (Kaise et al. 1989) and lung ornithine decarboxylase activity was reduced after ingestion of one or two doses of $720 \mathrm{mg} \mathrm{DMA} / \mathrm{kg}$ (Ahmad et al. 1999a). Localized lung hemorrhage was observed in dogs after a single oral dose of $14.2 \mathrm{mg} / \mathrm{kg}$ roxarsone in a capsule (Kerr et al. 1963). No respiratory effects were seen after intermediate or chronic exposure of rats, mice, or dogs exposed to $35 \mathrm{mg}$ MMA/kg/day (Waner and Nyska 1988), 7.8-94 mg DMA/kg/day (Arnold et al. 2006; Crown et al. 1987; Rubin et al. 1989; Zomber et al. 1989), or 4-136 mg/kg/day roxarsone (NTP 1989b).

\section{Cardiovascular Effects.}

Inorganic Arsenicals. A number of studies in humans indicate that arsenic ingestion may lead to serious effects on the cardiovascular system. Characteristic effects on the heart from both acute and long-term exposure include altered myocardial depolarization (prolonged QT interval, nonspecific ST segment changes) and cardiac arrhythmias (Cullen et al. 1995; Glazener et al. 1968; Goldsmith and From 1986; Heyman et al. 1956; Little et al. 1990; Mizuta et al. 1956; Moore et al. 1994b; Mumford et al. 2007). A significant dose-related increase in the prevalence of cardiac electrophysiologic abnormalites was observed in residents of Inner Mongolia, China; the incidences of QT prolongation were observed in 3.9, 11.1 , and $20.6 \%$ of the residents with drinking water levels of $<21,110-300$, and $430-690 \mu \mathrm{g} / \mathrm{L}$, respectively (Mumford et al. 2007). Hypertrophy of the ventricular wall was observed at autopsy after acute exposure to $93 \mathrm{mg}$ of arsenic (Quatrehomme et al. 1992). Long-term, low-level exposures may also lead to damage to the vascular system. The most dramatic example of this is "Blackfoot Disease," a condition that is endemic in an area of Taiwan where average drinking water levels of arsenic range from 0.17 to $0.80 \mathrm{ppm}$ (Tseng 1977), corresponding to doses of about $0.014-0.065 \mathrm{mg} \mathrm{As} / \mathrm{kg} / \mathrm{day}$ (IRIS 2007). The disease is characterized by a progressive loss of circulation in the hands and feet, leading ultimately to necrosis and gangrene (Chen et al. 1988b; Ch'i and Blackwell 1968; Tseng 1977, 1989; Tseng et al. 1968, 1995, 1996). Several researchers have presented evidence that other factors besides arsenic (e.g., other water contaminants, dietary deficits) may play a role in the etiology of this disease (Ko 1986; Lu et al. 1990; Yu et al. 1984). While this may be true, the clear association between the occurrence of Blackfoot Disease and the intake of elevated arsenic levels indicates that arsenic is at least a contributing factor. The results of a recent study suggested that individuals with a lower capacity to methylate 
inorganic arsenic to DMA have a higher risk of developing peripheral vascular disease in the Blackfoot Disease-hyperendemic area in Taiwan (Tseng et al. 2005).

Arsenic exposure in Taiwan has also been associated with an increased incidence of cerebrovascular and microvascular diseases (Chiou et al. 1997; Wang et al. 2002, 2003) and ischemic heart disease (Chang et al. 2004; Chen et al. 1996; Hsueh et al. 1998b; Tsai et al. 1999; Tseng et al. 2003). Moreover, effects of arsenic on the vascular system have also been reported in a number of other populations. For example, hypertension, defined as a systolic blood pressure of $\geq 140 \mathrm{~mm} \mathrm{Hg}$ in combination with a diastolic blood pressure of $\geq 90 \mathrm{~mm} \mathrm{Hg}$, was associated with estimated lifetime doses of approximately $0.055 \mathrm{mg}$ $\mathrm{As} / \mathrm{kg} /$ day $(0.25 \mathrm{mg} / \mathrm{L}$ in water) in a study of people in Bangladesh (Rahman et al. 1999); no significant association was found with estimated doses of $0.018 \mathrm{mg} \mathrm{As} / \mathrm{kg} /$ day $(0.75 \mathrm{mg} / \mathrm{L}$ in water $)$. Wang et al. (2003) found an increased incidence of microvascular and macrovascular disease among subjects in Taiwan living in an arseniasis-endemic area in which the water of artesian wells had arsenic concentrations $>0.35 \mathrm{mg} / \mathrm{L}$ (estimated doses of $>0.03 \mathrm{mg} \mathrm{As} / \mathrm{kg} /$ day). An additional study of Taiwanese subjects reported a significant increase in incidence of hypertension associated with concentrations of arsenic in the water $>0.7 \mathrm{mg} / \mathrm{L}$ (estimated doses of $>0.06 \mathrm{mg} \mathrm{As} / \mathrm{kg} /$ day) (Chen et al. 1995). Studies in Chile indicate that ingestion of $0.6-0.8 \mathrm{ppm}$ arsenic in drinking water (corresponding to doses of $0.02-$ $0.06 \mathrm{mg} \mathrm{As} / \mathrm{kg} /$ day, depending on age) increases the incidence of Raynaud's disease and of cyanosis of fingers and toes (Borgoño and Greiber 1972; Zaldívar 1974, 1977; Zaldívar and Guillier 1977). Autopsy of five children from this region who died of apparent arsenic toxicity showed a marked thickening of small and medium sized arteries in tissues throughout the body, especially the heart (Rosenberg 1974). In addition, cardiac failure, arterial hypotension, myocardial necrosis, and thrombosis have been observed in children who died from chronic arsenic ingestion (Zaldívar 1974), as well as adults chronically exposed to arsenic (Dueñas et al. 1998). Likewise, thickening and vascular occlusion of blood vessels were noted in German vintners exposed to arsenical pesticides in wine and in adults who drank arsenic-contaminated drinking water (Roth 1957; Zaldívar and Guillier 1977). A survey of Wisconsin residents using private wells for their drinking water found that residents exposed for at least 20 years to water concentrations of $>10 \mu \mathrm{g} \mathrm{As} / \mathrm{L}$ had increased incidences of cardiac bypass surgery, high blood pressure, and circulatory problems as compared with residents exposed to lower arsenic concentrations (Zierold et al. 2004). Similarly, Lewis et al. (1999) reported increased mortality from hypertensive heart disease in both men and women among a cohort exposed to arsenic in their drinking water in Utah, as compared with the general population of Utah. Limitations in the study included lack of evaluation of smoking as a confounder and of other dietary sources of arsenic, and the lack of a dose-response for hypertensive heart disease. Another ecological study (Engel and Smith 1994) found significant increases in deaths from 
arteriosclerosis, aortic aneurysm, and all other diseases of the arteries, arterioles, and capillaries among U.S. residents with arsenic drinking waters of $>20 \mu \mathrm{g} / \mathrm{L}$; the increase in deaths from congenital anomalies of the heart and other anomalies of the circulatory system also observed in this subpopulation limits the interpretation of the findings.

Similar alterations in vascular reactivity have been noted in rats given repeated oral doses of arsenic trioxide (11 mg As/kg/day) for several weeks (Bekemeier and Hirschelmann 1989), although no histological effects could be detected in the hearts of rats or dogs exposed to up to $30 \mathrm{mg} \mathrm{As} / \mathrm{kg} / \mathrm{day}$ as arsenate or arsenite for 2 years (Byron et al. 1967; Kroes et al. 1974; Schroeder et al. 1968). Acute exposure of rats to gallium arsenide at a dose of $1,040 \mathrm{mg} \mathrm{As} / \mathrm{kg}$ resulted in an increase in blood pressure and heart rate, while $520 \mathrm{mg} \mathrm{As} / \mathrm{kg}$ had no effect (Flora et al. 1997a). Guinea pigs exposed to arsenic trioxide for 1 day $(0,7.6,22.7$, or $37.9 \mathrm{mg} \mathrm{As} / \mathrm{kg}$ ) or 8 days ( 0 or $3.8 \mathrm{mg} \mathrm{As} / \mathrm{kg} /$ day) showed prolongation of the cardiac QT interval and action potential duration (Chiang et al. 2002).

Organic Arsenicals. No adverse cardiovascular effects were noted after acute human ingestion of $1,714 \mathrm{mg} \mathrm{MSMA} / \mathrm{kg}$ (Shum et al. 1995). However, sinus tachycardia was noted after acute ingestion of $73 \mathrm{mg} \mathrm{DMA} / \mathrm{kg}$ (as dimethyl arsenic acid and dimethyl arsenate) (Lee et al. 1995). No cardiovascular effects were seen after intermediate or chronic exposure of laboratory animals to 35-67.1 mg MMA/kg/day (Arnold et al. 2003; Waner and Nyska 1988), 7.8-94 mg DMA/kg/day (Arnold et al. 2006; Crown et al. 1987; Rubin et al. 1989; Zomber et al. 1989), or 4-136 mg/kg/day roxarsone (NTP 1989b).

\section{Gastrointestinal Effects.}

Inorganic Arsenicals. Clinical signs of gastrointestinal irritation, including nausea, vomiting, diarrhea, and abdominal pain, are observed in essentially all cases of short-term high-dose exposures to inorganic arsenic (e.g., Armstrong et al. 1984; Bartolome et al. 1999; Campbell and Alvarez 1989; Chakraborti et al. 2003a; Cullen et al. 1995; Fincher and Koerker 1987; Goebel et al. 1990; Kingston et al. 1993; LevinScherz et al. 1987; Lugo et al. 1969; Moore et al. 1994b; Muzi et al. 2001; Uede and Furukawa 2003; Vantroyen et al. 2004). Similar signs are also frequently observed in groups or individuals with longerterm, lower-dose exposures (e.g., Borgoño and Greiber 1972; Cebrián et al. 1983; Franzblau and Lilis 1989; Guha Mazumder et al. 1988, 1998a; Haupert et al. 1996; Holland 1904; Huang et al. 1985; Mizuta et al. 1956; Nagai et al. 1956; Silver and Wainman 1952; Wagner et al. 1979; Zaldívar 1974), but effects are usually not detectable at exposure levels below about $0.01 \mathrm{mg} \mathrm{As} / \mathrm{kg} / \mathrm{day}$ (Harrington et al. 1978; Valentine et al. 1985). These symptoms generally decline within a short time after exposure ceases. 
Gastrointestinal irritation symptoms form the basis (in part) for the acute oral MRL of $0.005 \mathrm{mg} / \mathrm{kg} / \mathrm{day}$ for inorganic arsenic, as described in footnote b in Table 3-3. More severe symptoms (hematemesis, hemoperitoneum, gastrointestinal hemorrhage, and necrosis) have been reported in some cases with acute exposure to $8 \mathrm{mg}$ As/kg or more (Civantos et al. 1995; Fincher and Koerker 1987; Levin-Scherz et al. 1987; Quatrehomme et al. 1992), and also in some people with long-term ingestion of 0.03-0.05 mg As/kg/day as a medicinal preparation (Lander et al. 1975; Morris et al. 1974).

Clinical signs of gastrointestinal irritation were observed in monkeys and rats given repeated oral doses of arsenic (6 and $11 \mathrm{mg} \mathrm{As/kg/day,} \mathrm{respectively)} \mathrm{for} 2$ weeks (Bekemeier and Hirschelmann 1989; Heywood and Sortwell 1979). Hemorrhagic gastrointestinal lesions have also been reported in animal studies. A monkey that died after repeated oral treatment with $6 \mathrm{mg} \mathrm{As} / \mathrm{kg} /$ day for approximately 1 month was found to have acute inflammation and hemorrhage of the small intestine upon necropsy (Heywood and Sortwell 1979). This lesion was not found in other monkeys that died in this study, or in the survivors. Two pregnant mice that died after repeated gavage treatment with $24 \mathrm{mg} \mathrm{As} / \mathrm{kg} / \mathrm{day}$ as arsenic acid had hemorrhagic lesions in the stomach (Nemec et al. 1998). Gross gastrointestinal lesions (stomach adhesions, eroded luminal epithelium in the stomach) were seen frequently in rats treated by gavage with $8 \mathrm{mg} \mathrm{As} / \mathrm{kg} / \mathrm{day}$ as arsenic trioxide starting before mating and continuing through the end of gestation (Holson et al. 2000). The lesions were not found in rats treated with $4 \mathrm{mg} \mathrm{As} / \mathrm{kg} /$ day in this study. No histological evidence of gastrointestinal injury was detected in rats exposed to arsenate or arsenite in the feed for 2 years at doses up to $30 \mathrm{mg} \mathrm{As} / \mathrm{kg} / \mathrm{day}$, but dogs fed a diet containing $2.4 \mathrm{mg} \mathrm{As} / \mathrm{kg} / \mathrm{day}$ as arsenite for 2 years had some bleeding in the gut (Byron et al. 1967; Kroes et al. 1974).

Organic Arsenicals. Vomiting was noted after ingestion of $793 \mathrm{mg} / \mathrm{kg}$ arsenic (as monosodium methanearsenate) in a suicide attempt (Shum et al. 1995). Ingestion of $78 \mathrm{mg}$ DMA/kg (as dimethyl arsenic acid and dimethyl arsenate) induced vomiting, abdominal pain, hyperactive bowel, and diarrhea (Lee et al. 1995).

The gastrointestinal tract appears to be the critical target of toxicity following oral exposure to MMA. Diarrhea/loose feces has been reported in mice and rabbits following a single gavage dose of 2,200 $\mathrm{mg}$ MMA/kg or $60 \mathrm{mg} \mathrm{MSMA/kg,} \mathrm{respectively} \mathrm{(Jaghabir} \mathrm{et} \mathrm{al.} \mathrm{1988;} \mathrm{Kaise} \mathrm{et} \mathrm{al.} \mathrm{1989),} \mathrm{pregnant} \mathrm{rabbits}$ administered $12 \mathrm{mg} \mathrm{MMA} / \mathrm{kg} /$ day via gavage (Irvine et al. 2006), rats exposed to $30.2 \mathrm{mg} \mathrm{MMA} / \mathrm{kg} / \mathrm{day}$ in the diet during the first year of a 2-year study (Arnold et al. 2003), dogs administered $2 \mathrm{mg}$ MMA/kg/day via capsule for 52 weeks (Waner and Nyska 1988), rats fed diets containing $25.7 \mathrm{mg}$ MMA/kg/day for 2 years (Arnold et al. 2003), and mice exposed to $67.1 \mathrm{mg} \mathrm{MMA} / \mathrm{kg} / \mathrm{day}$ in the diet for 
2 years (Arnold et al. 2003). However, the increased incidence of diarrhea is not always accompanied by macroscopic or histological alterations in the gastrointestinal tissues. For example, in the 2-year rat study (Arnold et al. 2003; incidence data reported in Crown et al. 1990), an increased incidence of diarrhea was observed at $25.7 \mathrm{mg} \mathrm{MMA} / \mathrm{kg} /$ day; macroscopic or histological alterations were observed in some animals, but the incidence was similar to controls. At the next highest dose level (72.4 mg MMA/kg/day), thickened wall and edema and hemorrhagic, necrotic, ulcerated, or perforated mucosa were observed in the large intestine and significant increases in the incidence of squamous metaplasia of the epithelial columnar absorptive cells were found in the cecum, colon, and rectum. Squamous metaplasia was also observed in the cecum and colon of mice chronically exposed to $67.1 \mathrm{mg}$ MMA/kg/day (Arnold et al. 2003; incidence data reported in Gur et al. 1991).

There are some reports of gastrointestinal effects in rats and dogs exposed to DMA; however, the LOAELs for these effects are higher than the LOAELs for MMA and most rodent studies do not report effects at nonlethal doses. Diarrhea with congestion and hemorrhagic gastrointestinal contents were observed in rats exposed to a lethal dose of $190 \mathrm{mg} \mathrm{DMA} / \mathrm{kg} /$ day in the diet for 4 weeks (Crown et al. 1987) and diarrhea and vomiting were reported in dogs administered $16 \mathrm{mg} \mathrm{DMA} / \mathrm{kg} /$ day via capsule 6 days/week (Zomber et al. 1989). No gastrointestinal effects were observed in rats or mice chronically exposed to 7.8 or $94 \mathrm{mg}$ DMA/kg/day, respectively (Arnold et al. 2006).

Vomiting and gastrointestinal hemorrhage were observed in dogs after a single capsulized dose of $50 \mathrm{mg} / \mathrm{kg}$ roxarsone (Kerr et al. 1963), although slightly higher doses administered for 13 weeks to rats and mice had no effect (NTP 1989b). No gastrointestinal effects were seen after chronic exposure of rats (4 mg/kg/day) or mice (43 mg/kg/day) to roxarsone (NTP 1989b).

\section{Hematological Effects.}

Inorganic Arsenicals. Anemia and leukopenia are common effects of arsenic poisoning in humans, and have been reported following acute (Armstrong et al. 1984; Goldsmith and From 1986; Mizuta et al. 1956; Muzi et al. 2001; Westhoff et al. 1975), intermediate (Franzblau and Lilis 1989; Heyman et al. 1956; Nagai et al. 1956; Wagner et al. 1979), and chronic oral exposures (Chakraborti et al. 2003a; Glazener et al. 1968; Guha Mazumder et al. 1988; Hopenhayn et al. 2006; Kyle and Pease 1965; Tay and Seah 1975) at doses of $0.002 \mathrm{mg} \mathrm{As} / \mathrm{kg} /$ day or more. These effects may be due to both a direct cytotoxic or hemolytic effect on the blood cells (Armstrong et al. 1984; Fincher and Koerker 1987; Goldsmith and From 1986; Kyle and Pease 1965; Lerman et al. 1980) and a suppression of erythropoiesis (Kyle and 
Pease 1965; Lerman et al. 1980). However, hematological effects are not observed in all cases of arsenic exposure (EPA 1981b; Harrington et al. 1978; Huang et al. 1985; Silver and Wainman 1952) or even all acute poisoning cases (Cullen et al. 1995; Moore et al. 1994b).

In an acute animal study, Tice et al. (1997) found that there was a decrease in polychromatic erythrocytes in the bone marrow of mice treated with $6 \mathrm{mg} \mathrm{As} / \mathrm{kg} /$ day for 1 or 4 days. There was no effect at $3 \mathrm{mg}$ $\mathrm{As} / \mathrm{kg} /$ day. Long-term studies found mild anemia in dogs fed arsenite or arsenate for 2 years at $2.4 \mathrm{mg}$ As/kg/day, but no hematological effect in dogs fed $1 \mathrm{mg} \mathrm{As} / \mathrm{kg} /$ day for 2 years or $1.9 \mathrm{mg} \mathrm{As} / \mathrm{kg} / \mathrm{day}$ for 26 weeks (Byron et al. 1967; Neiger and Osweiler 1989). Chronic rat studies found little or no evidence of anemia at doses up to $30 \mathrm{mg} \mathrm{As} / \mathrm{kg} /$ day, even with co-exposure to lead (Byron et al. 1967; Kroes et al. 1974). No hematological effects were found in monkeys exposed to arsenic doses of 3-6 mg As/ $\mathrm{kg} / \mathrm{day}$ for 1 year (Heywood and Sortwell 1979).

Rats exposed to arsenate for 6 weeks had decreased activities of several enzymes involved in heme synthesis, but data were not provided on whether this resulted in anemia (Woods and Fowler 1977, 1978). Exposure of rats to $\geq 5 \mathrm{ppm}$ of arsenic $(0.30 \mathrm{mg} \mathrm{As} / \mathrm{kg} /$ day as sodium arsenite) in the drinking water for 4 weeks resulted in increased platelet aggregation, while 10 or $25 \mathrm{ppm}(0.60$ or $1.5 \mathrm{mg} \mathrm{As} / \mathrm{kg} / \mathrm{day})$ was associated with increased P-selectin-positive cells and decreased occlusion time (Lee et al. 2002), representing a change in platelet function. Similarly, exposure of rats or guinea pigs to 10 or $25 \mathrm{ppm}$ of arsenic as arsenite (approximate doses of $0,0.92$, or $2.3 \mathrm{mg} \mathrm{As} / \mathrm{kg} /$ day for rats and 0, 0.69, or $1.7 \mathrm{mg}$ As/kg/day for guinea pigs) in the drinking water for 16 weeks (Kannan et al. 2001) resulted in decreases in erythrocyte and leukocyte numbers (rats and guinea pigs), increased blood mean corpuscular volume and corpuscular hemoglobin mass (guinea pigs only), and decreased mean corpuscular hemoglobin concentration (rats only). Gallium arsenide also disrupts heme synthesis in rats, although the evidence suggests that this effect is due primarily to the gallium moiety (Flora et al. 1997a).

Organic Arsenicals. No adverse hematological effects were noted in a man who ingested $78 \mathrm{mg} / \mathrm{kg}$ as dimethyl arsenic acid and dimethyl arsenate (Lee et al. 1995). No hematological effects were observed in rats exposed to 8.4 or $72.4 \mathrm{mg} \mathrm{MMA} / \mathrm{kg}$ /day for 2 years (Arnold et al. 2003; Shen et al. 2003) or dogs administered $35 \mathrm{mg} \mathrm{MMA} / \mathrm{kg} /$ day for 52 weeks (Waner and Nyska 1988); additionally, no alterations in total or differential leukocyte levels were observed in mice exposed to $67.1 \mathrm{mg}$ MMA/kg/day for 2 years (Arnold et al. 2003). Although some studies have reported hematological alterations following oral exposure to DMA, this is not a consistent finding. Observed alterations include decreased mean corpuscular hemoglobin concentration in rats exposed to $2.3 \mathrm{mg} \mathrm{DMA} / \mathrm{kg} /$ day for 10 weeks (Rubin et al. 
1989), decreased hemoglobin and erythrocyte levels in rats exposed to $4.5 \mathrm{mg}$ DMA/ $/ \mathrm{kg} / \mathrm{day}$ for 13 weeks (Crown et al. 1987), decreased erythrocyte levels and increased leukocyte levels in dogs administered capsules containing $40 \mathrm{mg} \mathrm{DMA} / \mathrm{kg}$ /day for 52 weeks (Zomber et al. 1989), and decreased lymphocyte and increased monocyte levels were observed in mice chronically exposed to $94 \mathrm{mg} \mathrm{DMA} / \mathrm{kg} / \mathrm{day}$ (Arnold et al. 2006). No hematological alterations have been observed in rats exposed to $7.8 \mathrm{mg}$ DMA $/ \mathrm{kg}$ /day for 2 years (Arnold et al. 2006). Similarly, no hematological effects were observed in rats (Kerr et al. 1963; NTP 1989b), mice (NTP 1989b), or dogs (Prier et al. 1963) exposed to 20-32, 68, or $5 \mathrm{mg} / \mathrm{kg} /$ day roxarsone, respectively, for intermediate or chronic durations

\section{Musculoskeletal Effects.}

Inorganic Arsenicals. No studies were located regarding musculoskeletal effects in humans or animals after oral exposure to inorganic arsenicals.

Organic Arsenicals. No studies were located regarding musculoskeletal effects in humans after oral exposure to organic arsenicals. No musculoskeletal effects were seen after intermediate or chronic exposure of rats and mice to MMA (Arnold et al. 2003), DMA (Arnold et al. 2006), or roxarsone (NTP 1989b).

\section{Hepatic Effects.}

Inorganic Arsenicals. A number of studies in humans exposed to inorganic arsenic by the oral route have noted signs or symptoms of hepatic injury. Clinical examination often reveals that the liver is swollen and tender (Chakraborty and Saha 1987; Franklin et al. 1950; Guha Mazumder et al. 1988, 1998a; Liu et al. 2002; Mizuta et al. 1956; Silver and Wainman 1952; Wade and Frazer 1953; Zaldívar 1974), and analysis of blood sometimes shows elevated levels of hepatic enzymes (Armstrong et al. 1984; Franzblau and Lilis 1989; Guha Mazumder 2005; Hernández-Zavala et al. 1998). These effects are most often observed after repeated exposure to doses of 0.01-0.1 mg As/kg/day (Chakraborty and Saha 1987; Franklin et al. 1950; Franzblau and Lilis 1989; Guha Mazumder et al. 1988; Mizuta et al. 1956; Silver and Wainman 1952; Wade and Frazer 1953), although doses as low as $0.006 \mathrm{mg} \mathrm{As} / \mathrm{kg} / \mathrm{day}$ have been reported to have an effect following chronic exposure (Hernández-Zavala et al. 1998). Hepatic effects have also been reported in acute bolus poisoning cases at doses of $2 \mathrm{mg} \mathrm{As} / \mathrm{kg} / \mathrm{day}$ or more (Hantson et al. 1996; Kamijo et al. 1998; Levin-Scherz et al. 1987; Quatrehomme et al. 1992; Vantroyen et al. 2004), although acute exposure to $19 \mathrm{mg} \mathrm{As} / \mathrm{kg}$ did not cause hepatic effects in an infant (Cullen et al. 1995). 
Histological examination of the livers of persons chronically exposed to similar doses has revealed a consistent finding of portal tract fibrosis (Guha Mazumder 2005; Guha Mazumder et al. 1988; Morris et al. 1974; Piontek et al. 1989; Szuler et al. 1979), leading in some cases to portal hypertension and bleeding from esophageal varices (Szuler et al. 1979); cirrhosis has also been reported at an increased frequency in arsenic-exposed individuals (Tsai et al. 1999). Several researchers consider that these hepatic effects are secondary to damage to the hepatic blood vessels (Morris et al. 1974; Rosenberg 1974), but this is not directly established.

Acute exposure of monkeys to $6 \mathrm{mg}$ As/ $\mathrm{kg} /$ day resulted in vacuolization of the hepatocytes (Heywood and Sortwell 1979). Studies in dogs or mice have not detected clinically significant hepatic injury following exposure to either arsenite or arsenate (Byron et al. 1967; Fowler and Woods 1979; Kerkvliet et al. 1980; Neiger and Osweiler 1989; Schroeder and Balassa 1967), although enlargement of the common bile duct was noted in rats fed either arsenate or arsenite in the diet for 2 years (Byron et al. 1967; Kroes et al. 1974) and lipid vacuolation and fibrosis were seen in the livers of rats exposed to $12 \mathrm{mg} \mathrm{As} / \mathrm{kg} / \mathrm{day}$ as arsenate in the drinking water for 6 weeks (Fowler et al. 1977). Similarly, fatty changes and inflammatory cell infiltration were seen in the livers of both normal and metallothionein-null mice exposed to $5.6 \mathrm{mg}$ arsenic/kg/day in the drinking water for 48 weeks (Liu et al. 2000). Increases in liver zinc and copper concentrations were noted in rats receiving a single oral dose of $10 \mathrm{mg} \mathrm{As} / \mathrm{kg}$ as sodium arsenite (Flora and Tripathi 1998) and hepatic levels of malondialdehyde were increased and glutathione levels were decreased in livers of rats receiving $200 \mathrm{mg} \mathrm{As} / \mathrm{kg}$ as GaAs (Flora et al. 1998). An increase in indices of peroxidation was reported in rats dosed with approximately $0.02 \mathrm{mg} \mathrm{As} / \mathrm{kg} / \mathrm{day}$ for 60 days from drinking water containing $2.5 \mathrm{mg}$ sodium arsenite/L (Bashir et al. 2006); absolute liver weight was also increased at this dose level. Elevated levels of serum aspartate aminotransferase (AST) were observed in rats administered a single oral dose of $100 \mathrm{mg} \mathrm{As} / \mathrm{kg}$ as GaAs (Flora et al. 1998). Exposure of guinea pigs to 0.69 or $1.7 \mathrm{mg} \mathrm{As} / \mathrm{kg} /$ day in the drinking water for 16 weeks, but not in rats exposed to 0.92 or $2.3 \mathrm{mg} \mathrm{As} / \mathrm{kg} /$ day, resulted in increases in delta-aminolevulinic acid synthetase (ALAS) levels (Kannan et al. 2001). Exposure of BALB/C mice to $0.7 \mathrm{mg}$ arsenic/kg/day in the drinking water for 15 months resulted in increased liver weights, changes in liver enzymes (glutathione S-transferase, glutathione reductase, catalase, glucose-6-phosphate dehydrogenase, glutathione peroxidase), fatty liver, and fibrosis (Santra et al. 2000).

Organic Arsenicals. No adverse hepatic effects were noted after ingestion of 1,714 mg/kg MSMA or $78 \mathrm{mg} \mathrm{DMA} / \mathrm{kg}$ (as dimethyl arsenic acid and dimethyl arsenate) in a suicide attempt (Lee et al. 1995; Shum et al. 1995). No other studies of the hepatic effects of organic arsenicals in humans were located. 
Histological examination of livers from rabbits given repeated oral doses of MMA showed diffuse inflammation and hepatocellular degeneration (Jaghabir et al. 1989), but the lesions were not severe. Male rats exposed to a time-weighted average (TWA) dose of $72.4 \mathrm{mg} \mathrm{MMA} / \mathrm{kg} /$ day for 104 weeks showed a decrease in absolute liver weight, while females exposed to $98.5 \mathrm{mg} \mathrm{MMA} / \mathrm{kg} /$ day showed histiocytic proliferation of the liver (Arnold et al. 2003); however, these effects were probably due to a decrease in body weight and secondary complications of perforation and ulceration of the gastrointestinal effect, respectively. Shen et al. (2003) reported increases in and the number of GST-P-positive foci in the livers of rats exposed to average concentrations of $8.4 \mathrm{mg} \mathrm{MMA} / \mathrm{kg} / \mathrm{day}$ in the diet for 104 weeks. No effects were observed in rats exposed to DMA (Siewicki 1981), but mice exposed to one or two oral doses of $720 \mathrm{mg}$ DMA/kg had decreased liver glutathione and cytochrome P-450 content and serum ornithine decarboxylase activity (Ahmad et al. 1999a). Generalized icterus was reported in dogs after acute exposure to roxarsone (Kerr et al. 1963). Some small fluctuations in liver weight have been noted in rats and mice after intermediate oral exposure to roxarsone, but the toxicological significance of this is not clear and is not observed after chronic exposure of rats and mice to lower doses (NTP 1989b).

\section{Renal Effects.}

Inorganic Arsenicals. Most case studies of acute and chronic arsenic toxicity do not report clinical signs of significant renal injury, even when other systems are severely impaired (e.g., Cullen et al. 1995; Franzblau and Lilis 1989; Jenkins 1966; Kersjes et al. 1987; Mizuta et al. 1956; Silver and Wainman 1952). In some cases, elevated serum levels of creatinine or bilirubin have been noted (Armstrong et al. 1984; Levin-Scherz et al. 1987; Moore et al. 1994b), and mild proteinuria may occur (Armstrong et al. 1984; Glazener et al. 1968; Tay and Seah 1975). Acute renal failure in some bolus poisoning episodes (e.g., Fincher and Koerker 1987; Goebel et al. 1990; Levin-Scherz et al. 1987; Lugo et al. 1969; Moore et al. 1994b) is probably a result of fluid imbalances or vascular injury (Rosenberg 1974; Zaldívar 1974). Glomerular congestion has been observed after an acute exposure to high doses (Quatrehomme et al. 1992).

Studies in animals also indicate that the kidney is not a major target organ for inorganic arsenic (Byron et al. 1967; Schroeder and Balassa 1967; Woods and Southern 1989), although some effects have been reported at high exposure levels. Mild histological changes in the renal tubules of monkeys exposed to arsenate for 2 weeks were noted by Heywood and Sortwell (1979), and some mild alterations in renal mitochondria in rats exposed to arsenate for 6 weeks were noted by Brown et al. (1976). Mild proteinuria 
(Flora et al. 1998) and an increase in kidney zinc concentration (Flora and Tripathi 1998) have also been noted in rats exposed orally to a single dose of $100 \mathrm{mg} \mathrm{As} / \mathrm{kg}$ as GaAs or $10 \mathrm{mg} \mathrm{As} / \mathrm{kg}$ as sodium arsenite, respectively. These data suggest that the kidney is relatively less sensitive to arsenic than most other organ systems, and renal effects are unlikely to be of concern except secondary to fluid imbalances or cardiovascular injury.

Organic Arsenicals. No adverse renal effects were noted after ingestion of 1,714 mg MSMA/kg in a suicide attempt (Shum et al. 1995). Animal studies have reported renal and urinary bladder effects following oral exposure to organic arsenicals; the available data suggest that the urinary system is a more sensitive target for DMA, than for MMA or roxarsone. A decrease in urine volume was observed in rabbits following a single gavage dose of $30 \mathrm{mg} \mathrm{MSMA} / \mathrm{kg} /$ day (Jaghabir et al. 1988) and a decrease in urine volume (35 mg MMA/kg/day) and an increase in urine specific gravity ( $8 \mathrm{mg} \mathrm{MMA} / \mathrm{kg} /$ day) were observed in dogs administered MMA via capsule for 52 weeks (Waner and Nyska 1988). However, these effects may be indicative of dehydration due to diarrhea rather than a direct effect on the kidney. In a 2-year study in rats (Arnold et al. 2003), an increase in the severity of progressive glomerulonephropathy was observed in females at $33.9 \mathrm{mg} \mathrm{MMA} / \mathrm{kg} /$ day. Hydronephrosis, pyelonephritis, cystitis, and decreases in urine volume and $\mathrm{pH}$ were also observed $72.4 \mathrm{mg} \mathrm{MMA} / \mathrm{kg} /$ day; however, the investigators noted that these lesions probably resulted from urinary tract obstruction, which was secondary to peritonitis caused by gastrointestinal tract ulcerations. An increased incidence of progressive glomerulonephropathy was also observed in male mice exposed to $\geq 6.0 \mathrm{mg} \mathrm{MMA} / \mathrm{kg} /$ day in the diet for 2 years (Arnold et al. 2003; incidence data reported in Gur et al. 1991); the investigators (Gur et al. 1991) noted that the kidney lesions were consistent with the normal spectrum of spontaneous lesions and that there were no differences in character or severity of the lesions between the different groups.

Exposure to DMA has resulted in kidney effects in rats and mice exposed to at least $3.1 \mathrm{or} 37 \mathrm{mg}$ $\mathrm{DMA} / \mathrm{kg} /$ day, respectively; no renal effects were observed in dogs exposed to doses as high as $40 \mathrm{mg}$ $\mathrm{As} / \mathrm{kg} /$ day for 52 weeks (Zomber et al. 1989). In rats, the renal damage is characterized by increased urine volume and $\mathrm{pH}$, decreased urine osmolarity and electrolyte (sodium, potassium, chlorine) levels, increased urinary calcium levels, and increased organ weight, nephrocalcinosis, and necrosis in the renal papillae and/or cortex; an increase in water consumption is also typically observed. The LOAELs for these effects are 5-57 mg DMA/kg/day in intermediate-duration studies (Arnold et al. 1999; Crown et al. 1987; Murai et al. 1993) and $3.1 \mathrm{mg} \mathrm{DMA} / \mathrm{kg} /$ day in a chronic-duration study (Arnold et al. 2006). Another study did not find renal effects in rats exposed to $16.5 \mathrm{mg} \mathrm{DMA} / \mathrm{kg} / \mathrm{day}$ (Rubin et al. 1989). This study involved exposure to Sprague-Dawley rats compared to Fischer 344 rats used in the studies with 
positive results; it is not known if this reflects a difference in strain sensitivity. In mice, progressive glomerulonephropathy was observed at $37 \mathrm{mg} \mathrm{DMA} / \mathrm{kg} /$ day and nephrocalcinosis was observed at $94 \mathrm{mg}$ DMA/kg/day (Arnold et al. 2006).

Increased kidney weights and minimal tubular epithelial cell degeneration, tubular casts, and focal mineralization were observed in rats exposed to $32 \mathrm{mg} / \mathrm{kg} /$ day roxarsone for 13 weeks (NTP 1989b). No adverse effects were observed in rats at doses as high as $20 \mathrm{mg} / \mathrm{kg} /$ day (Kerr et al. 1963; NTP 1989b) for 13 weeks or $10 \mathrm{mg} / \mathrm{kg} /$ day for 2 years (NTP 1989b; Prier et al. 1963). No adverse renal effects have been observed in mice exposed to roxarsone doses as high as $136 \mathrm{mg} / \mathrm{kg} /$ day (NTP 1989b) or $43 \mathrm{mg} / \mathrm{kg} / \mathrm{day}$ (NTP 1989b; Prier et al. 1963) for intermediate or chronic durations, respectively, or in dogs exposed to $5 \mathrm{mg} / \mathrm{kg} /$ day for a chronic duration (Prier et al. 1963).

Damage to the urinary bladder has been observed in several studies in which rats were exposed to DMA. The observed effects include altered bladder cell surface characteristics in rats exposed to $11 \mathrm{mg}$ DMA $/ \mathrm{kg} /$ day in the diet for 2 weeks (Cohen et al. 2001), increased bladder weight and regenerative proliferation (measured as an increase in BrdU labeling) in bladder epithelium at $5 \mathrm{mg} \mathrm{DMA} / \mathrm{kg} / \mathrm{day}$ for 10 weeks (Arnold et al. 1999), necrosis of bladder epithelium at $10 \mathrm{mg}$ DMA/kg/day for 10 weeks (Arnold et al. 1999), nodular or papillar hyperplasia at $3.4 \mathrm{mg} \mathrm{DMA} / \mathrm{kg} /$ day for 2 years (Wei et al. 2002), and urothelial vacuolar degeneration and hyperplasia of urothelial cells at $3.1 \mathrm{mg} \mathrm{DMA} / \mathrm{kg} /$ day for 2 years (Arnold et al. 2006). Vacuolization of the urothelium in the urinary bladder have also been observed in mice exposed to $7.8 \mathrm{mg} \mathrm{DMA} / \mathrm{kg} /$ day in the diet for 2 years (Arnold et al. 2006). Inconsistent results were found for MMA. Hyperplasia was observed in the bladders of rats exposed to $1 \mathrm{mg} \mathrm{As} / \mathrm{kg} / \mathrm{day}$ as MMA in drinking water for 2 years (Shen et al. 2003), but bladder effects were not observed in another 2-year study (Arnold et al. 2003) in which rats were exposed to doses as high as $34.8 \mathrm{mg} \mathrm{As} / \mathrm{kg} /$ day as MMA in the diet. No urinary bladder effects were found in rats and mice exposed to 64 or $136 \mathrm{mg} / \mathrm{kg} /$ day roxarsone for 13 weeks (NTP 1989b) or 4 or $43 \mathrm{mg} / \mathrm{kg} /$ day roxarsone for 2 years (NTP 1989b).

\section{Endocrine Effects.}

Inorganic Arsenicals. Very little has been written about the effects of oral exposure to arsenic on endocrine glands. In a report of the autopsies of five children who died in Chile after chronic exposure to arsenic in the drinking water, arterial thickening in the pancreas was noted (Rosenberg 1974). An association has been demonstrated between exposure to arsenic in drinking water and an increased 
incidence of diabetes mellitus (Lai et al. 1994; Rahman et al. 1998; Tsai et al. 1999; Tseng et al. 2000; Wang et al. 2003), although dose-response relationships are not available.

Exposure of rats to $2.3 \mathrm{mg} \mathrm{As} / \mathrm{kg} / \mathrm{day}$ as arsenic trioxide for 30 days resulted in reductions in the number of islet cells in the pancreas, as well as significant reductions in pancreatic superoxide dismutase (SOD) and catalase enzyme levels and increases in the production of nitric oxide and malondialdehyde (Mukherjee et al. 2004).

Organic Arsenicals. No studies of effects of organic arsenic compounds on endocrine glands in humans were found. Hypertrophy of thyroid epithelium was observed in rats exposed to $33.9 \mathrm{mg} \mathrm{MMA} / \mathrm{kg} / \mathrm{day}$ in the diet for 2 years (Arnold et al. 2003), $4.0 \mathrm{mg}$ DMA/kg/day in the diet for 13 weeks (Crown et al. 1987), $16.5 \mathrm{mg} \mathrm{DMA} / \mathrm{kg} /$ day in the diet for at least 10 weeks (Rubin et al. 1989), and $7.8 \mathrm{mg}$ $\mathrm{DMA} / \mathrm{kg} /$ day in the diet for 2 years (Arnold et al. 2006). No other biologically significant effects were observed in other endocrine tissues following exposure to MMA or DMA. No adverse effects were seen in the adrenal or pituitary glands, thyroid, or pancreas after intermediate or chronic exposure of rats (2064 or $4 \mathrm{mg} / \mathrm{kg} /$ day, respectively) and mice (136 or $43 \mathrm{mg} / \mathrm{kg} /$ day, respectively) to roxarsone (NTP 1989b).

\section{Dermal Effects.}

Inorganic Arsenicals. One of the most common and characteristic effects of arsenic ingestion is a pattern of skin changes that include generalized hyperkeratosis and formation of hyperkeratotic warts or corns on the palms and soles, along with areas of hyperpigmentation interspersed with small areas of hypopigmentation on the face, neck, and back. These and other dermal effects have been noted in a large majority of human studies involving repeated oral exposure (e.g., Ahmad et al. 1997, 1999b; Ahsan et al. 2000; Bickley and Papa 1989; Borgoño and Greiber 1972; Borgoño et al. 1980; Cebrián et al. 1983; Chakraborti et al. 2003a, 2003b; Chakraborty and Saha 1987; Foy et al. 1992; Franklin et al. 1950; Franzblau and Lilis 1989; Guha Mazumder et al. 1988, 1998a, 1998b, 1998c; Guo et al. 2001a; Haupert et al. 1996; Huang et al. 1985; Lander et al. 1975; Liu et al. 2002; Lüchtrath 1983; Milton et al. 2004; Mizuta et al. 1956; Morris et al. 1974; Nagai et al. 1956; Piontek et al. 1989; Rosenberg 1974; Saha and Poddar 1986; Silver and Wainman 1952; Szuler et al. 1979; Tay and Seah 1975; Tseng et al. 1968; Wade and Frazer 1953; Wagner et al. 1979; Wong et al. 1998a, 1998b; Zaldívar 1974, 1977). In cases of lowlevel chronic exposure (usually from water), these skin lesions appear to be the most sensitive indication of effect, so this end point is considered to be the most appropriate basis for establishing a chronic oral MRL. This is supported by the finding that other effects (hepatic injury, vascular disease, neurological 
effects) also appear to have similar thresholds. As shown in Table 3-3 and Figure 3-3, numerous studies in humans have reported dermal effects at chronic dose levels generally ranging from about 0.01 to $0.1 \mathrm{mg} \mathrm{As} / \mathrm{kg} /$ day (Ahmad et al. 1997; Bickley and Papa 1989; Borgoño and Greiber 1972; Borgoño et al. 1980; Cebrián et al. 1983; Chakraborty and Saha 1987; Foy et al. 1992; Franklin et al. 1950; Guha Mazumder et al. 1988; Huang et al. 1985; Lüchtrath 1983; Piontek et al. 1989; Silver and Wainman 1952; Tseng et al. 1968; Zaldívar 1974, 1977). However, in a study with detailed exposure assessment, all confirmed cases of skin lesions ingested water containing $>100 \mu \mathrm{g} / \mathrm{L}$ arsenic (approximately $0.0037 \mathrm{mg}$ $\mathrm{As} / \mathrm{kg} /$ day) and the lowest known peak arsenic concentration ingested by a case was $0.115 \mu \mathrm{g} / \mathrm{L}$ (approximately $0.0043 \mathrm{mg} \mathrm{As} / \mathrm{kg} /$ day) (Haque et al. 2003). Another large study reported increased incidence of skin lesions associated with estimated doses of $0.0012 \mathrm{mg} \mathrm{As} / \mathrm{kg} / \mathrm{day}(0.023 \mathrm{mg} \mathrm{As} / \mathrm{L}$ drinking water) (Ahsan et al. 2006). Several epidemiological studies of moderately sized populations (20-200 people) exposed to arsenic through drinking water have detected no dermal or other effects at average chronic doses of 0.0004-0.01 mg As/kg/day (Cebrián et al. 1983; EPA 1981b; Guha Mazumder et al. 1988; Harrington et al. 1978; Valentine et al. 1985), and one very large study detected no effects in any person at an average total daily intake (from water plus food) of $0.0008 \mathrm{mg} \mathrm{As} / \mathrm{kg} /$ day (Tseng et al. 1968). This value has been used to calculate a chronic oral MRL for inorganic arsenic of $0.0003 \mathrm{mg} / \mathrm{kg} / \mathrm{day}$, as described in footnote $\mathrm{c}$ in Table 3-3.

Another prominent dermal effect associated with chronic ingestion of inorganic arsenic is skin cancer. As discussed in greater detail in Section 3.2.2.7 (below), some of these skin cancers may evolve from the hyperkeratotic corns or warts, while the areas of altered pigmentation are not considered to be precancerous (EPA 1988d).

Dermal lesions similar to those observed in humans have not been noted in oral exposure studies in monkeys (Heywood and Sortwell 1979), dogs (Byron et al. 1967), or rodents (Schroeder et al. 1968). However, a hyperplastic response to oral arsenic exposure was reported in arsenic-exposed mice (Rossman et al. 2004).

Organic Arsenicals. No studies were located regarding dermal effects in humans after oral exposure to organic arsenicals. No gross or histological skin alterations were observed in rats or mice following intermediate- or chronic-duration exposure to MMA (Arnold et al. 2003; as reported in Crown et al. 1990; Gur et al. 1991), DMA (Arnold et al. 2006; as reported in Gur et al. 1989a, 1989b), or roxarsone (NTP $1989 b)$ 


\section{Ocular Effects.}

Inorganic Arsenicals. Periorbital swelling was reported in people drinking contaminated well water at an approximate dose of $0.2 \mathrm{mg}$ As/ $\mathrm{kg}$ for 1 week (Armstrong et al. 1984). Facial edema, generally involving the eyelids, was a prominent feature of arsenic poisoning among 220 cases associated with an episode of arsenic contamination of soy sauce in Japan (Mizuta et al. 1956). Exposure was to an estimated dose of $0.05 \mathrm{mg} / \mathrm{kg} /$ day and lasted for up to 2-3 weeks. The edema developed soon after the initial exposure and then subsided. This effect forms the basis (in part) for the acute oral MRL of $0.005 \mathrm{mg} / \mathrm{kg} / \mathrm{day}$ for inorganic arsenic, as described in footnote $b$ in Table 3-3. Nemec et al. (1998) noted the appearance of dried red material around the eyes of mice receiving daily oral doses of $24 \mathrm{mg} \mathrm{As} / \mathrm{kg}$ as arsenic acid for 10 days during gestation.

Organic Arsenicals. No studies were located regarding ocular effects in humans or animals after oral exposure to organic arsenicals. No gross or histological alterations in the eye were observed in rats or mice following intermediate- or chronic-duration exposure to MMA (Arnold et al. 2003; as reported in Crown et al. 1990; Gur et al. 1991), DMA (Arnold et al. 2006; as reported in Gur et al. 1989a, 1989b), or roxarsone (NTP 1989b).

\section{Body Weight Effects.}

Inorganic Arsenicals. A 41-year old woman exposed to arsenic in the drinking water for 4 months at an approximate dose of $0.06 \mathrm{mg} \mathrm{As} / \mathrm{kg} /$ day reported losing 40 pounds $(18 \mathrm{~kg})$ of body weight before seeking treatment (Wagner et al. 1979). Weight loss was also among the effects observed in a series of 475 chronic arsenism patients hospitalized in Antofagasto, Chile after receiving approximate doses of $0.02 \mathrm{mg} \mathrm{As} / \mathrm{kg} /$ day in the drinking water for an unspecified number of years (Zaldívar 1974).

Reductions in body weight gain are commonly seen in animal studies of ingested arsenic. In pregnant rats, body weight gain was reduced by gavage treatment with $23 \mathrm{mg} \mathrm{As} / \mathrm{kg} / \mathrm{day}$ as arsenic trioxide on day 9 of gestation (NOAEL=15 mg As/kg/day, Stump et al. 1999), and by repeated gavage treatment with $8 \mathrm{mg} \mathrm{As} / \mathrm{kg} /$ day as arsenic trioxide from 2 weeks prior to mating through gestation (NOAEL=4 $\mathrm{mg}$ $\mathrm{As} / \mathrm{kg} / \mathrm{day}$, Holson et al. 2000). Exposure of rats by gavage to $26.6 \mathrm{mg} \mathrm{As} / \mathrm{kg} /$ day as sodium arsenite, but not $13.3 \mathrm{mg} \mathrm{As} / \mathrm{kg} /$ day or lower, 5 days/week for 4 weeks resulted in a significant decrease in body weight (Schulz et al. 2002). In 6-week rat studies, body weight gain was decreased at 11-12 mg As/kg/day, but not at 6-9 mg As/kg/day (Brown et al. 1976; Fowler et al. 1977). In a 12-week oral 
gavage study, rats dosed with $1.5 \mathrm{mg} / \mathrm{kg} /$ day sodium arsenite had a median final body weight $18 \%$ lower than controls Dhar et al. (2005). A 60-day rat study with sodium arsenite in the drinking water reported a $13 \%$ reduction in final body weight in rats dosed with approximately $0.02 \mathrm{mg} \mathrm{As} / \mathrm{kg} /$ day (Bashir et al. 2006). In chronic rat studies of arsenate and arsenite, body growth decreases were found at doses as low as $2 \mathrm{mg} \mathrm{As} / \mathrm{kg}$ /day in feeding studies (Byron et al. 1967; Kroes et al. 1974), while rats exposed to lower levels of sodium arsenite in the drinking water $(0.6 \mathrm{mg} \mathrm{As} / \mathrm{kg} /$ day $)$ throughout their lifetimes grew normally (Schroeder et al. 1968). Rats given a single oral dose of $100 \mathrm{mg} \mathrm{As} / \mathrm{kg}$ as GaAs exhibited a $15 \%$ reduction in body weight compared to controls 7 days after exposure (Flora et al. 1998). Body weight gain was decreased in mice at $24 \mathrm{mg} \mathrm{As} / \mathrm{kg} /$ day in a gestation exposure study (Nemec et al. 1998), $10 \mathrm{mg} \mathrm{As} / \mathrm{kg} /$ day in a 6-week study (Fowler and Woods 1979), and $1 \mathrm{mg} \mathrm{As} / \mathrm{kg} /$ day in a 2-year study (Schroeder and Balassa 1967). Growth was unaffected in mice that received $12 \mathrm{mg} \mathrm{As} / \mathrm{kg} /$ day in the gestation exposure study (Nemec et al. 1998), $5 \mathrm{mg} \mathrm{As} / \mathrm{kg} /$ day in the 6-week study (Fowler and Woods 1979), or 0.7-0.8 mg As/kg/day in 1-3 month arsenate drinking water studies (Healy et al. 1998). Dogs chronically treated with $2.4 \mathrm{mg} \mathrm{As} / \mathrm{kg} /$ day as sodium arsenite lost $44-61 \%$ of their starting body weight and died, while lower doses had no effect on growth (Byron et al. 1967). Weight depression was also reported in dogs chronically treated with $2.4 \mathrm{mg} \mathrm{As} / \mathrm{kg} /$ day as sodium arsenate (Byron et al. 1967). Feed consumption and body weight gain were significantly reduced in a dose-related manner in dogs fed 1.5 or $1.9 \mathrm{mg} \mathrm{As} / \mathrm{kg} /$ day as sodium arsenite in the diet (Neiger and Osweiler 1989). Dogs in the high-dose group lost $25 \%$ of their body weight over the 17 -week study period. Pair-fed controls lost weight at the same rate as high-dose dogs, showing that the effect on body weight was due to reduced feed consumption, rather than a direct effect of arsenic.

Organic Arsenicals. No studies were located regarding body weight effects in humans after oral exposure to organic arsenicals. In animal studies of organic arsenicals, decreases in body weight gain were observed in rats and mice after acute, intermediate, and chronic duration exposure to MMA (Arnold et al. 2003; Waner and Nyska 1988), DMA (Murai et al. 1993), and roxarsone (NTP 1989b); decreases in body weight gain have also been reported in pregnant rats and rabbits exposed to MMA (Irvine et al. 2006) or DMA (Irvine et al. 2006; Rogers et al. 1981). For MMA, the decreases in body weight gain were observed following intermediate-duration exposure of rats and dogs to 106.9 or $8 \mathrm{mg} \mathrm{MMA} / \mathrm{kg} / \mathrm{day}$ (Arnold et al. 2003; Waner and Nyska 1988), respectively, and following chronic-duration exposure of rats, mice, and dogs to 25.7, 67.1, or $8 \mathrm{mg} \mathrm{MMA} / \mathrm{kg} /$ day, respectively (Arnold et al. 2003; Waner and Nyska 1988). The decreases in body weight gain occurred at doses that were associated with diarrhea and histological alterations in the gastrointestinal tract (Arnold et al. 2003; Waner and Nyska 1988). One DMA study in nonpregnant animals reported decreases in body weight gain in rats administered $57 \mathrm{mg}$ 
DMA/kg/day via gavage 5 days/week for 4 weeks (Murai et al. 1993); other DMA studies have not reported decreases in body weight gain in rats following exposure to $11 \mathrm{mg} \mathrm{DMA} / \mathrm{kg} /$ day for acute durations (Cohen et al. 2001), 3.7-60 mg DMA/kg/day for intermediate durations (Arnold et al. 1999; Crown et al. 1987; Rubin et al. 1989; Siewicki 1981; Wanibuchi et al. 1996; Yamamoto et al. 1995), or $0.77 \mathrm{mg} \mathrm{DMA} / \mathrm{kg} /$ day for chronic durations (Arnold et al. 2006). No alterations in body weight gain were observed in mice exposed to $94 \mathrm{mg}$ DMA/kg/day for 2 years (Arnold et al. 2006). The lowest doses of roxarsone to produce a decrease in growth were 32 and $16 \mathrm{mg} / \mathrm{kg} /$ day in rats following acute- or intermediate-duration exposure, respectively, and 168 and $136 \mathrm{mg} / \mathrm{kg} /$ day in mice following acute or intermediate exposure (NTP 1989b); at the highest dose tested in chronic studies, no significant alterations in body weight gain were observed in rats at $4 \mathrm{mg} / \mathrm{kg} / \mathrm{day}$ or in mice at $43 \mathrm{mg} / \mathrm{kg} / \mathrm{day}$ (NTP 1989b).

\subsubsection{Immunological and Lymphoreticular Effects}

Inorganic Arsenicals. No studies were located regarding immunological and lymphoreticular effects in humans after oral exposure to inorganic arsenicals. No evidence of immunosuppression was detected in mice exposed to arsenate at levels up to $100 \mathrm{ppm}(20 \mathrm{mg} \mathrm{As} / \mathrm{kg} /$ day) in drinking water (Kerkvliet et al. 1980). This NOAEL is shown in Table 3-3 and Figure 3-3. Gallium arsenide at doses of 52-260 mg $\mathrm{As} / \mathrm{kg} /$ day produced significant, dose-related decreases in relative spleen weight, spleen cellularity, humoral immune response (antibody forming cell response to sheep RBC), and delayed type hypersensitivity in rats (Flora et al. 1998). However, it is not clear to what extent these effects are due to the arsenic moiety.

Organic Arsenicals. No studies were located regarding immunological and lymphoreticular effects in humans or animals after oral exposure to organic arsenicals. No histological alterations were observed in immunological or lymphoreticular tissues following intermediate-duration exposure of rats to $43.2 \mathrm{mg}$ DMA $/ \mathrm{kg} /$ day in the diet (Crown et al. 1987) or rats and mice to 18.23 or $38.7 \mathrm{mg} \mathrm{As} / \mathrm{kg} /$ day as roxarsone, respectively (NTP 1989b) or following chronic-duration exposure of rats and mice to 72.4 or $67.1 \mathrm{mg}$ MMA/kg/day (Arnold et al. 2003), 7.8 or $94 \mathrm{mg} \mathrm{DMA} / \mathrm{kg} /$ day (Arnold et al. 2006), or 4 or $43 \mathrm{mg} / \mathrm{kg} /$ day roxarsone (NTP 1989b). No studies examined immune function following oral exposure to organic arsenicals. 


\subsubsection{Neurological Effects}

Inorganic Arsenicals. A large number of epidemiological studies and case reports indicate that ingestion of inorganic arsenic can cause injury to the nervous system. Acute, high-dose exposures $(2 \mathrm{mg} \mathrm{As} / \mathrm{kg} / \mathrm{day}$ or above) often lead to encephalopathy, with signs and symptoms such as headache, lethargy, mental confusion, hallucination, seizures, and coma (Armstrong et al. 1984; Bartolome et al. 1999; Civantos et al. 1995; Cullen et al. 1995; Danan et al. 1984; Fincher and Koerker 1987; Levin-Scherz et al. 1987; Quatrehomme et al. 1992; Uede and Furukawa 2003; Vantroyen et al. 2004). Repeated exposures to lower levels (0.03-0.1 mg As/kg/day) are typically characterized by a symmetrical peripheral neuropathy (Chakraborti et al. 2003a, 2003b; Foy et al. 1992; Franzblau and Lilis 1989; Guha Mazumder et al. 1988; Hindmarsh et al. 1977; Huang et al. 1985; Lewis et al. 1999; Mizuta et al. 1956; Muzi et al. 2001; Silver and Wainman 1952; Szuler et al. 1979; Wagner et al. 1979). This neuropathy usually begins as numbness in the hands and feet, but later may develop into a painful "pins and needles" sensation. Both sensory and motor nerves are affected, and muscle weakness often develops, sometimes leading to wrist-drop or ankle-drop (Chhuttani et al. 1967; Heyman et al. 1956). Diminished sensitivity to stimulation and abnormal patellar reflexes have also been reported (Mizuta et al. 1956). Histological examination of nerves from affected individuals reveals a dying-back axonopathy with demyelination (Goebel et al. 1990; Hindmarsh and McCurdy 1986). Some recovery may occur following cessation of exposure, but this is a slow process and recovery is usually incomplete (Fincher and Koerker 1987; Le Quesne and McLeod 1977; Murphy et al. 1981). Peripheral neuropathy is also sometimes seen following acute highdose exposures, with or without the previously described encephalopathy (Armstrong et al. 1984; Baker et al. 2005; Fincher and Koerker 1987; Goebel et al. 1990; Hantson et al. 1996; Kamijo et al. 1998). Neurological effects were not generally found in populations chronically exposed to doses of $0.006 \mathrm{mg}$ As/kg/day or less (EPA 1981b; Harrington et al. 1978; Hindmarsh et al. 1977), although fatigue, headache, dizziness, insomnia, nightmare, and numbness of the extremities were among the symptoms reported at 0.005 , but not $0.004 \mathrm{mg}$ As/ $/ \mathrm{kg} /$ day in a study of 31,141 inhabitants of 77 villages in Xinjiang, China (Lianfang and Jianzhong 1994), and depression was reported in some Wisconsin residents exposed to $2-10 \mu \mathrm{g} \mathrm{As} / \mathrm{L}$ in the drinking water for 20 years or longer (Zierold et al. 2004).

There is emerging evidence suggesting that exposure to arsenic may be associated with intellectual deficits in children. For example, Wasserman et al. (2004) conducted a cross-sectional evaluation of intellectual function in 201 children 10 years of age whose parents were part of a larger cohort in Bangladesh. Intellectual function was measured using tests drawn from the Wechsler Intelligence Scale for Children; results were assessed by summing related items into Verbal, Performance, and Full-Scale 
raw scores. The mean arsenic concentration in the water was $0.118 \mathrm{mg} / \mathrm{L}$. The children were divided into four exposure groups, representing $<5.5,5.6-50,50-176$, or 177-790 $\mu \mathrm{g} \mathrm{As} / \mathrm{L}$ drinking water. After adjustment for confounding factors, a dose-related inverse effect of arsenic exposure was seen on both Performance and Full-Scale subset scores; for both end points, exposure to $\geq 50 \mu \mathrm{g} / \mathrm{L}$ resulted in statistically significant differences $(p<0.05)$ relative to the lowest exposure group $(<5.5 \mu \mathrm{g} / \mathrm{L})$. In a later report, the same group of investigators examined 3016 -year-old children from the same area (Wasserman et al. 2007). In this case, the children were categorized into the following quartiles based on water arsenic concentration: $0.1-20.9,21-77.9,78-184.9$, and 185-864 $\mu \mathrm{g} / \mathrm{L}$. After adjustment for water Mn, blood lead, and sociodemographic features known to contribute to intellectual function, water arsenic was significantly negatively associated with both Performance and Processing speed raw scores. Analyses of the dose-response showed that compared to the first quartile, those in the second and third categories had significantly lower Performance raw scores ( $p<0.03$ and $p=0.05$, respectively). Those in the fourth category had marginally significantly lower Full-Scale and Processing Speed raw scores. It should be mentioned, however, that in general, arsenic in the water explained $<1 \%$ of the variance in test scores. Water arsenic made no contribution to IQ outcomes. A study of 351 children age 5-15 years from West Bengal, India, found significant associations between urinary arsenic concentrations and reductions in scores of tests of vocabulary, object assembly, and picture completion; the magnitude of the reductions varied between 12 and 21\% (von Ehrenstein et al. 2007). In this cohort, the average lifetime peak arsenic concentration in well water was $0.147 \mathrm{mg} / \mathrm{L}$. However, no clear pattern was found for increasing categories of peak arsenic water concentrations since birth and children's scores in the various neurobehavioral tests conducted. Furthermore, using peak arsenic as a continuous variable in the regression models also did not support an adverse effect on the tests results. Exposure to arsenic in utero also did not suggest an association with the tests scores. Von Ehrestein et al. (2007) concluded that the study provided little evidence for an effect of long-term arsenic concentrations in drinking water and that the lack of findings with past exposures via drinking water may be due to incomplete assessment of past exposure, particularly exposure originating from food. Wasserman's results are consistent with those of ecological studies in children in Taiwan (Tsai et al. 2003) and in China (Wang et al. 2007). In the former, adolescents exposed to low (0.0017-0.0018 mg As/ $\mathrm{kg} /$ day; $\mathrm{n}=20)$ levels of inorganic arsenic in the drinking water showed decreased performance in the switching attention task, while children in the high exposure group (0.0034-0.0042 mg As/kg/day; n=29) showed decreased performance in both the switching attention task and in tests of pattern memory, relative to unexposed controls $(n=60)$. In the study in China (age 8- 12 years), 87 children whose mean arsenic concentration in the drinking water was $0.190 \mathrm{mg} / \mathrm{L}$ had a mean IQ score of 95 compared with 101 for children ( $\mathrm{n}=253)$ with $0.142 \mathrm{mg} / \mathrm{L}$ arsenic in the water and 105 for control children $(n=196)$ with $0.002 \mathrm{mg} / \mathrm{L}$ arsenic in the drinking water (Wang et 
al. 2007). The differences in IQ scores between the two exposure groups and the control group were statistically significant.

Neurological effects have also been observed in animal studies. Rodriguez et al. (2001) evaluated neurobehavioral changes in male Sprague-Dawley rats exposed to $0,5,10$, or $20 \mathrm{mg} \mathrm{As} / \mathrm{kg} / \mathrm{day}$ as sodium arsenite by gavage for 2 or 4 weeks; significant effects were seen in spontaneous locomotor activity and the food pellet manipulation test in the high-dose animals, while no effects were seen in the low- or middose rats. Decreased performance in open field tests were also seen in rats exposed to $26.6 \mathrm{mg}$ As/ $/ \mathrm{kg} /$ day, but not to $13.3 \mathrm{mg} / \mathrm{kg} /$ day or less, as sodium arsenite for 4 weeks (Schulz et al. 2002); curiously, the behavioral changes were no longer present at 8 and 12 weeks of exposure, which may suggest an adaptive response. Heywood and Sortwell (1979) reported salivation and uncontrolled head shaking in two monkeys given several doses of $6 \mathrm{mg} \mathrm{As} / \mathrm{kg} /$ day as arsenate, while no such effects were noted in monkeys given $3 \mathrm{mg} \mathrm{As} / \mathrm{kg} /$ day for 2 weeks. Nemec et al. (1998) observed ataxia and prostration in pregnant female rabbits treated with $1.5 \mathrm{mg} \mathrm{As} / \mathrm{kg} /$ day repeatedly during gestation, but not in rabbits treated with $0.4 \mathrm{mg} \mathrm{As} / \mathrm{kg} / \mathrm{day}$. Some changes in levels of neurotransmitters (dopamine, norepinephrine, and 5-hydroxytryptamine) were seen in rats exposed to $2.3 \mathrm{mg} \mathrm{As} / \mathrm{kg} /$ day as sodium arsenite and guinea pigs exposed to $1.7 \mathrm{mg} \mathrm{As} / \mathrm{kg} / \mathrm{day}$ as sodium arsenite in the drinking water for 16 weeks (Kannan et al. 2001) or in rats exposed to $0.14 \mathrm{mg} \mathrm{As} / \mathrm{kg} /$ day as sodium arsenite by gavage for 28 days (Chattopadhyay et al. 2001), but the functional significance of these changes is not clear.

The highest NOAEL values and all reliable LOAEL values for neurological effects from inorganic arsenic in each species and duration category are recorded in Table 3-3 and plotted in Figure 3-3.

Organic Arsenicals. Numbness and tingling of the fingertips, toes, and circumoral region were reported by a women exposed to an unspecified amount of organic arsenic in bird's nest soup. Discontinuation of exposure resulted in the disappearance of symptoms (Luong and Nguyen 1999). Decreased absolute brain weights were seen in male rats exposed to $25.7 \mathrm{mg} \mathrm{MMA} / \mathrm{kg} /$ day and female rats exposed to $\geq 33.9 \mathrm{mg} \mathrm{MMA} / \mathrm{kg} /$ day, but decreased body weight also occurred at these exposure levels, and relative brain weights were increased in the males at $25.7 \mathrm{mg} \mathrm{MMA} / \mathrm{kg} / \mathrm{day}$ and the females at $\geq 33.9 \mathrm{mg}$ MMA/kg/day in this study (Arnold et al. 2003). No neurological clinical signs or brain lesions were observed following chronic exposure of rats to $72.4 \mathrm{mg} \mathrm{MMA} / \mathrm{kg} /$ day or mice to $67.1 \mathrm{mg} \mathrm{MMA} / \mathrm{kg} / \mathrm{day}$ (Arnold et al. 2003). Decreased spontaneous motility, increased startle response, and ataxia were observed in mice receiving a single gavage dose of 1,757 mg DMA/kg/day (Kaise et al. 1989); no other evidence (clinical signs or histological alterations) were observed in chronic studies of DMA in which 
rats and mice were exposed to 7.8 or $94 \mathrm{mg} \mathrm{DMA} / \mathrm{kg} / \mathrm{day}$, respectively (Arnold et al. 2006). Two studies in pigs indicate that repeated oral doses of roxarsone $(6.3-20 \mathrm{mg} / \mathrm{kg} / \mathrm{day}$ for $1 \mathrm{month})$ can cause significant neurotoxicity (Edmonds and Baker 1986; Kennedy et al. 1986; Rice et al. 1985). The main signs were time-dependent degenerations of myelin and axons (Kennedy et al. 1986; Rice et al. 1985). Evidence of neurological effects (hyperexcitability, ataxia, trembling) was noted in some rat and mouse studies (Kerr et al. 1963; NTP 1989b). Reliable NOAELs and LOAELs are presented in Tables 3-4, 3-5, and 3-6, and Figures 3-4, 3-5, and 3-6.

\subsubsection{Reproductive Effects}

Inorganic Arsenicals. Exposure to arsenic in drinking water has been associated with adverse reproductive outcomes in some studies. For example, a study of 96 women in Bangladesh who had been drinking water containing $\geq 0.10 \mathrm{mg} \mathrm{As} / \mathrm{L}$ (approximately $0.008 \mathrm{mg} \mathrm{As} / \mathrm{kg} /$ day) for 5-10 years reported a significant increase in spontaneous abortions $(p=0.008)$, stillbirth $(p=0.046)$, and preterm birth $(p=0.018)$ compared to a nonexposed group (Ahmad et al. 2001). Similar results were reported by Milton et al. (2005) who found a significant association between concentrations of arsenic in the water $>0.05 \mathrm{mg} / \mathrm{L}$ (approximately $0.006 \mathrm{mg} \mathrm{As} / \mathrm{kg} /$ day) and spontaneous abortion (odds ratio $[\mathrm{OR}]=2.5 ; 95 \% \mathrm{CI}=1.5-4.3$ ) in a study of 533 women, also from Bangladesh. A study of 202 women from West Bengal, India, reported that exposure to arsenic concentrations of arsenic $\geq 0.2 \mathrm{mg} / \mathrm{L}$ in drinking water (approximately $0.02 \mathrm{mg}$ $\mathrm{As} / \mathrm{kg} / \mathrm{day})$ during pregnancy were associated with a 6 -fold increased risk of stillbirth (OR $=6.1 ; 95 \%$ $C I=1.54-24.0$ ) after adjustment for confounders (von Ehrenstein et al. 2006). No association was found between arsenic exposure and risk of spontaneous abortion $(\mathrm{OR}=1.01 ; 95 \% \mathrm{CI}=0.73-10.8)$. An earlier study of 286 women in the United States also found no significant association between arsenic in the drinking water $(0.0016 \mathrm{mg} / \mathrm{L}$; approximately $0.00005 \mathrm{mg} \mathrm{As} / \mathrm{kg} /$ day $)$ and spontaneous abortion (OR=1.7; 95\% CI=0.7-4.2) (Aschengrau et al. 1989).

Lugo et al. (1969) reported a case of a 17-year-old mother who ingested inorganic arsenic (Cowley's Rat and Mouse Poison) at week 30 of pregnancy. Twenty-four hours after ingestion of approximately $30 \mathrm{~mL}$ of arsenic trioxide $(0.39 \mathrm{mg} \mathrm{As} / \mathrm{kg})$, she was admitted for treatment of acute renal failure. She went into labor and delivered a live female infant weighing 2 pounds, 7 ounces with a 1-minute Apgar score of 4. The infant's clinical condition deteriorated and she died at 11 hours of age.

Reproductive performance was not affected in female rats that received gavage doses of $8 \mathrm{mg}$ As/ $/ \mathrm{kg} / \mathrm{day}$ (as $\mathrm{As}_{2} \mathrm{O}_{3}$ ) from 14 days prior to mating through gestation day 19 (Holson et al. 2000). Reproductive 
indices that were evaluated included the precoital interval (time to mating), mating index (percentage of rats mated), and fertility index (percentage of matings resulting in pregnancy). In a 3-generation study in mice given sodium arsenite in drinking water at an average dose of $1 \mathrm{mg} \mathrm{As} / \mathrm{kg} / \mathrm{day}$, there was a significant increase in the incidence of small litters and a trend toward a decreased number of pups per litter in all three generations of the treated group (Schroeder and Mitchener 1971). This finding is consistent with the results of developmental toxicity studies reported in Section 3.2.2.6. Female rats exposed to $0.24 \mathrm{mg} \mathrm{As} / \mathrm{kg} /$ day (as arsenite) for 28 days showed changes in several reproductive system end points, including decreases in wet weights of the ovary and uterus, inhibition of steroidogenic enzymes, decreased ovarian and uterine peroxidase activities, and decreased estradiol levels relative to controls (Chattopadhyay et al. 2001). NOAEL and LOAEL values from these studies are shown in Table 3-3 and Figure 3-3.

Organic Arsenicals. No studies were located regarding reproductive effects in humans after oral exposure to organic arsenicals. No histological alterations in male or female reproductive tissues were observed in laboratory animals following exposure to MMA (Arnold et al. 2003), DMA (Arnold et al. 2006), or roxarsone (NTP 1989b) and no alterations in sperm parameters were observed in male rats exposed to $76 \mathrm{mg} \mathrm{MMA} / \mathrm{kg} /$ day for at least 14 weeks (Schroeder 1994). However, some functional alterations have been reported in animals exposed to MMA or DMA. A decrease in estrus was observed in dogs exposed to $35 \mathrm{mg}$ MMA/kg/day for 52 weeks (Waner and Nyska 1988); decreases in body weight gain (terminal body weight was 59\% lower than controls) were also observed at this dose level and the effect may have been secondary to systemic toxicity. Decreases in pregnancy rate and male fertility index were observed in $\mathrm{F}_{0}$ and $\mathrm{F}_{1}$ rats exposed to $76 \mathrm{mg} \mathrm{MMA} / \mathrm{kg} /$ day for 14 weeks prior to mating and during the mating, gestation, and lactation periods (Schroeder 1994). In the $F_{0}$ animals, the pregnancy rate and male fertility index were not statistically different from controls; however, the values were below historical controls and the investigators considered the effect to be treatment-related. In the $\mathrm{F}_{1}$ animals, the male fertility index was statistically different from controls but the pregnancy rate was not; both parameters were within the range found in historical controls, but the investigators considered the effect to be treatment-related due to the consistency of the findings in the $\mathrm{F}_{0}$ and $\mathrm{F}_{1}$ animals. Impaired fertility, as evidenced by a decreased number of litters, was observed in male mice dosed with MSMA $(119 \mathrm{mg} / \mathrm{kg} /$ day) during a 19-day mating period with unexposed females (Prukop and Savage 1986); the poor reporting of the study protocol and results precludes drawing conclusions from this study. An increase in the number of does with aborted fetuses was observed in rabbits exposed to $48 \mathrm{mg}$ DMA $/ \mathrm{kg} /$ day as DMA (Irvine et al. 2006); severe maternal toxicity (weight loss, reduced food intake, and 
diarrhea) was also observed at this dose level. No reproductive effects were observed in a 2-generation rat study in which rats were exposed to $16.5 \mathrm{mg}$ DMA/kg/day (Rubin et al. 1989).

\subsubsection{Developmental Effects}

Inorganic Arsenicals. Whether ingestion of inorganic arsenic may cause developmental effects in humans has not been extensively investigated. Lugo et al. (1969) reported a case of a mother who ingested inorganic arsenic (Cowley's Rat and Mouse Poison) at 30 weeks of gestation. Twenty-four hours after ingestion, she went into labor and delivered a live female infant weighing 2 pounds, 7 ounces with a 1-minute Apgar score of 4. The infant's clinical condition deteriorated with frequent episodes of apnea and bradycardia; subsequent venous blood gas determinations documented hypoxia, hypercapnea, and acidosis. The infant died at 11 hours of age. Autopsy performed 8 hours after death showed organ immaturity, generalized petechial hemorrhages, and hyaline membrane disease. Severe intra-alveolar pulmonary hemorrhage was remarkable. High arsenic levels were found in the infant's liver, kidney, and brain, demonstrating easy passage of inorganic arsenic across the placenta. The authors considered most of the findings in the neonate to be attributable to immaturity, but suggested that arsenic may have played a role in the severe intra-alveolar hemorrhaging that contributed to death.

Chronic exposure of women to arsenic in the drinking water has been associated with infants with low birth weights in Taiwan (Yang et al. 2003) and Chile (Hopenhayn et al. 2003a). Similar associations have been made between late fetal mortality, neonatal mortality, and postneonatal mortality and exposure to high levels of arsenic in the drinking water (up to $0.86 \mathrm{mg} / \mathrm{L}$ during over a decade), based on comparisons between subjects in low- and high-arsenic areas of Chile (Hopenhayn-Rich et al. 2000). More recently, von Ehrenstein et al. (2006) reported no significant association between exposure to concentrations of $\geq 0.1 \mathrm{mg} / \mathrm{L}$ arsenic in drinking water (approximately $0.008 \mathrm{mg} \mathrm{As} / \mathrm{kg} /$ day) ( $\mathrm{n}=117 ; 29$ women were exposed to $\geq 0.5 \mathrm{mg} / \mathrm{L}$ ) and increased risk for neonatal death or infant mortality during the first year of life in a study of a population in West Bengal, India. The same group of investigators reported significantly increased SMRs for lung cancer and bronchiectasis among subjects in a city in Chile who had probable exposure in utero (maternal exposure) or during childhood to high levels of arsenic (near $0.9 \mathrm{mg} / \mathrm{L}$ ) in the drinking water (Smith et al. 2006). For those exposed in early childhood, the SMR for lung cancer was $7.0(95 \% \mathrm{CI}=5.4-8.9, p<0.001)$ and for bronchiecstasis $12.4(95 \% \mathrm{CI}=3.3-31.7, p<0.001)$. For those born during the high-exposure period, the corresponding SMRs were $6.1(95 \% \mathrm{CI}=3.5-9.9, p<0.001)$ and $46.2(95 \% \mathrm{CI}=21.1-87.7, p<0.001)$. The mortality data analyzed were for the age range $30-49$ years. 
No overall association between arsenic in drinking water and congenital heart defects was detected in a case-control study in Boston (Zierler et al. 1988), although an association with one specific lesion (coarctation of the aorta) was noted $(\mathrm{OR}=3.4,95 \% \mathrm{CI}=1.3-8.9)$. A study of 184 women with neural tube defects in the offspring living in a Texas county bordering Mexico found that exposure to levels of arsenic in the drinking water $>0.010 \mathrm{mg} / \mathrm{L}$ (range or upper limit not specified) did not significantly increase the risk for neural tube defects $(\mathrm{OR}=2.0,95 \% \mathrm{CI}=0.1-3.1)$ (Brender et al. 2006).

Studies in animals, however, suggest that ingested inorganic arsenic may produce developmental effects at high doses that also produce overt maternal toxicity. Rats treated with a single gavage dose of $23 \mathrm{mg}$ $\mathrm{As} / \mathrm{kg}$ as arsenic trioxide on day 9 of gestation had a significant increase in postimplantation loss and a decrease in viable fetuses per litter, while those treated with $15 \mathrm{mg}$ As/ $\mathrm{kg}$ showed no effects (Stump et al. 1999). Rats treated by daily gavage with $8 \mathrm{mg} \mathrm{As} / \mathrm{kg} /$ day starting 14 days before mating and continuing through gestation had significantly reduced fetal body weights and significantly increased incidences of several skeletal variations (unossified sternebrae \#5 or \#6, slight or moderate sternebrae malalignment, 7 th cervical ribs) that the researchers considered to be consequences of developmental growth retardation (Holson et al. 2000). No developmental effects were found at $4 \mathrm{mg} \mathrm{As} / \mathrm{kg} /$ day in this study. Exposure of rats to $2.93-4.20 \mathrm{mg} \mathrm{As} / \mathrm{kg} /$ day throughout gestation and for 4 months postnatally resulted in alterations in neurobehavioral parameters in the offspring, including increased spontaneous locomotor activity and number of errors in a delayed alternation task; maternal behavior was not affected (Rodriguez et al. 2002). Studies in mice found increased fetal mortality, decreased fetal body weight, a low incidence of gross malformations (primarily exencephaly), and an increase in skeletal malformations in mice given single gavage doses of 23-48 mg As/kg during gestation (Baxley et al. 1981; Hood et al. 1978), with no effects at $11 \mathrm{mg} \mathrm{As} / \mathrm{kg}$. Similarly, in mice treated with $24 \mathrm{mg} \mathrm{As} / \mathrm{kg} /$ day as arsenic acid on days 6-15 of gestation, there was a significant increase in the number of resorptions per litter ( $42 \% \mathrm{vs} .4 \%$ in controls) and significant decreases in the number of live pups per litter (6.6 vs. 12.3 in controls) and mean fetal weight (1.0 g vs. $1.3 \mathrm{~g}$ in controls), while no developmental effects were found at $12 \mathrm{mg} \mathrm{As} / \mathrm{kg} / \mathrm{day}$ (Nemec et al. 1998). Hamsters treated with a single gavage dose of $14 \mathrm{mg} \mathrm{As} / \mathrm{kg}$ during gestation also had increased fetal mortality and decreased fetal body weight (Hood and Harrison 1982), with no effect at $11 \mathrm{mg} \mathrm{As} / \mathrm{kg}$. However, the most sensitive species was the rabbit, which had increased resorptions and decreased viable fetuses per litter at $1.5 \mathrm{mg} \mathrm{As} / \mathrm{kg} /$ day and a developmental NOAEL of $0.4 \mathrm{mg}$ $\mathrm{As} / \mathrm{kg} /$ day, following repeated gavage dosing with arsenic acid during gestation (Nemec et al. 1998). In each of these studies (except Hood et al. 1978, which failed to report maternal effects), overt maternal toxicity, including death in some cases, was found at the same or lower doses as the developmental 
effects (Baxley et al. 1981; Holson et al. 2000; Hood and Harrison 1982; Nemec et al. 1998; Stump et al. 1999).

It is noteworthy that the effect in the 3-generation reproduction study in mice by Schroeder and Mitchener (1971), decreased pups per litter (all generations), is consistent with the findings of many of these shorterterm studies (Baxley et al. 1981; Hood and Harrison 1982; Hood et al. 1978; Nemec et al. 1998; Stump et al. 1999). The dose in this long-term study was $1 \mathrm{mg} \mathrm{As} / \mathrm{kg} /$ day; in a 2-year study by these researchers, this dose produced effects such as decreased body weight gain and increased mortality (Schroeder and Balassa 1967).

A series of studies presented evidence that inorganic arsenic may be a transplacental carcinogen in animals. Waalkes et al. (2003, 2004a, 2004b, 2004c) exposed timed-pregnant AJ mice to 0, 42.5, or $85 \mathrm{ppm}$ of sodium arsenite in the drinking water from gestation day 8 through 18 and observed the offspring for 90 weeks following birth; the study authors estimated daily doses at 9.55 and $19.3 \mathrm{mg}$ $\mathrm{As} / \mathrm{kg} /$ day. A dose-related increase was reported in the incidence of hepatocellular carcinomas and adrenal tumors in the male offspring from both treatment levels, while male offspring from high-dose animals showed an increase in total number of tumors. In female offspring, an increase in uterine hyperplasia was seen in the offspring of both treated groups while the offspring of high-dose animals showed increased incidence of lung carcinomas. For both exposed groups, regardless of gender, the offspring showed a significant increase in the number of malignant tumors (Waalkes et al. 2003). More recent studies from the same group of investigators have suggested that aberrant estrogen signaling, potentially through inappropriate estrogen receptor- $\alpha(\mathrm{ER}-\alpha)$, may play a role in arsenic-induced liver tumors in male offspring (Waalkes et al. 2006a) and in arsenic-induced uterine and bladder carcinoma in female offspring (Waalkes et al. 2006b). The latter was based on the observation of over-expression of ER- $\alpha$ and $p S 2$, an estrogen-regulated gene, in the respective tissues.

These studies (shown in Table 3-3 and Figure 3-3) indicate that the fetus may be affected by ingested arsenic.

Organic Arsenicals. No studies were located regarding developmental effects in humans after oral exposure to organic arsenicals. The developmental toxicity of organic arsenicals has been investigated in rats and rabbits for MMA and in rats, mice, and rabbits for DMA. Decreased fetal weights and an increased incidence of fetuses with incomplete ossification of thoracic vertebrae were observed in the offspring of rats administered via gavage $500 \mathrm{mg}$ MMA/kg/day on gestational days 6-15; no 
developmental effects were observed at $100 \mathrm{mg} \mathrm{MMA} / \mathrm{kg} /$ day (Irvine et al. 2006). Decreases in maternal body weight gain were observed at 100 and $500 \mathrm{mg} \mathrm{MMA} / \mathrm{kg} / \mathrm{day}$. A decrease in pup survival was observed in $F_{1}$ and $F_{2}$ offspring of rats exposed to $76 \mathrm{mg} \mathrm{MMA} / \mathrm{kg} /$ day (Schroeder 1994); although pup survival was not statistically different from controls, the investigators considered the effect to be biologically significant because survival in the MMA pups was outside the lower range of survival in historical controls. Increases in the number of fetuses with supernumerary thoracic ribs and eight lumbar vertebrae were observed in the offspring of rabbits administered to $12 \mathrm{mg} \mathrm{MMA} / \mathrm{kg} /$ day on gestational days 7-19 (Irvine et al. 2006); the investigators noted that these effects were probably secondary to maternal stress.

No developmental effects were observed in the offspring of rats administered via gavage $15 \mathrm{mg}$ DMA/kg/day on gestational days 7-16 (Rogers et al. 1981). At $30 \mathrm{mg}$ DMA/kg/day, there was an increase in the percentage of fetuses with irregular palatine rugae; no maternal effects were observed at this dose level (Rogers et al. 1981). The investigators noted that the functional significance of aberrant rugae in rats is not known. Doses of $\geq 36 \mathrm{mg}$ DMA $/ \mathrm{kg} /$ day resulted in decreases in fetal weights and delays in ossification (Chernoff et al. 1990; Irvine et al. 2006; Rogers et al. 1981); decreases in maternal body weight gain were often observed at the same dose levels. Irvine et al. (2006) also reported an increase in the occurrence of diaphragmatic hernia in the offspring of rats exposed to $36 \mathrm{mg}$ DMA/kg/day as DMA on gestational days 6-15. Mice appear to be less sensitive than rats to the developmental toxicity of DMA. No developmental effects were observed in the offspring of mice administered $200 \mathrm{mg}$ DMA/kg/day on gestational days 7-16 (Rogers et al. 1981); at higher doses, decreases in fetal body weight, delays in ossification, and cleft palate were observed (Kavlock et al. 1985; Rogers et al. 1981). In rabbits, a NOAEL of $12 \mathrm{mg} \mathrm{DMA} / \mathrm{kg} /$ day was identified (Irvine et al. 2006); at $48 \mathrm{mg} \mathrm{DMA} / \mathrm{kg} / \mathrm{day}$, there were increased maternal deaths and abortions.

\subsubsection{Cancer}

Inorganic Arsenicals. There is convincing evidence from a large number of epidemiological studies and case reports that ingestion of inorganic arsenic increases the risk of developing skin cancer (Alain et al. 1993; Beane Freeman et al. 2004; Bickley and Papa 1989; Cebrián et al. 1983; Chen et al. 2003; Guo et al. 2001a; Haupert et al. 1996; Hsueh et al. 1995; Lewis et al. 1999; Lüchtrath 1983; Mitra et al. 2004; Morris et al. 1974; Piontek et al. 1989; Sommers and McManus 1953; Tay and Seah 1975; Tsai et al. 1998a, 1999; Tseng 1977; Tseng et al. 1968; Zaldívar 1974; Zaldívar et al. 1981). Lesions commonly observed are multiple squamous cell carcinomas, some of which appear to develop from the 
hyperkeratotic warts or corns described in Section 3.2.2.2. In addition, multiple basal cell carcinomas may occur, typically arising from cells not associated with hyperkeratinization. In most cases, skin cancer develops only after prolonged exposure, but one study has reported skin cancer in people exposed for $<1$ year (Reymann et al. 1978). Although both types of skin cancer can be removed surgically, they may develop into painful lesions that may be fatal if left untreated (Shannon and Strayer 1989).

A number of studies that identify CELs in exposed humans are summarized in Table 3-3 and shown in Figure 3-3. The EPA reviewed the studies that provided dose-response data on the risk of skin cancer (EPA 1988d) and concluded that the most useful study for the purposes of quantitative risk assessment was the ecologic epidemiology study by Tseng et al. (1968). In this study, the incidence of skin cancer was measured as a function of exposure level in over 40,000 people residing in 37 villages in Taiwan, and compared to a control group of over 7,500 people. Beyond the very large sample size, other strengths of this study include excellent case ascertainment (physical examination), inclusion of both males and females, and lifetime exposure duration. Weaknesses and uncertainties include poor nutritional status of the exposed populations, their genetic susceptibility, their exposure to inorganic arsenic from nonwater sources, and the applicability of extrapolating data from Taiwanese to the U.S. population because of different background rates of cancer, possibly genetically determined, and differences in diet other than arsenic (e.g., low protein and fat and high carbohydrate) (EPA 1988d). Because of a lack of information on the amount of individual exposure, subjects were classified into three exposure groups (i.e., high, medium, and low). Based upon pooled data for skin cancer incidence and average well concentrations for each village in the Tseng et al. (1968) study, the EPA calculated a unit risk (the upper-bound excess cancer risk from lifetime exposure to water containing $1 \mu \mathrm{g}$ As/L) of $5 \times 10^{-5}$ (IRIS 2007). The average daily doses (expressed as $\mathrm{mg} \mathrm{As} / \mathrm{kg} /$ day) that correspond to excess cancer risks of $1 \times 10^{-4}-1 \times 10^{-7}$ are shown in Figure 3-3.

The use of a cancer risk estimate derived from the Tseng et al. (1968) study for a U.S. population has been the source of intense debate. Some have argued and have provided data in support of the view that there is persuasive evidence that inorganic arsenic is a cause of human cancer at several sites (i.e., Smith et al. 1992, 1995, 2002). On the other hand, a number of concerns have been raised regarding the strength, or lack of strength, of the database, including: the adequacy of the model used by EPA and the accuracy and reliability of the exposure data (Brown et al. 1997a, 1997b); a number of host and environmental factors among the Taiwanese not applicable elsewhere (Carlson-Lynch et al. 1994); a possible threshold for arsenic carcinogenicity and nonlinearities in the dose-response curve (Abernathy et al. 1996; Slayton et al. 1996); differences in health and nutrition between Taiwan and the United States 
that might increase cancer risk in Taiwan (Beck et al. 1995); the possibility that lower doses of arsenic may be beneficial role in some physiological processes (EPA 1988d; FNB/IOM 2001; NRC 1999, 2001); and the possibility of significant exposure to arsenic from sources other than the well water (Chappell et al. 1997). Many of these factors were recognized by EPA (1988d). A report by NRC (2001) suggested that the risks calculated based on increases in incidence of lung and bladder cancers may be greater than those calculated by the EPA based on incidences of skin cancer.

Several early epidemiological studies performed in the United States did not report an increased frequency of skin cancer in small populations consuming water containing arsenic at levels of around 0.10.2 ppm (EPA 1981b; Goldsmith et al. 1972; Harrington et al. 1978; Morton et al. 1976). These early data suggested that arsenic-associated skin cancer is not a common problem in this country, but these studies lacked sufficient statistical power to detect small increases in skin cancer incidence that might have occurred at these low doses (EPA 1983g). Later studies in exposed U.S. populations from Utah (Lewis et al. 1999) and Iowa (Beane Freeman et al. 2004) have suggested that arsenic-exposed individuals within the United States may have increased incidence or risk of mortality from some skin cancers, melanoma in particular; however, exposure data from these studies are generally insufficient for dose-response analysis. Another study found a suggestion of an arsenic-induced effect on the development of skin cancer, but the association did not achieve statistical significance (Karagas et al. 2001). Therefore, the risk of arsenic-induced skin cancers in U.S. populations, while it may appear to be less than in some other evaluated populations, may be the reflection that, in most studies, exposures were lower.

In addition to the risk of skin cancer, there is mounting evidence that ingestion of arsenic may increase the risks of internal cancers as well. Many case studies have noted the occurrence of internal tumors of the liver and other tissues in patients with arsenic-induced skin cancer (Falk et al. 1981b; Kasper et al. 1984; Koh et al. 1989; Lander et al. 1975; Regelson et al. 1968; Sommers and McManus 1953; Tay and Seah 1975; Zaldívar et al. 1981). These studies are supported by large-scale epidemiological studies, where associations and/or dose response trends have been detected for tumors of the bladder, kidney, liver, lung, and prostate (Chen and Wang 1990; Chen et al. 1985, 1986, 1988a, 1988b, 1992; Chiou et al. 1995; Cuzick et al. 1992; Ferreccio et al. 1998; Guo et al. 1997; Hopenhayn-Rich et al. 1998; Kurttio et al. 1999; Lewis et al. 1999; Moore et al. 2002; Rivara et al. 1997; Smith et al. 1998; Tsuda et al. 1995a; Wu et al. 1989). The EPA has not yet calculated a unit risk value or slope factor for arsenic-induced internal tumors. 
There is increasingly convincing evidence that long-term exposure to arsenic can result in the development of bladder cancer (Bates et al. 2004; Chen et al. 1992, 2003; Chiou et al. 1995, 2001; Cuzick et al. 1992; Guo et al. 2001b; Karagas et al. 2004; Lamm et al. 2004; Michaud et al. 2004; Steinmaus et al. 2003), with transitional cell cancers being the most prevalent. Chiou et al. (1995) reported a doseresponse relationship between long-term arsenic exposure from drinking artesian well water and the incidence of lung cancer, bladder cancer, and cancers of all sites combined (after adjustment for age, sex, and cigarette smoking) in four townships in Taiwan exposed to inorganic arsenic in drinking water (0$1.14 \mathrm{mg} / \mathrm{L})$. In a later followup study of the same cohort, the increase in bladder cancer was found to be statistically significant only in subjects exposed for 40 years or longer (Chiou et al. 2001). Cuzick et al. (1992) evaluated a cohort treated with Fowler's solution (potassium arsenite) in Lancashire, England, during the period 1945-1969 and followed through 1991; the cohort of 478 patients showed a significant excess of bladder cancer, but no excess for other causes of death. Of a subcohort of 142 patients examined for signs of arsenicism around 1970 (Cuzick et al. 1992), all 11 subsequent cancer deaths occurred in those with signs of arsenicism $(p=0.0009)$. Hopenhayn-Rich et al. (1996a) investigated bladder cancer mortality for the years 1986-1991 in the 26 counties of Cordoba, Argentina, and reported that bladder cancer SMRs were consistently higher in counties with documented arsenic exposure; a later case-control study by the same authors (Bates et al. 2004) did not report statistically significant increases in bladder cancers resulting from arsenic exposure, except in individuals exposed for 50 years or longer. Guo et al. (2001a) reported significantly increased rate differences for bladder cancer in men and women in Taiwan exposed to $0.64 \mathrm{mg}$ arsenic/L in the drinking water, but not at lower exposure levels. The arsenic-induced bladder tumors do not appear to be histologically different than similar bladder tumor types of nonarsenic origin (Chow et al. 1997), although they tended to be more pronounced. In contrast, Michaud et al. (2004) reported no correlation between arsenic levels in toenails and the incidence of bladder cancers in Finnish workers. Among evaluated U.S. cohorts, there has generally been no association between arsenic exposure ( $\sim 60-100 \mu \mathrm{g}$ As/L) and the incidence of mortality from bladder cancers (Lamm et al. 2004; Steinmaus et al. 2003), although it is possible that smoking may render individuals more susceptible to arsenic-induced bladder tumors (Karagas et al. 2004; Steinmaus et al. 2003).

Studies have also suggested that chronic oral exposure to arsenic may result in the development of respiratory tumors and increased incidence of lung cancer (Ferreccio et al. 2000; Guo 2004; Nakadaira et al. 2002; Smith et al. 1998; Viren and Silvers 1999). A study of arsenic-exposed individuals in northern Chile reported significantly increased odds ratios for lung cancer among subjects with $\geq 30 \mu \mathrm{g} A s / \mathrm{L}$ of drinking water (Ferreccio et al. 2000), although when adjusted for socioeconomic status, smoking, and 
other factors, the increase was only significant at $60 \mu \mathrm{g}$ As/L or greater. Guo (2004) reported significantly increased rates differences (RD) for lung cancer for Taiwanese men and women exposed to $0.64 \mathrm{mg} \mathrm{As} / \mathrm{L}$ or greater, with those subjects $>50$ years of age being particularly at risk. Nakadaira et al. (2002) suggested that even comparatively short exposure durations ( $\leq 5$ years) may be sufficient for the development of arsenic-induced lung cancer.

Studies in U.S. populations exposed to arsenic in drinking water (EPA 1981b; Lamm et al. 2004; Lewis et al. 1999; Morton et al. 1976; Steinmaus et al. 2003; Valentine et al. 1992) have not yielded the cancer incidences and health effects noted in Taiwan, Mexico, and Chile. Whether this difference is due to a smaller population of subjects compared to Taiwan, to overall lower doses in exposed U.S. populations, or to differences in nutritional or socioeconomic conditions has not been resolved. It should be noted that exposed populations in Mexico and Chile are also smaller than those in Taiwan.

Most studies of animals exposed to arsenate or arsenite by the oral route have not detected any clear evidence for an increased incidence of skin cancer or other cancers (Byron et al. 1967; Kroes et al. 1974; Schroeder et al. 1968). Arsenic has sometimes been called a "paradoxical" human carcinogen because of this lack of animal data (Jager and Ostrosky-Wegman 1997). The basis for the lack of tumorigenicity in animals is not known, but could be related to species-specific differences in arsenic distribution, and induction of cell proliferation (Byrd et al. 1996) (see Section 3.5). As discussed in Section 3.5 below, the carcinogenic effects of arsenic may partially result from its function as a cocarcinogen, which would not manifest in most animal carcinogenicity studies.

One mouse study using transgenic mice (which carry the v-Ha-ras oncogene) administered $48 \mathrm{mg}$ $\mathrm{As} / \mathrm{kg} /$ day as sodium arsenite in drinking water for 4 weeks followed by dermal application of 12-O-tetradecanoylphorbol-13-acetage (TPA) to shaved back skin twice a day for 2 weeks showed an increase in the incidence of skin papillomas when compared to transgenic mice receiving only TPA treatment, only arsenic, or to wild-type mice receiving both TPA and arsenic (Germolec et al. 1998); arsenic treatment alone did not result in increased papilloma incidence. Increases in mRNA transcripts for the growth factors transforming growth factor- $\alpha$ (TGF- $\alpha$ ) and granulocyte/ macrophage-colony stimulating factor (GM-CSF) were detected in the epidermis of the arsenic-treated mice.

A few studies in mice have noted that arsenic ingestion may actually decrease the incidence of some tumor types. For example, arsenic exposure caused decreased incidence of urethane-induced pulmonary tumors (Blakley 1987), spontaneous mammary tumors (Schrauzer and Ishmael 1974; Schrauzer et al. 
1976), and tumors resulting from injection of mouse sarcoma cells (Kerkvliet et al. 1980). However, arsenic also increased the growth rate of the tumors that did occur, resulting in a net decrease in survival time in tumor-bearing animals (Kerkvliet et al. 1980; Schrauzer and Ishmael 1974). These observations suggest that arsenic may affect different types of neoplastic cells differently, perhaps acting mainly as a tumor promoter (Schrauzer and Ishmael 1974; Shirachi et al. 1983), although some studies have suggested that arsenic's actions are not consistent with tumor promotion (Baroni et al. 1963; Boutwell 1963).

There is evidence suggesting that inorganic arsenic can induce cancer in the offspring from mice exposed to arsenic during gestation (transplacental carcinogen) (Waalkes et al. 2003, 2004a, 2004b, 2004c, 2006a, 2006b). These studies are summarized in Section 3.2.2.6, Developmental Effects.

Organic Arsenicals. No studies were located regarding cancer in humans after oral exposure to organic arsenicals. Two lifetime carcinogenicity studies with MMA did not find significant increases in tumors in rats exposed to $72.4 \mathrm{mg} \mathrm{MMA} / \mathrm{kg} /$ day in the diet for 2 years (Arnold et al. 2003) or $8.4 \mathrm{mg} \mathrm{MMA} / \mathrm{kg} / \mathrm{day}$ in drinking water for 2 years (Shen et al. 2003). No significant increases in neoplastic lesions were observed in mice exposed to $67.1 \mathrm{mg} \mathrm{MMA} / \mathrm{kg} /$ day in the diet for 2 years (Arnold et al. 2003).

In contrast, significant increases in the incidence of urinary bladder tumors have been observed in rats exposed for 2 years to $7.8 \mathrm{mg} \mathrm{DMA} / \mathrm{kg} /$ day in the diet (Arnold et al. 2006) or $3.4 \mathrm{mg} \mathrm{DMA} / \mathrm{kg} / \mathrm{day}$ in drinking water (Wei et al. 1999, 2002). The incidence of bladder tumors was similar to controls in the rats exposed to $0.77 \mathrm{mg}$ DMA/kg/day (Arnold et al. 2006) or $0.75 \mathrm{mg}$ DMA $/ \mathrm{kg} /$ day (Wei et al. 1999, 2002). Neither study reported significant increases in the incidence of neoplastic lesions in other tissues. Arnold et al. (2006) did not find increases in the incidence of neoplastic lesions in mice exposed to doses as high as $94 \mathrm{mg}$ DMA/kg/day in the diet for 2 years. Hayashi et al. (1998) reported that exposure of $\mathrm{A} / \mathrm{J}$ mice (a strain susceptible to lung tumorigenesis) to $10.4 \mathrm{mg} \mathrm{DMA} / \mathrm{kg} / \mathrm{day}$ (but not 1.3 or $5.2 \mathrm{mg}$ $\mathrm{DMA} / \mathrm{kg} /$ day) in drinking water for 50 weeks resulted in an increased incidence of papillary adenomas and/or adenocarcinomas and an increased number of lung tumors per mouse.

The incidence of basophilic foci (believed to be a precancerous lesion) in the liver of rats initiated with diethylnitrosamine was increased by subsequent 6-month drinking water exposure to $11 \mathrm{mg} \mathrm{DMA} / \mathrm{kg} / \mathrm{day}$, suggesting that this compound could act as a cancer promoter (Johansen et al. 1984). Additional evidence for the possible role of DMA as a promoter comes from Yamamoto et al. (1995), who reported that 15 or $60 \mathrm{mg}$ DMA $/ \mathrm{kg} /$ day in the drinking water for 24 weeks significantly enhanced the tumor induction in the 
urinary bladder, kidney, liver, and thyroid gland in male F344 rats treated with a series of initiators. Wanibuchi et al. (1996) reported that treatment of F344 rats for 32 weeks with up to $14.3 \mathrm{mg}$ DMA $/ \mathrm{kg} /$ day DMA in the drinking water did not result in increased incidences of urinary bladder papillomas or carcinomas, but that incidence of these tumors was elevated if the animals were first pretreated with an initiating compound (BBN). A later study by Li et al. (1998) reported that NBR rats (which do not synthesize $\alpha_{2 \mu}$-globulin) exposed to an initiator for 4 weeks followed by DMA for 32 weeks, similar to the Wanibuchi et al. (1996) study, showed a statistically significant increase in simple hyperplasia and papillary or nodular hyperplasia of the bladder. A study by Salim et al. (2003) suggested that DMA primarily exerts its carcinogenic effects on spontaneous tumor development.

No increases in tumor incidence were observed in rats, mice, or dogs exposed to 10,13 , or $5 \mathrm{mg} / \mathrm{kg} / \mathrm{day}$ roxarsone, respectively, in the diet for 2 years (Prier et al. 1963). Similarly, no evidence of carcinogenicity was observed in female rats or male or female mice exposed to 4 or $43 \mathrm{mg} / \mathrm{kg} / \mathrm{day}$ as roxarsone in the diet for 2 years (NTP 1989b). However, a slight increase in pancreatic tumors was noted in male rats exposed to $4 \mathrm{mg} / \mathrm{kg} /$ day (NTP 1989b); this was considered to constitute equivocal evidence of carcinogenicity.

\subsubsection{Dermal Exposure}

Adverse effects from dermal exposure to inorganic or organic arsenicals have not been extensively investigated. Table 3-7 summarizes studies in animals and humans that provide quantitative data on dermal exposure-effect relationships for inorganic arsenicals. No quantitative data on dermal exposure to organic arsenicals were located. Available quantitative and qualitative data are discussed in greater detail below.

\subsubsection{Death}

Inorganic Arsenicals. No studies were located regarding death in humans after dermal exposure to inorganic arsenicals. In rats, no deaths resulted from dermal exposure to arsenate or arsenite at doses up to $1,000 \mathrm{mg}$ As $/ \mathrm{kg}$ (Gaines 1960). These data indicate that dermal exposure to inorganic arsenic compounds is very unlikely to result in death.

Organic Arsenicals. No studies were located regarding death in humans after dermal exposure to organic arsenicals. No deaths were observed in rabbits receiving daily dermal applications of $540 \mathrm{mg} \mathrm{As} / \mathrm{kg}$ as 
Table 3-7 Levels of Significant Exposure to Inorganic Arsenic - Dermal

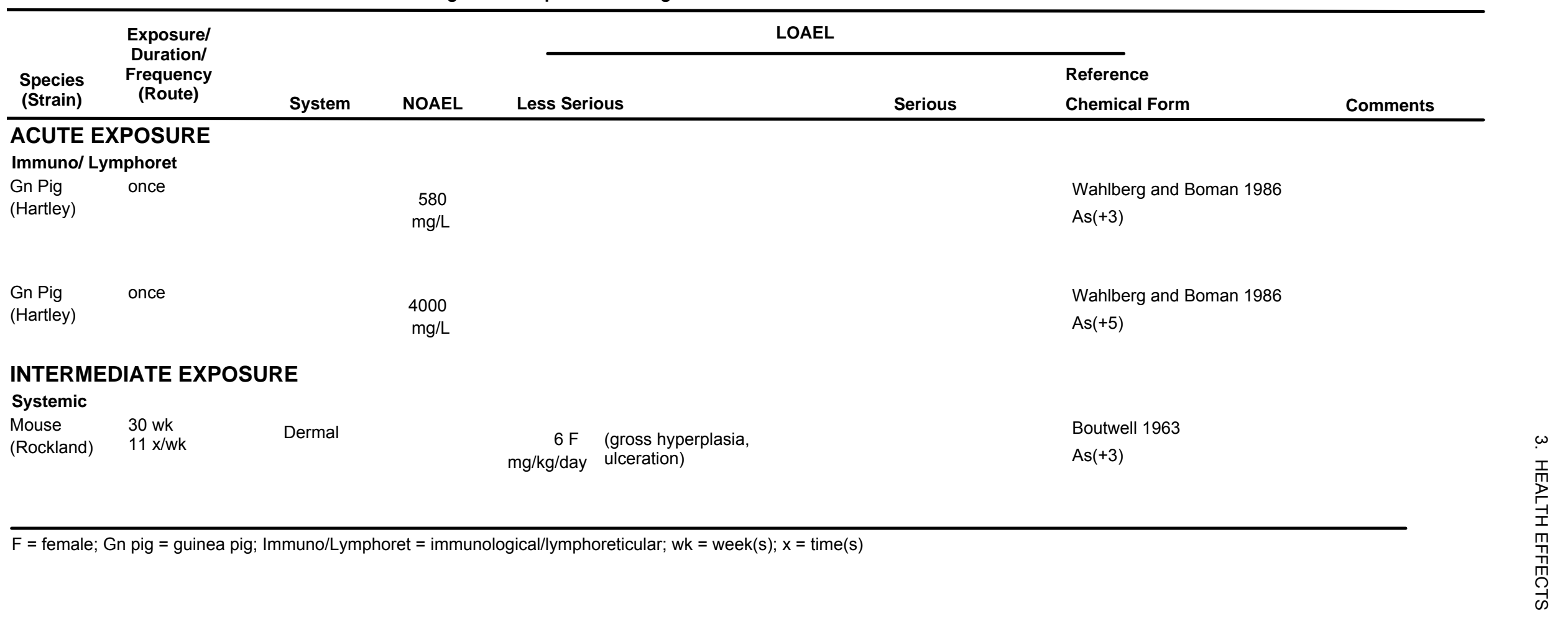


MMA 5 days/week for 21 days (Margitich and Ackerman 1991b) or 1,000 mg DMA/kg/day 5 days/week for 21 days (Margitich and Ackerman 1991a).

\subsubsection{Systemic Effects}

No studies were located that have associated respiratory, cardiovascular, gastrointestinal, hematological, musculoskeletal, hepatic, renal, endocrine, ocular, or body weight effects in humans or animals with dermal exposure to inorganic arsenicals.

Respiratory Effects. No studies were located regarding respiratory effects in humans after dermal exposure to organic arsenicals. No histological effects were observed in the respiratory tracts of rabbits following dermal application of $1,000 \mathrm{mg} / \mathrm{kg} /$ day MMA or DMA 5 days/week for 21 days (Margitich and Ackerman 1991a, 1991b).

Cardiovascular Effects. No studies were located regarding cardiovascular effects in humans after dermal exposure to organic arsenicals. No histological effects were observed in the hearts of rabbits following dermal application of $1,000 \mathrm{mg} / \mathrm{kg} /$ day MMA or DMA 5 days/week for 21 days (Margitich and Ackerman 1991a, 1991b).

Hematological Effects. No studies were located regarding hematological effects in humans after dermal exposure to organic arsenicals. No treatment-related hematological alterations were observed in rabbits receiving dermal applications of 1,000 mg MMA/kg/day (Margitich and Ackerman 1991a) or 1,000 mg DMA/kg/day 5 days/week for 21 days (Margitich and Ackerman 1991b).

Hepatic Effects. No studies were located regarding hepatic effects in humans after dermal exposure to organic arsenicals. No significant alterations in blood clinical chemistry, liver weights, or histopathology were observed in rabbits dermally exposed to $1,000 \mathrm{mg} / \mathrm{kg} / \mathrm{day}$ MMA or DMA 5 days/week for 21 days (Margitich and Ackerman 1991a, 1991b).

Renal Effects. No studies were located regarding renal effects in humans after dermal exposure to organic arsenicals. No significant alterations in urinalysis, kidney weights, or histopathology were observed in rabbits following dermal exposure to 1,000 mg MMA/kg/day (Margitich and Ackerman 1991a) or 1,000 mg DMA/kg/day 5 days/week for 21 days (Margitich and Ackerman 1991b). 
Endocrine Effects. No studies were located regarding endocrine effects in humans after dermal exposure to organic arsenicals. No alterations in adrenal gland weight or histopathology of the adrenal glands, pancreas, pituitary gland, thyroid gland, and parathyroid gland were observed in rabbits following dermal application of $1,000 \mathrm{mg} / \mathrm{kg} /$ day MMA or DMA 5 days/week for 21 days (Margitich and Ackerman 1991a, 1991b).

\section{Dermal Effects.}

Inorganic Arsenicals. Several studies of humans exposed to arsenic dusts in the workplace have reported that inorganic arsenic (usually arsenic trioxide) can cause contact dermatitis (Holmqvist 1951; Pinto and McGill 1953). Typical responses included erythema and swelling, with papules and vesicles in more severe cases (Holmqvist 1951). The dermal contact rates that cause these effects in humans have not been quantified, but a similar direct irritation of the skin has been noted in mice exposed to $4 \mathrm{mg} \mathrm{As} / \mathrm{kg} / \mathrm{day}$ as potassium arsenite for 30 weeks (Boutwell 1963). In contrast, no significant dermal irritation was noted in guinea pigs exposed to aqueous solutions containing 4,000 mg As/L as arsenate or $580 \mathrm{mg} \mathrm{As} / \mathrm{L}$ as arsenite (Wahlberg and Boman 1986). These studies indicate that direct contact may be of concern at high exposure levels, but do not suggest that lower levels are likely to cause significant irritation.

Studies on possible dermal sensitization by inorganic arsenicals are discussed in Section 3.2.3.3 below.

Organic Arsenicals. Contact dermatitis was reported in workers involved in the application of an organic arsenical herbicide, which is a mixture of DMA and its sodium salt (Peoples et al. 1979).

Application of an unspecified amount of MMA to the skin of rabbits was reported to result in mild dermal irritation in a Draize test (Jaghabir et al. 1988). No dermal irritation was reported in rabbits repeatedly exposed to 1,000 mg MMA/kg/day (Margitich and Ackerman 1991a) or 1,000 mg DMA/kg/day 5 days/week for 21 days (Margitich and Ackerman 1991b).

Ocular Effects. No studies were located regarding ocular effects in humans or animals after dermal exposure to organic arsenicals.

Body Weight Effects. No studies were located regarding body weight effects in humans after dermal exposure to organic arsenicals. No significant alterations in body weight gain were observed in rabbits 
following a 5 day/week exposure to $1,000 \mathrm{mg} / \mathrm{kg} /$ day MMA or DMA for 21 days (Margitich and Ackerman 1991a, 1991b).

\subsubsection{Immunological and Lymphoreticular Effects}

Inorganic Arsenicals. Examination of workers exposed to arsenic trioxide dusts in a copper smelter led Holmqvist (1951) to suspect that repeated dermal contact could lead to dermal sensitization. In support of this, Holmqvist (1951) found a positive patch test in $80 \%$ of the exposed workers compared to $30 \%$ in a control population. These data do suggest that workers may be sensitized to arsenic, but the high response rate in controls seems unusual. A much lower response rate $(0.5 \%)$ was noted in another patch test study of dermal sensitization (Wahlberg and Boman 1986), and the few positive responses seemed to be due to a cross-reactivity with nickel. Mohamed (1998) evaluated 11 male workers at a tin smelting factory where arsenic trioxide levels ranged from 5.2 to $14.4 \mathrm{mg} / \mathrm{m}^{3}$. The workers experienced symptoms of generalized itch, dry and hyperpigmented skin, folliculitis, and superficial ulcerations. The authors concluded that arsenic-containing dust collected on the sweat on the workers' skin, causing contact dermatitis. Studies in guinea pigs did not yield evidence of a sensitization reaction to inorganic arsenic (Wahlberg and Boman 1986).

Organic Arsenicals. Support for sensitization to DMA is provided in a case report of a 26-year-old woman who was occupationally exposed to DMA and experienced eczema on her face (Bourrain et al. 1998). Patch testing confirmed an allergic reaction to DMA, and avoidance of DMA resulted in disappearance of the symptoms. No studies were located regarding immunological or lymphoreticular effects in animals after dermal exposure to organic arsenicals.

No studies were located that have associated any of the following effects in humans or animals with dermal exposure to inorganic or organic arsenicals:

\subsubsection{Neurological Effects}

\subsubsection{Reproductive Effects}

\subsubsection{Developmental Effects}

\subsubsection{Cancer}

Inorganic Arsenicals. No studies were found that have associated cancer in humans with dermal exposure to arsenic. Application of arsenic acid to the skin of mice pretreated with dimethylbenz- 
anthracene did not result in any skin tumors (Kurokawa et al. 1989), suggesting that arsenic does not act as a promoter in this test system.

Organic Arsenicals. No studies were located regarding cancer in humans or animals after dermal exposure to organic arsenicals.

\subsection{GENOTOXICITY}

Inorganic Arsenicals. There have been a large number of studies of the genotoxic effects of arsenic. Tables 3-8 and 3-9 summarize a number of reports on the in vitro and in vivo genotoxicity of inorganic arsenicals, respectively. In general, in vitro studies in prokaryotic organisms have been negative for gene mutations (Lantzsch and Gebel 1997; Löfroth and Ames 1978; Nishioka 1975; Rossman et al. 1980; Ulitzur and Barak 1988). Studies in human fibroblasts, lymphocytes, and leukocytes, mouse lymphoma cells, Chinese hamster ovary cells, and Syrian hamster embryo cells demonstrate that in vitro arsenic exposure can induce chromosomal aberrations and sister chromatid exchange (see Table 3-8 for citations). I vitro studies in human, mouse, and hamster cells have also been positive for DNA damage and repair and enhancement or inhibition of DNA synthesis.

Studies of humans have detected a higher-than-average incidence of chromosomal aberrations in peripheral lymphocytes, both after inhalation exposure (Beckman et al. 1977; Nordenson et al. 1978) and oral exposure (Burgdorf et al. 1977; Nordenson et al. 1979). These studies must be interpreted with caution, since in most cases, there were only a small number of subjects and a number of other chemical exposures were possible (EPA 1984a). Human and animal data are available indicating that inhaled inorganic arsenic is clastogenic. Workers exposed to unspecified concentrations of arsenic trioxide at the Ronnskar copper smelter in Sweden were found to have a significant increase in the frequency of chromosomal aberrations in peripheral lymphocytes (Beckman et al. 1977; Nordenson et al. 1978). This result is supported by an animal study that found increased chromosomal aberrations in the livers of fetuses from pregnant mice exposed to 22, but not 2.2 or $0.20, \mathrm{mg} \mathrm{As} / \mathrm{m}^{3}$ as arsenic trioxide on days 912 of gestation (Nagymajtényi et al. 1985). Workers in the arsenic-based glass making industry in southern India had a significantly increased frequency of micronuclei in buccal cells and increased DNA damage in leukocytes compared to a control group (Vuyyuri et al. 2006). Exposure levels were not available, but the concentration of arsenic in the blood from workers was approximately 5 times higher than in the reference group. 
Table 3-8. Genotoxicity of Inorganic Arsenic In Vitro

\begin{tabular}{|c|c|c|c|c|c|}
\hline \multirow[b]{2}{*}{ Valence } & \multirow[b]{2}{*}{ Species (test system) } & \multirow[b]{2}{*}{ End point } & \multicolumn{2}{|c|}{ Results } & \multirow[b]{2}{*}{ Reference } \\
\hline & & & $\begin{array}{l}\text { With } \\
\text { activation }\end{array}$ & $\begin{array}{l}\text { Without } \\
\text { activation }\end{array}$ & \\
\hline \multicolumn{6}{|c|}{ Prokaryotic organisms: } \\
\hline $\mathrm{As}^{+3}$ & Escherichia coli & Reverse mutation & No data & + & Nishioka 1975 \\
\hline $\mathrm{As}^{+3}$ & E. coli PQ37 & Gene mutation & No data & - & $\begin{array}{l}\text { Lantzsch and } \\
\text { Gebel } 1997\end{array}$ \\
\hline $\mathrm{As}^{+3}$ & E. coli (six strains) & Reverse mutation & No data & - & $\begin{array}{l}\text { Rossman et al. } \\
1980\end{array}$ \\
\hline $\mathrm{As}^{+3}$ & Salmonella typhimurium & Gene mutation & No data & - & $\begin{array}{l}\text { Löfroth and } \\
\text { Ames } 1978\end{array}$ \\
\hline $\mathrm{As}^{+3}$ & Photobacterium fischeri & Gene mutation & No data & - & $\begin{array}{l}\text { Ulitzur and Barak } \\
1988\end{array}$ \\
\hline $\mathrm{As}^{+5}$ & S. typhimurium & Gene mutation & No data & - & $\begin{array}{l}\text { Löfroth and } \\
\text { Ames } 1978\end{array}$ \\
\hline $\mathrm{As}^{+5}$ & P. fischeri & Gene mutation & No data & + & $\begin{array}{l}\text { Ulitzur and Barak } \\
1988\end{array}$ \\
\hline \multicolumn{6}{|c|}{ Eukaryotic organisms: } \\
\hline \multicolumn{6}{|c|}{ Fungi: } \\
\hline $\begin{array}{l}\mathrm{As}^{+3} \\
\mathrm{As}^{+5}\end{array}$ & $\begin{array}{l}\text { Saccharomyces } \\
\text { cerevisiae }\end{array}$ & Gene mutation & No data & - & Singh 1983 \\
\hline & Mammalian cells: & & & & \\
\hline $\mathrm{As}^{+3}$ & Human fibroblasts & DNA repair inhibition & No data & + & $\begin{array}{l}\text { Okui and } \\
\text { Fujiwara } 1986\end{array}$ \\
\hline $\mathrm{As}^{+3}$ & Human fibroblasts & $\begin{array}{l}\text { DNA repair and mutant } \\
\text { frequencies }\end{array}$ & + & + & $\begin{array}{l}\text { Wiencke et al. } \\
1997\end{array}$ \\
\hline $\mathrm{As}^{+3}$ & Human fibroblasts & DNA repair inhibition & + & + & $\begin{array}{l}\text { Hartwig et al. } \\
1997\end{array}$ \\
\hline $\mathrm{As}^{+3}$ & $\begin{array}{l}\text { Human fibroblasts } \\
\text { (MRC5CV1) }\end{array}$ & DNA migration & No data & + & $\begin{array}{l}\text { Hartmann and } \\
\text { Speit } 1996\end{array}$ \\
\hline $\mathrm{As}^{+3}$ & $\begin{array}{l}\text { Human fibroblasts (HFW } \\
\text { cells) }\end{array}$ & Cytotoxicity & No data & + & Lee and Ho 1994 \\
\hline $\mathrm{As}^{+3}$ & $\begin{array}{l}\text { Human skin fibroblasts } \\
\text { (HFW) }\end{array}$ & $\begin{array}{l}\text { Chromosome } \\
\text { endoreduplication }\end{array}$ & No data & + & $\begin{array}{l}\text { Huang et al. } \\
1995\end{array}$ \\
\hline $\mathrm{As}^{+3}$ & Human skin fibroblasts & $\begin{array}{l}\text { Chromosomal } \\
\text { aberrations }\end{array}$ & No data & + & Yih et al. 1997 \\
\hline $\mathrm{As}^{+3}$ & $\begin{array}{l}\text { Human fetal lung } \\
\text { fibroblasts }\end{array}$ & DNA strand breaks & No data & + & $\begin{array}{l}\text { Dong and Luo } \\
1993\end{array}$ \\
\hline $\mathrm{As}^{+3}$ & $\begin{array}{l}\text { Human fetal lung } \\
\text { fibroblasts ( } 2 \mathrm{BS} \text { cells) }\end{array}$ & DNA damage and repair & No data & + & $\begin{array}{l}\text { Dong and Luo } \\
1994\end{array}$ \\
\hline $\begin{array}{l}\mathrm{As}^{+3} \\
\mathrm{As}^{+5}\end{array}$ & $\begin{array}{l}\text { Human umbilical cord } \\
\text { fibroblasts }\end{array}$ & $\begin{array}{l}\text { Chromosomal } \\
\text { aberrations }\end{array}$ & No data & + & $\begin{array}{l}\text { Oya-Ohta et al. } \\
1996\end{array}$ \\
\hline $\mathrm{As}^{+3}$ & Diploid human fibroblasts & $\begin{array}{l}\text { Morphological } \\
\text { transformation }\end{array}$ & No data & + & Landolph 1994 \\
\hline
\end{tabular}


Table 3-8. Genotoxicity of Inorganic Arsenic In Vitro

\begin{tabular}{|c|c|c|c|c|c|}
\hline \multirow[b]{2}{*}{ Valence } & \multirow[b]{2}{*}{ Species (test system) } & \multirow[b]{2}{*}{ End point } & \multicolumn{2}{|c|}{ Results } & \multirow[b]{2}{*}{ Reference } \\
\hline & & & $\begin{array}{l}\text { With } \\
\text { activation }\end{array}$ & $\begin{array}{l}\text { Without } \\
\text { activation }\end{array}$ & \\
\hline $\mathrm{As}^{+3}$ & Human leukocytes & Chromosomal aberration & No data & + & $\begin{array}{l}\text { Nakamuro and } \\
\text { Sayato } 1981\end{array}$ \\
\hline $\mathrm{As}^{+3}$ & Human lymphocytes & DNA protein cross-links & - & - & Costa et al. 1997 \\
\hline $\begin{array}{l}\mathrm{As}^{+3} \\
\mathrm{As}^{+5}\end{array}$ & Human lymphocytes & $\begin{array}{l}\text { Enhancement or } \\
\text { inhibition on DNA } \\
\text { synthesis }\end{array}$ & No data & + & Meng 1993a \\
\hline $\begin{array}{l}\mathrm{As}^{+3} \\
\mathrm{As}^{+5}\end{array}$ & Human lymphocytes & $\begin{array}{l}\text { Enhancement or } \\
\text { inhibition on DNA } \\
\text { synthesis }\end{array}$ & No data & + & Meng 1993b \\
\hline $\begin{array}{l}\mathrm{As}^{+3} \\
\mathrm{As}^{+5}\end{array}$ & Human lymphocytes & $\begin{array}{l}\text { Enhancement or } \\
\text { inhibition on DNA } \\
\text { synthesis }\end{array}$ & No data & + & Meng 1994 \\
\hline $\mathrm{As}^{+3}$ & Human lymphocytes & $\begin{array}{l}\text { Hyperdiploidy and } \\
\text { chromosomal breakage }\end{array}$ & No data & $(+)$ & Rupa et al. 1997 \\
\hline $\mathrm{As}^{+3}$ & Human lymphocytes & Hyperdiploid nuclei & No data & + & $\begin{array}{l}\text { Ramirez et al. } \\
1997\end{array}$ \\
\hline $\mathrm{As}^{+3}$ & Human lymphocytes & Chromosomal aberration & No data & + & $\begin{array}{l}\text { Beckman and } \\
\text { Nordenson } 1986\end{array}$ \\
\hline $\mathrm{As}^{+3}$ & Human lymphocytes & $\begin{array}{l}\text { Chromosomal } \\
\text { aberrations and sister } \\
\text { chromatid exchange }\end{array}$ & No data & + & $\begin{array}{l}\text { Nordenson et al. } \\
1981\end{array}$ \\
\hline $\mathrm{As}^{+3}$ & Human lymphocytes & Chromosomal aberration & No data & + & Sweins 1983 \\
\hline $\mathrm{As}^{+3}$ & Human lymphocytes & $\begin{array}{l}\text { Chromosomal } \\
\text { aberrations }\end{array}$ & No data & + & $\begin{array}{l}\text { Yager and } \\
\text { Wiencke } 1993\end{array}$ \\
\hline $\mathrm{As}^{+3}$ & Human lymphocytes & $\begin{array}{l}\text { Chromosomal } \\
\text { aberrations }\end{array}$ & No data & + & Vega et al. 1995 \\
\hline $\mathrm{As}^{+3}$ & Human lymphocytes & $\begin{array}{l}\text { Chromosomal } \\
\text { aberrations }\end{array}$ & No data & + & Wan et al. 1982 \\
\hline $\mathrm{As}^{+3}$ & Human lymphocytes & $\begin{array}{l}\text { Chromosomal } \\
\text { aberrations and sister } \\
\text { chromatic exchange }\end{array}$ & No data & + & $\begin{array}{l}\text { Wiencke and } \\
\text { Yager } 1992\end{array}$ \\
\hline $\mathrm{As}^{+3}$ & Human lymphocytes & $\begin{array}{l}\text { Chromosome aberrations } \\
\text { and sister chromatid } \\
\text { exchanges }\end{array}$ & No data & + & $\begin{array}{l}\text { Larramendy et al. } \\
1981\end{array}$ \\
\hline $\mathrm{As}^{+3}$ & Human lymphocytes & $\begin{array}{l}\text { Sister chromatid } \\
\text { exchange }\end{array}$ & No data & + & Gebel et al. 1997 \\
\hline $\mathrm{As}^{+5}$ & Human lymphocytes & $\begin{array}{l}\text { Sister chromatid } \\
\text { exchange }\end{array}$ & No data & - & Gebel et al. 1997 \\
\hline $\mathrm{As}^{+3}$ & Human lymphocytes & $\begin{array}{l}\text { Sister chromatid } \\
\text { exchange }\end{array}$ & No data & + & $\begin{array}{l}\text { Hartmann and } \\
\text { Speit } 1994\end{array}$ \\
\hline $\mathrm{As}^{+3}$ & Human lymphocytes & $\begin{array}{l}\text { Sister chromatid } \\
\text { exchange }\end{array}$ & No data & + & Jha et al. 1992 \\
\hline $\mathrm{As}^{+3}$ & Human lymphocytes & $\begin{array}{l}\text { Sister chromatid } \\
\text { exchange }\end{array}$ & No data & + & $\begin{array}{l}\text { Rasmussen and } \\
\text { Menzel } 1997\end{array}$ \\
\hline
\end{tabular}


Table 3-8. Genotoxicity of Inorganic Arsenic In Vitro

\begin{tabular}{|c|c|c|c|c|c|}
\hline \multirow[b]{2}{*}{ Valence } & \multirow[b]{2}{*}{ Species (test system) } & \multirow[b]{2}{*}{ End point } & \multicolumn{2}{|c|}{ Results } & \multirow[b]{2}{*}{ Reference } \\
\hline & & & $\begin{array}{l}\text { With } \\
\text { activation }\end{array}$ & $\begin{array}{l}\text { Without } \\
\text { activation }\end{array}$ & \\
\hline $\begin{array}{l}\mathrm{As}^{+3} \\
\mathrm{As}^{+5}\end{array}$ & $\begin{array}{l}\text { Human T-cell lymphoma- } \\
\text { derived cell line (Molt-3) }\end{array}$ & PARP activity inhibition & No data & + & $\begin{array}{l}\text { Yager and } \\
\text { Wiencke } 1997\end{array}$ \\
\hline $\mathrm{As}^{+3}$ & $\begin{array}{l}\text { Human cervix carcinoma } \\
\text { HeLa and cisplatin- } \\
\text { resistant HeLa/CPR } \\
\text { variant cells }\end{array}$ & DNA repair modification & + & + & Chao 1996 \\
\hline $\mathrm{As}^{+3}$ & $\begin{array}{l}\text { Human cervix carcinoma } \\
\text { cells (HeLa) }\end{array}$ & DNA damage recognition & No data & - & $\begin{array}{l}\text { Hartwig et al. } \\
1998\end{array}$ \\
\hline $\mathrm{As}^{+3}$ & $\begin{array}{l}\text { Human osteosarcoma } \\
\text { cells (HOS) }\end{array}$ & DNA repair & No data & + & Hu et al. 1998 \\
\hline $\mathrm{As}^{+3}$ & $\begin{array}{l}\text { Human osteosarcoma } \\
\text { cells (HOS) }\end{array}$ & Cell transformation & No data & + & Mure et al. 2003 \\
\hline $\mathrm{As}^{+3}$ & $\begin{array}{l}\text { Human-hamster hybrid } \\
A_{1} \text { cells }\end{array}$ & DNA adducts & No data & + & $\begin{array}{l}\text { Kessel et al. } \\
2002\end{array}$ \\
\hline $\mathrm{As}^{+3}$ & Mouse lymphoma cells & $\begin{array}{l}\text { Enhanced viral forward } \\
\text { mutation }\end{array}$ & No data & $(+)$ & $\begin{array}{l}\text { Oberly et al. } \\
1982\end{array}$ \\
\hline $\begin{array}{l}\mathrm{As}^{+3} \\
\mathrm{As}^{+5}\end{array}$ & $\begin{array}{l}\text { Mouse lymphoma cells } \\
{\left[\text { L5178Y/TK }{ }^{+}-(-3.7 .2 \mathrm{C})\right]}\end{array}$ & Chromosomal mutations & No data & + & $\begin{array}{l}\text { Moore et al. } \\
1997 a\end{array}$ \\
\hline $\mathrm{As}^{+3}$ & $\begin{array}{l}\text { Mouse lymphoma cells } \\
{\left[\text { L5178Y } \mathrm{tk}^{+} /(3.7 . \mathrm{sc})\right]}\end{array}$ & Mutagenicity & No data & + & $\begin{array}{l}\text { Oberly et al. } \\
1996\end{array}$ \\
\hline $\begin{array}{l}\mathrm{As}^{+3} \\
\mathrm{As}^{+5}\end{array}$ & Mouse lymphoma cells & $\begin{array}{l}\text { Chromosomal } \\
\text { aberrations }\end{array}$ & No data & + & $\begin{array}{l}\text { Moore et al. } \\
1994 a\end{array}$ \\
\hline $\mathrm{As}^{+3}$ & Mouse lymphoma cells & $\begin{array}{l}\text { Chromosomal } \\
\text { aberrations }\end{array}$ & No data & + & Sofuni et al. 1996 \\
\hline $\mathrm{As}^{+3}$ & Mouse 3 T6 cells & Gene amplification & No data & + & Lee et al. 1988 \\
\hline $\mathrm{As}^{+3}$ & $\begin{array}{l}\text { Mouse embryo } \\
\text { fibroblasts }(\mathrm{C} 3 \mathrm{H} / 10 \mathrm{~T} / 2 \\
\mathrm{Cl} 8)\end{array}$ & $\begin{array}{l}\text { Morphological } \\
\text { transformation }\end{array}$ & No data & + & Landolph 1994 \\
\hline $\mathrm{As}^{+3}$ & $\begin{array}{l}\text { Chinese hamster V79 } \\
\text { cells }\end{array}$ & Gene mutation & No data & - & $\begin{array}{l}\mathrm{Li} \text { and Rossman } \\
1991\end{array}$ \\
\hline $\mathrm{As}^{+3}$ & $\begin{array}{l}\text { Chinese hamster V79 } \\
\text { cells }\end{array}$ & Gene mutation & No data & - & $\begin{array}{l}\text { Rossman et al. } \\
1980\end{array}$ \\
\hline $\mathrm{As}^{+3}$ & $\begin{array}{l}\text { Chinese hamster V79 } \\
\text { cells }\end{array}$ & $\begin{array}{l}\text { DNA damage, DNA- } \\
\text { protein cross-linking, } \\
\text { micronucleus induction }\end{array}$ & No data & + & $\begin{array}{l}\text { Gebel et al. } \\
1998 a\end{array}$ \\
\hline $\mathrm{As}^{+3}$ & $\begin{array}{l}\text { Chinese hamster V79 } \\
\text { cells }\end{array}$ & $\begin{array}{l}\text { DNA repair and mutant } \\
\text { frequencies }\end{array}$ & No data & + & $\begin{array}{l}\mathrm{Li} \text { and Rossman } \\
1991\end{array}$ \\
\hline $\mathrm{As}^{+3}$ & $\begin{array}{l}\text { Chinese hamster V79 } \\
\text { cells }\end{array}$ & $\begin{array}{l}\text { Intrachromosomal } \\
\text { homologous } \\
\text { recombination }\end{array}$ & No data & + & $\begin{array}{l}\text { Helleday et al. } \\
2000\end{array}$ \\
\hline $\mathrm{As}^{+3}$ & $\begin{array}{l}\text { Chinese hamster ovary } \\
\text { cells (CHO-AL) }\end{array}$ & Gene mutation & No data & + & Hei et al. 1998 \\
\hline $\mathrm{As}^{+3}$ & $\begin{array}{l}\text { Chinese hamster ovary } \\
\text { cells (CHO-AS52) }\end{array}$ & Mutagenicity & No data & + & $\begin{array}{l}\text { Meng and Hsie } \\
1996\end{array}$ \\
\hline
\end{tabular}


Table 3-8. Genotoxicity of Inorganic Arsenic In Vitro

\begin{tabular}{|c|c|c|c|c|c|}
\hline \multirow[b]{2}{*}{ Valence } & \multirow[b]{2}{*}{ Species (test system) } & \multirow[b]{2}{*}{ End point } & \multicolumn{2}{|c|}{ Results } & \multirow[b]{2}{*}{ Reference } \\
\hline & & & $\begin{array}{l}\text { With } \\
\text { activation }\end{array}$ & $\begin{array}{l}\text { Without } \\
\text { activation }\end{array}$ & \\
\hline $\mathrm{As}^{+3}$ & $\begin{array}{l}\text { Chinese hamster ovary } \\
\text { cells }\end{array}$ & Gene mutation & No data & + & Yang et al. 1992 \\
\hline $\mathrm{As}^{+3}$ & $\begin{array}{l}\text { Chinese hamster ovary } \\
\text { cells }\end{array}$ & DNA repair inhibition & No data & + & $\begin{array}{l}\text { Lee-Chen et al. } \\
1993\end{array}$ \\
\hline $\mathrm{As}^{+3}$ & $\begin{array}{l}\text { Chinese hamster ovary } \\
\text { cells }\end{array}$ & DNA repair inhibition & No data & - & $\begin{array}{l}\text { Lee-Chen et al. } \\
1992\end{array}$ \\
\hline $\mathrm{As}^{+3}$ & $\begin{array}{l}\text { Chinese hamster ovary } \\
\text { cells (CHO-K1) }\end{array}$ & DNA strand breaks & + & + & $\begin{array}{l}\text { Lee-Chen et al. } \\
1994\end{array}$ \\
\hline $\mathrm{As}^{+3}$ & $\begin{array}{l}\text { Chinese hamster ovary } \\
\text { cells ( } \mathrm{CHO}-\mathrm{K} 1)\end{array}$ & DNA strand breaks & No data & + & Lynn et al. 1997 \\
\hline $\mathrm{As}^{+3}$ & $\begin{array}{l}\text { Chinese hamster ovary } \\
\text { cells }\end{array}$ & Aberrant metaphases & No data & + & Jan et al. 1986 \\
\hline $\mathrm{As}^{+3}$ & $\begin{array}{l}\text { Chinese hamster ovary } \\
\text { cells }\end{array}$ & Aberrant metaphases & No data & + & Lee et al. 1986 \\
\hline $\mathrm{As}^{+3}$ & $\begin{array}{l}\text { Chinese hamster ovary } \\
\text { cells }\end{array}$ & $\begin{array}{l}\text { Chromosomal } \\
\text { aberrations }\end{array}$ & + & + & $\begin{array}{l}\text { Huang et al. } \\
1992\end{array}$ \\
\hline $\mathrm{As}^{+3}$ & $\begin{array}{l}\text { Chinese hamster ovary } \\
\text { cells (CHO-K1) }\end{array}$ & $\begin{array}{l}\text { Chromosomal } \\
\text { aberrations }\end{array}$ & No data & + & $\begin{array}{l}\text { Huang et al. } \\
1993\end{array}$ \\
\hline $\begin{array}{l}\mathrm{As}^{+3} \\
\mathrm{As}^{+5}\end{array}$ & $\begin{array}{l}\text { Chinese hamster ovary } \\
\text { cells ( } \mathrm{CHO}-\mathrm{K} 1)\end{array}$ & $\begin{array}{l}\text { Chromosomal } \\
\text { aberrations and sister } \\
\text { chromatid exchange }\end{array}$ & No data & + & $\begin{array}{l}\text { Kochhar et al. } \\
1996\end{array}$ \\
\hline $\mathrm{As}^{+3}$ & $\begin{array}{l}\text { Chinese hamster ovary } \\
\text { cells }\end{array}$ & $\begin{array}{l}\text { Chromosomal } \\
\text { aberrations and sister } \\
\text { chromatid exchange }\end{array}$ & + & + & $\begin{array}{l}\text { Lin and Tseng } \\
1992\end{array}$ \\
\hline $\mathrm{As}^{+3}$ & $\begin{array}{l}\text { Chinese hamster ovary } \\
\text { cells }\end{array}$ & $\begin{array}{l}\text { Chromosomal } \\
\text { aberrations and sister } \\
\text { chromatid exchange }\end{array}$ & No data & + & Wan et al. 1982 \\
\hline $\mathrm{As}^{+3}$ & $\begin{array}{l}\text { Chinese hamster ovary } \\
\text { cells }\end{array}$ & $\begin{array}{l}\text { Sister chromatid } \\
\text { exchange and } \\
\text { micronucleus induction }\end{array}$ & No data & + & Fan et al. 1996 \\
\hline $\mathrm{As}^{+3}$ & $\begin{array}{l}\text { Chinese hamster ovary } \\
\text { cells }\end{array}$ & $\begin{array}{l}\text { Cell-killing and } \\
\text { micronucleus induction }\end{array}$ & No data & + & $\begin{array}{l}\text { Wang and Huang } \\
1994\end{array}$ \\
\hline $\mathrm{As}^{+3}$ & $\begin{array}{l}\text { Chinese hamster ovary } \\
\text { cells }\end{array}$ & Micronuclei & No data & + & $\begin{array}{l}\text { Liu and Huang } \\
1997\end{array}$ \\
\hline $\mathrm{As}^{+3}$ & $\begin{array}{l}\text { Chinese hamster ovary } \\
\text { cells }\end{array}$ & Micronuclei formation & No data & + & $\begin{array}{l}\text { Yee-Chien and } \\
\text { Haimei } 1996\end{array}$ \\
\hline $\mathrm{As}^{+3}$ & $\begin{array}{l}\text { Chinese hamster ovary } \\
\text { cells }\end{array}$ & Micronuclei induction & No data & + & Wang et al. 1997 \\
\hline $\mathrm{As}^{+3}$ & $\begin{array}{l}\text { Chinese hamster ovary } \\
\text { cells }\end{array}$ & Cytotoxicity & No data & - & Lee and Ho 1994 \\
\hline $\mathrm{As}^{+3}$ & $\begin{array}{l}\text { Syrian hamster embryo } \\
\text { cells }\end{array}$ & Gene mutation & No data & - & Lee et al. 1985 \\
\hline
\end{tabular}


Table 3-8. Genotoxicity of Inorganic Arsenic In Vitro

\begin{tabular}{|c|c|c|c|c|c|}
\hline \multirow[b]{2}{*}{ Valence } & \multirow[b]{2}{*}{ Species (test system) } & \multirow[b]{2}{*}{ End point } & \multicolumn{2}{|c|}{ Results } & \multirow[b]{2}{*}{ Reference } \\
\hline & & & $\begin{array}{l}\text { With } \\
\text { activation }\end{array}$ & $\begin{array}{l}\text { Without } \\
\text { activation }\end{array}$ & \\
\hline $\mathrm{As}^{+3}$ & $\begin{array}{l}\text { Syrian hamster embryo } \\
\text { cells }\end{array}$ & $\begin{array}{l}\text { Chromosome aberrations } \\
\text { and sister chromatid } \\
\text { exchanges }\end{array}$ & No data & + & $\begin{array}{l}\text { Larramendy et al. } \\
1981\end{array}$ \\
\hline $\mathrm{As}^{+3}$ & $\begin{array}{l}\text { Syrian hamster embryo } \\
\text { cells }\end{array}$ & Chromosomal aberration & No data & + & Lee et al. 1985 \\
\hline $\mathrm{As}^{+3}$ & $\begin{array}{l}\text { Syrian hamster embryo } \\
\text { cells }\end{array}$ & $\begin{array}{l}\text { Sister chromatid } \\
\text { exchange }\end{array}$ & No data & + & Lee et al. 1985 \\
\hline $\mathrm{As}^{+3}$ & $\begin{array}{l}\text { Syrian hamster embryo } \\
\text { cells }\end{array}$ & Micronuclei induction & No data & - & $\begin{array}{l}\text { Gibson et al. } \\
1997\end{array}$ \\
\hline $\mathrm{As}^{+3}$ & $\begin{array}{l}\text { Syrian hamster embryo } \\
\text { cells }\end{array}$ & Micronuclei induction & No data & - & $\begin{array}{l}\text { Gibson et al. } \\
1997\end{array}$ \\
\hline $\mathrm{As}^{+3}$ & $\begin{array}{l}\text { Syrian hamster embryo } \\
\text { cells }\end{array}$ & $\begin{array}{l}\text { Morphological } \\
\text { transformation }\end{array}$ & No data & + & $\begin{array}{l}\text { Kerckaert et al. } \\
1996\end{array}$ \\
\hline $\mathrm{As}^{+3}$ & $\begin{array}{l}\text { Syrian hamster embryo } \\
\text { cells }\end{array}$ & $\begin{array}{l}\text { Morphological } \\
\text { transformation }\end{array}$ & No data & + & Lee et al. 1985 \\
\hline $\mathrm{As}^{+3}$ & $\begin{array}{l}\text { Syrian hamster embryo } \\
\text { cells }\end{array}$ & $\begin{array}{l}\text { Morphological } \\
\text { transformation }\end{array}$ & No data & + & Casto et al. 1979 \\
\hline $\mathrm{As}^{+5}$ & Human fibroblasts & DNA repair inhibition & No data & - & $\begin{array}{l}\text { Okui and } \\
\text { Fujiwara } 1986\end{array}$ \\
\hline $\mathrm{As}^{+5}$ & Human leukocytes & $\begin{array}{l}\text { Chromosomal } \\
\text { aberrations }\end{array}$ & No data & $(+)$ & $\begin{array}{l}\text { Nakamuro and } \\
\text { Sayato } 1981\end{array}$ \\
\hline $\mathrm{As}^{+5}$ & Human lymphocytes & $\begin{array}{l}\text { Chromosomal } \\
\text { aberrations }\end{array}$ & No data & - & $\begin{array}{l}\text { Nordenson et al. } \\
1981\end{array}$ \\
\hline $\mathrm{As}^{+5}$ & Human lymphocytes & $\begin{array}{l}\text { Chromosome aberrations } \\
\text { and sister chromatid } \\
\text { exchanges }\end{array}$ & No data & + & $\begin{array}{l}\text { Larramendy et al. } \\
1981\end{array}$ \\
\hline $\mathrm{As}^{+5}$ & Human lymphocytes & $\begin{array}{l}\text { Sister chromatid } \\
\text { exchange }\end{array}$ & No data & - & $\begin{array}{l}\text { Rasmussen and } \\
\text { Menzel } 1997\end{array}$ \\
\hline $\mathrm{As}^{+5}$ & $\begin{array}{l}\text { Human peripheral } \\
\text { lymphocytes }\end{array}$ & $\begin{array}{l}\text { Sister chromatid } \\
\text { exchange }\end{array}$ & No data & + & $\begin{array}{l}\text { Zanzoni and } \\
\text { Jung } 1980\end{array}$ \\
\hline $\mathrm{As}^{+5}$ & $\begin{array}{l}\text { Human keratinocyte line } \\
\text { SCC- } 9 \text { cells }\end{array}$ & $\begin{array}{l}\text { Keratinocyte } \\
\text { programming and } \\
\text { transcriptional activity }\end{array}$ & No data & + & $\begin{array}{l}\text { Kachinskas et al. } \\
1997\end{array}$ \\
\hline $\mathrm{As}^{+5}$ & Mouse lymphoma cells & Gene mutation & No data & - & $\begin{array}{l}\text { Amacher and } \\
\text { Paillet } 1980\end{array}$ \\
\hline $\mathrm{As}^{+5}$ & Mouse lymphoma cells & Gene mutation & No data & - & $\begin{array}{l}\text { Amacher and } \\
\text { Paillet } 1980\end{array}$ \\
\hline $\mathrm{As}^{+5}$ & $\begin{array}{l}\text { Chinese hamster ovary } \\
\text { cells }\end{array}$ & $\begin{array}{l}\text { Chromosomal } \\
\text { aberrations }\end{array}$ & No data & + & Wan et al. 1982 \\
\hline $\mathrm{As}^{+5}$ & $\begin{array}{l}\text { Syrian hamster embryo } \\
\text { cells }\end{array}$ & Gene mutation & No data & - & Lee et al. 1985 \\
\hline $\mathrm{As}^{+5}$ & $\begin{array}{l}\text { Syrian hamster embryo } \\
\text { cells }\end{array}$ & $\begin{array}{l}\text { Chromosome aberrations } \\
\text { and sister chromatid } \\
\text { exchanges }\end{array}$ & No data & + & $\begin{array}{l}\text { Larramendy et al. } \\
1981\end{array}$ \\
\hline
\end{tabular}


3. HEALTH EFFECTS

Table 3-8. Genotoxicity of Inorganic Arsenic In Vitro

\begin{tabular}{llllll}
\hline & & & \multicolumn{2}{c}{ Results } \\
\cline { 3 - 4 } Valence & Species (test system) & End point & \multicolumn{2}{l}{$\begin{array}{l}\text { With } \\
\text { activation }\end{array}$} & $\begin{array}{l}\text { Without } \\
\text { activation Reference }\end{array}$ \\
\hline $\mathrm{As}^{+5}$ & $\begin{array}{l}\text { Syrian hamster embryo } \\
\text { cells }\end{array}$ & $\begin{array}{l}\text { Chromosomal } \\
\text { aberrations }\end{array}$ & No data & + & Lee et al. 1985 \\
$\mathrm{As}^{+5}$ & $\begin{array}{l}\text { Syrian hamster embryo } \\
\text { cells }\end{array}$ & $\begin{array}{l}\text { Sister chromatid } \\
\text { exchange }\end{array}$ & No data & + & Lee et al. 1985 \\
$\mathrm{As}^{+5}$ & $\begin{array}{l}\text { Syrian hamster embryo } \\
\text { cells }\end{array}$ & $\begin{array}{l}\text { Morphological } \\
\text { transformation }\end{array}$ & No data & + & Lee et al. 1985 \\
$\mathrm{As}^{+5}$ & $\begin{array}{l}\text { Syrian hamster embryo } \\
\text { cells }\end{array}$ & $\begin{array}{l}\text { Morphological } \\
\text { transformation }\end{array}$ & No data & + & $\begin{array}{l}\text { DiPaolo and } \\
\text { Casto 1979 }\end{array}$ \\
\hline
\end{tabular}

$(+)=$ weakly positive or marginal result; $-=$ negative result; + = positive result; DNA = deoxyribonucleic acid 
Table 3-9. Genotoxicity of Inorganic Arsenic In Vivo

\begin{tabular}{|c|c|c|c|c|c|}
\hline Valence & $\begin{array}{l}\text { Exposure } \\
\text { route }\end{array}$ & $\begin{array}{l}\text { Species (test } \\
\text { system) }\end{array}$ & End point & Results & Reference \\
\hline \multicolumn{6}{|c|}{ Nonmammalian } \\
\hline $\mathrm{As}^{+3} \mathrm{As}^{+5}$ & Injection & $\begin{array}{l}\text { Drosophila } \\
\text { melanogaster }\end{array}$ & $\begin{array}{l}\text { Somatic mutations and } \\
\text { mitotic recombination }\end{array}$ & + & $\begin{array}{l}\text { Ramos-Morales and } \\
\text { Rodriguez-Arnaiz } \\
1995\end{array}$ \\
\hline $\mathrm{As}^{+3} \mathrm{As}^{+5}$ & Larval feeding & D. melanogaster & $\begin{array}{l}\text { Somatic mutations and } \\
\text { mitotic recombination }\end{array}$ & + & $\begin{array}{l}\text { Ramos-Morales and } \\
\text { Rodriguez-Arnaiz } \\
1995\end{array}$ \\
\hline $\mathrm{As}^{+5}$ & Larvae & D. melanogaster & Mitotic recombinations & + & de la Rosa et al. 1994 \\
\hline \multicolumn{6}{|c|}{ Mammalian } \\
\hline $\mathrm{As}^{+3}$ & Inhalation & Human (lymphocytes) & $\begin{array}{l}\text { Chromosomal } \\
\text { aberrations }\end{array}$ & - & Beckman et al. 1977 \\
\hline $\mathrm{As}^{+3}$ & Inhalation & Human (lymphocytes) & $\begin{array}{l}\text { Chromosomal } \\
\text { aberrations }\end{array}$ & + & Nordenson et al. 1978 \\
\hline $\mathrm{As}^{+3}$ & Oral & Human (lymphocytes) & $\begin{array}{l}\text { Chromosomal } \\
\text { aberrations }\end{array}$ & - & Burgdorf et al. 1977 \\
\hline No data & Oral & Human (lymphocytes) & $\begin{array}{l}\text { Chromosomal } \\
\text { aberrations }\end{array}$ & - & Vig et al. 1984 \\
\hline No data & Oral & Human (skin) & DNA adducts & + & Matsui et al. 1999 \\
\hline $\mathrm{As}^{+3}$ & Oral & Human (lymphocytes) & $\begin{array}{l}\text { Sister chromatid } \\
\text { exchange }\end{array}$ & - & Burgdorf et al. 1977 \\
\hline $\mathrm{As}^{+3}$ & Oral & Human (lymphocytes) & $\begin{array}{l}\text { Sister chromatid } \\
\text { exchange }\end{array}$ & + & Hsu et al. 1997 \\
\hline No data & Oral & Human (lymphocytes) & $\begin{array}{l}\text { Sister chromatid } \\
\text { exchange }\end{array}$ & + & Lerda 1994 \\
\hline No data & Oral & Human (lymphocytes) & $\begin{array}{l}\text { Sister chromatid } \\
\text { exchange }\end{array}$ & + & Liou et al. 1999 \\
\hline No data & Oral & Human (lymphocytes) & $\begin{array}{l}\text { Sister chromatid } \\
\text { exchange }\end{array}$ & + & Mahata et al. 2003 \\
\hline $\mathrm{As}^{+3}$ & Oral & Human (lymphocytes) & $\begin{array}{l}\text { Sister chromatid } \\
\text { exchange }\end{array}$ & - & Nordenson et al. 1978 \\
\hline No data & Oral & Human (lymphocytes) & $\begin{array}{l}\text { Sister chromatid } \\
\text { exchange }\end{array}$ & - & Vig et al. 1984 \\
\hline No data & Oral & $\begin{array}{l}\text { Human skin } \\
\text { carcinoma }\end{array}$ & $\begin{array}{l}\text { Mutation and } \\
\text { overexpression of p53 }\end{array}$ & + & Hsu et al. 1999 \\
\hline $\mathrm{As}^{+3}$ & Oral & $\begin{array}{l}\text { Exfoliated human } \\
\text { epithelial cells }\end{array}$ & Micronuclei & + & Moore et al. 1996 \\
\hline $\mathrm{As}^{+3}$ & Oral & $\begin{array}{l}\text { Exfoliated human } \\
\text { epithelial cells }\end{array}$ & Micronuclei & + & Tian et al. 2001 \\
\hline No data & Oral & $\begin{array}{l}\text { Human (bladder } \\
\text { cells) }\end{array}$ & Micronuclei & + & Moore et al. 1995 \\
\hline No data & Oral & Human (lymphocytes) & Micronuclei & + & Martínez et al. 2004 \\
\hline No data & Oral & Human (lymphocytes) & Micronuclei & + & Basu et al. 2004 \\
\hline No data & Oral & $\begin{array}{l}\text { Human (oral mucosa } \\
\text { cells) }\end{array}$ & Micronuclei & + & Basu et al. 2004 \\
\hline
\end{tabular}


Table 3-9. Genotoxicity of Inorganic Arsenic In Vivo

\begin{tabular}{|c|c|c|c|c|c|}
\hline Valence & $\begin{array}{l}\text { Exposure } \\
\text { route }\end{array}$ & $\begin{array}{l}\text { Species (test } \\
\text { system) }\end{array}$ & End point & Results & Reference \\
\hline No data & Oral & $\begin{array}{l}\text { Human (urothelial } \\
\text { cells) }\end{array}$ & Micronuclei & + & Basu et al. 2004 \\
\hline $\mathrm{As}^{+5}$ & Oral & $\begin{array}{l}\text { Rat (bone marrow } \\
\text { cells) }\end{array}$ & $\begin{array}{l}\text { Chromosomal } \\
\text { aberrations }\end{array}$ & + & Datta et al. 1986 \\
\hline $\mathrm{As}^{+3}$ & Inhalation & Mouse (fetal liver) & $\begin{array}{l}\text { Chromosomal } \\
\text { aberrations }\end{array}$ & $(+)$ & $\begin{array}{l}\text { Nagymajtényi et al. } \\
1985\end{array}$ \\
\hline $\mathrm{As}^{+3}$ & Oral & $\begin{array}{l}\text { Mouse (bone marrow } \\
\text { cells) }\end{array}$ & $\begin{array}{l}\text { Chromosomal } \\
\text { aberrations }\end{array}$ & + & Das et al. 1993 \\
\hline $\mathrm{As}^{+3}$ & Oral & $\begin{array}{l}\text { Mouse (bone marrow } \\
\text { cells) }\end{array}$ & $\begin{array}{l}\text { Chromosomal } \\
\text { aberrations }\end{array}$ & + & Poddar et al. 2000 \\
\hline $\mathrm{As}^{+3}$ & Oral & $\begin{array}{l}\text { Mouse (bone marrow } \\
\text { cells) }\end{array}$ & $\begin{array}{l}\text { Chromosomal breaks, } \\
\text { exchanges }\end{array}$ & - & Poma et al. 1987 \\
\hline $\mathrm{As}^{+3}$ & Oral & $\begin{array}{l}\text { Mouse } \\
\text { (spermatogonia) }\end{array}$ & $\begin{array}{l}\text { Chromosomal } \\
\text { aberrations }\end{array}$ & - & Poma et al. 1987 \\
\hline $\mathrm{As}^{+3}$ & Oral & Mouse (leukocytes) & Chromosomal breaks & + & McDorman et al. 2002 \\
\hline $\mathrm{As}^{+3}$ & Intraperitoneal & $\begin{array}{l}\text { Mouse (bone marrow } \\
\text { cells) }\end{array}$ & $\begin{array}{l}\text { Chromosomal breaks, } \\
\text { exchanges }\end{array}$ & - & Poma et al. 1981 \\
\hline $\mathrm{As}^{+3}$ & Intraperitoneal & $\begin{array}{l}\text { Mouse (bone marrow } \\
\text { cells) }\end{array}$ & Micronuclei & + & DeKnudt et al. 1986 \\
\hline $\mathrm{As}^{+3}$ & Intraperitoneal & $\begin{array}{l}\text { Mouse } \\
\text { (spermatogonia) }\end{array}$ & Spermatongonia & - & Poma et al. 1981 \\
\hline $\mathrm{As}^{+3}$ & Intraperitoneal & $\begin{array}{l}\text { Mouse } \\
\text { (spermatogonia) }\end{array}$ & Sperm morphology & - & DeKnudt et al. 1986 \\
\hline $\mathrm{As}^{+3}$ & Intraperitoneal & $\begin{array}{l}\text { Mouse } \\
\text { (spermatogenesis) }\end{array}$ & $\begin{array}{l}\text { Dominant lethal } \\
\text { mutations }\end{array}$ & - & DeKnudt et al. 1986 \\
\hline
\end{tabular}

$(+)$ = weakly positive or marginal result; $-=$ negative result; + = positive result; DNA = deoxyribonucleic acid 
Investigations of genotoxic effects of ingested arsenic have yielded mixed results possibly due to the different types of cells examined and the different exposure levels experienced by the populations studied. A study of p53 mutations in arsenic-related skin cancers from patients in Taiwan exposed to arsenic from drinking water found a high rate of p53 mutations and different types of p53 mutations compared with those seen in UV-induced skin cancers (Hsu et al. 1999); similar results have been found in mice (Salim et al. 2003). In humans exposed to Fowler's solution (potassium arsenite, usually taken at a dose of about $0.3 \mathrm{mg} \mathrm{As} / \mathrm{kg} /$ day [Holland 1904]), increased sister chromatid exchanges, but no increase in chromosomal aberrations, was reported in one study (Burgdorf et al. 1977), while just the converse (increased aberrations but no increase in sister chromatid exchange) was reported in another (Nordenson et al. 1979). Moore et al. (1997a) reported an exposure-dependent increase in the occurrence of micronucleated cells in epithelial cells from the bladder in a male population in northern Chile chronically exposed to high and low arsenic levels in their drinking water (average concentrations, 600 and $15 \mu \mathrm{g}$ As/L, respectively), and noted that chromosome breakage was the major cause of micronucleus (MN) formation. Similar results were reported by Martínez et al. (2004) who evaluated micronuclei formation in peripheral lymphocytes from people in northern Chile exposed to up to $0.75 \mathrm{mg} \mathrm{As} / \mathrm{L}$ in their drinking water. In contrast, Martínez et al. (2005) did not find a significant increase in micronuclei in buccal cells from subjects from the same area relative to a low exposure group. Vig et al. (1984) found no significant differences in the frequency of chromosomal aberrations or sister chromatid exchanges between two populations in Nevada with differing levels of arsenic in their drinking water (mean concentrations of 5 and $109 \mu \mathrm{g} / \mathrm{L}$ ). In animal studies, an increased incidence of chromosomal abnormalities was detected in rats given oral doses of sodium arsenate (4 mg As/kg/day) for 2-3 weeks (Datta et al. 1986), but no consistent increase in chromosomal aberrations was detected in bone marrow cells or spermatogonia from mice given sodium arsenite (about $50 \mathrm{mg} \mathrm{As} / \mathrm{kg} /$ day) for up to 8 weeks (Poma et al. 1987). These studies suggest that ingested arsenic may cause chromosomal effects, but these data are too limited to draw a firm conclusion.

Organic Arsenicals. The genotoxicity of the organic arsenicals has been investigated in a number of studies (see Table 3-10). Several tests indicate that DMA and roxarsone may be able to cause chromosome aberrations, mutations, and deoxyribonucleic acid (DNA) strand breaks; in vitro studies with MMA did not find significant increases in the occurrence of chromosome aberrations, forward or reverse mutations, unscheduled DNA synthesis (Chun and Killeen 1989a, 1989b, 1989c, 1989d). An increased number of DNA strand breaks were detected in lung and other tissues of mice and rats given oral doses of $\sim 1,500 \mathrm{mg} / \mathrm{kg}$ DMA (Okada and Yamanaka 1994; Yamanaka et al. 1989a); this effect appeared to be related to the formation of some active oxygen species. These breaks were largely repaired within 24 hours, so the relevance with respect to health risk is uncertain. 


\section{Table 3-10. Genotoxicity of Organic Arsenic}

\begin{tabular}{|c|c|c|c|c|c|}
\hline \multirow[b]{2}{*}{ Chemical form } & \multirow[b]{2}{*}{ Species (test system) } & \multirow[b]{2}{*}{ End point } & \multicolumn{2}{|c|}{ Results } & \multirow[b]{2}{*}{ Reference } \\
\hline & & & $\begin{array}{l}\text { With } \\
\text { activation }\end{array}$ & $\begin{array}{l}\text { Without } \\
\text { activation }\end{array}$ & \\
\hline \multicolumn{6}{|c|}{ Prokaryotic organisms (in vitro): } \\
\hline MMA & Salmonella typhimurium & Gene mutation & - & - & $\begin{array}{l}\text { Chun and } \\
\text { Killeen 1989c }\end{array}$ \\
\hline DMA & Escherichia coli & Gene mutation & No data & + & $\begin{array}{l}\text { Yamanaka et } \\
\text { al. } 1989 \mathrm{~b}\end{array}$ \\
\hline Roxarsone & S. typhimurium & Gene mutation & - & - & NTP 1989b \\
\hline \multicolumn{6}{|c|}{ Eukaryotic organisms (in vitro): } \\
\hline MMA & $\begin{array}{l}\text { Chinese hamster ovary } \\
\text { cells }\end{array}$ & $\begin{array}{l}\text { Chromosome } \\
\text { aberrations }\end{array}$ & - & - & $\begin{array}{l}\text { Chun and } \\
\text { Killeen 1989a }\end{array}$ \\
\hline MMA & $\begin{array}{l}\text { Mouse lymphoma cells } \\
\left(\mathrm{L} 178 \mathrm{Y} / \mathrm{TK}^{+-}\right)\end{array}$ & Forward mutation & - & - & $\begin{array}{l}\text { Chun and } \\
\text { Killeen 1989b }\end{array}$ \\
\hline MMA & Rat heptocytes & $\begin{array}{l}\text { Unscheduled DNA } \\
\text { synthesis }\end{array}$ & No data & - & $\begin{array}{l}\text { Chun and } \\
\text { Killeen 1989d }\end{array}$ \\
\hline DMA & $\begin{array}{l}\text { Human peripheral } \\
\text { lymphocytes }\end{array}$ & $\begin{array}{l}\text { Mitogenesis } \\
\text { inhibited }\end{array}$ & No data & - & $\begin{array}{l}\text { Endo et al. } \\
1992\end{array}$ \\
\hline DMA & Human lymphocytes & $\begin{array}{l}\text { Sister chromatid } \\
\text { exchange }\end{array}$ & No data & - & $\begin{array}{l}\text { Rasmussen } \\
\text { and Menzel } \\
1997\end{array}$ \\
\hline DMA & $\begin{array}{l}\text { Human alveolar (L-132) } \\
\text { cells }\end{array}$ & $\begin{array}{l}\text { Lung-specific DNA } \\
\text { damage }\end{array}$ & No data & + & $\begin{array}{l}\text { Kato et al. } \\
1994\end{array}$ \\
\hline DMA & $\begin{array}{l}\text { Human alveolar type II } \\
\text { (L-132) cells }\end{array}$ & $\begin{array}{l}\text { DNA single-strand } \\
\text { breaks }\end{array}$ & + & + & $\begin{array}{l}\text { Kawaguchi et } \\
\text { al. } 1996\end{array}$ \\
\hline DMA & $\begin{array}{l}\text { Human diploid L-132 } \\
\text { epithelial cells }\end{array}$ & $\begin{array}{l}\text { DNA single-strand } \\
\text { breaks }\end{array}$ & No data & + & Rin et al. 1995 \\
\hline DMA & $\begin{array}{l}\text { Human alveolar type II } \\
\text { (L-132) cells }\end{array}$ & DNA strand breaks & No data & + & $\begin{array}{l}\text { Tezuka et al. } \\
1993\end{array}$ \\
\hline DMA & $\begin{array}{l}\text { Human embryonic cell } \\
\text { line of type II alveolar } \\
\text { epithelial cells (L-132) }\end{array}$ & $\begin{array}{l}\text { DNA single-strand } \\
\text { breaks and DNA- } \\
\text { protein crosslinks }\end{array}$ & No data & + & $\begin{array}{l}\text { Yamanaka et } \\
\text { al. } 1993\end{array}$ \\
\hline DMA & $\begin{array}{l}\text { Human alveolar } \\
\text { epithelial (L-132) cells }\end{array}$ & $\begin{array}{l}\text { DNA single-strand } \\
\text { breaks and DNA- } \\
\text { protein crosslinks }\end{array}$ & No data & + & $\begin{array}{l}\text { Yamanaka et } \\
\text { al. } 1995\end{array}$ \\
\hline DMA & $\begin{array}{l}\text { Human pulmonary } \\
\text { epithelial (L-132) cells }\end{array}$ & $\begin{array}{l}\text { DNA single-strand } \\
\text { breaks }\end{array}$ & No data & + & $\begin{array}{l}\text { Yamanaka et } \\
\text { al. } 1997\end{array}$ \\
\hline DMA & $\begin{array}{l}\text { Human umbilical cord } \\
\text { fibroblasts }\end{array}$ & $\begin{array}{l}\text { Chromosomal } \\
\text { aberrations }\end{array}$ & No data & + & $\begin{array}{l}\text { Oya-Ohta et al. } \\
1996\end{array}$ \\
\hline DMA & $\begin{array}{l}\text { Mouse lymphoma cells } \\
\left(\mathrm{L} 5178{\mathrm{Y} / \mathrm{TK}^{+} /}^{\prime}-3.7 .2 \mathrm{C}\right)\end{array}$ & $\begin{array}{l}\text { Chromosomal } \\
\text { mutations }\end{array}$ & No data & + & $\begin{array}{l}\text { Moore et al. } \\
1997 a\end{array}$ \\
\hline DMA & $\begin{array}{l}\text { Chinese hamster lung } \\
\text { and diploid cells (V79) }\end{array}$ & $\begin{array}{l}\text { Mitotic arrest and } \\
\text { tetraploid } \\
\text { formation }\end{array}$ & No data & + & $\begin{array}{l}\text { Endo et al. } \\
1992\end{array}$ \\
\hline
\end{tabular}




\section{Table 3-10. Genotoxicity of Organic Arsenic}

\begin{tabular}{|c|c|c|c|c|c|}
\hline \multirow[b]{2}{*}{ Chemical form } & \multirow[b]{2}{*}{ Species (test system) } & \multirow[b]{2}{*}{ End point } & \multicolumn{2}{|c|}{ Results } & \multirow[b]{2}{*}{ Reference } \\
\hline & & & $\begin{array}{l}\text { With } \\
\text { activation }\end{array}$ & $\begin{array}{l}\text { Without } \\
\text { activation }\end{array}$ & \\
\hline DMA & $\begin{array}{l}\text { Chinese hamster V79 } \\
\text { cells }\end{array}$ & $\begin{array}{l}\text { Chromosomal } \\
\text { aberrations }\end{array}$ & No data & + & $\begin{array}{l}\text { Ueda et al. } \\
1997\end{array}$ \\
\hline DMA & $\begin{array}{l}\text { Chinese hamster lung } \\
\text { and diploid cells (V79) }\end{array}$ & $\begin{array}{l}\text { Chromosomal } \\
\text { aberrations }\end{array}$ & No data & + & $\begin{array}{l}\text { Kitamura et al. } \\
2002\end{array}$ \\
\hline DMA & $\begin{array}{l}\text { Chinese hamster lung } \\
\text { and diploid cells (V79) }\end{array}$ & $\begin{array}{l}\text { Chromosomal } \\
\text { aberrations }\end{array}$ & + & + & $\begin{array}{l}\text { Kuroda et al. } \\
2004\end{array}$ \\
\hline DMA & $\begin{array}{l}\text { Chinese hamster V79 } \\
\text { cells }\end{array}$ & $\begin{array}{l}\text { Tetraploids and } \\
\text { mitotic arrest }\end{array}$ & No data & + & $\begin{array}{l}\text { Eguchi et al. } \\
1997\end{array}$ \\
\hline MMA & $\begin{array}{l}\text { Human umbilical cord } \\
\text { fibroblasts }\end{array}$ & $\begin{array}{l}\text { Chromosomal } \\
\text { aberrations }\end{array}$ & No data & + & $\begin{array}{l}\text { Oya-Ohta et al. } \\
1996\end{array}$ \\
\hline MMA & $\begin{array}{l}\text { Chinese hamster V79 } \\
\text { cells }\end{array}$ & $\begin{array}{l}\text { Tetraploids and } \\
\text { mitotic arrest }\end{array}$ & No data & + & $\begin{array}{l}\text { Eguchi et al. } \\
1997\end{array}$ \\
\hline Roxarsone & $\begin{array}{l}\text { Drosophila } \\
\text { melanogaster }\end{array}$ & $\begin{array}{l}\text { Sex linked } \\
\text { recessive }\end{array}$ & No data & - & NTP 1989b \\
\hline Roxarsone & Rat hepatocyte & $\begin{array}{l}\text { DNA double- } \\
\text { strand breaks }\end{array}$ & No data & + & $\begin{array}{l}\text { Storer et al. } \\
1996\end{array}$ \\
\hline Roxarsone & $\begin{array}{l}\text { A31-1-13 clone of } \\
\text { BALB/c-3T3 cells }\end{array}$ & $\begin{array}{l}\text { Transformation } \\
\text { response and } \\
\text { mutagenicity }\end{array}$ & No data & - & $\begin{array}{l}\text { Matthews et al. } \\
1993\end{array}$ \\
\hline Roxarsone & $\begin{array}{l}\text { Mouse lymphoma } \\
\text { (L5178Y) cells }\end{array}$ & $\begin{array}{l}\text { Trifluorothymidine } \\
\text { resistance }\end{array}$ & No data & + & NTP 1989b \\
\hline \multicolumn{6}{|c|}{ Eukaryotic organisms (in vivo): } \\
\hline DMA & Rat (oral exposure) & $\begin{array}{l}\text { DNA single-stand } \\
\text { breaks in lung }\end{array}$ & No data & + & $\begin{array}{l}\text { Yamanaka and } \\
\text { Okada } 1994\end{array}$ \\
\hline DMA & Mouse (oral exposure) & $\begin{array}{l}\text { DNA strand breaks } \\
\text { in tissues }\end{array}$ & No data & + & $\begin{array}{l}\text { Yamanaka et } \\
\text { al. 1989b }\end{array}$ \\
\hline DMA & Mouse (oral exposure) & $\begin{array}{l}\text { DNA single-stand } \\
\text { breaks in lung }\end{array}$ & No data & + & $\begin{array}{l}\text { Yamanaka et } \\
\text { al. } 1993\end{array}$ \\
\hline DMA & Mouse (oral exposure) & $\begin{array}{l}\text { DNA single-strand } \\
\text { breaks in lung }\end{array}$ & No data & - & $\begin{array}{l}\text { Yamanaka et } \\
\text { al. } 1989 a\end{array}$ \\
\hline DMA & Mouse (oral exposure) & $\begin{array}{l}\text { DNA adduct } \\
\text { formation }\end{array}$ & No data & + & $\begin{array}{l}\text { Yamanaka et } \\
\text { al. } 2001\end{array}$ \\
\hline DMA & Mouse (injection) & $\begin{array}{l}\text { Aneuploidy in } \\
\text { bone marrow cells }\end{array}$ & No data & + & $\begin{array}{l}\text { Kashiwada et } \\
\text { al. } 1998\end{array}$ \\
\hline
\end{tabular}

- = negative result; + = positive result; DMA = dimethylarsinic acid; DNA = deoxyribonucleic acid; MMA = monomethylarsonic acid 


\subsection{TOXICOKINETICS}

There is an extensive database on the toxicokinetics of inorganic arsenic. Most studies have been performed in animals, but there are a number of studies in humans as well. These studies reveal the following main points:

- Both arsenate and arsenite are well absorbed by both the oral and inhalation routes. Absorption by the dermal route has not been well characterized, but is low compared to the other routes. Inorganic arsenic in soil is absorbed to a lesser extent than solutions of arsenic salts.

- The rate of absorption of arsenic in highly insoluble forms (e.g., arsenic sulfide, lead arsenate) is much lower than that of more soluble forms via both oral and inhalation routes.

- Once absorbed, arsenites are oxidized to arsenates and methylated. This process may then be repeated to result in dimethylated arsenic metabolites.

- Distribution of arsenic in the rat is quite different from other animal species, suggesting that the rat is probably not an appropriate toxicokinetic model for distribution, metabolism, or excretion of arsenic by humans.

- The As(+3) form undergoes enzymic methylation primarily in the liver to form MMA and DMA. The rate and relative proportion of methylation production varies among species. The rate of methylation varies considerably among tissues.

- Most arsenic is promptly excreted in the urine as a mixture of As $(+3), \operatorname{As}(+5)$, MMA, and DMA; DMA is usually the primary form in the urine. Smaller amounts are excreted in feces. Some arsenic may remain bound to tissues, depending inversely on the rate and extent of methylation.

Less information is available for the organic arsenicals. It appears that both MMA and DMA are well absorbed, but are rapidly excreted in the urine and feces. MMA may be methylated to DMA, but neither MMA nor DMA are demethylated to yield inorganic arsenic.

A review of the evidence that supports these conclusions is presented below.

\subsubsection{Absorption}

\subsubsection{Inhalation Exposure}

Inorganic Arsenicals. Since arsenic exists in air as particulate matter, absorption across the lung involves two processes: deposition of the particles onto the lung surface, and absorption of arsenic from the deposited material. In lung cancer patients exposed to arsenic in cigarette smoke, deposition was 
estimated to be about $40 \%$ and absorption was $75-85 \%$ (Holland et al. 1959). Thus, overall absorption (expressed as a percentage of inhaled arsenic) was about 30-34\%. In workers exposed to arsenic trioxide dusts in smelters, the amount of arsenic excreted in the urine (the main route of excretion; see Section 3.4.4) was about 40-60\% of the estimated inhaled dose (Pinto et al. 1976; Vahter et al. 1986). Absorption of arsenic trioxide dusts and fumes (assessed by measurement of urinary metabolites) correlated with time weighted average arsenic air concentrations from personal breathing zone air samplers (Offergelt et al. 1992). Correlations were best immediately after a shift and just before the start of the next shift. Although the percent deposition was not measured in these cases, it seems likely that nearly all of the deposited arsenic was absorbed. This conclusion is supported by intratracheal instillation studies in rats and hamsters, where clearance of oxy compounds of arsenic (sodium arsenite, sodium arsenate, arsenic trioxide) from the lung was rapid and nearly complete (60-90\% within 1 day) (Marafante and Vahter 1987; Rhoads and Sanders 1985). In contrast, arsenic sulfide and lead arsenate were cleared more slowly (Marafante and Vahter 1987), indicating that the rate of absorption may be lower if the inhaled arsenic is in a highly insoluble form. There are no data to suggest that absorption of inhaled arsenic in children differs from that in adults.

Organic Arsenicals. No studies were located regarding absorption of organic arsenicals in humans or animals after inhalation exposure. However, DMA instilled in the lungs of rats was absorbed very rapidly (half-time of 2.2 minutes) and nearly completely (at least 92\%) (Stevens et al. 1977). This indicates that organic arsenicals are likely to be well absorbed by the inhalation route.

\subsubsection{Oral Exposure}

Inorganic Arsenicals. Several studies in humans indicate that arsenates and arsenites are well absorbed across the gastrointestinal tract. The most direct evidence is from a study that evaluated the 6-day elimination of arsenic in healthy humans who were given water from a high-arsenic sampling site (arsenic species not specified) and that reported approximately 95\% absorption (Zheng et al. 2002). A similar absorption efficiency can be estimated from measurements of fecal excretion in humans given oral doses of arsenite, where $<5 \%$ was recovered in the feces (Bettley and O'Shea 1975). This indicates absorption was at least $95 \%$. These results are supported by studies in which urinary excretion in humans was found to account for $55-87 \%$ of daily oral intakes of arsenate or arsenite (Buchet et al. 1981b; Crecelius 1977; Kumana et al. 2002; Mappes 1977; Tam et al. 1979b). In contrast, ingestion of arsenic triselenide $\left(\mathrm{As}_{2} \mathrm{Se}_{3}\right)$ did not lead to a measurable increase in urinary excretion (Mappes 1977), indicating that 


\section{HEALTH EFFECTS}

gastrointestinal absorption may be much lower if highly insoluble forms of arsenic are ingested. There are no data to suggest that absorption of arsenic from the gut in children differs from that in adults.

These observations in humans are supported by a number of studies in animals. Fecal excretion of arsenates and arsenites ranged from 2 to $10 \%$ in monkeys and mice, with $70 \%$ or more appearing in urine (Charbonneau et al. 1978; Roberts et al. 2002; Vahter 1981; Vahter and Norin 1980). Oral absorption of $\left[{ }^{73} \mathrm{As}\right]$ labeled sodium arsenate in mice was unaffected by dose $(0.0005-5 \mathrm{mg} / \mathrm{kg})$ as reflected in percentage of dose excreted in feces over 48 hours (Hughes et al. 1994). Absorption ranged from 82 to $89 \%$ at all doses. Gonzalez et al. (1995) found that the percentage of arsenate that was absorbed in rats decreased as the dose increased from 6 to $480 \mu \mathrm{g}$, suggesting saturable, zero-order absorption of arsenate in this species. Hamsters appear to absorb somewhat less than humans, monkeys, and mice, since fecal excretion usually ranges from 10 to 40\% (Marafante and Vahter 1987; Marafante et al. 1987a; Yamauchi and Yamamura 1985). Rabbits also appear to absorb less arsenate than humans, monkeys, or mice after oral exposure (Freeman et al. 1993). After a gavage dose of $1.95 \mathrm{mg} / \mathrm{kg}$ sodium arsenate, $45 \%$ of the arsenate was recovered in feces in males and $52 \%$ in females. As in humans, when highly insoluble arsenic compounds are administered (arsenic trisulfide, lead arsenate), gastrointestinal absorption is reduced 20-30\% (Marafante and Vahter 1987).

Bioavailability of arsenic was measured in rabbits ingesting doses of smelting soils that contained arsenic primarily in the form of sulfides (Freeman et al. 1993). Bioavailability was assessed by comparing the amounts of arsenic that was excreted after ingestion of the soil to that excreted after an intravenous dose of sodium arsenate. The bioavailability of the arsenic in the ingested soil was $24 \pm 3.2 \%$ and that of sodium arsenate in the gavage dose was $50 \pm 5.7 \%$. Approximately $80 \%$ of the arsenic from ingested soil was eliminated in the feces compared with $50 \%$ of the soluble oral dose and $10 \%$ of the injected dose. In another study, rabbits dosed with sodium arsenite $(0.8 \mathrm{mg} \mathrm{As} / \mathrm{kg})$ had 5 times greater blood arsenic concentrations than rabbits dosed with arsenic-containing soil (2.8 mg As/kg), suggesting a lower bioavailability of the arsenic in soil (Davis et al. 1992).

Studies of the bioavailability of arsenic suggest that absorption of arsenic in ingested dust or soil is likely to be considerably less than absorption of arsenic from ingested salts (Davis et al. 1992, 1996; EPA 1997g; Freeman et al. 1993, 1995; Pascoe et al. 1994; Roberts et al. 2002, 2007; Rodriguez et al. 1999). Oral absorption of arsenic in a group of three female Cynomolgus monkeys from a soluble salt, soil, and household dust was compared with absorption of an intravenous dose of sodium arsenate (Freeman et al. 1995). Mean absolute percentage bioavailability based on urine arsenic excretion was reported at 
$67.6 \pm 2.6 \%$ (gavage), $19.2 \pm 1.5 \%$ (oral dust), and $13.8 \pm 3.3 \%$ (oral soil). Mean absolute percentage bioavailability based on blood arsenic levels was reported at $91.3 \pm 12.4 \%$ (gavage), $9.8 \pm 4.3 \%$ (oral dust), and $10.9 \pm 5.2 \%$ (oral soil). The arsenic in the dust and soil was approximately $3.5-5$-fold (based on levels in the urine) and 8-9-fold (based on levels in the blood) less bioavailable than arsenic in solution. Two other studies in monkeys reported relative bioavailability of arsenic in soil from a number of locations (electrical substation, wood preserving sites, pesticide sites, cattle-dip sites, volcanic soil, and mining sites) ranged from 5 to $31 \%$ (Roberts et al. 2002, 2007). A study in beagle dogs fed with soil containing $\mathrm{As}_{2} \mathrm{O}_{5}$ or treated with intravenous soluble arsenic found that compared to injection the bioavailability of arsenic from ingested soil was $8.3 \pm 2.0 \%$ (Groen et al. 1993). The bioavailability of arsenic in soil has been studied in juvenile swine that received daily oral doses of soil or sodium arsenate (in food or by gavage) for 15 days (EPA 1997g). The soils were obtained from various mining and smelting sites and contained, in addition to arsenic at concentrations of 100-300 $\mu \mathrm{g} / \mathrm{g}$, lead at concentrations of 3,000 $14,000 \mu \mathrm{g} / \mathrm{g}$. The arsenic doses ranged from 1 to $65.4 \mu \mathrm{g} / \mathrm{kg} /$ day. The fraction of the arsenic dose excreted in urine was measured on days 7 and 14 and the relative bioavailability of the soil-borne arsenic was estimated as the ratio of urinary excretion fractions, soil arsenic:sodium arsenate. The mean relative bioavailability of soil-borne arsenic ranged from 0 to $98 \%$ in soils from seven different sites (mean $\pm \mathrm{SD}$, $45 \% \pm 32$ ). Estimates for relative bioavailability of arsenic in samples of smelter slag and mine tailings ranged from 7 to $51 \%($ mean $\pm \mathrm{SD}, 35 \% \pm 27)$. Rodriguez et al. (1999) used a similar approach to estimate the relative bioavailability of arsenic in mine and smelter wastes (soils and solid materials) in juvenile swine. Samples included iron slag deposits and calcine deposits and had arsenic concentrations that ranged from 330 to $17,500 \mu \mathrm{g} / \mathrm{g}$. Relative bioavailability (waste:sodium arsenate) ranged from 3 to $43 \%$ for 13 samples (mean, 21\%) and was higher in iron slag wastes (mean, 25\%) than in calcine wastes (mean, 13\%).

Bioavailability of arsenic from soil is reduced by low solubility and inaccessibility due to the presence of secondary reaction products or insoluble matrix components (Davis et al. 1992). This is supported by studies conducted with in vitro simulations of the gastric and/or intestinal fluids (Hamel et al. 1998; Pouschat and Zagury 2006; Rodriguez et al. 1999; Ruby et al. 1996, 1999; Williams et al. 1998). When soils containing arsenic are incubated in simulated gastrointestinal fluids, only a fraction of the arsenic becomes soluble. Estimates of the soluble, or bioaccessible, arsenic fraction have ranged from 3 to $50 \%$ for various soils and mining and smelter waste materials (Pouschat and Zagury 2006; Rodriguez et al. 1999; Ruby et al. 1996); these estimates are similar to in vivo estimates of the relative bioavailability of arsenic in these same materials (Ruby et al. 1999). 
Organic Arsenicals. Based on urinary excretion studies in volunteers, it appears that both MMA and DMA are well absorbed (at least 75-85\%) across the gastrointestinal tract (Buchet et al. 1981a; Marafante et al. 1987b). This is supported by studies in animals, where at least $75 \%$ absorption has been observed for DMA (Marafante et al. 1987b; Stevens et al. 1977; Vahter et al. 1984; Yamauchi and Yamamura 1984) and MMA (Hughes et al. 2005; Yamauchi et al. 1988). In mice, the relative bioavailability of MMA appears to be dose-dependent; $81 \%$ was absorbed following a single gavage dose of $0.4 \mathrm{mg}$ MMA $/ \mathrm{kg} /$ day compared to $60 \%$ following administration of $4 \mathrm{mg} \mathrm{MMA} / \mathrm{kg} /$ day (Hughes et al. 2005).

\subsubsection{Dermal Exposure}

Inorganic Arsenicals. No quantitative studies were located on absorption of inorganic arsenicals in humans after dermal exposure. Percutaneous absorption of $\left[{ }^{73} \mathrm{As}\right]$ as arsenic acid $\left(\mathrm{H}_{3} \mathrm{AsO}_{4}\right)$ alone and mixed with soil has been measured in skin from cadavers (Wester et al. 1993). Labeled arsenic was applied to skin in diffusion cells and transit through the skin into receptor fluid measured. After 24 hours, $0.93 \%$ of the dose passed through the skin and $0.98 \%$ remained in the skin after washing. Absorption was lower with $\left[{ }^{73} \mathrm{As}\right]$ mixed with soil: $0.43 \%$ passed through the skin over 24 hours and $0.33 \%$ remained in the skin after washing.

Dermal absorption of arsenic has been measured in Rhesus monkeys (Lowney et al. 2005; Wester et al. 1993). After 24 hours, $6.4 \%$ of $\left[{ }^{73} \mathrm{As}\right]$ as arsenic acid was absorbed systemically, as was $4.5 \%$ of $\left[{ }^{73} \mathrm{As}\right]$ mixed with soil (Wester et al. 1993). Similarly, $2.8 \%$ of soluble arsenic in water was detected in the urine 24 hours after exposure (Lowney et al. 2005). However, arsenic from soil was poorly absorbed; $0.12 \%$ was detected in the urine after 24 hours. Differences between the Wester et al. (1993) and Lowney et al. (2005) studies in terms of uptake from soil may be due to the differences in forms of arsenic in the soil. In the Wester et al. (1993) study, soil was mixed with radiolabelled arsenic acid in water; Lowney et al. (2005) used soil samples from a pesticide manufacturing facility that historically manufactured arsenical pesticides (the arsenic was primarily in the iron oxide and iron silicate mineral phases). Lowney et al. (2005) also measured urinary levels of arsenic following dermal application of CCA residues and found that the levels did not increase from background. Uptake of arsenic into blood or tissues was undetectable for up to 24 hours in rats whose tails were immersed in solutions of sodium arsenate for 1 hour. However, arsenic began to increase in blood, liver, and spleen over the next 5 days (Dutkiewicz 1977). The rate of uptake was estimated to be $1-33 \mu \mathrm{g} / \mathrm{cm}^{2} /$ hour. These findings suggest that dermal exposure leads initially to arsenic binding to skin, and that the bound arsenic may slowly be taken up into the blood, even after exposure ends. 
Organic Arsenicals. No studies were located on absorption of organic arsenicals in humans or animals after dermal exposure.

\subsubsection{Distribution}

\subsubsection{Inhalation Exposure}

Inorganic Arsenicals. No studies were located on the distribution of arsenic in humans or animals after inhalation exposure, but intratracheal administration of arsenic trioxide to rats resulted in distribution of arsenic to the liver, kidney, skeleton, gastrointestinal tract, and other tissues (Rhoads and Sanders 1985). This is consistent with data from oral and parenteral studies (below), which indicate that absorbed arsenic is distributed throughout the body.

Organic Arsenicals. No studies were located regarding the distribution of organic arsenicals in humans or animals after inhalation exposure. However, DMA administered to rats by the intratracheal route was distributed throughout the body (Stevens et al. 1977), suggesting that inhalation of organic arsenicals would also lead to widespread distribution.

\subsubsection{Oral Exposure}

Inorganic Arsenicals. Analysis of tissues taken at autopsy from people who were exposed to background levels of arsenic in food and water revealed that arsenic is present in all tissues of the body (Liebscher and Smith 1968). Most tissues had about the same concentration level (0.05-0.15 ppm), while levels in hair $(0.65 \mathrm{ppm})$ and nails $(0.36 \mathrm{ppm})$ were somewhat higher. This indicates that there is little tendency for arsenic to accumulate preferentially in any internal organs. However, exposure levels may not have been high enough to cause elevated levels in tissues. Arsenic exposure may have been low enough that the methylation process in the body resulted in limited accumulation in internal organs. Tissue analysis of organs taken from an individual following death from ingestion of $8 \mathrm{~g}$ of arsenic trioxide (about $3 \mathrm{~g}$ of arsenic) showed a much higher concentration of arsenic in liver $(147 \mu \mathrm{g} / \mathrm{g})$ than in kidney $(27 \mu \mathrm{g} / \mathrm{g})$ or muscle, heart, spleen, pancreas, lungs, or cerebellum (11-12 $\mu \mathrm{g} / \mathrm{g}$ ) (Benramdane et al. 1999a). Small amounts were also found in other parts of the brain $(8 \mu \mathrm{g} / \mathrm{g})$, skin $(3 \mu \mathrm{g} / \mathrm{g})$, and hemolyzed blood $(0.4 \mu \mathrm{g} / \mathrm{g})$. Many studies have been performed where arsenic levels in hair and nails have been measured and correlations with exposure analyzed. Some of these studies are discussed in Section 3.8, Biomarkers of Exposure. 
Inorganic arsenic passes easily through the placenta. High levels of arsenic were found in the liver, kidney, and brain during autopsy of an infant prematurely born to a young mother who had ingested inorganic arsenic at week 30 of gestation (Lugo et al. 1969). Arsenic was detected in human breast milk at concentrations of $0.00013-0.00082 \mathrm{ppm}$ in a World Health Organization study (Somogyi and Beck 1993). Arsenic concentrations were $0.0001-0.0044 \mathrm{ppm}$ in human milk sampled from 88 mothers on the Faroe Islands whose diets were predominantly seafood (Grandjean et al. 1995). Exposures to arsenic from the seafood diet in this population was most likely to organic "fish arsenic." In a population of Andean women exposed to high concentrations (about $200 \mathrm{ppb}$ ) of inorganic arsenic in drinking water, concentrations of arsenic in breast milk ranged from about 0.0008 to $0.008 \mathrm{ppm}$ (Concha et al. 1998b).

Studies in mice and hamsters given oral doses of arsenate or arsenite have found elevated levels of arsenic in all tissues examined (Hughes et al. 2003; Vahter and Norin 1980; Yamauchi and Yamamura 1985), including the placenta and fetus of pregnant females (Hood et al. 1987, 1988). Inorganic arsenic crosses the placental barrier and selectively accumulates in the neuroepithelium of the developing animal embryo (Hanlon and Ferm 1977; Lindgren et al. 1984). In mice, radiolabel from orally administered 74-As was widely distributed to all tissues, with the highest levels in skin, kidney, and liver (Hughes et al. 2003). No obvious differences between $\mathrm{As}(+3)$ and $\mathrm{As}(+5)$ were found, although residual levels after 24 hours tended to be higher for $\mathrm{As}(+3)$ than $\mathrm{As}(+5)$ (Vahter and Norin 1980). However, in vitro studies have found that the cellular uptake of $\mathrm{As}(+3)$ was higher than that of $\mathrm{As}(+5)$ (Bertolero et al. 1987; Dopp et al. 2004); in mouse cells, the difference was 4-fold (Bertolero et al. 1987). In hamsters, increases in tissue levels were noted after oral treatment with $\mathrm{As}(+3)$ for most tissues (hair, kidney, liver, lung, skin, muscle), with the largest increases in liver and lung (Yamauchi and Yamamura 1985). Liver and kidney arsenic concentrations increased with dose in dogs fed arsenite in the diet for 6 months (Neiger and Osweiler 1992). A study examining the speciation of arsenic following a single dose exposure to sodium arsenate to mice (Kenyon et al. 2005) found that the levels of inorganic arsenic and DMA were similar in the blood, liver, and kidney; much lower levels of MMA were found in these tissues. The concentration of DMA in the lungs exceeded inorganic arsenic and the levels of inorganic arsenic and MMA were similar; the DMA concentration was about 6 times higher than that of inorganic arsenic.

Inorganic arsenic crosses the placental barrier and selectively accumulates in the neuroepithelium of the developing animal embryo (Hanlon and Ferm 1977; Lindgren et al. 1984). Following maternal exposure to arsenite or arsenate throughout gestation and lactation, inorganic arsenic and DMA were detected in the 
newborn mouse brains (Jin et al. 2006). The levels of inorganic arsenic in the brain were similar to those in the newborn livers; however, the levels of DMA in the brain were about twice as high as in the liver.

Organic Arsenicals. No studies were located on the distribution of organic arsenicals in humans following oral exposure. Studies in animals found MMA and DMA distributed to all tissues after acute oral doses (Hughes et al. 2005; Stevens et al. 1977; Vahter et al. 1984; Yamauchi and Yamamura 1984; Yamauchi et al. 1988). In mice, MMA is rapidly distributed throughout the body with peak tissue concentrations occurring between 0.25 and 4 hours after administration of a single gavage dose of 0.4 or $4 \mathrm{mg}$ MMA/kg (Hughes et al. 2005). The peak levels of MMA in the bladder, kidneys, and lungs were higher than blood, with the highest levels occurring in the bladder. The terminal half-lives of MMA were 4.2-4.9 hours in the liver, lung, and blood, 9.0 hours in the urinary bladder, and 15.9 hours in the kidney in mice dosed with $0.4 \mathrm{mg} \mathrm{MMA} / \mathrm{kg}$; similar half-lives were measured in the $4.0 \mathrm{mg} \mathrm{MMA} / \mathrm{kg}$ mice. Two hours after dosing, most of the methylated arsenic in the tissues was in the form of MMA. In rats exposed to $100 \mathrm{mg} / \mathrm{kg}$ DMA in the diet for 72 days, high levels of arsenic was detected in the blood ( $\mathrm{Lu}$ et al. 2004a). The arsenic was primarily found in the erythrocyte; the concentration in the erythrocyte was 150 times higher than the arsenic concentration in the plasma.

\subsubsection{Dermal Exposure}

No studies were located regarding distribution of inorganic or organic arsenicals in humans or animals after dermal exposure.

\subsubsection{Other Routes of Exposure}

Inorganic Arsenicals. Studies in mice, rabbits, and monkeys injected intravenously with solutions of arsenite or arsenate confirm that arsenic is widely distributed throughout the body (Lindgren et al. 1982; Marafante and Vahter 1986; Vahter and Marafante 1983; Vahter et al. 1982). Shortly after exposure, the concentration of arsenic tends to be somewhat higher in liver, kidney, lung, and gastrointestinal epithelium (Hughes et al. 2000; Lindgren et al. 1982; Vahter and Marafante 1983; Vahter et al. 1982), but levels tend to equilibrate over time. Arsenate shows a tendency to deposit in skeletal tissue that is not shared by arsenite (Lindgren et al. 1982, 1984), presumably because arsenate is an analog of phosphate.

The distribution of arsenic in the rat is quite different from other animal species. Following intramuscular injection of carrier-free radio-arsenate in rats, most of the injected arsenic became bound to hemoglobin in red blood cells, and very little reached other tissues (Lanz et al. 1950). However, similar experiments 
in dogs, mice, guinea pigs, rabbits, and chicks found very little uptake of arsenic into the blood in these species (cats gave intermediate results).

Organic Arsenicals. Following intravenous administration of DMA in mice, DMA is rapidly distributed throughout the body (Hughes et al. 2000). In the blood, the DMA was initially detected in the plasma, but fairly rapidly equilibrated between the plasma and erythrocytes. Blood, plasma, erythrocyte, liver, and kidney distribution and elimination of DMA did not differ in groups of mice administered 1.11 or $111 \mathrm{mg}$ DMA/kg. However, a significant difference in DMA elimination from the lungs was observed; the elimination half-time increased from 91 minutes in the $1.11 \mathrm{mg} \mathrm{DMA} / \mathrm{kg}$ group to 6,930 minutes in the $111 \mathrm{mg}$ DMA/kg group.

\subsubsection{Metabolism}

Inorganic Arsenicals. The metabolism of inorganic arsenic has been extensively studied in humans and animals, and is diagrammed in Figure 3-7. Two basic processes are involved: (1) reduction/oxidation reactions that interconvert $\mathrm{As}(\mathrm{III})$ and $\mathrm{As}(\mathrm{V})$, and (2) methylation reactions, which convert arsenite to MMA and DMA. The resulting series of reactions results in the reduction of inorganic arsenate to arsenite (if necessary), methylation to MMA(V), reduction to MMA(III), and methylation to DMA(V). These processes appear to be similar whether exposure is by the inhalation, oral, or parenteral route. The human body has the ability to change inorganic arsenic to organic forms (i.e., by methylation) that are more readily excreted in urine. In addition, inorganic arsenic is also directly excreted in the urine. It is estimated that by means of these two processes, $>75 \%$ of the absorbed arsenic dose is excreted in the urine (Marcus and Rispin 1988), although this may vary with the dose and exposure duration. This mechanism is thought to have an upper-dose limit which, when overwhelmed, results in a higher incidence of arsenic toxicity. This is supported by a case report of an individual who died 3 days after ingesting $8 \mathrm{~g}$ of arsenic trioxide (about $3 \mathrm{~g}$ of arsenic) (Benramdane et al. 1999a). Only 20\% of the total arsenic in all tissues analyzed was methylated (14\% MMA, 6\% DMA), while 78\% remained as arsenite and $2 \%$ as arsenate.

The majority of the evidence characterizing the metabolic pathways of arsenic is derived from analysis of urinary excretion products. Exposure of humans to either arsenates or arsenites results in increased levels of inorganic As(+3), inorganic As(+5), MMA, and DMA in urine (Aposhian et al. 2000a, 2000b; Buchet et al. 1981a, 1981b; Concha et al. 1998a, 1998b; Crecelius 1977; Kurttio et al. 1998; Lovell and Farmer 
Figure 3-7. Inorganic Arsenic Biotransformation Pathway

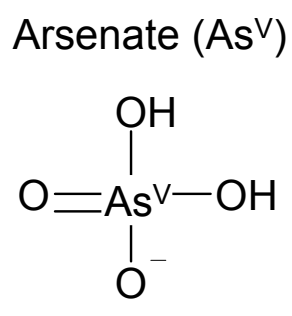

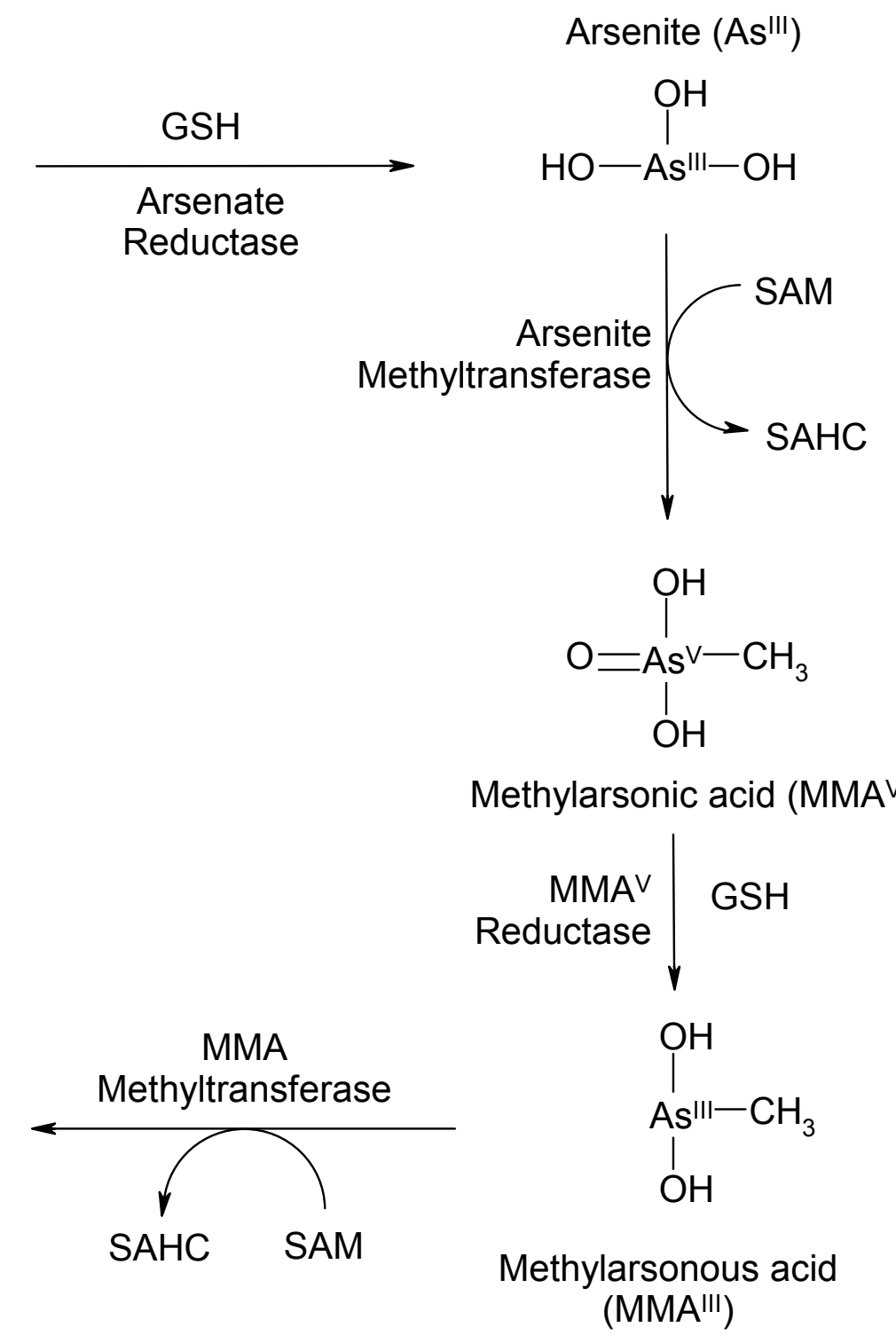

Arsenite (As'II)

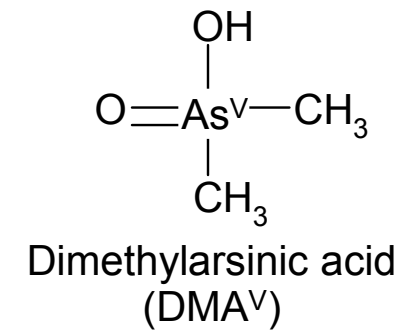

Dimethylarsinic acid $\left(\mathrm{DMA}^{\mathrm{v}}\right)$

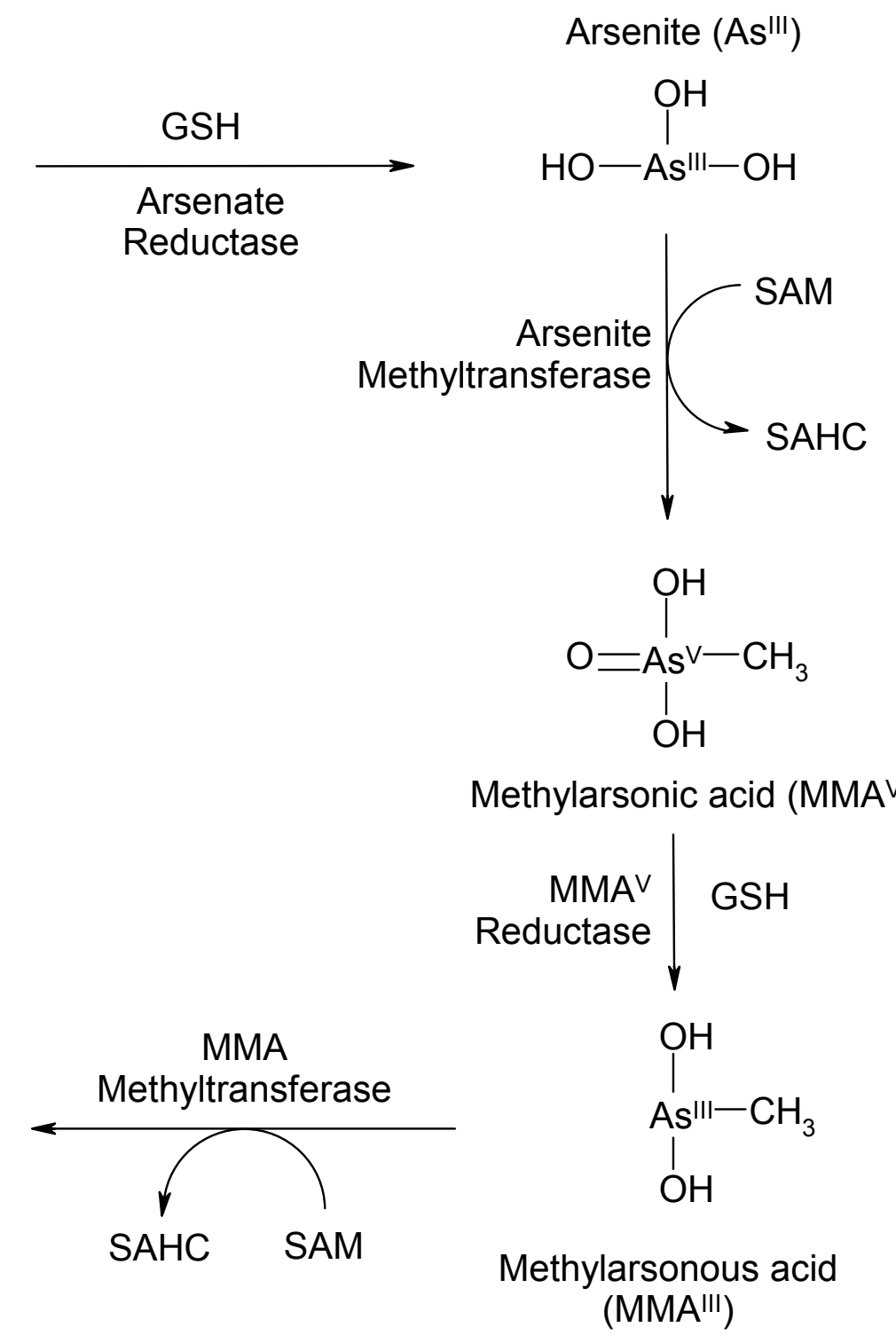

Methylarsonic acid $\left(\mathrm{MMA}^{\mathrm{V}}\right)$

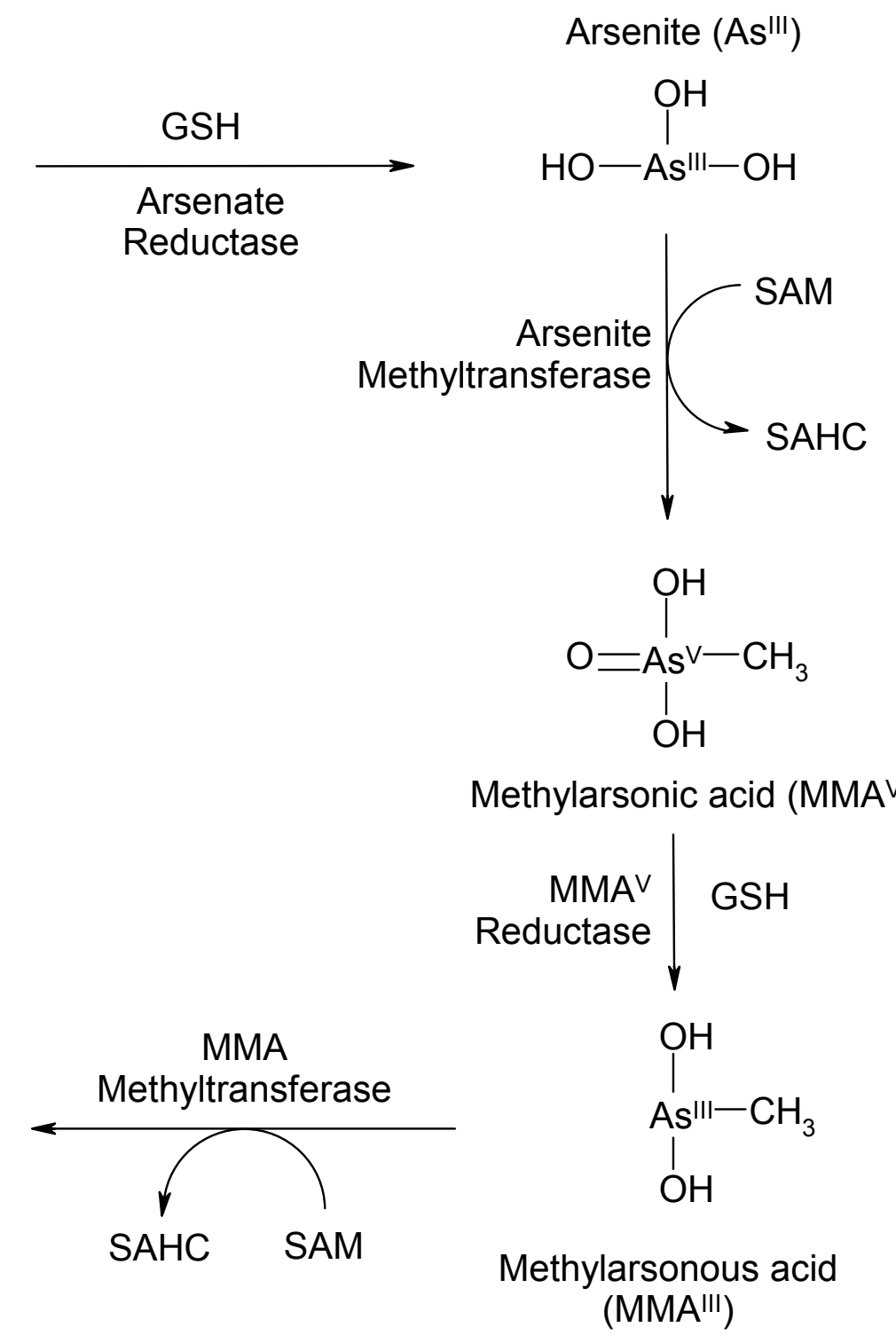

SAHC = S-adenosylhomocysteine $;$ SAM = S-adenosylmethionine

Source: adapted from Aposhian et al. 2000b 
1985; Smith et al. 1977; Tam et al. 1979b; Vahter 1986). Similar results are obtained from studies in mice (Vahter 1981; Vahter and Envall 1983), hamsters (Hirata et al. 1988; Marafante and Vahter 1987; Takahashi et al. 1988), and rabbits (Maiorino and Aposhian 1985; Marafante et al. 1985; Vahter and Marafante 1983). Historically, little distinction was made between MMA(V) and MMA(III) in the urine in most studies, and the assumption was that the majority of MMA in the urine was MMA(V); however, Aposhian et al. (2000a, 2000b) demonstrated that the methylated arsenic atom may be in either valance state.

The relative proportions of $\mathrm{As}(+3), \mathrm{As}(+5), \mathrm{MMA}$, and DMA in urine can vary depending upon the chemical administered, time after exposure, route of exposure, dose level, and exposed species. In general, however, DMA is the principal metabolite following long-term exposure, with lower levels of inorganic arsenic $[\mathrm{As}(+3)$ and $\mathrm{As}(+5)]$ and MMA. In humans, the relative proportions are usually about 40-75\% DMA, 20-25\% inorganic arsenic, and 15-25\% MMA (Buchet et al. 1981a; Hopenhayn et al. 2003b; Loffredo et al. 2003; Mandal et al. 2001; Smith et al. 1977; Tam et al. 1979b; Tokunaga et al. 2002; Vahter 1986). With relatively constant exposure levels, these metabolic proportions remain similar over time (Concha et al. 2002), and appear to be similar among family members (Chung et al. 2002). One study of groups of women and children in two villages in Argentina showed that children ingesting large amounts of arsenic in their drinking water $(200 \mu \mathrm{g} / \mathrm{L})$ excreted about $49 \%$ inorganic arsenic and $47 \%$ DMA (Concha et al. 1998b). This compared to 32\% inorganic arsenic and 66\% DMA for the women in the study. This may indicate that metabolism of arsenic in children is less efficient than in adults. The rabbit has a ratio of metabolites similar to human adults (Maiorino and Aposhian 1985), suggesting that this may be a good animal model for toxicokinetics in humans. Mice may also be a good human toxicokinetic model based on the similarity of arsenic metabolism and deposition (Vahter et al. 2002). In contrast, the guinea pig, marmoset, and tamarin monkey do not methylate inorganic arsenic (Healy et al. 1998; Vahter and Marafante 1985; Vahter et al. 1982; Zakharyan et al. 1996); thus, they may be poor models for humans.

Reduction of arsenate to arsenite can be mediated by glutathione (Menzel et al. 1994). Scott et al. (1993) showed that glutathione forms complexes with both arsenate and arsenite in vitro, and that glutathione is oxidized (and arsenate reduced) in the glutathione-arsenate reaction. Studies in vitro indicate that the substrate for methylation is $\mathrm{As}(+3)$, and that $\mathrm{As}(+5)$ is not methylated unless it is first reduced to $\mathrm{As}(+3)$ (Buchet and Lauwerys 1985, 1988; Lerman et al. 1983). The main site of methylation appears to be the liver, where the methylation process is mediated by enzymes that utilize S-adenosylmethionine as cosubstrate (Buchet and Lauwerys 1985, 1988). Under normal conditions, the availability of methyl 
donors (e.g., methionine, choline, cysteine) does not appear to be rate limiting in methylating capacity, either in humans (Buchet et al. 1982) or in animals (Buchet and Lauwerys 1987; Buchet et al. 1981a). However, severe dietary restriction of methyl donor intake can result in significant decreases in methylating capacity (Buchet and Lauwerys 1987; Vahter and Marafante 1987).

Arsenic methyltransferase and MMA methyltransferase activities have been purified to homogeneity from cytosol of rabbit liver (Zakharyan et al. 1995), Rhesus monkey liver (Zakharyan et al. 1996), and rat liver (Thomas et al. 2004). It appears that a single protein catalyzes both activities. This activity transfers a methyl group from S-adenosylmethionine to $\mathrm{As}(+3)$ yielding MMA, which is then further methylated to DMA. Reduced glutathione is probably a co-factor in vivo, but other thiols can substitute in vitro (L-cysteine, dithiothreitol). The substrate saturation concentration for rabbit arsenite methyltransferase is $50 \mu \mathrm{M}$, for MMA methyltransferase it is $1,000 \mu \mathrm{M}$. The purified activity is specific for arsenite and MMA; selenite, selenate, selenide, and catechols do not serve as substrates. Thomas et al. (2004) reported cloning the gene for an S-adenosylmethionine-dependent methyltransferase from rat liver cytosol that catalyzes the conversion of arsenic to methylated and dimethylated species. It bears a high similarity to translations of cyt19 genes in both the mouse and the human; both this gene and protein are now termed arsenic ( +3 oxidation state) methyltransferase (AS3MT).

Studies in mice indicate that exposure to arsenic does not induce arsenic methylation activity (Healy et al. 1998). Mice receiving up to $0.87 \mathrm{mg} \mathrm{As} / \mathrm{kg} /$ day as sodium arsenate in drinking water for 91 days had the same arsenic methylating activity as unexposed controls. Specific activities were highest in testis $(1.45 \mathrm{U} / \mathrm{mg})$ followed by kidney $(0.70 \mathrm{U} / \mathrm{mg})$, liver $(0.40 \mathrm{U} / \mathrm{mg})$, and lung $(0.20 \mathrm{U} / \mathrm{mg})$. None were affected by arsenic exposure.

An alternative biotransformation pathway (Figure 3-8) has recently been proposed for arsenic (Hayakawa et al. 2005) based on the nonenzymatic formation of glutathione complexes with arsenite resulting in the formation of arsenic triglutathione. The arsenic triglutathione is subsequently methylated by AS3MT to form monomethyl arsenic glutathione. At low glutathione levels (1 mM), the monomethyl arsenic glutathione is hydrolyzed to form MMA(III). At high glutathione levels (5 mM), the monomethyl arsenic glutathione is methylated to dimethylarsinic glutathione by AS3MT. Dimethylarsinic glutathione is quickly hydrolyzed to form DMA(III) (Hayakawa et al. 2005; Thomas et al. 2007). In the classical inorganic arsenic biotransformation pathway (Figure 3-7), MMA(V) is converted to the more toxic MMA(III); in contrast, in the alternative pathway, MMA(III) is converted to the less toxic MMA(V). 
Figure 3-8. Alternative Inorganic Arsenic Biotransformation Pathway

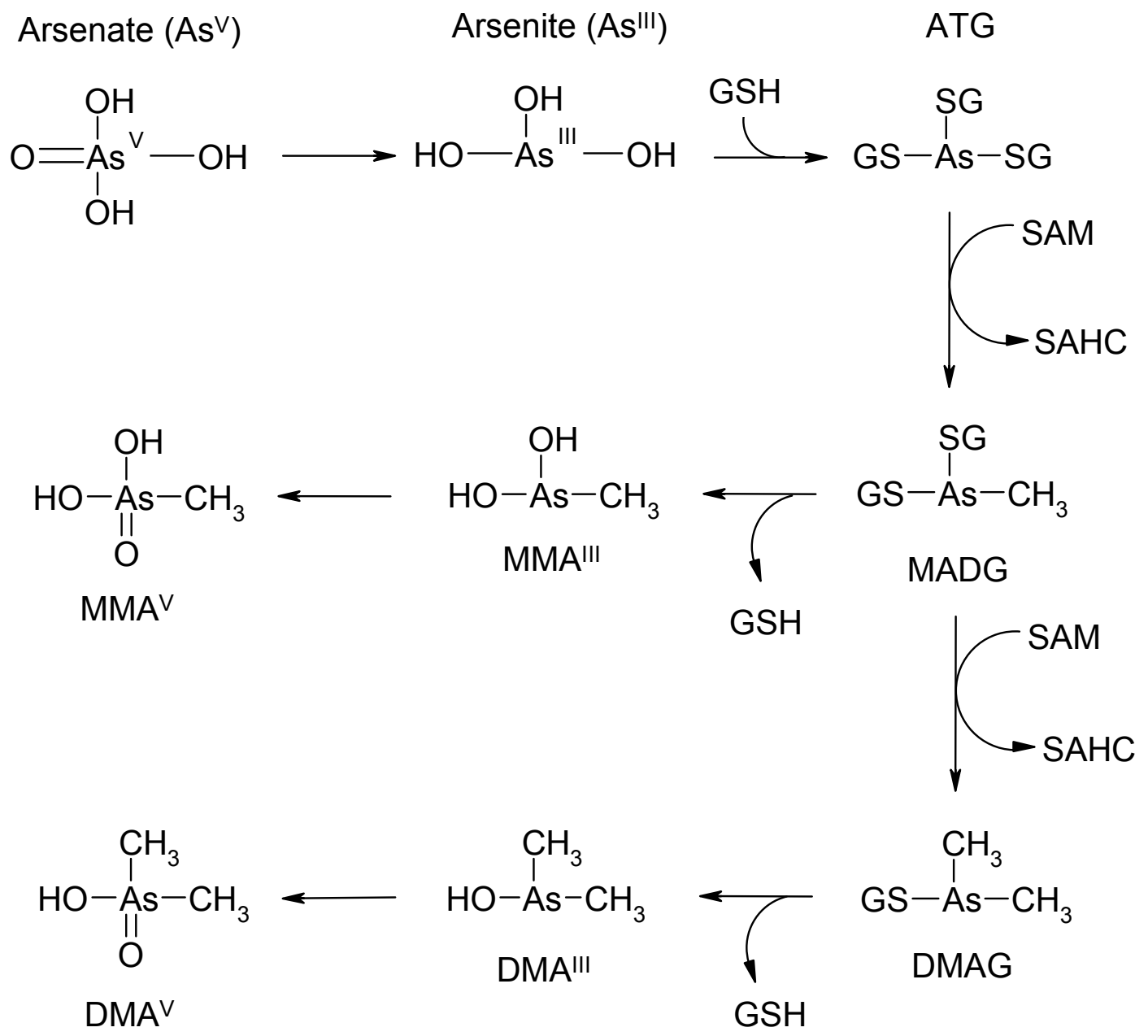

ATG = arsenic triglutathione; DMAG = dimethylarsinic glutathione; GSH = glutathione; $\mathrm{MADG}=$ monomethyl arsenic glutathione; SAHC = S-adenosylhomocysteine; SAM = S-adenosylmethionine 
Since methylation tends to result in lower tissue retention of inorganic arsenic (Marafante and Vahter 1984, 1986; Marafante et al. 1985; Vahter and Marafante 1987), the methylation process is usually viewed as a detoxification mechanism. However, several studies showing an elevated toxicity of MMA(III) relative even to As(III) in cultured human liver cells (Petrick et al. 2000, 2001) have called this assumption into question. Because methylation is an enzymic process, an important issue is the dose of arsenic that saturates the methylation capacity of an organism, resulting in a possible increased level of the more toxic As(III) in tissues, or whether or not such a dose exists. Limited data from studies in humans suggest that methylation may begin to become limiting at doses of about $0.2-1 \mathrm{mg} /$ day $(0.003-$ $0.015 \mathrm{mg} / \mathrm{kg} /$ day) (Buchet et al. 1981b; Marcus and Rispin 1988). However, these observations are relatively uncertain since they are based on data from only a few subjects, and the pattern of urinary excretion products in humans who ingested high (near lethal) oral doses or were exposed to elevated levels in the workplace is not much different from that in the general population (Lovell and Farmer 1985; Vahter 1986). Furthermore, the nutrient intakes reported by Engel and Receveur (1993) were sufficient to accommodate the body stores of methyl groups needed for arsenic biomethylation. At the highest arsenic level reported in the endemic area, the biomethylation process required only a few percent of the total daily methyl intake (Mushak and Crocetti 1995). Thus, the dose rate at which methylation capacity becomes saturated cannot be precisely defined with current data.

Organic Arsenicals. With the exception of arsenosugars, which may undergo extensive metabolism, organic arsenicals appear to undergo little metabolism. Humans who ingested a dose of MMA converted a small amount (about 13\%) to DMA (Buchet et al. 1981a). Similarly, in mice and hamsters, DMA and MMA are primarily excreted unchanged in the urine (Hughes et al. 2005; Marafante et al. 1987b; Vahter et al. 1984). In mice, a small percentage of MMA is methylated to DMA and some is further methylated to trimethylarsine oxide (TMAO) (Hughes et al. 2005). In contrast, administration of MMA(III) to mice resulted in the excretion of mostly DMA(V) and smaller amounts of MMA(V), MMA(III), and DMA(III) (Hughes et al. 2005). As with MMA, only a small percentage $(<10 \%)$ of the DMA is methylated to TMAO (Hughes et al. 2005; Marafante et al. 1987b; Yamauchi and Yamamura 1984; Yamauchi et al. 1988).

MMA and DMA are more extensively methylated in rats compared to other animal species. After 1 week of exposure to $100 \mathrm{mg} \mathrm{As} / \mathrm{kg} /$ day as MMA in drinking water, rats excreted $50.6 \%$ of the total arsenic in urine as MMA, $19.0 \%$ as DMA, $6.9 \%$ as TMAO, and $0.4 \%$ as tetramethylarsonium (Yoshida et al. 1998). In contrast, mice exposed to a single dose of $40 \mathrm{mg}$ As $/ \mathrm{kg}$ as MMA excreted $89.6 \%$ of the dose as MMA, $6.2 \%$ as DMA, and $1.9 \%$ as TMAO (Hughes et al. 2005). Similarly, 24 hours after administration of a 
single oral dose of $50 \mathrm{mg} \mathrm{As} / \mathrm{kg}$ as MMA in hamsters, $26.9 \%$ was excreted in urine as MMA, $1.43 \%$ as DMA, and $0.07 \%$ as trimethylarsenic compound (Yamauchi et al. 1988). As with MMA, oral exposure of mice and hamsters to DMA results in most of the dose being excreted in the urine in the form of DMA (or DMA complex) (Marafante et al. 1987b); in rats, the levels of DMA and TMAO are about equal (Yoshida et al. 1998).

The available data suggest that the methylarsenates are not demethylated to inorganic arsenic either in humans (Buchet et al. 1981a; Marafante et al. 1987b) or in animals (rats and hamsters) (Stevens et al. 1977; Yamauchi and Yamamura 1984; Yoshida et al. 2001).

\subsubsection{Elimination and Excretion}

\subsubsection{Inhalation Exposure}

Inorganic Arsenicals. As noted previously (see Section 3.4.1.1), urinary excretion of arsenic appears to account for 30-60\% of the inhaled dose (Holland et al. 1959; Pinto et al. 1976; Vahter et al. 1986). Since the deposition fraction usually ranges from about 30 to $60 \%$ for most respirable particles (EPA 1989b), this suggests that nearly all arsenic that is deposited in the lung is excreted in the urine. The time course of excretion in humans exposed by inhalation has not been thoroughly investigated, but urinary arsenic levels in workers in a smelter rose within hours after they came to work on Monday and then fell over the weekend (Vahter et al. 1986). This implies that excretion is fairly rapid, and this is supported by intratracheal studies in rats (Rhoads and Sanders 1985) and hamsters (Marafante and Vahter 1987), where whole-body clearance of administered arsenate or arsenite occurred with a half-time of 1 day or less. However, the study in rats (Rhoads and Sanders 1985) found that the clearance of arsenic trioxide was biphasic, with 95\% cleared with a half-time of 29 minutes and the remaining arsenic cleared with a halftime of 75 days. For sodium arsenate and sodium arsenite, $<0.1 \%$ of the dose was retained in the lung 3 days after exposure of hamsters; $1.3 \%$ of the arsenic trisulfide dose was retained after 3 days (Marafante and Vahter 1987). The Marafante and Vahter (1987) study suggested that lung clearance was influenced by compound solubility. The primary forms of arsenic found in the urine of inhalation-exposed humans are DMA and MMA, with inorganic arsenic comprising $<25 \%$ of the total urinary arsenic (Apostoli et al. 1999).

Organic Arsenicals. No studies were located regarding the excretion of organic arsenicals by humans or animals after inhalation exposure. However, rats that were given a single intratracheal dose of DMA 
excreted about $60 \%$ in the urine and about $8 \%$ in the feces within 24 hours (Stevens et al. 1977). This indicates that organic arsenicals are likely to be promptly excreted after inhalation exposure.

\subsubsection{Oral Exposure}

Inorganic Arsenicals. Direct measurements of arsenic excretion in humans who ingested known amounts of arsenite or arsenate indicate that very little is excreted in the feces (Bettley and O'Shea 1975), and that $45-85 \%$ is excreted in urine within 1-3 days (Apostoli et al. 1999; Buchet et al. 1981a; Crecelius 1977; Mappes 1977; Tam et al. 1979b). At low exposure levels, urinary arsenic levels generally increase linearly with increasing arsenic intake (Calderon et al. 1999). During lactation, a very small percent of ingested arsenic may also be excreted in the breast milk (Concha et al. 1998a). A similar pattern of urinary and fecal excretion is observed in hamsters (Marafante and Vahter 1987; Yamauchi and Yamamura 1985) and mice (Vahter and Norin 1980); this pattern is typically modeled as a biphasic process (e.g., Hughes et al. 2003). Generally, whole body clearance is fairly rapid, with half-times of 40 60 hours in humans (Buchet et al. 1981b; Mappes 1977). Clearance is even more rapid in mice and hamsters, with 90\% removed in 2 days (Hughes et al. 2003; Marafante and Vahter 1987; Vahter 1981; Vahter and Norin 1980).

A study in pregnant women exposed to elevated levels of inorganic arsenic in drinking water found that most of the ingested arsenic was excreted in the urine as DMA (79-85\%), with smaller amounts excreted as inorganic arsenic (8-16\%) or MMA (5-6\%) (Christian et al. 2006). Similarly, in mice, arsenate is primarily excreted in the urine as DMA, with lesser amounts of inorganic arsenic and MMA (Kenyon et al. 2005). Following a single oral dose of $10 \mu \mathrm{mol} / \mathrm{kg}$ sodium arsenate, $78.4 \%$ was excreted as DMA, $20.2 \%$ as inorganic arsenic, and $1.45 \%$ as MMA; at a 10 -fold higher dose, the ratio of DMA to inorganic arsenic decreased (57.7\% DMA, 39.8\% inorganic arsenic, and 2.59\% MMA).

Arsenic is also excreted in the bile via the formation of two arsenic-glutathione complexes (arsenic triglutathione and methylarsenic diglutathione) (Kala et al. 2000). In rats administered $5.0 \mathrm{mg} / \mathrm{kg}$ sodium arsenite, equal amounts of arsenic triglutathione and methylarsenic diglutathione were found in the bile 18-20 minutes after exposure. At a lower arsenic dose $(0.5 \mathrm{mg} / \mathrm{kg})$, only methylarsenic diglutathione was found. As discussed in Section 3.4.4.4, biliary excretion of arsenic has also been detected in mice, hamsters, guinea pigs, and rabbits following parenteral exposure (Csanaky and Gregus 2002). 
Organic Arsenicals. Studies in humans indicate that ingested MMA and DMA are excreted mainly in the urine (75-85\%), and this occurs mostly within 1 day (Buchet et al. 1981a; Marafante et al. 1987b). This is supported by studies in rats, mice, and hamsters, although in animals, excretion is more evenly distributed between urine and feces (Hughes et al. 2005; Marafante et al. 1987b; Stevens et al. 1977; Yamauchi and Yamamura 1984; Yamauchi et al. 1988). In mice administered $40 \mathrm{mg}$ As $/ \mathrm{kg}$ as DMA, $56.4 \%$ was excreted in the urine as DMA, 7.7\% as a DMA complex, and 3.5\% as TMAO during a 48-hour period after dosing; in the feces, $24.3 \%$ was DMA and 4.9\% as DMA complex (Marafante et al. 1987b). In hamsters, $38.7 \%$ was DMA, $11.2 \%$ as DMA complex, and 6.4\% as TMAO in the urine; in the feces, $37.3 \%$ as DMA and $4.9 \%$ as DMA complex. As with DMA, most MMA is excreted in the urine and feces as parent compound. In the urine of mice administered $0.4 \mathrm{mg}$ As $/ \mathrm{kg}$ as MMA, $98.2 \%$ of the urinary arsenicals was in the form of MMA(V) and 1.8\% as MMA(III) (Hughes et al. 2005); at a 10-fold higher dose, $89.6 \%$ was excreted as MMA(V), 1.2\% as MMA(III), 6.2\% as DMA(V), 1.1\% as DMA(III), and $1.9 \%$ as TMAO. As discussed previously, exposure of rats to MMA or DMA results in the excretion of a higher percentage of metabolites. After 1 week exposure to MMA, 50.6\% of the dose was excreted as MMA, $19.0 \%$ as DMA, and 6.9\% of TMAO (Yoshida et al. 1998). A 1-week exposure to DMA, $44.9 \%$ was excreted as DMA in the urine and $40.0 \%$ as TMAO (Yoshida et al. 1998). A longer-term exposure to DMA ( $>7$ months) resulted in a higher percentage of the amount of parent compound excreted; 56-65\% as DMA and 23-35\% as TMAO ( $\mathrm{Li}$ et al. 1998; Wanibuchi et al. 1996; Yoshida et al. 1998).

In mice and hamsters, DMA and MMA are rapidly cleared from the body (Hughes et al. 2005; Marafante et al. 1987b; Vahter et al. 1984). In mice, $85 \%$ of the initial oral dose of DMA was eliminated from the body with a half-life of 2.5 hours (Vahter et al. 1984). In contrast to the mouse data, $45 \%$ on the initial DMA dose to rats was eliminated with a half-time of 13 hours and the remaining 55\% had an elimination half-time of 50 days (Vahter et al. 1984).

\subsubsection{Dermal Exposure}

Inorganic Arsenicals. No studies were located regarding excretion of inorganic arsenicals in humans or animals following dermal exposure. In rats, arsenic absorbed through the tail was excreted approximately equally in urine and feces, similar to the excretion pattern following oral exposure (Dutkiewicz 1977).

Organic Arsenicals. No studies were located regarding excretion of organic arsenicals in humans or animals following dermal exposure. 


\subsubsection{Other Routes of Exposure}

Inorganic Arsenicals. Excretion of arsenate and arsenite following parenteral exposure of animals is similar to that seen following oral exposure. In rabbits and mice, urinary excretion within 8 hours usually accounts for about 50-80\% of the dose (Maehashi and Murata 1986; Maiorino and Aposhian 1985; Vahter and Marafante 1983). Somewhat lower levels (30-40\%) are excreted in the urine of marmoset monkeys (Vahter and Marafante 1985; Vahter et al. 1982), probably because of the absence of methylation in this species. Whole-body clearance studies in mice indicate that arsenate is over $65 \%$ removed within 24 hours, while arsenite is about $86 \%$ removed at 24 hours (Lindgren et al. 1982). A relatively small proportion of an injected dose of arsenic $\mathrm{V}$ ( $10 \%$ for rats, $4 \%$ for mice, and $<2 \%$ for hamsters, guinea pigs, and rabbits) was found to be excreted into the bile within the first 2 hours postinjection (Csanaky and Gregus 2002). Following arsenic III injection, a much greater percentage ( $92 \%$ for guinea pigs and $75 \%$ for rats) of the arsenic was found in the bile in the first 2 hours after administration (Csanaky and Gregus 2002). Similarly, approximately $40 \%$ of an intravenous dose of sodium arsenite was excreted into the bile of rats, most of it occurring during the first hour after exposure (Kala et al. 2000). Kala et al. (2000) determined that the biliary transport of arsenic was dependent on the formation of arsenic-glutathione complexes, which were transported out of hepatocytes by multidrug resistance associated protein 2 (MRP2/cMOAT); most of the arsenic in bile was in the form of arsenic triglutathione or methylarsenic diglutathione.

\subsubsection{Physiologically Based Pharmacokinetic (PBPK)/Pharmacodynamic (PD) Models}

Physiologically based pharmacokinetic (PBPK) models use mathematical descriptions of the uptake and disposition of chemical substances to quantitatively describe the relationships among critical biological processes (Krishnan et al. 1994). PBPK models are also called biologically based tissue dosimetry models. PBPK models are increasingly used in risk assessments, primarily to predict the concentration of potentially toxic moieties of a chemical that will be delivered to any given target tissue following various combinations of route, dose level, and test species (Clewell and Andersen 1985). Physiologically based pharmacodynamic (PBPD) models use mathematical descriptions of the dose-response function to quantitatively describe the relationship between target tissue dose and toxic end points.

PBPK/PD models refine our understanding of complex quantitative dose behaviors by helping to delineate and characterize the relationships between: (1) the external/exposure concentration and target tissue dose of the toxic moiety, and (2) the target tissue dose and observed responses (Andersen and 
Krishnan 1994; Andersen et al. 1987). These models are biologically and mechanistically based and can be used to extrapolate the pharmacokinetic behavior of chemical substances from high to low dose, from route to route, between species, and between subpopulations within a species. The biological basis of PBPK models results in more meaningful extrapolations than those generated with the more conventional use of uncertainty factors.

The PBPK model for a chemical substance is developed in four interconnected steps: (1) model representation, (2) model parameterization, (3) model simulation, and (4) model validation (Krishnan and Andersen 1994). In the early 1990s, validated PBPK models were developed for a number of toxicologically important chemical substances, both volatile and nonvolatile (Krishnan and Andersen 1994; Leung 1993). PBPK models for a particular substance require estimates of the chemical substancespecific physicochemical parameters, and species-specific physiological and biological parameters. The numerical estimates of these model parameters are incorporated within a set of differential and algebraic equations that describe the pharmacokinetic processes. Solving these differential and algebraic equations provides the predictions of tissue dose. Computers then provide process simulations based on these solutions.

The structure and mathematical expressions used in PBPK models significantly simplify the true complexities of biological systems. If the uptake and disposition of the chemical substance(s) are adequately described, however, this simplification is desirable because data are often unavailable for many biological processes. A simplified scheme reduces the magnitude of cumulative uncertainty. The adequacy of the model is, therefore, of great importance, and model validation is essential to the use of PBPK models in risk assessment.

PBPK models improve the pharmacokinetic extrapolations used in risk assessments that identify the maximal (i.e., the safe) levels for human exposure to chemical substances (Andersen and Krishnan 1994). PBPK models provide a scientifically sound means to predict the target tissue dose of chemicals in humans who are exposed to environmental levels (for example, levels that might occur at hazardous waste sites) based on the results of studies where doses were higher or were administered in different species. Figure 3-9 shows a conceptualized representation of a PBPK model.

If PBPK models for arsenic exist, the overall results and individual models are discussed in this section in terms of their use in risk assessment, tissue dosimetry, and dose, route, and species extrapolations. 


\section{Figure 3-9. Conceptual Representation of a Physiologically Based Pharmacokinetic (PBPK) Model for a Hypothetical Chemical Substance}

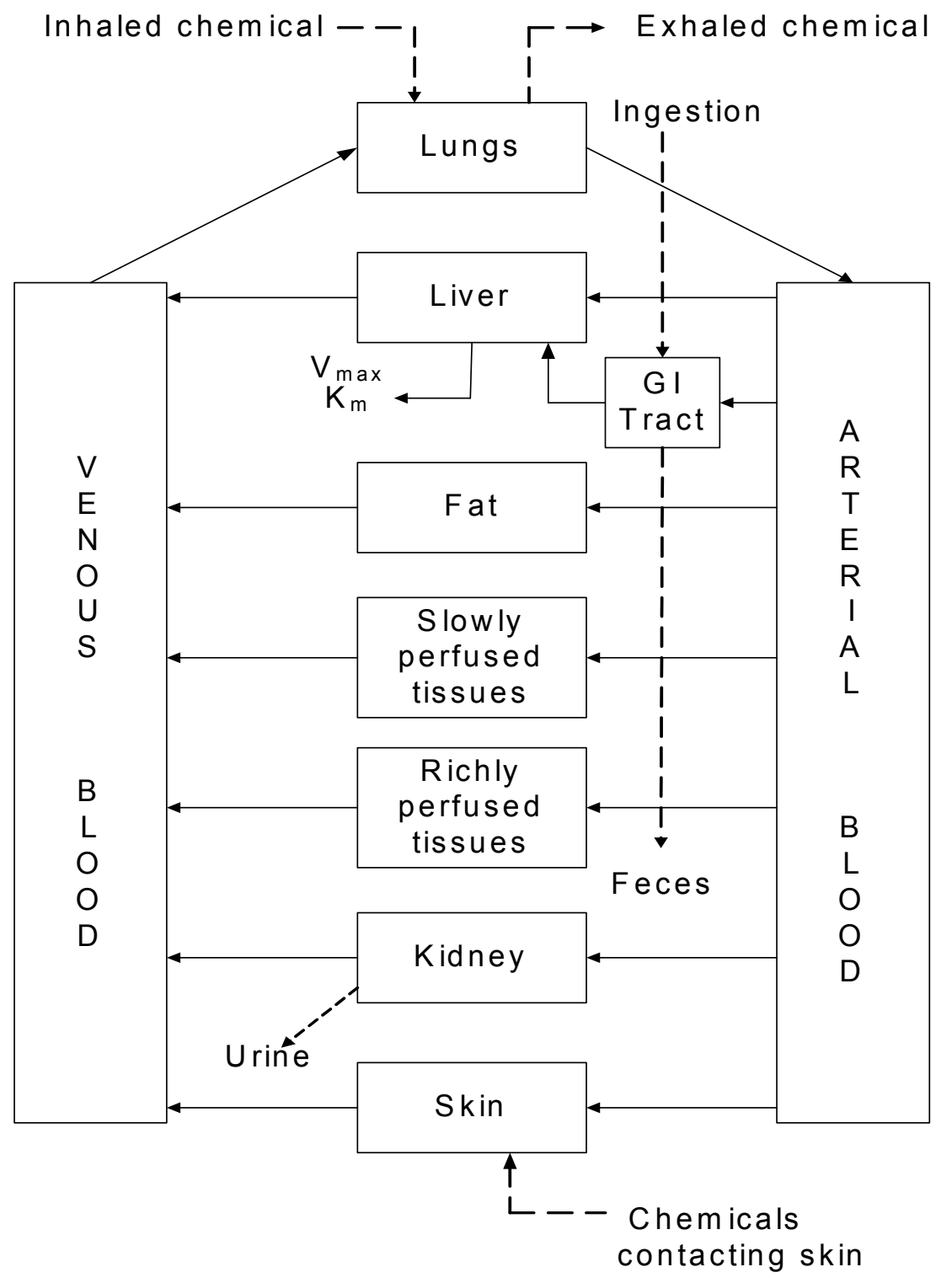

Note: This is a conceptual representation of a physiologically based pharmacokinetic (PBPK) model for a hypothetical chemical substance. The chemical substance is shown to be absorbed via the skin, by inhalation, or by ingestion, metabolized in the liver, and excreted in the urine or by exhalation.

Source: adapted from Krishnan and Andersen 1994 
Several PBPK models have been developed for inorganic arsenic; the Mann, Yu, and Menzel models are discussed below. A joint research effort between the Chemical Industry Institute of Toxicology (CIIT) Centers for Health Research, EPA, ENVIRON International, and the Electric Power Research Institute (EPRI) is underway to develop a biologically based dose response model of carcinogenicity. Part of this effort involves refining the existing PBPK models (Clewell et al. 2007).

\subsubsection{Summary of PBPK Models}

The Mann model (Gentry et al. 2004; Mann et al. 1996a, 1996b), Yu model (Yu 1998a, 1998b; Yu 1999a, 1999b), and Menzel model (Menzel et al. 1994) are the PBPK models for arsenic currently available. The Mann model simulates the absorption, distribution, metabolism, elimination, and excretion of $\mathrm{As}(+3)$, As(+5), MMA, and DMA after oral and inhalation exposure in mice, hamsters, rabbits, and humans. The Yu model simulates the absorption, distribution, metabolism, elimination, and excretion of $\mathrm{As}(+3)$, As(+5), MMA, and DMA after oral exposure to inorganic arsenic in mice, rats, or humans. The Menzel model is a preliminary model that predicts internal organ burden of arsenic during specific oral exposures, simulating the metabolism, distribution to organs and binding to organs in mice, rats, and humans.

\subsubsection{Arsenic PBPK Model Comparison}

The Mann model is a well-derived model, consisting of multiple compartments and metabolic processes, and modeling four chemical forms of arsenic (two organic and two inorganic), which has been validated using experimental data. The Yu model has more compartments than the Mann model, also models metabolism and fate of four forms of arsenic, and has likewise been validated using experimental data. The Menzel model is still preliminary and has not been validated.

\subsubsection{Discussion of Models}

\section{The Mann Model}

Risk assessment. The Mann model was not used for risk assessment.

Description of the model. The Mann model was initially developed to simulate oral, intratracheal, and intravenous exposure to arsenic in rabbits and hamsters (Mann et al. 1996a). In a companion paper, the model was expanded to include inhalation exposure and extrapolated and applied to humans (Mann et al. 1996b). A subsequent paper further expanded the model to include mice (Gentry et al. 2004). 
The model consists of six tissue compartments: blood, liver, kidneys, lungs, skin, and other tissues. The blood compartment is divided into plasma and red blood cell subcompartments, considered to be at equilibrium. Three routes of exposure are considered in the model. Oral exposure is considered to enter the liver from the gastrointestinal tract via first-order kinetics. Intratracheal exposure results in deposition into the pulmonary and tracheo-bronchial regions of the respiratory tract. Uptake into blood from the pulmonary region is considered to be via first order kinetics into plasma, uptake from the tracheobronchial region is by both transfer into plasma and transport into the gastrointestinal tract. Intravenous injection results in a single bolus dose into the plasma compartment.

Metabolism in the model consists of oxidation/reduction and two methylation reactions. The oxidation/reduction of inorganic arsenic was modeled as a first order process in the plasma, with reduction also included in the kidneys. Methylation of As(+3) was modeled as a two-step process occurring in the liver according to Michaelis-Menton kinetics.

Most physiological parameters were derived by scaling to body weight. In cases where parameters were not available (absorption rates, tissue affinity, biotransformation), estimates were obtained by fitting. This was done by duplicating the initial conditions of published experiments in the model, varying the unknown parameters and comparing the results of the simulation to the reported results. Tissue affinity constants were estimated using reported arsenic levels in tissues at various times after exposure. Metabolic rate constants and absorption rate constants were estimated using data for excretion of arsenic metabolites in urine and feces. Figure 3-10 shows the animal model and Tables 3-11, 3-12, 3-13, and 3-14 provide the parameters used in the animal model. The human model is similar to the animal models with adjustments for body weight and absorption and metabolic rates. A naso-pharynx compartment is included in the human model, which was not present in the animal models. Penetration and deposition in the respiratory tract are based on the log-normal particle size distribution of the aerosol. Metabolic and absorption rate constants were fitted using experimental data on urinary excretion of arsenic following a single oral dose of As(+3) (Buchet et al. 1981a) or As(+5) (Tam et al. 1979b) in volunteers. The lung absorption rate constant was obtained by fitting the total urinary excretion of arsenic as predicted with the model to experimental data obtained from occupational exposure to arsenic trioxide (Offergelt et al. 1992). Figure 3-11 shows the human model, and Tables 3-15 and 3-16 provide the data and constants used in the human model. 
Figure 3-10. Parameters Used in the Mann PBPK Model for Animals

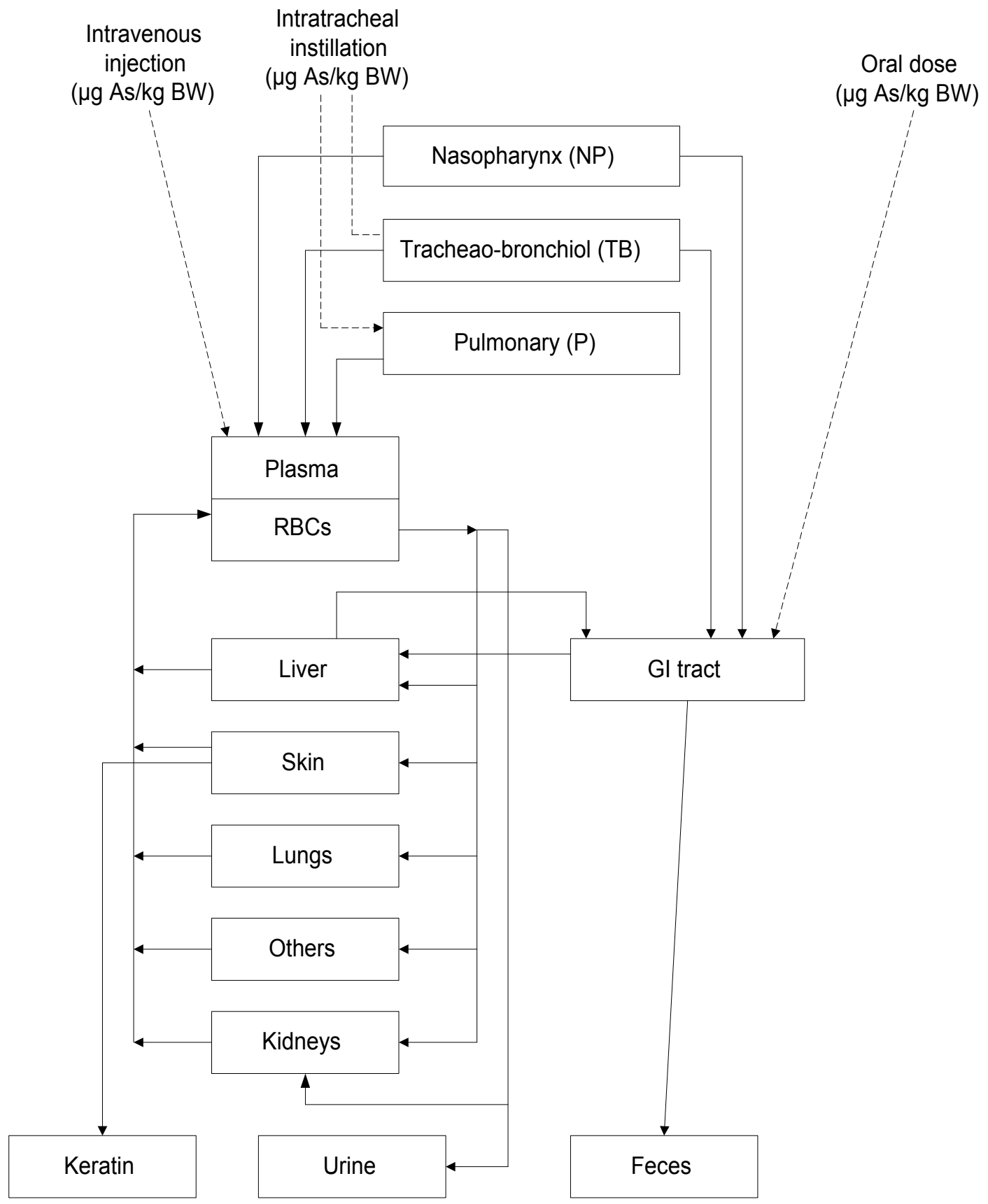

Source: Mann et al. 1996b 


\section{Table 3-11. Parameters Used in the Mann PBPK Model for Animals}

\begin{tabular}{|c|c|c|}
\hline Physiological parameter & Rabbit (body weight=3.5 kg) & Hamster (body weight $=0.100 \mathrm{~kg}$ ) \\
\hline Blood volume $(\mathrm{mL})$ & 253 & 7.0 \\
\hline \multicolumn{3}{|l|}{ Organ weight $(\mathrm{g})$} \\
\hline Liver & 121 & 4.8 \\
\hline Kidneys & 25 & 1.2 \\
\hline Lungs & 31 & 1.0 \\
\hline Skin & 420 & 17.1 \\
\hline \multicolumn{3}{|l|}{ Organ volume $(\mathrm{mL})$} \\
\hline Others & 2,386 & 62.0 \\
\hline \multicolumn{3}{|l|}{ Lumen volume $(\mathrm{mL})$} \\
\hline Stomach & 15 & 0.5 \\
\hline Small intestine & 20 & 0.6 \\
\hline \multicolumn{3}{|l|}{ Blood flow (mL/minute) } \\
\hline Cardiac output & 556 & 38.3 \\
\hline Liver, hepatic & 25 & 1.2 \\
\hline Liver, splanchic & 98 & 6.0 \\
\hline Kidneys & 100 & 7.0 \\
\hline Lungs & 13 & 0.7 \\
\hline Skin & 38 & 2.6 \\
\hline Others & 282 & 20.8 \\
\hline \multicolumn{3}{|l|}{ Clearance (mL/minute) } \\
\hline Glomerular Filtration Rate & 10 & 0.6 \\
\hline Small intestine length $(\mathrm{cm})$ & 180 & 56.0 \\
\hline Total capillary surface area $\left(\mathrm{cm}^{2}\right)$ & 93,835 & $2,681.0$ \\
\hline
\end{tabular}

PBPK = physiologically based pharmacokinetic

Source: Mann et al. 1996a 
Table 3-12. Tissue Affinity Constants $\left(\mathrm{K}_{\mathrm{ij}}\right)$ Obtained for the Mann PBPK Model for Animals by Fitting for Rabbits and Hamsters

\begin{tabular}{lrrrr}
\hline & \multicolumn{3}{c}{$K_{i j}$ (unitless) } \\
\cline { 2 - 5 } Tissue $(i)$ & As $(\mathrm{V})$ & As $($ III $)$ & \multicolumn{1}{c}{ MMA } & DMA \\
\hline Liver & 1 & 200 & 10 & 1 \\
Kidneys & 40 & 20 & 100 & 5 \\
Lungs & 1 & 1 & 1 & 20 \\
Skin & 1 & 60 & 50 & 1 \\
Others & 10 & 40 & 1 & 1 \\
\hline
\end{tabular}

DMA = dimethylarsinic acid; $\mathrm{MMA}=$ monomethylarsonic acid; $\mathrm{PBPK}=$ physiologically based pharmacokinetic

Source: Mann et al. 1996a 
Table 3-13. Metabolic Rate Constants for the Mann PBPK Model for Animals Obtained by Fitting for Rabbits and Hamsters

\begin{tabular}{llrr}
\hline Oxidation/reduction & First order & \multicolumn{1}{c}{ Rabbit } & \multicolumn{1}{c}{ Hamster } \\
\hline Reduction & $(1 /$ hour $)$ & $3,000.00$ & 100.00 \\
Oxidation & $(1 /$ hour $)$ & $6,000.00$ & 400.00 \\
Kidney reduction & $(1 /$ hour $)$ & 30.00 & 1.00 \\
Methylation & Michaelis-Menten & & \\
1st step & $K_{\text {MMMA }}(\mu \mathrm{mol} / \mathrm{mL})$ & 0.05 & 0.12 \\
& $V_{\text {MAX }}^{\text {MMA }}(\mu \mathrm{mol} / \mathrm{mL}$-hour $)$ & 4.00 & 0.12 \\
2nd step & $K_{\text {MMA }}(\mu \mathrm{mol} / \mathrm{mL})$ & 0.90 & 0.08 \\
& $V_{\text {MAX }}(\mu \mathrm{mol} / \mathrm{mL}$-hour $)$ & 1.50 & 0.12 \\
\hline
\end{tabular}

$\mathrm{DMA}=$ dimethylarsinic acid; $\mathrm{MMA}=$ monomethylarsonic acid; PBPK = physiologically based pharmacokinetic

Source: Mann et al. 1996a 
Table 3-14. Fitted Gastrointestinal Tract and Lung Absorption Half-time for the Hamster for the Mann PBPK Model

\begin{tabular}{lcc}
\hline & \multicolumn{2}{c}{ Absorption, half-time (hour) } \\
\cline { 2 - 3 } Arsenic compound & Gastrointestinal tract & Lung \\
\hline $\mathrm{As}(\mathrm{V})$ & 0.08 & 12 \\
$\mathrm{Na}_{3}\left(\mathrm{AsO}_{4}\right)$ & 0.39 & 690 \\
$\mathrm{~Pb}_{3}\left(\mathrm{AsO}_{4}\right)$ & 0.28 & - \\
$\mathrm{As}_{2} \mathrm{O}_{5}$ & & 12 \\
$\mathrm{As}(\mathrm{III})$ & 0.08 & 12 \\
$\mathrm{NaAsO}_{2}$ & 0.48 & - \\
$\mathrm{As}_{2} \mathrm{~S}_{3}$ & 0.02 & - \\
$\mathrm{As}_{2} \mathrm{O}_{3}$ & 0.09 & \\
$\mathrm{DMA}$ & &
\end{tabular}

DMA = dimethylarsinic acid; PBPK = physiologically based pharmacokinetic

Source: Mann et al. 1996a 
Figure 3-11. Parameters Used in the Mann PBPK Model for Humans

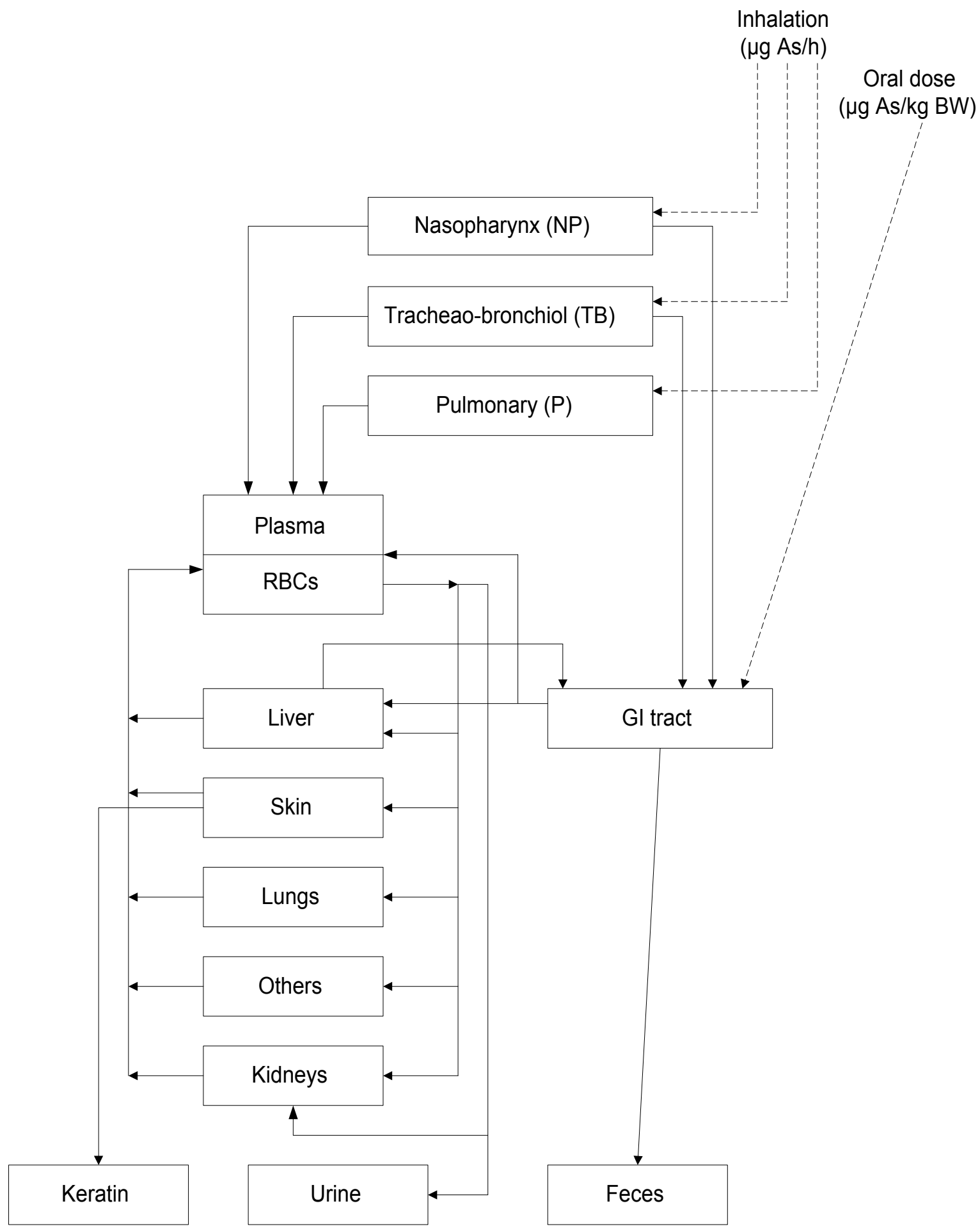


Table 3-15. Physiological Data Used in the Mann PBPK Model for Humans

\begin{tabular}{|c|c|c|c|}
\hline Physiological parameter & Organ & Units & $\begin{array}{l}\text { Human } \\
\text { (body weight=70 kg) }\end{array}$ \\
\hline Blood volume & & $\mathrm{mL}$ & 5,222 \\
\hline \multirow[t]{5}{*}{ Organ weight } & Liver & $g$ & 1,856 \\
\hline & Kidneys & $g$ & 314 \\
\hline & Lungs & $g$ & 584 \\
\hline & Skin & $g$ & 6,225 \\
\hline & Others & $g$ & 55,277 \\
\hline \multirow[t]{2}{*}{ Lumen volume } & Stomach & $\mathrm{mL}$ & 274 \\
\hline & Small intestine & $\mathrm{mL}$ & 393 \\
\hline \multirow[t]{7}{*}{ Blood flow } & Cardiac output & L/minute & 5.29 \\
\hline & Liver, hepatic & L/minute & 0.32 \\
\hline & Liver, splanchic & L/minute & 1.02 \\
\hline & Kidneys & L/minute & 0.95 \\
\hline & Lungs & $\mathrm{L} /$ minute & 0.16 \\
\hline & Skin & $\mathrm{L} /$ minute & 0.35 \\
\hline & Others & $\mathrm{L} /$ minute & 2.49 \\
\hline \multicolumn{4}{|l|}{ Creatinine } \\
\hline Male & & g/day & 1.7 \\
\hline Female & & g/day & 1.0 \\
\hline \multicolumn{4}{|l|}{ Clearance } \\
\hline Glomerular filtration rate & & $\mathrm{mL} /$ minute & 156 \\
\hline Small intestine length & & $\mathrm{cm}$ & 481 \\
\hline Nasopharynx area & & $\mathrm{cm}^{2}$ & 177 \\
\hline Tracheobronchial area & & $\mathrm{cm}^{2}$ & 5,036 \\
\hline Pulmonary area & & $\mathrm{cm}^{2}$ & 712,471 \\
\hline Total capillary surface area & & $\mathrm{cm}^{2}$ & $1,877 \times 10^{6}$ \\
\hline
\end{tabular}

$\mathrm{DMA}=$ dimethylarsinic acid; $\mathrm{MMA}=$ monomethylarsonic acid; $\mathrm{PBPK}=$ physiologically based pharmacokinetic Source: Mann et al. 1996b 
Table 3-16. Tissue Affinity Constants $\left(K_{i j}\right)$ Obtained by Fitting the Mann PBPK Animal Model for Use with Humans

\begin{tabular}{lrrrc}
\hline & \multicolumn{4}{c}{$\mathrm{K}_{\mathrm{ij}}$ (unitless) } \\
\cline { 2 - 5 } Tissue $(\mathrm{i})$ & $\mathrm{As}(\mathrm{V})$ & $\mathrm{As}(\mathrm{III})$ & $\mathrm{MMA}$ & $\mathrm{DMA}$ \\
\hline Liver & 1 & 200 & 10 & 1 \\
Kidneys & 40 & 20 & 100 & 5 \\
Lungs & 1 & 1 & 1 & 20 \\
Skin & 1 & 60 & 50 & 1 \\
Red blood cells & 0.2 & 1.5 & 0.2 & 0.2 \\
Others & 10 & 40 & 1 & 1 \\
\hline
\end{tabular}

$\mathrm{DMA}=$ dimethylarsinic acid; $\mathrm{MMA}=$ monomethylarsonic acid; PBPK = physiologically based pharmacokinetic Source: Mann et al. 1996b 
Validation of the model. The model was generally successful in describing the disposition of an intravenous dose of sodium arsenate in rabbits over a 24-hour period (Marafante et al. 1985).

Discrepancies included a 6-7-fold overestimation of levels in skin at 24 hours and underestimation of As $(+5)$ in plasma in the hour following injection. A statistical assessment of how well the model fit the empirical data was not presented. In hamsters, the model was also generally predictive of oral and intratracheal exposures (Marafante and Vahter 1987). Generally, predictions were better for the exposures to $\mathrm{As}(+5)$ than for those to $\mathrm{As}(+3)$.

The human model was validated using data from studies of repeated oral intake of sodium arsenite in volunteers (Buchet et al. 1981b), occupational exposure to arsenic trioxide and elemental arsenic (Vahter et al. 1986), and community exposure to $\mathrm{As}(+5)$ via drinking water (Harrington et al. 1978; Valentine et al. 1979). Simulations were generally in good agreement with the experimental data.

The predictions of tissue distribution, metabolism, and elimination of arsenic compounds from the mouse model were compared with experimental data, and showed generally good agreement. The model tended to overpredict the concentration of organic arsenicals in the lungs, and to a lesser extent in the kidneys and liver, while for inorganic arsenic, the model overpredicted the levels of arsenic (V) present in the urine of acutely-exposed mice.

Target tissues. Levels in skin were not well predicted by this model in animals. Results for the lung were not presented, except for the mouse model, which tended to overpredict lung levels. The human model was only used to predict urinary metabolites.

Species extrapolation. Species extrapolation was not attempted in this model. However, tissue affinities derived for the rabbit and hamster models were used in the human model.

Interroute extrapolation. Interroute extrapolation was not attempted in this model.

\section{The Menzel Model}

Risk assessment. The Menzel model was not used for risk assessment. 
Description of the model. The Menzel model was developed to simulate oral exposure to arsenic from drinking water and food. Inhalation of arsenic in the particulate phase or as arsine gas is not considered. The chemical species in drinking water is assumed to be As $(+5)$.

The model consists of two sets of compartments: those in which the pools of arsenic are not influenced by blood perfusion, and those in which blood perfusion does determine arsenic burden. The former set of compartments includes the gut, feces, hair, bladder, and urine. The latter set of compartments included lung, liver, fat, skin, kidney, and other tissues. Oral exposure is considered to enter the liver from the gastrointestinal tract.

The model followed that of Andersen and coworkers (Andersen et al. 1987; Ramsey and Andersen 1984). Data from mice were used to test predictions of absorption. Excretion is considered to be rapid and complete into the urine, with no reabsorption from the kidney. Fecal arsenic content accounts for unabsorbed arsenic excreted in the bile, and complex arsenic species from food. Metabolism includes reduction by glutathione and methylation. Arsenic accumulation in the skin, hair and nails was included by assuming that arsenic binds irreversibly to protein sulfide groups in hair and nails.

Validation of the model. The model was preliminary and has not been validated.

Target tissues. Target tissues have not yet been modeled.

Species extrapolation. Species extrapolation was not attempted in this model.

Interroute extrapolation. Interroute extrapolation was not attempted in this model.

\section{The Yu Model}

Risk assessment. The Yu model was not used for risk assessment.

Description of the model. The Yu model was developed to simulate oral exposure to arsenic in mice and rats (Yu 1998a, 1998b), and was later adapted for oral exposures in humans (Yu 1999a, 1999b). Inhalation of arsenic in the particulate phase or as arsine gas is not considered. $\mathrm{As}(+3), \mathrm{As}(+5)$, MMA, and DMA were all considered in the model, though the movements of MMA and DMA were not considered. 
The model consists of eight tissue compartments: intestine, skin, muscle, fat, kidney, liver, lung, and vessel-rich group (VRG, e.g., brain); in the human model, the VRG and kidney compartments were combined. Only oral exposure was considered. Absorption is based on absorption to the stomach, which then passes the arsenic to the gastrointestinal tract. From the gastrointestinal tract, arsenic is either transferred to the blood or excreted in the feces.

The physiological parameters for the model were obtained from published values in the literature. Tissue/blood partition coefficients were based on the postmortem blood and tissue concentrations from a fatal human poisoning case study (Saady et al. 1989). Tissue volumes and blood flow rates were based on published values from a number of sources (EPA 1988e; Reitz et al. 1990). Absorption and excretion rate constants were based on experimental observations of blood concentrations and urinary and fecal excretion following oral administration of inorganic arsenic (Odanaka et al. 1980; Pomroy et al. 1980). Metabolic rate constants for the methylation and dimethylation of inorganic arsenic were also based on experimental observations (Buchet et al. 1981a; Crecelius 1977). Figure 3-12 shows the model and Table 3-17 provides the parameters used for each species.

Validation of the model. The model was generally successful at predicting the urinary excretion 48 hours after administration of $5 \mathrm{mg} / \mathrm{kg}$ inorganic arsenic in both rats and mice. After 48 hours, the observed/predicted ratios associated with excreted doses ranged from 0.78 to 1.11 for the mouse and from 0.85 to 0.93 for the rat. However, the model overpredicted the amount of inorganic arsenic found in the feces of mice at 24 and 48 hours, and overpredicted the amount of DMA formed by exposed mice at 48 hours. In rats, the model overestimated the urinary and fecal excretion of inorganic arsenic at 24 hours postexposure, though at 48 hours, measured values all fell within the predicted ranges. The human model was also generally successful at predicting the urinary excretion of arsenic compounds following oral exposure, based on results of controlled human exposure studies (Buchet et al. 1981a; Vahter 1983). In general, however, the model underpredicts excretion at early time points and overpredicts at later time points, with 24 hours being the time at which its predictive capabilities agreed most strongly with available data.

The ability of the model to predict tissue burdens was not compared to actual data for any species. 
Figure 3-12. Parameters Used in the Yu PBPK Model for Animals

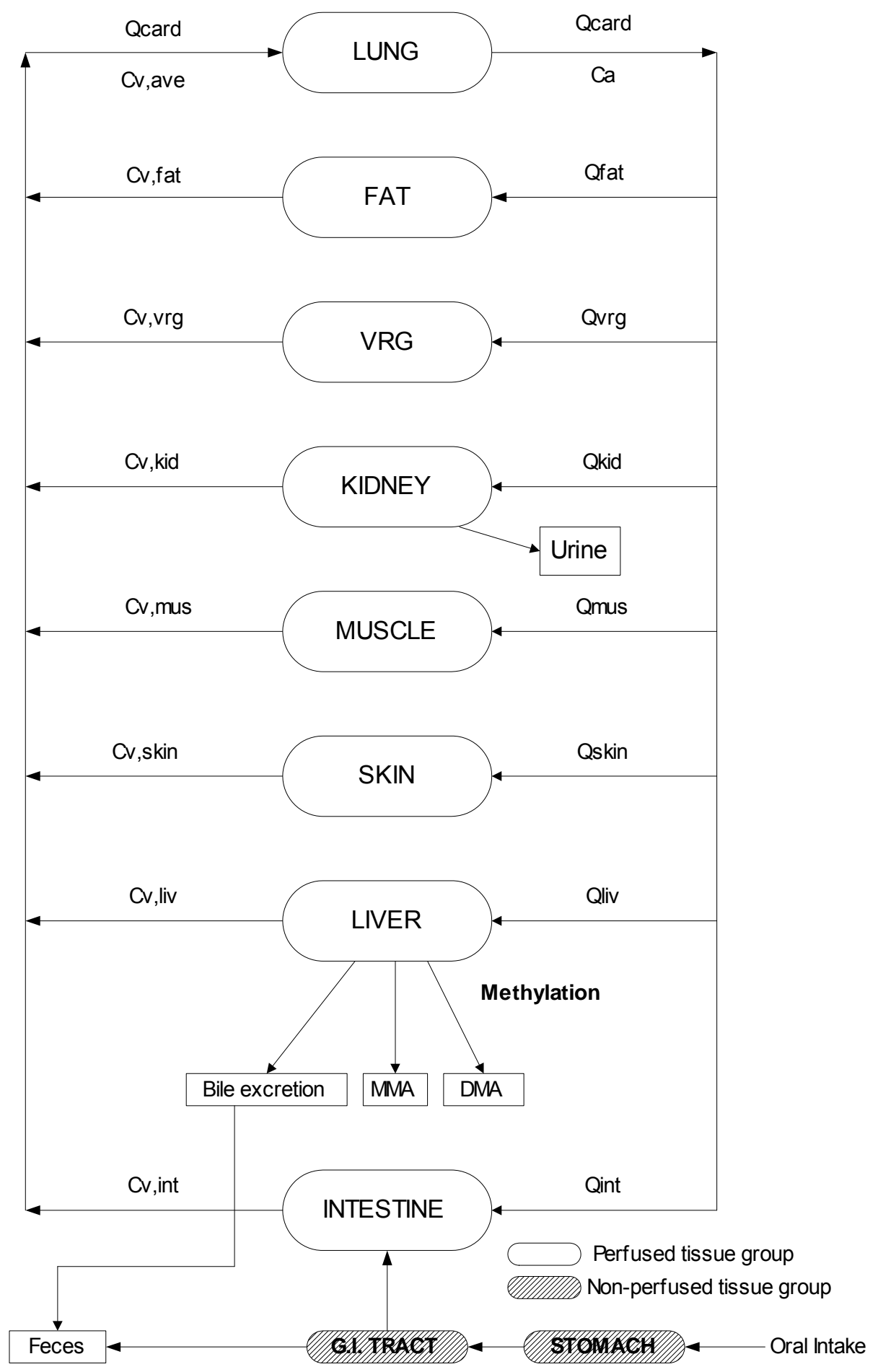


Table 3-17. Parameters Used in the Yu PBPK Model

\begin{tabular}{|c|c|c|c|}
\hline & Mouse & Rat & Human \\
\hline Partition coefficients & & & (As $\left.{ }^{I I I} / \mathrm{As}^{\mathrm{V}} / \mathrm{MMA} / \mathrm{DMA}\right)$ \\
\hline Intestine & 6.0 & 6.0 & $2.8 / 2.8 / 1.2 / 1.4$ \\
\hline Skin & 5.0 & 5.0 & $2.5 / 2.5 / 1.25 / 1.25$ \\
\hline VRG & 6.0 & 6.0 & Combined with kidney \\
\hline Muscle & 5.0 & 10.0 & $2.6 / 2.6 / 1.8 / 2.8$ \\
\hline Fat & - & 0.5 & $0.3 / 0.3 / 0.3 / 0.3$ \\
\hline Kidney & 8.5 & 7.5 & $4.15 / 4.15 / 1.8 / 2.075$ \\
\hline Liver & 10.0 & 10.0 & $5.5 / 5.3 / 2.35 / 2.65$ \\
\hline Lung & 4.0 & 4.0 & $4.15 / 4.15 / 1.8 / 2.075$ \\
\hline \multicolumn{4}{|l|}{ Blood flow rate (mL/hour) } \\
\hline Intestine & 100 & 528 & 1,810 \\
\hline Skin & 7.68 & 37.8 & 130 \\
\hline VRG & 157 & 960 & $\mathrm{~N} / \mathrm{A}$ \\
\hline Muscle & 153 & 1,260 & 25,850 \\
\hline Fat & - & 253.2 & 6,467 \\
\hline Kidney & 255 & 255 & 45,240 \\
\hline Liver & 255 & 1,260 & 32,320 \\
\hline Lung & N/R & N/R & 129,000 \\
\hline \multicolumn{4}{|l|}{ Tissue volume $(\mathrm{mL})$} \\
\hline Intestine & 1.94 & 6.9 & 558 \\
\hline Skin & 1.83 & 15.4 & 606 \\
\hline VRG & 0.81 & 23.0 & $\mathrm{~N} / \mathrm{A}$ \\
\hline Muscle & 19.9 & 162 & 6,989 \\
\hline Fat & - & 14.5 & 2,328 \\
\hline Kidney & 0.484 & 1.63 & 248 \\
\hline Liver & 1.67 & 5.82 & 422 \\
\hline Lung & 0.124 & 1.0 & 400 \\
\hline \multicolumn{4}{|l|}{ Metabolism constants } \\
\hline $\operatorname{Vmax}_{(\mathrm{MMA})}(\mu \mathrm{mol} / \mathrm{hour})$ & 0.45 & 0.15 & 11.25 \\
\hline $\operatorname{Vmax}_{(\mathrm{DMA})}(\mu \mathrm{mol} /$ hour $)$ & 0.375 & 0.06 & 22.25 \\
\hline $\mathrm{Km}_{(\mathrm{MMA})}(\mu \mathrm{mol} /$ hour $)$ & 1.0 & 0.2 & 0.01 \\
\hline $\mathrm{Km}_{(\mathrm{DMA})}(\mu \mathrm{mol} /$ hour $)$ & 0.2 & 0.2 & 0.01 \\
\hline
\end{tabular}


3. HEALTH EFFECTS

Table 3-17. Parameters Used in the Yu PBPK Model

\begin{tabular}{lccl}
\hline & Mouse & Rat & Human \\
\hline First-order rate constants & & & $($ As \\
$\mathrm{K}_{\mathrm{SI}}\left(\right.$ hour $\left.^{-1}\right)$ & 0.3 & 0.3 & $-/ 1.2 /-/-$ \\
$\mathrm{K}_{\mathrm{Al}}\left(\right.$ hour $\left.^{-1}\right)$ & 1.5 & 3.6 & $-/ 1.2 /-/-$ \\
$\mathrm{K}_{\text {fecal }}\left(\right.$ hour $\left.^{-1}\right)$ & 0.33 & 0.048 & $-/ 0.0012 / 0.0 / 0.0$ \\
$\mathrm{~K}_{\text {urinary }}\left(\right.$ hour $\left.^{-1}\right)$ & 1.32 & 0.9 & $0.05 / 0.075 / 0.07 / 0.04$ \\
$\mathrm{~K}_{\text {biliary }}\left(\right.$ hour $\left.^{-1}\right)$ & 0.33 & 0.3 & $-/ 0.018 /-/-$ \\
\hline
\end{tabular}

$\mathrm{DMA}=$ dimethylarsinic acid; $\mathrm{MMA}=$ monomethylarsonic acid; N/A = not applicable; N/R = not reported

Source: Yu 1998a, 1998b, 1999a, 1999b 
Target tissues. Model predictions of tissue burdens were not compared to actual data. The model accurately predicted, with a few exceptions, the urinary and fecal excretion of inorganic arsenic and its metabolites in rats, mice, and humans.

Species extrapolation. Species extrapolation beyond rats and mice was not attempted using this model. The human model has not been compared to, or linked with, either of the rodent models.

Interroute extrapolation. Interroute extrapolation was not attempted using this model.

\subsection{MECHANISMS OF ACTION}

\subsubsection{Pharmacokinetic Mechanisms}

Arsenic absorption depends on its chemical form. In humans, As(+3), As(+5), MMA, and DMA are orally absorbed $\geq 75 \%$. Arsenic is also easily absorbed via inhalation. Absorption appears to be by passive diffusion in humans and mice, although there is evidence (Gonzalez et al. 1995) for a saturable carrier-mediated cellular transport process for arsenate in rats (for review, see Rosen 2002). Dermal absorption appears to be much less than by the oral or inhalation routes. Bioavailability of arsenic from soil appears to be lower via the oral route than it is for sodium salts of arsenic. Arsenic in soil may form water insoluble compounds (e.g., sulfides), which are poorly absorbed.

Arsenic and its metabolites distribute to all organs in the body; preferential distribution has not been observed in human tissues at autopsy or in experiments with animal species other than rat (in which arsenic is concentrated in red blood cells). Since the liver is a major site for the methylation of inorganic arsenic, a "first-pass" effect is possible after gastrointestinal absorption; however, this has not been investigated in animal models.

Arsenic and its metabolites are largely excreted via the renal route. This excretion mechanism is not likely to be saturated within the dose range expected from human exposure. Excretion can also occur via feces after oral exposure; a minor excretion pathway is nails and hair. The methylation of inorganic arsenic is the major metabolism pathway. The proportion of metabolites recovered in urine $(\operatorname{As}(+3)$, As(+5), MMA, DMA) are roughly consistent in humans regardless of the exposure scenario. However, interindividual variation is great enough that it cannot be determined if capacity limitation may occur in some individuals. 
The manifestation of arsenic toxicity depends on dose and duration of exposure. Single oral doses in the range of $2 \mathrm{mg} \mathrm{As} / \mathrm{kg}$ and higher have caused death in humans. Doses as low as $0.05 \mathrm{mg} \mathrm{As} / \mathrm{kg} / \mathrm{day}$ over longer periods (weeks to months) have caused gastrointestinal, hematological, hepatic, dermal, and neurological effects. These effects appear to be a result of direct cytotoxicity. Long-term exposure (years) to drinking water at levels as low as $0.001 \mathrm{mg} \mathrm{As} / \mathrm{kg} /$ day have been associated with skin diseases and skin, bladder, kidney, and liver cancer. Long-term inhalation exposure to arsenic has also been associated with lung cancer at air levels as low as $0.05-0.07 \mathrm{mg} / \mathrm{m}^{3}$. It is not clear at this time why longterm toxicity is different between the oral and inhalation routes, given that arsenic is easily absorbed into the systemic circulation by both routes.

Studies in mice and rats have shown that arsenic compounds induce metallothionein, a metal-binding protein thought to detoxify cadmium and other heavy metals, in vivo (Albores et al. 1992; Hochadel and Waalkes 1997; Kreppel et al. 1993; Maitani et al. 1987a). The potency of arsenic compounds in inducing metallothionein parallels their toxicity (i.e., $\mathrm{As}(+3)>\mathrm{As}(+5)>\mathrm{MMA}>\mathrm{DMA})$. For cadmium, it is thought that metallothionein binds the metal, making it biologically inactive. For arsenic, however, only a small percentage of the administered arsenic is actually bound to metallothionein (Albores et al. 1992; Kreppel et al. 1994; Maitani et al. 1987a). In vitro studies have shown that affinity of arsenic for metallothionein is much lower than that of cadmium or zinc (Waalkes et al. 1984). It has been proposed that metallothionein might protect against arsenic toxicity by acting as an antioxidant against oxidative injury produced by arsenic (NRC 1999).

\subsubsection{Mechanisms of Toxicity}

Mechanisms of arsenic-induced toxicity and carcinogenicity have not been clearly identified. However, recent efforts to elucidate mechanisms of arsenic toxicity and carcinogenicity have resulted in numerous in vitro and in vivo reports. Whereas these mechanistic studies typically employed relatively high arsenic exposure levels, some of the most recent studies were performed using more environmentally-relevant exposure levels. Due to the extremely large amount of mechanistic data for arsenic, it is not feasible to include all pertinent primary studies that address issues concerning proposed mechanisms of arsenic toxicity and carcinogenicity. Therefore, the following discussion of mechanisms of arsenic toxicity represents a summary of information from several recent review articles (Chen et al. 2004, 2005; Florea et al. 2005; Hughes 2002; Kitchin 2001; Lantz and Hays 2006; Navas-Acien et al. 2005; Rossman 2003; Roy and Saha 2002; Thomas et al. 2007; Vahter 2002). 
It is becoming increasingly evident that the toxicity and carcinogenicity of arsenic is likely to be closely associated with metabolic processes. Absorbed pentavalent arsenic (AsV) is rapidly reduced to trivalent arsenic (AsIII) at least partially in the blood. Much of the formed AsIII is distributed to tissues and taken up by cells (particularly hepatocytes). Many cell types appear to accumulate AsIII more rapidly than $\mathrm{AsV}$. Because AsIII (as arsenite) is known to be more highly toxic than AsV (as arsenate), the reduction step may be considered bioactivation rather than detoxification. Glutathione appears to play a role in the reduction of $\mathrm{AsV}$ to AsIII, which is required prior to methylation. Methylation of arsenic ultimately forms relatively less toxic MMA and DMA; this process is accomplished by alternating between the reduction of $\mathrm{AsV}$ to AsIII and the addition of a methyl group; S-adenosylmethionine is considered to be the source of the methyl group. Both MMA and DMA are less reactive with tissue constituents than inorganic arsenic and both are readily excreted in the urine. The methylation process appears to include multiple intermediates, some of which are more reactive than inorganic arsenic. For example, reactive trivalent metabolites, MMAIII and DMAIII, have been detected in the urine of human subjects chronically exposed to arsenic in drinking water, and in vitro studies have demonstrated MMAIII to be more toxic than arsenite or arsenate to human hepatocytes, epidermal keratinocytes, and bronchial epithelial cells. Additional in vitro studies have demonstrated genotoxic and DNA damaging properties of both MMAIII and DMAIII.

AsV (as arsenate) has been demonstrated to: (1) replace phosphate in glucose-6-phosphate and 6-phosphogluconate in vitro, (2) replace phosphate in the sodium pump and the anion exchange transport system of human red blood cells, (3) diminish the in vitro formation of adenosine-t'-triphosphate (ATP) by replacing phosphate in enzymatic reactions, and (4) deplete ATP in some cellular systems, but not in human erythrocytes. However, it is becoming more apparent that the major source of arsenic toxicity and carcinogenicity is related to its reduction to arsenite.

AsIII (as arsenite) is known to react with thiol-containing molecules such as glutathione and cysteine in vitro. Methylated trivalent arsenics such as MMAIII are potent inhibitors of glutathione reductase and thioredoxin reductase. It has been suggested that binding of arsenite and methylated trivalent arsenicals to critical thiol groups could lead to the inhibition of essential biochemical reactions, alteration of cellular redox status, and eventual cytotoxicity. Binding of MMAIII and DMAIII to protein has also been demonstrated in vitro. Arsenite inhibits pyruvate dehydrogenase (PDH), a complex that oxidizes pyruvate to acetyl-CoA, a precursor to intermediates of the citric acid cycle that provides reducing equivalents to the electron transport system for ATP production. This property may explain the depletion of carbohydrates in arsenite-treated rats. 
Evidence that arsenic may induce alterations in nitric oxide metabolism and endothelial function includes findings that persons exposed to high levels of arsenic in drinking water had decreased serum and urine concentrations of nitric oxide metabolites, which was reversed upon intervention with drinking water containing lower levels of arsenic. Urinary arsenic levels have been inversely associated with nitric oxide production in activated monocytes. Arsenite concentrations of 1-25 $\mu \mathrm{M}$ inhibited endothelial nitric oxide synthase activity and resulting decreased cell growth in human endothelial cells, although lower concentrations up-regulated the expression of constitutive nitric oxide synthase 3 , which might serve as an explanation for observed arsenic-induced cell growth and angiogenesis.

Although epidemiological studies demonstrate the carcinogenicity of arsenic in humans, early animal cancer bioassays failed to demonstrate a carcinogenic effect following lifetime exposure to inorganic arsenic. However, more recent focus has resulted in the development of animal models that exhibit carcinogenic activity in skin, urinary bladder, liver, and lung, tissues implicated in arsenic-induced cancer in humans. This concordance in target sites among animal models and humans indicates that common mechanisms of action may be applicable to humans and laboratory animals.

Several modes of action have been proposed to explain, at least in part, the carcinogenicity of arsenic. It is likely that multiple mechanisms are involved, some of which may relate to noncancer effects as well.

Oxidative Stress. Mechanistic studies of arsenic toxicity have suggested a role of the generation of reactive oxygen species in the toxicity of inorganic arsenic. Results of both in vivo and in vitro studies of arsenic-exposed humans and animals suggest the possible involvement of increased lipid peroxidation, superoxide production, hydroxyl radical formation, blood nonprotein sulfhydrals, and/or oxidant-induced DNA damage. Reduction of cellular oxidant defense by treatment with glutathione-depleting agents results in an increased sensitivity of cells to arsenic toxicity. Support for mechanisms of toxicity that involves arsenic-induced oxidative stress includes findings that inhaled arsenic can predispose the lung to oxidative damage, chronic low-dose arsenic alters genes and proteins that are associated with oxidative stress and inflammation, and major transcriptional regulators of altered genes are redox sensitive.

Genotoxicity. Collectively, in vitro and in vivo genotoxicity assays have demonstrated that arsenics cause single strand breaks, formation of apurinic/apyrimidinic sites, DNA base and oxidative base damage, DNA-protein crosslinks, chromosomal aberrations, aneuploidy, sister chromatid exchanges, and micronuclei. Chromosomal aberrations, characterized by chromatid gaps, breaks and fragmentation, 
endoreduplication, and chromosomal breaks, are dose-dependent and arsenite is more potent than arsenate. Both MMAIII and DMAIII are directly genotoxic and are many times more potent than arsenite at inducing DNA damage. Inorganic arsenic can potentiate the mutagenicity observed with other chemicals, although arsenic itself does not appear to induce point mutations. Arsenic-induced genotoxicity may involve oxidants or free radical species.

Altered Growth Factors $\rightarrow$ Cell Proliferation $\rightarrow$ Promotion of Carcinogenesis. Increased concentrations of growth factors can lead to cell proliferation and eventual promotion of carcinogenesis. Arsenicinduced cell death can also lead to compensatory cell regeneration and carcinogenesis. Altered growth factors, cell proliferation, and promotion of carcinogenesis have all been demonstrated in one or more systems exposed to arsenics. Altered growth factors and mitogenesis were noted in human keratinocytes. Cell death was observed in human hepatocytes and rat bladder epithelium. Cell proliferation was demonstrated in human keratinocytes and intact human skin and rodent bladder cells. Promotion of carcinogenesis was noted in rat bladder, kidney, liver, and thyroid, and mouse skin and lung.

Additional Mechanisms of Toxicity Data. Inorganic arsenic exposure has been shown to modify the expression of a variety of genes related to cell growth and defense, including the tumor suppressor gene $\mathrm{p} 53$, as well as to alter the binding of nuclear transcription factors. Carcinogenic effects of arsenic may result from a cocarcinogenic effect. Whereas arsenic exposure alone did not elicit skin tumors in mice, co-exposure to arsenic and ultraviolet light resulted in skin tumors that were greater in number and larger in size than those produced by ultraviolet light alone. Arsenate and arsenite enhanced the amplification of a gene that codes for the enzyme dihydrofolate reductase, arsenate being more potent than arsenite. Furthermore, inhibition of DNA repair has been demonstrated in arsenic-treated cells.

\subsubsection{Animal-to-Human Extrapolations}

The usefulness of animal models for toxicity studies with arsenic is significantly limited by two major factors. First and most importantly, no animal model exists for the health effect of greatest concern for human exposure: carcinogenicity in skin and other organs after oral exposure. Second, the pattern of metabolism in humans (significant excretion of the methylated forms of arsenic) is unlike that of most other mammalian species (the mouse and rabbit may be exceptions). The ratios of inorganic to organic arsenic excreted also vary between species. The rat sequesters arsenic in its erythrocytes and is not a suitable model for human toxicity. 


\subsection{TOXICITIES MEDIATED THROUGH THE NEUROENDOCRINE AXIS}

Recently, attention has focused on the potential hazardous effects of certain chemicals on the endocrine system because of the ability of these chemicals to mimic or block endogenous hormones. Chemicals with this type of activity are most commonly referred to as endocrine disruptors. However, appropriate terminology to describe such effects remains controversial. The terminology endocrine disruptors, initially used by Thomas and Colborn (1992), was also used in 1996 when Congress mandated the EPA to develop a screening program for “...certain substances [which] may have an effect produced by a naturally occurring estrogen, or other such endocrine effect[s]...”. To meet this mandate, EPA convened a panel called the Endocrine Disruptors Screening and Testing Advisory Committee (EDSTAC), and in 1998, the EDSTAC completed its deliberations and made recommendations to EPA concerning endocrine disruptors. In 1999, the National Academy of Sciences released a report that referred to these same types of chemicals as hormonally active agents. The terminology endocrine modulators has also been used to convey the fact that effects caused by such chemicals may not necessarily be adverse. Many scientists agree that chemicals with the ability to disrupt or modulate the endocrine system are a potential threat to the health of humans, aquatic animals, and wildlife. However, others think that endocrine-active chemicals do not pose a significant health risk, particularly in view of the fact that hormone mimics exist in the natural environment. Examples of natural hormone mimics are the isoflavinoid phytoestrogens (Adlercreutz 1995; Livingston 1978; Mayr et al. 1992). These chemicals are derived from plants and are similar in structure and action to endogenous estrogen. Although the public health significance and descriptive terminology of substances capable of affecting the endocrine system remains controversial, scientists agree that these chemicals may affect the synthesis, secretion, transport, binding, action, or elimination of natural hormones in the body responsible for maintaining homeostasis, reproduction, development, and/or behavior (EPA 1997h). Stated differently, such compounds may cause toxicities that are mediated through the neuroendocrine axis. As a result, these chemicals may play a role in altering, for example, metabolic, sexual, immune, and neurobehavioral function. Such chemicals are also thought to be involved in inducing breast, testicular, and prostate cancers, as well as endometriosis (Berger 1994; Giwercman et al. 1993; Hoel et al. 1992).

There is little evidence to suggest that arsenic functions as an endocrine disruptor. An association has been demonstrated between exposure to arsenic in drinking water and increased incidence of diabetes mellitus (Rahman et al. 1998; Tsai et al. 1999; Tseng et al. 2000; Wang et al. 2003), although doseresponse relationships are not available and the mechanism of action for this response has not been characterized. Studies by Waalkes and coworkers (Waalkes et al. 2006a, 2006b) have suggested that in 
mice, arsenic may interact with estrogens to enhance production of female urogenital cancers and male hepatocellular cancer following exposure to arsenic in utero. The mechanism by which this might happen has not been elucidated. No other relevant data were located in humans or animals. Data on general effects of arsenic compounds on the endocrine system are presented in Sections 3.2.1.2 and 3.2.2.2 above.

In vitro studies provide suggestive evidence that arsenic may act as an endocrine disruptor. Studies by Bodwell et al. $(2004,2006)$ and Davey et al. (2007) demonstrate that arsenic can alter gene regulation of steroid hormone receptors for glucocorticoids, mineralocorticoids, progesterone, and estrogen.

\subsection{CHILDREN'S SUSCEPTIBILITY}

This section discusses potential health effects from exposures during the period from conception to maturity at 18 years of age in humans, when all biological systems will have fully developed. Potential effects on offspring resulting from exposures of parental germ cells are considered, as well as any indirect effects on the fetus and neonate resulting from maternal exposure during gestation and lactation. Relevant animal and in vitro models are also discussed.

Children are not small adults. They differ from adults in their exposures and may differ in their susceptibility to hazardous chemicals. Children's unique physiology and behavior can influence the extent of their exposure. Exposures of children are discussed in Section 6.6, Exposures of Children.

Children sometimes differ from adults in their susceptibility to hazardous chemicals, but whether there is a difference depends on the chemical (Guzelian et al. 1992; NRC 1993). Children may be more or less susceptible than adults to health effects, and the relationship may change with developmental age (Guzelian et al. 1992; NRC 1993). Vulnerability often depends on developmental stage. There are critical periods of structural and functional development during both prenatal and postnatal life, and a particular structure or function will be most sensitive to disruption during its critical period(s). Damage may not be evident until a later stage of development. There are often differences in pharmacokinetics and metabolism between children and adults. For example, absorption may be different in neonates because of the immaturity of their gastrointestinal tract and their larger skin surface area in proportion to body weight (Morselli et al. 1980; NRC 1993); the gastrointestinal absorption of lead is greatest in infants and young children (Ziegler et al. 1978). Distribution of xenobiotics may be different; for example, infants have a larger proportion of their bodies as extracellular water, and their brains and livers are proportionately larger (Altman and Dittmer 1974; Fomon 1966; Fomon et al. 1982; Owen and Brozek 
1966; Widdowson and Dickerson 1964). The infant also has an immature blood-brain barrier (Adinolfi 1985; Johanson 1980) and probably an immature blood-testis barrier (Setchell and Waites 1975). Many xenobiotic metabolizing enzymes have distinctive developmental patterns. At various stages of growth and development, levels of particular enzymes may be higher or lower than those of adults, and sometimes unique enzymes may exist at particular developmental stages (Komori et al. 1990; Leeder and Kearns 1997; NRC 1993; Vieira et al. 1996). Whether differences in xenobiotic metabolism make the child more or less susceptible also depends on whether the relevant enzymes are involved in activation of the parent compound to its toxic form or in detoxification. There may also be differences in excretion, particularly in newborns who all have a low glomerular filtration rate and have not developed efficient tubular secretion and resorption capacities (Altman and Dittmer 1974; NRC 1993; West et al. 1948). Children and adults may differ in their capacity to repair damage from chemical insults. Children also have a longer remaining lifetime in which to express damage from chemicals; this potential is particularly relevant to cancer.

Certain characteristics of the developing human may increase exposure or susceptibility, whereas others may decrease susceptibility to the same chemical. For example, although infants breathe more air per kilogram of body weight than adults breathe, this difference might be somewhat counterbalanced by their alveoli being less developed, which results in a disproportionately smaller surface area for alveolar absorption (NRC 1993).

Arsenic has been recognized as a human toxicant for many centuries, and the symptoms of acute poisoning are well known. Children who are exposed to high levels of arsenic exhibit symptoms similar to those seen in adults, including respiratory, cardiovascular, dermal, and neurological effects, and vomiting if the arsenic is ingested (Borgoño et al. 1980; Foy et al. 1992; Kersjes et al. 1987; Muzi et al. 2001; Rosenberg 1974; Zaldívar 1974; Zaldívar and Guillier 1977). Arterial thickening of the pancreas was observed in five children who died in Chile after chronic exposure to arsenic (Rosenberg 1974). Foy et al. (1992) described systemic effects of chronic arsenic exposure in children in a village near a tin and tungsten mining operation in Thailand. The arsenic concentration in water samples from 35 shallow wells averaged $0.82 \mathrm{mg} \mathrm{As} / \mathrm{L}$ (range, 0.02-2.7 $\mathrm{mg} \mathrm{As} / \mathrm{L}$ ). Piped water (available in some homes) had a concentration of $0.07 \mathrm{mg} \mathrm{As} / \mathrm{L}$. A survey of skin manifestations of arsenic poisonings was conducted in the autumn of 1987. The case reports of four children were presented. All of the children had hyperkeratosis and hyperpigmentation of the extremities, including tibia, palms, and soles. In addition, one child had developed weakness 3 years previously and had anorexia and a chronic cough for 1 year. She had been held back twice in school as a slow learner. On examination, she had a runny nose and 
weakness of her wrist joints. The liver was about 4 finger-breadths below the right costal margin with a sharp but tender edge. Blood arsenic levels ranged from 0.087 to $0.46 \mu \mathrm{g} / \mathrm{mL}$ and the arsenic level in hair ranged from 14.4 to $20 \mu \mathrm{g} / \mathrm{g}$. The authors concluded that the finding of typical skin manifestations of chronic arsenic poisoning suggests that it may take a considerably shorter period of time to develop these manifestations than previously thought. However, it is not known what effect co-exposure to tin and tungsten might have had on skin manifestations in these children. Exposure to high arsenic levels during gestation and/or during early childhood also was associated with significant increases in SMRs for lung cancer and bronchiectasis during adulthood in a study of residents in a city in Chile with high arsenic levels in the drinking water (near $0.9 \mathrm{mg} / \mathrm{L}$ ) during several years (Smith et al. 2006).

As previously mentioned in Sections 3.2.1.4 and 3.2.2.4, exposure of children to arsenic also has been associated with neurological deficits in children. Studies by Wasserman et al. $(2004,2007)$ of $6-$ and 10-year-old children from Bangladesh reported small but significant decreases in some tests of cognitive function associated with levels of arsenic in the water $\geq 0.05 \mathrm{mg} / \mathrm{L}$. A study of pre-school age children in West Bengal, India, reported an association between current urinary arsenic concentrations, but not longterm water arsenic, and small decrements in intellectual tests (von Ehrenstein et al. 2007). Similar results were reported in a study of children in Taiwan (Tsai et al. 2003) and in China (Wang et al. 2007). Neurological effects have also been associated with elevated levels of arsenic in the air. For example, Bencko et al. (1977) reported that children of approximately 10 years of age living near a power plant burning coal of high arsenic content showed significant hearing losses (increased threshold) compared to a control group of children living outside the polluted area (Bencko et al. 1977). Also, in a study of Mexican children, Calderón et al. (2001) reported that children living near a smelter complex had poor performance on tests evaluating verbal IQ than children who lived farther from the smelter. Thus, the limited data available suggest that exposure of children to inorganic arsenic may result in detrimental effects on neurobehavioral parameters.

Wulff et al. (1996) conducted a retrospective study of a cohort of children born between 1961 and 1990 in the municipality of Skelleftea, Sweden, where a smelter released arsenic and other pollutants including lead, copper, cadmium, and sulfur dioxide. Childhood cancer incidences among children born in the vicinity of the smelter (i.e., within $20 \mathrm{~km})$ and distant from the smelter $(>20 \mathrm{~km})$ were compared with expected incidences based on Swedish national statistics. There appeared to be an increased risk of childhood cancer (all types combined) among children born in the vicinity of the smelter (SIR=195, 95\% $\mathrm{CI}=88-300$, based on 13 cases observed and 6.7 expected), but the increase was not statistically significant, and in any event, the role of arsenic in any finding from this study is confounded by the 
presence of other metals. The number of cases $(n=42)$ was very close to the expected number $(n=41.8)$ among children born distant from the smelter. Similar results were reported in a study by Moore et al. (2002), which did not find increased incidence ratios for all childhood cancers or for childhood leukemias in children from an area of Nevada with high arsenic exposures.

Inorganic arsenic has been characterized as a developmental toxicant. It is known to cross the placental barrier and selectively accumulate in the neuroepithelium of the developing animal embryo (Hanlon and Ferm 1977; Lindgren et al. 1984). Studies in animals have also revealed that various fetal malformations occur after embryonic exposure to arsenic in vitro; neural tube defects are the predominant and consistent malformation in these studies (Chaineau et al. 1990; Mirkes and Cornel 1992; Morrissey and Mottet 1983; Mottet and Ferm 1983; Tabacova et al. 1996; Willhite and Ferm 1984; Wlodarczyk et al. 1996). In vivo studies have shown that high doses of ingested arsenic can produce developmental effects (fetal mortality, skeletal defects), but generally only at maternally toxic doses (Baxley et al. 1981; Holson et al. 1999, 2000; Hood and Harrison 1982; Hood et al. 1978; Nemec et al. 1998; Stump et al. 1999). A series of studies showed an increased incidence of tumors in the offspring of mice exposed to arsenic from gestational day 8 through day 18(Waalkes et al. 2003, 2004a, 2004b, 2004c, 2006a, 2006b) (see

Section 3.2.2.6 for further details). In humans, acute prenatal exposure to high doses of inorganic arsenic can result in miscarriage and early neonatal death (Bolliger et al. 1992; Lugo et al. 1969). Although several studies have reported marginal associations between prolonged low-dose human arsenic exposure and adverse reproductive outcomes, including spontaneous abortion, stillbirth, developmental impairment, and congenital malformation (Ahmad et al. 2001; Aschengrau et al. 1989; Chakraborti et al. 2003c; Hopenhayn-Rich et al. 2000; Nordström et al. 1978a, 1979b; Yang et al. 2003; Zierler et al. 1988), none of these studies have provided convincing evidence for such effects or information concerning possible dose-response relationships.

There is no evidence for differences in absorption of arsenic in children and adults. Ingestion of arsenic in dirt may be an important route of exposure for young children. A study that used a synthetic gastric juice designed to mimic gastric conditions in a 2-year-old child found that absorption of arsenic from contaminated soil was likely to be up to 5 times lower than the total concentration of arsenic in the soil (Williams et al. 1998). As previously mentioned, arsenic crosses the placenta and preferentially accumulates in the embryonic neuroepithelium. In addition, arsenic is known to be present in breast milk at low concentrations. Arsenic concentrations were low in human milk sampled from 88 mothers in the Faroe Islands (0.0001-0.0044 ppm), where the diet is predominantly seafood (exposures were primarily to "fish arsenic" [Grandjean et al. 1995]), in a population of Andean women (0.0008-0.008 ppm) exposed 
to high concentrations of inorganic arsenic in drinking water (Concha et al. 1998b), and in a World Health Organization survey (0.00013-0.00082 ppm) (Somogyi and Beck 1993). There is no information in the literature describing storage of arsenic in maternal tissues. There is some evidence that metabolism of arsenic in children is less efficient than in adults. Children in two villages in Argentina ingesting large amounts of arsenic in their drinking water $(200 \mu \mathrm{g} / \mathrm{L})$ excreted about $49 \%$ inorganic arsenic and $47 \%$ DMA, compared to 32\% inorganic arsenic and 66\% DMA for the women in the study (Concha et al. 1998b). No PBPK models specifically targeted at fetuses, infants, or children, or pregnant or lactating women were found in the literature. There are no biomarkers that have been specifically identified for children exposed to arsenic. In addition, no unique interactions of arsenic with other chemicals have been identified in children.

The mechanism of toxic action of arsenic in the mammalian cell may involve inhibition of proliferation of cells (Dong and Luo 1993; Jha et al. 1992; Petres et al. 1977). In addition, high-dose arsenic impairs assembly and disassembly of microtubules, thus interfering with mitotic spindle formation and embryonal cell division (Léonard and Lauwerys 1980; Li and Chou 1992; Mottet and Ferm 1983). Arsenic compounds also cause chromosomal aberrations (Jha et al. 1992; Léonard and Lauwerys 1980), which may disrupt cell cycling. The direct toxic effects of high levels of arsenic in the developing embryo result not from a difference in the mechanism of toxicity during development, but rather from the existence of a unique target tissue, the neuroepithelium. The process of neurulation involves cell shape changes, cytokinesis, and cell adhesion, which are dependent upon cytoskeletal elements that are functionally affected by arsenic (Dallaire and Béliveau 1992; Edelman 1992; Gunn et al. 1992; Li and Chou 1992; Moriss-Kay et al. 1994; Schoenwolf and Smith 1990; Taubeneck et al. 1994). However, since arsenic is known to affect vasculature, and since altered placental and/or embryonal vasculature has been suggested as a mechanism leading to neural tube defects, the embryo may be sensitive to this manifestation of arsenic toxicity.

\subsection{BIOMARKERS OF EXPOSURE AND EFFECT}

Biomarkers are broadly defined as indicators signaling events in biologic systems or samples. They have been classified as markers of exposure, markers of effect, and markers of susceptibility (NAS/NRC 1989).

Due to a nascent understanding of the use and interpretation of biomarkers, implementation of biomarkers as tools of exposure in the general population is very limited. A biomarker of exposure is a xenobiotic 
substance or its metabolite(s) or the product of an interaction between a xenobiotic agent and some target molecule(s) or cell(s) that is measured within a compartment of an organism (NAS/NRC 1989). The preferred biomarkers of exposure are generally the substance itself, substance-specific metabolites in readily obtainable body fluid(s), or excreta. However, several factors can confound the use and interpretation of biomarkers of exposure. The body burden of a substance may be the result of exposures from more than one source. The substance being measured may be a metabolite of another xenobiotic substance (e.g., high urinary levels of phenol can result from exposure to several different aromatic compounds). Depending on the properties of the substance (e.g., biologic half-life) and environmental conditions (e.g., duration and route of exposure), the substance and all of its metabolites may have left the body by the time samples can be taken. It may be difficult to identify individuals exposed to hazardous substances that are commonly found in body tissues and fluids (e.g., essential mineral nutrients such as copper, zinc, and selenium). Biomarkers of exposure to arsenic are discussed in Section 3.8.1.

Biomarkers of effect are defined as any measurable biochemical, physiologic, or other alteration within an organism that, depending on magnitude, can be recognized as an established or potential health impairment or disease (NAS/NRC 1989). This definition encompasses biochemical or cellular signals of tissue dysfunction (e.g., increased liver enzyme activity or pathologic changes in female genital epithelial cells), as well as physiologic signs of dysfunction such as increased blood pressure or decreased lung capacity. Note that these markers are not often substance specific. They also may not be directly adverse, but can indicate potential health impairment (e.g., DNA adducts). Biomarkers of effects caused by arsenic are discussed in Section 3.8.2.

A biomarker of susceptibility is an indicator of an inherent or acquired limitation of an organism's ability to respond to the challenge of exposure to a specific xenobiotic substance. It can be an intrinsic genetic or other characteristic or a preexisting disease that results in an increase in absorbed dose, a decrease in the biologically effective dose, or a target tissue response. If biomarkers of susceptibility exist, they are discussed in Section 3.10, Populations That Are Unusually Susceptible.

\subsubsection{Biomarkers Used to Identify or Quantify Exposure to Arsenic}

Arsenic levels in blood, urine, hair, and nails have all been investigated and used as biological indicators of exposure to arsenic. Since arsenic is cleared from blood within a few hours (Tam et al. 1979b; Vahter 1983), measurements of blood arsenic reflect exposures only within the very recent past. Typical values in nonexposed individuals are $<1 \mu \mathrm{g} / \mathrm{L}$ (Heydorn 1970; Hindmarsh and McCurdy 1986; Valentine et al. 
1979). Consumption of medicines containing arsenic is associated with blood values of $100-250 \mu \mathrm{g} / \mathrm{L}$, while blood levels in acutely toxic and fatal cases may be $1,000 \mu \mathrm{g} / \mathrm{L}$ or higher (Driesback 1980).

However, blood levels do not appear to be reliable indicators of chronic exposure to low levels of arsenic. For example, there was no correlation between the level of arsenic in blood of residents and the level of arsenic in drinking water in several U.S. communities where water levels ranged from about 6 to $125 \mu \mathrm{g} / \mathrm{L}$ (Valentine et al. 1979, 1981). Consequently, measurement of blood arsenic is not generally considered to be a reliable means of monitoring human populations for arsenic exposure.

As discussed in Section 3.4.4, most arsenic that is absorbed from the lungs or the gastrointestinal tract is excreted in the urine, mainly within 1-2 days. For this reason, measurement of urinary arsenic levels is generally accepted as the most reliable indicator of recent arsenic exposure, and this approach has proved useful in identifying above-average exposures in populations living near industrial point sources of arsenic (e.g., Milham and Strong 1974; Polissar et al. 1990). By the inhalation route, several researchers have found that there is a good quantitative correlation between the concentration of arsenic in workplace $\operatorname{air}\left(\mathrm{C}_{\text {air }}, \mu \mathrm{g} / \mathrm{m}^{3}\right)$ and the concentration in the urine $\left(\mathrm{C}_{\text {urine }}, \mu \mathrm{g} / \mathrm{L}\right)$ of exposed workers. For example, Pinto et al. (1976) found a linear relationship for exposures ranging up to $150 \mu \mathrm{g} / \mathrm{m}^{3}$, given by the following equation:

$$
\mathrm{C}_{\text {air }}=0.3 \mathrm{C}_{\text {urine }}
$$

Enterline et al. (1987a) reinvestigated this relationship over a wider range of exposures (up to $3,500 \mu \mathrm{g} / \mathrm{m}^{3}$ ), and found that the curve tended to be concave upward, as given by the following equation:

$$
\mathrm{C}_{\text {air }}=0.0064\left(\mathrm{C}_{\text {urine }}\right)^{1.94}
$$

This indicates that at higher exposure levels, a higher fraction of the dose is excreted in urine, although the toxicokinetic basis for this is not certain. Numerous studies have used above-average urinary levels (i.e., higher than about $100 \mu \mathrm{g} / \mathrm{L}$ ) as evidence of recent arsenic ingestion (e.g., Borgoño et al. 1980; Fincher and Koerker 1987; Franzblau and Lilis 1989; Goldsmith and From 1986; Kyle and Pease 1965; Valentine et al. 1981). Calderon et al. (1999) found a quantitative correlation between the log of the mean total urinary arsenic concentration/creatinine (TAs/c, $\mu \mathrm{g} / \mathrm{mg}$ ) of people living in areas with arseniccontaminated drinking water sources and the $\log$ of the inorganic arsenic concentration in the drinking water $(\operatorname{InAs}, \mu \mathrm{g} / \mathrm{L})$. The equation for the regression line is: 


$$
\mathrm{TAs} / \mathrm{c}=10^{-2.57} \mathrm{x}(\mathrm{InAs})^{0.63}
$$

where -2.57 and 0.63 are the intercept and slope, respectively, for the regression of the log 10 -transformed data. Mixed model regression analysis showed that the log of estimated arsenic intake from drinking water ( $\mu \mathrm{g} /$ day) is also a good predictor of TAs/c excretion (Calderon et al. 1999).

There is some indication that speciation of urinary arsenic may indicate the extent of past cumulative exposure to arsenic. Hsueh et al. (1998a) reported higher levels of DMA and MMA in the urine of individuals with higher cumulative past exposure to inorganic arsenic. Speciated urinary arsenic is also a recommended biomarker for recent inorganic arsenic exposure. Walker and Griffin (1998) used the EPA Exposure Assessment Model and a number of site-specific data covering environmental and biological factors to predict total and speciated urinary arsenic concentrations for children living near high levels of arsenic-contaminated soil. There was reasonable agreement between the measured and predicted speciated urinary arsenic concentrations.

An important limitation to the use of total urinary arsenic as a biomarker of exposure is that arsenobetaine is excreted (unmetabolized) in urine after ingestion of certain seafoods (Brown et al. 1990; Kalman 1987; Tam et al. 1982). Since "fish arsenic" is essentially nontoxic, analytical methods based on total urinary arsenic content may overestimate exposures to arsenic species that are of health concern. As discussed in Section 7.1, there are adequate methods for distinguishing arsenobetaine from other forms of arsenic in urine (inorganic, MMA, DMA), although these are not convenient to use as a routine screening method.

Arsenic tends to accumulate in hair and nails, and measurement of arsenic levels in these tissues may be a useful indicator of past exposures. Normal levels in hair and nails are 1 ppm or less (Choucair and Ajax 1988; Franzblau and Lilis 1989). These values may increase from several-fold to over 100-fold following arsenic exposure (Agahian et al. 1990; Bencko 2005; Bencko et al. 1986; de Peyster and Silvers 1995; EPA 1977a, 1981b; Karagas et al. 1996; Milham and Strong 1974; Valentine et al. 1979; Yamauchi et al. 1989) and remain elevated for 6-12 months (Choucair and Ajax 1988). Minimum exposure levels that produce measurable increases in arsenic levels in hair and nails have not been precisely defined. For hair, ingestion of 50-120 ppb of arsenic in drinking water produced only a marginal effect, but a clear increase was noted at $393 \mathrm{ppb}$ (Valentine et al. 1979). A study of children living in a region polluted with arsenic derived from a power plant burning coal with a high arsenic content found a significant correlation between arsenic levels in hair and distance from the source of emission (Bencko and Symon 1977). 
Inhalation exposure of workers to about $0.6 \mu \mathrm{g} / \mathrm{m}^{3}$ of arsenic in air significantly increased average levels in nails (Agahian et al. 1990), although there was wide variation between individuals.

Analysis of hair may yield misleading results due to the presence of arsenic adsorbed to the external surface, but this can be minimized by collecting samples from close to the scalp or from unexposed areas and by washing the hair before analysis (e.g., Paschal et al. 1989). Similarly, extensive washing of nails is required to remove exogenous contamination (Agahian et al. 1990). The relationship between consumption of food items and levels of arsenic in toenails has been evaluated by MacIntosh et al. (1997) using standard multivariate regression models. This approach does not appear to be highly reliable, but may be sufficient for exploring associations between diet and disease. Kurttio et al. (1998) used linear regression models to show that there is a good association between arsenic concentration in hair $(\mathrm{mg} / \mathrm{kg})$ and total arsenic concentration in urine $(\mu \mathrm{g} / \mathrm{L})$, arsenic concentration in drinking water $(\mu \mathrm{g} / \mathrm{L})$ or daily intake of arsenic ( $\mu \mathrm{g} /$ day). A $10 \mu \mathrm{g} / \mathrm{L}$ increase in the drinking water concentration or a $10-20 \mu \mathrm{g} / \mathrm{day}$ increase in daily arsenic intake corresponded to a $0.1 \mathrm{mg} / \mathrm{kg}$ increase in the arsenic concentration in hair. It is also important to note that the measurement of arsenic in hair and fingernails is a process not readily accessible to many clinical offices.

\subsubsection{Biomarkers Used to Characterize Effects Caused by Arsenic}

As discussed in Section 3.2, the characteristic pattern of skin changes caused by arsenic (hyperkeratinization, hyperpigmentation) is probably the most sensitive and diagnostic clinical indicator of chronic exposure to arsenic. However, no means has been developed for detecting these effects except by routine dermatological examination.

Peripheral neuropathy is another characteristic effect of arsenic exposure, and several researchers have investigated decreased nerve conduction velocity or amplitude as a biomarker for peripheral neuropathy. While effects can usually be detected in individuals with clinical signs of neuropathy (e.g., Goebel et al. 1990; Jenkins 1966; Le Quesne and McLeod 1977; Morton and Caron 1989; Murphy et al. 1981), effects are only marginal (EPA 1977a; Hindmarsh et al. 1977; Valentine et al. 1981) or undetectable (EPA 1981b; Kreiss et al. 1983) in exposed populations without obvious clinical signs of toxicity. This indicates that this approach is probably not sufficiently sensitive to detect neurological effects earlier than by standard neurological examination (Hindmarsh and McCurdy 1986). Also, decreases in nerve conduction velocity or amplitude are not specific for arsenic-induced neuropathy. 
Arsenic is known to affect the activity of a number of enzymes, and some of these may have potential as biomarkers of effect. Most promising is the spectrum of effects caused by arsenic on the group of enzymes responsible for heme synthesis and degradation, including inhibition of coproporphyrinogen oxidase and heme synthetase (Woods and Fowler 1978; Woods and Southern 1989) and activation of heme oxygenase (Sardana et al. 1981). Menzel et al. (1998) has examined the in vitro induction of human lymphocyte heme oxygenase $1(\mathrm{HO} 1)$ as a biomarker of arsenite exposure. Arsenite did induce de novo synthesis of HO1 in human lymphoblastoid cells, but it has not been determined if the same response is induced in vivo. It has been shown in animals that these arsenic-induced enzymic changes result in increased urinary levels of uroporphyrin, coproporphyrin, and bilirubin (Albores et al. 1989; Woods and Fowler 1978), and it has been shown that these effects can be detected in the urine of arsenic-exposed humans (García-Vargas and Hernández-Zavala 1996). Therefore, altered urinary levels of these hemerelated compounds could serve as a biomarker of effect. However, it is known that numerous other toxic metals also have similar effects on heme metabolism (Albores et al. 1989; Sardana et al. 1981; Woods and Southern 1989), so it is likely that these effects would not be specific for arsenic.

For more information on biomarkers for renal and hepatic effects of chemicals, see ATSDR/CDC Subcommittee Report on Biological Indicators of Organ Damage (Agency for Toxic Substances and Disease Registry 1990b) and for information on biomarkers for neurological effects, see OTA (1990).

\subsection{INTERACTIONS WITH OTHER CHEMICALS}

A number of researchers have found that arsenic compounds tend to reduce the effects of selenium (Hill 1975; Howell and Hill 1978; Kraus and Ganther 1989; Levander 1977; Miyazaki et al. 2003; Moxon et al. 1945; Schrauzer 1987; Schrauzer et al. 1978). Likewise, selenium can decrease the effects of arsenic, including clastogenicity (Beckman and Nordenson 1986; Biswas et al. 1999; Sweins 1983), delayed mutagenesis (Rossman and Uddin 2004), cocarcinogenesis (Uddin et al. 2005), cytotoxicity (Babich et al. 1989; Rössner et al. 1977; Styblo and Thomas 2001), and teratogenicity (Holmberg and Ferm 1969). The mechanism of this mutual inhibition of effects is not known, but may be related to the formation of a selenium-arsenic complex (seleno-bis [S-gluthionyl] arsinium ion; Gailer et al. 2002) that is excreted more rapidly than either arsenic or selenium alone (Cikrt et al. 1988; Hill 1975; Levander 1977; Levander and Baumann 1966) or due to selenium-induced changes in arsenic methylation (Styblo and Thomas 2001; Walton et al. 2003). There is little direct evidence that variations in selenium exposure in humans lead to significant increases or decreases in arsenic toxicity, although copper smelter workers who developed lung cancer had lower tissue levels of selenium than workers who did not develop lung tumors 
(Gerhardsson et al. 1985, 1988). This suggests that selenium deficiency could significantly increase the risk of lung cancer following inhalation exposure to arsenic, but it is difficult to distinguish cause from effect in such a study. However, there is evidence that administration of selene can facilitate recovery from arsenic poisoning. In residents living in an area of Inner Mongolia with high levels of arsenic in drinking water, administration of 100-200 $\mu \mathrm{g}$ selenium/day in the form of selenium yeast and exposure to arsenic-free water for 14 months resulted in a greater improvement in clinical signs and symptoms, liver function, and EKG readings as compared to residents administered arsenic-free water only (Wuyi et al. 2001; Yang et al. 2002). An improvement in skin lesions was observed in 67 and 21\% of the subjects in the selenium-supplemented and control groups (Yang et al. 2002). Additionally, the levels of arsenic in blood, hair, and urine were significantly lower after the 14-month period only in the selenium supplemented group.

The interaction between cigarette smoking, inhalation of arsenic, and the risk of lung cancer has not been extensively investigated. Smoking appeared to increase lung cancer risk synergistically (multiplicatively) in one study of smelter workers (Pershagen et al. 1981), although the data are not adequate to exclude a simple additive interaction (Thomas and Whittemore 1988). Cigarette smoking has been shown to increase the occurrence of lung cancer in people with high levels of arsenic in the drinking water (Chiou et al. 1995; Tsuda et al. 1995a). Suggestive evidence of a positive interaction between arsenic and benzo(a)pyrene has also been noted for induction of lung adenocarcinomas in hamsters (Pershagen et al. 1984).

Co-exposure to ethanol and arsenic may exacerbate the toxic effects of arsenic. Simultaneous exposure of rats to ethanol (10\% in drinking water) and arsenic (dose not stated) for 6 weeks produced a significant increase in the concentration of arsenic in the kidney, a nonsignificant increase of arsenic in the liver and a significant increase in the concentration of glutathione in the liver, compared to rats treated with either ethanol or arsenic alone (Flora et al. 1997a, 1997b). Histological damage to the liver, but not the kidneys, was increased in rats treated with both ethanol and arsenic compared to those receiving only arsenic.

Studies of rats exposed to arsenic, lead, and cadmium, alone or in combination, have revealed mainly additive or subadditive effects on body weight, hematological parameters, and enzymes of heme synthesis (Mahaffey and Fowler 1977; Mahaffey et al. 1981). Similarly, studies of the tissue levels of arsenic in rats fed arsenic with or without lead or cadmium revealed only limited evidence of any toxicokinetic interactions (Mahaffey et al. 1981). Pretreatment of rats with a nontoxic dose of cadmium had no effect on the lethality of a high dose of arsenic and did not reduce arsenic-induced hepatotoxicity (Hochadel and 
Waalkes 1997). These data do not suggest that arsenic toxicity is likely to be significantly influenced by concomitant exposure to these metals. However, supplementation with zinc or chromium may be useful in reducing chronic arsenism. Arsenic has been shown to cause an increase in total plasma cholesterol; co-administration of chromium(III) counteracts this effect (Aguilar et al. 1997). Pretreatment of mice with zinc, at least 24 hours before injection with arsenic-73, reduced arsenic retention compared to controls that did not receive the zinc pretreatment or received it only a short time before the administration of arsenic (Kreppel et al. 1994). Zinc is an inducer of metallothionein, but this induction does not appear to be the mechanism that reduces arsenic toxicity because other inducers of metallothionein did not reduce arsenic toxicity and arsenic elimination was increased by the zinc pretreatment.

Since methylation of arsenic is a detoxification mechanism, it is possible that chemicals that interfere with the methylation process could increase toxicity. This is supported by studies in animals in which reagents that inhibit methylation enzymes (e.g., periodate-oxidized adenosine) caused an increase in tissue levels of inorganic arsenic (Marafante and Vahter 1986; Marafante et al. 1985). Similarly, cellular glutathione levels appear to play a role in the methylation process, and treatment with reagents (e.g., phorone) that decrease glutathione levels increases arsenic toxicity (Buchet and Lauwerys 1987). Inadequate dietary intake of methionine, choline, or protein may also exacerbate arsenic toxicity. Rabbits pretreated with diets low in choline, methionine, or protein showed a significant increase in tissue retention of arsenic and a significant decrease in the excretion of dimethylarsinic acid (Vahter and Marafante 1987). The increased retention of arsenic in rabbits fed these deficient diets is likely to be due to a reduction in arsenic methylation. Thus, the toxic effects of chronic arsenic ingestion may be increased in populations that are also subject to malnutrition.

\subsection{POPULATIONS THAT ARE UNUSUALLY SUSCEPTIBLE}

A susceptible population will exhibit a different or enhanced response to arsenic than will most persons exposed to the same level of arsenic in the environment. Reasons may include genetic makeup, age, health and nutritional status, and exposure to other toxic substances (e.g., cigarette smoke). These parameters result in reduced detoxification or excretion of arsenic, or compromised function of organs affected by arsenic. Populations who are at greater risk due to their unusually high exposure to arsenic are discussed in Section 6.7, Populations with Potentially High Exposures. 
No studies were located that identified an unusual susceptibility of any human subpopulation to arsenic. Several studies have evaluated possible sex-related differences in arsenic toxicity and carcinogenesis (Aposhian et al. 2000a, 2000b; Calderon et al. 1999; Loffredo et al. 2003; Mandal et al. 2001; Watanabe et al. 2001), but have not consistently identified differences. However, since the degree of arsenic toxicity may be influenced by the rate and extent of its methylation in the liver (see Section 3.4.3), it seems likely that some members of the population might be especially susceptible because of lower than normal methylating capacity. Studies of exposed humans in Taiwan suggested that subjects with lower secondary methylation indices have an increased risk of bladder cancer (Chen et al. 2003) and peripheral vascular disease (Tseng et al. 2005), particularly in subjects with high exposure levels. Reduced hepatic methylation could result from dietary deficiency of methyl donors such as choline or methionine (Buchet and Lauwerys 1987; Vahter and Marafante 1987), although this is unlikely to be a concern for most people in the United States. There is evidence that methylation capacity can vary greatly among individuals (e.g., Buchet et al. 1981a; Foà et al. 1984; Hopenhayn-Rich et al. 1996b; Tam et al. 1979b), but the basis of this variation and its impact on human susceptibility have not been fully established. There is some evidence that low dietary protein intake and possibly other nutritional deficiencies can decrease arsenic methylation (Steinmaus et al. 2005a). Recently, Heck et al. (2007) examined whether the capacity to methylate arsenic differs by nutrient intake in a cohort of 1,016 Bangladeshi adults exposed to arsenic in drinking water. The results showed that higher intakes of cysteine, methionine, calcium, protein, and vitamin B-12 were associated with lower percentages of inorganic arsenic and higher ratios of MMA to inorganic arsenic in urine. In addition, higher intakes of niacin and choline were associated with higher DMA/MMA ratios, after adjustment for sex, age, smoking, total urinary arsenic, and total energy intake. The issue of increased susceptibility to arsenic due to poor nutrition was discussed by NRC (2001), it was concluded that, with regard to skin effects, studies of cohorts from India, Bangladesh, and Taiwan suggest that nutrition plays an important role in arsenic toxicity. On the other hand, studies in other regions of the world (i.e., Chile) involving populations with much better nutrition argue against poor nutrition having a major impact on arsenic toxicity.

Various genetic polymorphisms also seem to play a role in arsenic-induced toxicity. For example, a study of 85 lung cancer patients and 108 healthy controls in northern Chile reported that there was a nonstatistically significant difference for the frequency of the GSTM1 null genotype between the healthy and lung cancer patients stratified by gender and smoking status. The same results were observed for the MspI CYP450 1A1 polymorphism (Adonis et al. 2005). Hsueh et al. (2005) examined the association of four polymorphisms: $\mathrm{NAD}(\mathrm{P}) \mathrm{H}$ oxidase, manganese superoxide dismutase (MnSOD), catalase, and endothelial nitric oxide synthase (eNOS) with arsenic related hypertension risk among 79 hypertensive 
cases and 213 controls in an arseniasis-hyperendemic area in Taiwan. The results showed that MnSOD polymorphism significantly increased the risk of hypertension regardless of exposure to arsenic. NAD(P)H oxidase and eNOS polymorphisms were significantly associated with increased risk of hypertension in subjects with higher cumulative arsenic exposure ( $\geq 10.5 \mathrm{mg} / \mathrm{L}$ x year), whereas catalase polymorphism was not associated with hypertension. The results also showed that the association between MnSOD, NAD(P)H oxidase, and eNOS polymorphisms and risk of hypertension were more pronounced in subjects with high triglyceride level. A study of a population of West Bengal, India, exposed to arsenic via drinking water reported that the frequencies of null genotype in GSTT1 were 13.52 and $12.92 \%$ in skin-symptomatic and skin-asymptomatic individuals, and GSTM1 null genotype were 13.90 and $22.47 \%$ in skin-symptomatic and skin-asymptomatic individuals, respectively (Ghosh et al. 2006). Compared to those with GSTM1 null genotype, subjects with GST1-positive (at least one allele) had significantly higher risk of arsenic-induced skin lesions. Recently, Steinmaus et al. (2007) investigated urinary arsenic methylation patterns and genetic polymorphisms in methylenetetrahydrofolate reductase (MTHFR) and GST in 170 subjects (139 males) from an arsenic-exposed region in Argentina. MTHFR is a key enzyme in the metabolism of folate and has been linked to arsenic metabolism and toxicity (NRC 1999). Steinmaus et al. (2007) found that subjects with the TT/AA variant of MTHFR 677/1298 (associated with lower MTHFR activity) excreted a significantly higher proportion on ingested arsenic as inorganic arsenic and a smaller proportion as DMA(V). The study also reported that women with null genotype of GSTM1 excreted a significantly higher proportion of arsenic as monomethylarsenate than women with the active genotype. The study also found no association between polymorphisms in GSTT1 and arsenic methylation.

There is a report that described severe arsenic-induced neuropathy that developed only in a 5,10-methylenetetrahydrofolate-reductase (MTHFR) deficient member of a family that had been exposed to arsenic (Brouwer et al. 1992). The authors suggest that the MTHFR deficiency in this girl might explain the fact that of all the family members exposed to arsenic, only she developed severe clinical signs of arsenic poisoning. Liver disease does not appear to decrease methylation capacity in humans, at least at low levels of arsenic exposure (Buchet et al. 1982; Geubel et al. 1988).

\subsection{METHODS FOR REDUCING TOXIC EFFECTS}

This section will describe clinical practice and research concerning methods for reducing toxic effects of exposure to arsenic. However, because some of the treatments discussed may be experimental and unproven, this section should not be used as a guide for treatment of exposures to arsenic. When specific 
exposures have occurred, poison control centers and medical toxicologists should be consulted for medical advice. The following texts provide specific information about treatment following exposures to arsenic:

Tintinalli JE, Ruiz E, Krone RL, eds. 1996. Emergency medicine. A comprehensive study. American College of Emergency Physicians. 4th ed. New York, NY: The McGraw-Hill Companies, Inc.

Goldfrank RL, Flomenbaum NE, Lewin NA, et al., eds. 1998. Goldfrank's toxicologic emergencies. 6th ed. Stamford, CT: Appleton and Lange.

Ellenhorn MJ. 1997. Ellenhorn's medical toxicology. Diagnosis and treatment of human poisoning. Baltimore, MD: Williams \& Wilkins.

\subsubsection{Reducing Peak Absorption Following Exposure}

No data were located regarding the reduction of absorption after inhalation exposure to arsenic.

There are a number of methods for reducing absorption of arsenic following oral exposure. In cases of acute high-dose exposure, the removal of arsenic from the gastrointestinal tract may be facilitated by gastric lavage, stomach intubation, induced emesis, or use of cathartics (saline, sorbitol) within a few hours after ingestion (Agency for Toxic Substances and Disease Registry 1990a; Aposhian and Aposhian 1989; Campbell and Alvarez 1989; Driesback 1980; Ellenhorn and Barceloux 1988; EPA 1989e; Haddad and Winchester 1990; Kamijo et al. 1998; Stutz and Janusz 1988). However, the efficacy of several of these methods has been questioned by some authors, and in some cases, the treatments may be contraindicated. For example, vomiting and diarrhea often occur soon after ingesting arsenic, and therefore, use of an emetic or cathartic may not be necessary. Also, emesis should not be induced in obtunded, comatose, or convulsing patients (Campbell and Alvarez 1989; Ellenhorn and Barceloux 1988; EPA 1989e), and saline cathartics should be used with caution in patients with impaired renal function (Campbell and Alvarez 1989). Vantroyen et al. (2004) described a case of a massive arsenic trioxide overdose that was successfully treated by continuous gastric irrigation with sodium bicarbonate, forced diuresis, and administration of BAL and DMSA. Treatments of this sort are unlikely to be required following low-level exposures.

Another possible approach for reducing absorption following oral exposure is to administer substances that bind the arsenic in the gastrointestinal tract. For example, activated charcoal is sometimes used for this purpose (Campbell and Alvarez 1989; EPA 1989e; Stutz and Janusz 1988), although the effectiveness of this treatment is not well established. Because pentavalent arsenic is a phosphate analogue, 
administration of phosphate-binding substance such as aluminum hydroxide might possibly be useful, but this has not been investigated. Sulfhydryl compounds might be given to bind trivalent arsenic, but it seems unlikely that these would be effective under the acid conditions in the stomach, and it is not clear that such complexes would have reduced gastrointestinal absorption.

Following dermal or ocular exposure to arsenic, several measures can be taken to minimize absorption. All contaminated clothing should be removed, and contacted skin should be immediately washed with soap and water. Eyes that have come in contact with arsenic should be flushed with copious amounts of clean water (EPA 1989e; Stutz and Janusz 1988).

\subsubsection{Reducing Body Burden}

Acute arsenic intoxication may require treatment with chelating agents such as dimercaprol (BAL) and D-penicillamine. Although body burden is not necessarily reduced, these chelators bind free arsenic and serve to reduce the body's pool of biologically active arsenic. Chelation therapy is most effective when instituted within a few hours after exposure, and efficacy decreases as time after exposure increases (Agency for Toxic Substances and Disease Registry 1990a; Kamijo et al. 1998; McFall et al. 1998; Peterson and Rumack 1977).

In general, chelating agents should be used with caution, since they may have serious side effects such as pain, fever, hypotension, and nephrotoxicity (Ellenhorn and Barceloux 1988). Some water-soluble and less toxic analogues of BAL such as dimercaptosuccinic acid (DMSA), dimercaptopropyl phthalamadic acid (DMPA), and dimercaptopropane sulfonic acid (DMPS) are currently under investigation and may prove to be promising treatments for arsenic poisoning (Agency for Toxic Substances and Disease Registry 1990a; Aposhian and Aposhian 1989; Aposhian et al. 1997; Guha Mazumder 1996; Kreppel et al. 1995). However, a randomized placebo trial of 2,3-dimercaptosuccinic acid as a therapy for chronic arsenosis due to drinking contaminated water found no significant difference between patients treated with 2,3-dimercaptosuccinic acid and those treated with a placebo (Guha Mazumder et al. 1998a). $\mathrm{N}$-acetylcysteine has been used in animals to chelate arsenic (Haddad and Winchester 1990), and a human case study reported $\mathrm{N}$-acetylcysteine to be successful in treating a case of arsenic poisoning that was not responding well to BAL treatment (Martin et al. 1990). Vantroyen et al. (2004) described a case of a massive arsenic trioxide overdose that was successfully treated by continuous gastric irrigation with sodium bicarbonate, forced diuresis, and administration of BAL and DMSA. 
As discussed in Section 3.4.3, once arsenic has been absorbed into the blood stream, it undergoes methylation to yield MMA and DMA. These forms of arsenic are less toxic than inorganic arsenic and are cleared from the body by excretion in the urine. Therefore, if it were possible to enhance arsenic methylation, both body burden and toxicity of arsenic might be reduced. However, experimental evidence in animals and humans suggests that arsenic methylation is not enhanced to any significant degree by supplementation with methylation cofactors (Buchet and Lauwerys 1987; Buchet et al. 1982), presumably because it is enzyme level and not cofactor availability that is rate limiting in arsenic methylation.

\subsubsection{Interfering with the Mechanism of Action for Toxic Effects}

It is generally thought that trivalent arsenic exerts its toxic effects mainly by complexing with sulfhydryl groups in key enzymes within the body, thereby inhibiting critical functions such as gluconeogenesis and DNA repair (Aposhian and Aposhian 1989; Li and Rossman 1989). Therefore, administration of sulfhydryl-containing compounds soon after exposure could provide alternative target molecules for arsenic, and prevent inhibition of enzyme functions. In fact, many of the chelating agents discussed above (BAL, DMSA, DMPA, DMPS, N-acetylcysteine) contain sulfhydryl groups, and this may account for their efficacy.

The mechanism by which pentavalent arsenic acts is less certain. Since pentavalent arsenic is reduced in the body to the trivalent state, pentavalent arsenic may act in a similar manner as described above for trivalent arsenic. If this is the case, efforts to inhibit the reduction of pentavalent arsenic would decrease its toxicity. However, no methods are currently recognized for blocking this reduction. Pentavalent arsenic may also exert effects by acting as a phosphate analogue. As a phosphate analogue, pentavalent arsenic could potentially affect a number of biological processes, including ATP production, bone formation, and DNA synthesis. However, any effort to interfere in normal phosphate metabolism could produce serious side effects, and no method is known for selectively interfering with arsenate metabolism.

\subsection{ADEQUACY OF THE DATABASE}

Section 104(I)(5) of CERCLA, as amended, directs the Administrator of ATSDR (in consultation with the Administrator of EPA and agencies and programs of the Public Health Service) to assess whether adequate information on the health effects of arsenic is available. Where adequate information is not available, ATSDR, in conjunction with the National Toxicology Program (NTP), is required to assure the 
initiation of a program of research designed to determine the health effects (and techniques for developing methods to determine such health effects) of arsenic.

The following categories of possible data needs have been identified by a joint team of scientists from ATSDR, NTP, and EPA. They are defined as substance-specific informational needs that if met would reduce the uncertainties of human health assessment. This definition should not be interpreted to mean that all data needs discussed in this section must be filled. In the future, the identified data needs will be evaluated and prioritized, and a substance-specific research agenda will be proposed.

\subsubsection{Existing Information on Health Effects of Arsenic}

The existing data on health effects of inhalation, oral, and dermal exposure of humans and animals to inorganic and organic arsenic are summarized in Figures 3-13 and 3-14, respectively. The purpose of this figure is to illustrate the existing information concerning the health effects of arsenic. Each dot in the figure indicates that one or more studies provide information associated with that particular effect. The dot does not necessarily imply anything about the quality of the study or studies, nor should missing information in this figure be interpreted as a "data need". A data need, as defined in ATSDR's Decision Guide for Identifying Substance-Specific Data Needs Related to Toxicological Profiles (Agency for Toxic Substances and Disease Registry 1989), is substance-specific information necessary to conduct comprehensive public health assessments. Generally, ATSDR defines a data gap more broadly as any substance-specific information missing from the scientific literature.

As shown in Figure 3-13, there is a substantial database on the toxicity of inorganic arsenicals, both in humans and in animals. The oral route has been most thoroughly investigated, and reports are available on most end points of concern following acute, intermediate, and chronic exposure. The inhalation route has also been studied extensively, mainly in humans, with special emphasis on lung cancer. A number of noncancer end points have also been studied following inhalation exposure, but information on these effects is less extensive. Limited information on the effects of dermal exposure is also available in both humans and animals, focusing mainly on direct irritancy and dermal sensitization reactions. The absence of studies on other effects of inorganic arsenic following dermal exposure is probably not a critical data need, since dermal uptake of inorganic arsenic appears to be sufficiently limited that other routes of exposure (oral or inhalation) would almost always be expected to be of greater concern. 
Figure 3-13. Existing Information on Health Effects of Inorganic Arsenic

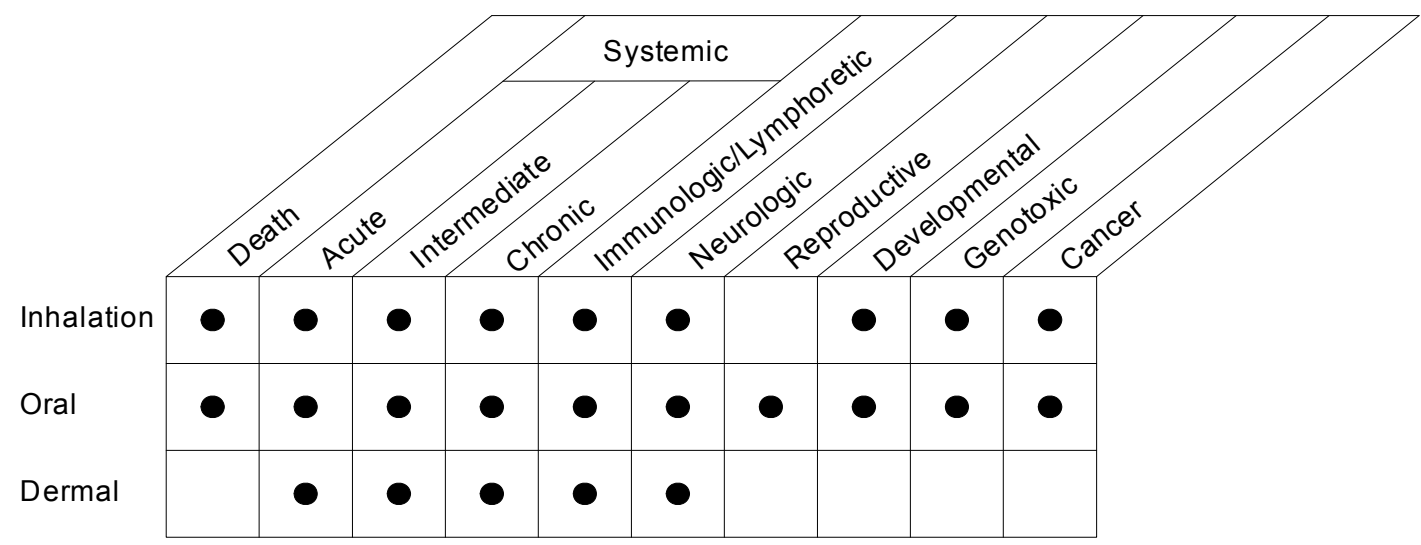

Human

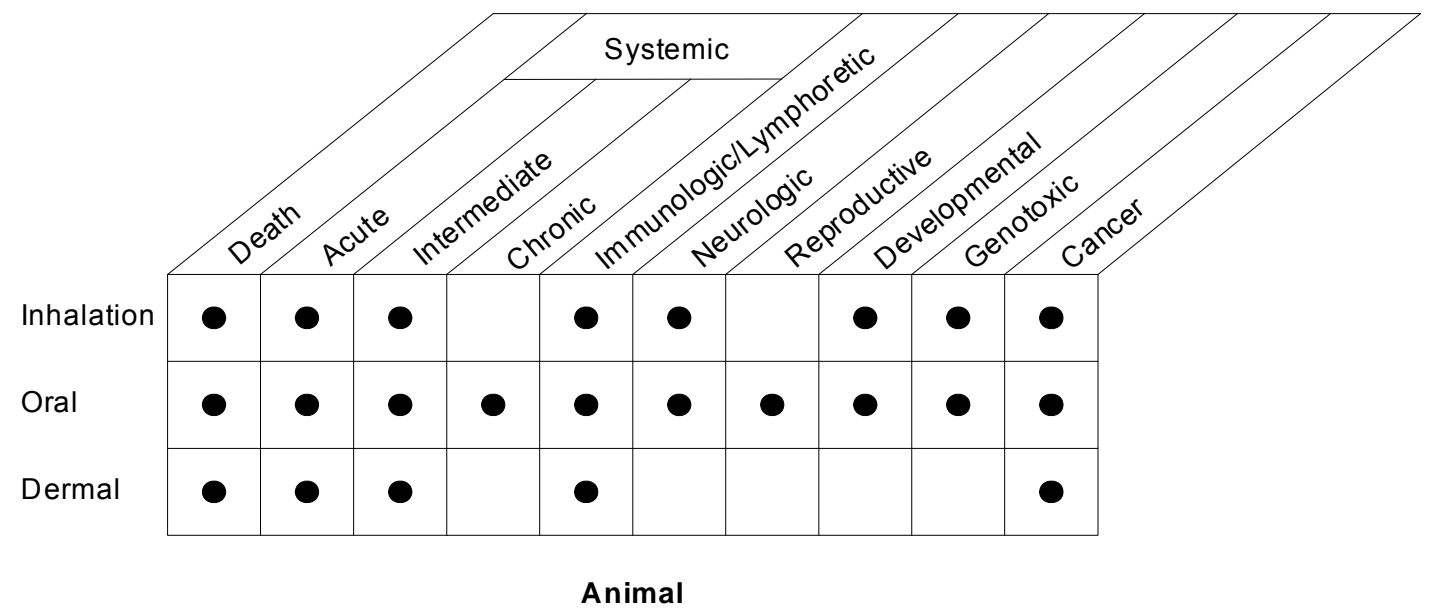

Existing Studies 
Figure 3-14. Existing Information on Health Effects of Organic Arsenic

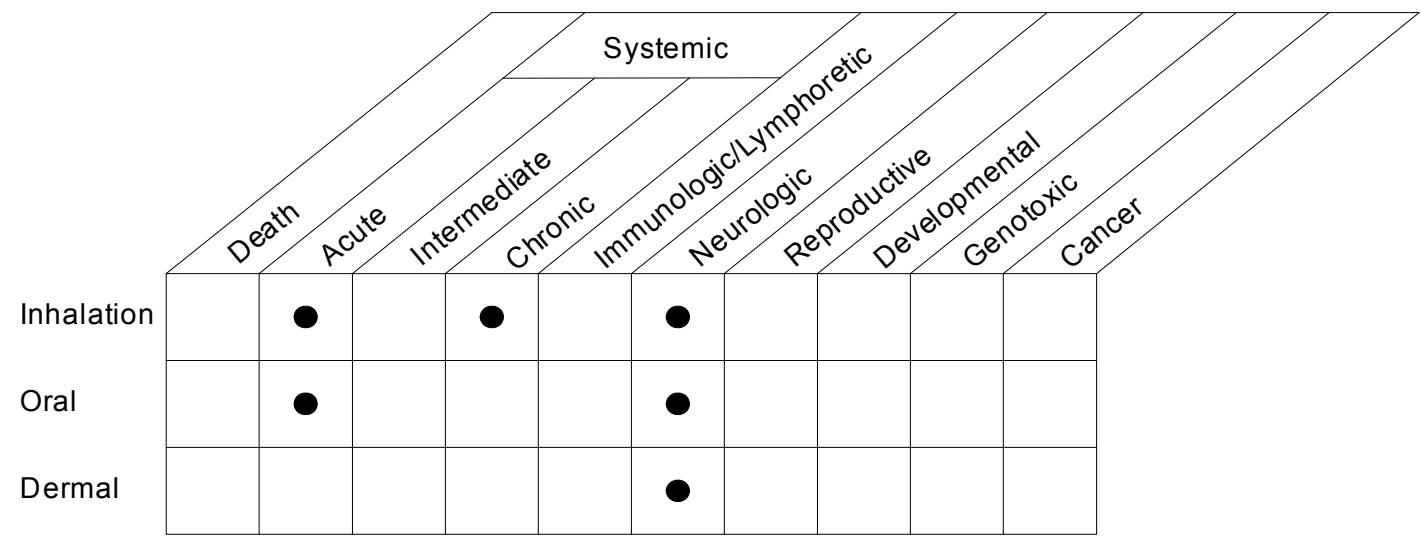

Human

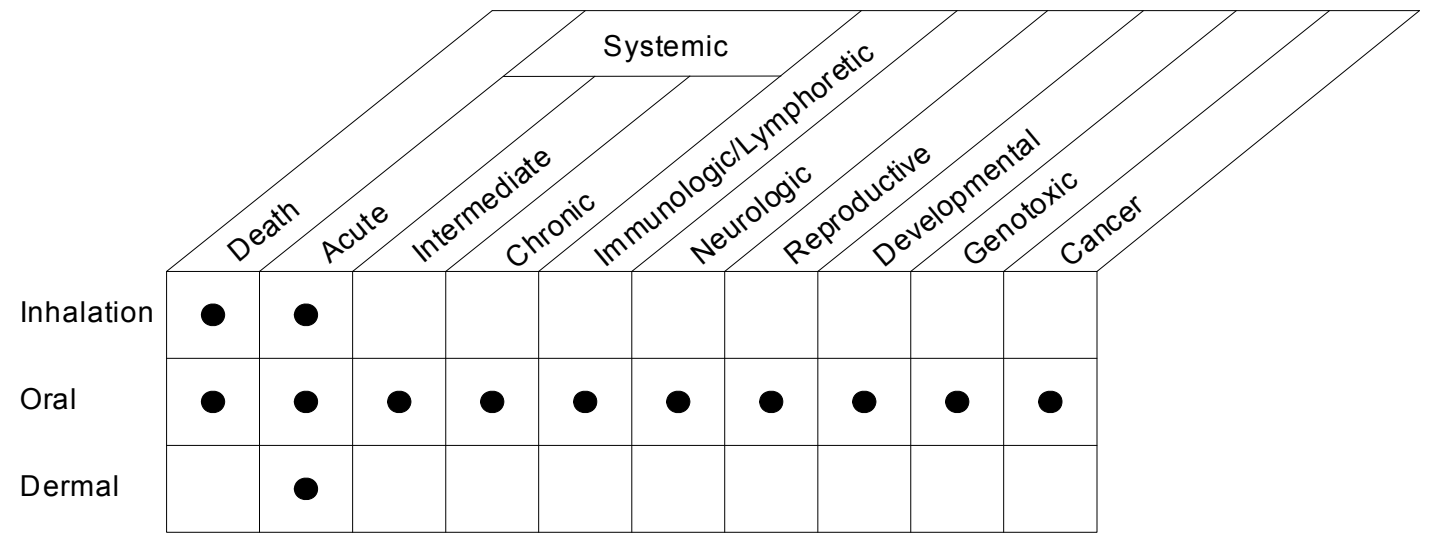

Animal

Existing Studies 
As shown in Figure 3-14, very little information is available on the effects of organic arsenic compounds in humans, although there are a number of studies in animals. These studies mainly involve the oral route, since all of these compounds are nonvolatile solids, although a few acute inhalation studies have been performed. Limited information is available on acute dermal lethality and dermal irritancy of some organic arsenicals, but data are lacking on other effects of organic arsenicals following dermal exposure. As discussed previously, in evaluating the adequacy of the database on arsenic, it is important to keep in mind that most studies in animals indicate that they are quantitatively less sensitive to arsenic than humans. For this reason, data from animal studies should be used to draw inferences about effects in humans only with caution.

\subsubsection{Identification of Data Needs}

\section{Acute-Duration Exposure.}

Inorganic Arsenicals. There is only limited information on the effects of acute inhalation exposure to arsenic in humans, but the chief symptoms appear to be irritation of the respiratory and gastrointestinal tracts (Beckett et al. 1986; Bolla-Wilson and Bleecker 1987; Dunlap 1921; Ide and Bullough 1988; Morton and Caron 1989; Pinto and McGill 1953). Quantitative data are lacking, but effects generally appear to be mild even at high-exposure levels. On this basis, it seems that risks of acute effects are probably low for inhalation exposures in the environment or near waste sites. Research to obtain a quantitative acute inhalation NOAEL value that could be used to derive an acute inhalation MRL would, therefore, be useful but not critical. There are numerous case studies in humans on the acute oral toxicity of arsenic, and the main end points (gastrointestinal irritation, pancytopenia, hepatic injury, neuropathy) are well characterized (Armstrong et al. 1984; Fincher and Koerker 1987). An acute oral MRL of $0.005 \mathrm{mg} \mathrm{As} / \mathrm{kg}$ /day was derived for inorganic arsenic based on a LOAEL for gastrointestinal symptoms and facial edema reported by Mizuta et al. (1956). Additional studies to define an acute oral NOAEL would be useful to reduce uncertainty in the MRL derivation. Acute dermal exposure is unlikely to cause serious systemic injury, but it can lead to contact dermatitis and skin sensitization (Holmqvist 1951; Pinto and McGill 1953). However, available data do not permit a quantitative estimate of the concentration of arsenic on the skin or in air, dust, soil, or water that causes these effects. Further research would be valuable to obtain a quantitative NOAEL for direct dermal effects, since humans may have dermal contact with contaminated soil or water near hazardous waste sites. 
Organic Arsenicals. Information on the acute toxicity of organic arsenicals in humans is limited to reports of gastrointestinal irritation in individuals ingesting pesticides containing organic arsenicals (Lee et al. 1995; Shum et al. 1995); these case reports provide limited dosing information. Acute lethality and systemic toxicity data exist for several compounds by inhalation, oral, and dermal exposure of animals. Inhalation data are limited to a lethality study of rats and mice exposed to MMA or DMA that reported respiratory and ocular irritation (Stevens et al. 1979). The oral acute studies consist of lethality studies for MMA (Gur and Nyska 1990; Jaghabir et al. 1988; Kaise et al. 1989), DMA (Kaise et al. 1989), and roxarsone (Kerr et al. 1963; NTP 1989b), systemic toxicity studies (or longer-term studies reporting effects within the first 2 weeks of exposure) for MMA (Irvine et al. 2006), DMA (Ahmad et al. 1999a; Chernoff et al. 1990; Cohen et al. 2001; Crown et al. 1987; Irvine et al. 2006; Kavlock et al. 1985; Rogers et al. 1981; Zomber et al. 1989), or roxarsone (NTP 1989b). For MMA, the available data suggest that the gastrointestinal tract may be the most sensitive target of toxicity; however, the study identifying the lowest LOAEL (Irvine et al. 2006) involved bolus administration and this is not an appropriate exposure route to estimate human risk for gastrointestinal effects following environmental exposure to MMA. The available animal studies for DMA have examined urinary bladder (Cohen et al. 2001) and developmental toxicity (Chernoff et al. 1990, Irvine et al. 2006; Kavlock et al. 1985; Rogers et al. 1981). For DMA, acute-duration studies in rats suggest that the urinary bladder is the most sensitive target of toxicity in rats (Cohen et al. 2001); however, there is evidence from longer-term studies that rats may be more sensitive than humans and other species for bladder effects. Thus, rat data were not considered as the basis of an acute-duration oral MRL for DMA. Other effects observed following acute exposure to DMA include developmental and maternal effects in mice (Kavlock et al. 1985; Rogers et al. 1981) and rabbits (Irvine et al. 2006) and diarrhea and vomiting in dogs receiving a bolus dose of DMA (Zomber et al. 1989). An acute-duration oral MRL was not derived for DMA because it is not known if systemic effects would occur at lower doses than the developmental effects. For roxarsone, the available data suggest that the most sensitive effect following acute oral exposure is neuropathy observed in pigs (Kennedy et al. 1986; Rice et al. 1985). At the only dose tested in this study, tremors, clonic convulsions, and equivocal evidence of myelin degeneration were observed; these were considered serious effects and not suitable for the derivation of an acute-duration oral MRL for roxarsone. Additional studies are needed for MMA, DMA, and roxarsone that examine a variety of end points in several species; studies for roxarsone should also include examination of neurological end points, which would be useful for identifying the critical targets of toxicity and establishing dose-response relationships. 


\section{HEALTH EFFECTS}

\section{Intermediate-Duration Exposure.}

Inorganic Arsenicals. Intermediate-duration inhalation exposure of humans to arsenic appears to result in respiratory tract irritation (occasionally including perforation of the nasal septum) and mild gastrointestinal tract irritation (Ide and Bullough 1988). Quantitative data are too limited (only one study, of one individual) to derive an intermediate-duration inhalation MRL. Further studies to define the NOAEL for intermediate-duration inhalation exposure of humans would be valuable, since humans could be exposed to arsenic-containing airborne dusts near smelters, chemical plants, or waste sites. Effects of intermediate-duration oral exposure are similar to those of acute oral exposure, but may also include development of vascular injury and a characteristic group of skin changes (Franzblau and Lilis 1989; Holland 1904; Wagner et al. 1979). Most studies indicate that these effects occur at doses of about $0.05 \mathrm{mg} \mathrm{As} / \mathrm{kg} /$ day or higher, but the data do not provide a firm basis for identifying the intermediateduration NOAEL. For this reason, no intermediate-duration oral MRL has been derived. Further studies to establish the NOAEL would be valuable, since humans could have intermediate-duration oral exposures to arsenic through ingestion of contaminated soil or water near smelters, chemical factories, or waste sites. Since dermal effects appear to be restricted to acute irritancy, intermediate-duration dermal studies are probably not essential.

Organic Arsenicals. No information was located on the intermediate-duration toxicity of organic arsenicals in humans. Several studies have examined the intermediate-duration oral toxicity of MMA; dietary exposure studies in rats and mice (Arnold et al. 2003) identify the gastrointestinal tract as the most sensitive target. Diarrhea and lesions in the cecum, colon, and rectum have been observed. The rat 13-week study (Arnold et al. 2003) was used as the basis of the MRL. Because rats appear to be more sensitive to the toxicity of DMA, rat studies were not considered for MRL derivation. The only non-rat study was a chronic-duration dog study reporting effects during the first 51 weeks of exposure (Zomber et al. 1989); these effects included diarrhea and vomiting. However, because DMA was administered via capsule, this study was not considered adequate for derivation of an MRL. Additional studies are needed for DMA to identify critical targets of toxicity and establish dose-response relationships in non-rat species. The available data for roxarsone suggest that neurotoxicity in pigs is the most sensitive end point. One of the two available neurotoxicity studies in pigs (Edmonds and Baker 1986) did not include sensitive tests of toxicity and was not considered for MRL derivation; the other study identified a serious LOAEL at the only dose tested and thus, was not suitable for MRL derivation. Several comprehensive studies examined the toxicity of roxarsone in rats and mice (NTP 1989b). Renal tubular damage in rats was the most sensitive end point (NTP 1989b); however, the LOAEL for this effect was 9 times higher 
than the dose associated with neurotoxicity in pigs. Additional studies are needed to establish a no effect level for neurotoxicity in pigs, which could be used to derive an intermediate duration MRL for roxarsone. Further studies on the intermediate-duration inhalation and dermal toxicity of these compounds would be valuable, especially in humans, since people may be exposed to organic arsenicals during their manufacture or use, or from materials deposited in waste sites.

\section{Chronic-Duration Exposure and Cancer.}

Inorganic Arsenicals. The target tissues of chronic-duration exposure of humans to inorganic arsenic are the same as for intermediate-duration exposure for both the oral and inhalation routes. Effects of dermal exposure appear to be restricted to direct irritation of exposed surfaces. Therefore, chronic-duration studies are probably not essential for the dermal route. Quantitative data from one study identify an inhalation exposure level of about $0.1 \mathrm{mg} \mathrm{As} / \mathrm{m}^{3}$ as the LOAEL for skin changes (Perry et al. 1948), but because there are no additional supporting studies and a NOAEL is not clearly established, a chronicduration inhalation MRL has not been derived. Additional studies in humans to define the chronic inhalation NOAEL for dermal or other effects would be valuable, since humans may be chronically exposed to arsenic dusts in air near smelters, chemical factories, or waste sites. Chronic oral exposure data from studies in humans indicate that the LOAEL for skin lesions and other effects is probably about 0.01-0.02 mg As/kg/day (10-20 $\mu \mathrm{g} \mathrm{As/kg/day),} \mathrm{and} \mathrm{that} \mathrm{the} \mathrm{NOAEL} \mathrm{is} \mathrm{probably} \mathrm{between} 0.0004$ and 0.0009 mg As/kg/day (0.4-0.9 $\mu \mathrm{g}$ As/kg/day) (Cebrián et al. 1983; EPA 1981b; Hindmarsh et al. 1977; Tseng 1977; Tseng et al. 1968). The NOAEL of $0.0008 \mathrm{mg} \mathrm{As} / \mathrm{kg} /$ day from the study by Tseng et al. (1968) is appropriate for derivation of a chronic-duration oral MRL, but an uncertainty factor of 3 was required to account for the fact that the population that constituted the no-effect group were relatively young (possibly decreasing the ability to detect dermal or other effects that increase in prevalence with age). Another issue that needs to be acknowledged, which is common to ecological studies and contributes to uncertainty, is the fact that individual doses were not available and were calculated from group mean arsenic concentrations in well water using estimated water intake parameters. For this reason, further epidemiological studies that do not rely on an ecological-based exposure assessment that would provide additional support for the threshold dose for arsenic in humans would be valuable.

There are numerous studies in humans that support the carcinogenic effects of inorganic arsenic from inhalation exposure (Enterline et al. 1987a, 1987b, 1995; Järup and Pershagen 1991; Järup et al. 1989; Lee-Feldstein 1986; Welch et al. 1982) and oral exposure (Chen et al. 1986, 1988b, 1992; Chiou et al. 1995; Ferreccio et al. 1996; Hsueh et al. 1995; Lander et al. 1975; Liu and Chen 1996; Lüchtrath 1983; 
Smith et al. 1992; Tseng 1977; Tseng et al. 1968; Yu et al. 1992; Zaldívar 1974; Zaldívar et al. 1981). Quantitative slope factors have been derived for both routes. There is a noticeable absence, however, of 2-year animal carcinogenicity studies for either the inhalation or oral route of exposure (Chan and Huff 1997). In light of the ongoing controversy over the reasons for the absence of a carcinogenic effect in animals, it seems prudent to firmly establish a negative effect in a 2-year study. The carcinogenic effects of chronic dermal exposure to inorganic arsenicals have not been studied, but dermal exposure is a relatively minor route of exposure, and these studies would not be a top priority.

The mechanism of arsenic carcinogenicity is not known, although the current view is that it functions mainly as a promoter or cocarcinogen. Further studies on the mechanism of arsenic toxicity would be particularly valuable to improve our ability to evaluate human cancer risks from inhalation or oral exposures that might occur near waste sites. Also, mechanistic studies could help in the evaluation of cancer risks from organic derivatives (see below).

Organic Arsenicals. There is very little information on the chronic toxicity of organic arsenicals in humans. One study of workers exposed to arsanilic acid did not identify any adverse effects, but no systematic, clinical, or toxicological examinations of exposed people were performed (Watrous and McCaughey 1945). Chronic toxicity studies are available for rats, mice, and dogs exposed to MMA (Arnold et al. 2003; Waner and Nyska 1988), DMA (Arnold et al. 2006; Zomber et al. 1989), and roxarsone (NTP 1989b; Prier et al. 1963). Chronic exposure to MMA results in diarrhea in rats, mice, and dogs (Arnold et al. 2003; Waner and Nyska 1988) and an increase in progressive nephropathy in rats and mice (Arnold et al. 2003). The increased incidence of progressive nephropathy was used as the basis of the chronic-duration oral MRL for MMA. For DMA, chronic exposure also resulted in an increased incidence of diarrhea and vomiting in dogs (Zomber et al. 1989) and an increased incidence of vacuolization in the urinary bladder and progressive nephropathy in mice (Arnold et al. 2006). The vacuolization in the urinary bladder was used as the basis of a chronic-duration oral MRL for DMA. The available data for chronic-exposure to roxarsone were considered inadequate for derivation of an MRL. The highest doses tested in the rat, mouse, and dog studies (NTP 1989b; Prier et al. 1963) were NOAELs. Intermediate-duration studies identify neurotoxicity in pigs as the most sensitive end point; this has not been adequately examined following chronic exposure and studies are needed.

No information was located on carcinogenic effects of organic arsenicals in humans. The carcinogenic potential of MMA (Arnold et al. 2003), DMA (Arnold et al. 2006), and roxarsone (NTP 1989b) following oral exposure has been investigated in rats and mice. No evidence of carcinogenicity was observed 
following oral exposure to MMA (Arnold et al. 2003) and equivocal evidence of carcinogenicity was found in male rats, with no evidence of carcinogenicity in female rats or in male or female mice orally exposed to roxarsone (NTP 1989b). Oral exposure to DMA resulted in an increased incidence of urinary bladder tumors in rats and no evidence of carcinogenicity in mice (Arnold et al. 2006). However, there is concern that the rat is not a good model to assess the carcinogenic potential of DMA in humans due to species differences in the toxicokinetic properties of DMA. No information was located on the carcinogenicity of organic arsenicals following inhalation or dermal exposure. Studies of humans exposed in the workplace would provide valuable information on the carcinogenic potential of organic arsenicals, particularly DMA. Studies on cancer risk following inhalation and dermal exposure to organic arsenicals are would be useful since these are possible routes of exposure for humans.

\section{Genotoxicity.}

Inorganic Arsenicals. There are several studies that suggest that inorganic arsenic may cause genotoxicity (mainly chromosomal effects) in exposed humans (Burgdorf et al. 1977; Nordenson et al. 1978), and this is supported by numerous studies in animals (Datta et al. 1986; DeKnudt et al. 1986; Nagymajtényi et al. 1985) and cultured cells (Beckman and Nordenson 1986; Casto et al. 1979; DiPaolo and Casto 1979; Lee et al. 1985; Nakamuro and Sayato 1981; Nishioka 1975; Oberly et al. 1982; Okui and Fujiwara 1986; Sweins 1983; Ulitzur and Barak 1988; Zanzoni and Jung 1980). The mechanism of genotoxicity is not known, but may be due to the ability of arsenite to interfere with DNA repair (Li and Rossman 1989) or to alter apoptosis (Pi et al. 2005) or the ability of arsenate to act as a phosphate analog. Further studies to improve our understanding of the mechanism of genotoxicity would be valuable, since this could aid in the understanding of arsenic-induced cancer risk.

Organic Arsenicals. For organic arsenicals, in vitro genotoxicity studies are available for arsenobetaine (Eguchi et al. 1997; Oya-Ohta et al. 1996), MMA (Chun and Killeen 1989a, 1989b, 1989c, 1989d; Eguchi et al. 1997; Oya-Ohta et al. 1996), DMA (Eguchi et al. 1997; Endo et al. 1992; Kato et al. 1994; Kawaguchi et al. 1996; Kitamura et al. 2002; Kuroda et al. 2004; Moore et al. 1997a; Oya-Ohta et al. 1996; Rasmussen and Menzel 1997; Rin et al. 1995; Tezuka et al. 1993; Ueda et al. 1997; Yamanaka et al. 1989b, 1993, 1995, 1997), and roxarsone (Matthews et al. 1993; NTP 1989b; Storer et al. 1996) and in vivo studies are available for DMA (Kashiwada et al. 1998; Yamanaka and Okada 1994; Yamanaka et al. 1989a, 1989b, 1993, 2001). The results of these studies suggest that DMA and roxarsone are clastogenic and can cause DNA strand breaks. Additional in vivo studies are needed to evaluate the genotoxic potential of MMA and roxarsone. 


\section{Reproductive Toxicity.}

Inorganic Arsenicals. Several studies have examined reproductive function in populations living in Bangladesh or India exposed to high levels of arsenic in drinking water and found increases in spontaneous abortions/stillbirths or preterm births (Ahmad et al. 2001; von Ehrenstein et al. 2006); another study in U.S. women did not find an increase in adverse reproductive outcomes (Aschengrau et al. 1989). Available animal studies did not find evidence for reproductive effects following inhalation or oral exposure (Holson et al. 1999, 2000), except for a trend toward decreased pups per litter in mice in a 3-generation study (Schroeder and Mitchener 1971) that is consistent with embryolethality observed in developmental studies of inorganic arsenic. Studies on spermatogenesis and reproductive success in arsenic-exposed workers would be valuable in evaluating whether there are significant reproductive risks of arsenic in humans, and this could be further strengthened by studies including histopathological examination of reproductive tissues (which was not done in the existing studies) in animals.

Organic Arsenicals. No information was located on reproductive effects of organic arsenicals in humans and no inhalation or dermal exposure animal studies were located. Intermediate- and chronic-duration oral studies for MMA (Arnold et al. 2003), DMA (Arnold et al. 2006), and roxarsone (NTP 1989b) have not reported histological damage to reproductive tissues. Decreases in pregnancy rate and male fertility index were observed in a two-generation study in rats (Schroeder 1994) and a single generation study in mice (Prukop and Savage 1986) exposed to MMA; the poor reporting in the Prukop and Savage (1986) study limits its usefulness in assessing reproductive toxicity. However, in the two-generation study, the differences between control and exposed rats were not statistically different; the effect was considered biologically significant because effects observed in the exposed rats were outside the range found in historical controls. Another reproductive performance study to confirm these results would be useful. No reproductive effects were observed in a two generation study in rats exposed to DMA (Rubin et al. 1989).

\section{Developmental Toxicity.}

Inorganic Arsenicals. There are several epidemiological studies that suggest that inhalation (Ihrig et al. 1998; Nordström et al. 1978a, 1978b, 1979a, 1979b) or oral (Hopenhayn et al. 2003a; Yang et al. 2003) exposure to inorganic arsenic might increase the risk of low birth weight, congenital defects, or abortion in exposed women. These studies do not establish that arsenic was responsible, since all involved exposures to other chemicals or risk factors, but do suggest that additional studies on developmental 
parameters in humans exposed to arsenic would be valuable in determining whether this is an effect of concern. Other human studies have not found significant associations between arsenic levels in drinking water and increased neonatal deaths or infant mortality (von Ehrenstein et al. 2006) or the increase in congenital heart defects (Zierler et al. 1988) or neural tube defects (Brender et al. 2006). Studies in animals support the view that oral, inhalation, and parenteral exposure to inorganic arsenic can all increase the incidence of fetotoxicity and teratogenicity, although this appears to occur only at doses that are toxic or even lethal to the dams (Baxley et al. 1981; Beaudoin 1974; Carpenter 1987; Ferm and Carpenter 1968; Ferm et al. 1971; Hanlon and Ferm 1986; Holson et al. 1999, 2000; Hood and Bishop 1972; Hood and Harrison 1982; Hood et al. 1978; Mason et al. 1989; Nagymajtényi et al. 1985; Nemec et al. 1998; Stump et al. 1999; Willhite 1981). There are also some data to suggest that it may increase the risk of transplacental cancer in humans (Smith et al. 2006) and animals (Waalkes et al. 2003). Thus, additional studies in animals may be useful in defining the mechanisms of these developmental effects and in identifying the time of maximum susceptibility of the fetus, but such studies probably will not help identify a safe exposure level for humans.

Organic Arsenicals. No information was located regarding developmental effects in humans after oral or inhalation exposure to organic arsenicals. Animal studies conducted in rats (Chernoff et al. 1990; Irvine et al. 2006; Rogers et al. 1981), mice (Kavlock et al. 1985; Rogers et al. 1981), and rabbits (Irvine et al. 2006) have examined the developmental toxicity of organic arsenicals. Decreases in fetal body weights and delays in ossification were commonly reported at maternally toxic (decreases in body weight gain) doses of DMA (Irvine et al. 2006; Kavlock et al. 1985; Rogers et al. 1981). However, one study found increases in the percentage of fetuses with irregular palatine rugae at DMA doses not associated with maternal toxicity (Rogers et al. 1981). This effect has not been reported in other studies and additional developmental studies are needed to confirm the finding. In view of the apparent differences in susceptibility between animals and humans, it would be valuable to investigate whether there are any measurable effects on development in humans exposed to organic arsenicals in the workplace or the environment.

\section{Immunotoxicity.}

Inorganic Arsenicals. No studies were located on immunotoxic effects in humans after oral exposure to inorganic arsenic. One inhalation study in humans (Bencko et al. 1988), an inhalation study in animals (Aranyi et al. 1985), one oral study in animals (Kerkvliet et al. 1980), and one intratracheal instillation study in animals (Sikorski et al. 1989) suggest that arsenic causes little or no functional impairment of the 
immune system, but one inhalation study in animals found decreased pulmonary bactericidal activity and increased susceptibility to streptococcal infection in exposed mice (Aranyi et al. 1985). Additional studies (both in humans and animals) would be valuable to investigate this end point further. Dermal exposure of humans to high levels of arsenic dusts may cause dermal sensitization (Holmqvist 1951), but the dose and time dependence of this phenomenon are not known. Studies to determine whether dermal sensitization occurs in people with low level dermal exposures to arsenic in dust or soil, such as might occur for residents near an arsenic-containing waste site, would be valuable in assessing the significance of this effect to nonoccupationally exposed populations.

Organic Arsenicals. No information was located on the effect of organic arsenicals exposure in humans or animals on immune function. Since there are suggestions that inorganic arsenic may cause some changes in the immune system, studies on possible immune effects of the common organic arsenicals might be helpful.

\section{Neurotoxicity.}

Inorganic Arsenicals. There is convincing evidence from studies in humans that inorganic arsenic can cause serious neurological effects, both after inhalation (Beckett et al. 1986; Bencko et al. 1977; Blom et al. 1985; Buchancová et al. 1998; Calderón et al. 2001; Danan et al. 1984; Feldman et al. 1979; Gerr et al. 2000; Lagerkvist and Zetterlund 1994; Morton and Caron 1989) and oral exposure (Armstrong et al. 1984; Bartolome et al. 1999; Chakraborti et al. 2003a, 2003b; Civantos et al. 1995; Cullen et al. 1995; Danan et al. 1984; EPA 1977a; Feldman et al. 1979; Fincher and Foy et al. 1992; Franzblau and Lilis 1989; Guha Mazumder et al. 1988; Hindmarsh et al. 1977; Huang et al. 1985; Fincher and Koerker 1987; Levin-Scherz et al. 1987; Lewis et al. 1999; Mizuta et al. 1956; Muzi et al. 2001; Quatrehomme et al. 1992; Silver and Wainman 1952; Szuler et al. 1979; Tsai et al. 2003; Uede and Furukawa 2003; Vantroyen et al. 2004; Wagner et al. 1979). This is based mainly on clinical observations and neurological examinations of exposed persons. Available studies provide a reasonable estimate of LOAEL and NOAEL values by the oral route, but similar data are lacking for the inhalation route. Further studies designed to identify the threshold for neurological effects in humans exposed by the inhalation route would be valuable, since humans may be exposed to arsenic dusts in air from smelters, chemical factories, or waste sites. Adult animals appear to be much less susceptible than humans to the neurological effects of inorganic arsenic, so studies in adult animals would probably not help in estimation of a safe exposure limit. However, in light of recent findings of possible associations between arsenic in drinking water and neurobehavioral alterations in children (Tsai et al. 2003; von Ehrenstein et 
al. 2007; Wang et al. 2007; Wasserman et al. 2004, 2007), studies in animals, in which confounding can be eliminated, may be warranted.

Organic Arsenicals. Information on neurological effects of organic arsenicals in humans is limited to an occupational study that did not find increases in the frequency of central or peripheral nervous system complaints (Watrous and McCaughey 1945) and a case report of a women of a women reporting numbness and tingling of the fingertips, toes, and circomoral region who was exposed to organic arsenic in soup (Luong and Nguyen 1999). Neurological effects have also been observed in some animal studies. Decreases spontaneous motility, ataxia, and increased startle response were observed in mice exposed to a single high dose of DMA (Kaise et al. 1989). Degeneration of myelin and axons were observed in several studies involving oral exposure of pigs to roxarsone (Edmonds and Baker 1986; Kennedy et al. 1986; Rice et al. 1985). Hyperexcitability, ataxia, and trembling have also been observed in rats and mice orally exposed to roxarsone (Kerr et al. 1963; NTP 1989b). These findings suggest that more extensive investigations of the neurotoxic potential of roxarsone and other organic arsenicals would be valuable to determine the potential human health risk from these compounds, since humans could be exposed during the manufacture or use of these compounds, or near waste sites where they have been deposited.

Epidemiological and Human Dosimetry Studies. Numerous epidemiologic studies of humans exposed to inorganic arsenic by the oral and inhalation routes constitute the database on arsenic-related cancer and noncancer human health effects. As with virtually all epidemiologic investigations, these studies are limited by possible confounding from factors such as smoking, exposure to other chemicals, and differences in population characteristics (e.g., nutritional state, metabolism, and toxicokinetics) that inhibit extrapolation of study results to a wider population. Moreover, many of these studies lack good dose estimates for study participants. Some studies lack quantitative data altogether. For this reason, improved data on confounding factors and improved methods of human dosimetry would be valuable in any further human epidemiologic studies of arsenic, either in the workplace or in the general environment. Recent work has broadened the qualitative dose-response information beyond the highly exposed Taiwanese population, but additional studies of persons with lower exposure levels would be especially valuable for risk assessments for the U.S. population. From a public health standpoint, well designed studies of common noncancer health outcomes (e.g., cardiovascular disease and diabetes) could be more important than additional studies of cancer. Availability of methods for biomonitoring of exposure are discussed below. 


\section{Biomarkers of Exposure and Effect.}

Exposure. There are sensitive and specific methods for measuring arsenic in blood, urine, hair, nails, and other tissues, and this is the approach normally employed for measuring arsenic exposure in humans. Usually total arsenic is measured, but methods are available for measuring inorganic arsenic and each of the organic derivatives separately. Urinary levels are generally considered to be the most reliable indication of recent exposures (Enterline et al. 1987a; Milham and Strong 1974; Pinto et al. 1976; Polissar et al. 1990), but if a high urinary level is present, care must be taken to account for the presence of nontoxic forms of arsenic from the diet. Blood levels are sometimes used to evaluate the status of acutely poisoned individuals (Driesback 1980; Heydorn 1970; Hindmarsh and McCurdy 1986; Valentine et al. $1979,1981)$, but this approach is not generally useful for biomonitoring of long-term exposure to low levels. Hair and nails provide a valuable indication of exposures that occurred 1-10 months earlier (Agahian et al. 1990; Bencko et al. 1986; Choucair and Ajax 1988; EPA 1977a, 1981b; Milham and Strong 1974; Valentine et al. 1979; Yamauchi et al. 1989), although care must be taken to exclude external contamination of these samples. Cumulative urinary arsenic levels may be used to derive a quantitative estimate of exposure (Enterline et al. 1987a; Pinto et al. 1976), but data on the quantitative relation between exposure and arsenic levels in nails and hair were not located. Efforts to establish an algorithm for estimating past exposure levels from hair or nail levels would be valuable in quantifying average long-term exposure levels in people where repeated urinary monitoring is not feasible.

Effect. The effects of arsenic are mainly nonspecific, but the combined presence of several of the most characteristic clinical signs (e.g., nausea, diarrhea, peripheral neuropathy, anemia, vascular lesions, hyperkeratinization, hyperpigmentation) is usually adequate to suggest arsenic intoxication. Although there are standard clinical methods for detecting and evaluating each of these effects, there are no recognized methods for identifying early (preclinical) effects in exposed persons. Neurophysiological measurements of nerve conduction velocity or amplitude have been investigated (Goebel et al. 1990; Jenkins 1966; Le Quesne and McLeod 1977; Morton and Caron 1989; Murphy et al. 1981), but at present, this approach does not seem to offer much advantage over a standard neurological examination. Changes in urinary excretion levels of several heme-related metabolites appear to be a good indication of preclinical effects of arsenic toxicity in animals (Albores et al. 1989; Sardana et al. 1981; Woods and Fowler 1978; Woods and Southern 1989), but this has not been established in humans and is not specific for arsenic-induced effects. Further efforts to develop these approaches and to identify other more specific biochemical or physiological indicators of arsenic-induced effects would be very valuable in monitoring the health of persons exposed to low levels of arsenic in the environment or near waste sites. 
Absorption, Distribution, Metabolism, and Excretion. Available data from toxicokinetic studies in humans reveal that arsenates and arsenites are well absorbed following both oral and inhalation exposure. Data on distribution are limited, but it appears that arsenic is transported to nearly all tissues. Metabolism involves mainly reduction-oxidation reactions that interconvert $\mathrm{As}(+5)$ and $\mathrm{As}(+3)$ and methylation of As(+3) to yield MMA and DMA. Most arsenic is rapidly excreted in the urine as a mixture of inorganic arsenics, MMA, and DMA, although some may remain bound in tissues (especially skin, hair, and fingernails). These findings are strongly supported by numerous studies in animals. Because methylation represents a detoxification pathway, an area of special interest is the capacity of the human body to methylate inorganic arsenic. Limited data suggest that the methylation system might begin to become saturated at intakes of about 0.2-1 mg As/day (Buchet et al. 1981b; Marcus and Rispin 1988), but this is uncertain. Further studies to define the rate and saturation kinetics of whole-body methylation in humans would be especially helpful in evaluating human health risk from the low levels of arsenic intake that are usually encountered in the environment. Along the same line, further studies to determine the nature and magnitude of individual variations in methylation capacity and how this depends on diet, age, and other factors would be very useful in understanding and predicting which members of a population are likely to be most susceptible.

The toxicokinetics of dermal exposure have not been studied. It is usually considered that dermal uptake of arsenates and arsenites is sufficiently slow that this route is unlikely to be of health concern (except that due to direct irritation), but studies to test the validity of this assumption would be valuable. Also, dermal uptake of organic arsenicals could be of concern, and quantitative data on the rate and extent of this would be helpful in evaluating risks from application of arsenical pesticides or exposures to organic arsenicals in waste sites.

Comparative Toxicokinetics. Available toxicity data indicate that arsenic causes many of the same effects in animals that are observed in humans, but that animals are significantly less sensitive. The basis for this difference in susceptibility is not certain but is probably mainly a result of differences in absorption, distribution, metabolism, or excretion. For example, rats strongly retain arsenic in red blood cells (Lanz et al. 1950), while humans (and most other species) do not. Similarly, marmoset monkeys do not methylate inorganic arsenic (Vahter and Marafante 1985; Vahter et al. 1982), while humans and other animal species do. Because of these clear differences in toxicity and toxicokinetics between species, further comparative toxicokinetic studies that focus on the mechanistic basis for these differences would be very valuable. At a minimum, this would help clarify which laboratory species are the most useful 
models for humans and could ultimately lead to development of a PBPK model that would permit reliable extrapolation of observations across species.

Methods for Reducing Toxic Effects. There are a number of general methods for reducing the absorption of arsenic in the gastrointestinal tract and skin, but there are currently no methods for reducing the absorption of arsenic from the lungs. The removal of arsenic from the gastrointestinal tract is usually facilitated by the use of emetics, cathartics, lavages, or activated charcoal (Agency for Toxic Substances and Disease Registry 1990a; Aposhian and Aposhian 1989; Campbell and Alvarez 1989; Driesback 1980; Ellenhorn and Barceloux 1988; EPA 1989e; Haddad and Winchester 1990; Mitra et al. 2004; Stutz and Janusz 1988). Studies that investigate the effects of phosphate-binding chemicals (aluminum hydroxide) and nonabsorbable sulfhydryl compounds on the absorption of pentavalent and trivalent arsenic, respectively, may be useful in developing treatments that are more specific to arsenic intoxication. Once arsenic is in the body, treatment usually involves the use of one or more chelators, such as BAL or penicillamine. However, these agents often exhibit adverse side effects (Agency for Toxic Substances and Disease Registry 1990a; Ellenhorn and Barceloux 1988), and are generally only applied following high-dose acute exposure. Further studies investigating the efficacy of less toxic arsenic chelators, such as DMSA, DMPA, DMPS, and N-acetyl cysteine, may lead to the development of safer treatment methods. Studies on the efficacy of chelators and agents to enhance methylation and elimination in treatment of chronic arsenic exposure would also be helpful, as available treatment methods for chronic arsenic exposure are limited. Trivalent arsenic is generally believed to exert toxic effects by binding to the vicinal sulfhydryl group of key enzymes, thereby interfering with a number of biological processes, such as gluconeogenesis and DNA repair (Li and Rossman 1989; Szinicz and Forth 1988). Since pentavalent arsenic may need to be reduced in the body to the trivalent state before it can exert toxic effects, studies that investigate methods for blocking this conversion may lead to a method for interfering with the mechanism of action for pentavalent arsenic. The insufficient intake of calcium, animal protein, folate, selenium, and fiber may enhance the toxic effects of inorganic arsenic (Mitra et al. 2004), but it is not known if dietary supplementation will prove effective in patients who already show arsenic-induced symptoms.

Children's Susceptibility. Data needs relating to both prenatal and childhood exposures, and developmental effects expressed either prenatally or during childhood, are discussed in detail in the Developmental Toxicity subsection above. 
A majority of the data on the effects of exposure of humans to arsenic has focused on adults. Although a few studies of acute poisoning and chronic exposure specifically describe children (Borgoño et al. 1980; Concha et al. 1998a, 1998b, 1999; Foy et al. 1992; Kersjes et al. 1987; Rosenberg 1974; Zaldívar 1974; Zaldívar and Guillier 1977), in general, data are lacking. Specifically, although there is a substantial database on the effect of arsenic on animal development, there are few data describing developmental effects in humans. Additional research in this area, using populations in areas of endemic arsenic exposure, would be useful.

Although there is no reason to suspect that the pharmacokinetics of arsenic differs in children and adults, there are few data available on this topic. Research on absorption, distribution, metabolism, and excretion in children would aid in determining if children are at an increased risk, especially in areas where chronic exposure to an environmental source occurs. With regard to exposure during development, additional research on maternal kinetics, and transfer via breast milk would be useful in obtaining a more complete picture of prenatal and neonatal development, especially with regard to neural development and the possible development of childhood cancer.

Child health data needs relating to exposure are discussed in Section 6.8.1, Identification of Data Needs: Exposures of Children.

\subsubsection{Ongoing Studies}

A number of researchers are continuing to investigate the toxicity and toxicokinetics of arsenic.

Table 3-18 summarizes studies being sponsored by agencies of the U.S. federal government (FEDRIP 2007). Additional research is being sponsored by industry groups and other agencies, and research is also ongoing in a number of foreign countries. 


\section{Table 3-18. Ongoing Studies on Health Effects of Arsenic, Federally Funded}

\begin{tabular}{|c|c|c|c|}
\hline Investigator & Affiliation & Title & Sponsor \\
\hline Ahsan, $\mathrm{H}$ & $\begin{array}{l}\text { Columbia University, New } \\
\text { York, New York }\end{array}$ & $\begin{array}{l}\text { Chemoprevention of arsenic-induced skin } \\
\text { cancer }\end{array}$ & $\mathrm{NCl}$ \\
\hline Ahsan, $\mathrm{H}$ & $\begin{array}{l}\text { Columbia University, New } \\
\text { York, New York }\end{array}$ & $\begin{array}{l}\text { Genetic susceptibility to arsenic-induced } \\
\text { skin cancer }\end{array}$ & $\mathrm{NCl}$ \\
\hline Andrew, A & $\begin{array}{l}\text { Darmouth College, } \\
\text { Hanover, New Hampshire }\end{array}$ & Bladder cancer prognostic indicators & $\mathrm{NCl}$ \\
\hline Beckman, $\mathrm{K}$ & $\begin{array}{l}\text { Children's Hospital and } \\
\text { Research Center, Oakland, } \\
\text { California }\end{array}$ & $\begin{array}{l}\text { Fetal arsenic-nutrient interaction in adult- } \\
\text { onset cancer }\end{array}$ & NIEHS \\
\hline Bodwell, J & $\begin{array}{l}\text { Darmouth College, } \\
\text { Hanover, New Hampshire }\end{array}$ & $\begin{array}{l}\text { Arsenic effects on glucocorticoid receptor } \\
\text { action }\end{array}$ & NIEHS \\
\hline Calderon, $\mathrm{R}$ & $\begin{array}{l}\text { EPA, Research Triangle } \\
\text { Park, North Carolina }\end{array}$ & $\begin{array}{l}\text { Arsenic-induced skin conditions identified in } \\
\text { Southwest United States }\end{array}$ & HEERL \\
\hline Christiani, D & $\begin{array}{l}\text { Harvard University, Boston, } \\
\text { Massachusetts }\end{array}$ & Arsenic and health in Bangladesh & NIEHS \\
\hline Dong, Z & $\begin{array}{l}\text { University of Minnesota, } \\
\text { Minneapolis, Minnesota }\end{array}$ & $\begin{array}{l}\text { Molecular basis of arsenic-induced cell } \\
\text { transformation }\end{array}$ & $\mathrm{NCl}$ \\
\hline Finnell, R & $\begin{array}{l}\text { Texas A \& M University } \\
\text { College Station, Texas }\end{array}$ & $\begin{array}{l}\text { Sensitive genotypes to arsenic as a model } \\
\text { environmental toxicant }\end{array}$ & NIEHS \\
\hline Frenkel, K & $\begin{array}{l}\text { New York University, New } \\
\text { York, New York }\end{array}$ & $\begin{array}{l}\text { Metal induced inflammatory factors, } \\
\text { oxidative stress, and suppression }\end{array}$ & NIEHS \\
\hline Futscher, B & $\begin{array}{l}\text { University of Arizona, } \\
\text { Tucson, Arizona }\end{array}$ & $\begin{array}{l}\text { Epigenetic remodeling by environmental } \\
\text { arsenicals }\end{array}$ & $\mathrm{NCl}$ \\
\hline Gamble, M & $\begin{array}{l}\text { Columbia University, New } \\
\text { York, New York }\end{array}$ & Nutritional influences on arsenic toxicity & NIEHS \\
\hline Germolec, D & $\begin{array}{l}\text { NIH, Research Triangle } \\
\text { Park, North Carolina }\end{array}$ & $\begin{array}{l}\text { The role of growth factors and inflammatory } \\
\text { mediators in arsenic-induced } \\
\text { dermatotoxicity }\end{array}$ & NIEHS \\
\hline Guallar, E & $\begin{array}{l}\text { Johns Hopkins University, } \\
\text { Baltimore, Maryland }\end{array}$ & $\begin{array}{l}\text { Mercury, arsenic, and carotid } \\
\text { atherosclerosis }\end{array}$ & NIEHS \\
\hline $\mathrm{He}, \mathrm{K}$ & $\begin{array}{l}\text { Northwestern University, } \\
\text { Chicago, Illinois }\end{array}$ & $\begin{array}{l}\text { Trace elements and CVD risks factors } \\
\text { among young adults }\end{array}$ & NHLBI \\
\hline Hei, T & $\begin{array}{l}\text { Columbia University, New } \\
\text { York, New York }\end{array}$ & Mechanisms of arsenic carcinogenesis & NIEHS \\
\hline Huang, C & $\begin{array}{l}\text { New York University, New } \\
\text { York, New York }\end{array}$ & $\begin{array}{l}\text { Effects of arsenic on } \mathrm{PI}-3 \mathrm{~K} \text { signaling } \\
\text { pathway }\end{array}$ & $\mathrm{NCl}$ \\
\hline Hudgens, E & $\begin{array}{l}\text { EPA, Research Triangle } \\
\text { Park, North Carolina }\end{array}$ & $\begin{array}{l}\text { Study of individuals chronically exposed to } \\
\text { arsenic in drinking water }\end{array}$ & HEERL \\
\hline Hughes, M & $\begin{array}{l}\text { EPA, Research Triangle } \\
\text { Park, North Carolina }\end{array}$ & $\begin{array}{l}\text { Biomarkers of exposure: a case study with } \\
\text { inorganic arsenic }\end{array}$ & HEERL \\
\hline Hughes, M & $\begin{array}{l}\text { EPA, Research Triangle } \\
\text { Park, North Carolina }\end{array}$ & $\begin{array}{l}\text { Tissue dosimetry, metabolism, and } \\
\text { excretion of pentavalent arsenic }\end{array}$ & HEERL \\
\hline Jing, $Y$ & $\begin{array}{l}\text { New York University, New } \\
\text { York, New York }\end{array}$ & \multicolumn{2}{|l|}{ Arsenic trioxide and acute myeloid leukemia $\mathrm{NCl}$} \\
\hline
\end{tabular}




\section{Table 3-18. Ongoing Studies on Health Effects of Arsenic, Federally Funded}

\begin{tabular}{|c|c|c|c|}
\hline Investigator & Affiliation & Title & Sponsor \\
\hline Jung, $\mathrm{M}$ & $\begin{array}{l}\text { Georgetown University, } \\
\text { Washington, DC }\end{array}$ & $\begin{array}{l}\text { Epigenetic regulation by poly(ADP-ribose) } \\
\text { in response to arsenite }\end{array}$ & NIEHS \\
\hline Karin, M & $\begin{array}{l}\text { University of California San } \\
\text { Diego, La Jolla, California }\end{array}$ & $\begin{array}{l}\text { Interaction of heavy metal ions with the } \\
\text { human genome }\end{array}$ & NIEHS \\
\hline Kelsey, K & $\begin{array}{l}\text { Harvard University, Boston, } \\
\text { Massachusetts }\end{array}$ & $\begin{array}{l}\text { Arsenic mode of action in cancer-models } \\
\text { of epigenetic mechanism }\end{array}$ & NIEHS \\
\hline Liu, K & $\begin{array}{l}\text { University of New Mexico, } \\
\text { Albuquerque, New Mexico }\end{array}$ & $\begin{array}{l}\text { Oxidative mechanisms of arsenic-induced } \\
\text { carcinogenesis }\end{array}$ & NIEHS \\
\hline Markowski, V & $\begin{array}{l}\text { University of Southern } \\
\text { Maine, Portland, Maine }\end{array}$ & $\begin{array}{l}\text { Developmental arsenic exposure produces } \\
\text { cognitive impairment }\end{array}$ & NIEHS \\
\hline Martin, M & $\begin{array}{l}\text { Georgetown University, } \\
\text { Washington, DC }\end{array}$ & $\begin{array}{l}\text { Arsenic and epigenetic regulation of gene } \\
\text { expression }\end{array}$ & NIEHS \\
\hline Muscarella, D & $\begin{array}{l}\text { Cornell University Ithaca, } \\
\text { Ithaca, New York }\end{array}$ & $\begin{array}{l}\text { Arsenite effects on CD40 signaling and B- } \\
\text { cell apoptosis }\end{array}$ & NIEHS \\
\hline Nichols, $\mathrm{R}$ & $\begin{array}{l}\text { Dartmouth College, } \\
\text { Hanover, New Hampshire }\end{array}$ & Effect of arsenic on cytochrome P450 & NIEHS \\
\hline Nriagu, J & $\begin{array}{l}\text { University of Michigan, Ann } \\
\text { Harbor, Michigan }\end{array}$ & $\begin{array}{l}\text { Arsenic exposure and bladder cancer in } \\
\text { Michigan }\end{array}$ & $\mathrm{NCl}$ \\
\hline Rosen, B & $\begin{array}{l}\text { Wayne State University, } \\
\text { Detroit, Michigan }\end{array}$ & Mechanisms of arsenical transport & NIGMS \\
\hline Rosen, B & $\begin{array}{l}\text { Wayne State University, } \\
\text { Detroit, Michigan }\end{array}$ & $\begin{array}{l}\text { Metal binding domains in metallo-regulatory } \\
\text { proteins }\end{array}$ & NIAID \\
\hline Rosenblatt, A & $\begin{array}{l}\text { University of Miami, Coral } \\
\text { Gables, Florida }\end{array}$ & $\begin{array}{l}\text { Environmental arsenic and androgen } \\
\text { receptor regulation }\end{array}$ & NIEHS \\
\hline Rossman, $\mathrm{T}$ & $\begin{array}{l}\text { New York University, New } \\
\text { York, New York }\end{array}$ & $\begin{array}{l}\text { Investigation and genetic analysis of the } \\
\text { human arsenite efflux pump }\end{array}$ & NIEHS \\
\hline Schwartz, J & $\begin{array}{l}\text { Harvard University, Boston } \\
\text { Massachusetts }\end{array}$ & $\begin{array}{l}\text { Epigenetic effects of particles and metals } \\
\text { on cardiac health of an aging cohort }\end{array}$ & NIEHS \\
\hline Self, W & $\begin{array}{l}\text { University of Central } \\
\text { Florida, Orlando, Florida }\end{array}$ & $\begin{array}{l}\text { Impact of arsenicals on selenoprotein } \\
\text { synthesis }\end{array}$ & NIEHS \\
\hline Sens, D & $\begin{array}{l}\text { University of North Dakota, } \\
\text { Grand Forks, North Dakota }\end{array}$ & $\begin{array}{l}\text { Metallothionein isoform-3 urinary marker } \\
\text { bladder cancer }\end{array}$ & NIEHS \\
\hline Sheldon, L & $\begin{array}{l}\text { Dartmouth College, } \\
\text { Hanover, New Hampshire }\end{array}$ & $\begin{array}{l}\text { Arsenic, histone modification, and } \\
\text { transcription }\end{array}$ & NIEHS \\
\hline Shi, X & $\begin{array}{l}\text { University of Kentucky, } \\
\text { Lexington, Kentucky }\end{array}$ & $\begin{array}{l}\text { Mechanism of arsenic-induced } \\
\text { carcinogenesis }\end{array}$ & $\mathrm{NCl}$ \\
\hline Smith, A & $\begin{array}{l}\text { University of California, } \\
\text { Berkeley, California }\end{array}$ & Arsenic biomarker epidemiology & NIEHS \\
\hline Spallholz, J & $\begin{array}{l}\text { Texas Tech University, } \\
\text { Lubbock, Texas }\end{array}$ & $\begin{array}{l}\text { Selenium against arsenic toxicity and skin } \\
\text { lesions }\end{array}$ & $\mathrm{NCl}$ \\
\hline States, C & $\begin{array}{l}\text { University of Louisville, } \\
\text { Louisville, Kentucky }\end{array}$ & $\begin{array}{l}\text { Arsenic induced miotic arrest associated } \\
\text { apoptosis }\end{array}$ & NIEHS \\
\hline Styblo, M & $\begin{array}{l}\text { University of North } \\
\text { Carolina, Chapel Hill, North } \\
\text { Carolina }\end{array}$ & $\begin{array}{l}\text { Metabolism and toxicity of arsenic in human } \\
\text { liver }\end{array}$ & NIEHS \\
\hline
\end{tabular}


3. HEALTH EFFECTS

\section{Table 3-18. Ongoing Studies on Health Effects of Arsenic, Federally Funded}

\begin{tabular}{|c|c|c|c|}
\hline Investigator & Affiliation & Title & Sponsor \\
\hline Taylor, $\mathrm{P}$ & $\begin{array}{l}\text { Division of Cancer } \\
\text { Epidemiology and } \\
\text { Genetics, NCl, Bethesda, } \\
\text { Maryland }\end{array}$ & $\begin{array}{l}\text { Biologic specimen bank for early lung } \\
\text { cancer markers in Chinese tin miners }\end{array}$ & $\mathrm{NCl}$ \\
\hline Taylor, B & $\begin{array}{l}\text { University of Louisville, } \\
\text { Louisville, Kentucky }\end{array}$ & Arsenite inhibition of mitotic progression & NIEHS \\
\hline Vaillancourt, $\mathrm{R}$ & $\begin{array}{l}\text { University of Arizona, } \\
\text { Tucson, Arizona }\end{array}$ & Modulation of Prostaglandins by Arsenic & NIEHS \\
\hline Willett, W & $\begin{array}{l}\text { Harvard University, Boston, } \\
\text { Massachusetts }\end{array}$ & $\begin{array}{l}\text { Prospective studies of diet and cancer in } \\
\text { men and women }\end{array}$ & $\mathrm{NCl}$ \\
\hline Wright, R & $\begin{array}{l}\text { Brigham and Women's } \\
\text { Hospital, Boston, } \\
\text { Massachusetts }\end{array}$ & Metal mixtures and neurondevelopment & NIEHS \\
\hline Zhang, D & $\begin{array}{l}\text { University of Arizona, } \\
\text { Tucson, Arizona }\end{array}$ & $\begin{array}{l}\text { The protective role of Nrf } 2 \text { in arsenic- } \\
\text { induced toxicity and carcinogenicity }\end{array}$ & NIEHS \\
\hline
\end{tabular}

EPA = Environmental Protection Agency; NHEERL = National Health and Environmental Effects Research Laboratory; $\mathrm{NCI}=$ National Cancer Institute; $\mathrm{NHLBI}=$ National Heart, Lung, and Blood Institute; NIEHS = National Institute of Environmental Health Sciences; NIAID= National Institute of Allergy and Infectious Diseases;

NIGMS = National Institute of General Medical Sciences; NIH = National Institute of Health

Source: FEDRIP 2007 
This page is intentionally blank. 


\section{CHEMICAL AND PHYSICAL INFORMATION}

\subsection{CHEMICAL IDENTITY}

Information regarding the chemical identity of arsenic and some common inorganic and organic arsenic compounds are located in Tables 4-1 and 4-2, respectively.

\subsection{PHYSICAL AND CHEMICAL PROPERTIES}

Information regarding the physical and chemical properties of arsenic and some common inorganic and organic arsenic compounds is located in Tables 4-3 and 4-4, respectively.

Arsenic appears in Group $15(\mathrm{~V})$ of the periodic table, below nitrogen and phosphorus. Arsenic is classified chemically as a metalloid, having both properties of a metal and a nonmetal; however, it is frequently referred to as a metal. Elemental arsenic, which is also referred to as metallic arsenic, $(\operatorname{As}(0))$ normally occurs as the $\alpha$-crystalline metallic form, which is a steel gray and brittle solid. The $\beta$-form is a dark gray amorphous solid. Other allotropic forms of arsenic may also exist. In compounds, arsenic typically exists in one of three oxidation states, $-3,+3$, and +5 (Carapella 1992). Arsenic compounds can be categorized as inorganic, compounds without an arsenic-carbon bond, and organic, compounds with an arsenic-carbon bond. 


\section{Table 4-1. Chemical Identity of Arsenic and Selected Inorganic Arsenic Compounds $^{a}$}

\begin{tabular}{|c|c|c|c|c|}
\hline Characteristic & Arsenic & Arsenic acid & Arsenic pentoxide & Arsenic trioxide \\
\hline Synonym(s) & $\begin{array}{l}\text { Arsenic black; } \\
\text { colloidal arsenic; } \\
\text { gray arsenic, metallic } \\
\text { arsenic }\end{array}$ & Orthoarsenic acid & $\begin{array}{l}\text { Arsenic(V) oxide; } \\
\text { arsenic acid } \\
\text { anhydride; diarsenic } \\
\text { pentoxide }\end{array}$ & $\begin{array}{l}\text { Arsenic(III) oxide; } \\
\text { arsenious acid; } \\
\text { arsenious oxide; } \\
\text { white arsenic }\end{array}$ \\
\hline $\begin{array}{l}\text { Registered trade } \\
\text { name(s) }\end{array}$ & No data & $\begin{array}{l}\text { Zotox; Hi-Yield } \\
\text { Desiccant H-10; } \\
\text { Desiccant L-10; } \\
\text { Crab Grass Killer }\end{array}$ & No data & $\begin{array}{l}\text { White Arsenic; } \\
\text { Arsenicum Album }\end{array}$ \\
\hline \multirow{2}{*}{$\begin{array}{l}\text { Chemical formula } \\
\text { Chemical structure }\end{array}$} & As & $\mathrm{H}_{3} \mathrm{AsO}_{4}$ & $\mathrm{As}_{2} \mathrm{O}_{5}$ & $\mathrm{As}_{2} \mathrm{O}_{3}$ \\
\hline & As & 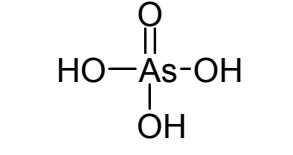 & {$\left[\mathrm{As}^{5+}\right]_{2}\left[\mathrm{O}^{2-}\right]_{5}$} & {$\left[\mathrm{As}^{3+}\right]_{2}\left[\mathrm{O}^{2-}\right]_{3}$} \\
\hline \multicolumn{5}{|l|}{ Identification numbers: } \\
\hline CAS registry & $7440-38-2$ & 7778-39-4 & $1303-28-2$ & $1327-53-3$ \\
\hline NIOSH RTECS ${ }^{b}$ & CG0525000 & CG0700000 & CG2275000 & CG3325000 \\
\hline EPA hazardous waste & D004 & D004, P010 & D004, P011 & D004, P012 \\
\hline OHM/TADS & No data & No data & No data & No data \\
\hline $\begin{array}{l}\text { DOT/UN/NA/IMDG } \\
\text { shipping }\end{array}$ & UN1558/IMDG 6.1 & $\begin{array}{l}\text { UN1553 (liquid)/ } \\
\text { UN1554 (solid)/ } \\
\text { IMDG } 6.1 \text { (liquid } \\
\text { and solid) }\end{array}$ & UN1559/IMDG 6.1 & $\begin{array}{l}\text { UN1561/ } \\
\text { IMDG } 6.1\end{array}$ \\
\hline HSDB & 509 & 431 & 429 & 419 \\
\hline EINECS & $231-148-6$ & $231-901-9$ & $215-116-9$ & $215-481-4$ \\
\hline $\mathrm{NCl}$ & No data & No data & No data & No data \\
\hline
\end{tabular}




\section{Table 4-1. Chemical Identity of Arsenic and Selected Inorganic Arsenic Compounds ${ }^{a}$}

\begin{tabular}{|c|c|c|c|c|}
\hline Characteristic & Calcium arsenate & $\begin{array}{l}\text { Gallium } \\
\text { arsenide }\end{array}$ & Sodium arsenate & Sodium arsenite \\
\hline Synonym(s) & $\begin{array}{l}\text { Calcium ortho- } \\
\text { arsenate; arsenic } \\
\text { acid, calcium salt }\end{array}$ & $\begin{array}{l}\text { Gallium mono- } \\
\text { arsenide }\end{array}$ & $\begin{array}{l}\text { Disodium arsenate, } \\
\text { dibasic; disodium } \\
\text { hydrogen arsenate; } \\
\text { arsenic acid, } \\
\text { disodium salt }\end{array}$ & $\begin{array}{l}\text { Arsenenous acid, } \\
\text { sodium salt; } \\
\text { sodium meta- } \\
\text { arsenite }\end{array}$ \\
\hline $\begin{array}{l}\text { Registered trade } \\
\text { name(s) }\end{array}$ & $\begin{array}{l}\text { Pencal; Security; } \\
\text { Turf-Cal; Chip-Cal; } \\
\text { SPRA-Cal }\end{array}$ & No data & No data & $\begin{array}{l}\text { Atlas "A"; Penite; } \\
\text { Kill-All; Chem- } \\
\text { Sen 56; Chem } \\
\text { Pels C; } \\
\text { Progalumnol } \\
\text { Double }\end{array}$ \\
\hline Chemical formula & $\mathrm{Ca}_{3}\left(\mathrm{AsO}_{4}\right)_{2}$ & GaAs & $\mathrm{Na}_{2} \mathrm{HAsO}_{4}$ & $\mathrm{NaAsO}_{2}$ \\
\hline Chemical structure & {$\left[\mathrm{Ca}^{2+}\right]_{3}\left[\begin{array}{c}\mathrm{O} \\
\mathrm{O}=\stackrel{\mathrm{As}-\mathrm{O}^{-}}{\mathrm{l}} \\
\mathrm{O}^{-}\end{array}\right]_{2}$} & Ga:As & 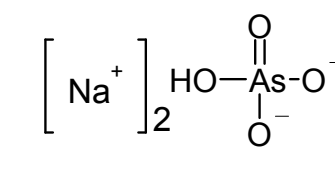 & $\|_{\mathrm{As}^{-} \mathrm{O}^{-} \mathrm{Na}^{+}}$ \\
\hline \multicolumn{5}{|l|}{ Identification numbers: } \\
\hline CAS registry & $7778-44-1$ & $1303-00-0$ & $7778-43-0$ & $7784-46-5$ \\
\hline NIOSH RTECS ${ }^{b}$ & CG0830000 & LW8800000 & CG0875000 & CG3675000 \\
\hline EPA hazardous waste & D004 & D004 & D004 & D004 \\
\hline OHM/TADS & No data & No data & No data & 7800057 \\
\hline $\begin{array}{l}\text { DOT/UN/NA/IMDG } \\
\text { shipping }\end{array}$ & UN1573/IMDG 6.1 & $\begin{array}{l}\text { UN 2803; } \\
\text { Gallium/ } \\
\text { IMDG 8.0; } \\
\text { Gallium }\end{array}$ & UN 1685/IMDG 6.1 & $\begin{array}{l}\text { UN1686 } \\
\text { (aqueous } \\
\text { solution)/UN2027 } \\
\text { (solid)/IMDG } 6.1\end{array}$ \\
\hline HSDB & 1433 & 4376 & 1675 & 693 \\
\hline EINECS & $233-287-8$ & $215-114-8$ & $231-902-4$ & $232-070-5$ \\
\hline $\mathrm{NCl}$ & No data & No data & No data & No data \\
\hline
\end{tabular}

${ }^{a}$ All information obtained from HSDB 2007 and CHEMIDplus 2007, except where noted.

${ }^{\mathrm{b}} \mathrm{RTECS} 2007$

CAS $=$ Chemical Abstracts Service; DOT/UN/NA/IMDG $=$ Department of Transportation/United Nations/North America/Intergovernmental Maritime Dangerous Goods Code; EINECS = European Inventory of Existing Chemical Substances; EPA = Environmental Protection Agency; HSDB = Hazardous Substances Data Bank; $\mathrm{NCl}=\mathrm{National}$ Cancer Institute; NIOSH = National Institute for Occupational Safety and Health; OHM/TADS = Oil and Hazardous Materials/Technical Assistance Data System; RTECS = Registry of Toxic Effects of Chemical Substances 
Table 4-2. Chemical Identity of Selected Organic Arsenic Compounds ${ }^{a}$

\begin{tabular}{|c|c|c|c|}
\hline Characteristic & Arsanilic acid & Arsenobetaine & Dimethylarsinic acid \\
\hline Synonym(s) & $\begin{array}{l}\text { (4-Aminophenyl)arsonic } \\
\text { acid; antoxylic acid; } \\
\text { atoxylic acid, Pro-Gen }\end{array}$ & $\begin{array}{l}\text { Arsonium, } \\
\text { (carboxymethyl)- } \\
\text { trimethyl-, hydroxide, } \\
\text { inner salt }\end{array}$ & $\begin{array}{l}\text { Cacodylic acid; hydroxydi- } \\
\text { methyl-arsine oxide; DMA; } \\
\text { DMAA }\end{array}$ \\
\hline $\begin{array}{l}\text { Registered trade } \\
\text { name(s) }\end{array}$ & No data & No data & $\begin{array}{l}\text { 510; Arsan; Phytar 560; } \\
\text { Rad-E-Cate } 35\end{array}$ \\
\hline Chemical formula & $\mathrm{C}_{6} \mathrm{H}_{8} \mathrm{AsNO}_{3}$ & $\mathrm{C}_{5} \mathrm{H}_{11} \mathrm{AsO}_{2}$ & $\mathrm{C}_{2} \mathrm{H}_{7} \mathrm{AsO}_{2}$ \\
\hline Chemical structure & $\mathrm{O}$ & $\mathrm{CH}_{3}$ & 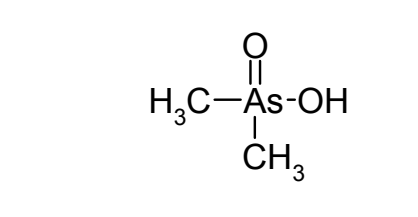 \\
\hline \multicolumn{4}{|l|}{ Identification numbers: } \\
\hline CAS registry & $98-50-0$ & $64436-13-1$ & $75-60-5$ \\
\hline NIOSH RTECS ${ }^{b}$ & CF7875000 & $\mathrm{CH} 9750000$ & $\mathrm{CH} 7525000$ \\
\hline EPA hazardous waste & D004 & No data & U136/D004 \\
\hline OHM/TADS & No data & No data & No data \\
\hline $\begin{array}{l}\text { DOT/UN/NA/IMDG } \\
\text { shipping }\end{array}$ & No data & No data & UN1572/IMDG 6.1 \\
\hline HSDB & 432 & No data & 360 \\
\hline EINECS & $202-674-3$ & No data & $200-883-4$ \\
\hline $\mathrm{NCl}$ & No data & No data & No data \\
\hline
\end{tabular}


4. CHEMICAL AND PHYSICAL INFORMATION

Table 4-2. Chemical Identity of Selected Organic Arsenic Compounds ${ }^{a}$

\begin{tabular}{|c|c|c|c|}
\hline Characteristic & $\begin{array}{l}\text { Disodium methane- } \\
\text { arsonate }\end{array}$ & Methanearsonic acid & $\begin{array}{l}\text { 3-Nitro-4-hydroxy-phenyl- } \\
\text { arsonic acid }\end{array}$ \\
\hline Synonym(s) & $\begin{array}{l}\text { DSMA; disodium } \\
\text { monomethane arsonate }\end{array}$ & $\begin{array}{l}\text { Arsonic acid, methyl-; } \\
\text { monomethylarsonic acid }\end{array}$ & $\begin{array}{l}\text { Roxarsone; 3-nitro- } \\
\text { 4-hydroxyphenylarsonic acid; } \\
\text { 3-Nitro-10 }\end{array}$ \\
\hline $\begin{array}{l}\text { Registered trade } \\
\text { name(s) }\end{array}$ & $\begin{array}{l}\text { Ansar 8100; Arrhenal; } \\
\text { Ansar DSMA Liquid; } \\
\text { Dinate; Crab-E-Rad; } \\
\text { Chipco Crab Kleen; } \\
\text { Arsinyl; Sodar; Methar; } \\
\text { Drexel DSMA Liquid; Di- } \\
\text { Tac; Ansar 184; Weed-E- } \\
\text { Rad; Versar DSMA-LQ; } \\
\text { Calar-E-Rad; Dal-E-Rad; } \\
\text { Jon-Trol; Namate }\end{array}$ & No data & No data \\
\hline Chemical formula & $\mathrm{CH}_{3} \mathrm{AsO}_{3} \mathrm{Na}_{2}$ & $\mathrm{CH}_{5} \mathrm{AsO}_{3}$ & $\mathrm{C}_{6} \mathrm{H}_{6} \mathrm{AsNO}_{6}$ \\
\hline Chemical structure & $\mathrm{H}_{3} \mathrm{C}-{\stackrel{\mathrm{As}}{\mathrm{O}}-\mathrm{O}^{-} \mathrm{Na}^{+}}_{\mathrm{O}^{-} \mathrm{Na}^{+}}$ & $\underset{\substack{\mathrm{O} \\
\mathrm{H}_{3} \mathrm{C}-\stackrel{\mathrm{A} s}{\mathrm{l}}-\mathrm{OH} \\
\mathrm{OH}}}{\mathrm{OH}}$ & $\begin{array}{c}\mathrm{O} \\
\mathrm{HO}-\mathrm{As}-1 \\
\mathrm{OH}\end{array}$ \\
\hline \multicolumn{4}{|l|}{$\begin{array}{l}\text { Identification } \\
\text { numbers: }\end{array}$} \\
\hline CAS registry & $144-21-8$ & $124-58-3$ & $121-19-7$ \\
\hline NIOSH RTECS ${ }^{b}$ & PA2275000 & PA1575000 & CY5250000 \\
\hline $\begin{array}{l}\text { EPA hazardous } \\
\text { waste }\end{array}$ & D004 & D004 & D004 \\
\hline OHM/TADS & No data & No data & No data \\
\hline $\begin{array}{l}\text { DOT/UN/NA/IMDG } \\
\text { shipping }\end{array}$ & No data & No data & No data \\
\hline HSDB & 1701 & 845 & 4296 \\
\hline EINECS & $205-620-7$ & $204-705-6$ & $204-453-7$ \\
\hline $\mathrm{NCl}$ & No data & No data & C5608 \\
\hline
\end{tabular}




\section{Table 4-2. Chemical Identity of Selected Organic Arsenic Compounds ${ }^{a}$}

\begin{tabular}{|c|c|c|c|}
\hline Characteristic & Sodium arsanilate & Sodium dimethylarsinate & $\begin{array}{l}\text { Sodium } \\
\text { methanearsonate }\end{array}$ \\
\hline Synonym(s) & $\begin{array}{l}\text { (4-Aminophenyl)arsonic acid } \\
\text { sodium salt; arsanilic acid } \\
\text { sodium salt; arsamin; atoxyl; } \\
\text { soamin; trypoxyl }\end{array}$ & $\begin{array}{l}\text { Sodium cacodylate; cacodylic } \\
\text { acid, sodium salt; sodium } \\
\text { dimethylarsonate }\end{array}$ & $\begin{array}{l}\text { Arsonic acid, methyl-, } \\
\text { monosodium salt; } \\
\text { monosodium acid } \\
\text { metharsonate; MSMA }\end{array}$ \\
\hline $\begin{array}{l}\text { Registered trade } \\
\text { name(s) }\end{array}$ & No data & $\begin{array}{l}\text { Ansar 160; Ansar 560; Bolls- } \\
\text { Eye; Chemaid; Phytar 560, } \\
\text { component of (with 012501); } \\
\text { Rad-E-Cate } 25 .\end{array}$ & $\begin{array}{l}\text { Ansar 529; Ansar 170; } \\
\text { Target MSMA; Phyban } \\
\text { H.C.; Deconate; } \\
\text { Mesamate; Bueno; } \\
\text { Monate Merge 823; } \\
\text { Dal-E-Rad; Weed-S- } \\
\text { Rad; Arsanote liquid; } \\
\text { Silvisar 550. }\end{array}$ \\
\hline Chemical formula & $\mathrm{C}_{6} \mathrm{H}_{7} \mathrm{AsNO}_{3} \mathrm{Na}$ & $\mathrm{C}_{2} \mathrm{H}_{6} \mathrm{AsO}_{2} \mathrm{Na}$ & $\mathrm{CH}_{4} \mathrm{AsO}_{3} \mathrm{Na}$ \\
\hline Chemical structure & $\mathrm{Na}^{+} \mathrm{O}=\prod_{\substack{\mathrm{O} \\
\mathrm{O}}}^{\mathrm{O}}$ & 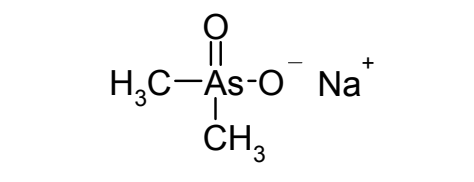 & 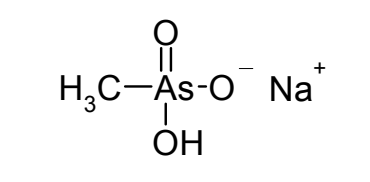 \\
\hline \multicolumn{4}{|l|}{$\begin{array}{l}\text { Identification } \\
\text { numbers: }\end{array}$} \\
\hline CAS registry & $127-85-5$ & $124-65-2$ & $2163-80-6$ \\
\hline NIOSH RTECS ${ }^{b}$ & CF9625000 & $\mathrm{CH} 7700000$ & PA2625000 \\
\hline $\begin{array}{l}\text { EPA hazardous } \\
\text { waste }\end{array}$ & D004 & D004 & D004 \\
\hline OHM/TADS & No data & No data & No data \\
\hline $\begin{array}{l}\text { DOT/UN/NA/IMDG } \\
\text { shipping }\end{array}$ & UN2473/IMDG 6.1 & UN1688/IMDG 6.1 & No data \\
\hline HSDB & 5189 & 731 & 754 \\
\hline EINECS & $204-869-9$ & 204-708-2 & $218-495-9$ \\
\hline $\mathrm{NCl}$ & C61176 & No data & C60071 \\
\hline
\end{tabular}

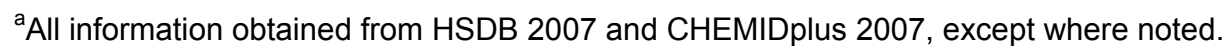

${ }^{\mathrm{b}}$ RTECS 2007

CAS = Chemical Abstracts Service; DOT/UN/NA/IMDG = Dept. of Transportation/United Nations/North America/Intergovernmental Maritime Dangerous Goods Code; EINECS = European Inventory of Existing Chemical Substances; EPA = Environmental Protection Agency; $\mathrm{HSDB}=$ Hazardous Substances Data Bank; $\mathrm{NCl}=\mathrm{National}$ Cancer Institute; NIOSH = National Institute for Occupational Safety and Health; OHM/TADS = Oil and Hazardous Materials/Technical Assistance Data System; RTECS = Registry of Toxic Effects of Chemical Substances 
Table 4-3. Physical and Chemical Properties of Arsenic and Selected Inorganic Arsenic Compounds ${ }^{\mathrm{a}}$

\begin{tabular}{|c|c|c|c|c|}
\hline Property & Arsenic & Arsenic acid & $\begin{array}{l}\text { Arsenic } \\
\text { pentoxide }\end{array}$ & Arsenic trioxide \\
\hline Molecular weight & 74.9216 & 141.944 & 229.840 & 197.841 \\
\hline Color & $\begin{array}{l}\text { Silver-gray or tin- } \\
\text { white }\end{array}$ & White $^{b}$ & White & White \\
\hline Physical state & Solid & Solid $^{b}$ & Solid & Solid \\
\hline Melting point & $817^{\circ} \mathrm{C}$ (triple point) & $35^{\circ} \mathrm{C}$ & $\begin{array}{l}\text { Decomposes at } \\
\sim 300^{\circ} \mathrm{C}\end{array}$ & $\begin{array}{l}313^{\circ} \mathrm{C} \text { (claudetite) } \\
274^{\circ} \mathrm{C} \text { (arsenolite) }\end{array}$ \\
\hline Boiling point & $614^{\circ} \mathrm{C}$ sublimes & $\begin{array}{l}\text { Loses } \mathrm{H}_{2} \mathrm{O} \text { at } \\
160^{\circ} \mathrm{C}^{\mathrm{b}}\end{array}$ & No data & $460^{\circ} \mathrm{C}$ \\
\hline Density & $\begin{array}{l}5.778 \mathrm{~g} / \mathrm{cm}^{3} \text { at } \\
25{ }^{\circ} \mathrm{C}\end{array}$ & $\sim 2.2 \mathrm{~g} / \mathrm{cm}^{3}$ & $4.32 \mathrm{~g} / \mathrm{cm}^{3}$ & $\begin{array}{l}3.865 \mathrm{~g} / \mathrm{cm}^{3} \text { (cubes) } \\
4.15 \mathrm{~g} / \mathrm{cm}^{3} \text { (rhombic } \\
\text { crystals) }\end{array}$ \\
\hline Odor & Odorless & No data & No data & Odorless \\
\hline \multicolumn{5}{|l|}{ Odor threshold: } \\
\hline Water & No data & No data & No data & No data \\
\hline Air & No data & No data & No data & No data \\
\hline \multicolumn{5}{|l|}{ Solubility: } \\
\hline Water & Insoluble & $302 \mathrm{~g} / \mathrm{L}$ at $12.5^{\circ} \mathrm{C}^{\mathrm{b}}$ & $2,300 \mathrm{~g} / \mathrm{L}$ at $20^{\circ} \mathrm{C}$ & $17 \mathrm{~g} / \mathrm{L}$ at $16^{\circ} \mathrm{C}$ \\
\hline $\begin{array}{l}\text { Organic } \\
\text { solvent(s) }\end{array}$ & No data & $\begin{array}{l}\text { Soluble in alcohol, } \\
\text { glycerol }^{b}\end{array}$ & Soluble in alcohol & $\begin{array}{l}\text { Practically insoluble in } \\
\text { alcohol, chloroform, } \\
\text { ether; soluble in } \\
\text { glycerol }\end{array}$ \\
\hline Other & $\begin{array}{l}\text { Insoluble in caustic } \\
\text { and nonoxidizing } \\
\text { acids }\end{array}$ & No data & $\begin{array}{l}\text { Soluble in acid, } \\
\text { alkali }\end{array}$ & $\begin{array}{l}\text { Soluble in dilute } \\
\text { hydrochloric acid, } \\
\text { alkali hydroxide, } \\
\text { carbonate solution }\end{array}$ \\
\hline \multicolumn{5}{|l|}{$\begin{array}{l}\text { Partition } \\
\text { coefficients: }\end{array}$} \\
\hline $\log \mathrm{K}_{\mathrm{ow}}$ & No data & No data & No data & No data \\
\hline $\log \mathrm{K}_{\mathrm{oc}}$ & No data & No data & No data & No data \\
\hline $\mathrm{pK}_{\mathrm{a}}$ & No data & $\begin{array}{l}\mathrm{pK}_{\mathrm{a} 1}=2.22 ; \mathrm{pK}_{\mathrm{a} 2}=6.98 \\
\mathrm{pK}_{\mathrm{a} 3}=11.53^{\mathrm{c}}\end{array}$ & No data & No data \\
\hline Vapor pressure & $\begin{array}{l}7.5 \times 10^{-3} \mathrm{mmHg} \text { at } \\
280^{\circ} \mathrm{C}\end{array}$ & No data & No data & $\begin{array}{l}2.47 \times 10^{-4} \mathrm{mmHg} \text { at } \\
25^{\circ} \mathrm{C}\end{array}$ \\
\hline $\begin{array}{l}\text { Autoignition } \\
\text { temperature }\end{array}$ & No data & No data & No data & Not flammable \\
\hline Flashpoint & No data & No data & No data & No data \\
\hline $\begin{array}{l}\text { Flammability } \\
\text { limits in air }\end{array}$ & No data & No data & No data & No data \\
\hline $\begin{array}{l}\text { Conversion } \\
\text { factors }\end{array}$ & No data & No data & No data & No data \\
\hline Explosive limits & No data & No data & No data & No data \\
\hline
\end{tabular}


Table 4-3. Physical and Chemical Properties of Arsenic and Selected Inorganic Arsenic Compounds ${ }^{\mathrm{a}}$

\begin{tabular}{|c|c|c|c|c|}
\hline Property & Calcium arsenate & Gallium arsenide & $\begin{array}{l}\text { Disodium } \\
\text { arsenate }\end{array}$ & Sodium arsenite \\
\hline Molecular weight & 398.072 & 144.64 & 185.91 & 130.92 \\
\hline Color & Colorless & Dark gray & Colorless $^{d}$ & White to gray-white \\
\hline Physical state & Solid & Solid & Solid $^{d}$ & Solid \\
\hline Melting point & $\begin{array}{l}\text { Decomposes on } \\
\text { heating }\end{array}$ & $1,238^{\circ} \mathrm{C}$ & $57^{\circ} \mathrm{C}^{\mathrm{d}}$ & No data \\
\hline Boiling point & No data & No data & No data & No data \\
\hline Density & $3.620 \mathrm{~g} / \mathrm{cm}^{3}$ & $\begin{array}{l}5.3176 \mathrm{~g} / \mathrm{cm}^{3} \text { at } \\
25^{\circ} \mathrm{C}\end{array}$ & $1.87 \mathrm{~g} / \mathrm{cm}^{\mathrm{d}}$ & $1.87 \mathrm{~g} / \mathrm{cm}^{3}$ \\
\hline Odor & Odorless & Garlic odor & Odorless $^{d}$ & No data \\
\hline \multicolumn{5}{|l|}{ Odor threshold: } \\
\hline Water & No data & No data & No data & No data \\
\hline Air & No data & No data & No data & No data \\
\hline \multicolumn{5}{|l|}{ Solubility: } \\
\hline Water & $0.13 \mathrm{~g} / \mathrm{L}$ at $25^{\circ} \mathrm{C}$ & $<1 \mathrm{mg} / \mathrm{mL}$ at $20^{\circ} \mathrm{C}$ & $\begin{array}{l}\text { Soluble } 1: 3 \text { parts } \\
\text { in water }^{d}\end{array}$ & Freely soluble in water \\
\hline $\begin{array}{l}\text { Organic } \\
\text { solvents }\end{array}$ & Insoluble & $\begin{array}{l}<1 \mathrm{mg} / \mathrm{mg} \text { dimethyl } \\
\text { sulfoxide, ethanol, } \\
\text { methanol, acetone }\end{array}$ & $\begin{array}{l}\text { Slightly soluble in } \\
\text { alcohol; soluble in } \\
\text { glycerol }^{d}\end{array}$ & $\begin{array}{l}\text { Slightly soluble in } \\
\text { alcohol }\end{array}$ \\
\hline Other & $\begin{array}{l}\text { Soluble in dilute } \\
\text { acids }\end{array}$ & $\begin{array}{l}\text { Soluble in } \\
\text { hydrochloric acid }\end{array}$ & $\begin{array}{l}\text { Slightly soluble in } \\
\text { alkaline solution }{ }^{d}\end{array}$ & No data \\
\hline \multicolumn{5}{|l|}{$\begin{array}{l}\text { Partition } \\
\text { coefficients: }\end{array}$} \\
\hline Log $\mathrm{K}_{\mathrm{ow}}$ & No data & No data & No data & No data \\
\hline $\log K_{o c}$ & No data & No data & No data & No data \\
\hline $\mathrm{pK}_{\mathrm{a}}$ & No data & No data & & \\
\hline Vapor pressure & $\sim 0 \mathrm{mmHg}$ at $20^{\circ} \mathrm{C}$ & No data & No data & No data \\
\hline $\begin{array}{l}\text { Autoignition } \\
\text { temperature }\end{array}$ & Not combustible & No data & No data & Not combustible \\
\hline Flashpoint & No data & No data & No data & No data \\
\hline $\begin{array}{l}\text { Flammability } \\
\text { limits in air }\end{array}$ & No data & No data & No data & No data \\
\hline $\begin{array}{l}\text { Conversion } \\
\text { factors }\end{array}$ & No data & No data & No data & No data \\
\hline Explosive limits & No data & No data & No data & No data \\
\hline
\end{tabular}

${ }^{a}$ All information from HSDB 2007, except where noted.

${ }^{b} V$ alue for arsenic acid hemihydrate

${ }^{\mathrm{C} N R C} 1999$

${ }^{d}$ Value for disodium arsenate heptahydrate 


\section{Table 4-4. Physical and Chemical Properties of Selected Organic Arsenic Compounds $^{\mathrm{a}}$}

\begin{tabular}{|c|c|c|c|}
\hline Property & Arsenilic acid & Arsenobetaine & Dimethylarsinic acid \\
\hline Molecular weight & 217.06 & $196.1^{\mathrm{b}}$ & 138.00 \\
\hline Color & White & No data & Colorless \\
\hline Physical state & Solid & Solid $^{\mathrm{b}}$ & Solid \\
\hline Melting point & $232^{\circ} \mathrm{C}$ & $\begin{array}{l}203-210^{\circ} \mathrm{C} \\
\text { (decomposes) }^{\mathrm{b}}\end{array}$ & $195^{\circ} \mathrm{C}$ \\
\hline Boiling point & & No data & $>200^{\circ} \mathrm{C}$ \\
\hline Density & $1.9571 \mathrm{~g} / \mathrm{cm}^{3}$ at $10^{\circ} \mathrm{C}$ & No data & No data \\
\hline Odor & Practically odorless & No data & Odorless \\
\hline \multicolumn{4}{|l|}{ Odor threshold: } \\
\hline Water & No data & No data & No data \\
\hline Air & No data & No data & No data \\
\hline \multicolumn{4}{|l|}{ Solubility: } \\
\hline Water & $\begin{array}{l}\text { Slightly soluble in cold } \\
\text { water; soluble in hot water }\end{array}$ & No data & $2,000 \mathrm{~g} / \mathrm{L}$ at $25^{\circ} \mathrm{C}$ \\
\hline Organic solvent(s) & $\begin{array}{l}\text { Slightly soluble in alcohol; } \\
\text { soluble in amyl alcohol; } \\
\text { insoluble in ether, acetone, } \\
\text { benzene, chloroform }\end{array}$ & No data & $\begin{array}{l}\text { Soluble in alcohol; } \\
\text { insoluble in diethyl } \\
\text { ether }\end{array}$ \\
\hline Acids & $\begin{array}{l}\text { Slightly soluble in acetic } \\
\text { acid; soluble in alkaki } \\
\text { carbonates; moderately } \\
\text { soluble in concentrated } \\
\text { mineral acids; insoluble in } \\
\text { dilute mineral acids }\end{array}$ & No data & Soluble in acetic acid \\
\hline \multicolumn{4}{|l|}{ Partition coefficients: } \\
\hline $\log \mathrm{K}_{\mathrm{ow}}$ & No data & No data & No data \\
\hline $\log \mathrm{K}_{\mathrm{oc}}$ & No data & No data & No data \\
\hline $\mathrm{pK}_{\mathrm{a}}$ & No data & $2.2^{\mathrm{C}}$ & 1.57 \\
\hline Vapor pressure & No data & No data & No data \\
\hline Henry's law constant & No data & No data & No data \\
\hline Autoignition temperature & No data & No data & No data \\
\hline Flashpoint & No data & No data & No data \\
\hline Flammability & No data & No data & Nonflammable \\
\hline Conversion factors & No data & No data & No data \\
\hline Explosive limits & No data & No data & No data \\
\hline
\end{tabular}




\section{Table 4-4. Physical and Chemical Properties of Selected Organic Arsenic Compounds $^{\mathrm{a}}$}

\begin{tabular}{|c|c|c|c|}
\hline Property & Methanearsonic acid & $\begin{array}{l}\text { 3-Nitro-4-hydroxy- } \\
\text { phenylarsonic acid }\end{array}$ & Sodium arsanilate \\
\hline Molecular weight & 139.97 & 263.03 & 239.04 \\
\hline Color & White & Pale yellow & White or creamy white \\
\hline Physical state & Solid & Solid & Solid \\
\hline Melting point & $160.5^{\circ} \mathrm{C}$ & No data & No data \\
\hline Boiling point & No data & No data & No data \\
\hline Density & No data & No data & No data \\
\hline Odor & No data & No data & Odorless \\
\hline \multicolumn{4}{|l|}{ Odor threshold: } \\
\hline Water & No data & No data & No data \\
\hline Air & No data & No data & No data \\
\hline \multicolumn{4}{|l|}{ Solubility: } \\
\hline Water & $256 \mathrm{~g} / \mathrm{L}$ at $20^{\circ} \mathrm{C}$ & $\begin{array}{l}\text { Slightly soluble in cold } \\
\text { water; soluble in about } \\
30 \text { parts boiling water }\end{array}$ & $\begin{array}{l}\text { Soluble } 1 \text { part in } 3 \text { parts } \\
\text { water }\end{array}$ \\
\hline Organic solvents & Soluble in ethanol & $\begin{array}{l}\text { Soluble in methanol, } \\
\text { ethanol, acetone; } \\
\text { insoluble in ether, ethyl } \\
\text { acetate }\end{array}$ & $\begin{array}{l}\text { Soluble } 1 \text { part in } \\
150 \text { parts alcohol; } \\
\text { practically insoluble in } \\
\text { chloroform, ether }\end{array}$ \\
\hline Acids & No data & $\begin{array}{l}\text { Soluble in acetic acid, } \\
\text { alkalies; sparingly } \\
\text { soluble in dilute mineral } \\
\text { acids }\end{array}$ & No data \\
\hline \multicolumn{4}{|l|}{ Partition coefficients: } \\
\hline $\log \mathrm{K}_{\mathrm{ow}}$ & No data & No data & No data \\
\hline $\log K_{o c}$ & No data & No data & No data \\
\hline $\mathrm{pK}_{\mathrm{a}}$ & $\mathrm{pK}_{\mathrm{a} 1}=4.1 ; \mathrm{pK}_{\mathrm{a} 2}=9.02$ & No data & No data \\
\hline Vapor pressure at $25^{\circ} \mathrm{C}$ & $<7.5 \times 10^{-8} \mathrm{mmHg}$ & No data & No data \\
\hline Henry's law constant & No data & No data & No data \\
\hline Autoignition temperature & No data & No data & No data \\
\hline Flashpoint & No data & No data & No data \\
\hline Flammability & No data & No data & No data \\
\hline Conversion factors & No data & No data & No data \\
\hline Explosive limits & No data & No data & No data \\
\hline
\end{tabular}



Table 4-4. Physical and Chemical Properties of Selected Organic Arsenic
Compounds $^{\mathrm{a}}$

\begin{tabular}{|c|c|c|c|}
\hline Property & $\begin{array}{l}\text { Disodium } \\
\text { methanearsonate }\end{array}$ & $\begin{array}{l}\text { Sodium } \\
\text { dimethylarsinate }\end{array}$ & $\begin{array}{l}\text { Sodium } \\
\text { methanearsonate }\end{array}$ \\
\hline Molecular weight & 183.93 & 159.98 & 161.95 \\
\hline Color & White & Colorless to light yellow & White \\
\hline Physical state & Solid & Solid & Solid \\
\hline Melting point & $>355^{\circ} \mathrm{C}$ & $200^{\circ} \mathrm{C}$ & $130-140^{\circ} \mathrm{C}$ \\
\hline Boiling point & No data & No data & No data \\
\hline Density & $1.04 \mathrm{~g} / \mathrm{cm}^{3}$ & $>1 \mathrm{~g} / \mathrm{cm}^{3}$ at $20^{\circ} \mathrm{C}$ & $1.55 \mathrm{~g} / \mathrm{mL}^{\mathrm{d}}$ \\
\hline Odor & No data & Odorless & Odorless \\
\hline \multicolumn{4}{|l|}{ Odor threshold: } \\
\hline Water & No data & No data & No data \\
\hline Air & No data & No data & No data \\
\hline \multicolumn{4}{|l|}{ Solubility: } \\
\hline Water & $432 \mathrm{~g} / \mathrm{L}$ at $25^{\circ} \mathrm{C}$ & $200 \mathrm{~g} / \mathrm{L}$ at $25^{\circ} \mathrm{C}$ & $580 \mathrm{~g} / \mathrm{L}$ at $20^{\circ} \mathrm{C}$ \\
\hline Organic solvents & $\begin{array}{l}\text { Soluble in methanol; } \\
\text { practically insoluble in most } \\
\text { organic solvents }\end{array}$ & No data & $\begin{array}{l}\text { Insoluble in most } \\
\text { organic solvents }\end{array}$ \\
\hline Acids & No data & No data & No data \\
\hline \multicolumn{4}{|l|}{ Partition coefficients: } \\
\hline $\log \mathrm{K}_{\mathrm{ow}}$ & $<1$ & No data & -3.10 \\
\hline $\log \mathrm{K}_{\mathrm{oc}}$ & No data & No data & No data \\
\hline $\mathrm{pK}_{\mathrm{a}}$ & $\mathrm{pK}_{\mathrm{a} 1}=4.1 ; \mathrm{pK}_{\mathrm{a} 2}=8.94$ & 6.29 & $\mathrm{pK}_{\mathrm{a} 1}=4.1 ; \mathrm{pK}_{\mathrm{a} 2}=9.02$ \\
\hline Vapor pressure at $25^{\circ} \mathrm{C}$ & $10^{-7} \mathrm{mmHg}$ & No data & $7.8 \times 10^{-8} \mathrm{mmHg}$ \\
\hline Henry's law constant & No data & No data & No data \\
\hline Autoignition temperature & No data & No data & No data \\
\hline Flashpoint & No data & No data & No data \\
\hline Flammability & Nonflammable & No data & Nonflammable \\
\hline Conversion factors & No data & No data & No data \\
\hline Explosive limits & No data & No data & No data \\
\hline
\end{tabular}

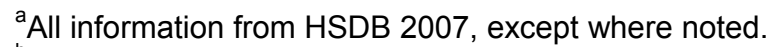

${ }^{\mathrm{b}}$ Cannon et al. 1981 (arsenobetaine as monohydrate)

${ }^{\mathrm{c}}$ Teräsahde et al. 1996

dValue for Ansar 6.6 
This page is intentionally blank. 


\section{PRODUCTION, IMPORT/EXPORT, USE, AND DISPOSAL}

\subsection{PRODUCTION}

Arsenic is presently obtained as a byproduct of the smelting of copper, lead, cobalt, and gold ores.

Arsenic trioxide is volatilized during smelting and accumulates in the flue dust, which may contain up to $30 \%$ arsenic trioxide. The crude flue dust is further refined by mixing with small amounts of galena or pyrite to prevent the formation of arsensites and roasting to yield arsenic trioxide of $90-95 \%$ purity. By successive sublimations, a purity of $99 \%$ can be obtained. Elemental arsenic can be prepared by the reduction of arsenic oxide with charcoal. Demand for elemental arsenic is limited and thus, about $95 \%$ of arsenic is marketed and consumed in combined form, principally as arsenic trioxide, which is subsequently converted to arsenic acid (Carapella 1992; Hanusch et al. 1985; USGS 2006a).

Since 1985, when the ASARCO smelter in Tacoma, Washington ceased operation, there has been no domestic production of arsenic trioxide or elemental arsenic and consequently, the United States remains entirely dependent on imports (U.S. Bureau of Mines 1988, 1990; USGS 2006a). Prior to its cessation, U.S. production of arsenic trioxide had been 7,300 metric tons in 1983, 6,800 metric tons in 1984, and 2,200 metric tons in 1985 (U.S. Bureau of Mines 1988). In 2005, arsenic trioxide was obtained from the treatment of nonferrous ores or concentrates in 14 countries. In 2005, the world's largest producer of arsenic trioxide was China, followed by Chile and Peru. China is the world leader in the production of commercial-grade arsenic followed by Japan. The United States, with an apparent demand of 8,800 metric tons in 2005 , is the world's leading consumer of arsenic, mainly for CCA. This is an increase over 2004 with an apparent demand of 6,800 metric tons, but far less than that of 2003, 21,600 metric tons (USGS 2006a).

Tables 5-1 and 5-2 list facilities in each state that manufacture or process arsenic and arsenic compounds, respectively, as well as the intended use and the range of maximum amounts of arsenic or arsenic compounds that are stored on site. In 2004, there were 58 and 361 reporting facilities that produced, processed, or used arsenic and arsenic compounds, respectively, in the United States. The data listed in Tables 5-1 and 5-2 are derived from the Toxics Release Inventory (TRI04 2006). Only certain types of facilities were required to report. Therefore, this is not an exhaustive list. Current U.S. manufacturers of selected arsenic compounds are given in Table 5-3. 


\section{Table 5-1. Facilities that Produce, Process, or Use Arsenic}

\begin{tabular}{|c|c|c|c|c|}
\hline State $^{a}$ & $\begin{array}{l}\text { Number of } \\
\text { facilities }\end{array}$ & $\begin{array}{l}\text { Minimum } \\
\text { amount on site } \\
\text { in pounds }\end{array}$ & $\begin{array}{l}\text { Maximum } \\
\text { amount on site } \\
\text { in pounds }\end{array}$ & 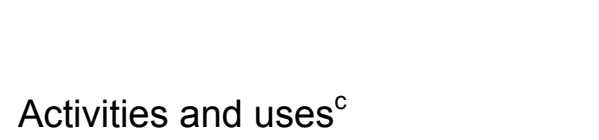 \\
\hline AK & 1 & $1,000,000$ & $9,999,999$ & 1,13 \\
\hline $\mathrm{AL}$ & 18 & 0 & $9,999,999$ & $1,2,3,5,7,8,11,12,13,14$ \\
\hline AR & 4 & 1,000 & 999,999 & 7,8 \\
\hline$A Z$ & 9 & 0 & 99,999 & $1,3,4,5,8,12,13$ \\
\hline CA & 31 & 0 & $9,999,999$ & $1,2,3,4,5,6,7,8,9,11,12,13$ \\
\hline $\mathrm{CO}$ & 8 & 0 & 999,999 & $2,7,8,11,12$ \\
\hline $\mathrm{FL}$ & 10 & 1,000 & 999,999 & $1,3,5,7,8,11,12$ \\
\hline GA & 16 & 100 & $49,999,999$ & $2,3,4,6,7,8,11,12,13,14$ \\
\hline $\mathrm{HI}$ & 1 & 10,000 & 99,999 & 8 \\
\hline IA & 4 & 100 & 99,999 & $6,7,8$ \\
\hline ID & 7 & 0 & $49,999,999$ & $1,2,3,5,6,7,9,12,13$ \\
\hline IL & 16 & 0 & 999,999 & $1,3,4,5,6,7,8,12,14$ \\
\hline IN & 17 & 0 & 999,999 & $1,3,5,6,7,8,9,12,13$ \\
\hline KY & 9 & 0 & 999,999 & $1,2,3,5,6,7,8,11$ \\
\hline LA & 8 & 0 & 999,999 & $1,2,3,7,8,12,13$ \\
\hline MA & 5 & 1,000 & 999,999 & $3,7,8$ \\
\hline MD & 9 & 0 & 999,999 & $1,2,4,5,6,7,8$ \\
\hline MI & 10 & 0 & 999,999 & $3,7,8,12,13$ \\
\hline MN & 5 & 100 & 99,999 & $1,7,8,13$ \\
\hline MO & 6 & 100 & 999,999 & $1,2,3,4,5,6,7,8$ \\
\hline MS & 9 & 1,000 & $49,999,999$ & $2,3,4,7,8,9$ \\
\hline NC & 21 & 0 & $49,999,999$ & $1,2,3,4,5,6,7,8,9,12,13,14$ \\
\hline ND & 2 & 0 & 99,999 & 8 \\
\hline NE & 1 & 0 & 99 & 8 \\
\hline NJ & 9 & 0 & 99,999 & $1,2,3,5,7,8,9$ \\
\hline NM & 2 & 10,000 & 999,999 & 7,12 \\
\hline NV & 6 & 1,000 & $99,999,999$ & $1,2,4,5,6,7,8,11,12,13$ \\
\hline NY & 4 & 0 & 99,999 & $7,8,12$ \\
\hline $\mathrm{OH}$ & 15 & 0 & 999,999 & $1,2,3,4,5,8,9,12,13$ \\
\hline OK & 9 & 0 & 99,999 & $1,2,5,6,7,9,11,12,13$ \\
\hline OR & 6 & 10,000 & 999,999 & $1,5,7,8,12$ \\
\hline PA & 24 & 0 & 999,999 & $1,2,3,5,6,7,8,10,11,12,13$ \\
\hline PR & 3 & 1,000 & 99,999 & 8,11 \\
\hline SC & 9 & 0 & 9,999,999 & $1,2,3,5,6,8,12$ \\
\hline SD & 1 & $10,000,000$ & $49,999,999$ & $1,7,11,13$ \\
\hline TN & 11 & 0 & 999,999 & $1,2,3,6,7,8,11,12,14$ \\
\hline $\mathrm{TX}$ & 29 & 0 & $49,999,999$ & $1,2,3,4,5,6,7,8,10,11,12,13,14$ \\
\hline VA & 8 & 0 & 999,999 & $2,3,7,8,10$ \\
\hline
\end{tabular}


Table 5-1. Facilities that Produce, Process, or Use Arsenic

\begin{tabular}{lcrrl}
\hline & $\begin{array}{l}\text { Minimum } \\
\text { Number of } \\
\text { facilities }^{\text {amount on site }}\end{array}$ & $\begin{array}{l}\text { Maximum } \\
\text { in pounds }^{\mathrm{b}}\end{array}$ & $\begin{array}{l}\text { amount on site } \\
\text { in pounds }^{\mathrm{b}}\end{array}$ & Activities and uses $^{\mathrm{c}}$ \\
\hline WA & 3 & 0 & 99,999 & $5,7,8$ \\
WI & 9 & 0 & 99,999 & $1,2,3,4,5,6,7,8,12$ \\
WV & 19 & 100 & 999,999 & $1,2,3,5,7,8,10,11,12$ \\
WY & 1 & 100 & 999 & 1,13 \\
\hline
\end{tabular}

${ }^{a}$ Post office state abbreviations used

${ }^{\mathrm{b}}$ Amounts on site reported by facilities in each state ${ }^{\mathrm{c}}$ Activities/Uses:
1. Produce
6. Impurity
11. Chemical Processing Aid
2. Import
7. Reactant
12. Manufacturing Aid
3. Onsite use/processing
8. Formulation Component
13. Ancillary/Other Uses
4. Sale/Distribution
9. Article Component
14. Process Impurity
5. Byproduct
10. Repackaging

Source: TRI04 2006 (Data are from 2004) 
Table 5-2. Facilities that Produce, Process, or Use Arsenic Compounds

\begin{tabular}{|c|c|c|c|c|}
\hline State $^{a}$ & $\begin{array}{l}\text { Number of } \\
\text { facilities }\end{array}$ & $\begin{array}{l}\text { Minimum } \\
\text { amount on site } \\
\text { in pounds }\end{array}$ & $\begin{array}{l}\text { Maximum } \\
\text { amount on site } \\
\text { in pounds }{ }^{b}\end{array}$ & Activities and uses ${ }^{c}$ \\
\hline AK & 6 & 1,000 & $49,999,999$ & $1,5,7,12,13,14$ \\
\hline $\mathrm{AL}$ & 37 & 0 & $499,999,999$ & $1,2,3,4,5,7,8,9,11,12,13$ \\
\hline AR & 20 & 1,000 & $99,999,999$ & $1,2,3,7,8,9,11,12,13,14$ \\
\hline$A Z$ & 29 & 100 & $499,999,999$ & $1,2,3,4,5,6,7,8,9,10,11,12,13,14$ \\
\hline CA & 40 & 100 & $99,999,999$ & $1,2,3,4,5,6,7,8,9,11,12,13,14$ \\
\hline $\mathrm{CO}$ & 9 & 1,000 & $49,999,999$ & $1,2,3,4,5,6,7,8,9,11,12$ \\
\hline $\mathrm{DE}$ & 1 & 10,000 & 99,999 & $1,5,9$ \\
\hline FL & 30 & 0 & 999,999 & $1,3,4,5,7,8,9,11,12,13,14$ \\
\hline GA & 50 & 0 & $49,999,999$ & $1,2,3,4,5,6,7,8,9,11,12,13,14$ \\
\hline $\mathrm{HI}$ & 6 & 1,000 & 99,999 & $7,8,11$ \\
\hline IA & 22 & 0 & $9,999,999$ & $1,3,4,5,6,7,8,9,11,12,13$ \\
\hline ID & 6 & 10,000 & $9,999,999$ & $1,3,5,6,7,8,9,12,13$ \\
\hline IL & 44 & 0 & 999,999 & $1,2,3,4,5,6,7,8,9,10,11,12,13,14$ \\
\hline IN & 54 & 0 & $9,999,999$ & $1,2,3,4,5,6,7,8,9,11,12,13,14$ \\
\hline KS & 14 & 0 & 999,999 & $1,3,4,5,6,7,8,12,13,14$ \\
\hline KY & 29 & 0 & 999,999 & $1,2,3,4,5,6,7,8,9,10,11,12,13,14$ \\
\hline LA & 32 & 0 & $499,999,999$ & $1,2,3,4,5,6,7,8,9,11,12,13$ \\
\hline MA & 9 & 0 & 999,999 & $1,4,5,6,7,8$ \\
\hline MD & 19 & 0 & 999,999 & $1,4,5,7,8,9,11,12,13$ \\
\hline ME & 2 & 1,000 & 99,999 & 7 \\
\hline MI & 32 & 0 & 999,999 & $1,2,3,4,5,6,7,8,9,11,12,13,14$ \\
\hline MN & 13 & 0 & 999,999 & $1,3,4,5,7,8,9,11,12,13$ \\
\hline MO & 32 & 0 & $499,999,999$ & $1,2,3,4,5,6,7,8,9,11,12,13,14$ \\
\hline MS & 28 & 1,000 & $49,999,999$ & $1,2,3,4,5,6,7,8,9,11,12,13$ \\
\hline MT & 8 & 1,000 & $10,000,000,000$ & $1,2,3,4,5,6,7,12,13,14$ \\
\hline NC & 65 & 0 & $9,999,999$ & $1,2,3,4,5,6,7,8,9,10,11,12,13,14$ \\
\hline ND & 11 & 1,000 & 99,999 & $1,5,8,9,11,12,13,14$ \\
\hline NE & 6 & 1,000 & 999,999 & $1,2,3,4,5,6,8,9,12,13$ \\
\hline $\mathrm{NH}$ & 2 & 1,000 & 99,999 & 8,11 \\
\hline NJ & 35 & 0 & 999,999 & $1,2,3,4,5,6,7,8,9,12,13$ \\
\hline NM & 11 & 1,000 & $499,999,999$ & $1,5,7,12,13$ \\
\hline NV & 31 & 1,000 & $10,000,000,000$ & $1,2,3,5,6,7,9,11,12,13,14$ \\
\hline NY & 27 & 0 & $9,999,999$ & $1,2,3,4,5,7,8,9,12,13$ \\
\hline $\mathrm{OH}$ & 50 & 0 & 999,999 & $1,2,3,4,5,6,7,8,9,11,12,13,14$ \\
\hline OK & 14 & 100 & $9,999,999$ & $1,2,3,4,5,6,8,12,13,14$ \\
\hline OR & 12 & 100 & 99,999 & $1,2,3,7,8,12$ \\
\hline PA & 53 & 0 & 999,999 & $1,2,3,4,5,6,7,8,9,11,12,13,14$ \\
\hline PR & 8 & 1,000 & 99,999 & $1,2,3,5,8,11$ \\
\hline RI & 7 & 100 & 99,999 & 7,8 \\
\hline
\end{tabular}


Table 5-2. Facilities that Produce, Process, or Use Arsenic Compounds

\begin{tabular}{|c|c|c|c|c|}
\hline State $^{a}$ & $\begin{array}{l}\text { Number of } \\
\text { facilities }\end{array}$ & $\begin{array}{l}\text { Minimum } \\
\text { amount on site } \\
\text { in pounds }\end{array}$ & $\begin{array}{l}\text { Maximum } \\
\text { amount on site } \\
\text { in pounds }{ }^{b}\end{array}$ & Activities and uses $^{c}$ \\
\hline SC & 34 & 0 & $49,999,999$ & $1,2,3,4,5,6,7,8,9,11,12,13,14$ \\
\hline SD & 6 & 1,000 & $99,999,999$ & $1,5,6,7,8,11,12,13$ \\
\hline TN & 29 & 0 & $99,999,999$ & $1,2,3,4,5,6,7,8,9,10,11,12$ \\
\hline TX & 54 & 0 & $499,999,999$ & $1,2,3,4,5,6,7,8,9,11,12,13,14$ \\
\hline UT & 23 & 0 & $499,999,999$ & $1,3,4,5,6,7,8,9,10,12,13$ \\
\hline VA & 24 & 0 & $499,999,999$ & $1,2,3,4,5,7,8,9,11,12,13,14$ \\
\hline WA & 14 & 0 & 999,999 & $1,2,3,4,5,7,8,9,11,12,13$ \\
\hline WI & 13 & 100 & 99,999 & $1,2,3,4,5,6,7,8,9,11$ \\
\hline WV & 26 & 0 & 999,999 & $1,2,3,4,5,6,7,8,9,12,13,14$ \\
\hline WY & 9 & 1,000 & 99,999 & $1,3,4,5,7,8,9,12,13$ \\
\hline
\end{tabular}

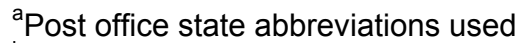

${ }^{\mathrm{b}}$ Amounts on site reported by facilities in each state ${ }^{\mathrm{C}}$ Activities/Uses:
1. Produce
6. Impurity
2. Import
7. Reactant
3. Onsite use/processing
8. Formulation Component
11. Chemical Processing Aid
4. Sale/Distribution
9. Article Component
12. Manufacturing Aid
13. Ancillary/Other Uses
5. Byproduct
10. Repackaging
14. Process Impurity

Source: TRI04 2006 (Data are from 2004) 
Table 5-3. Current U.S. Manufacturers of Selected Arsenic Compounds ${ }^{a}$

\begin{tabular}{|c|c|}
\hline Company & Location(s) \\
\hline \multicolumn{2}{|l|}{ Arsenic acid } \\
\hline Arch Wood Protection, Inc. & Conley, Georgia \\
\hline Osmose Wood Preserving, Inc. & Millington, Tennessee \\
\hline \multicolumn{2}{|l|}{ Arsanilic acid } \\
\hline Fleming Laboratories, Inc. & Charlotte, North Carolina \\
\hline \multicolumn{2}{|l|}{ Copper Chromated Arsenic (CCA) } \\
\hline Arch Wood Protection, Inc. & $\begin{array}{l}\text { Conley, Georgia; Kalama, Washington; Smyrna, Georgia }{ }^{\text {b }} \text {, } \\
\text { Valparaiso, Indiana }\end{array}$ \\
\hline Chemical Specialties, Inc. $^{\mathrm{b}}$ & Charlotte, North Carolina \\
\hline Osmose Wood Preserving, Inc. ${ }^{b}$ & Buffalo, New York \\
\hline \multicolumn{2}{|l|}{ Calcium acid methanearsonate (CAMA) } \\
\hline Drexel Chemical Company (formulator) ${ }^{\mathrm{c}}$ & No information provided \\
\hline \multicolumn{2}{|c|}{ Disodium methanearsonate (DSMA) } \\
\hline Drexel Chemical Company & Tunica, Mississippi \\
\hline \multicolumn{2}{|l|}{ Monosodium methyl arsonate (MSMA) } \\
\hline Drexel Chemical Company & Tunica, Mississippi \\
\hline \multicolumn{2}{|l|}{ Gallium arsenide } \\
\hline Atomergic Chemetals Corporation & Farmingdale, New York \\
\hline
\end{tabular}

${ }^{a}$ Derived from Stanford Research Institute (SRI 2006), except where otherwise noted. SRI reports production of chemicals produced in commercial quantities (defined as exceeding 5,000 pounds or $\$ 10,000$ in value annually) by the companies listed.

${ }^{\mathrm{b}}$ USGS 2006a

${ }^{\mathrm{c}}$ Meister et al. 2006 


\subsection{IMPORT/EXPORT}

Since U.S. production ceased in 1985, all arsenic consumed in the United States is imported. Imports of arsenic (metal and compounds combined) have increased substantially since the mid-1980s, reaching 8,810 metric tons (as arsenic content) in 2005 , of which 812 metric tons was as elemental arsenic. In 2005, 11,000 metric tons of arsenic trioxide was imported into the United States. China is the major import source for elemental arsenic from 2001 to 2004, supplying 81\%, followed by Japan (15\%) and Hong Kong (2\%). China is also the major import source in 2001-2004 for arsenic trioxide, supplying $59 \%$ to the United States, followed by Morocco (22\%), Chile (7\%), and Mexico (5\%) (USGS 2006a, 2006b).

U.S. exports of elemental arsenic were 220 metric tons in 2004 and are estimated to be 200 metric tons in 2005 (USGS 2006b). In 2005, U.S. import of arsenic was approximately $8.1 \times 10^{5}$ kilograms (810 metric tons) (ITA 2007a, 2007b).

\subsection{USE}

In 2003, the United States was the world's largest consumer of arsenic, with an apparent demand of 21,600 metric tons. In 2005, the Unites States was still the world's largest consumer of arsenic, mainly for CCA. Production of wood preservatives, primarily $\mathrm{CCA}, \mathrm{CrO}_{3} \cdot \mathrm{CuO} \bullet \mathrm{As}_{2} \mathrm{O}_{5}$, accounted for $>90 \%$ of domestic consumption of arsenic trioxide prior to 2004. In 2005, about $65 \%$ of domestic consumption of arsenic trioxide was used for the production of CCA. The remainder was used for the production of agricultural chemicals, including herbicides, and insecticides. The major U.S. producers of CCA in 2005 included Arch Wood Protection, Inc., Smyrna Georgia; Chemical Specialties Inc., Charlotte, North Carolina; and Osmose Wood Preserving, Inc., Buffalo, New York (USGS 2006a). CCA is the most widely used wood preservative in the world. Wood treated with CCA is referred to as 'pressure treated' wood (American Wood Preservers Association 2007; Page and Loar 1993). In 1997, approximately 727.8 million cubic feet (20.6 million cubic meters) of wood products were pressure treated in the United States. CCA is a water-based product that protects several commercially available species of western lumber from decay and insect attack. It is widely used in treating utility poles, building lumber, and wood foundations. CCA comes in three types, $\mathrm{A}, \mathrm{B}$, and $\mathrm{C}$, which contain different proportions of chromium, copper, and arsenic oxides. Type $\mathrm{C}$, the most popular type, contains $\mathrm{CrO}_{3}, \mathrm{CuO}$, and $\mathrm{As}_{2} \mathrm{O}_{5}$ in the proportions $47.5,18.5$, and $34.0 \%$, respectively. The retention levels are 0.25 pounds per cubic feet (pcf) for above ground use such as fencing and decking, $0.40 \mathrm{pcf}$ for lumber used in ground contact such as 
fence posts and deck posts, and 0.60 pcf for all weather wood foundations (Chicago Flameproof 2000; Permapost 2000). Piling used for fresh and saltwater contact should contain 0.80 and $2.5 \mathrm{pcf}$ of CCA, respectively. Ammoniacal copper zinc arsenate (ACZA) is another arsenic containing preservative used to treat wood; however, it is not as widely used as CCA-C (Lebow et al. 2000).

In 2003, U.S. manufacturers of arsenical wood preservatives began a voluntary transition from CCA to other wood preservatives in wood products for certain residential uses, such as play structures, picnic tables, decks, fencing, and boardwalks. This phase out was completed on December 31, 2003; wood treated prior to this date could still be used and structures made with CCA-treated wood would not be affected. CCA-treated wood products continue to be used in industrial applications (EPA 2003a).

Elemental arsenic is used as an alloying element in ammunition and solders, as an anti-friction additive to metals used for bearings, and to strengthen lead-acid storage battery grids. In the past, the predominant use of arsenic was in agriculture. The uses of lead arsenate as a growth regulator on citrus, calcium arsenate as an herbicide on turf, sodium arsenite as a fungicide on grapes, and arsenic acid as a desiccant on okra for seed and cotton were voluntarily cancelled in the late 1980s and the early 1990s (EPA 2006). The herbicides, MSMA and DSMA, are registered for weed control on cotton, for turf grass and lawns, and under trees, vines, and shrubs; calcium acid methanearsonate (CAMA) is registered for postemergent weed control on lawns. Cacodylic acid, a defoliant and herbicide, is registered for weed control under nonbearing citrus trees, around buildings and sidewalks, and for lawn renovation (EPA 2006). Approximately 3 million pounds of MSMA or DSMA, and 100,000 pounds of cacodylic acid are applied in the U.S. annually based on EPA's Screening Level Use Analysis data. Data were not available for CAMA. Application to cotton and turf (residential and golf courses) are the major uses of organic arsenical herbicides. Currently, there are approximately 90, 25, 4, and 35 end-use products containing MSMA, DSMA, CAMA, and cacodylic acid, respectively (EPA 2006).

Other organic arsenicals used in agriculture include arsanilic acid, sodium arsanilate, and 3-nitro4-hydroxyphenylarsonic acid (roxarsone), which are antimicrobials used in animal and poultry feeds (Beerman 1994). While the U.S. Food and Drug Administration (FDA) has authorized the used of these compounds as medicinal feed additives, only one of the arsenical compounds may be used at a time as the sole source of organic arsenic in the feed (EPA 1998k). In 1999-2000, about 70\% of the broiler industry added roxarsone to broiler poultry feed; concentrations of roxarsone in feed range from 22.7 to $45.4 \mathrm{~g} / \mathrm{ton}$ (Garbarino et al. 2003). 
From the mid-nineteenth century to the introduction of organic pesticides in the 1940s, inorganic arsenic compounds were the dominant pesticides available to farmers and fruit growers. Calcium arsenate was formerly used to control the boll weevil and cotton worm and was used as an herbicide. Lead arsenate was used on apple and other fruit orchards as well as on potato fields. Sodium arsenite was used to control weeds on railroad right-of-ways, potato fields, and in industrial areas, as well as in baits and to debark trees. Sodium arsenate had some application in ant traps. The use of inorganic arsenic compounds in agriculture has virtually disappeared beginning around the 1960s (Azcue and Nriagu 1994; Meister 1987; Merwin et al. 1994; Sanok et al. 1995). Food uses were voluntarily cancelled in 1993 as was the use of arsenic acid as a defoliant on cotton plants; inorganic arsenic's remaining allowable uses are in ant baits and wood preservatives (EPA 1999h). In 1987, EPA issued a preliminary decision to cancel the registration of most inorganic arsenicals used as nonwood pesticides (Loebenstein 1994) (see Chapter 8). According to the California Department of Pesticide Regulation, arsenic acid, arsenic pentoxide, and arsenic trioxide are registered currently as pesticides in the United States; there are no active registrants listed for calcium arsenate, lead arsenate, or sodium arsenite (NPIRS 2007).

High-purity arsenic (99.9999\%) is used by the electronics industry for gallium-arsenide semiconductors for telecommunications, solar cells, and space research (USGS 2006b). Arsenic trioxide and arsenic acid were used as a decolorizer and fining agent in the production of bottle glass and other glassware (Carapella 1992).

Arsenic compounds have a long history of use in medicine. Inorganic arsenic was used as a therapeutic agent through the mid-twentieth century, primarily for the treatment of leukemia, psoriasis, and chronic bronchial asthma; organic arsenic antibiotics were extensively used in the treatment of spirochetal and protozoal disease (NRC 1999). The availability of inorganic arsenicals in Western medicines ended in the 1970s, although they may still be encountered in non-Western traditional medicines. By the 1980s, the only remaining medicinal organic arsenical was melarsoprol for treatment of the meningoencephalitic stage of African trypanosomiasis. There has been renewed interest in arsenic as a therapeutic agent, namely the use of arsenic trioxide in the treatment of acute promyelocytic leukemia (APL) (Gallagher 1998; Kroemer and de Thé 1999; Miller 1998; Wang 2001). In 2000, the FDA approved arsenic trioxide for this use (FDA 2000). 


\subsection{DISPOSAL}

Wastes containing arsenic are considered hazardous wastes, and as such, their treatment, storage, and disposal are regulated by law (see Chapter 8). The main route of disposal of solid wastes containing arsenic is landfilling. EPA has promulgated rules and treatment standards for landfilling liquid arsenical wastes (EPA 1990e). Arsenic-containing electronic components such as relays, switches, and circuit boards are disposed of at hazardous waste sites, and the elemental arsenic is not reclaimed. Process water at wood treatment plants that contained arsenic contained was reused. Gallium-arsenide scrap from the manufacture of semiconductor devices was reprocessed for arsenic recovery. Arsenic was not recovered from arsenical residues and dusts at domestic nonferrous smelters (USGS 2006b).

CCA-treated wood is classified as nonhazardous waste under the Federal Resource Conservation and Recovery Act (RCRA). CCA-treated wood is disposed of with regular municipal trash (i.e., municipal solid waste, not yard waste). It should not be burned in open fires, stoves, residential boilers, or fire places and should not be composted or used as mulch. Treated wood from commercial or industrial applications may only be burned in commercial or industrial incinerators in accordance with state and federal regulations (Adobe Lumber 2002; EPA 2005a).

Arsenic is listed as a toxic substance under Section 313 of the Emergency Planning and Community Right to Know Act (EPCRA) under Title III of the Superfund Amendments and Reauthorization Act (SARA) (EPA 1995c). Disposal of wastes containing arsenic is controlled by a number of federal regulations (see Chapter 8). 


\section{POTENTIAL FOR HUMAN EXPOSURE}

\subsection{OVERVIEW}

Arsenic has been identified in at least 1,149 of the 1,684 hazardous waste sites that have been proposed for inclusion on the EPA National Priorities List (NPL) (HazDat 2006). However, the number of sites evaluated for arsenic is not known. The frequency of these sites can be seen in Figure 6-1. Of these sites, 1,134 are located within the United States and 11, 2, and 2 are located in the Commonwealth of Puerto Rico, the Virgin Islands, and Guam (not shown).

Arsenic is widely distributed in the Earth's crust, which contains about 3.4 ppm arsenic (Wedepohl 1991). It is mostly found in nature in minerals, such as realgar $\left(\mathrm{As}_{4} \mathrm{~S}_{4}\right)$, orpiment $\left(\mathrm{As}_{2} \mathrm{~S}_{3}\right)$, and arsenolite $\left(\mathrm{As}_{2} \mathrm{O}_{3}\right)$, and only found in its elemental form to a small extent. There are over 150 arsenic-bearing minerals (Budavari et al. 2001; Carapella 1992). While arsenic is released to the environment from natural sources such as wind-blown soil and volcanoes, releases from anthropogenic sources far exceed those from natural sources. Anthropogenic sources of arsenic include nonferrous metal mining and smelting, pesticide application, coal combustion, wood combustion, and waste incineration. Most anthropogenic releases of arsenic are to land or soil, primarily in the form of pesticides or solid wastes. However, substantial amounts are also released to air and water.

Arsenic found in soil either naturally occurring or from anthropogenic releases forms insoluble complexes with iron, aluminum, and magnesium oxides found in soil surfaces, and in this form, arsenic is relatively immobile. However, under reducing conditions, arsenic can be released from the solid phase, resulting in soluble mobile forms of arsenic, which may potentially leach into groundwater or result in runoff of arsenic into surface waters. In aquatic systems, inorganic arsenic occurs primarily in two oxidation states, $\mathrm{As}(\mathrm{V})$ and $\mathrm{As}(\mathrm{III})$. Both forms generally co-exist, although $\mathrm{As}(\mathrm{V})$ predominates under oxidizing conditions and As(III) predominates under reducing conditions. Arsenic may undergo a variety of reactions in the environment, including oxidation-reduction reactions, ligand exchange, precipitation, and biotransformation (EPA 1979, 1984a; Pongratz 1998; Welch et al. 1988). These reactions are influenced by Eh (the oxidation-reduction potential), $\mathrm{pH}$, metal sulfide and sulfide ion concentrations, iron concentration, temperature, salinity, and distribution and composition of the biota (EPA 1979; Wakao et al. 1988). Much of the arsenic will adsorb to particulate matter and sediment. Arsenic released to air exists mainly in the form of particulate matter. Arsenic released from combustion processes will 
6. POTENTIAL FOR HUMAN EXPOSURE

Figure 6-1. Frequency of NPL Sites with Arsenic Contamination

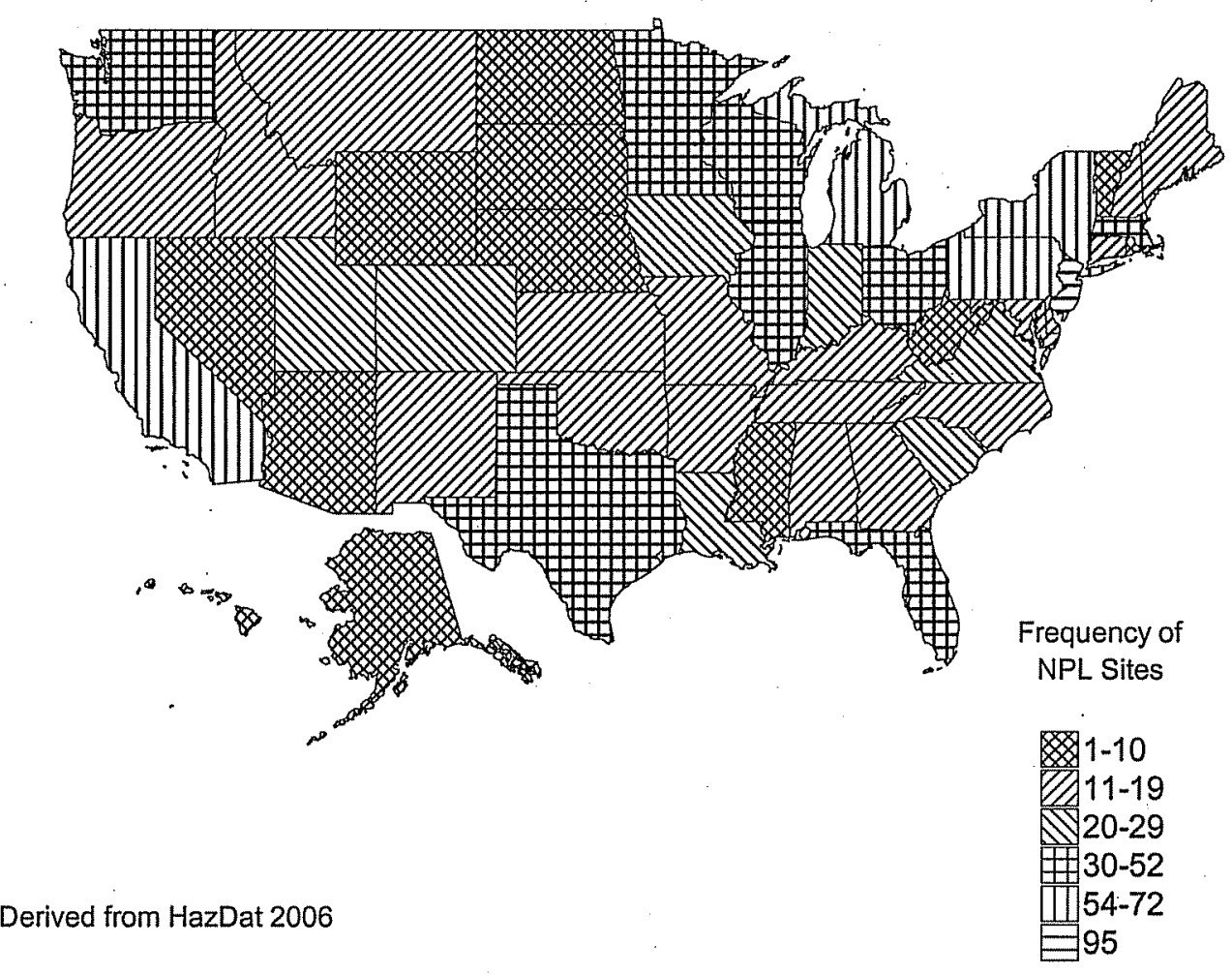


generally occur as highly soluble oxides. These particles are dispersed by the wind and returned to the earth in wet or dry deposition. Arsines that are released to the atmosphere as a result of microbial action are oxidized to nonvolatile species that settle back to the ground.

Because arsenic is a natural component of the Earth's crust, low levels of the element are found in all environmental media. Atmospheric levels of arsenic in remote locations (away from human releases) range from 1 to $3 \mathrm{ng} / \mathrm{m}^{3}$, while concentrations in urban areas may range from 20 to $100 \mathrm{ng} / \mathrm{m}^{3}$. Concentrations in water are usually $<10 \mu \mathrm{g} / \mathrm{L}$, although higher levels may occur near natural mineral deposits or anthropogenic sources. Natural levels of arsenic in soil usually range from 1 to $40 \mathrm{mg} / \mathrm{kg}$, with a mean of $5 \mathrm{mg} / \mathrm{kg}$, although much higher levels may occur in mining areas, at waste sites, near high geological deposits of arsenic-rich minerals, or from pesticide application. Arsenic is also found in many foods, at concentrations that usually range from 20 to $140 \mu \mathrm{g} / \mathrm{kg}$. Total arsenic concentrations may be substantially higher in certain seafoods. However, the general consensus in the literature is that about 85$>90 \%$ of the arsenic in the edible parts of marine fish and shellfish is organic arsenic (e.g., arsenobetaine, arsenochloline, dimethylarsinic acid) and that approximately 10\% is inorganic arsenic (EPA 2003b). Drinking water in the United States generally contains an average of $2 \mu \mathrm{g} / \mathrm{L}$ of arsenic (EPA 1982c), although $12 \%$ of water supplies from surface water sources in the north Central region of the United States and $12 \%$ of supplies from groundwater sources in the western region have levels exceeding $20 \mu \mathrm{g} / \mathrm{L}$ (Karagas et al. 1998). In January 2001, EPA adopted a new standard that arsenic levels in drinking water were not to exceed $10 \mu \mathrm{g} / \mathrm{L}$, replacing the previous standard of $50 \mu \mathrm{g} / \mathrm{L}$. The date for compliance with the new MCL was January 23, 2006 (EPA 2001).

For most people, diet is the largest source of exposure to arsenic. Mean dietary intakes of total arsenic of $50.6 \mu \mathrm{g} /$ day (range of $1.01-1,081 \mu \mathrm{g} /$ day) and $58.5 \mu \mathrm{g} /$ day (range of $0.21-1,276 \mu \mathrm{g} /$ day) has been reported for females and males (MacIntosh et al. 1997). U.S. dietary intake of inorganic arsenic has been estimated to range from 1 to $20 \mu \mathrm{g} /$ day, with grains and produce expected to be significant contributors to dietary inorganic arsenic intake (Schoof et al. 1999a, 1999b). The predominant dietary source of arsenic is generally seafood. Inorganic arsenic in seafood sampled in a market basket survey of inorganic arsenic in food ranged from $<0.001$ to $0.002 \mu \mathrm{g} / \mathrm{g}$ (Schoof et al. 1999a, 1999b). Intake of arsenic from air and soil are usually much smaller than that from food and water (Meacher et al. 2002).

People who produce or use arsenic compounds in occupations such as nonferrous metal smelting, pesticide manufacturing or application, wood preservation, semiconductor manufacturing, or glass production may be exposed to substantially higher levels of arsenic, mainly from dusts or aerosols in air. 
Exposure at waste sites may occur by a variety of pathways, including inhalation of dusts in air, ingestion of contaminated soil or water, or through the food chain. The magnitude of the exposures can only be evaluated on a site-by-site basis; however, exposures generally do not exceed background intakes from food and drinking water.

Tables 4-1, 4-2, 4-3, and 4-4 summarize all of the names, abbreviations, and structures of the various arsenic compounds that are discussed in Chapter 6.

\subsection{RELEASES TO THE ENVIRONMENT}

The Toxics Release Inventory (TRI) data should be used with caution because only certain types of facilities are required to report (EPA 2005k). This is not an exhaustive list. Manufacturing and processing facilities are required to report information to the TRI only if they employ 10 or more full-time employees; if their facility is included in Standard Industrial Classification (SIC) Codes 10 (except 1011, 1081, and 1094), 12 (except 1241), 20-39, 4911 (limited to facilities that combust coal and/or oil for the purpose of generating electricity for distribution in commerce), 4931 (limited to facilities that combust coal and/or oil for the purpose of generating electricity for distribution in commerce), 4939 (limited to facilities that combust coal and/or oil for the purpose of generating electricity for distribution in commerce), 4953 (limited to facilities regulated under RCRA Subtitle C, 42 U.S.C. section 6921 et seq.), 5169, 5171, and 7389 (limited S.C. section 6921 et seq.), 5169, 5171, and 7389 (limited to facilities primarily engaged in solvents recovery services on a contract or fee basis); and if their facility produces, imports, or processes $\geq 25,000$ pounds of any TRI chemical or otherwise uses $>10,000$ pounds of a TRI chemical in a calendar year (EPA 2005k).

\subsubsection{Air}

Estimated releases of 4,800 pounds ( $\sim 2.2$ metric tons) of arsenic to the atmosphere from 58 domestic manufacturing and processing facilities in 2004 , accounted for about $0.52 \%$ of the estimated total environmental releases from facilities required to report to the TRI (TRI04 2006). Estimated releases of 0.13 million pounds ( $\sim 59$ metric tons) of arsenic compounds to the atmosphere from 361 domestic manufacturing and processing facilities in 2004 , accounted for about $0.11 \%$ of the estimated total environmental releases from facilities required to report to the TRI (TRI04 2006). These releases for arsenic and arsenic compounds are summarized in Table 6-1 and 6-2, respectively. 
Table 6-1. Releases to the Environment from Facilities that Produce, Process, or Use Arsenic ${ }^{a}$

\begin{tabular}{|c|c|c|c|c|c|c|c|c|c|}
\hline \multirow[b]{3}{*}{ State $^{c}$} & \multirow[b]{3}{*}{$\mathrm{RF}^{\mathrm{d}}$} & \multicolumn{8}{|c|}{ Reported amounts released in pounds per year ${ }^{b}$} \\
\hline & & \multirow[b]{2}{*}{ Air ${ }^{\mathrm{e}}$} & \multirow[b]{2}{*}{ Water $^{f}$} & \multirow[b]{2}{*}{$\mathrm{UI}^{\mathrm{g}}$} & \multirow[b]{2}{*}{ Land $^{\text {h }}$} & \multirow[b]{2}{*}{ Other ${ }^{i}$} & \multicolumn{3}{|c|}{ Total release } \\
\hline & & & & & & & On-site & Off-site ${ }^{k}$ & $\begin{array}{l}\text { On- and } \\
\text { off-site }\end{array}$ \\
\hline $\mathrm{AL}$ & 1 & 51 & 162 & 0 & 110,264 & 0 & 110,425 & 52 & 110,477 \\
\hline AR & 2 & 0 & 0 & No data & 0 & 0 & No data & 0 & 0 \\
\hline$A Z$ & 2 & 10 & 0 & 0 & 20,717 & 0 & 20,727 & 0 & 20,727 \\
\hline $\mathrm{CA}$ & 3 & 13 & 14 & 0 & 5,482 & 0 & 13 & 5,497 & 5,510 \\
\hline $\mathrm{FL}$ & 2 & 4 & 0 & 0 & 0 & 4,950 & 4 & 4,950 & 4,954 \\
\hline GA & 4 & 8 & 10 & 0 & 1,603 & 5 & 13 & 1,613 & 1,626 \\
\hline IA & 1 & 0 & 1 & 0 & 0 & 0 & 0 & 1 & 1 \\
\hline ID & 1 & 39 & 0 & 0 & 361,252 & 0 & 361,291 & 0 & 361,291 \\
\hline IL & 2 & 250 & 129 & 0 & 14,087 & 0 & 379 & 14,087 & 14,466 \\
\hline IN & 1 & 5 & 5 & 0 & 13,250 & 250 & 5 & 13,505 & 13,510 \\
\hline KS & 1 & 0 & 0 & No data & 0 & 0 & No data & 0 & 0 \\
\hline KY & 1 & 0 & 1 & 0 & 0 & 6 & 1 & 6 & 7 \\
\hline MI & 2 & 0 & 5 & 0 & 0 & 750 & 5 & 750 & 755 \\
\hline MN & 1 & 15 & 47 & 0 & 14,504 & 0 & 15 & 14,551 & 14,566 \\
\hline MO & 1 & 5 & 0 & 0 & 0 & 4,040 & 5 & 4,040 & 4,045 \\
\hline MS & 2 & 0 & 0 & 0 & 0 & 0 & 0 & 0 & 0 \\
\hline NC & 4 & 35 & 8 & 0 & 1 & 1 & 43 & 2 & 45 \\
\hline NV & 1 & 0 & 0 & 0 & 0 & 0 & 0 & 0 & 0 \\
\hline NY & 4 & 0 & 1 & 0 & 26,525 & 1 & 26,401 & 126 & 26,527 \\
\hline $\mathrm{OH}$ & 2 & 13 & 0 & 0 & 0 & 0 & 13 & 0 & 13 \\
\hline OR & 1 & 0 & 0 & 0 & 92,606 & 0 & 92,606 & 0 & 92,606 \\
\hline PA & 5 & 166 & 8 & 0 & 14,362 & 26,140 & 199 & 40,477 & 40,676 \\
\hline SC & 3 & 10 & 10 & 0 & 0 & 1,002 & 15 & 1,007 & 1,022 \\
\hline TN & 3 & 3,988 & 0 & 0 & 0 & 0 & 3,988 & 0 & 3,988 \\
\hline $\mathrm{TX}$ & 5 & 139 & 376 & 168,563 & 12,600 & 0 & 181,636 & 42 & 181,678 \\
\hline
\end{tabular}




\section{Table 6-1. Releases to the Environment from Facilities that Produce, Process, or} Use Arsenic ${ }^{\mathrm{a}}$

\begin{tabular}{|c|c|c|c|c|c|c|c|c|c|}
\hline \multirow[b]{3}{*}{ State $^{c}$} & \multirow[b]{3}{*}{$\mathrm{RF}^{\mathrm{d}}$} & \multicolumn{8}{|c|}{ Reported amounts released in pounds per year ${ }^{b}$} \\
\hline & & \multirow[b]{2}{*}{ Air $^{\mathrm{e}}$} & \multirow[b]{2}{*}{ Water ${ }^{f}$} & \multirow[b]{2}{*}{$U I^{g}$} & \multirow[b]{2}{*}{ Land $^{\mathrm{h}}$} & \multirow[b]{2}{*}{ Other $^{i}$} & \multicolumn{3}{|c|}{ Total release } \\
\hline & & & & & & & On-site & Off-site ${ }^{k}$ & $\begin{array}{l}\text { On- and } \\
\text { off-site }\end{array}$ \\
\hline$\overline{\mathrm{WI}}$ & 2 & 15 & 0 & 0 & 760 & 0 & 15 & 760 & 776 \\
\hline WV & 1 & 0 & 0 & 0 & 10,135 & 0 & 10,135 & 0 & 10,135 \\
\hline Total & 58 & 4,766 & 778 & 168,563 & 698,149 & 37,145 & 807,935 & 101,466 & 909,401 \\
\hline
\end{tabular}

${ }^{a}$ The TRI data should be used with caution since only certain types of facilities are required to report. This is not an exhaustive list. Data are rounded to nearest whole number.

${ }^{\mathrm{b}}$ Data in TRI are maximum amounts released by each facility.

${ }^{\mathrm{C}}$ Post office state abbreviations are used.

${ }^{\mathrm{d}}$ Number of reporting facilities.

${ }^{\mathrm{e}}$ The sum of fugitive and point source releases are included in releases to air by a given facility.

f Surface water discharges, waste water treatment-(metals only), and publicly owned treatment works (POTWs) (metal and metal compounds).

${ }^{\mathrm{g}}$ Class I wells, Class II-V wells, and underground injection.

${ }^{h}$ Resource Conservation and Recovery Act (RCRA) subtitle C landfills; other on-site landfills, land treatment, surface impoundments, other land disposal, other landfills.

'Storage only, solidification/stabilization (metals only), other off-site management, transfers to waste broker for disposal, unknown

${ }^{\mathrm{j} T}$ The sum of all releases of the chemical to air, land, water, and underground injection wells.

kTotal amount of chemical transferred off-site, including to POTWs.

$\mathrm{RF}=$ reporting facilities; $\mathrm{UI}=$ underground injection

Source: TRI04 2006 (Data are from 2004) 
Table 6-2. Releases to the Environment from Facilities that Produce, Process, or Use Arsenic Compounds ${ }^{a}$

\begin{tabular}{|c|c|c|c|c|c|c|c|c|c|}
\hline \multirow[b]{3}{*}{ State $^{c}$} & \multirow[b]{3}{*}{$\mathrm{RF}^{\mathrm{d}}$} & \multicolumn{8}{|c|}{ Reported amounts released in pounds per year ${ }^{\mathrm{b}}$} \\
\hline & & \multirow[b]{2}{*}{ Air $^{\mathrm{e}}$} & \multirow[b]{2}{*}{ Water ${ }^{f}$} & \multirow[b]{2}{*}{$\mathrm{UI}^{\mathrm{g}}$} & \multirow[b]{2}{*}{ Land $^{\mathrm{h}}$} & \multirow[b]{2}{*}{ Other ${ }^{i}$} & \multicolumn{3}{|c|}{ Total release } \\
\hline & & & & & & & On-site & Off-site ${ }^{k}$ & $\begin{array}{l}\text { On- and off- } \\
\text { site }\end{array}$ \\
\hline$\overline{\mathrm{AK}}$ & 1 & 511 & 0 & $1,400,000$ & $1,200,000$ & 0 & $2,600,511$ & 0 & $2,600,511$ \\
\hline$A L$ & 19 & 4,299 & 18,127 & 0 & 853,469 & 7,555 & 875,876 & 7,574 & 883,450 \\
\hline AR & 12 & 0 & 0 & 0 & 133 & 26,435 & 0 & 26,568 & 26,568 \\
\hline$A Z$ & 5 & 5,421 & 0 & 0 & 402,335 & 422 & 394,749 & 13,429 & 408,178 \\
\hline $\mathrm{CA}$ & 5 & 65 & 14 & 0 & 355,660 & 86,396 & 160,673 & 281,461 & 442,134 \\
\hline $\mathrm{CO}$ & 1 & 11 & 0 & 0 & 4,094 & 0 & 4,105 & 0 & 4,105 \\
\hline $\mathrm{CT}$ & 1 & 0 & 0 & 0 & 0 & 0 & No data & 0 & 0 \\
\hline $\mathrm{FL}$ & 15 & 3,208 & 503 & 0 & 343,508 & 4,057 & 346,310 & 4,966 & 351,276 \\
\hline GA & 23 & 8,643 & 7,823 & 0 & 422,124 & 5,127 & 437,496 & 6,221 & 443,717 \\
\hline $\mathrm{HI}$ & 1 & 0 & 0 & 0 & 0 & 0 & No data & 0 & 0 \\
\hline IA & 4 & 1,291 & 482 & 0 & 0 & 35,324 & 1,773 & 35,324 & 37,097 \\
\hline ID & 3 & 332 & 20 & 0 & $1,056,904$ & 0 & $1,057,256$ & 0 & $1,057,256$ \\
\hline IL & 11 & 3,960 & 3,110 & 0 & 96,093 & 21,038 & 71,819 & 52,382 & 124,202 \\
\hline IN & 21 & 13,786 & 8,282 & 0 & 768,297 & 42,808 & 632,704 & 200,470 & 833,174 \\
\hline KS & 4 & 924 & 0 & 0 & 12,082 & 1 & 13,006 & 1 & 13,007 \\
\hline KY & 18 & 14,406 & 8,427 & 0 & 616,074 & 95,285 & 578,080 & 156,112 & 734,192 \\
\hline LA & 7 & 265 & 23 & 0 & 25,426 & 0 & 25,563 & 151 & 25,714 \\
\hline MA & 1 & 0 & 0 & 0 & 0 & 500 & 0 & 500 & 500 \\
\hline MD & 8 & 1,870 & 291 & 0 & 34,130 & 114,115 & 2,661 & 147,745 & 150,406 \\
\hline MI & 10 & 1,123 & 2,310 & 68,924 & 101,857 & 1,059 & 77,505 & 97,769 & 175,274 \\
\hline MN & 2 & 10 & 130 & 0 & 19,270 & 0 & 19,410 & 0 & 19,410 \\
\hline MO & 6 & 462 & 116 & 0 & 27,855 & 936 & 10,026 & 19,343 & 29,369 \\
\hline MS & 6 & 61 & 121 & 0 & 11,676 & 46 & 11,228 & 676 & 11,904 \\
\hline MT & 3 & 630 & 0 & 0 & $2,138,190$ & 37 & $2,138,820$ & 37 & $2,138,857$ \\
\hline $\mathrm{NC}$ & 15 & 5,626 & 4,732 & 0 & 168,030 & 2,429 & 178,388 & 2,429 & 180,818 \\
\hline ND & 6 & 6,326 & 5 & 0 & 318,175 & 0 & 137,961 & 186,545 & 324,506 \\
\hline NE & 2 & 180 & 0 & 0 & 11,000 & 0 & 11,180 & 0 & 11,180 \\
\hline NJ & 2 & 0 & 1 & 0 & 0 & 8 & 0 & 9 & 9 \\
\hline NM & 2 & 130 & 0 & 0 & 18,326 & 0 & 18,456 & 0 & 18,456 \\
\hline NV & 10 & 3,041 & 30,017 & 0 & $98,894,564$ & 0 & $98,927,328$ & 294 & $98,927,622$ \\
\hline NY & 3 & 67 & 36 & 0 & 27,059 & 802 & 27,141 & 823 & 27,964 \\
\hline $\mathrm{OH}$ & 17 & 8,595 & 8,352 & 81,024 & 741,730 & 274 & 668,157 & 171,818 & 839,975 \\
\hline OK & 4 & 115 & 13 & 0 & 25,000 & 4,202 & 115 & 29,215 & 29,330 \\
\hline OR & 4 & 0 & 5 & 0 & 0 & 4,012 & 5 & 4,012 & 4,017 \\
\hline PA & 23 & 18,963 & 2,166 & 0 & 666,753 & 69,053 & 403,582 & 353,353 & 756,935 \\
\hline PR & 3 & 0 & 0 & 0 & 0 & 0 & No data & 0 & 0 \\
\hline RI & 1 & 0 & 8 & 0 & 0 & 1,006 & 8 & 1,006 & 1,014 \\
\hline
\end{tabular}




\section{Table 6-2. Releases to the Environment from Facilities that Produce, Process, or Use Arsenic Compounds ${ }^{\mathrm{a}}$}

\begin{tabular}{|c|c|c|c|c|c|c|c|c|c|}
\hline \multirow[b]{3}{*}{ State $^{c}$} & \multirow[b]{3}{*}{$\mathrm{RF}^{\mathrm{d}}$} & \multicolumn{8}{|c|}{ Reported amounts released in pounds per year ${ }^{b}$} \\
\hline & & \multirow[b]{2}{*}{ Air $^{\mathrm{e}}$} & \multirow[b]{2}{*}{ Water $^{f}$} & \multirow[b]{2}{*}{$\mathrm{UI}^{\mathrm{g}}$} & \multirow[b]{2}{*}{ Land $^{\mathrm{h}}$} & \multirow[b]{2}{*}{ Other ${ }^{i}$} & \multicolumn{3}{|c|}{ Total release } \\
\hline & & & & & & & On-site & Off-site ${ }^{k}$ & $\begin{array}{l}\text { On- and off- } \\
\text { site }\end{array}$ \\
\hline$\overline{S C}$ & 13 & 2,178 & 1,443 & 0 & 25,817 & 22,705 & 29,438 & 22,705 & 52,143 \\
\hline SD & 1 & 0 & 0 & 0 & 0 & 0 & No data & 0 & 0 \\
\hline TN & 13 & 3,379 & 25,878 & 0 & 292,914 & 17,219 & 258,643 & 80,746 & 339,389 \\
\hline TX & 17 & 4,616 & 199 & 33,148 & 196,385 & 31,557 & 226,751 & 39,155 & 265,906 \\
\hline UT & 5 & 6,715 & 4,500 & 0 & $6,368,500$ & 3,500 & $6,379,715$ & 3,500 & $6,383,215$ \\
\hline VA & 11 & 1,911 & 2,773 & 0 & 160,154 & 8,463 & 164,789 & 8,512 & 173,301 \\
\hline WA & 4 & 0 & 0 & 0 & 0 & 0 & No data & 0 & 0 \\
\hline WI & 4 & 94 & 21 & 0 & 1,313 & 9,216 & 223 & 10,421 & 10,644 \\
\hline WV & 12 & 2,693 & 2,417 & 0 & 536,628 & 10,000 & 441,237 & 110,501 & 551,738 \\
\hline WY & 2 & 3,300 & 0 & 0 & 10,800 & 0 & 14,100 & 0 & 14,100 \\
\hline Total & 361 & 129,205 & 132,347 & $1,583,096$ & $116,952,326$ & 625,588 & $117,346,787$ & $2,075,775$ & $119,422,562$ \\
\hline
\end{tabular}

${ }^{a}$ The TRI data should be used with caution since only certain types of facilities are required to report. This is not an exhaustive list. Data are rounded to nearest whole number.

${ }^{\mathrm{b}}$ Data in TRI are maximum amounts released by each facility.

${ }^{c}$ Post office state abbreviations are used.

${ }^{\mathrm{d}}$ Number of reporting facilities.

${ }^{\mathrm{e}}$ The sum of fugitive and point source releases are included in releases to air by a given facility.

fSurface water discharges, waste water treatment-(metals only), and publicly owned treatment works (POTWs) (metal and metal compounds).

${ }^{9}$ Class I wells, Class II-V wells, and underground injection.

${ }^{h}$ Resource Conservation and Recovery Act (RCRA) subtitle C landfills; other on-site landfills, land treatment, surface impoundments, other land disposal, other landfills.

'Storage only, solidification/stabilization (metals only), other off-site management, transfers to waste broker for disposal, unknown

j'The sum of all releases of the chemical to air, land, water, and underground injection wells.

kTotal amount of chemical transferred off-site, including to POTWs.

$\mathrm{RF}=$ reporting facilities; $\mathrm{UI}=$ underground injection

Source: TRI04 2006 (Data are from 2004) 
Arsenic naturally occurs in soil and will be present in the atmosphere as airborne dust. It is also emitted from volcanoes and in areas of dormant volcanism (e.g., fumaroles). Gaseous alkyl arsenic compounds may be released from soil that has been treated with inorganic arsenic compounds as a result of biogenic processes (Schroeder et al. 1987; Tamaki and Frankenberger 1992). Arsenic naturally occurs in sea water and vegetation and is released into the atmosphere in sea salt spray and forest fires. Anthropogenic sources of arsenic include nonferrous metal smelting, coal, oil and wood combustion, and municipal waste incineration. Arsenic naturally occurs in coal and oil and therefore, coal- and oil-fired power plants release arsenic to the atmosphere in their emissions (Pacyna 1987). Arsenic's use in agriculture and industrial processes also contributes to its emissions. One important source of arsenic emissions is cotton ginning in which the cotton seeds are removed from the raw cotton.

The National Air Toxics Assessment reported that total anthropogenic emissions for arsenic compounds in the United States in 1996 were 355 tons/year (EPA 2005b). EPA conducted a modeling study with the Assessment System for Population Exposure Nationwide (ASPEN) in which estimates of emissions of hazardous air pollutants were used to estimate air quality (Rosenbaum et al. 1999). Using 1990 data, the total emissions of arsenic in the conterminous 48 states, excluding road dust or windblown dust from construction or agricultural tilling was estimated to be 3.0 tons/day with $90 \%$ of emissions coming from point sources and 5\% each from area and mobile sources. U.S. emissions of arsenic to the atmosphere were estimated as 3,300 metric tons per year between 1979 and 1986 (Pacyna et al. 1995). There is evidence that anthropogenic emissions, at least from smelters, are lower than they had been in the early 1980s. It is likely that air releases of arsenic decreased during the 1980s due to regulations on industrial emissions (EPA 1986f), improved control technology for coal-burning facilities, and decreased use of arsenical pesticides.

Nriagu and Pacyna (1988) and Pacyna et al. (1995) estimated worldwide emissions of arsenic to the atmosphere for 1983. Estimates of yearly emissions from anthropogenic sources ranged from 12,000 to 25,600 metric tons with a median value of 18,800 metric tons. Natural sources contributed 1,100 23,500 metric tons annually. Chilvers and Peterson (1987) estimated global natural and anthropogenic arsenic emissions to the atmosphere as 73,500 and 28,100 metric tons per year, respectively. Copper smelting and coal combustion accounted for $65 \%$ of anthropogenic emissions. A U.S. Bureau of Mines study on the flow of mineral commodities estimated that global emissions of arsenic from metal smelting, coal burning, and other industrial uses ranged from 24,000 to 124,000 metric tons per year compared to natural releases, mostly from volcanoes, ranging from 2,800 to 8,000 metric tons per year (Loebenstein 1994). 
Pirrone and Keeler (1996) compared trends of trace element emissions from major anthropogenic sources in the Great Lakes region with ambient concentrations observed in urban areas of the region. They found that arsenic emissions increased about 2.8\% per year from 1982 to 1988 and then decreased steadily by about $1.4 \%$ per year to 1993 . Coal combustion in electric utilities and in residential, commercial, and industrial facilities was an important source of arsenic in the region, accounting for about $69 \%$ of the total emissions. Iron-steel manufacturing accounted for about 13\% of the region wide arsenic emissions and nonferrous metals production for $17 \%$.

Arsenic in the particulate phase is the predominant (89-98.6\%) form of arsenic in the troposphere (Matschullat 2000). Inorganic species, most commonly trivalent arsenic, is the dominant form of arsenic in the air over emission areas; methylated forms of arsenic are probably of minor significance. Arseniccontaining air samples of smelter or coal-fired power plant origin consist largely of trivalent arsenic in both vapor and particulate form (Pacyna 1987). Oxides are the primary species evolved from fossil fuel and industrial processes. Additionally, arsenic trisulfide has also been reported from coal combustion, organic arsines from oil combustion, and arsenic trichloride from refuse incineration.

Arsenic has been identified in 59 air samples collected from 1,684 current or former NPL hazardous waste sites where it was detected in some environmental media (HazDat 2006).

\subsubsection{Water}

Estimated releases of 780 pounds ( $\sim 0.35$ metric tons) of arsenic to surface water from 58 domestic manufacturing and processing facilities in 2004 , accounted for about $0.09 \%$ of the estimated total environmental releases from facilities required to report to the TRI (TRI04 2006). Estimated releases of $1.3 \times 105$ pounds ( $\sim 59$ metric tons) of arsenic compounds to surface water from 361 domestic manufacturing and processing facilities in 2004 , accounted for about $0.11 \%$ of the estimated total environmental releases from facilities required to report to the TRI (TRI04 2006). These releases for arsenic and arsenic compounds are summarized in Tables 6-1 and 6-2, respectively.

Arsenic may be released to water from the natural weathering of soil and rocks, and in areas of vulcanism. Arsenic may also leach from soil and minerals into groundwater. Anthropogenic sources of arsenic releases to water include mining, nonferrous metals, especially copper, smelting, waste water, dumping of sewage sludge, coal burning power plants, manufacturing processes, urban runoff, atmospheric deposition and poultry farms (Garbarino et al. 2003; Nriagu and Pacyna 1988; Pacyna et al. 1995). A contributory 
part of mining and coal burning power plants is leaching from abandoned mine tailing and fly ash waste piles. Significant amounts of arsenic are released in liquid effluents from gold-milling operations using cyanide (Environment Canada 1993). Nriagu and Pacyna (1988) and Pacyna et al. (1995) estimated global anthropogenic inputs of arsenic into rivers, lakes, and oceans for 1983; annual estimated inputs ranged from 11,600 to 70,300 metric tons with a median value of 41,800 metric tons. Arsenic was detected in $58 \%$ of samples of urban storm water runoff from 8 of 15 cities surveyed in the National Urban Runoff Program at concentrations ranging from 1 to $50.5 \mu \mathrm{g} / \mathrm{L}$ (Cole et al. 1984).

Leaching of arsenic from soil, landfills, or slag deposits is a source of arsenic in groundwater (Francis and White 1987; Wadge and Hutton 1987). The arsenic in soil may be naturally-occurring or a result of the application of arsenic-containing pesticides or sludge. Wood treated with CCA is used in piers, piling and bulkheads and arsenic can leach from the treated wood (Breslin and Adler-Ivanbrook 1998; Brooks 1996; Cooper 1991; Sanders et al. 1994; Weis et al. 1998). Ammoniacal copper zinc arsenate (ACZA) is another arsenic-containing waterborne preservative; however, it is not as widely used as CCA (Lebow et al. 2000).

Arsenic has been identified in 846 groundwater and 414 surface water samples collected from 1,684 NPL hazardous waste sites, where it was detected in some environmental media (HazDat 2006).

\subsubsection{Soil}

Estimated releases of 0.70 million pounds ( $\sim 320$ metric tons) of arsenic to soils from 58 domestic manufacturing and processing facilities in 2004 , accounted for about $77 \%$ of the estimated total environmental releases from facilities required to report to the TRI (TRI04 2006). An additional 0.17 million pounds ( $\sim 77$ metric tons), constituting about $19 \%$ of the total environmental emissions, were released via underground injection (TRI04 2006). Estimated releases of 117 million pounds ( $\sim 5.3 \times 104$ metric tons) of arsenic compounds to soils from 361 domestic manufacturing and processing facilities in 2004 , accounted for about $98 \%$ of the estimated total environmental releases from facilities required to report to the TRI (TRI04 2006). An additional 1.6 million pounds ( $\sim 20$ metric tons), constituting about $1.3 \%$ of the total environmental emissions, were released via underground injection (TRI04 2006). These releases for arsenic and arsenic compounds are summarized in Tables 6-1 and 6-2, respectively.

The soil receives arsenic from a variety of anthropogenic sources, including ash residue from power plants, smelting operations, mining wastes, and municipal, commercial, and industrial waste. Ash from 
power plants is often incorporated into cement and other materials that are used for roads and construction. Arsenic may be released from such material into soil. Nriagu and Pacyna (1988) and Pacyna et al. (1995) estimated global anthropogenic inputs of arsenic into soil for 1983. Excluding mine tailings and smelter slag, annual estimated inputs ranged from 52,000 to 112,000 metric tons with a median value of 82,000 metric tons. Mine tailings and smelter slag were estimated to add an additional 7,200-11,000 and 4,500-9,000 metric tons, respectively. Old abandoned mine tailings undoubtedly contribute still more. Wood treated with CCA used in foundations or posts could potentially release arsenic into the surrounding soil. CCA preservatives have been shown to leach to varying degrees from wood, as well as through soils in both field and laboratory studies (Chirenje et al. 2003a; Hingston et al. 2001; Lebow et al. 2000; Rahman et al. 2004; Stilwell and Graetz 2001; USDA/USDT 2000). Arsenic may also be released on land through the application of pesticides and fertilizer. Senesi et al. (1999) reported the range of arsenic in 32 fertilizers as $2.2-322 \mathrm{ng} / \mathrm{g}$. Roxarsone (3-nitro-4-hydroxyphenylarsonic acid), which was used to treat poultry feed in approximately $70 \%$ of the broiler poultry operations in 1999-2000, is excreted unchanged in the manure. Poultry litter (manure and bedding) is routinely used as fertilizer to cropland and pasture. In 2000 , assuming $70 \%$ of the 8.3 billion broiler poultry produced in the United States were fed roxarsone-treated feed, the resulting manure would contain approximately $2.5 \times 10^{5} \mathrm{~kg}$ of arsenic (Garbarino et al. 2003). Land application of sewage sludge is another source of arsenic in soil. Arsenic was detected in sewage sludge samples from 23 cities at concentrations of $0.3-$ $53 \mu \mathrm{g} / \mathrm{g}$ (Mumma et al. 1984).

Arsenic has been identified in 758 soil and 515 sediment samples collected from 1,684 NPL hazardous waste sites, where it was detected in some environmental media (HazDat 2006).

\subsection{ENVIRONMENTAL FATE}

\subsubsection{Transport and Partitioning}

Arsenic in soil may be transported by wind or in runoff or may leach into the subsurface soil. However, because many arsenic compounds tend to partition to soil or sediment under oxidizing conditions, leaching usually does not transport arsenic to any great depth (EPA 1982c; Moore et al. 1988; PantsarKallio and Manninen 1997; Welch et al. 1988). Arsenic is largely immobile in agricultural soils; therefore, it tends to concentrate and remain in upper soil layers indefinitely. Downward migration has been shown to be greater in a sandy soil than in a clay loam (Sanok et al. 1995). Arsenic from lead arsenate that was used for pest control did not migrate downward below $20 \mathrm{~cm}$ in one fruit orchard; in another orchard, 15 years after sludge amendments and deep plowing, essentially all arsenic residues 
remained in the upper $40 \mathrm{~cm}$ of soil (Merwin et al. 1994). Leaching of arsenic in polluted wetland soil was low; leaching was correlated with the amount of dissolved organic matter in the soil (Kalbitz and Wennrich 1998). The effect of soil characteristics, namely $\mathrm{pH}$, organic matter content, clay content, iron oxide content, aluminum oxide content, and cation exchange capacity (CEC), on the adsorption of various metals, including the metalloid arsenic, to 20 Dutch surface soils was assessed by regression analysis (Janssen et al. 1997). The most influential parameter affecting arsenic adsorption was the iron content of the soil.

Arsenic that is adsorbed to iron and manganese oxides may be released under reducing conditions, which may occur in sediment or flooding conditions (LaForce et al. 1998; McGeehan 1996; Mok and Wai 1994). In addition to reductive dissolution, when nutrient levels are adequate, microbial action can also result in dissolution (LaForce et al. 1998). Interestingly, drying of the previously flooded soil increases arsenic adsorption, possibly due to alterations in iron mineralogy (McGeehan et al. 1998).

Darland and Inskeep (1997) conducted a study to determine the effects of $\mathrm{pH}$ and phosphate competition on the transport of arsenate $\left(\mathrm{H}_{x} \mathrm{AsO}_{4}{ }^{x-3}\right)$ through saturated columns filled with sand containing free iron oxides. At $\mathrm{pH} 4.5$ and 6.5 , arsenate transport was strongly retarded, while at $\mathrm{pH} 8.5$, it was rapid. The enhanced transport of arsenate at $\mathrm{pH} 8$ is consistent with the $\mathrm{pH}$ dependence of surface complexation reactions describing arsenate sorption by metal oxide minerals that can be categorized as a ligand exchange mechanism. Phosphate was shown to compete effectively with arsenate for adsorption sites on the sand, but the competition was not sufficient to desorb all of the arsenate in batch column experiments, even when the applied phosphate exceeded the column adsorption capacity by a factor of two. The researchers concluded that arsenate desorption kinetics may play an important role in the transport of arsenate through porous media. In a study looking at the effect of competing anions on the adsorption of arsenite and arsenate on ferrihydrite, the effect of phosphate on arsenate adsorption was greater at higher $\mathrm{pH}$ than at low $\mathrm{pH}$ and the opposite trend was observed for arsenite. While sulfate did not change the affinity of arsenate for ferrihydrite, sulfate reduced the adsorption of arsenite at pHs below 7.0 (Jain and Loeppert 2000).

Smith et al. (1999) investigated the sorption properties of both As(V) and As(III) in 10 Australian soils of widely different chemistry and mineralogy at commonly found arsenic levels. Adsorption of both arsenate and arsenite was rapid (1 hour). The amount of $\mathrm{As}(\mathrm{V})$ sorbed varied widely $(1.7-62.0 \mathrm{~L} / \mathrm{kg})$; soils with lower amounts of oxidic material adsorbed much less arsenic than those with higher amounts of these minerals. Arsenate sorption was highly correlated with the iron oxide content of the soil and this 
factor probably accounts for much of the variation in soil adsorptivity. Considerable leaching of arsenic occurred at a separate site where cattle were treated with a dip containing arsenic (cattle dip site) and that contained similar soil properties to that studied by Smith et al. (1999). Arsenite adsorption, which was investigated in four of the Australian soils, was sorbed to a lesser extent than was arsenate. This was attributed to soil minerology and the species of $\mathrm{As}(\mathrm{V})$ (arsenate) and $\mathrm{As}(\mathrm{III})$ (arsenite) present in solution; at $\mathrm{pH} 5-7$, the dominant $\mathrm{As}(\mathrm{V})$ species are $\mathrm{H}_{2} \mathrm{AsO}_{4}{ }^{-}$and $\mathrm{HAsO}_{4}{ }^{2-}$ and neutral $\mathrm{H}_{3} \mathrm{AsO}_{3}$ is the dominant As(III) species. For soils containing low amounts of oxidic minerals, $\mathrm{pH}$ had little effect on As(V) sorption, while for oxidic soils, a decrease in sorption was evident as the $\mathrm{pH}$ increased. In contrast, As(III) sorption increased with increasing pH (Smith et al. 1999). Jain et al. (1999) reported similar results where arsenite were both found to bind strongly to iron oxides; however, the adsorption of arsenate decreases with increasing $\mathrm{pH}$, while the adsorption of arsenite increases with increasing $\mathrm{pH}$ (Jain et al. 1999). As(III), which exists in a neutral form as arsenous acid, $\mathrm{H}_{3} \mathrm{AsO}_{3}\left(\mathrm{pK}_{\mathrm{a}}=9.23,12.13,13.4\right)$, is less strongly adsorbed on mineral surfaces than the oxyanions of arsenic acid, $\mathrm{H}_{3} \mathrm{AsO}_{4},\left(\mathrm{pK}_{\mathrm{a}}=2.22,6.98\right.$, 11.53) (NRC 1999). Based on its $\mathrm{pK}_{\mathrm{a}}$ values, arsenic acid would exist as a mixture of arsenate anions, $\mathrm{H}_{2} \mathrm{AsO}_{4}{ }^{-}$and $\mathrm{HAsO}_{4}{ }^{2-}$, under most environmental conditions ( $\mathrm{pH}$ 5-9).

The practice of liming to remediate contaminated soils and mine tailings has the potential to mobilize arsenic. Experiments performed by Jones et al. (1997) indicate that the increased mobility appears to be consistent with the $\mathrm{pH}$ dependence of sorption reactions of arsenic on iron oxide minerals rather than dissolution-precipitation reactions involving arsenic. They recommend that remediation of acidic mine tailings or other arsenic-contaminated soils be carefully evaluated with respect to potential arsenic mobilization, especially at contaminated sites hydraulically connected to surface or groundwaters.

Transport and partitioning of arsenic in water depends upon the chemical form (oxidation state and counter ion) of the arsenic and on interactions with other materials present. Soluble forms move with the water, and may be carried long distances through rivers (EPA 1979). However, arsenic may be adsorbed from water onto sediments or soils, especially clays, iron oxides, aluminum hydroxides, manganese compounds, and organic material (EPA 1979, 1982c; Welch et al. 1988). Under oxidizing and mildly reducing conditions, groundwater arsenic concentrations are usually controlled by adsorption rather than by mineral precipitation. The extent of arsenic adsorption under equilibrium conditions is characterized by the distribution coefficient, $\mathrm{K}_{\mathrm{d}}$, which measures the equilibrium partitioning ratio of adsorbed to dissolved contaminant. The value of $\mathrm{K}_{\mathrm{d}}$ depends strongly upon the $\mathrm{pH}$ of the water, the arsenic oxidation state, and the temperature. In acidic and neutral waters, $\mathrm{As}(\mathrm{V})$ is extensively adsorbed, while $\mathrm{As}(\mathrm{III})$ is relatively weakly adsorbed. Trivalent inorganic arsenic exists predominantly as arsenous acid $\left(\mathrm{H}_{3} \mathrm{AsO}_{3}\right)$ 
at environmental $\mathrm{pH}$ and is not strongly adsorbed to suspended solids and sediments in the water column. Pentavalent inorganic arsenic exists predominantly as $\mathrm{H}_{2} \mathrm{AsO}_{4}{ }^{-}$and $\mathrm{HAsO}_{4}{ }^{2-}$ in most environmental waters, which has considerably greater adsorption characteristics than arsenous acid. While in acidic and neutral waters, $\mathrm{As}(\mathrm{V})$ is more strongly adsorbed relative to $\mathrm{As}(\mathrm{III})$, in high-pH waters $(\mathrm{pH}>9)$ aquifer $\mathrm{K}_{\mathrm{d}}$ values are considerably lower for both oxidation states (Mariner et al. 1996). Sediment-bound arsenic may be released back into the water by chemical or biological interconversions of arsenic species (see Section 6.3.2).

Arsenic enters rivers from where mining operations occurred and is transported downstream, moving from water and sediment into biofilm (attached algae, bacterial, and associated fine detrital material), and then into invertebrates and fish. The source of arsenic in the water column may be resuspended sediment. While arsenic bioaccumulates in animals, it does not appear to biomagnify between tropic levels (Eisler 1994; Farag et al. 1998; Williams et al. 2006).

Most anthropogenic arsenic emitted to the atmosphere arises from high temperature processes (e.g., coal and oil combustion, smelting operations, and refuse incineration) and occurs as fine particles with a mass median diameter of about $1 \mu \mathrm{m}$ (Coles et al. 1979; Pacyna 1987). These particles are transported by wind and air currents until they are returned to earth by wet or dry deposition. Their residence time in the atmosphere is about 7-9 days, in which time the particles may be transported thousands of kilometers (EPA 1982b; Pacyna 1987). Long-range transport was evident in analyzing deposition of arsenic in countries like Norway; there was no indication that the marine environment contributed significantly to the deposition (Steinnes et al. 1992). Atmospheric fallout can be a significant source of arsenic in coastal and inland waters near industrial areas. Scudlark et al. (1994) determined the average wet depositional flux of arsenic as $49 \mu \mathrm{g} \mathrm{As} / \mathrm{m}^{2} /$ year for two sites in Chesapeake Bay, Maryland from June 1990 to July 1991. They found a high degree of spatial and temporal variability. The elemental fluxes derived predominantly from anthropogenic sources. Golomb et al. (1997) report average total (wet + dry) deposition rates to Massachusetts Bay of $132 \mu \mathrm{g} / \mathrm{m}^{2} /$ year, of which $21 \mu \mathrm{g} / \mathrm{m}^{2} /$ year was wet deposition during the period September 15, 1992-September 16, 1993. Hoff et al. (1996) estimated the following arsenic loadings into the Great Lakes for 1994 (lake, wet deposition, dry deposition): Superior, $11,000 \mathrm{~kg} /$ year, 3,600 kg/year; Michigan, 5,000 kg/year, 1,800 kg/year; Erie, 5,500 kg/year, $1,800 \mathrm{~kg} /$ year; and Ontario, 3,000 kg/year, $580 \mathrm{~kg} /$ year. The measured dry deposition fluxes of arsenic at four sampling sites around Lake Michigan ranged approximately from 0.01 to $1.5 \mu \mathrm{g} \mathrm{As} / \mathrm{m}^{2} /$ day; estimated inputs of arsenic into Lake Michigan were reported to be $1.4 \times 10^{3} \mathrm{~kg} / \mathrm{year}$ (Shahin et al. 2000). 
Terrestrial plants may accumulate arsenic by root uptake from the soil or by absorption of airborne arsenic deposited on the leaves, and certain species may accumulate substantial levels (EPA 1982b). Yet, even when grown on highly polluted soil or soil naturally high in arsenic, the arsenic level taken up by the plants is comparatively low (Gebel et al. 1998b; Pitten et al. 1999). Kale, lettuce, carrots, and potatoes were grown in experimental plots surrounding a wood preservation factory in Denmark where waste wood was incinerated to investigate the amount and pathways for arsenic uptake by plants (Larsen et al. 1992). On incineration, the arsenate in the wood preservative was partially converted to arsenite; the arsenic emitted from the stack was primarily particle bound. Elevated levels of inorganic arsenic were found in the test plants and in the soil around the factory. Statistical analyses revealed that the dominating pathway for transport of arsenic from the factory to the leafy vegetables (kale) was by direct atmospheric deposition, while arsenic in the root crops (potatoes and carrots) was a result of both soil uptake and atmospheric deposition. Arsenic accumulation by plants is affected by arsenic speciation. Uptake of four arsenic species (arsenite, arsenate, methylarsonic acid, and dimethylarsinic acid) by turnips grown under soilless culture conditions showed that while uptake increased with increasing arsenic concentration in the nutrient, the organic arsenicals showed higher upward translocation than the inorganic arsenical (Carbonell-Barrachina et al. 1999). The total amount of arsenic taken up by the turnip plants (roots and shoots) followed the trend methylarsenate (MMA) $<$ dimethylarsinic acid (DMA) $<$ arsenite $<$ arsenate. In a similar experiment, conducted with tomato plants, the total amount of arsenic taken up by the tomato plants followed the trend DMA $<$ MMA $<$ arsenate $\approx$ arsenite, with arsenic concentrations in the plants increasing with increasing arsenic concentration in the nutrient solution. Arsenic was mainly accumulated in the root system (85\%) with smaller amounts translocating to the fruit (1\%). However, plants treated with MMA and DMA had higher arsenic concentrations in the shoots and fruit than those treated with arsenite or arsenate (Burlo et al. 1999). Terrestrial plants growing on land bordering arsenic-contaminated waters show relatively little arsenic content, even though the sediments have arsenic concentrations as high as $200 \mu \mathrm{g} / \mathrm{g}$ (Tamaki and Frankenberger 1992). Arsenic concentrations in vegetables grown in uncontaminated soils and contaminated soils containing arsenic, as well as other metals and organic contaminants, were generally $<12 \mu \mathrm{g} / \mathrm{kg}$ wet weight. A maximum arsenic concentration of $18 \mu \mathrm{g} / \mathrm{kg}$ wet weight was found in unpeeled carrots grown in soil, which contained a mean arsenic concentration of $27 \mathrm{mg} / \mathrm{kg}$ dry weight (Samsøe-Petersen et al. 2002).

In a study by Rahman et al. (2004), CCA-treated lumber was used to construct raised garden beds to determine how far the components of CCA migrated in the soil and the uptake of these components by crops grown in the soil. Arsenic was found to diffuse laterally into the soil from the CCA-treated wood, with the highest concentrations found at $0-2 \mathrm{~cm}$ from the treated wood and a steady decline in 
concentration with increased distance. The highest average arsenic concentrations found in soil closest $(0-2 \mathrm{~cm})$ to the CCA-treated wood were 56 and $46 \mu \mathrm{g} / \mathrm{g}$ in loamy sand and sandy loam soils, respectively. At a distance of 30-35 $\mathrm{cm}$ from the CCA-treated wood, arsenic concentrations were approximately $7 \mu \mathrm{g} / \mathrm{g}$ in both soils. All samples were of the top $0-15 \mathrm{~cm}$ of soil. Crops grown in both soil types within 0-2 $\mathrm{cm}$ of the CCA-treated wood contained higher concentrations of arsenic, 0.186 and $10.894 \mu \mathrm{g} / \mathrm{g}$ for carrots without peal and bean leaves and stems, respectively, than those grown at $1.5 \mathrm{~m}$ from the CCA-treated wood, 0.006 and $0.682 \mu \mathrm{g} / \mathrm{g}$ for bean pods and bean leaves and stems, respectively. However, based on FDA guidelines on tolerance limits, these crops would be considered approved for human consumption. Studies by Chirenje et al. (2003a) also showed that elevated arsenic concentrations were found in surface $(0-5 \mathrm{~cm})$ soils immediately surrounding, within the first $0.3 \mathrm{~m}$, of utility poles, fences, and decks made with CCA-treated wood. Factors such as the preservative formula, fixation temperature, post treatment handling, and timber dimensions of CCA-treated wood, as well as the $\mathrm{pH}$, salinity, and temperature of the leaching media can affect the leach rates from CCA-treated wood (Hingston et al. 2001). Studies of leaching of the components of CCA- and ACZA-treated wood used to construct a boardwalk in wetland environments reported elevated arsenic levels in soil and sediment below and adjacent to these structures. Generally, these levels decreased with increasing distance from the structure (Lebow et al. 2000). Increased concentrations of arsenic were also observed under CCA-treated bridges. Arsenic levels declined with distance from the bridge and were near background levels at $1.8-3 \mathrm{~m}$ from the bridge's perimeter (USDA/USDT 2000).

In a study by Lebow et al. (2003), the use of a water repellent finish on CCA-treated wood significantly reduces the amount of arsenic, as well as copper and chromium, in the run-off water. It was also observed the exposure to UV radiation caused a significant increase in leaching from both finished and unfinished samples of CCA-treated wood. Small amounts of arsenic can be transferred from CCA-treated wood to skin from touching CCA-treated wood surfaces (Hemond and Solo-Gabriele 2004; Kwon et al. 2004; Shalat et al. 2006; Ursitti et al. 2004; Wang et al. 2005).

Breslin and Adler-Ivanbrook (1998) examined the leaching of the copper, chromium, and arsenic from CCA-treated wood in laboratory studies using samples of treated southern yellow pine in solutions simulating estuarine waters. The tank leaching solutions were frequently sampled and replaced to approximate field conditions. Initial 12-hour fluxes ranging from $0.2 \times 10^{-10}$ to $5.2 \times 10^{-10} \mathrm{~mol} / \mathrm{mm}^{2} \mathrm{~d}$ was reported for arsenic. After 90 days, arsenic fluxes decreased to $0.5 \times 10^{-11}-3.1 \times 10^{-11} \mathrm{~mol} / \mathrm{mm}^{2} \mathrm{~d}$. A study by Cooper (1991) demonstrated that the buffer system used in leaching studies of components from CCAtreated wood can significantly change the amount arsenic released from treated wood. Samples of four 
species of CCA-treated wood were exposed to four acidic leaching solutions. In the samples exposed to water adjusted to $\mathrm{pHs}$ of $3.5,4.5$, and 5.5 , losses of arsenic after 13 days were generally $<7 \%$. However, when a leaching solution of sodium hydroxide and citric acid buffer $(\mathrm{pH} 5.5)$ was used, the percent of arsenic leached ranged from 27.4 to $46.7 \%$ (Cooper 1991).

Arsenic bioaccumulation depends on various factors, such as environmental setting (marine, estuarine, freshwater), organism type (fish, invertebrate), trophic status within the aquatic food chain, exposure concentrations, and route of uptake (Williams et al. 2006). Bioaccumulation refers to the net accumulation of a chemical by aquatic organisms as a result of uptake from all environmental sources, such as water, food, and sediment, whereas bioconcentration refers to the uptake of a chemical by an aquatic organism through water (EPA 2003b). Biomagnification in aquatic food chains does not appear to be significant (EPA 1979, 1982b, 1983e, 2003b; Mason et al. 2000; Williams et al. 2006).

Bioconcentration of arsenic occurs in aquatic organisms, primarily in algae and lower invertebrates. Both bottom-feeding and predatory fish can accumulate contaminants found in water. Bottom-feeders are readily exposed to the greater quantities of metals, including the metalloid arsenic, which accumulate in sediments. Predators may bioaccumulate metals from the surrounding water or from feeding on other fish, including bottom-feeders, which can result in the biomagnification of the metals in their tissues. An extensive study of the factors affecting bioaccumulation of arsenic in two streams in western Maryland in 1997-1998 found no evidence of biomagnification since arsenic concentrations in organisms tend to decrease with increasing tropic level (Mason et al. 2000). Arsenic is mainly accumulated in the exoskeleton of invertebrates and in the livers of fish. No differences were found in the arsenic levels in different species of fish, which included herbivorous, insectivorous, and carnivorous species. The major bioaccumulation transfer is between water and algae, at the base of the food chain and this has a strong impact on the concentration in fish. National Contaminant Biomonitoring data produced by the Fish and Wildlife Service were used to test whether differences exist between bottom-feeders and predators in tissue levels of metals and other contaminants. No differences were found for arsenic (Kidwell et al. 1995). The bioconcentration factors (BCFs) of bryophytes, invertebrates, and fish (livers) in Swedish lakes and brooks impacted by smelter emissions were 8,700, 1,900-2,200, and 200-800, respectively (Lithner et al. 1995). EPA (2003b) assessed a large dataset of bioaccumulation data for various fish and invertebrate species. BCF values in this dataset ranged from 0.048 to 1,390 .

Williams et al. (2006) reviewed 12 studies of arsenic bioaccumulation in freshwater fish, and proposed that $\mathrm{BCF}$ and bioaccumulation factor $(\mathrm{BAF})$ values are not constant across arsenic concentrations in 
water. BCF or BAF values from these 12 studies ranged from 0.1 to 3,091. Williams et al. (2006) found that $\mathrm{BCF}$ and $\mathrm{BAF}$ values appear to be the highest within the range of ambient arsenic concentrations, and decline steeply to relatively low levels as the arsenic concentrations in water increase. Based on this analysis, arsenic concentrations in tissue and BAF values may be a power function of arsenic concentrations in water. EPA (2007b) also reported that for many nonessential metals, including arsenic, accumulation is nonlinear with respect to exposure concentration.

\subsubsection{Transformation and Degradation}

\subsubsection{Air}

Arsenic is released into the atmosphere primarily as arsenic trioxide or, less frequently, in one of several volatile organic compounds, mainly arsines (EPA 1982b). Trivalent arsenic and methyl arsines in the atmosphere undergo oxidation to the pentavalent state (EPA 1984a), and arsenic in the atmosphere is usually a mixture of the trivalent and pentavalent forms (EPA 1984a; Scudlark and Church 1988). Photolysis is not considered an important fate process for arsenic compounds (EPA 1979).

\subsubsection{Water}

Arsenic in water can undergo a complex series of transformations, including oxidation-reduction reactions, ligand exchange, precipitation, and biotransformation (EPA 1979, 1984a; Sanders et al. 1994; Welch et al. 1988). Rate constants for these various reactions are not readily available, but the factors most strongly influencing fate processes in water include $\mathrm{Eh}, \mathrm{pH}$, metal sulfide and sulfide ion concentrations, iron concentrations, temperature, salinity, distribution and composition of the biota, season, and the nature and concentration of natural organic matter (EPA 1979; Farago 1997; Redman et al. 2002; Wakao et al. 1988). Organic arsenical pesticides, such as MSMA, DSMA, and DMA do not degrade by hydrolysis or by aquatic photolysis (EPA 2006). No formation of arsine gas from marine environments has been reported (Tamaki and Frankenberger 1992).

Inorganic species of arsenic are predominant in the aquatic environment. In the $\mathrm{pH}$ range of natural waters, the predominant aqueous inorganic $\mathrm{As}(\mathrm{V})$ species are the arsenate ions, $\mathrm{H}_{2} \mathrm{AsO}_{4}{ }^{-}$and $\mathrm{HAsO}_{4}{ }^{2-}$; the predominant inorganic As(III) species is $\mathrm{As}(\mathrm{OH})_{3}$ (Aurillo et al. 1994; EPA 1982c). As(V) generally dominates in oxidizing environments such as surface water and As(III) dominates under reducing conditions such as may occur in groundwater containing high levels of arsenic. However, the reduction of arsenate to arsenite is slow, so arsenate can be found in reducing environments. Conversely, the oxidation of arsenite in oxidizing environments is moderately slow (half-life, 0.4-7 days in coastal 
systems) and therefore, arsenite can be found in oxidizing environments (Mariner et al. 1996; Sanders et al. 1994). The main organic species in fresh water are MMA and DMA; however, these species are usually present at lower concentrations than inorganic arsenic species (Eisler 1994). (The toxicities of MMA and DMA are discussed in Chapter 3.) Aquatic microorganisms may reduce the arsenate to arsenite, as well as methylate arsenate to its mono- or dimethylated forms (Aurillo et al. 1994; Benson 1989; Braman and Foreback 1973; Edmonds and Francesconi 1987; Sanders et al. 1994). Methylated species are also produced by the biogenic reduction of more complex organoarsenic compounds like arsenocholine or arsenobetaine. Water samples from a number of lakes and estuaries, mostly in California, show measurable concentrations of methylated arsenic (equivalent to 1-59\% of total arsenic) (Anderson and Bruland 1991). Within the oxic photic zone, arsenate and DMA were the dominant species. A seasonal study of one lake demonstrated that DMA was the dominant form of arsenic in surface waters during late summer and fall. Methylated species declined and arsenate species increased when the lake turned over in late fall. Mono Lake, a highly alkaline body of water, and four rivers did not have measurable concentrations of methylated arsenic. It was hypothesized that the reason why methylated forms were not detected in Mono Lake was that the extremely high inorganic arsenic concentrations in the lake, $230 \mu \mathrm{M}(17 \mathrm{mg} / \mathrm{L})$, could overwhelm the analysis of small amounts of organic forms. Other possibilities are that the high alkalinity or very high phosphate levels in the water, $260 \mu \mathrm{M}$ (25 mg/L), are not conducive to biogenic methylation (Anderson and Bruland 1991). Both reduction and methylation of $\mathrm{As}(\mathrm{V})$ may lead to increased mobilization of arsenic, since $\mathrm{As}(\mathrm{III})$, dimethylarsinates, and monomethylarsonates are much less particle-reactive than As(V) (Aurillo et al. 1994). In the estuarial Patuxet River, Maryland, arsenate concentrations peaked during the summer, at $1.0 \mu \mathrm{g} / \mathrm{L}$ in 1988 1989 (Sanders et al. 1994). In contrast, winter to spring levels were around $0.1 \mu \mathrm{g} / \mathrm{L}$. Arsenite concentrations were irregularly present at low levels during the year. Peaks of DMA occurred at various times, particularly in the winter and late spring and appeared to be linked with algal blooms. The DMA peak declined over several months that was followed by a rise in MMA. The MMA was thought to be occurring as a degradation product of DMA. A similar seasonal pattern of arsenic speciation was observed in Chesapeake Bay. Arsenite methylation took place during the warmer months leading to changes down the main stem of the bay; arsensite production dominated the upper reaches of the bay and methylated species dominated the more saline lower reaches. In coastal waters, reduced and methylated species are present in lower concentrations, around 10-20\% of total arsenic (Sanders et al. 1994). In groundwater, arsenic generally exists as the oxyanion of arsenate $\left(\mathrm{H}_{\mathrm{x}} \mathrm{AsO}_{4}{ }^{3-\mathrm{x}}\right)$ or arsenite $\left(\mathrm{H}_{\mathrm{x}} \mathrm{AsO}_{3}{ }^{3-\mathrm{x}}\right)$, or both; however, the distribution between arsenite and arsenate is not always predictable based on oxidation-reduction potential (Robertson 1989; Welch et al. 1988). 


\subsubsection{Sediment and Soil}

In soil, arsenic is found as a complex mixture of mineral phases, such as co-precipitated and sorbed species, as well as dissolved species (Roberts et al. 2007). The degree of arsenic solubility in soil will depend on the amount of arsenic distributed between these different mineral phases. The dissolution of arsenic is also affected by particle size. The distribution between these phases may reflect the arsenic source (e.g., pesticide application, wood treatment, tanning, or mining operations), and may change with weathering and associations with iron and manganese oxides and phosphate minerals in the soil (Roberts et al. 2007; Ruby et al. 1999). Davis et al. (1996) reported that in soil in Anaconda, Montana, a smelting site from 1860 to 1980 , contained arsenic that is only in a sparingly soluble form, consisting of primarily arsenic oxides and phosphates.

The arsenic cycle in soils is complex, with many biotic and abiotic processes controlling its overall fate and environmental impact. Arsenic in soil exists in various oxidation states and chemical species, depending upon soil $\mathrm{pH}$ and oxidation-reduction potential. Under most environmental conditions, inorganic $\mathrm{As}(\mathrm{V})$ will exist as a mixture of arsenate anions, $\mathrm{H}_{2} \mathrm{AsO}_{4}{ }^{-}$and $\mathrm{HAsO}_{4}{ }^{2-}$, and inorganic $\mathrm{As}(\mathrm{III})$ will exist as $\mathrm{H}_{3} \mathrm{AsO}_{3}$. The arsenate and arsenite oxyanions have various degrees of protonation depending upon pH (EPA 1982b; McGeehan 1996). As(V) predominates in aerobic soils, and As(III) predominates in slightly reduced soils (e.g., temporarily flooded) or sediments (EPA 1982b; Sanders et al. 1994). As(III) commonly partitions to the aqueous phase in anoxic environments, and would be more mobile. $\operatorname{As}(\mathrm{V})$ usually remains bound to minerals, such as ferrihydrite and alumina, limiting its mobility and bioavailability (Rhine et al. 2006).

Arsenite is moderately unstable in the presence of oxygen; however, it can be found under aerobic conditions as well (Sanders et al. 1994). While arsenate is strongly sorbed by soils under aerobic conditions, it is rapidly desorbed as the system becomes anaerobic. Once it is desorbed, arsenate can be reduced to arsenite, which exhibits greater mobility in soils (McGeehan 1996). Transformations between the various oxidation states and species of arsenic occur as a result of biotic or abiotic processes (Bhumbla and Keefer 1994). While degradation of an organic compound is typically considered complete mineralization, in the case of organic arsenic compounds, the element arsenic itself cannot be degraded. However, the organic portion of the molecule can be metabolized (Woolson 1976).

Arsenicals applied to soils may be methylated by microorganisms to arsines, which are lost through volatilization, and organic forms may be mineralized to inorganic forms. Gao and Burau (1997) reported 
that the overall percentage of DMA and MMA minerialized after 70 days ranged from 3 to $87 \%$ in air-dry soil and a soil near saturation, respectively. The rate of demethylation of DMA increased with soil moisture. Over the same 70-day period, arsenic losses as volatile arsines were much lower than minerialization, ranging from 0.001 to $0.4 \%$. Arsine evolution rates followed the order: DMA $>$ MMA $>$ arsenite $=$ arsenate (Gao and Burau 1997). Woolson and Kearney (1973) reported that ${ }^{14} \mathrm{C}$-labeled DMA degraded differently in soils under aerobic and anaerobic conditions. Under anaerobic conditions, $61 \%$ of the applied DMA was converted to a volatile alkyl arsine after 24 weeks, and lost from the soil system. Under aerobic conditions, 35\% was converted to a volatile organo-arsenic compound, possibly dimethyl arsine, and $41 \%$ was converted to ${ }^{14} \mathrm{CO}_{2}$ and arsenate after 24 weeks. Similar to microorganisms in soils, Reimer (1989) reported that microorganisms found in natural marine sediments and sediments contaminated with mine-tailings are also capable of methylating arsenic under aerobic and anaerobic conditions. Von Endt et al. (1968) reported that the degradation of ${ }^{14} \mathrm{C}$-labelled monosodium methanearsonate (MSMA) was found to range from 1.7 to $10 \%$ in Dundee silty clay loam soil and Sharkey clay soil after 60 days, respectively. MSMA decomposition to $\mathrm{CO}_{2}$ was a slow process without a lag period. Sterilized soils were found to produce essentially no ${ }^{14} \mathrm{CO}_{2}(0.7 \%)$ after 60 days, indicating that soil bacteria contributed to the decomposition of MSMA (Von Endt et al. 1968). Akkari et al. (1986) studied the degradation of MSMA in various soils. At $20 \%$ water content, half-lives of 144,88 , and 178 days were reported in Sharkey clay, Taloka silt loam, and Steele-Crevasse sand loam, respectively. The Sharkey soil with the highest clay content was expected to have the greatest adsorptive capacity for both water and MSMA, reducing the amount of MSMA available in the soil solution to microorganisms that degrade the MSMA. The half-lives were 25, 41, and 178 days under anaerobic (flooded) conditions in Sharkey clay, Taloka silt loam, and Steele-Crevasse sand loam, respectively. Under flooded conditions, MSMA degradation occurs by reductive methylation to form arsinite and alkylarsine gas. The authors attributed the longer half-lives for MSMA degradation in the SteeleCrevasse sand loam soil to its low organic matter content, which may have supported fewer microbial populations needed for oxidation demethylation under aerobic conditions. Under flooded conditions, anaerobiosis is expected to be slowest in low organic matter sandy loam soils (Akkari et al. 1986).

Organic arsenical pesticides, such as MSMA, DSMA, and DMA, do not degrade by hydrolysis or by soil photolysis (EPA 2006).

Roxarsone (3-nitro-4-hydroxyphenylarsonic acid) used in poultry feed is found excreted unchanged in poultry litter (bedding and manure). Roxarsone found in poultry litter, which is used to amend agricultural soil, was found to degrade to arsenate in approximately $3-4$ weeks upon composting 
(Garbarino et al. 2003). In addition, the arsenic in poultry litter was found to be easily mobilized by water; however, its leach rate from amended soils was slow enough that it accumulated in soils (Rutherford et al. 2003).

A sequential fractionation scheme was used to assess the chemical nature, and thus the potential bioavailability, of arsenic at cattle dip sites in Australia where sodium arsenite was used extensively in cattle dips from the turn of the century until the early 1950s (McLaren et al. 1998). Most sites contained substantial amounts, $13 \%$ on the average, of arsenic in the two most labile fractions indicating a high potential for bioaccessibility and leaching. The bulk of the arsenic appeared to be associated with amorphous iron and aluminum minerals in soil. Similarly, arsenic in soil and mine waste in the Tamar Valley in England was found to be concentrated in a fraction associated with iron and organic-iron (Kavanagh et al. 1997). Laboratory studies were performed to assess the phase partitioning of trace metals, including the metalloid arsenic, to sediment from the Coeur d'Alene River, a mining area of Idaho, and the release of metals under simulated minor and major flooding events (LaForce et al. 1998). Arsenic was primarily associated with the iron and manganese oxides as seen by its large release when these oxides were reduced. Arsenic levels were comparatively low in the organic fraction and remaining residual fraction and negligible in the extractible fractions.

\subsubsection{Other Media}

Carbonell-Barrachina et al. (2000) found the speciation and solubility of arsenic in sewage sludge suspensions to be affected by $\mathrm{pH}$ and $\mathrm{Eh}$. Under oxidizing conditions, the solubility of arsenic was low, with a major portion of the soluble arsenic present as organic arsenic compounds, mainly dimethylarsinic acid (approximately $74 \%$ of the total arsenic in solution). Under moderately reducing conditions ( 0 $100 \mathrm{mV}$ ), inorganic arsenic accounted for the majority (90\%) of the total arsenic in solution, and the solubility of arsenic was increased due to dissolution of iron oxyhydroxides. Under strongly reducing conditions $(-250 \mathrm{mV})$, arsenic solubility was decreased by the formation of insoluble sulfides. The $\mathrm{pH}$ of the solution was also found to influence the speciation and solubility of arsenic. At neutral $\mathrm{pH}$, the solubility of arsenic was at its maximum, and decreased under acidic or alkaline conditions. Inorganic arsenic species were the dominant species at $\mathrm{pH} 5.0$; at $\mathrm{pH}$ 6.5, the major soluble forms were organic arsenic species. The biomethylation of arsenic was limited at acidic $\mathrm{pH}$, and was at its maximum at near neutral pH (Carbonell-Barrachina et al. 2000). 


\subsection{LEVELS MONITORED OR ESTIMATED IN THE ENVIRONMENT}

Reliable evaluation of the potential for human exposure to arsenic depends in part on the reliability of supporting analytical data from environmental samples and biological specimens. Concentrations of arsenic in unpolluted atmospheres and in pristine surface waters are often so low as to be near the limits of current analytical methods. In reviewing data on arsenic levels monitored or estimated in the environment, it should also be noted that the amount of chemical identified analytically is not necessarily equivalent to the amount that is bioavailable. The analytical methods available for monitoring arsenic in a variety of environmental media are detailed in Chapter 7.

\subsubsection{Air}

Arsenic in ambient air is usually a mixture of particulate arsenite and arsenate; organic species are of negligible importance except in areas of substantial methylated arsenic pesticide application or biotic activity (EPA 1984a). Mean levels in ambient air in the United States have been reported to range from $<1$ to $3 \mathrm{ng} / \mathrm{m}^{3}$ in remote areas and from 20 to $30 \mathrm{ng} / \mathrm{m}^{3}$ in urban areas (Davidson et al. 1985; EPA 1982c; IARC 1980; NAS 1977a). EPA conducted a modeling study with the Assessment System for Population Exposure Nationwide (ASPEN) in which estimates of emissions of hazardous air pollutants were used to estimate ambient concentrations (Rosenbaum et al. 1999). Using 1990 data to estimate total emissions of arsenic in the conterminous 48 states, excluding road dust or windblown dust from construction or agricultural tilling, the 25 th percentile, median, and 75 th percentile arsenic concentration were estimated to be 9,20 , and $30 \mathrm{ng} / \mathrm{m}^{3}$, respectively. Maps illustrating the amount of toxic air pollutant emissions, including arsenic compounds, by county in 1996 for the 48 coterminous states of the United States as well as Puerto Rico and the Virgin Islands are available on the internet at http://www.epa.gov/ttn/atw/nata/mapemis.html, as of March 2005. Schroeder et al. (1987) listed ranges of arsenic concentrations in air of $0.007-1.9,1.0-28$, and $2-2,320 \mathrm{ng} / \mathrm{m}^{3}$ in remote, rural, and urban areas, respectively. The average annual arsenic concentration in air at Nahant, Massachusetts, just north of Boston, between September 1992 and September 1993 , was $1.2 \mathrm{ng} / \mathrm{m}^{3} ; 75 \%$ of the arsenic was associated with fine $(<2.5 \mu \mathrm{m})$ particles. The long-term means of the ambient concentrations of arsenic measured in urban areas of the Great Lakes region from 1982 to 1993 ranged from 4.2 to $9.6 \mathrm{ng} / \mathrm{m}^{3}$ (Pirrone and Keeler 1996). Large cities generally have higher arsenic air concentrations than smaller ones due to emissions from coal-fired power plants (IARC 1980), but maximum 24-hour concentrations generally are $<100 \mathrm{ng} / \mathrm{m}^{3}$ (EPA 1984a). In the spring of 1990, aerosols and cloud water that were sampled by aircraft at an altitude of $1.2-3 \mathrm{~km}$ above the Midwestern United States had a mean mixed layer arsenic concentration of $1.6 \pm 0.9 \mathrm{ng} / \mathrm{m}^{3}$ (Burkhard et al. 1994). A mean arsenic concentration of $1.0 \pm 0.5 \mathrm{ng} / \mathrm{m}^{3}$ was reported at 
Mayville, New York, a site $400 \mathrm{~km}$ to the northwest of the sampling area and directly downwind on most days.

Arsenic was monitored at an application site in the San Joaquin Valley, California and at four sites in nearby communities in 1987 where sodium arsenite was used as a fungicide on tokay grapes (Baker et al. 1996). The maximum arsenic concentration measured 15-20 meters from the edge of the field was $260 \mathrm{ng} / \mathrm{m}^{3}$. The maximum arsenic concentration at four community sites in the area was $76 \mathrm{ng} / \mathrm{m}^{3}$. The concentration at an urban background site was $3 \mathrm{ng} / \mathrm{m}^{3}$ (Baker et al. 1996). Sodium arsenite is no longer registered in California (Baker et al. 1996). The highest historic arsenic levels detected in the atmosphere were near nonferrous metal smelters, with reported concentrations up to $2,500 \mathrm{ng} / \mathrm{m}^{3}$ (IARC 1980; NAS 1977a; Schroeder et al. 1987).

Arsenic air concentrations measured in several indoor public places (e.g., cafeteria, coffee house, music club, Amtrak train, and several restaurants) with environmental tobacco smoke (ETS) ranged from $<0.1$ to $1 \mathrm{ng} / \mathrm{m}^{3}$, with a mean of $0.4 \pm 0.3 \mathrm{ng} / \mathrm{m}^{3}$. Sites that were ETS-free (university office and library) had arsenic concentrations $<0.13 \mathrm{ng} / \mathrm{m}^{3}$ (Landsberger and $\mathrm{Wu}$ 1995). The Toxic Exposure Assessment at Columbia/Harvard (TEACH) study measured levels of various toxics in New York City air in 1999. Exposures were assessed in a group of 46 high school students in West Central Harlem. Mean arsenic concentrations in summer home outdoor, home indoor, and personal air of the participants were 0.37 , 0.40 , and $0.45 \mathrm{ng} / \mathrm{m}^{3}$, respectively (Kinney et al. 2002). Detected arsenic concentrations in indoor and outdoor air collected as part of the National Human Exposure Assessment Survey (NHEXAS) in Arizona ranged from 3.4 to 22.3 and from 3.5 to $25.7 \mathrm{ng} / \mathrm{m}^{3}$, respectively, with 71 and $68 \%$ below the detection limit (1.8-14.3 ng/m³ $)\left(\mathrm{O}^{\prime}\right.$ Rourke et al. 1999).

\subsubsection{Water}

Arsenic is widely distributed in surface water, groundwater, and finished drinking water in the United States. A survey of 293 stations in two nationwide sampling networks on major U.S. rivers found median arsenic levels to be $1 \mu \mathrm{g} / \mathrm{L}$; the 75th percentile level was $3 \mu \mathrm{g} / \mathrm{L}$ (Smith et al. 1987). Arsenic was detected in 1,298 of 3,452 surface water samples recorded in the STORET database for 2004 at concentrations ranging from 0.138 to $1,700 \mu \mathrm{g} / \mathrm{L}$ in samples where arsenic was detected (EPA 2005c). Two streams in western Maryland that were the focus of a major bioaccumulation study in 1997-1998 had arsenic concentrations of $0.370 \pm 0.200$ and $0.670 \pm 0.460 \mu \mathrm{g} / \mathrm{L}$ (Mason et al. 2000). Surface water will be impacted by runoff from polluted sites. An average arsenic concentration of $5.12 \mu \mathrm{g} / \mathrm{L}$ was reported in 
water from Moon Lake, a Mississippi River alluvial floodplain in northwest Mississippi. Intensive cultivation has occurred in this area, including cotton, soybeans, and rice (Cooper and Gillespie 2001). Hard-rock mining activities occurred in the southern part of Colorado and New Mexico north of Taos since the latter part of the 19th century until recently, which have impacted the Rio Grande and its tributaries. A mean arsenic concentration of approximately $0.8 \mu \mathrm{g} / \mathrm{L}$ was reported for the main stem of the Rio Grande sampled in June and September 1994. Arsenic concentrations in the Alamosa River, Colorado were 0.11 and $0.14 \mu \mathrm{g} / \mathrm{L}$ in June and September 1994, respectively, and 1.4 $\mu \mathrm{g} / \mathrm{L}$ in Big Arsenic Spring, New Mexico in September 1994 (Taylor et al. 2001). Arsenic concentrations in water from watersheds in Black Hills, South Dakota, an area impacted by gold mining activities ranged from 2.5 to $55 \mu \mathrm{g} / \mathrm{L}$ and from 1.7 to $51 \mu \mathrm{g} / \mathrm{L}$ in unfiltered and filtered samples, respectively; concentrations from reference areas ranged from 1.1 to $3.4 \mu \mathrm{g} / \mathrm{L}$ and from 0.9 to $1.9 \mu \mathrm{g} / \mathrm{L}$ in unfiltered and filtered samples, respectively (May et al. 2001). Arsenic concentrations ranged from 0.29 to $34.0 \mu \mathrm{g} / \mathrm{L}$ in water samples from Wakulla River and St. Joseph Bay North, along the Florida Panhandle; arsenic contamination in this area is likely to result from nonpoint source pollution (Philp et al. 2003).

Data on total arsenic in surface water from a number of seas and oceans show levels of $<1 \mu \mathrm{g} / \mathrm{L}$, except in the Antarctic Ocean and Southwest Pacific Oceans where the levels are 1.1 and $1.2 \mu \mathrm{g} / \mathrm{L}$, respectively. Levels in coastal waters and estuaries are generally somewhat higher, in the range of $1-3 \mu \mathrm{g} / \mathrm{L}$. However, estuarine water in Salinas, California had arsenic levels of $7.42 \mu \mathrm{g} / \mathrm{L}$ (Francesconi et al. 1994). The dissolved arsenic concentration in water at 40 sites in the Indian River Lagoon System in Florida ranged from 0.35 to $1.6 \mu \mathrm{g} / \mathrm{L}$ with a mean of $0.89 \pm 0.34 \mu \mathrm{g} / \mathrm{L}$ (Trocine and Trefry 1996). Thermal waters generally have arsenic levels of $20-3,800 \mu \mathrm{g} / \mathrm{L}$, although levels as high as $276,000 \mu \mathrm{g} / \mathrm{L}$ have been recorded (Eisler 1994).

Arsenic levels in groundwater average about 1-2 $\mu \mathrm{g} / \mathrm{L}$, except in some western states with volcanic rock and sulfidic mineral deposits high in arsenic, where arsenic levels up to $3,400 \mu \mathrm{g} / \mathrm{L}$ have been observed (IARC 1980; Page 1981; Robertson 1989; Welch et al. 1988). In western mining areas, groundwater arsenic concentrations up to $48,000 \mu \mathrm{g} / \mathrm{L}$ have been reported (Welch et al. 1988). Arsenic concentrations in groundwater samples collected form 73 wells in 10 counties in southeast Michigan in 1997 ranged from 0.5 to $278 \mu \mathrm{g} / \mathrm{L}$, with an average of $29 \mu \mathrm{g} / \mathrm{L}$. Most (53-98\%) of the arsenic was detected as arsenite (Kim et al. 2002). The U.S. Geological Survey mapped concentrations of arsenic in approximately 31,350 groundwater samples collected between 1973 and 2001; the counties in which at least 25\% of wells exceed various levels are shown in Figure 6-2 (USGS 2007a). Most arsenic in natural waters is a 
Figure 6-2. Counties in Which at Least 25\% of Wells Exceed Different Arsenic Levels

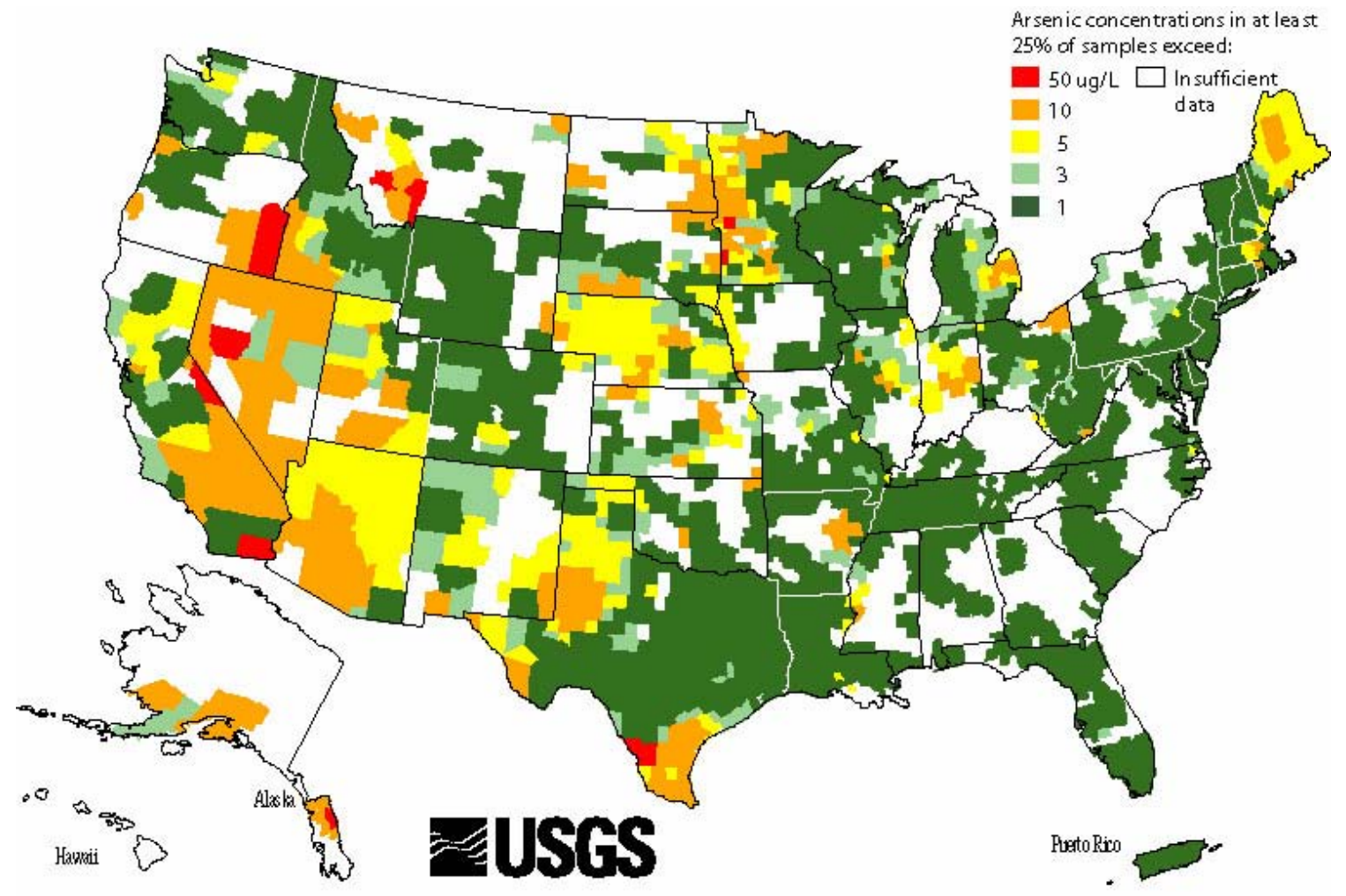

Source: USGS 2007a 
mixture of arsenate and arsenite, with arsenate usually predominating (Braman and Foreback 1973; EPA 1982c, 1984a). Methylated forms have also been detected in both surface water and groundwater, at levels ranging from 0.01 to $7.4 \mu \mathrm{g} / \mathrm{L}$ (Braman and Foreback 1973; Hood 1985), with most values below $0.3 \mu \mathrm{g} / \mathrm{L}$ (Hood 1985). In a survey of shallow groundwater quality in the alluvial aquifer beneath a major urban center, Denver, Colorado, arsenic levels in the 30 randomly-chosen wells sampled had median levels of $<1 \mu \mathrm{g} / \mathrm{L}$; the maximum level was $33 \mu \mathrm{g} / \mathrm{L}$ (Bruce and McMahon 1996). Arsenic levels in groundwater sometimes exceeded the EPA maximum contaminant level (MCL), which was $50 \mu \mathrm{g} / \mathrm{L}$ at the time, in the Willamette Valley, Oregon and a nine-county region of southeastern Michigan (USGS 1999b, 1999c).

Arsenic has also been detected in rainwater at average concentrations of $0.2-0.5 \mu \mathrm{g} / \mathrm{L}$ (Welch et al. 1988). This range is consistent with that found in a 1997-1998 study in western Maryland, which was the focus of a major bioaccumulation study (Mason et al. 2000). Arsenic levels in wet deposition in the watershed as well as throughfall into the two streams were $0.345 \pm 0.392,0.400 \pm 0.400$, and $0.330 \pm 0.250 \mu \mathrm{g} / \mathrm{L}$, respectively. Median arsenic concentrations in 30-day rainwater composite samples collected May-September 1994 from eight arctic catchments in northern Europe at varying distances and wind directions from the emissions of a Russian nickel ore mining, roasting, and smelting industry on the Kola Peninsula ranged from 0.07 to $12.3 \mu \mathrm{g} / \mathrm{L}$ (Reimann et al. 1997). Rain and snow samples were collected during the fall of 1996 and winter of 1997 at eight locations in a semi-circular pattern radiating out (2$15 \mathrm{~km}$ ) in the direction of the prevailing wind from the Claremont incinerator located in New Hampshire. This incinerator processes 200 tons of solid waste per day. Arsenic concentrations in rainwater and snow ranged from 0.020 to $0.079 \mu \mathrm{g} / \mathrm{L}$ and from 0.80 to $1.28 \mu \mathrm{g} / \mathrm{L}$, respectively (Feng et al. 2000).

Drinking water is one of the most important sources of arsenic exposure. Surveys of drinking water in the United States have found that $>99 \%$ of public water supplies have arsenic concentrations below the EPA MCL, which was $50 \mu \mathrm{g} / \mathrm{L}$ at the time (EPA 1984a). In an EPA study of tap water from 3,834 U.S. residences, the average value was $2.4 \mu \mathrm{g} / \mathrm{L}$ (EPA 1982c).

Before the MCL for arsenic in drinking water was lowered from 50 to $10 \mu \mathrm{g} / \mathrm{L}$, studies were undertaken to ascertain how different standards would affect compliance. One such survey sponsored by the Water Industry Technical Action Fund was the National Arsenic Occurrence Survey (NAOS). NAOS was based on a representational survey of public water systems defined by source type, system size, and geographical location. Additionally, it included a natural occurrence factor, a stratifying variable that could qualitatively describe the likelihood of arsenic occurrence in the supply. To predict finished water 
arsenic concentrations, data on the water treatment options, efficiency, and frequency of use were factored in. The results of the NAOS are presented in Table 6-3. The NAOS results are in general agreement with two older and more limited national surveys, EPA's National Inorganics and Radionuclides Survey (NIRS) and the Metropolitan Water District of Southern California Survey (MWDSC). The percentages of water systems that would be out of compliance are estimated to be 1.7, 3.6, 9.3, and 20.7\% for arsenic MCLs of 20,10, 5, and $2 \mu \mathrm{g} / \mathrm{L}$, respectively. Arsenic concentrations were determined in drinking in EPA Region V (Indiana, Illinois, Michigan, Minnesota, Ohio, and Wisconsin) as part of the NHEXAS; mean arsenic concentration in flushed and standing tap water were both $1.1 \mu \mathrm{g} / \mathrm{L}$ (Thomas et al. 1999). A review by Frost et al. (2003) of existing data from the EPA Arsenic Occurrence and Exposure Database, as well as additional data from state health and environmental departments and water utilities found that 33 counties in 11 states had estimated mean drinking water arsenic concentrations of $10 \mu \mathrm{g} / \mathrm{L}$ or greater. Eleven counties had mean arsenic concentrations of $\geq 20 \mu \mathrm{g} / \mathrm{L}$, and two counties had mean arsenic concentrations of $\geq 50 \mu \mathrm{g} / \mathrm{L}$ (Frost et al. 2003).

The north central region and the western region of the United States have the highest arsenic levels in surface water and groundwater sources, respectively. In a study of drinking water from New Hampshire, arsenic concentrations ranged from $<0.01$ to $180 \mu \mathrm{g} / \mathrm{L}$ in the 793 households tested. More than $10 \%$ of the private wells had arsenic concentrations $>10 \mu \mathrm{g} / \mathrm{L}$, and $2.5 \%$ had levels $>50 \mu \mathrm{g} / \mathrm{L}$ (Karagas et al. 1998). In New Hampshire, 992 randomly selected household water samples were analyzed for arsenic levels and the results for domestic well users were compared with those for users of municipal water supplies (Peters et al. 1999). The concentrations ranged from $<0.0003$ to $180 \mu \mathrm{g} / \mathrm{L}$, with water from domestic wells containing significantly more arsenic than water from municipal supplies; the median concentration of the former was about $0.5 \mu \mathrm{g} / \mathrm{L}$ and the latter was $0.2 \mu \mathrm{g} / \mathrm{L}$. None of the municipal supplies exceeded an arsenic concentration of $50 \mu \mathrm{g} / \mathrm{L}$, and $2 \%$ of the domestic wells were found to have arsenic concentrations that exceeded $50 \mu \mathrm{g} / \mathrm{L}$. Approximately $2 \%$ of the municipal water users have water with arsenic levels exceeding $10 \mu \mathrm{g} / \mathrm{L}$ compared with $13 \%$ of domestic wells. Twenty-five percent of domestic wells and 5\% of municipal supplies were found to have arsenic concentrations exceeding $2 \mu \mathrm{g} / \mathrm{L}$. The highest arsenic levels in New Hampshire are associated with bedrock wells in the south eastern and south central part of the state (Peters et al. 1999). In a study of arsenic in well water supplies in Saskatchewan, Canada, 13\% of samples were $>20 \mu \mathrm{g} / \mathrm{L}$ and one sample exceeded $100 \mu \mathrm{g} / \mathrm{L}$ (Thompson et al. 1999). It was noted that the samples with high arsenic levels were derived from sites that were in near proximity to each other, indicating the presence of 'hot spots' with similar geological characteristics. As part of an epidemiological study, Engel and Smith (1994) investigated the levels of arsenic in drinking 


\section{Table 6-3. Regional Occurrence of Arsenic in U.S. Water Sources and Finished} Drinking Water

\begin{tabular}{|c|c|c|c|c|}
\hline \multirow[b]{2}{*}{ Geographical region } & \multicolumn{4}{|c|}{ Arsenic concentration in $\mu \mathrm{g} / \mathrm{L}$} \\
\hline & $<1$ & $1-5$ & $5-20$ & $>20$ \\
\hline \multicolumn{5}{|c|}{ Occurrence in U.S. surface water sources } \\
\hline Region 1. New England & 50 & 50 & 0 & 0 \\
\hline Region 2. Mid-Atlantic & 84 & 12 & 4 & 0 \\
\hline Region 3. South East & 93 & 7 & 0 & 0 \\
\hline Region 4. Midwest & 24 & 76 & 0 & 0 \\
\hline Region 5. South Central & 32 & 55 & 13 & 0 \\
\hline Region 6. North Central & 33 & 22 & 33 & 0 \\
\hline Region 7. Western & 42 & 58 & 0 & 0 \\
\hline \multicolumn{5}{|c|}{ Occurrence in U.S. groundwater sources } \\
\hline Region 1. New England & 71 & 21 & 7 & 0 \\
\hline Region 2. Mid-Atlantic & 81 & 4 & 11 & 4 \\
\hline Region 3. South East & 82 & 14 & 2 & 0 \\
\hline Region 4. Midwest & 40 & 40 & 15 & 5 \\
\hline Region 5. South Central & 68 & 27 & 15 & 0 \\
\hline Region 6. North Central & 30 & 40 & 30 & 0 \\
\hline Region 7. Western & 24 & 34 & 28 & 14 \\
\hline \multicolumn{5}{|c|}{ Occurrence in U.S. finished surface water supplies } \\
\hline Region 1. New England & 88 & 12 & 0 & 0 \\
\hline Region 2. Mid-Atlantic & 92 & 8 & 0 & 0 \\
\hline Region 3. South East & 100 & 0 & 0 & 0 \\
\hline Region 4. Midwest & 73 & 27 & 0 & 0 \\
\hline Region 5. South Central & 74 & 19 & 7 & 0 \\
\hline Region 6. North Central & 44 & 44 & 0 & 12 \\
\hline Region 7. Western & 42 & 58 & 0 & 0 \\
\hline \multicolumn{5}{|c|}{ Occurrence in U.S. finished groundwater supplies } \\
\hline Region 1. New England & 79 & 21 & 0 & 0 \\
\hline Region 2. Mid-Atlantic & 81 & 4 & 11 & 4 \\
\hline Region 3. South East & 94 & 4 & 2 & 0 \\
\hline Region 4. Midwest & 58 & 27 & 12 & 3 \\
\hline Region 5. South Central & 61 & 27 & 12 & 0 \\
\hline Region 6. North Central & 40 & 50 & 10 & 0 \\
\hline Region 7. Western & 20 & 40 & 22 & 12 \\
\hline
\end{tabular}

Source: National Arsenic Occurrence Survey (Frey and Edwards 1997) 
water throughout the United States between 1968 and 1984. They found that 30 counties in 11 states had mean arsenic levels of $>5 \mu \mathrm{g} / \mathrm{L}$, with a range of 5.4-91.5 $\mu \mathrm{g} / \mathrm{L} ; 15$ counties had mean levels from 5 to $10 \mu \mathrm{g} / \mathrm{L} ; 10$ counties had mean levels from 10 to $20 \mu \mathrm{g} / \mathrm{L}$; and 5 counties had levels $>20 \mu \mathrm{g} / \mathrm{L}$. The highest levels were found in Churchill County, Nevada, where $89 \%$ of the population was exposed to a mean arsenic concentration of $100 \mu \mathrm{g} / \mathrm{L}$ and $11 \%$ to a mean of $27 \mu \mathrm{g} / \mathrm{L}$. A study by Frost et al. (2003) identified 33 counties from 11 states in which the average arsenic concentration of at least $75 \%$ of public wells was $>10 \mu \mathrm{g} / \mathrm{L}$. Arsenic concentrations in drinking water from these counties ranged from 10.3 to $90.0 \mu \mathrm{g} / \mathrm{L}$ in Pinal, Arizona and Churchill, Nevada, respectively (Frost et al. 2003).

Many communities have high levels of arsenic in their drinking water because of contamination or as a result of the geology of the area. In Millard County, Utah, seven towns had median and maximum arsenic levels of 18.1-190.7 and 125-620 $\mu \mathrm{g} / \mathrm{L}$, respectively, in their drinking water (Lewis et al. 1999). The mean arsenic concentration in tap water from homes in Ajo, Arizona, about 2 miles from an open pit copper mine and smelter was $90 \mu \mathrm{g} / \mathrm{L}$ (Morse et al. 1979). The town's water was supplied from five deep wells.

Countries such as Mexico, Bangladesh, India, Chile, Argentina, and Vietnam have highly elevated levels of arsenic in drinking water in some regions (Bagla and Kaiser 1996; Berg et al. 2001; Tondel et al. 1999; WHO 2001; Wyatt et al. 1998a, 1998b). In Bangladesh and West Bengal, the soil naturally contains high levels of arsenic, which leaches into the shallow groundwater that is tapped for drinking water. In West Bengal, India, it is estimated that more than one million Indians are drinking arsenic-laced water and tens of millions more could be at risk in areas that have not been tested for contamination. Analysis of 20,000 tube-well waters revealed that $62 \%$ have arsenic at levels above the World Health Organization (WHO) permissible exposure limit (PEL) in drinking water of $10 \mu \mathrm{g} / \mathrm{L}$, with some as high as 3,700 $\mu \mathrm{g} / \mathrm{L}$ (Bagla and Kaiser 1996). Analysis of 10,991 and 58,166 groundwater samples from 42 and 9 arsenicaffected districts in Bangladesh and West Bengal were found to have arsenic levels that were 59 and 34\%, respectively, above $50 \mu \mathrm{g} / \mathrm{L}$ (Chowdhury et al. 2000). Berg et al. (2001), studied the arsenic contamination of the Red River alluvial tract in Hanoi, Vietnam and the surrounding rural areas. Arsenic concentrations in groundwater from private small-scale tube-wells averaged $159 \mu \mathrm{g} / \mathrm{L}$, ranging from 1 to $3,050 \mu \mathrm{g} / \mathrm{L}$. Arsenic concentrations ranged from 37 to $320 \mu \mathrm{g} / \mathrm{L}$ in raw groundwater pumped from the lower aquifer for the Hanoi water supply (Berg et al. 2001). Several investigators have noticed a correlation between high levels of arsenic and fluoride in drinking water (Wyatt et al. 1998a, 1998b). Arsenic concentrations in drinking water from four villages in Bangladesh ranged from 10 to 2,040 $\mu \mathrm{g} / \mathrm{L}$ (Tondel et al. 1999). 


\subsubsection{Sediment and Soil}

Arsenic is widely distributed in the Earth's crust, which contains about 3.4 ppm arsenic (Wedepohl 1991). It is mostly found in nature minerals, such as realgar $\left(\mathrm{As}_{4} \mathrm{~S}_{4}\right)$, orpiment $\left(\mathrm{As}_{2} \mathrm{~S}_{3}\right)$, and arsenolite $\left(\mathrm{As}_{2} \mathrm{O}_{3}\right)$, and only found in its elemental form to a small extent. There are over 150 arsenic-bearing minerals (Budavari et al. 2001; Carapella 1992). Arsenic concentrations in soils from various countries can range from 0.1 to $50 \mu \mathrm{g} / \mathrm{g}$ and can vary widely among geographic regions. Typical arsenic concentrations for uncontaminated soils range from 1 to $40 \mu \mathrm{g} / \mathrm{g}$, with the lowest concentrations in sandy soils and soils derived from granites. Higher arsenic concentrations are found in alluvial soils and soils with high organic content (Mandal and Suzuki 2002). Arsenic in soil may originate from the parent materials that form the soil, industrial wastes, or use of arsenical pesticides. Geological processes that may lead to high arsenic concentrations in rock and subsequently the surrounding soil include hydrothermic activity and pegmatite formation (Peters et al. 1999). In the first case, thermal activity results in the dissolution and transport of metals, including the metalloid arsenic, which are precipitated in fractures in rocks. In the second process, cooling magmas may concentrate metals that are injected into rocks, crystallizing as pegmatites. Areas of volcanic activity include large areas of California, Hawaii, Alaska, Iceland, and New Zealand.

The U.S. Geological Survey reports the mean and range of arsenic in soil and other surficial materials as 7.2 and $<0.1-97 \mu \mathrm{g} / \mathrm{g}$, respectively (USGS 1984). The concentrations of arsenic in 445 Florida surface soils ranged from 0.01 to $50.6 \mu \mathrm{g} / \mathrm{g}$ (Chen et al. 1999). The median, arithmetic mean, and geometric mean were $0.35,1.34 \pm 3.77$, and $0.42 \pm 4.10 \mu \mathrm{g} / \mathrm{g}$, respectively. Chirenje et al. (2003b) reported a geometric mean arsenic concentrations of $0.40(0.21-660)$ and $2.81(0.32-110) \mu \mathrm{g} / \mathrm{g}$ in surface soil samples $(0-20 \mathrm{~cm})$ collected in May-June 2000 from Gainesville and Miami, Florida, respectively. The geometric mean arsenic concentration in 50 California soils was $2.8 \mu \mathrm{g} / \mathrm{g}$ (Chen et al. 1999). In the Florida surface soils, arsenic was highly correlated $(\alpha=0.0001)$ with the soil content of clay, organic carbon, CEC, total iron, and total aluminum. Arsenic tends to be associated with clay fractions and iron and manganese oxyhydroxides. Soils of granitic origin are generally low in arsenic, about $4 \mu \mathrm{g} / \mathrm{g}$, whereas arsenic in soils derived from sedimentary rocks may be as high as 20-30 $\mu \mathrm{g} / \mathrm{g}$ (Yan-Chu 1994). Soils overlying arsenic-rich geologic deposits, such as sulfide ores, may have soil concentrations two orders of magnitude higher (NAS 1977a). 
Soils in mining areas or near smelters may contain high levels of arsenic. Arsenic concentrations up to $27,000 \mu \mathrm{g} / \mathrm{g}$ were reported in soils contaminated with mine or smelter wastes (EPA 1982b). Soils at an abandoned mining site in the Tamar Valley in southwest England have arsenic concentrations that may exceed 50,000 $\mu \mathrm{g} / \mathrm{g}$ (Erry et al. 1999). The average arsenic levels in the top $2 \mathrm{~cm}$ of different soil types in the vicinity of a former copper smelter in Anaconda, Montana, ranged from 121 to $236 \mu \mathrm{g} / \mathrm{g}$; levels were significantly related to proximity and wind direction to the smelter site (Hwang et al. 1997a). Smelter fallout can contaminate land miles from the source. Soils in mainland southern King County were studied for the presence of arsenic and lead (WSDOE 2005). Soil samples were collected in the fall of 1999 and the spring of 2001 from locations around the ASARCO smelter, which operated in Ruston from the 1890s to 1986 . The study area ran roughly from the I-90 corridor south to the King-Pierce county line, from the Puget Sound shore to the Cascade foothills. Almost all of the contamination was found was in the 0 6 -inch depths of the cores samples; 62 of the 75 samples were found to have arsenic levels above $20 \mathrm{ppm}$ (WSDOE 2005).

Soil on agricultural lands treated with arsenical pesticides may retain substantial amounts of arsenic. One study reported an arsenic concentration of $22 \mu \mathrm{g} / \mathrm{g}$ in treated soil compared to $2 \mu \mathrm{g} / \mathrm{g}$ for nearby untreated soil (EPA 1982b). Arsenic was measured in soil samples taken from 10 potato fields in Suffolk County on Long Island, New York, where sodium arsenite had been used for vine control and fall weed control for many years. Lead arsenate also may have been used as an insecticide in certain areas. The mean arsenic levels taken at a depth of $0-18 \mathrm{~cm}$ from each of the 10 fields ranged from $27.8 \pm 5.44 \mu \mathrm{g} / \mathrm{g}$ dry weight $(\mathrm{n}=10)$ to $51.0 \pm 7.40 \mu \mathrm{g} / \mathrm{g}$ dry weight $(\mathrm{n}=10)$. These levels were markedly higher than the level of $2.26 \pm 0.33 \mu \mathrm{g} / \mathrm{g}(\mathrm{n}=10)$ for untreated control soils (Sanok et al. 1995). A survey was conducted in 1993 to determine the concentrations of arsenic and lead in soil samples from 13 old orchards in New York State. Lead arsenate was used for pest control in fruit orchards for many years, mainly from the 1930s to 1960s, and residues remain in the soil. Concentrations of arsenic ranged from 1.60 to $141 \mu \mathrm{g} / \mathrm{g}$ dry weight (Merwin et al. 1994). Arsenic and lead concentrations were also measured in former orchard soils contaminated by lead arsenate from the Hanford site in Washington State. The mean arsenic concentration in surface $(5-10 \mathrm{~cm})$ and subsurface $(10-50 \mathrm{~cm})$ soils were $30(2.9-270)$ and $74(32-$ 180) $\mu \mathrm{g} / \mathrm{g}$ dry weight, respectively (Yokel and Delistraty 2003). Average arsenic concentration of 5.728, 5.614 , and $6.746 \mu \mathrm{g} / \mathrm{g}$ were reported in soils, lake sediments, and wetland sediments, respectively, from Moon Lake, a Mississippi River alluvial floodplain in northwest Mississippi. Intensive cultivation has occurred in this area, including cotton, soybeans, and rice (Cooper and Gillespie 2001). A geometric mean arsenic concentration of $20.6 \mathrm{mg} / \mathrm{kg}$ (range $4.6-340 \mathrm{mg} / \mathrm{kg}$ ) was reported soil collected during the 
summer and fall of 2003 from 85 homes in Middleport, New York, where historical pesticide manufacturing was associated with arsenic in the soil (Tsuji et al. 2005).

The Washington State Area-wide Soil Contamination Project provides various data on arsenic contamination in soils across Washington State (Washington State 2006). Arsenic concentrations within areas affected by area-wide soil contamination are highly variable, ranging from natural background levels to $>3,000 \mathrm{ppm}$ in smelter areas. Generally, average arsenic concentrations in soil at developed properties are $<100 \mathrm{ppm}$. Areas affected by smelter emissions in King, Pierce, Snohomish, and Stevens Counties have a higher likelihood of arsenic soil contamination than other areas of the State due to historical emissions from metal smelters located in Tacoma, Harbor Island, Everett, Northport, and Trail, British Columbia. Areas where apples and pears were historically grown, such as Chelan, Spokane, Yakima, and Okanogan Counties, also have a higher likelihood of arsenic soil contamination than other areas due to the past use of lead arsenate pesticides. Generally, arsenic contamination in soils from historical smelter emissions and historical use of lead-arsenate pesticides is found in the upper 618 inches of soil (Washington State 2006).

The New Jersey Department of Environmental Protection (Historic Pesticide Contamination Task Force 1999) reported on the analysis of soil samples collected from 18 sites for various pesticide residues, including arsenic, from current and former agricultural sites in New Jersey in order to assess contamination from historic pesticide use. Arsenic was detected in all 463 samples, with concentrations ranging from 1.4 to $310 \mathrm{ppm}$.

Natural concentrations of arsenic in sediments are usually $<10 \mu \mathrm{g} / \mathrm{g}$ dry weight, but can vary widely around the world (Mandal and Suzuki 2002). Sediment arsenic concentrations reported for U.S. rivers, lakes, and streams range from about 0.1 to $4,000 \mu \mathrm{g} / \mathrm{g}$ (Eisler 1994; Heit et al. 1984; NAS 1977a; Welch et al. 1988). During August through November 1992 and August 1993, bed sediment in the South Platte River Basin (Colorado, Nebraska, and Wyoming) was sampled and analyzed for 45 elements, including arsenic. The range of arsenic found was $2.8-31 \mu \mathrm{g} / \mathrm{g}$ dry weight and the geometric mean $(\mathrm{n}=23)$ was $5.7 \mu \mathrm{g} / \mathrm{g}$ (Heiny and Tate 1997). The arsenic concentration in surface sediment $(0-2 \mathrm{~cm})$ at 43 sites in the Indian River Lagoon System in Florida ranged from 0.6 to $15 \mu \mathrm{g} / \mathrm{g}$ dry weight with a mean of $5.0 \pm 3.9 \mu \mathrm{g} / \mathrm{g}$ (Trocine and Trefry 1996). Arsenic levels were well correlated with those of aluminum. Correlation with aluminum levels is used to normalize sediment level concentrations to natural levels in Florida estuaries. Surficial sediments collected from 18 locations in 3 major tributaries to Newark Bay, New Jersey, were analyzed for 7 toxic metals, including arsenic (Bonnevie et al. 1994). The highest 
concentrations of arsenic were found in the Rahway River adjacent to a chemical plant, $58 \mu \mathrm{g} / \mathrm{g}$ dry weight, and in the Hackensack River adjacent to a coal-fired power plant, $49 \mu \mathrm{g} / \mathrm{g}$. The average arsenic concentration for all sediments was $17 \pm 16 \mu \mathrm{g} / \mathrm{g}$. Sediments collected from seven sites in Baltimore Harbor, Maryland, at five seasonal periods between June 1987 and June 1988 had a geometric mean maximum of $7.29 \mu \mathrm{g} / \mathrm{g}$ dry weight and a geometric mean minimum of $1.25 \mu \mathrm{g} / \mathrm{g}$ (Miles and Tome 1997). This harbor is one of two sub-tributaries of the Chesapeake Bay where contaminants have been discharged on a large scale.

The upper Clark Fork River basin in western Montana is widely contaminated by metals from past mining, milling, and smelting activities. In a 1991 study, arsenic levels were determined in sediment along the river and in a reservoir $205 \mathrm{~km}$ downstream. Total arsenic in sediments from Clark Fork River decreased from $404 \mu \mathrm{g} / \mathrm{g}$ dry weight at the farthest upstream sampling station to $11 \mu \mathrm{g} / \mathrm{g}, 201 \mathrm{~km}$ downstream. Sediment samples from the Milltown Reservoir had arsenic concentrations ranging from 6 to $56 \mu \mathrm{g} / \mathrm{g}$ (Brumbaugh et al. 1994). Total recoverable arsenic in nonfiltered pore water from the Clark Fork River decreased from $1,740 \mu \mathrm{g} / \mathrm{L}$ at the farthest upstream sampling station to $31 \mu \mathrm{g} / \mathrm{L}$ at the $201 \mathrm{~km}$ station (Brumbaugh et al. 1994). The Coeur d'Alene river basin in northern Idaho has been contaminated with heavy metals from mining and smelting operations since 1885 (Farag et al. 1998). A 1994 study determined the metal content of sediment, biofilm, and invertebrates at 13 sites in the basin, 10 with historic mining activity and 3 reference sites. The mean arsenic levels in sediment at the mining sites ranged from 8.3 to $179.0 \mu \mathrm{g} / \mathrm{g}$ dry weight, compared to $2.4-13.1 \mu \mathrm{g} / \mathrm{g}$ dry weight at the reference sites. The mean arsenic levels in biofilm adhering to rock in the water at the mining sites ranged from 7.5 to $155.8 \mu \mathrm{g} / \mathrm{g}$ dry weight, compared to $7.2-27.3 \mu \mathrm{g} / \mathrm{g}$ dry weight at the reference sites. In Whitewood Creek, South Dakota, where as much as 100 million tons of mining and milling waste derived from gold mining activities were discharged between 1876 and 1977, mean and maximum sediment arsenic concentrations were 1,920 and $11,000 \mu \mathrm{g} / \mathrm{g}$, respectively (USGS 1987). Uncontaminated sediment had mean arsenic levels of $9.2 \mu \mathrm{g} / \mathrm{g}$. Arsenic concentrations in surface $(0-5 \mathrm{~cm})$ sediments from watersheds in Black Hills, South Dakota, an area impacted by gold mining activities, ranged from 23 to $1,951 \mu \mathrm{g} / \mathrm{g}$ dry weight; concentrations from reference areas ranged from 10 to $58 \mu \mathrm{g} / \mathrm{g}$ dry weight (May et al. 2001). Swan Lake, a sub-bay of Galveston Bay in Texas is a highly industrial area that received runoff from a tin smelter in the 1940s and 1950s. Surface sediments at 17 sites where oysters and mussels were collected ranged from 4.53 to $103 \mu \mathrm{g} / \mathrm{g}$ (Park and Presley 1997). A site in the channel leading from the old smelter had arsenic levels of $568 \mu \mathrm{g} / \mathrm{g}$. Surface sediment was less contaminated than deeper sediment, indicating less arsenic input recently than in the past as a result of the smelter closing. 
It has been suggested that the wood preservative most commonly used in dock pilings and bulkheads, CCA, can be toxic to estuarine organisms. Wendt et al. (1996) measured arsenic in surface sediments and oysters from creeks with high densities of docks and from nearby reference creeks with no docks. The average concentrations in the sediments ranged from 14 to $17 \mu \mathrm{g} / \mathrm{g}$ throughout the study area, which is within the range of natural background levels. Weis et al. (1998) sampled sediments along a 10-m transect from CCA-treated wood bulkheads from four Atlantic coast estuaries. Arsenic concentrations were highest in the fine-grained portion of the sediments near the CCA-treated bulkhead $(0-1 \mathrm{~m})$; arsenic concentrations were generally at reference levels at distances $>1 \mathrm{~m}$ from the bulkheads (Weis et al. 1998).

Soils below and around play structures constructed from CCA-treated wood in the City of Toronto, Canada were sampled and analyzed for inorganic arsenic (Ursitti et al. 2004). A mean arsenic concentration of $2.1 \mu \mathrm{g} / \mathrm{g}$ (range $0.5-10 \mu \mathrm{g} / \mathrm{g}$ ) was reported in soil samples taken within $1 \mathrm{~m}$ of the CCAtreated wood for all play structures. Soil samples that were collected $10 \mathrm{~m}$ from the play structures served as a background had arsenic concentration of $2.4 \mu \mathrm{g} / \mathrm{g}$ (range $0.5-13 \mu \mathrm{g} / \mathrm{g})$. A mean arsenic concentration of $6.2 \mu \mathrm{g} / \mathrm{g}$ (range $0.5-47.5 \mu \mathrm{g} / \mathrm{g}$ ) was reported in soil samples taken below CCA-treated wood for all play structures. Of the 217 play structures in the study, 32 had arsenic concentrations under the play structures that exceeded the Canadian federal soil guidelines with arsenic concentrations ranging from 12.4 to $47.5 \mu \mathrm{g} / \mathrm{g}$. From this study, the authors concluded that arsenic does not migrate laterally, but does accumulate in soil under elevated platforms constructed from CCA-treated wood (Ursitti et al. 2004).

\subsubsection{Other Environmental Media}

Low levels of arsenic are commonly found in food; the highest levels are found in seafood, meats, and grains. Typical U.S. dietary levels of arsenic in these foods range from $0.02 \mathrm{mg} / \mathrm{kg}$ in grains and cereals to $0.14 \mathrm{mg} / \mathrm{kg}$ in meat, fish, and poultry (Gartrell et al. 1986). Shellfish and other marine foods contain the highest arsenic concentrations and are the largest dietary source of arsenic (Gunderson 1995a; Jelinek and Corneliussen 1977; Tao and Bolger 1999). Arsenic levels in various fish and shellfish are presented in Table 6-4. In the U.S. Food and Drug Administration (FDA) Total Diet Study, 1991-1997, seafood contained the highest levels of arsenic, followed by rice/rice cereal, mushrooms, and poultry. Concentrations in canned tuna (in oil), fish sticks, haddock (pan-cooked), and boiled shrimp were 0.609$1.470,0.380-2.792,0.510-10.430$, and 0.290-2.681 mg/kg, respectively (Tao and Bolger 1999). Typically, arsenic levels in foods in the Total Diet Study, 1991-1996 were low, $<0.03 \mathrm{mg} / \mathrm{kg}$; only 63 of the 264 foods contained arsenic above this level. Similar results were reported in the Total Diet Study, 
Table 6-4. Levels of Arsenic in Fish and Shellfish-Recent Studies

\begin{tabular}{|c|c|c|c|}
\hline Sample type & $\begin{array}{l}\text { Arsenic concentration }{ }^{a} \\
(\mu \mathrm{g} / \mathrm{g})\end{array}$ & Comments & Reference \\
\hline \multicolumn{2}{|l|}{ Yellowtail flounder } & \multirow{4}{*}{$\begin{array}{l}\text { Samples collected from } \\
\text { Northwest Atlantic } 1993\end{array}$} & \multirow[t]{4}{*}{ Hellou et al. 1998} \\
\hline Muscle $(n=8)$ & $8-37$ & & \\
\hline Liver $(n=6)$ & $7-60$ & & \\
\hline Gonad $(n=6)$ & $1.2-9.4$ & & \\
\hline \multicolumn{2}{|l|}{ Marine organisms } & \multirow{7}{*}{$\begin{array}{l}\text { Belgian fish markets in } \\
1991 \text {; inorganic arsenic } \\
\text { ranged from } 0.003 \text { to } \\
0.2 \mu \mathrm{g} / \mathrm{g}\end{array}$} & \multirow{7}{*}{$\begin{array}{l}\text { Buchet and Lison } \\
1998\end{array}$} \\
\hline Ray $(n=8)$ & 16.4 & & \\
\hline $\operatorname{Cod}(n=8)$ & 4.7 & & \\
\hline Plaice $(n=8)$ & 19.8 & & \\
\hline Sole $(n=8)$ & 5.1 & & \\
\hline Sea-bream $(n=8)$ & 2.4 & & \\
\hline Mussell $(n=8)$ & 3.5 & & \\
\hline $\begin{array}{l}\text { Bluefin tuna (Thunnus thynus) } \\
(n=14)\end{array}$ & 3.2 & $\begin{array}{l}\text { Virgin Rocks, Grand Banks } \\
\text { of Newfoundland, Canada, } \\
1990\end{array}$ & Hellou et al. 1992 \\
\hline \multicolumn{2}{|l|}{ Fish } & \multirow{3}{*}{$\begin{array}{l}\text { National Contaminant } \\
\text { Biomonitoring Program, } \\
\text { 1984-1985, } 112 \text { stations }\end{array}$} & \multirow[t]{3}{*}{ Kidwell et al. 1995} \\
\hline Bottom feeding $(n=2,020)$ & $0.16 \pm 0.23$ wet weight & & \\
\hline Predatory $(n=12)$ & $0.16 \pm 0.140$ wet weight & & \\
\hline \multicolumn{2}{|l|}{ Oysters } & \multirow{4}{*}{$\begin{array}{l}\text { South Carolina, private } \\
\text { residential docks on tidal } \\
\text { creeks, } 1994\end{array}$} & \multirow[t]{4}{*}{ Wendt et al. 1996} \\
\hline$<1 \mathrm{~m}$ from docks $(\mathrm{n}=10)$ & $8.3 \pm 1.1$ & & \\
\hline$>10 m$ from docks $(n=10)$ & $7.6 \pm 0.9$ & & \\
\hline $\begin{array}{l}\text { Reference (no docks) } \\
(n=10\end{array}$ & $8.4 \pm 1.3$ & & \\
\hline Clams $(n=22)$ & $12 \pm 1.1$ & $\begin{array}{l}\text { Indian River Lagoon, } \\
\text { Florida, } 22 \text { sites, } 1990\end{array}$ & $\begin{array}{l}\text { Trocine and Trefry } \\
1996\end{array}$ \\
\hline \multicolumn{2}{|l|}{ Marine organisms } & \multirow{8}{*}{$\begin{array}{l}\text { Swan Lake, Galveston Bay, } \\
\text { Texas, } 1993\end{array}$} & \multirow{8}{*}{$\begin{array}{l}\text { Park and Presley } \\
1997\end{array}$} \\
\hline Snails & $13.3 \pm 17.0$ & & \\
\hline Blue crab & 6.61 & & \\
\hline Fish & 0.82 & & \\
\hline Shrimp & $1.37 \pm 0.64$ & & \\
\hline Whole crab & $5.35 \pm 2.51$ & & \\
\hline Oysters $(n=10$, pooled $)$ & $7.28 \pm 1.32$ & & \\
\hline Mussels ( $n=7$, pooled) & $7.75 \pm 2.15$ & & \\
\hline \multicolumn{2}{|l|}{ Marine organisms } & \multirow{3}{*}{$\begin{array}{l}\text { GPNEP, 1992, Galveston } \\
\text { Bay, Texas }\end{array}$} & \multirow{3}{*}{$\begin{array}{l}\text { Park and Presley } \\
1997\end{array}$} \\
\hline Blue crab & $2.31 \pm 2.15$ & & \\
\hline Fish & 2.46 & & \\
\hline \multicolumn{2}{|l|}{ Oysters, two areas } & $\begin{array}{l}\text { NOAA NS\&T Program, } \\
1986-1990\end{array}$ & \multirow[t]{3}{*}{$\begin{array}{l}\text { Park and Presley } \\
1997\end{array}$} \\
\hline $\mathrm{n}=78$, pooled & $4.50 \pm 1.08$ & Galveston Bay & \\
\hline $\mathrm{n}=874$, pooled & $9.67 \pm 7.00$ & Gulf of Mexico & \\
\hline
\end{tabular}


Table 6-4. Levels of Arsenic in Fish and Shellfish-Recent Studies

\begin{tabular}{|c|c|c|c|}
\hline Sample type & $\begin{array}{l}\text { Arsenic concentration }{ }^{a} \\
(\mu \mathrm{g} / \mathrm{g})\end{array}$ & Comments & Reference \\
\hline \multicolumn{4}{|l|}{ Marine crustaceans } \\
\hline $\begin{array}{l}\text { Parapenaeus longirostris } \\
\text { (pink shrimp) }(\mathrm{n}=826 \\
10 \text { pools }\end{array}$ & $\begin{array}{l}34.84 \pm 19.21 \\
(12.01-62.60)\end{array}$ & \multirow[t]{4}{*}{$\begin{array}{l}\text { Commercial crustaceans } \\
\text { from the Mediterranean } \\
\text { Sea (Italy) }\end{array}$} & \multirow[t]{4}{*}{$\begin{array}{l}\text { Storelli and } \\
\text { Marcotrigiano } 2001\end{array}$} \\
\hline $\begin{array}{l}\text { Aristeus antennatus (red } \\
\text { shrimp) }(n=387,8 \text { pool) }\end{array}$ & $\begin{array}{l}17.09 \pm 3.49 \\
(10.45-20.82)\end{array}$ & & \\
\hline $\begin{array}{l}\text { Plesionika martia (shrimp) } \\
\text { ( } \mathrm{n}=456,7 \text { pools) }\end{array}$ & $\begin{array}{l}40.82 \pm 2.50 \\
(36.37-44.06)\end{array}$ & & \\
\hline $\begin{array}{l}\text { Nephrops norvegicus } \\
\text { (Norway lobster) }(\mathrm{n}=270 \\
5 \text { pools) }\end{array}$ & $\begin{array}{l}43.48 \pm 14.21 \\
(35.63-69.15)\end{array}$ & & \\
\hline \multicolumn{4}{|l|}{ Freshwater fish } \\
\hline $\begin{array}{l}\text { Sabalo (Brycon } \\
\text { melanopterus) }(\mathrm{n}=3)\end{array}$ & $0.015-0.101$ & \multirow{5}{*}{$\begin{array}{l}\text { Fish samples (muscle) } \\
\text { were collected in August } \\
1997 \text { from the Candamo } \\
\text { River, Peru; a pristine } \\
\text { rainforest valley prior to the } \\
\text { start of oil-drilling activities }\end{array}$} & \multirow{5}{*}{ Gutleb et al. 2002} \\
\hline $\begin{array}{l}\text { Huazaco (Hoplias } \\
\text { malabaracus) }(n=4)\end{array}$ & nd -0.005 & & \\
\hline $\begin{array}{l}\text { Bagre (Pimelodus ornatus) } \\
(n=8)\end{array}$ & nd-0.201 & & \\
\hline $\begin{array}{l}\text { Boquichio (Prochilodus } \\
\text { nigricans) }(n=1)\end{array}$ & 0.063 & & \\
\hline $\begin{array}{l}\text { Doncello (Pseudo- } \\
\text { platystoma sp.) }(\mathrm{n}=1)\end{array}$ & 0.055 & & \\
\hline \multicolumn{4}{|l|}{ Freshwater fish } \\
\hline Bowfin (n=59) & $0.32 \pm 0.04$ wet weight & \multirow{11}{*}{$\begin{array}{l}\text { Savannah River, along and } \\
\text { below the Department of } \\
\text { Energy's Savannah River } \\
\text { Site (SRS); samples } \\
\text { analyzed were edible fillets }\end{array}$} & \multirow{11}{*}{ Burger et al. 2002} \\
\hline Bass $(n=47)$ & $0.03 \pm 0$ wet weight & & \\
\hline Channel catfish $(n=50)$ & $0.09 \pm 00.02$ wet weight & & \\
\hline Chain pickerel $(n=19)$ & $0.05 \pm 0.01$ wet weight & & \\
\hline Yellow perch $(n=51)$ & $0.05 \pm 0.01$ wet weight & & \\
\hline Black crappie $(n=52)$ & $0.04 \pm 0.01$ wet weight & & \\
\hline American eel $(n=24)$ & $0.04 \pm 0.01$ wet weight & & \\
\hline Shellcracker n=52) & $0.06 \pm 0$ wet weight & & \\
\hline Bluegill $(n=52)$ & $0.05 \pm 0.02$ wet weight & & \\
\hline Redbreast $(n=43)$ & $0.07 \pm 0.01$ wet weight & & \\
\hline Spotted sucker $(n=35)$ & $0.03 \pm 0$ wet weight & & \\
\hline
\end{tabular}


Table 6-4. Levels of Arsenic in Fish and Shellfish-Recent Studies

\begin{tabular}{|c|c|c|c|}
\hline Sample type & $\begin{array}{l}\text { Arsenic concentration }{ }^{a} \\
(\mu \mathrm{g} / \mathrm{g})\end{array}$ & Comments & Reference \\
\hline \multicolumn{4}{|l|}{ Horseshoe crabs } \\
\hline Apodeme $(n=74)$ & $7.034 \pm 0.65$ wet weight & \multirow{3}{*}{$\begin{array}{l}\text { Overall mean in tissues of } \\
\text { crabs collected from New } \\
\text { Jersey in } 2000\end{array}$} & \multirow[t]{6}{*}{ Burger et al. 2003} \\
\hline Egg $(n=63)$ & $5.924 \pm 0.345$ wet weight & & \\
\hline Leg $(n=74)$ & $\begin{array}{l}14.482 \pm 0.685 \text { wet } \\
\text { weight }\end{array}$ & & \\
\hline Apodeme $(n=40)$ & $7.513 \pm 0.835$ wet weight & \multirow{3}{*}{$\begin{array}{l}\text { Overall mean in tissues of } \\
\text { crabs collected from } \\
\text { Delaware in } 2000\end{array}$} & \\
\hline Egg $(n=35)$ & $6.766 \pm 0.478$ wet weight & & \\
\hline Leg $(n=40)$ & $\begin{array}{l}18.102 \pm 1.489 \text { wet } \\
\text { weight }\end{array}$ & & \\
\hline
\end{tabular}

${ }^{\mathrm{a} C}$ Concentrations are means \pm standard deviation, unless otherwise stated. Concentrations are in a dry weight basis, unless otherwise stated.

$\mathrm{GM}=$ geometric mean; GPNEP = Galveston Bay National Estuary Program; nd = not detected; NOAA NS\&T = National Oceanic and Atmospheric Administration National Status and Trends 
1991-1997, where the mean arsenic concentration in all foods was $0.036 \mathrm{mg} / \mathrm{kg}$ dry weight and arsenic was not detectable in about $88 \%$ of the foods and was detected at trace levels in another $7.8 \%$ of foods. The foods with the highest mean arsenic levels were haddock, canned tuna, fish sticks, shrimp, and fish sandwiches, with arsenic concentrations ranging from 5.33 to $0.568 \mathrm{mg} / \mathrm{kg}$ dry weight (Capar and Cunningham 2000). Nriagu and Lin (1995) analyzed 26 brands of wild rice sold in the United States and found arsenic levels ranging from 0.006 to $0.142 \mu \mathrm{g} / \mathrm{g}$ dry weight. Arsenic concentrations ranging from 0.05 to $0.4 \mu \mathrm{g} / \mathrm{g}$ are typically reported for rice from North America, Europe, and Taiwan (Meharg and Rahman 2003).

During a comprehensive total diet study extending from 1985 to 1988, foods were collected in six Canadian cities and processed into 112 composite food samples (Dabeka et al. 1993). The mean, median, and range of total arsenic in all samples were $0.0732,0.0051$, and $<0.0001-4.840 \mu \mathrm{g} / \mathrm{g}$, respectively. Food groups containing the highest mean arsenic levels were fish $(1.662 \mu \mathrm{g} / \mathrm{g})$, meat and poultry $(0.0243 \mu \mathrm{g} / \mathrm{g})$, bakery goods and cereals $(0.0245 \mu \mathrm{g} / \mathrm{g})$, and fats and oils $(0.0190 \mu \mathrm{g} / \mathrm{g})$. Of the individual samples, marine fish had the highest arsenic levels, with a mean of $3.048 \mu \mathrm{g} / \mathrm{g}$ for the cooked composites and $2.466 \mu \mathrm{g} / \mathrm{g}$ for the raw samples. Canned fish $(1.201 \mu \mathrm{g} / \mathrm{g})$ and shellfish $(2.041 \mu \mathrm{g} / \mathrm{g})$ also contained high means. Cooked poultry, raw mushrooms, and chocolate bars contained 0.100, 0.084, and $0.105 \mu \mathrm{g} / \mathrm{g}$, respectively.

National monitoring data from the Food Safety and Inspection Service National Residue Program (NRP) (1994-2000) found that the mean total arsenic concentration in livers of young chickens ranged from 0.33 to $0.43 \mu \mathrm{g} / \mathrm{g}$, with an overall mean of $0.39 \mu \mathrm{g} / \mathrm{g}$ (Lasky et al. 2004). The mean arsenic concentrations in liver for mature chickens, turkeys, hogs, and all other species over the same time period ranged from 0.10 to $0.16 \mu \mathrm{g} / \mathrm{g}$. Lasky et al. (2004) used the NRP arsenic data in livers of young chickens to estimate the concentrations of arsenic in muscle tissue, the most commonly consumed part of the chicken. Assuming that $65 \%$ of the arsenic in poultry and meat is inorganic, at a mean level of chicken consumption of $60 \mathrm{~g} /$ person/day, people may ingest an estimated 1.38-5.24 $\mu \mathrm{g} /$ day of inorganic arsenic from chicken.

A Danish study (Pedersen et al. 1994) reports the arsenic levels in beverages as the mean (range) in $\mu \mathrm{g} / \mathrm{L}$ as follows: red wine, $9(<2-25)$; white wine, $11(<2-33)$; fortified wine, $5(<2-11)$; beer, 7 (4-11); soft drinks, $3(<2-8)$; miscellaneous juices, 8 (3-13); instant coffee, 4 (0.7-7); and instant cocoa, 5.6 (1.612.8). 
In a study of dietary arsenic exposure in the Indigenous Peoples of the western Northwest Territories, Canada, fish contained the highest arsenic concentrations in foods consumed by the Dene and Métis populations with the highest concentration, $1.960 \mu \mathrm{g} / \mathrm{g}$, found in smoked/dried cisco (fish). Other foods derived from land mammals, birds, and plants contained lower arsenic concentrations. A mean arsenic intake of $<1.0 \mu \mathrm{g} / \mathrm{kg} / \mathrm{day}$ was reported for this population (Berti et al. 1998).

The general consensus in the literature is that about $85->90 \%$ of the arsenic in the edible parts of marine fish and shellfish is organic arsenic (e.g., arsenobetaine, arsenochloline, dimethylarsinic acid) and that approximately 10\% is inorganic arsenic (EPA 2003b). However, the inorganic arsenic content in seafood may be highly variable. For example, a study in the Netherlands reported that inorganic arsenic comprised $0.1-41 \%$ of the total arsenic in seafood (Vaessen and van Ooik 1989). Buchet et al. (1994) found that, on the average, $3 \%$ of the total arsenic in mussels was inorganic in form. Some commercially available seaweeds, especially brown algae varieties, may have high percentages of the total arsenic present as inorganic arsenic ( $>50 \%$ ) (Almela et al. 2002; Laparra et al. 2003). Arsenic concentrations ranging from 17 to $88 \mathrm{mg} / \mathrm{kg}$ dry weight were found in commercially available seaweeds (van Netten et al. 2000). Other arsenic compounds that may be found in seafood are arsenic-containing ribose derivatives called arsenosugars. Arsenosugars are the common organoarsenicals found in marine algae; they are also found in mussels, oysters, and clams (Le et al. 2004). Less information about the forms of arsenic in freshwater fish is known at this time (EPA 2003b).

Schoof et al. (1999a) reported on the analysis of 40 commodities anticipated to account for $90 \%$ of dietary inorganic arsenic intake. In this study, the amount of inorganic arsenic was measured in these foods. Consistent with earlier studies, total arsenic concentrations were highest in the seafood sampled (ranging from $160 \mathrm{ng} / \mathrm{g}$ in freshwater fish to $2,360 \mathrm{ng} / \mathrm{g}$ in marine fish). In contrast, average inorganic arsenic in seafood ranged from $<1$ to $2 \mathrm{ng} / \mathrm{g}$. The highest inorganic arsenic concentrations were found in raw rice (74 ng/g), followed by flour (11 ng/g), grape juice ( $9 \mathrm{ng} / \mathrm{g})$, and cooked spinach (6 ng/g).

Tobacco contains an average arsenic concentration of $1.5 \mathrm{ppm}$, or about $1.5 \mu \mathrm{g}$ per cigarette (EPA 1998j). Before arsenical pesticides were banned, tobacco contained up to $52 \mathrm{mg} \mathrm{As} / \mathrm{kg}$, whereas after the ban, maximum arsenic levels were reduced to $3 \mu \mathrm{g} / \mathrm{g}$ (Kraus et al. 2000). An international literature survey reports arsenic yields of $0-1.4 \mu \mathrm{g} /$ cigarette for mainstream (inhaled) cigarette smoke (Smith et al. 1997). The wide range of arsenic yields for flue-cured cigarettes suggests that the field history, soil, and fertilizer conditions under which the tobacco is grown will affect the arsenic concentration (Smith et al. 1997). 
Arsenic emission factors of $0.015-0.023 \mu \mathrm{g} /$ cigarette (mean $0.018 \pm 0.003 \mu \mathrm{g} /$ cigarette) have been measured for sidestream smoke from a burning cigarette (Landsberger and Wu 1995).

A median arsenic concentration of $2.1 \mu \mathrm{g} / \mathrm{g}$ and a deposition rate of $0.008 \mu \mathrm{g} / \mathrm{m}^{2} /$ day was reported in house dust in homes evaluated as part of the German Environmental Survey in 1990-1992. A mean arsenic concentration of $7.3 \mu \mathrm{g} / \mathrm{g}$ was reported in house dust from 48 residences in Ottawa, Canada (Butte and Heinzow 2002). These arsenic concentrations are expected to be representative of background levels. In general high arsenic concentrations were found in household dust collected from homes in areas with known arsenic contamination. Mean arsenic concentrations of 12.6 (2.6-57) and $10.8(1.0-49) \mu \mathrm{g} / \mathrm{g}$ were reported in house dust collected from the entryway and child play areas, respectively, from homes in a community in Washington State with a history of lead arsenate use (Wolz et al. 2003). Arsenic was detected in all 135 indoor floor dust samples collected as part of the NHEXAS from Arizona mining communities, ranging from $0.3-50.6 \mu \mathrm{g} / \mathrm{g}$, (O'Rourke et al. 1999). A geometric mean arsenic concentration of $10.8 \mu \mathrm{g} / \mathrm{g}$ (range 1.0-172 $\mu \mathrm{g} / \mathrm{g}$ ) was reported in house dust from 96 homes in Middleport, New York, with historical pesticide manufacture, collected during the summer and fall of 2003 (Tsuji et al. 2005).

Arsenic has also been detected in several homeopathic medicines at concentrations up to $650 \mu \mathrm{g} / \mathrm{g}$ (Kerr and Saryan 1986). Some Asian proprietary medicines that are manufactured in China, Hong Kong, and other Asian countries have been reported to contain levels of inorganic arsenic ranging from 25 to $107,000 \mu \mathrm{g} / \mathrm{g}$ (Chan 1994). Fifty medicinally important leafy samples that were analyzed for elemental concentrations contained arsenic at levels ranging from 0.12 to $7.36 \mu \mathrm{g} / \mathrm{g}$, with a mean of $2.38 \pm 1.2 \mu \mathrm{g} / \mathrm{g}$ (Reddy and Reddy 1997). Arsenic concentrations ranged from 0.005 to $3.77 \mu \mathrm{g} / \mathrm{g}$ in 95 dietary supplements purchased from retail stores in the Washington, DC area in 1999 (Dolan et al. 2003). Commercially available samples of Valarian, St. John's Wort, Passion Flower, and Echinacea were purchased in the United States and analyzed for various contaminants; arsenic concentrations were $0.0016-0.0085,0.0065-0.017 .8,0.0024-0.0124$, and $0.0021-0.0102 \mu \mathrm{g} / \mathrm{g}$, respectively, in these samples (Huggett et al. 2001). Concentrations of heavy metals including the metalloid arsenic were evaluated in 54 samples of Asian remedies that were purchased in stores in Vietnam and Hong Kong that would be easily accessible to travelers, as well as in health food and Asian groceries in Florida, New York, and New Jersey. Four remedies were found to contain daily doses exceeding $0.1 \mathrm{mg}$. Two of these contained what would have been a potentially significant arsenic dose, with daily doses of 16 and $7.4 \mathrm{mg}$ of arsenic (Garvey et al. 2001). 
The possible presence of toxic compounds in waste materials has raised concerns about the fate of these compounds either during the composting process or when the composted product is applied to soils. Three waste compost products generated at the Connecticut Agricultural Experiment Station had arsenic levels of 12.8, 9.8, and $13 \mu \mathrm{g} / \mathrm{g}$ dry weight, respectively (Eitzer et al. 1997). The arsenic levels in municipal solid waste composts from 10 facilities across the United States ranged from 0.9 to $15.6 \mu \mathrm{g} / \mathrm{g}$ dry weight with a mean of $6.7 \mu \mathrm{g} / \mathrm{g}$ (He et al. 1995). These are lower than the EPA 503 regulatory limit for arsenic of $41 \mu \mathrm{g} / \mathrm{g}$ for agricultural use of sewage sludge (EPA 1993b). Concentrations of arsenic in U.S. sewage sludges, which are sometimes spread on soil, were $<1 \mu \mathrm{g} / \mathrm{g}$. Arsenic is a common impurity in minerals used in fertilizers. A comprehensive Italian study found that the arsenic content in a number of mineral and synthetic fertilizers ranged from 2.2 to $322 \mathrm{mg} / \mathrm{kg}$ with a sample of triple superphosphate having the highest level (Senesi et al. 1999). Arsenic naturally occurs in coal and crude oil at levels of 0.34-130 and 0.0024-1.63 ppm, respectively, which would account for its presence in flue gas, fly ash, and bottom ash from power plants (Pacyna 1987).

Background arsenic levels in living organisms are usually $<1 \mu \mathrm{g} / \mathrm{g}$ wet weight (Eisler 1994). Levels are higher in areas with mining and smelting activity or where arsenical pesticides were used. Eisler (1994) has an extensive listing of arsenic levels in terrestrial and aquatic flora and fauna from literature sources to about 1990. The U.S. Fish and Wildlife Service's National Contaminant Biomonitoring Program have analyzed contaminants in fish at 116 stations (rivers and the Great Lakes) across the United States. The geometric mean concentration of arsenic for the five collection periods starting in 1976 were (period, concentration wet weight basis): 1976-1977, $0.199 \mu \mathrm{g} / \mathrm{g} ; 1978-1979,0.129 \mu \mathrm{g} / \mathrm{g} ; 1980-1981$, $0.119 \mu \mathrm{g} / \mathrm{g} ; 1984,0.106 \mu \mathrm{g} / \mathrm{g}$; and 1986, $0.083 \mu \mathrm{g} / \mathrm{g}$ (Schmitt et al. 1999). In 1986, the maximum and 85th percentile arsenic levels were 1.53 and $0.24 \mu \mathrm{g} / \mathrm{g}$, respectively. The highest concentrations of arsenic for all five collection periods were in bloaters from Lake Michigan at Sheboygan, Wisconsin. Arsenic levels declined by 50\% at this site between 1976-1997 and 1984. The major source of arsenic into Lake Michigan was a facility at Marinette, Wisconsin, which manufactured arsenic herbicides. Table 6-4 contains arsenic levels in aquatic organisms from more recent studies. The Coeur d'Alene river basin in northern Idaho has been contaminated with heavy metals from mining and smelting operations since 1885 (Farag et al. 1998). A 1994 study determined the metal content of sediment, biofilm, and invertebrates at 13 sites in the basin, 10 with historic mining activity, and 3 reference sites. The mean arsenic levels in benthic macroinvertebrates at the mining sites ranged from 2.2 to $97.0 \mu \mathrm{g} / \mathrm{g}$ dry weight, compared to $2.1-2.4 \mu \mathrm{g} / \mathrm{g}$ dry weight at the reference sites. A study of aquatic organism in Swan Lake, a highly polluted sub-bay of Galveston Bay, Texas showed that arsenic concentrations were in the order snail $>$ oyster $>$ crab $>$ shrimp $>$ fish (Park and Presley 1997). In contrast to metals like silver, cadmium, 
copper, and zinc, arsenic concentrations in oysters and mussels were less than in the sediment from which they were collected. No significant correlation was found between levels of arsenic in clams in the Indian River Lagoon in Florida with those found in sediment or water samples (Trocine and Trefry 1996). Small animals living at mining sites ingest more arsenic in their diet and have higher arsenic levels in their bodies than those living on uncontaminated sites (Erry et al. 1999). Seasonal variations in both arsenic intake and dietary composition may affect the amount of arsenic taken up by the body and transferred to predator animals. Tissue arsenic content of wood mice and bank voles living on both arseniccontaminated mining sites and uncontaminated sites were greater in autumn than spring. The lower tissue arsenic levels in spring of rodents living on contaminated sites suggest that there is no progressive accumulation of arsenic in overwintering animals.

\subsection{GENERAL POPULATION AND OCCUPATIONAL EXPOSURE}

Exposure to arsenic may include exposure to the more toxic inorganic forms of arsenic, organic forms of arsenic, or both. While many studies do not indicate the forms of arsenic to which people are exposed, this information may often be inferred from the source of exposure (e.g., fish generally contain arsenic as arsenobetaine). Yost et al. (1998) reported that the estimated daily dietary intake of inorganic arsenic for various age groups ranged from 8.3 to $14 \mu \mathrm{g} /$ day and from 4.8 to $12.7 \mu \mathrm{g} / \mathrm{day}$ in the United States and Canada, respectively, with $21-40 \%$ of the total dietary arsenic occurring in inorganic forms.

Drinking water may also be a significant source of arsenic exposure in areas where arsenic is naturally present in groundwater. While estimates of arsenic intake for typical adults drinking $2 \mathrm{~L}$ of water per day average about $5 \mu \mathrm{g} /$ day (EPA 1982c), intake can be much higher (10-100 $\mu \mathrm{g} / \mathrm{day})$ in geographical areas with high levels of arsenic in soil or groundwater (see Figure 6-2). It is assumed that nearly all arsenic in drinking water is inorganic (EPA 2001).

In the United States, food intake of arsenic has been estimated to range from $2 \mu \mathrm{g} / \mathrm{day}$ in infants to $92 \mu \mathrm{g} /$ day in 60-65-year-old men (see Table 6-5) (Tao and Bolger 1999). The average intake of inorganic arsenic are estimated to range from $1.34 \mu \mathrm{g} /$ day in infants to $12.54 \mu \mathrm{g} /$ day in $60-65$-year-old men. Tao and Bolger (1999) assumed that $10 \%$ of the total arsenic in seafood was inorganic and that $100 \%$ of the arsenic in all other foods was inorganic. The greatest dietary contribution to total arsenic was seafood (76-96\%) for all age groups, except infants. For infants, seafood and rice products contributed 42 and $31 \%$, respectively. Adult dietary arsenic intakes reported for other countries range 
Table 6-5. Mean Daily Dietary Intake of Arsenic for Selected U.S. Population Groups

\begin{tabular}{lccc}
\hline & \multicolumn{3}{c}{ Date of study } \\
\cline { 2 - 4 } Mean daily intake $(\mu \mathrm{g} / \mathrm{kg}$ body weight/day $)$ & $1984-1986^{\mathrm{a}}$ & 1986-1991 $^{\mathrm{b}}$ & 1991-1997 $^{\mathrm{c}}$ \\
\hline Provisional tolerable daily intake (PTDI) & $\mathrm{d}$ & 2.1 & 2.1 \\
6-11 months & 0.82 & 0.5 & 0.31 \\
2 years & 1.22 & 0.81 & 1.80 \\
14-16 years, female & 0.54 & 0.36 & 0.41 \\
14-16 years, male & 0.60 & 0.39 & 0.24 \\
25-30 years, female & 0.66 & 0.44 & 0.44 \\
25-30 years, male & 0.76 & 0.51 & 0.72 \\
60-65 years, female & 0.71 & 0.46 & 1.08 \\
60-65 years, male & 0.74 & 0.48 & 1.14 \\
\hline
\end{tabular}

${ }^{\mathrm{a}}$ Gunderson $1995 \mathrm{a}$

${ }^{\mathrm{b}}$ Gunderson 1995b

${ }^{\mathrm{C}}$ Tao and Bolger 1999

${ }^{\mathrm{d}}$ No agreement has been reached on a maximum acceptable intake for total arsenic; the FAO/WHO has assigned a PTDI for inorganic arsenic of $2.1 \mu \mathrm{g} / \mathrm{kg}$ body weight for adults. Data from FDA studies. FDA does not recommend daily intake levels for Arsenic. 
from 11.7 to $280 \mu \mathrm{g} /$ day (Tao and Bolger 1999). Schoof et al. (1999b) estimated that intake of inorganic arsenic in the U.S. diet ranges from 1 to $20 \mu \mathrm{g}$ /day, with a mean of $3.2 \mu \mathrm{g} / \mathrm{day}$. In contrast, these estimates of inorganic arsenic intakes are based on measured inorganic arsenic concentrations from a market basket survey.

The FDA conducted earlier Total Diet Studies in 1984-1986 and 1986-1991. For the sampling period of June 1984 to April 1986, the total daily intake of arsenic from foods was $58.1 \mu \mathrm{g}$ for a $25-30$-year-old male with seafood contributing $87 \%$ of the total (Gunderson 1995a). For the sampling period from July 1986 to April 1991, the total daily intake of arsenic from foods was lower, $38.6 \mu \mathrm{g}$ for a 25-30-year-old male. Seafood again was the major source of arsenic, contributing $88 \%$ of the total (Gunderson 1995b). Results of the two Total Diet Studies for selected population groups are shown in Table 6-5. The Total Diet Study for the sampling period from September 1991 to December 1996, shows that arsenic, at $\geq 0.03 \mu \mathrm{g} / \mathrm{g}$, was found in $55(21 \%)$ of the $261-264$ foods/mixed dishes analyzed. The highest concentrations again were found in seafood, followed by rice/rice cereal, mushrooms, and poultry. The estimated total daily intake of arsenic from foods was $56.6 \mu \mathrm{g}$ for a $25-30$-year-old male. Seafood was the major contributor, accounting for $88-96 \%$ of the estimated total arsenic intake of adults.

Average daily dietary exposures to arsenic were estimated for approximately 120,000 U.S. adults by combining data on annual diet, as measured by a food frequency questionnaire, with residue data for table-ready foods that were collected for the annual FDA Total Diet Study. Dietary exposures to arsenic were highly variable, with a mean of $50.6 \mu \mathrm{g} /$ day (range, $1.01-1,081 \mu \mathrm{g} /$ day) for females and $58.5 \mu \mathrm{g} / \mathrm{day}$ (range, 0.21-1,276 $\mu \mathrm{g}$ /day) for males (MacIntosh et al. 1997). Inorganic arsenic intake in 969 men and women was assessed by a semi-quantitative food frequency questionnaire in combination with a database for total arsenic content in foods and by toenail concentrations of arsenic. The mean estimated average daily consumption of inorganic arsenic was $10.22 \mu \mathrm{g}$ /day with a range of $0.93-104.89 \mu \mathrm{g} / \mathrm{day}$. An assumption of $1.5 \%$ of the total arsenic in fish and $20 \%$ of the total arsenic in shellfish was inorganic arsenic was used in this assessment (MacIntosh et al. 1997).

During a comprehensive total diet study extending from 1985 to 1988, the estimated daily dietary ingestion of total arsenic by the average Canadian was $38.1 \mu \mathrm{g}$ and varied from $14.9 \mu \mathrm{g}$ for the $1-4$ yearold-age group to $59.2 \mu \mathrm{g}$ for 20-39-year-old males (Dabeka et al. 1993). Daily intakes of arsenic from food by women in the Shiga Prefecture, Japan, were investigated by the duplicate portion method and by the market basket method. In 1991 and 1992, the daily intakes determined by the duplicate portion 
method were 206 and $210 \mu \mathrm{g}$, respectively. Those determined by the market basket method were 160 and $280 \mu \mathrm{g}$, respectively (Tsuda et al. 1995b).

Arsenic concentrations in human breast milk have been reported to range from 4 to $<10 \mu \mathrm{g} / \mathrm{L}$ in pooled human milk samples from Scotland and Finland to $200 \mu \mathrm{g} / \mathrm{L}$ in samples from Antofagasta, Chile, where there is a high natural environmental concentration of arsenic (Broomhall and Kovar 1986). The arsenic concentration in the breast milk of 35 women in Ismir, Turkey, a volcanic area with high thermal activity ranged from 3.24 to $5.41 \mu \mathrm{g} / \mathrm{L}$, with a median of $4.22 \mu \mathrm{g} / \mathrm{L}$ (Ulman et al. 1998). Sternowsky et al. (2002) analyzed breast milk from 36 women from three different regions in Germany. These regions included the city of Hamburg, a rural area, Soltau, Lower Saxony, and Munster, the potentially contaminated area. Arsenic was not detected $(<0.3 \mu \mathrm{g} / \mathrm{L})$ in 154 of 187 samples, with the highest concentration, $2.8 \mu \mathrm{g} / \mathrm{L}$, found in a sample from the rural area. The geometric means from the three areas were comparable.

The mean arsenic levels in three groups of cows in the region that grazed on land impacted by lava and thermal activity were $4.71,4.46$, and $4.93 \mu \mathrm{g} / \mathrm{L}$, compared to $5.25 \mu \mathrm{g} / \mathrm{L}$ for cows kept in sheds and fed commercial pellet feed and municipal water (Ulman et al. 1998). Mean arsenic concentrations in cow's milk ranging from 18.6 to $17.1 \mu \mathrm{g} / \mathrm{L}$ and from 16.7 to $18.0 \mu \mathrm{g} / \mathrm{L}$ were reported for cow's grazing in nonindustrial and an industrial regions, respectively, in Turkey (Erdogan et al. 2004).

A Danish study found that carrots grown in soil containing $30 \mu \mathrm{g} / \mathrm{g}$ of arsenic, which is somewhat above the $20 \mu \mathrm{g} / \mathrm{g}$ limit for total arsenic set by Denmark for growing produce, contained $0.014 \mu \mathrm{g} / \mathrm{g}$ fresh weight of arsenic, all in the form of inorganic As(III) and As(V) (Helgesen and Larsen 1998). An adult consuming 376 grams of vegetables a day $\left(90^{\text {th }}\right.$ percentile) represented solely by carrots would consume $5.3 \mu \mathrm{g}$ of arsenic a day. The study concluded that the estimated intake of arsenic from produce grown in soil meeting regulatory limits was low compared with other food sources and water.

If vegetables are grown in planters made of wood treated with CCA, arsenic may leach out of the wood and be taken up by the vegetables. In a study by Rahman et al. (2004), arsenic was found to diffuse into the soil from the CCA-treated wood, with the highest concentrations found at $0-2 \mathrm{~cm}$ from the CCAtreated wood and a steady decline in concentration with increased distance from the wood. Crops grown within $0-2 \mathrm{~cm}$ of the CCA-treated wood contained higher concentrations of arsenic than those grown at $1.5 \mathrm{~m}$ from the treated wood. However, the concentrations are below U.S. FDA tolerance limits that have been set for arsenic in select food items. In addition, food grown in this manner is unlikely to constitute a significant part of a person's diet. 
In 2003, U.S. manufacturers of arsenical wood preservatives began a voluntary transition from CCA to other wood preservatives in wood products for certain residential uses, such as play structures, picnic tables, decks, fencing, and boardwalks. This phase out was completed on December 31, 2003; wood treated prior to this date could still be used and structures made with CCA-treated wood would not be affected. CCA-treated wood products continue to be used in industrial applications (EPA 2003a). EPA's Consumer Awareness Program (CAP) for CCA is a voluntary program established by the manufacturers of CCA products to inform consumers about the proper handling, use, and disposal of CCA-treated wood. Additional information about this program can be found from EPA (2007a).

The arsenic content in the human body is $3-4 \mathrm{mg}$ and tends to increase with age. Arsenic concentrations in most tissues of the human body are $<0.3$ to $147 \mu \mathrm{g} / \mathrm{g}$ dry weight, excluding hair, nails, and teeth. Mammals tend to accumulate arsenic in keratin-rich tissues such as hair and nails. The normal concentrations of arsenic range from about 0.08 to $0.25 \mu \mathrm{g} / \mathrm{g}$ in hair, and $0.34 \mu \mathrm{g} / \mathrm{g}$ in nails. The normal concentration of arsenic in urine can range from 5 to $40 \mu \mathrm{g}$ per day (total) (Mandal and Suzuki 2002). Table 6-6 contains arsenic levels in various human tissues.

A German study investigated the transfer of arsenic from the environment to humans in the northern Palatine region, a former mining area characterized by high soil levels of arsenic $(<2-605 \mu \mathrm{g} / \mathrm{g})$ in residential areas compared to a region in southern lower Saxony with nonelevated levels of arsenic in soil (Gebel et al. 1998a). None of the residents were occupationally exposed to arsenic and the arsenic levels in drinking water were generally below $0.015 \mathrm{mg} / \mathrm{L}$. The mean levels of arsenic in urine and hair were lower in the reference area than in the former mining area (see Table 6-6), although within the mining area, there was a slight increase in arsenic levels in hair and arsenic excreted in urine with increasing arsenic content in soil. Children in the Palatine region did not have higher contents of arsenic in their hair or urine. The most significant factor contributing to elevated levels of arsenic in hair and urine was seafood consumption. In the combined population of people living in mining areas containing high levels of arsenic in soil and other areas, the level of arsenic in urine was positively associated with the extent of seafood consumption. However, the study also showed that seafood consumption does not lead to an extreme increase in excretion of arsenic in the urine. There are apparently other, unidentified factors affecting the urine levels. Only arsenic in urine, not in hair, was significantly correlated with age. The level of arsenic in urine was very slightly, but significantly correlated with the consumption of homegrown produce. Tobacco smoking had no correlation with the arsenic content of either hair or urine (Gebel et al. 1998a). 
Table 6-6. Levels of Arsenic in Human Tissue and Urine-Recent Studies

\begin{tabular}{|c|c|c|c|c|c|}
\hline \multirow[b]{2}{*}{ Site population } & \multirow[b]{2}{*}{ Sample } & \multicolumn{2}{|c|}{ Concentration } & \multirow[b]{2}{*}{ Units } & \multirow[b]{2}{*}{ Reference } \\
\hline & & Mean $^{\mathrm{a}}$ & Range & & \\
\hline \multicolumn{6}{|c|}{ Fort Valley, Georgia, Pesticide manufacturing facility (Superfund site) } \\
\hline \multirow{4}{*}{$\begin{array}{l}40 \text { workers (samples collected } \\
\text { at end of work week) }\end{array}$} & Urine, random & 11.6 & $<1-57$ & $\mu g / L$ & \multirow{4}{*}{$\begin{array}{l}\text { Hewitt et al. } \\
1995\end{array}$} \\
\hline & Urine, 24-hour & 11.0 & $<1-54$ & $\mu g / L$ & \\
\hline & Hair & 0.78 & $<0.01-6.3$ & $\mu g / g$ & \\
\hline & Fingernails & 0.79 & $<0.01-6.1$ & $\mu g / g$ & \\
\hline \multicolumn{6}{|l|}{ Hermosa, Sonora, Mexico } \\
\hline $\begin{array}{l}\text { Children, ages } 7-11, \text { exposed } \\
\text { to arsenic in water (mean } \\
\text { concentration [mean dose]): }\end{array}$ & Urine, 24-hour & & & & \multirow{4}{*}{$\begin{array}{l}\text { Wyatt et al. } \\
\text { 1998a, } \\
\text { 1998b } \\
y \\
y\end{array}$} \\
\hline $9 \mu \mathrm{g} / \mathrm{L}[0.481 \mu \mathrm{g} / \mathrm{kg} / \mathrm{day}]$ & & 10.26 & $4.05-19.68$ & $\mu g /$ day & \\
\hline $15 \mu \mathrm{g} / \mathrm{L}[0.867 \mu \mathrm{g} / \mathrm{kg} / \mathrm{day}]$ & & 10.54 & $2.82-20.44$ & $\mu g /$ day & \\
\hline 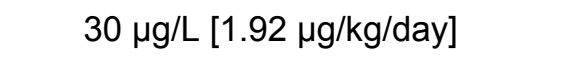 & & 25.18 & $5.44-93.28$ & $\mu g /$ day & \\
\hline \multicolumn{6}{|l|}{ Glasgow, Scotland } \\
\hline Adults, normal $(n=1,250)$ & Hair & 0.650 & $0.20-8.17$ & $\mu g / g$ & \multirow[t]{7}{*}{ Raie 1996} \\
\hline Adults, postmortem $(n=9)$ & Liver & $0.048[0.024]$ & $0.011-0.152$ & $\mu g / g$ & \\
\hline Infants, postmortem $(n=9)$ & Liver & $0.0099[0.007]$ & $0.0034-0.019$ & $\mu g / g$ & \\
\hline Adults, postmortem $(\mathrm{n}=8)$ & Lung & $0.044[0.022]$ & $0.0121-0.125$ & $\mu g / g$ & \\
\hline Infants, postmortem $(\mathrm{n}=9)$ & Lung & $0.007[0.0055]$ & $0.0011-0.015$ & $\mu g / g$ & \\
\hline Adults, postmortem $(n=9)$ & Spleen & $0.015[0.008]$ & $0.001-0.063$ & $\mu \mathrm{g} / \mathrm{g}$ & \\
\hline Infants, postmortem $(n=8)$ & Spleen & $\begin{array}{l}0.0049 \\
{[0.0045]}\end{array}$ & $\begin{array}{l}0.0011- \\
0.0088\end{array}$ & $\mu g / g$ & \\
\hline \multicolumn{6}{|c|}{ Palatinate Region, Germany (high As) ${ }^{b}$} \\
\hline Residents $(n=199)$ & Urine, 24-hour & $3.96[3.21]$ & $<0.1-18.32$ & $\mu g / g$ & \multirow{2}{*}{$\begin{array}{l}\text { Gebel et al. } \\
1998 a\end{array}$} \\
\hline Residents $(n=211)$ & Hair & $0.028[0.016]$ & $<0.005-0.154$ & $\mu \mathrm{g} / \mathrm{g}$ & \\
\hline \multicolumn{6}{|c|}{ Saxony, Germany (low As_reference) ${ }^{b}$} \\
\hline Residents $(n=75)$ & Urine, 24-hour & $7.58[6.20]$ & $0.29-23.78$ & $\mu g / g$ & \multirow{2}{*}{$\begin{array}{l}\text { Gebel et al. } \\
1998 a\end{array}$} \\
\hline Residents $(n=74)$ & Hair & $0.069[0.053]$ & $0.013-0.682$ & $\mu \mathrm{g} / \mathrm{g}$ & \\
\hline \multicolumn{6}{|c|}{ Ismir, Turkey, (volcanic area with high thermal activity) } \\
\hline $\begin{array}{l}\text { Nonoccupationally exposed } \\
\text { women }(n=35)\end{array}$ & Breast milk & $4.23[4.26]$ & $3.24-5.41$ & $\mu g / L$ & $\begin{array}{l}\text { Ulman et al. } \\
1998\end{array}$ \\
\hline \multicolumn{6}{|c|}{ Erlangen-Nurenberg Germany 1/92-12/93 } \\
\hline \multirow[t]{2}{*}{$\begin{array}{l}\text { Nonoccupationally exposed } \\
\text { people }(n=50)\end{array}$} & Lung & 5.5 & $<1-13.0$ & $\begin{array}{l}\mathrm{ng} / \mathrm{g} \\
\mathrm{ww}\end{array}$ & \multirow[t]{2}{*}{$\begin{array}{l}\text { Kraus et al. } \\
2000\end{array}$} \\
\hline & & 28.4 & $<1-73.6$ & $\begin{array}{l}\mathrm{ng} / \mathrm{g} \\
\mathrm{dw}\end{array}$ & \\
\hline
\end{tabular}


Table 6-6. Levels of Arsenic in Human Tissue and Urine-Recent Studies

\begin{tabular}{|c|c|c|c|c|c|}
\hline \multirow[b]{2}{*}{ Site population } & \multirow[b]{2}{*}{ Sample } & \multicolumn{2}{|c|}{ Concentration } & \multirow[b]{2}{*}{ Units } & \multirow[b]{2}{*}{ Reference } \\
\hline & & Mean $^{a}$ & Range & & \\
\hline \multicolumn{6}{|c|}{ Tarragona (Catalonia, Spain) 1997-1999 } \\
\hline \multirow[t]{5}{*}{$\begin{array}{l}\text { Nonoccupationally exposed } \\
\text { people }(n=78)\end{array}$} & Lung & $<0.05$ & & \multirow[t]{5}{*}{$\begin{array}{l}\mu g / g \\
w w\end{array}$} & \multirow[t]{5}{*}{$\begin{array}{l}\text { Garcia et al. } \\
2001\end{array}$} \\
\hline & Bone & $<0.05$ & & & \\
\hline & Kidney & $<0.05$ & & & \\
\hline & Liver & $<0.05$ & & & \\
\hline & Lung & $<0.05$ & & & \\
\hline \multicolumn{6}{|l|}{ West Bengal, India } \\
\hline \multirow[t]{2}{*}{$\begin{array}{l}\text { Residents consuming arsenic- } \\
\text { contaminated water }(n=47)\end{array}$} & Fingernail & 7.32 & $2.14-40.25$ & \multirow[t]{4}{*}{$\mu g / g$} & \multirow[t]{4}{*}{$\begin{array}{l}\text { Mandal et } \\
\text { al. } 2003\end{array}$} \\
\hline & Hair & 4.46 & $0.70-16.17$ & & \\
\hline \multirow{2}{*}{$\begin{array}{l}\text { Residents consuming } \\
\text { nonarsenic-contaminated water } \\
(n=15)\end{array}$} & Fingernail & 0.19 & $0.11-0.30$ & & \\
\hline & Hair & 0.07 & $0.03-0.12$ & & \\
\hline \multicolumn{6}{|l|}{ Middleport, NY, USA } \\
\hline Children $<7$ years $(n=77)$ & Urine & $15.1^{\mathrm{c}}$ & $2.1-59.6$ & \multirow[t]{4}{*}{$\mu g / L$} & \multirow{4}{*}{$\begin{array}{l}\text { Tsuji et al. } \\
2005\end{array}$} \\
\hline Children $<13$ years $(n=142)$ & Urine & $15.7^{\mathrm{c}}$ & $2.1-59.9$ & & \\
\hline $\begin{array}{l}\text { Children } \geq 7 \text { years and adults } \\
(n=362)\end{array}$ & Urine & $15.8^{c}$ & $3.9-773$ & & \\
\hline All participants & Urine & $15.7^{\mathrm{c}}$ & $2.1-773$ & & \\
\hline
\end{tabular}

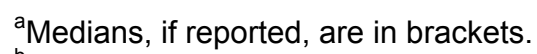

${ }^{\mathrm{b}}$ The reference group (Saxony) had significantly higher levels of arsenic in urine and hair. However, data from both groups correspond to normal range reference data.

${ }^{\mathrm{C}}$ Geometric mean, total arsenic

$\mathrm{dw}=$ dry weight; $w \mathrm{w}=$ wet weight 
A study was performed to look at the arsenic levels, as well as the arsenic species present, in hair and nail samples from individuals in an arsenic-affected area in West Bengal, India. Mean arsenic concentrations in hair and fingernails of the chronically arsenic exposed population were 4.46 and $7.32 \mu \mathrm{g} / \mathrm{g}$, respectively and were 0.07 and $0.19 \mu \mathrm{g} / \mathrm{g}$ in a control population. Fingernail samples were found to contain mostly inorganic arsenic $(>80 \%)$ as a mixture of $\mathrm{As}(\mathrm{III})$ and $\mathrm{As}(\mathrm{V})$, as well as DMA(III) and DMA(V). Hair samples also mostly contained inorganic arsenic (>90\%), as well as MMA(V) and DMA(V) (Mandal et al. 2003).

Arsenic in soil in communities surrounding former smelters is a public health concern, especially for infants and children who may consume significant quantities of soil. Since lead arsenate was used in apple and other fruit orchards, often at very high application rates, and this compound would be expected to accumulate and persist in surface soil, there are concerns to human health when these when old orchards are converted into subdivisions or when they are used to grow food crops or forage. However, arsenic in soil may be imbedded in minerals or occur as insoluble compounds such as sulfides and therefore, not be taken up by the body from the gastrointestinal tract. In addition, oxidation of mineral surfaces may result in armoring the primary mineral grain by a secondary reaction product. Arsenicbearing solids are often encapsulated in insoluble matrices such as silica, further diminishing arsenic availability (Davis et al. 1992).

Sarkar and Datta (2004) examined the bioavailability of arsenic from two soils with different arsenic retention capacities. In this study, Immokalee (Florida) and Orelia (Texas) soils were incubated after spiking with sodium arsenate for 4 months. The Immokalee soil is a sandy spodosol with low Fe/Al, $\mathrm{Ca} / \mathrm{Mg}$, and $\mathrm{P}$ contents and is likely to have minimal arsenic retention capacity. The Orelia soil is a sandy clay that is expected to have strong arsenic retention capacity. Arsenic speciation and bioavailability were studied immediately after spiking and after 4 months of incubation. Approximately $85 \%$ of the total arsenic (soluble and exchangeable fractions) was considered bioavailable and phytoavailable immediately after pesticide application for the Immokalee soil; after 4 months of incubation, this decreased to approximately $46 \%$. Immediately after pesticide application, the amounts of arsenic extracted in the soluble/exchangeable and Fe/Al-bound fractions were similar that of the Immokalee soil. After 4 months, the soluble arsenic decreased to approximately $45 \%$ and the Fe/Al-bound arsenic increased to about $40 \%$. Experiments looking at the bioavailability of arsenic from these two soils indicated that the potentially irreversible adsorption of arsenic by the Orelia soil rendered a significant portion of the total arsenic unavailable for absorption by the human gastrointestinal system. Initially after pesticide application, 
$100 \%$ of the arsenic was bioavailable; after 4 months, the bioavailable fraction was found to decrease to 88 and 69\% in the Immokalee and Orelia soils, respectively (Sarkar and Datta 2004).

Hamel et al. (1998) used synthetic gastric juice to estimate the bioaccessible fraction of metals in the stomach with varying liquid to solid ratios. They found that the bioaccessibility may vary in different soils and with varying liquid to solid ratios. Bioaccessibility was defined as the amount of metal that is soluble in synthetic gastric juice and therefore, potentially available for uptake across the intestinal lumen, while bioavailability was defined as the amount that was actually taken across the cell membranes. Arsenic bioaccessibility for National Institute of Standards and Technology (NIST) Montana Soil SRM 2710 , with a certified arsenic concentration of $626 \mu \mathrm{g} / \mathrm{g}$, was fairly consistent across the liquid-to-solid ratios and ranged from $41.8 \pm 18$ to $56 \pm 21 \%$. The extractability of a hazardous waste contaminated soil from Jersey City, New Jersey, was different than that observed for the Montana NIST soil. For the Jersey City soil, which had an arsenic concentration of $1,120 \mu \mathrm{g} / \mathrm{g}$, there was an increase in the bioaccessible arsenic as the liquid-to-solid ratio increased. Bioaccessible arsenic ranged from $4.5 \pm 0.8$ (at a liquid-tosolid ratio of 100:1) to $25 \pm 9 \%$ (at a ratio of 5,000:1). Similarly, smelter impacted soils from Anaconda, Montana contain metal-arsenic oxides and phosphates whose bioaccessibility is limited by solubility restraints for residence times typical of the gastrointestinal tract (Davis et al. 1992, 1996).

Inhalation of arsenic from ambient air is usually a minor exposure route for the general population. For example, the dose to a person who breathes $20 \mathrm{~m}^{3} /$ day of air containing 20-30 ng/m $/ \mathrm{m}^{3}$ (see Section 6.4.1) would be about $0.4-0.6 \mu \mathrm{g} /$ day. However, smokers may be exposed to arsenic by inhalation of mainstream smoke. Assuming that $20 \%$ of the arsenic in cigarettes is present in smoke, an individual smoking two packs of cigarettes per day would inhale about $12 \mu \mathrm{g}$ of arsenic (EPA 1984a). However, a German study of the arsenic levels in lung tissue of 50 unexposed deceased people (see Table 6-6) found no significant difference in lung arsenic concentrations of smokers versus nonsmokers, nor were there any significant age- or sex-related differences (Kraus et al. 2000). Before arsenical pesticides were banned, tobacco contained up to $52 \mu \mathrm{g} \mathrm{As} / \mathrm{g}$, whereas after the ban, maximum arsenic levels were reduced to $3 \mu \mathrm{g} / \mathrm{g}$.

Occupational exposure to arsenic may be significant in several industries, mainly nonferrous smelting, arsenic production, wood preservation, glass manufacturing, and arsenical pesticide production and application. Since arsenic compounds are used as a desiccant for cotton, workers involved in harvesting and ginning cotton may be exposed to arsenic. Occupational exposure would be via inhalation and dermal contact. Should any arsenic be retained in the cotton, workers handling the fabric and the general 
public would be exposed. The electronics industry is expanding the use of gallium arsenide in the production of electro-optical devices and integrated circuits, and workers in the industry where gallium arsenide is used may be exposed to hazardous substances such as arsenic, arsine, and various acids (Sheehy and Jones 1993). Occupational exposure to arsenic is generally assessed by measuring urinary excretion of arsenic. Past exposure is commonly assessed by arsenic levels in hair. Different types of occupational exposures may result in different uptakes of arsenic because of the bioavailability of the form of arsenic to which workers are exposed. For example, maintenance workers at a Slovak coal-fired power plant exposed to 8-hour TWA arsenic air concentrations of $48.3 \mu \mathrm{g} / \mathrm{m}^{3}$ (range, $0.17-375.2$ ) had urinary total arsenic levels of $16.9 \mu \mathrm{g} \mathrm{As} / \mathrm{g}$ creatinine (range, 2.6-50.8), suggesting that bioavailability of arsenic from airborne coal fly ash is about one-third that from in copper smelters and similar settings (Yager et al. 1997). Approximately $90 \%$ of the arsenic-containing particulates were $\geq 3.5 \mu \mathrm{m}$. Apostoli et al. (1999) monitored 51 glass workers exposed to arsenic trioxide by measuring dust in the breathing zone. The mean concentration of arsenic in air was $82.9 \mu \mathrm{g} / \mathrm{m}^{3}\left(1.5-312 \mu \mathrm{g} / \mathrm{m}^{3}\right)$; exposure was higher for workers involved in handling the particulate matter. The occupation exposures to principal contaminants, including arsenic, at five coal-fired power plants were evaluated during June-August 2002. Eight air samples were collected per similar exposure group at four of the five facilities; inorganic arsenic concentrations in all samples were below the limit of detection $\left(0.37-0.72 \mu \mathrm{g} / \mathrm{m}^{3}\right)$, as well as being below the OSHA permissible exposure limit (PEL) of $10 \mu \mathrm{g} / \mathrm{m}^{3}$ (Bird et al. 2004).

NIOSH researchers conducted a study of arsenic exposures and control systems for gallium arsenide operations at three microelectronics facilities during 1986-1987 (Sheehy and Jones 1993). Results at one plant showed that in all processes evaluated but one, the average arsenic exposures were at or above the OSHA action level of $5 \mu \mathrm{g} / \mathrm{m}^{3}$, with a maximum exposure of $8.2 \mu \mathrm{g} / \mathrm{m}^{3}$. While cleaning the Liquid Encapsulated Czochralski (LEC) pullers, the average potential arsenic exposure of the cleaning operators was 100 times the OSHA PEL of $10 \mu \mathrm{g} / \mathrm{m}^{3}$. Area arsenic samples collected at the plant in break-rooms and offices, 20-60 feet from the process rooms, had average arsenic concentrations of $1.4 \mu \mathrm{g} / \mathrm{m}^{3}$. At the other two plants, personal exposures to arsenic were well controlled for all processes evaluated.

A study has been conducted to examine the relationship between total arsenic levels in hair of employees in a semiconductor fabrication facility and job responsibility, a surrogate variable for arsenic exposure (de Peyster and Silvers 1995). Airborne arsenic was found in areas where equipment was cleaned but not in administrative areas. The highest airborne arsenic level found in the study, $15 \mu \mathrm{g} / \mathrm{m}^{3}$, was collected from the breathing zone of a maintenance employee who was cleaning a source housing over a period of 2 hours in an area with local exhaust ventilation. A concentration of $2 \mu \mathrm{g} / \mathrm{m}^{3}$ was found during the 
remainder of the cleaning period ( $\sim 53$ minutes). Workers in maintenance who were regularly assigned to cleaning equipment, and therefore presumed to have the highest exposure potential, had a mean hair arsenic level of $0.042 \mu \mathrm{g} / \mathrm{g}$. This was higher than the mean of $0.033 \mu \mathrm{g} / \mathrm{g}$ observed in administrative controls, but the difference was not significant. Maintenance workers who only occasionally cleaned and maintained arsenic-contaminated equipment had a mean hair arsenic level of $0.034 \mu \mathrm{g} / \mathrm{g}$, which was comparable to the controls. The highest group mean hair arsenic level of $0.044 \mu \mathrm{g} / \mathrm{g}$, surprisingly, was found in supervisors and engineers who were presumed to have the lowest exposure potential of all workers in the process areas. However, the highest concentrations of hair arsenic in engineers, 0.076 and $0.106 \mu \mathrm{g} / \mathrm{g}$, were observed in two heavy smokers who smoked 1-2 packs of cigarettes per day. A 2-way analysis of variance indicated that smoking appeared to be a significant contributing factor whereas occupational exposure was not.

Hwang and Chen (2000) evaluated arsenic exposure in 21 maintenance engineers (exposed group) and 10 computer programmers (control group) at 3 semiconductor manufacturing facilities. Samples of air, wipe, and urine, as well as used cleaning cloths and gloves were collected to determine arsenic exposure. Arsenic was undetectable in 46 of the 93 air samples, and most samples were generally below the recommended occupational exposure limit $\left(10 \mu \mathrm{g} / \mathrm{m}^{3}\right)$ in work areas during ion implanter maintenance. Arsine was detectable in 22 of the 45 area air samples and in 15 of the 35 personal air samples; however, all concentrations were well below the occupational exposure limit of $50 \mathrm{ppb}\left(160 \mu \mathrm{g} / \mathrm{m}^{3}\right)$. Mean arsine concentrations ranged from not detected to $4.0 \mathrm{ppb}\left(15 \mu \mathrm{g} / \mathrm{m}^{3}\right)$ in area air samples, and the mean arsine concentration of personal air for maintenance engineers was $4.3 \mathrm{ppb}\left(14 \mu \mathrm{g} / \mathrm{m}^{3}\right)$. Arsenic concentrations in wipe samples, used cleaning cloths, and gloves, varied from not detected to $146 \mu \mathrm{g} / \mathrm{cm}^{2}$. During ion implanter maintenance, urinary arsenic levels were found to increase (1.0-7.8 $\mu \mathrm{g} / \mathrm{g}$ creatinine) in the maintenance engineers, from a mean baseline concentration of $3.6 \mu \mathrm{g} / \mathrm{g}$ creatinine. The average urinary arsenic level for the computer programmers was $3.8 \mu \mathrm{g} / \mathrm{g}$ creatinine (Hwang and Chen 2000). Mean arsenic concentrations in blood of 103 workers in the optoelectronic industry and 67 controls were 8.58 and $7.85 \mu \mathrm{g} / \mathrm{L}$, respectively (Liao et al. 2004).

Concentrations of various metals, including arsenic, were measured in autopsy tissues (liver, lung, kidney, brain, and bone) collected from 78 nonoccupationally exposed subjects from Tarragona County, Spain between 1997 and 1999. In general, arsenic concentrations were under the analytical detection limit $(0.05 \mu \mathrm{g} / \mathrm{g}$ wet weight) in all tissues (Garcia et al. 2001). 
CCA preservatives are commonly used for treating timber used in constructions in marine and other humid environments or in contact with the ground. Exposure to CCA compounds may occur through dermal contact and inhalation of dust while working with the treated timber. Nygren et al. (1992) investigated the occupational exposure to airborne dust, chromium, copper, and arsenic in six joinery shops in Sweden where impregnated wood was used for most of their production. The mean airborne concentration of arsenic around various types of joinery machines ranged from 0.54 to $3.1 \mu \mathrm{g} / \mathrm{m}^{3}$. No increased concentrations of arsenic were found in the workers' urine. A study was carried out in Denmark to evaluate arsenic exposure in taxidermists, workers impregnating wood with CCA solutions, fence builders, construction workers, and workers impregnating electric pylons with arsenic solution (Jensen and Olsen 1995). Airborne arsenic exposure was documented in 19 of 27 individuals working with products containing arsenic. The maximum exposure concentration was $17.3 \mu \mathrm{g} / \mathrm{m}^{3}$, found for a single worker who was filling an impregnation container with CCA paste. Median exposures for indoor workers producing garden fences and weekend cottages were 3.7 and $0.9 \mu \mathrm{g} / \mathrm{m}^{3}$, respectively. The maximum urine concentration reported in the study was 294.5 nanomoles arsenic per millimole creatinine (195 $\mu \mathrm{g} \mathrm{As} / \mathrm{g}$ creatinine) and was from the injector impregnating electric pylons. The median concentration in workers on electric pylons was 80 nanomoles arsenic per millimole creatinine $(53 \mu \mathrm{g}$ As/g creatinine), which was 6 times the concentration in reference individuals. Urine arsenic levels in workers producing garden fences and in taxidermists were 2.9 and 1.8 times the reference level, respectively.

The NIOSH National Occupational Exposure Survey (NOES) conducted in 1981-1983 estimated that about 55,000 workers were potentially exposed to arsenic (NOES 1990). The NOES was based on field surveys of 4,490 facilities that included virtually all workplace environments, except mining and agriculture, where eight or more persons are employed. The principal exposure pathway is probably inhalation of arsenic adsorbed to particulates, but ingestion and possibly dermal exposure may also be common. Since arsenic is no longer produced in the United States (see Section 5.1) and many arsenical pesticide uses have been banned (see Chapter 8), it is likely that the number of workers occupationally exposed to arsenic has decreased markedly in more recent years.

\subsection{EXPOSURES OF CHILDREN}

This section focuses on exposures from conception to maturity at 18 years in humans. Differences from adults in susceptibility to hazardous substances are discussed in Section 3.7, Children's Susceptibility. 
Children are not small adults. A child's exposure may differ from an adult's exposure in many ways. Children drink more fluids, eat more food, breathe more air per kilogram of body weight, and have a larger skin surface in proportion to their body volume. A child's diet often differs from that of adults. The developing human's source of nutrition changes with age: from placental nourishment to breast milk or formula to the diet of older children who eat more of certain types of foods than adults. A child's behavior and lifestyle also influence exposure. Children crawl on the floor, put things in their mouths, sometimes eat inappropriate things (such as dirt or paint chips), and spend more time outdoors. Children also are closer to the ground, and they do not use the judgment of adults to avoid hazards (NRC 1993).

As with adults, most children are exposed to arsenic largely through their diet. Since the greatest dietary intake of arsenic is from fish and seafood, infants and young children for whom a substantial part of their food is milk, would not be exposed to arsenic from dietary sources as much as older children. Even when mothers consumer large amounts of seafood, there does not appear to be any major transfer of arsenobetaine, the major form of arsenic in seafood, from seafood to milk (Grandjean et al. 1995). Arsenic concentrations were very low in human milk sampled from 88 mothers in the Faroe Islands, where the seafood diet includes pilot whale meat and blubber. The total arsenic concentrations ranged from 0.1 to $4.4 \mu \mathrm{g} / \mathrm{kg}$, with a median of $1.6 \mu \mathrm{g} / \mathrm{kg}$ (Grandjean et al. 1995). The arsenic concentration in the breast milk of 35 women in Ismir, Turkey, a volcanic area with high thermal activity ranged from 3.24 to $5.41 \mu \mathrm{g} / \mathrm{L}$, with a median of $4.22 \mu \mathrm{g} / \mathrm{L}$ (Ulman et al. 1998). The mean arsenic levels in three groups of cows in the region that grazed on land impacted by lava and thermal activity were 4.71, 4.46, and $4.93 \mu \mathrm{g} / \mathrm{L}$, compared to $5.25 \mu \mathrm{g} / \mathrm{L}$ for cows kept in sheds and fed commercial pellet feed and municipal water. The arsenic levels in the urine of pregnant women and the cord blood of their infants were $0.625 \pm 0.027$ and $0.825 \pm 0.079 \mu \mathrm{g} / \mathrm{L}$, respectively. The authors concluded that there was no harmful exposure to arsenic in volcanic areas with high arsenic levels from suckling infants or feeding them local cow's milk, nor was there harm to the newborns from their mother's diet. Sternowsky et al. (2002) analyzed breast milk from 36 women from three different regions in Germany. These regions included the city of Hamburg, a rural area, Soltau, Lower Saxony, and Munster, the potentially contaminated area. Arsenic was not detected $(<0.3 \mu \mathrm{g} / \mathrm{L})$ in 154 of 187 samples, with the highest concentration, $2.8 \mu \mathrm{g} / \mathrm{L}$, found in a sample from the rural area. The geometric mean arsenic concentrations from the three areas were comparable. Calculated oral intakes of arsenic were between 0.12 and $0.37 \mu \mathrm{g} /$ day for an infant at 3 months of age and weighing $6 \mathrm{~kg}$.

According to the FDA study of 1986-1991, the mean daily intakes of arsenic are 0.5 and $0.81 \mu \mathrm{g} / \mathrm{kg}$ body weight per day for a 6-11-month-old infant and 2-year-old child, respectively (Gunderson 1995b). This 
can be compared to a mean daily intake of $0.51 \mu \mathrm{g} / \mathrm{kg}$-body weight per day for a $25-30$-year-old male (see Table 6-5). A Total Diet Study, from September 1991 to December 1996, estimated that the average inorganic arsenic intake for children of various age/sex groups were (age-sex group, total arsenic intake in $\mu \mathrm{g} /$ day, inorganic arsenic intake in $\mu \mathrm{g} /$ day): $6-11$ months, $2.15,1.35 ; 2$ years, $23.4,4.41 ; 6$ years, 30.3, 4.64; 10 years, 13.3, 4.21; and 14-16 years (females), 21.8, 5.15; 14-16 years (males), 15.4, 4.51 (Tao and Bolger 1999). The greatest dietary contribution (76-96\%) of total arsenic intake for all age groups other than infants was seafood. For infants, 41 and $34 \%$ of the estimated total arsenic intakes are from seafood and rice/rice cereals, respectively (Tao and Bolger 1999). Only for toddlers does the intake approach the World Health Organization's (WHO) provisional tolerable daily intake (PTDI) for inorganic arsenic (see Table 6-5). A 1985-1988 Canadian total diet study estimated that 1-4-year-olds ingested $14.9 \mu \mathrm{g}$ of total arsenic per day compared with $38.1 \mu \mathrm{g}$ by the average Canadian and $59.2 \mu \mathrm{g}$ for 20 39-year-old males (Dabeka et al. 1993). Yost et al. (2004) estimated the mean dietary intake for inorganic arsenic for children (1-6 years of age) to be $3.2 \mu \mathrm{g} /$ day, with a range of $1.6-6.2 \mu \mathrm{g} / \mathrm{day}$ for the $10^{\text {th }}$ and $95^{\text {th }}$ percentiles, respectively. Inorganic arsenic intake was predominantly contributed by grain and grain products, fruits and fruit juices, rice and rice products, and milk (Yost et al. 2004). Total arsenic and arsenobentaine concentrations were measured in 16 baby food samples obtained from manufactures in Spain; total arsenic concentrations ranged from 2.042 to $0.270 \mu \mathrm{g} / \mathrm{g}$ in plaice with vegetables and sole with white sauce, respectively. Arsenobetaine, which is the arsenical commonly found in fish, accounted for essentially $100 \%$ of the arsenic present in the samples (Vinas et al. 2003).

Arsenic exposure from drinking water may be elevated especially in groundwater from areas where arsenic occurs naturally in soil such as the western and north central sections of the United States (see Table 6-3 and Figure 6-2).

Arsenic exposure in communities near mining and smelting facilities or where arsenic had formerly been applied to agricultural land are a public health concern, especially for infants and children. Since arsenic remains in the surface soil indefinitely and long past land uses may be forgotten, people may not realize that they are living in areas where high levels of arsenic may occur in soil. Contaminated soils pose a particular hazard to children because of both hand-to-mouth behavior and intentional ingestion of soil (pica) that contains metals and other contaminants (Hamel et al. 1998). In these communities, arsenic may contaminate carpeting or may have been tracked in from outside. Children may be exposed to this arsenic while crawling around or playing on contaminated carpeting. Exposure may also result from dermal contact with the soil, or by inhaling the dust and then swallowing it after mucociliary transport up 
out of the lungs. Because much of the arsenic in soil is embedded in or adsorbed to soil particles or insoluble, it may not be in a form accessible for uptake by the body.

Hwang et al. (1997b) studied the arsenic exposure of children in Anaconda, Montana, in the vicinity of a former copper smelter from the summer of 1992 through the summer of 1993. Environmental samples and first morning voided urine samples from 414 children $<72$ months old were collected. Attention was focused on that fraction of the environmental source that was thought to be of the greatest risk to the child (i.e., arsenic in small particles $[<250 \mu \mathrm{m}]$ ) that could most readily adhere to hands and toys and could be inadvertently ingested. Average arsenic levels in different types of soil ranged from 121 to $236 \mu \mathrm{g} / \mathrm{g}$. Several studies have reported mean soil ingestion values for children ranging from 9 to $1,834 \mu \mathrm{g} / \mathrm{day}$. Assuming that high arsenic exposure areas have average arsenic levels in soil from 60 to $150 \mu \mathrm{g} / \mathrm{g}$, the resulting daily arsenic intake from soil could range from 1 to $275 \mu \mathrm{g} / \mathrm{day}$ per child. The geometric mean of speciated urinary arsenic (combined As(III), As(V), MMA and DMA) was $8.6 \pm 1.7 \mu \mathrm{g} / \mathrm{L}(\mathrm{n}=289)$ in the Hwang study. A nationwide survey on arsenic exposure in the vicinity of smelter sites revealed that children without excess arsenic exposure had average total urinary arsenic levels ranging from 5 to $10 \mu \mathrm{g} / \mathrm{L}$ (Hwang et al. 1997a). Compared to these values, the mean total urinary arsenic values found in the Hwang study were markedly higher, but they were still well below the WHO-recommended maximum excretion level for total arsenic of $100 \mu \mathrm{g} / \mathrm{L}$ as an action level for intervention. The investigators hypothesized that the relatively low urinary arsenic levels found in the study were probably a reflection of the low bioavailability of some forms of arsenic in contaminated soil. Hwang et al. (1997a) stated that arsenic intake through skin contact is insignificant and may be neglected in the assessment of childhood arsenic exposure. They recommend that parents or guardians pay more attention to their children's activity, especially hand-to-mouth behavior, even though the environmental contaminants might be elevated only slightly. Children in the northern Palatine region of German study, a former mining area characterized by high levels of arsenic $(<2-605 \mu \mathrm{g} / \mathrm{g})$ in residential areas did not show higher arsenic levels in their hair or urine than children from a reference area of Germany (Gebel et al. 1998a).

While CCA registrants voluntarily canceled the production of CCA-treated wood for residential use in 2003, there is a potential for exposure to arsenic from existing structures (Zartarian et al. 2006). Based on a review of existing studies, Hemond and Solo-Gabriele (2004) estimated that children with contact with CCA-treated wood may be subjected to doses in the range of tens of micrograms of arsenic per day. The most important route of exposure appeared to be by hand-to-mouth activities after contact with the CCAtreated wood. Kwon et al. (2004) compared the amounts of water-soluble arsenic on hands of children in 
contact with CCA-treated wood structures or sand in playgrounds. The mean amount of water-soluble arsenic on children's hands from playgrounds without CCA-treated wood was $0.095 \mu \mathrm{g}$ (range $0.011-$ $0.41 \mu \mathrm{g})$. A mean amount of water-soluble arsenic on children's hands from playgrounds with CCAtreated wood was $0.5 \mu \mathrm{g}$ (range $0.0078-3.5 \mu \mathrm{g}$ ) (Kwon et al. 2004). Additional data from the study by Kwon et al. (2004) showed that total arsenic collected in hand-washing water (insoluble arsenic on the filter combined with the water-soluble arsenic in the filtrate) was 0.934 and $0.265 \mu \mathrm{g}$ for the CCA playgrounds and the non-CCA playgrounds, respectively (Wang et al. 2005). Two wood surface swab samples collected from 217 play structures constructed from CCA-treated wood in the City of Toronto, Canada were sampled and analyzed for inorganic arsenic (Ursitti et al. 2004). Dislodgeable arsenic concentrations were found to vary widely from nondetectable $\left(0.08-0.25 \mu \mathrm{g} / 100 \mathrm{~cm}^{2}\right)$ to $521 \mu \mathrm{g} / 100 \mathrm{~cm}^{2}$ (mean $=41.6 \mu \mathrm{g} / 100 \mathrm{~cm}^{2}$ ), and were found to not be a useful predictor of soil arsenic levels (Ursitti et al. 2004).

Shalat et al. (2006) evaluated postexposure hand rinses and urine for total arsenic for 11 children (1371 months) in homes in Miami-Dade County, Florida, with and without CCA-treated playgrounds. Seven playgrounds were included in this study, and five of these contained either CCA-treated or partially CCAtreated wood. In addition, samples of wood, soil (5-8 cm from the base of the playground structure), mulch (when present), and synthetic wipes were analyzed for total arsenic. Wood and soil arsenic concentration were $<2.0$ and $<3 \mathrm{mg} / \mathrm{kg}$ for the non-CCA-treated playgrounds, respectively. Mean arsenic concentrations of 2,380 mg/kg (range 1,440-3,270 mg/kg) and $19 \mathrm{mg} / \mathrm{kg}(4.0-42 \mathrm{mg} / \mathrm{kg}$ ) were reported for wood and soil, respectively, in the playgrounds with CCA-treated wood. An arsenic concentration in mulch at one playground without CCA-treated wood was $0.4 \mathrm{mg} / \mathrm{kg}$, and arsenic concentrations were 0.6 and $69 \mathrm{mg} / \mathrm{kg}$ in mulch at two of the playgrounds with CCA-treated wood. The amount of arsenic removed by synthetic wipes from the non-CCA-treated wood was $<0.5 \mu \mathrm{g}$, while the mean amount of arsenic removed from the CCA-treated wood was $117 \mu \mathrm{g}$ (range 1.0-313 $\mu \mathrm{g}$ ). The amount of arsenic in hand rinses from children who played at the playgrounds with non-CCA-treated wood and at the playgrounds with CCA-treated wood were $<0.2$ and $0.6 \mu \mathrm{g}$ (range $<0.2-1.9 \mu \mathrm{g}$ ), respectively. The mean urinary total arsenic concentration was $0.0136 \mu \mathrm{g} / \mathrm{L}$ (range $0.0072-0.0231 \mu \mathrm{g} / \mathrm{L}$ ) for all children. No association between assess to CCA-treated playgrounds and urinary arsenic levels was found (Shalat et al. 2006).

The potential exposure children may receive from playing in play structures constructed from CCAtreated wood is generally smaller than that they would receive from food and water. For comparison, Yost et al. (2004) estimated the mean dietary intake for inorganic arsenic for children (1-6 years of age) 
to be $3.2 \mu \mathrm{g} /$ day, with a range of $1.6-6.2 \mu \mathrm{g} /$ day for the $10^{\text {th }}$ and $95^{\text {th }}$ percentiles, respectively. In a Total Diet Study, from September 1991 to December 1996, estimated average total intakes for children aged 611 months, 2 years, 6 years, and 10 years were 2.15, 23.4,30.3, and $13.3 \mu \mathrm{g} /$ day, respectively. Average inorganic arsenic intakes for the same age groups were estimated as $1.35,4.41,4.64$, and $4.21 \mu \mathrm{g} / \mathrm{day}$, respectively, based on data for total arsenic in foods and the assumption that $10 \%$ of the total arsenic in seafood was inorganic and that $100 \%$ of the arsenic in all other foods was inorganic (Tao and Bolger 1999). Hand washing after play would reduce the potential exposure to children to arsenic after playing on play structures constructed with CCA-treated wood, since most of the arsenic on the children's hands was removed with water (Kwon et al. 2004).

Concentrations of several toxic metals, including the metalloid arsenic, were measured in the placentas of 200 women in two urban cities in Ukraine, Kyiv and Dniprodzerzhinsk. Arsenic was detected in only 5\% of the samples with concentrations ranging from $<0.156$ to $0.378 \mu \mathrm{g} / \mathrm{g}$. In a study in Bulgaria, placental arsenic concentrations of 7 and $23 \mu \mathrm{g} / \mathrm{g}$ were reported in a control and smelter area, respectively. A placental arsenic concentration of $34 \mu \mathrm{g} / \mathrm{g}$ was reported in a region of Argentina with high concentrations of arsenic in drinking water (Zadorozhnaja et al. 2000).

Parents can inadvertently carry hazardous materials home from work on their clothes, skin, hair, and tools, and in their vehicles (DHHS 1995). Falk et al. (1981b) reported a case of hepatic angiosarcoma in a child that could be associated with arsenic contamination of a parent's clothing, the water supply, and the environment. The father worked in a copper mine and smelter area where his clothing was contaminated with dust containing arsenic. His daughter, who exhibited a high degree of pica, ate soil from the yard, and licked soil off her father's shoes. In a study of arsenic levels in homes in Hawaii, Klemmer et al. (1975) found higher levels in homes of employees of firms that used arsenic for pesticides or wood preservation, compared to homes where residents' work did not involve arsenic. The concentration of arsenic in dust from the homes of workers exposed to arsenic ranged from 5.2 to $1,080 \mu \mathrm{g} / \mathrm{g}$, compared to concentrations of $1.1-31 \mu \mathrm{g} / \mathrm{g}$ in dust from control homes.

While the harmful effects of many components of tobacco smoke are well known, those due to heavy metals in the smoke have not been sufficiently emphasized. The adverse health effects of these toxic metals on the fetus through maternal smoking are of special concern (Chiba and Masironi 1992). The concentration of arsenic in tobacco is relatively low, usually below detectable limits $(<1 \mu \mathrm{g} / \mathrm{g})$. Although the concentrations of inorganic and organic arsenic in the urine of adults do not appear to be influenced by smoking, a positive association was found between urinary arsenic levels in children and parental 
smoking habits. As detailed in a WHO report, the mean arsenic level in the urine of children of nonsmoking parents was $4.2 \mu \mathrm{g} / \mathrm{g}$ creatinine, in children with one smoking parent, it was $5.5 \mu \mathrm{g} / \mathrm{g}$, and in children with both parents smoking, it was $13 \mu \mathrm{g} / \mathrm{g}$ (Chiba and Masironi 1992). Tsuji et al. (2005) reported geometric mean concentrations of total arsenic of 15.1 and $15.7 \mu \mathrm{g} / \mathrm{L}$ in children $<7$ and $<13$ years old, respectively, from households in Middleport, New York, where historical pesticide manufacture was associated with arsenic in soil. Geometric mean concentrations of inorganic arsenic, MMA, and DMA were $0.81,0.54$, and $2.5 \mu \mathrm{g} / \mathrm{L}$, respectively, in children $<7$ years old and $0.83,0.55$, and $3.0 \mu \mathrm{g} / \mathrm{L}$, respectively, in children $<13$ years old (Tsuji et al. 2005).

The use of Chinese herbal medicines (CHM) appears to be common among Chinese women. Both CHM and Chinese proprietary medicines (CPM) are used for treatment of minor ailments in babies and children. Herbal medicines are available in capsule or tablet form in drug stores, supermarkets, and by mail. The CPM "Sin Lak Pill," "Lu Shen Wan," and other anti-asthma preparations have been found to contain inorganic arsenic levels ranging from 25 to $107,000 \mu \mathrm{g} / \mathrm{g}$, and cases of acute arsenic poisoning have been found in children and adults using these CPM (Chan 1994). Babies and children are particularly at risk because they may be given higher doses of these preparations per $\mathrm{kg}$ of body weight than adults would normally consume. They may also lack the hepatic enzymes responsible for drug biotransformation and detoxification (Chan 1994). Concentrations of heavy metals, including arsenic, were evaluated in 54 samples of Asian remedies that were purchased in stores in Vietnam and Hong Kong that would be easily accessible to travelers, as well as in health food and Asian groceries in Florida, New York, and New Jersey. One remedy that was recommended to treat children's fever would expose a $15 \mathrm{~kg}$ child to approximately $5.0 \mathrm{mg}$ of arsenic per day (Garvey et al. 2001). A folk remedy, purchased in California, for the treatment of chicken pox, flu-like symptoms, and nasal congestion, which had been given to two children in Wisconsin, was found to contain 36\% arsenic acid. One-half teaspoon of this powder (about $500 \mathrm{mg}$ of arsenic) was dissolved in hot water and taken 2-3 times per day (Werner et al. 2001).

Various metallic pigments and colors in the form of salts or lakes are used in toy production. Therefore, children may be exposed to toxic metals while playing with toys, especially when they lick, suck, or swallow a toy or a piece of a toy. Toys produced in European Union Markets must conform to restrictions concerning the bioavailability of toxic metals, including arsenic. The maximum limit for bioavailability of arsenic from the accessible parts of a toy is set to $0.1 \mu \mathrm{g} / \mathrm{day}$. This corresponds to an arsenic migration limit of $25 \mu \mathrm{g} / \mathrm{g}$ for all toy material, including modeling clay and paints (Rastogi and Pritzl 1996). A study was carried out to determine whether crayons, water colors, and water-based paints 
conform with the migration limits for toxic metals (Rastogi and Pritzl 1996). For the analysis, 94 samples representing 48 products were obtained from China, Taiwan, Japan, the United States, and European countries. Fifty-two samples showed migration of arsenic, ranging from 0.01 to $3.75 \mu \mathrm{g} / \mathrm{g}$.

\subsection{POPULATIONS WITH POTENTIALLY HIGH EXPOSURES}

In addition to individuals who are occupationally exposed to arsenic (see Section 6.5), there are several groups within the general population that have potentially high exposures (higher than background levels) to arsenic. These populations include individuals living in proximity to sites where arsenic was produced, used (e.g., as a pesticide), or disposed, and individuals living near one of the 1,684 NPL hazardous waste sites where arsenic has been found at elevated levels in some environmental media (HazDat 2006). It also includes point sources such as smelters, coal-fired power plants, and municipal incinerators. People living in areas of volcanic activity may be exposed to higher levels of arsenic since high levels are more likely to be present in the environment. Other populations at risk of potentially high levels of exposure include those whose water supply contains high levels of arsenic and those consuming large amounts of seafood or seaweed. However, as pointed out previously (see Section 6.4.4), arsenic in fish and shellfish, is largely in the form of the less harmful organic arsenical, arsenobetaine; however, some commercially available seaweeds, especially brown algae varieties, may have high percentages of the total arsenic present as inorganic arsenic ( $>50 \%$ ) (Almela et al. 2002; Laparra et al. 2003). While elevated urinary arsenic excretion levels have been associated with the consumption of fish and seafood, in a study of 32 sport fish consumers from Lakes Erie, Huron, and Michigan, only $6(19 \%)$ had detectable urine arsenic concentrations, $>4 \mu \mathrm{g} / \mathrm{L}$, and 5 of these consumed fish from Lake Huron (Anderson et al. 1998). Exposure of high levels of arsenic in drinking water is more apt to be absorbed by the body and be harmful than exposure to arsenic in seafood. For example, a group of 36 people in Zimapán, Mexico who consumed water from an aquifer with $1.0 \mathrm{mg} \mathrm{As} / \mathrm{L}$ had hair arsenic levels of $2.6-14.1 \mu \mathrm{g} / \mathrm{g}(10 \mu \mathrm{g} / \mathrm{g}$ average), compared with $2.4-13.9 \mu \mathrm{g} / \mathrm{g}(6.19 \mu \mathrm{g} / \mathrm{g}$ average) for a reference population that consumed bottled water with $<0.014 \mathrm{mg} / \mathrm{L}$ arsenic (Armienta et al. 1997).

A study was conducted to determine if significant arsenic exposure was occurring at a Superfund site in Fort Valley, Georgia (Hewitt et al. 1995). Random urine, 24-hour urine, hair, and fingernail samples were collected at the end of the workweek from 40 employees at an active pesticide manufacturing facility where arsenical pesticides had been produced for over 50 years prior to the mid-1970s.

Measurement of arsenic in the urine is considered to be the best method for monitoring recent exposure in industrial populations. Hair and fingernail analyses may provide an indication of exposures that occurred 
up to several months prior to testing, but both can adsorb and strongly retain arsenic from external sources. Since arsenic is rapidly cleared from the blood (half-life of 3-4 hours), blood arsenic levels are not considered suitable for monitoring populations for chronic low-level arsenic exposure. Results of the Hewitt study are summarized in Table 6-6. Urinary arsenic levels for all workers were well within the commonly accepted normal range of $<100 \mu \mathrm{g} / \mathrm{L}$.

As noted above, workers in a number of industries may have high exposures to arsenic, especially if proper safety procedures are not followed. For members of the general population, above-average exposure to arsenic from drinking water is possible in areas of high natural arsenic levels in groundwater or elevated arsenic levels in drinking water due to industrial discharges, pesticide applications, or leaching from hazardous waste facilities. Individuals living in the vicinity of large smelters and other industrial emitters of arsenic may be exposed to above-average arsenic levels both in the air, and as a result of atmospheric deposition, in water and soil and subsequent uptake into crops.

People sawing or drilling arsenic-treated wood without protective masks or burning this wood may be exposed to elevated levels of arsenic in air.

Recreational and subsistence fishers who consume appreciably higher amounts of locally caught fish from contaminated bodies of water may be exposed to higher levels of arsenic associated with dietary intake. Arsenic contamination has triggered the issuance of several human health advisories (EPA 1998g). As of December 1997, arsenic was identified as the causative pollutant in a restricted consumption advisory for the general population for all fish in a 7-mile area including Devil's Swamp Lake and Bayou Baton Rouge in Louisiana. A public health advisory has been issued for consumption of fish and shellfish from the Duwamish River, Seattle, Washington due to arsenic and other chemicals (WSDOE 2005).

\subsection{ADEQUACY OF THE DATABASE}

Section 104(i)(5) of CERCLA, as amended, directs the Administrator of ATSDR (in consultation with the Administrator of EPA and agencies and programs of the Public Health Service) to assess whether adequate information on the health effects of arsenic is available. Where adequate information is not available, ATSDR, in conjunction with NTP, is required to assure the initiation of a program of research designed to determine the health effects (and techniques for developing methods to determine such health effects) of arsenic. 
The following categories of possible data needs have been identified by a joint team of scientists from ATSDR, NTP, and EPA. They are defined as substance-specific informational needs that if met would reduce the uncertainties of human health assessment. This definition should not be interpreted to mean that all data needs discussed in this section must be filled. In the future, the identified data needs will be evaluated and prioritized, and a substance-specific research agenda will be proposed.

\subsubsection{Identification of Data Needs}

Physical and Chemical Properties. The chemical and physical properties of the arsenic species of chief toxicological and environmental concern are sufficiently well characterized to allow estimation of the environmental fates of these compounds. However, more information regarding the $\mathrm{K}_{\mathrm{ow}}$ and $\mathrm{K}_{\mathrm{oc}}$ values of the organic arsenicals would help predict the fate of these compounds in the environment.

Production, Import/Export, Use, Release, and Disposal. According to the Emergency Planning and Community Right-to-Know Act of 1986, 42 U.S.C. Section 11023, industries are required to submit substance release and off-site transfer information to the EPA. The TRI, which contains this information for 2004, became available in May of 2006. This database is updated yearly and should provide a list of industrial production facilities and emissions.

While arsenic has not been produced in the United States since 1985, the United States is the largest consumer of arsenic and substantial quantities of arsenic are imported, primarily as arsenic trioxide (USGS 2006a). The agricultural use of inorganic arsenic pesticides have been discontinued in the United States. However, some organic arsenicals still may be used in agriculture. Current production and use data for individual arsenical pesticides and other arsenic compounds would help to estimate human exposure to the various arsenic species. Because arsenical pesticides are so persistent, a more complete picture of past use of these products would enable us to predict what areas may contain high levels of arsenic in soil.

Comprehensive estimates on emissions of arsenic date to the early 1980s (Nriagu and Pacyna 1988). The industrial picture has changed considerably since then and emission controls are being mandated more and more. For example, emission factors for Canadian smelters calculated in 1993 were grossly lower than those estimated in 1983 (Skeaff and Dubreuil 1997). There is a need for accurate and up-to-date measurements of atmospheric arsenic releases from both natural and anthropogenic sources to better assess human exposure to arsenic and guide environmental protection measures. 
Environmental Fate. The interconversion of the various arsenic species and transport among the environmental media is complex and not all aspects are well-studied. Additional quantitative data on the rates of oxidation, reduction, and biotransformation reactions of arsenic compounds, and how these depend on environmental conditions would be useful in evaluating and predicting the fate and transport of arsenic at hazardous waste sites and other areas.

Bioavailability from Environmental Media. Toxicokinetic and toxicity studies establish that bioaccessible (e.g., soluble, not strongly adsorbed to soil or embedded in minerals) arsenic is highly absorbed following inhalation and oral exposure (see Sections 3.4.1.2 and 3.4.1.1). Some work has been done on the effect of environmental matrix (soil, food) on accessibility and absorption of arsenic (Davis et al. 1992, 1996; Hamel et al. 1998; Roberts et al. 2002, 2007), but additional data would be valuable. Limited data suggests that dermal absorption of arsenic is very low (see Section 3.4.1.3) (Lowney et al. 2005), further data would be useful to establish whether arsenic uptake occurs from contact with contaminated soil or water, since humans may be exposed by these routes near hazardous waste sites.

Food Chain Bioaccumulation. Bioconcentration factors have been measured for several freshwater and marine species. While some species (mainly marine algae and shellfish) tend to bioconcentrate arsenic (EPA 1980a; Roper et al. 1996), it is not biomagnified through the food chain (Eisler 1994; EPA 1979, 1982b, 1983e, 2003b; Williams et al. 2006).

Carrots growing on land containing somewhat more than the permissible of arsenic in crop land did not contain levels of arsenic that were harmful (Helgesen and Larsen 1998). However, further research on the uptake of arsenic by a variety of plants in a wide range of arsenic polluted sites (e.g., mining area, orchards previously treated with lead arsenate) would be valuable in assessing human exposure near such sites through the consumption of vegetables from home gardens.

Exposure Levels in Environmental Media. Additional reliable monitoring data for the levels of arsenic in contaminated media at hazardous waste sites are needed or need to be made available, so that the information obtained on levels of arsenic in the environment can be used in combination with the known body burden of arsenic to assess the potential risk of adverse health effects in populations living in the vicinity of hazardous waste sites. 
Extensive monitoring data are available for total arsenic in all environmental media. Additional monitoring studies, specifically those that include identification of arsenic species, would allow more precise estimation of current exposure levels and possible human health risks.

Exposure Levels in Humans. Arsenic has been detected in human tissues, including blood, urine, hair, nails, and internal organs. Data are available for populations exposed in the workplace and for the general population (de Peyster and Silvers 1995; Jensen and Olsen 1995; Nygren et al. 1992), and some studies have been published on exposures near waste sites (Hwang et al. 1997a; Tsuji et al. 2005). Additional biomonitoring studies of residents near waste sites that contain arsenic would be helpful in evaluating the likely human health risks from these sites.

While some data are available on the speciation of arsenic in food, additional data on the particular species of arsenic, rather than just the total arsenic concentration, present in foods, especially seafood, are needed to better estimate the potential hazards to human health by the consumption of these foods (Ryan et al. 2001).

This information is necessary for assessing the need to conduct health studies on these populations.

Exposures of Children. Contaminated soils pose a particular hazard to children because of pica and hand-to-mouth activities. Some studies have been performed on exposure and body burden (Hwang et al. 1997a), but additional studies, including investigations of unique pathways for exposures of children and the amount of soil a child ingests, would provide valuable data. Small amounts of arsenic were found to be transferred to hands of children playing on play structures constructed from CCA-treated wood (Hemond and Solo-Gabriele 2004; Kwon et al. 2004; Shalat et al. 2006; Ursitti et al. 2004; Wang et al. 2005). Based on a review of existing studies, Hemond and Solo-Gabriele (2004) estimated that children with contact with CCA-treated wood may be subjected to doses in the range of tens of micrograms of arsenic per day and suggested that exposure by this route warrants further study. The PTDI assigned by the Food and Agriculture Organization of the United Nations and the World Health Organization (FAO/WHO) applies to adults. Studies are needed to assess whether children are different in their weight adjusted intake of arsenic. No childhood-specific means for reducing exposure were identified.

Child health data needs relating to susceptibility are discussed in Section 3.12.2, Identification of Data Needs: Children's Susceptibility. 
Exposure Registries. No exposure registries for arsenic were located. This substance is not currently one of the compounds for which a sub-registry has been established in the National Exposure Registry. The substance will be considered in the future when chemical selection is made for subregistries to be established. The information that is amassed in the National Exposure Registry facilitates the epidemiological research needed to assess adverse health outcomes that may be related to exposure to this substance.

\subsubsection{Ongoing Studies}

The Federal Research in Progress (FEDRIP 2006) database provides additional information obtainable from a few ongoing studies that may fill in some of the data needs identified in Section 6.8.1. These studies are summarized in Table 6-7.

The U.S. Geological Survey, along with other federal and state agencies, industry, and academia, is conducting the National Geochemical Survey (NGS) in order to produce a body of geochemical data for the United States based primarily on stream sediments that have been analyzed using a consistent set of analytical methods. The goal of the NGS is to analyze at least one stream sediment sample in every $289 \mathrm{~km}^{2}$ area by a single analytical method across the entire United States (USGS 2007b).

EPA is conducting a 4-year (2000-2003) national screening-level study of contaminants in freshwater fish, referred to as the National Fish Tissue Study (EPA 2004c). This study will allow the EPA to develop national estimates of the mean concentrations of 268 chemicals in tissues of fish from lakes and reservoirs of the coterminous United States. EPA analysis of the data from this study was scheduled to begin in January 2005, with the final report scheduled to be released in 2006. Interim raw data have been released each year, and are available from EPA. Fish samples have been analyzed for total inorganic $\operatorname{arsenic}(\mathrm{As}(\mathrm{III})$ and As(V) combined), arsenic(III), arsenic(V), MMA(V), and DMA(V). Analysis for total arsenic was not performed as part of this study.

The American Water Works Association Research Foundation (AWWARF) supports research on arsenic in drinking water. 


\section{Table 6-7. Ongoing Studies on the Environmental Fate and Exposure of Humans to Arsenic}

\begin{tabular}{|c|c|c|c|}
\hline Investigator & Affiliation & Research description & Sponsor \\
\hline Basta, NT & $\begin{array}{l}\text { Ohio State University, } \\
\text { School of Natural } \\
\text { Resources, Columbus, Ohio }\end{array}$ & $\begin{array}{l}\text { Heavy metal and trace element } \\
\text { biogeochemistry in soils; } \\
\text { chemical speciation, } \\
\text { bioavailability, and toxicity }\end{array}$ & USDA \\
\hline Blum, CB & $\begin{array}{l}\text { Columbia University Health } \\
\text { Sciences, New York, New } \\
\text { York }\end{array}$ & $\begin{array}{l}\text { Bioavailability lead and arsenic } \\
\text { in soil to humans }\end{array}$ & NIEHS \\
\hline Hamilton, JW & $\begin{array}{l}\text { Dartmouth College, } \\
\text { Hanover, New Hampshire }\end{array}$ & $\begin{array}{l}\text { Toxic metals-biological and } \\
\text { environmental implications }\end{array}$ & NIEHS \\
\hline Hoppin, J & Not specified & $\begin{array}{l}\text { Monitoring of arsenic and other } \\
\text { compounds in the blood and } \\
\text { urine of a cohort of pregnant } \\
\text { women in Norway }\end{array}$ & NIEHS \\
\hline $\begin{array}{l}\text { Kpomblekou, AK; } \\
\text { Ankumah, RO }\end{array}$ & $\begin{array}{l}\text { Tuskegee University, } \\
\text { Agriculture and Home } \\
\text { Economics, Tuskegee, } \\
\text { Alabama }\end{array}$ & $\begin{array}{l}\text { Biochemical processes in soils } \\
\text { treated with trace-element- } \\
\text { enriched broiler litter; to } \\
\text { determine total arsenic and } \\
\text { other metal concentrations and } \\
\text { the distribution of their chemical } \\
\text { forms in soils under long-term } \\
\text { broiler litter treatments }\end{array}$ & USDA \\
\hline Loeppert, RH & $\begin{array}{l}\text { Texas A\&M University, Soil } \\
\text { and Crop Sciences, College } \\
\text { Station, Texas }\end{array}$ & $\begin{array}{l}\text { Inorganic chemical processes } \\
\text { influencing soil and water } \\
\text { quality }\end{array}$ & USDA \\
\hline Peryea, FJ & $\begin{array}{l}\text { Wenatchee Tree Fruit } \\
\text { Research \& Extension } \\
\text { Center Washington State } \\
\text { University, Pullman, } \\
\text { Washington }\end{array}$ & $\begin{array}{l}\text { Quantification of } \\
\text { biogeochemical processes in } \\
\text { lead arsenate-contaminated } \\
\text { orchard soils and development } \\
\text { of soil and plant management } \\
\text { practices to minimize the } \\
\text { toxicity risks that these soils } \\
\text { impose on agricultural crops } \\
\text { and to human and } \\
\text { environmental health }\end{array}$ & USDA \\
\hline $\begin{array}{l}\text { Miller, DM; DeLaune, } \\
\text { P; Miller, WP }\end{array}$ & $\begin{array}{l}\text { University of Arkansas, } \\
\text { Crop, Soil and } \\
\text { Environmental Sciences, } \\
\text { Fayetteville, Arkansas }\end{array}$ & $\begin{array}{l}\text { Arsenic levels in soils of } \\
\text { northwest Arkansas }\end{array}$ & USDA \\
\hline van Geen, A & $\begin{array}{l}\text { Columbia University, } \\
\text { Lamont-Doherty Earth } \\
\text { Observatory, Palisades, } \\
\text { New York }\end{array}$ & $\begin{array}{l}\text { Studies on arsenic in } \\
\text { groundwater in Bangladesh }\end{array}$ & NSF \\
\hline
\end{tabular}


Table 6-7. Ongoing Studies on the Environmental Fate and Exposure of Humans to Arsenic

\begin{tabular}{llll}
\hline Investigator & Affiliation & Research description & Sponsor \\
\hline Walker, MJ et al. & University of Nevada, & Arsenic in Churchill County, & USDA \\
& $\begin{array}{l}\text { Natural Resources and } \\
\text { Environmental Sciences, }\end{array}$ & $\begin{array}{l}\text { Nevada domestic water } \\
\text { supplies }\end{array}$ & \\
& $\begin{array}{l}\text { Reno, Nevada } \\
\text { Columbia University Health }\end{array}$ & Arsenic mobilization in & NIEHS \\
& $\begin{array}{l}\text { Coiences, New York, New } \\
\text { Bheng, Y }\end{array}$ & Bangladesh groundwater & \\
& York & & \\
\hline
\end{tabular}

NIEHS = National Institute of Environmental Health Sciences; NSF = National Science Foundation; USDA = U.S. Department of Agriculture

Source: FEDRIP 2006 
This page is intentionally blank. 


\section{ANALYTICAL METHODS}

The purpose of this chapter is to describe the analytical methods that are available for detecting, measuring, and/or monitoring arsenic, its metabolites, and other biomarkers of exposure and effect to arsenic. The intent is not to provide an exhaustive list of analytical methods. Rather, the intention is to identify well-established methods that are used as the standard methods of analysis. Many of the analytical methods used for environmental samples are the methods approved by federal agencies and organizations such as EPA and the National Institute for Occupational Safety and Health (NIOSH). Other methods presented in this chapter are those that are approved by groups such as the Association of Official Analytical Chemists (AOAC) and the American Public Health Association (APHA). Additionally, analytical methods are included that modify previously used methods to obtain lower detection limits and/or to improve accuracy and precision.

\subsection{BIOLOGICAL MATERIALS}

Atomic absorption spectrophotometry (AAS) is the most common analytical procedure for measuring arsenic in biological materials (Curatola et al. 1978; Foà et al. 1984; Johnson and Farmer 1989; Mushak et al. 1977; Norin and Vahter 1981; Sotera et al. 1988). In AAS analysis, the sample is heated in a flame or in a graphite furnace until the element atomizes. The ground-state atomic vapor absorbs monochromatic radiation from a source and a photoelectric detector measures the intensity of transmitted radiation (APHA 1989b). Inductively-coupled plasma atomic emission spectrometry (ICP-AES) and ICP-mass spectrometry (ICP-MS) are increasingly common techniques for the analysis of arsenic; both methods can generally provide lower detection limits than absorbance detection methods.

Samples may be prepared for AAS in a variety of ways. Most often, the gaseous hydride procedure is employed (Curatola et al. 1978; Foà et al. 1984; Johnson and Farmer 1989; Norin and Vahter 1981). In this procedure, arsenic in the sample is reduced to arsine $\left(\mathrm{AsH}_{3}\right)$, a gas that is then trapped and introduced into the flame. This approach measures total inorganic arsenic, but may not detect all organic forms unless preceded by a digestion step. Digestion or wet-ashing with nitric, sulfuric, and/or perchloric acids degrades the organic arsenic species to inorganic arsenic so that recovery of total arsenic from biological materials can be achieved (Maher 1989; Mushak et al. 1977; Versieck et al. 1983). In microwave assisted digestion, harsh oxidation conditions are used in conjunction with microwave heating (Benramdane et al. 1999b). For accurate results, it is important to check the completeness of the oxidation; however, this is seldom done (WHO 1981). 
The arsenic concentration in biological fluids and tissues may also be determined by neutron activation analysis (NAA) (Landsberger and Simsons 1987; Versieck et al. 1983). In this approach, the sample is irradiated with a source of neutrons that converts a portion of the arsenic atoms to radioactive isotopes, which can be quantified after separation from radioisotopes of other chemicals. Neutron activation has limited use because of the limited number of nuclear reactors in the United States providing this service and the need to dispose of radioactive waste. X-ray fluorescence is also capable of measuring arsenic in biological materials (Bloch and Shapiro 1986; Clyne et al. 1989; Nielson and Sanders 1983) and environmental samples (see Section 7.2). This method has the advantage that no sample digestion or separation steps are required. Hydride generation combined with atomic fluorescence spectroscopy (HGAFS) is a relatively new technique that provides freedom from interference offered by hydride generation with sensitivity better than to 20 parts per trillion and linearity up to $10 \mathrm{ppm}$ (PSA 2000).

Speciation of arsenic (i.e., analysis of organic arsenic compounds or different inorganic species, rather than total arsenic) is usually accomplished by employing separation procedures prior to introduction of the sample material into a detection system. Various types of chromatography or chelation-extraction techniques are most commonly used in combination with AAS, ICP-AES, or ICP-MS detection methods (Dix et al. 1987; Foà et al. 1984; Johnson and Farmer 1989; Mushak et al. 1977; Norin et al. 1987; Thomas and Sniatecki 1995). In one method, high performance liquid chromatography (HPLC) is combined with HG-AFS to quantify As(III), dimethylarsinic acid (DMA), momomethyl arsonic acid (MMA), and As(V) (PSA 2000). Another approach involves selective reduction of arsenate and arsenite (permitting quantification of individual inorganic arsenic species), and selective distillation of methyl arsines to quantify MMA and DMA (Andreae 1977; Braman et al. 1977; Crecelius 1978). Most methods for measuring arsenic in biological samples are unable to measure arsenobetaine with any accuracy because it does not form a hydride and it gives a different response from inorganic arsenic in electrothermal AAS. Ebdon et al. (1999) successfully employed HPLC coupled with ICP-MS to determine arsenic speciation in blood plasma, which was entirely arsenobetaine. Øygard et al. (1999) developed a simple method to determine inorganic arsenic in biological samples. Their method, which involves initially distilling inorganic arsenic from the sample as $\mathrm{AsCl}_{3}$ using $\mathrm{HCl}$, avoids separating and quantifying all of the different arsenic species, which is both costly and time-consuming.

Table 7-1 summarizes a variety of methods for measuring total arsenic and individual arsenic species in biological materials. None of these methods have been standardized by EPA or other federal agencies. 


\section{Table 7-1. Analytical Methods for Determining Arsenic in Biological Samples}

\begin{tabular}{|c|c|c|c|c|c|}
\hline $\begin{array}{l}\text { Sample } \\
\text { matrix }\end{array}$ & Preparation method & $\begin{array}{l}\text { Analytical } \\
\text { method }\end{array}$ & $\begin{array}{l}\text { Sample } \\
\text { detection limit }\end{array}$ & $\begin{array}{l}\text { Percent } \\
\text { recovery }\end{array}$ & Reference \\
\hline \multicolumn{6}{|c|}{ Methods for total arsenic: } \\
\hline Blood & $\begin{array}{l}\text { Digestion with nitric acid and } \\
\text { hydrogen peroxide; dry ash with } \\
\text { magnesium oxide/magnesium } \\
\text { nitrate; reduction with sodium } \\
\text { borohydride }\end{array}$ & HGAAS & $0.5 \mu \mathrm{g} / \mathrm{L}$ & $95-102$ & Foà et al. 1984 \\
\hline Blood, hair & $\begin{array}{l}\text { Wet ash with nitric/perchloric } \\
\text { acids; reduction with sodium } \\
\text { borohydride }\end{array}$ & HGAAS & $0.1 \mu \mathrm{g} / \mathrm{L}^{\mathrm{a}}$ & 95-105 & $\begin{array}{l}\text { Valentine et al. } \\
1979\end{array}$ \\
\hline Serum & $\begin{array}{l}\text { Irradiation; digestion with nitric/ } \\
\text { perchloric/sulfuric acids; } \\
\text { extraction with toluene }\end{array}$ & NAA & $0.088 \mathrm{ng} / \mathrm{mL}^{\mathrm{a}}$ & 94-98 & $\begin{array}{l}\text { Versieck et al. } \\
1983\end{array}$ \\
\hline Urine & Irradiate epithermally & NAA & $40-100 \mathrm{ng} / \mathrm{g}$ & 93-109 & $\begin{array}{l}\text { Landsberger and } \\
\text { Simsons } 1987\end{array}$ \\
\hline Urine & $\begin{array}{l}\text { Digestion with nitric and } \\
\text { perchloric acid; reduction with tin } \\
\text { chloride; generation arsine by } \\
\text { addition of zinc; reaction with } \\
\text { SDDC }\end{array}$ & $\begin{array}{l}\text { Colorimetric } \\
\text { photometry }\end{array}$ & $0.5 \mu \mathrm{g} / \mathrm{sample}$ & $90-110$ & Pinto et al. 1976 \\
\hline Urine & $\begin{array}{l}\text { Pretreatment with L-cysteine; } \\
\text { reduction with potassium iodide/ } \\
\text { ascorbic acid }\end{array}$ & $\begin{array}{l}\text { Flow injection } \\
\text { HGAAS }\end{array}$ & $0.1 \mu \mathrm{g} / \mathrm{L}$ & $95-100$ & Guo et al. 1997 \\
\hline Urine & $\begin{array}{l}\text { Drying sample; irradiation with } \\
\text { x-rays }\end{array}$ & $\mathrm{XRF}$ & $0.2 \mu \mathrm{g} / \mathrm{L}^{\mathrm{a}}$ & $92-108$ & Clyne et al. 1989 \\
\hline Hair & $\begin{array}{l}\text { Wet ashing with nitric/sulfuric } \\
\text { acids and hydrogen peroxide; } \\
\text { reduction to arsine with sodium } \\
\text { borohydride }\end{array}$ & HGAAS & $0.06 \mu \mathrm{g} / \mathrm{g}$ & 93 & $\begin{array}{l}\text { Curatola et al. } \\
1978\end{array}$ \\
\hline Soft tissue & $\begin{array}{l}\text { Digestion with nitric/sulfuric } \\
\text { acids; complexation with DDDC } \\
\text { in potassium iodide; extraction } \\
\text { with chloroform }\end{array}$ & GFAAS & $0.2 \mathrm{ppm}$ & 79.8 & $\begin{array}{l}\text { Mushak et al. } \\
1977\end{array}$ \\
\hline Nails & $\begin{array}{l}\text { Wet ashing with nitric/sulfuric } \\
\text { acids and hydrogen peroxide; } \\
\text { reduction to arsine with sodium } \\
\text { borohydride }\end{array}$ & HGAAS & $1.5 \mu \mathrm{g} / \mathrm{g}$ & No data & $\begin{array}{l}\text { Agahian et al. } \\
1990\end{array}$ \\
\hline \multicolumn{6}{|c|}{ Methods for arsenic speciation: } \\
\hline Urine & $\begin{array}{l}\text { Separation of } \mathrm{As}^{+3}, \mathrm{As}^{+5}, \mathrm{MMA} \text {, } \\
\text { and DMA on anion/cation } \\
\text { exchange resin column; } \\
\text { reduction to respective arsines } \\
\text { with sodium borohydride }\end{array}$ & IEC/HGAAS & $0.5 \mu \mathrm{g} / \mathrm{L}$ & $93-106$ & $\begin{array}{l}\text { Johnson and } \\
\text { Farmer } 1989\end{array}$ \\
\hline Urine & $\begin{array}{l}\text { Reduction of } \mathrm{As}^{+3}, \mathrm{As}^{+5}, \mathrm{MMA}, \\
\text { and DMA to arsines with sodium } \\
\text { borohydride }\end{array}$ & HGAAS & $0.08 \mu \mathrm{g} / \mathrm{L}$ & $97-104$ & $\begin{array}{l}\text { Norin and Vahter } \\
1981\end{array}$ \\
\hline
\end{tabular}




\section{Table 7-1. Analytical Methods for Determining Arsenic in Biological Samples}

\begin{tabular}{|c|c|c|c|c|c|}
\hline $\begin{array}{l}\text { Sample } \\
\text { matrix }\end{array}$ & Preparation method & $\begin{array}{l}\text { Analytical } \\
\text { method }\end{array}$ & $\begin{array}{l}\text { Sample } \\
\text { detection limit }\end{array}$ & $\begin{array}{l}\text { Percent } \\
\text { recovery }\end{array}$ & Reference \\
\hline Urine & $\begin{array}{l}\text { Reduction of } \mathrm{As}^{+3}, \mathrm{As}^{+5}, \mathrm{MMA}, \\
\text { and DMA to arsines; collection in } \\
\text { cold trap; selective distillation by } \\
\text { slow warming }\end{array}$ & $\begin{array}{l}\text { Atomic } \\
\text { emission } \\
\text { (direct- } \\
\text { current } \\
\text { plasma) }\end{array}$ & $\begin{array}{l}\leq 1 \mathrm{ng} \text { for all } \\
\text { four species }\end{array}$ & No data & $\begin{array}{l}\text { Braman et al. } \\
1977\end{array}$ \\
\hline Urine & $\begin{array}{l}\text { Extraction with chloroform/ } \\
\text { methanol; column separation } \\
\text { with chloroform/methanol; elution } \\
\text { on cation exchange column with } \\
\text { ammonium hydroxide }\end{array}$ & $\begin{array}{l}\text { HGAAS/TLC/ } \\
\text { HRMS }\end{array}$ & $\begin{array}{l}0.34 \mathrm{mg} / \\
\text { sample }^{\mathrm{a}}\end{array}$ & No data & Tam et al. 1982 \\
\hline $\begin{array}{l}\text { Blood/ } \\
\text { tissue }\end{array}$ & $\begin{array}{l}\text { Acidification with hydrochloric } \\
\text { acid; complexation with TGM; } \\
\text { extraction into cyclohexane; } \\
\text { separation on capillary column }\end{array}$ & GLC/ECD & $0.1 \mathrm{mg} / \mathrm{mL}$ & No data & Dix et al. 1987 \\
\hline $\begin{array}{l}\text { Blood } \\
\text { plasma }\end{array}$ & Separation by HPLC & $\begin{array}{l}\text { HPLC/ICP- } \\
\text { MS }\end{array}$ & $2.5 \mathrm{ng}$ As/mL & $\sim 100$ & $\begin{array}{l}\text { Ebdon et al. } \\
1999\end{array}$ \\
\hline Urine & $\begin{array}{l}\text { Separation by anion exchange } \\
\text { chromatography; detection by } \\
\text { direct coupling of column to ICP- } \\
\text { MS }\end{array}$ & IEC/ICP-MS & $\begin{array}{l}<0.45 \mu \mathrm{g} / \mathrm{L} \text { for } \\
\text { all species }\end{array}$ & No data & Inoue et al. 1994 \\
\hline $\begin{array}{l}\text { Marine } \\
\text { biota }\end{array}$ & $\begin{array}{l}\text { Extraction with methanol-water; } \\
\text { removal of fats by liquid-liquid } \\
\text { extraction or solid-phase } \\
\text { cartridge }\end{array}$ & $\begin{array}{l}\text { HPLC/ICP- } \\
\text { MS }\end{array}$ & $6-25 \mathrm{ng} / \mathrm{mL}$ & $\begin{array}{l}94.6 \text { (fish } \\
\text { muscle } \\
\text { CRM) }\end{array}$ & Sniatecki 1994 \\
\hline $\begin{array}{l}\text { Marine } \\
\text { biota }\end{array}$ & $\begin{array}{l}\text { Separation by anion exchange } \\
\text { coupled with HPLC; on-line } \\
\text { microwave oxidation }\end{array}$ & $\begin{array}{l}\text { HPLC/ } \\
\text { HGAAS }\end{array}$ & $0.3-0.9 \mathrm{ng}$ & $\begin{array}{l}95-110 \\
\text { (recovery } \\
\text { of spike } \\
\text { in fish } \\
\text { tissue) }\end{array}$ & $\begin{array}{l}\text { López- } \\
\text { Gonzálvez et al. } \\
1994\end{array}$ \\
\hline $\begin{array}{l}\text { Biological } \\
\text { samples- } \\
\text { Inorganic } \\
\text { arsenic }\end{array}$ & $\begin{array}{l}\text { Distill inorganic arsenic as } \mathrm{AsCl}_{3} \\
\text { using } \mathrm{HCl} \text { after prereduction of } \\
\mathrm{As}(\mathrm{V}) \text { with } \mathrm{KI} / \mathrm{HCl}\end{array}$ & $\begin{array}{l}\text { Flow-injection } \\
\text { HGAAS }\end{array}$ & $\begin{array}{l}0.045 \mathrm{mg} / \mathrm{kg} \\
\text { (dry matter) }\end{array}$ & No data & $\begin{array}{l}\text { Øygard et al. } \\
1999\end{array}$ \\
\hline
\end{tabular}

${ }^{a}$ Lowest reported concentration

$\mathrm{CRM}=$ certified reference material $; \mathrm{DDDC}=$ diethylammonium diethyldithiocarbamate; $\mathrm{DMA}=$ dimethylarsinate; $\mathrm{ECD}=$ electron capture detector; GFAAS = graphite furnace atomic absorption spectrometry; GLC = gas-liquid chromatography; HGAAS = hydride generation atomic absorption spectrometry; HRMS = high resolution mass spectrometry; ICP-MS = inductively-coupled plasma mass spectrometry; IEC = ion exchange chromatography; HPLC = high-performance liquid chromatography; MMA = monomethylarsonate; NAA = neutron activation analysis; SDDC = silver diethyldithiocarbamate; TGM = thioglycolic acid methylester; TLC = thin layer chromatography; $\mathrm{XRF}=\mathrm{x}$-ray fluorescence 
Detection limits in blood and urine are about $0.1-1 \mathrm{ppb}$ for most techniques; limits for hair and tissues are usually somewhat higher.

\subsection{ENVIRONMENTAL SAMPLES}

Arsenic in environmental samples is also measured most often by AAS techniques, with samples prepared by digestion with nitric, sulfuric, and/or perchloric acids (Dabeka and Lacroix 1987; EPA 1983b, 1994a, 1994b; Hershey et al. 1988). Other methods employed include a spectrophotometric technique in which a soluble red complex of arsine and silver diethyldithiocarbamate (SDDC) is formed (APHA 1977; EPA 1983c, 1983d), ICP-AES (EPA 2000c; NIOSH 2003), graphite furnace AAS (EPA 1983b, 1994b; NIOSH 1994b), ICP-MS (EPA 1991, 1994a, 1998j), and x-ray fluorescence (Khan et al. 1989; Nielson and Sanders 1983).

HPLC is currently the most common technique for separation of the species of arsenic found in seafood (Benramdane et al. 1999b; Guerin et al. 1999; Kumaresan and Riyazuddin 2001). An advantage of HPLC over other separation methods (e.g., gas chromatography [GC]) is that the arsenic species do not need to be derivatized prior to separation, avoiding concerns over complete conversion to the derivative for detection.

Since arsenic in air is usually associated with particulate matter, standard methods involve collection of air samples on glass fiber or membrane filters, acid extraction of the filters, arsine generation, and analysis by SDDC spectrophotometry or AAS (APHA 1977; NIOSH 1984).

Methods standardized by the EPA for measuring total arsenic in water and waste water, solid wastes, soil, and sediments include: ICP-MS (EPA 1998j, 1994a, 1991), ICP-AES (EPA 1996d), graphite furnace AAS (EPA 1994b), quartz furnace hydride generation AAS (EPA 1996h), and an electrochemical method using anodic stripping voltammetry (ASV) (EPA 1996e). A modification using cryogenic GC to EPA Method 1632 (HG/AAS) allows the technique to be adopted for the species As(III), As(V), MMA, and DMA to the $0.003 \mathrm{ppb}$ level (EPA 19981). Similar methods are recommended by APHA for water using AAS/hydride generation (APHA 1989c), AAS/graphite furnace technique (APHA 1989b), ICP (APHA 1989d), or SDDC spectrophotometry (APHA 1989a). The AAS/hydride generation method is generally resistant to matrix and chemical interferences (APHA 1989a). Techniques to compensate for these interferences have been described by EPA (1982b). 
Analysis for arsenic in foods is also most frequently accomplished by AAS techniques (Arenas et al. 1988; Dabeka and Lacroix 1987; Hershey et al. 1988; Tam and Lacroix 1982). Hydride generation is the sample preparation method most often employed (Arenas et al. 1988; Hershey et al. 1988), but interferences must be evaluated and minimized.

Speciation of inorganic arsenic in environmental samples is usually accomplished by chromatographic separation, chelation-extraction or elution of $\mathrm{As}(\mathrm{III})$, and then reduction of $\mathrm{As}(\mathrm{V})$ with subsequent similar treatment (Butler 1988; López-Gonzálvez et al. 1994; Mok et al. 1988; Rabano et al. 1989).

Methods are also available for quantifying organic arsenicals in environmental media, including arsenobetaine in fish (Beauchemin et al. 1988; Cannon et al. 1983) and other organic forms of arsenic in water, soil, and foods using hyphenated methods of separation and detection (HPLC/ICP-MS, HPLC/HGAAS, IC/ICP-MS) (Andreae 1977; Braman et al. 1977; Comber and Howard 1989; Crecelius 1978; Heitkemper et al. 1994; López-Gonzálvez et al. 1994; Odanaka et al. 1983; Teräsahde et al. 1996).

Methods have been developed for extraction of arsenic species from solid seafood samples that included treatment of the sample with mixtures of organic solvents (alcohols or chloroform) and water to extract the arsenic compounds that are soluble in water or polar organic solvents. These extracts can be subsequently analyzed by HPLC. Enzymatic digestion using trypsin has also been used to extract arsenic compounds from seafood samples (Benramdane et al. 1999b). These extraction techniques are used in place of digestion when speciated data are needed.

A summary of selected methods for analysis of total arsenic and individual inorganic and organic arsenic species in environmental samples is presented in Table 7-2.

\subsection{ADEQUACY OF THE DATABASE}

Section 104(i)(5) of CERCLA, as amended, directs the Administrator of ATSDR (in consultation with the Administrator of EPA and agencies and programs of the Public Health Service) to assess whether adequate information on the health effects of arsenic is available. Where adequate information is not available, ATSDR, in conjunction with NTP, is required to assure the initiation of a program of research designed to determine the health effects (and techniques for developing methods to determine such health effects) of arsenic. 
Table 7-2. Analytical Methods for Determining Arsenic in Environmental Samples

\begin{tabular}{|c|c|c|c|c|c|}
\hline $\begin{array}{l}\text { Sample } \\
\text { matrix }\end{array}$ & Preparation method & $\begin{array}{l}\text { Analytical } \\
\text { method }\end{array}$ & $\begin{array}{l}\text { Sample } \\
\text { detection limit }\end{array}$ & $\begin{array}{l}\text { Percent } \\
\text { recovery }\end{array}$ & Reference \\
\hline \multicolumn{6}{|c|}{ Methods for total arsenic: } \\
\hline $\begin{array}{l}\text { Air } \\
\text { (particulates) }\end{array}$ & $\begin{array}{l}\text { Collection on cellulose ester } \\
\text { membrane filter; digestion } \\
\text { with nitric acid, sulfuric acid, } \\
\text { and perchloric acid }\end{array}$ & $\begin{array}{l}\text { NIOSH Method } \\
\text { 7900; HGAAS }\end{array}$ & $0.02 \mu \mathrm{g} / \mathrm{sample}$ & No data & $\begin{array}{l}\mathrm{NIOSH} \\
1994 a\end{array}$ \\
\hline $\begin{array}{l}\text { Air } \\
\text { (particulate } \\
\text { arsenic and } \\
\text { arsenic } \\
\text { trioxide } \\
\text { vapor) }\end{array}$ & $\begin{array}{l}\text { Collection on } \\
\mathrm{Na}_{2} \mathrm{CO}_{3} \text {-impregnated } \\
\text { cellulose ester membrane } \\
\text { filter and } \mathrm{H}_{2} \mathrm{O}_{2}\end{array}$ & $\begin{array}{l}\text { NIOSH Method } \\
\text { 7901; GFAAS }\end{array}$ & $0.06 \mu \mathrm{g} / \mathrm{sample}$ & No data & $\begin{array}{l}\mathrm{NIOSH} \\
1994 b\end{array}$ \\
\hline Air & $\begin{array}{l}\text { Collection on cellulose ester } \\
\text { membrane filter; digestion } \\
\text { with nitric acid, sulfuric acid, } \\
\text { and perchloric acid }\end{array}$ & $\begin{array}{l}\text { NIOSH Method } \\
\text { 7300; ICP-AES }\end{array}$ & $0.140 \mu \mathrm{g} /$ filter & No data & $\begin{array}{l}\text { NIOSH } \\
2003\end{array}$ \\
\hline $\begin{array}{l}\text { Water/waste } \\
\text { water/solid } \\
\text { wastes }\end{array}$ & Acid digestion & $\begin{array}{l}\text { EPA Method } \\
\text { 6010C; ICP- } \\
\text { AES }\end{array}$ & $35 \mu \mathrm{g} / \mathrm{L}$ & 86 & EPA 2000c \\
\hline $\begin{array}{l}\text { Water/waste } \\
\text { water/solid } \\
\text { wastes }\end{array}$ & $\begin{array}{l}\text { Digestion with nitric and } \\
\text { hydrochloric acids }\end{array}$ & $\begin{array}{l}\text { EPA Method } \\
\text { 200.7; ICP- } \\
\text { AES }\end{array}$ & $8 \mu \mathrm{g} / \mathrm{L}$ & 106 & EPA 1994c \\
\hline $\begin{array}{l}\text { Water/soil/ } \\
\text { solid waste }\end{array}$ & $\begin{array}{l}\text { Digestion with nitric acid and } \\
\text { hydrogen peroxide }\end{array}$ & $\begin{array}{l}\text { EPA Methods } \\
206.2 \text { and } \\
7060 \mathrm{~A} ; \mathrm{GFAAS} \\
\text { with } \mathrm{Ni}\left(\mathrm{NO}_{3}\right)_{2} \\
\text { modifier }\end{array}$ & $1 \mu \mathrm{g} / \mathrm{L}$ & $85-106$ & $\begin{array}{l}\text { EPA 1983b, } \\
1994 b\end{array}$ \\
\hline $\begin{array}{l}\text { Water/waste } \\
\text { water/solid } \\
\text { waste }\end{array}$ & Digestion with nitric acid & $\begin{array}{l}\text { EPA Methods } \\
200.8,6020 \\
\text { and } 6020 A \\
\text { ICP-MS }\end{array}$ & $0.4 \mu \mathrm{g} / \mathrm{L}$ & $97-114$ & $\begin{array}{l}\text { EPA 1991, } \\
\text { 1994a, } \\
\text { 1998j }\end{array}$ \\
\hline $\begin{array}{l}\text { Water/soil/ } \\
\text { solid waste }\end{array}$ & $\begin{array}{l}\text { Digestion with nitric/sulfuric } \\
\text { acid; reduction to } \mathrm{As}^{+3} \text { with } \\
\text { tin chloride; reduction to } \\
\text { arsine with zinc in acid } \\
\text { solution }\end{array}$ & $\begin{array}{l}\text { EPA Method } \\
206.3\end{array}$ & $2 \mu \mathrm{g} / \mathrm{L}$ & $85-94$ & EPA 1983c \\
\hline Water & $\begin{array}{l}\text { Reduction to arsine in acid } \\
\text { solution; reaction with SDDC }\end{array}$ & $\begin{array}{l}\text { EPA Method } \\
206.4 ; \text { SDDC } \\
\text { colorimetric } \\
\text { spectrophoto- } \\
\text { metry at } \\
510 \mathrm{~nm}\end{array}$ & $10 \mu \mathrm{g} / \mathrm{L}$ & 100 & EPA 1983d \\
\hline Water & $\begin{array}{l}\text { Digestion with } 6 \mathrm{M} \mathrm{HCl} \text {; } \\
\text { reduction to arsine with } \\
\text { sodium borohydride; cold } \\
\text { trap and desorption into } \\
\text { quartz furnace }\end{array}$ & $\begin{array}{l}\text { EPA Method } \\
\text { 1632; HGAAS }\end{array}$ & $2 \mathrm{ng} / \mathrm{L}$ & No data & EPA 1998I \\
\hline
\end{tabular}


Table 7-2. Analytical Methods for Determining Arsenic in Environmental Samples

\begin{tabular}{|c|c|c|c|c|c|}
\hline $\begin{array}{l}\text { Sample } \\
\text { matrix }\end{array}$ & Preparation method & $\begin{array}{l}\text { Analytical } \\
\text { method }\end{array}$ & $\begin{array}{l}\text { Sample } \\
\text { detection limit }\end{array}$ & $\begin{array}{l}\text { Percent } \\
\text { recovery }\end{array}$ & Reference \\
\hline Food & $\begin{array}{l}\text { Digestion with nitric acid; dry } \\
\text { ashing with magnesium } \\
\text { oxide; reduction with } \\
\text { ascorbic acid; precipitation } \\
\text { with APDC in presence of } \\
\text { nickel carrier }\end{array}$ & GFAAS & $10 \mathrm{ng}$ & $86-107$ & $\begin{array}{l}\text { Dabeka and } \\
\text { Lacroix } \\
1987\end{array}$ \\
\hline Food & $\begin{array}{l}\text { Digestion with nitric/sulfuric/ } \\
\text { perchloric acids; reduction to } \\
\text { trivalent arsenic with potas- } \\
\text { sium iodide; reduction to } \\
\text { arsine with sodium boro- } \\
\text { hydride }\end{array}$ & HGAAS & $0.1 \mu \mathrm{g} / \mathrm{g}$ & $98-110$ & $\begin{array}{l}\text { Hershey et } \\
\text { al. } 1988\end{array}$ \\
\hline $\begin{array}{l}\text { Soil, rock, } \\
\text { coal }\end{array}$ & Preparation of pellet & $\begin{array}{l}\text { XRF } \\
\text { (backscatter) }\end{array}$ & $4 \mathrm{mg} / \mathrm{kg}$ & $\begin{array}{l}\text { SRM } \\
\text { recoveries: } \\
110 \pm 4 \text { in } \\
\text { soil; } \\
100 \pm 1 \text { in } \\
\text { rock; } 97 \pm 18 \\
\text { in coal }\end{array}$ & $\begin{array}{l}\text { Nielson and } \\
\text { Sanders } \\
1983\end{array}$ \\
\hline \multicolumn{6}{|c|}{ Methods for species of arsenic: } \\
\hline $\begin{array}{l}\text { Air } \\
\text { (particulate } \\
\text { organo- } \\
\text { arsenals) }\end{array}$ & Collection on PTFE filter & $\begin{array}{l}\text { NIOSH Method } \\
5022 ; \text { ion } \\
\text { chromato- } \\
\text { graphy/HGAAS }\end{array}$ & $\begin{array}{l}0.2 \mu \mathrm{g} \\
\text { As/sample }\end{array}$ & No data & $\begin{array}{l}\mathrm{NIOSH} \\
1994 \mathrm{C}\end{array}$ \\
\hline Air (arsine) & $\begin{array}{l}\text { Collection on coconut shell } \\
\text { charcoal; digestion with nitric } \\
\text { acid }\end{array}$ & $\begin{array}{l}\text { NIOSH Method } \\
\text { 6001; GFAAS }\end{array}$ & $0.004 \mu \mathrm{g} / \mathrm{sample}$ & No data & $\begin{array}{l}\text { NIOSH } \\
1994 d\end{array}$ \\
\hline $\begin{array}{l}\text { Air } \\
\text { particulates } \\
\left(\mathrm{As}^{+3} \text { and }\right. \\
\left.\mathrm{As}^{+5} \text { only }\right)\end{array}$ & $\begin{array}{l}\text { Collection on PFTE filter in } \\
\text { high volume dichotomous } \\
\text { virtual impactor; desorption } \\
\text { with ethanolic hydrochloric } \\
\text { acid; selective reduction of } \\
\mathrm{As}^{+3} \text { to arsine with zinc in } \\
\text { acid and reduction of } \mathrm{As}^{+5} \text { to } \\
\text { arsine with sodium tetra- } \\
\text { hydrodiborate }\end{array}$ & HGAAS & $1 \mathrm{ng} / \mathrm{m}^{3}$ & $\begin{array}{l}95 \pm 7\left(\mathrm{As}^{+3}\right) \\
100 \pm 8 \\
\left(\mathrm{As}^{+5}\right) \text { on } \\
\text { spiked } \\
\text { materials }\end{array}$ & $\begin{array}{l}\text { Rabano et } \\
\text { al. } 1989\end{array}$ \\
\hline Water & $\begin{array}{l}\text { Selective elution of } \mathrm{As}^{+3} \text { with } \\
\text { orthophosphoric acid; elution } \\
\text { and conversion of } \mathrm{As}^{+5} \text { to } \\
\mathrm{As}^{+3} \text { with sulfur dioxide }\end{array}$ & $\begin{array}{l}\text { IEC/ampero- } \\
\text { metric detector } \\
\text { (detects } \mathrm{As}^{+3} \\
\text { only) }\end{array}$ & $0.9 \mu \mathrm{g} / \mathrm{L}$ & $\begin{array}{l}95 \% \text { of } \\
\text { converted } \\
\mathrm{As}^{+5} \\
\text { recovered }\end{array}$ & Butler 1988 \\
\hline Water/soil & $\begin{array}{l}\text { Selective complexation of } \\
\mathrm{As}^{+5} \text { with ammonium } \\
\text { molybdate; extraction with } \\
\text { isoamyl alcohol to separate } \\
\text { from } \mathrm{As}^{+3}\end{array}$ & $\begin{array}{l}\text { Colorimetric } \\
\text { spectrometry at } \\
712 \mathrm{~nm}\end{array}$ & No data & No data & $\begin{array}{l}\text { Brown and } \\
\text { Button } 1979\end{array}$ \\
\hline
\end{tabular}


Table 7-2. Analytical Methods for Determining Arsenic in Environmental Samples

\begin{tabular}{|c|c|c|c|c|c|}
\hline $\begin{array}{l}\text { Sample } \\
\text { matrix }\end{array}$ & Preparation method & $\begin{array}{l}\text { Analytical } \\
\text { method }\end{array}$ & $\begin{array}{l}\text { Sample } \\
\text { detection limit }\end{array}$ & $\begin{array}{l}\text { Percent } \\
\text { recovery }\end{array}$ & Reference \\
\hline Water & $\begin{array}{l}\text { Selective extraction of } \mathrm{As}^{+3} \\
\text { with APDC into chloroform; } \\
\text { back extraction with nitric } \\
\text { acid; reduction of } \mathrm{As}^{+5} \text { to } \\
\mathrm{As}^{+3} \text { with thiosulfate and } \\
\text { extract }\end{array}$ & NAA & $0.01 \mathrm{ppb}$ & No data & $\begin{array}{l}\text { Braman et } \\
\text { al. } 1977\end{array}$ \\
\hline $\begin{array}{l}\text { Food (arseno- } \\
\text { betaine in } \\
\text { fish) }\end{array}$ & $\begin{array}{l}\text { Extraction of arsenobetaine } \\
\text { with methanol/chloroform; } \\
\text { digestion with nitric acid/ } \\
\text { magnesium nitrate for } \\
\text { remainder of As species }\end{array}$ & HPLC/ICP-MS & $\begin{array}{l}0.3 \mathrm{ng} \text { as } \\
\text { arsenobetaine }\end{array}$ & $\begin{array}{l}101 \pm 4 \\
\text { recovery of } \\
\text { arseno- } \\
\text { betaine }\end{array}$ & $\begin{array}{l}\text { Beauchemin } \\
\text { et al. } 1988\end{array}$ \\
\hline $\begin{array}{l}\text { Water/waste } \\
\text { water/soil } \\
\text { (inorganic } \\
\text { species) }\end{array}$ & $\begin{array}{l}\text { Acidification or digestion with } \\
\text { hydrochloric acid }\end{array}$ & $\begin{array}{l}\text { EPA Method } \\
\text { 7063; ASV }\end{array}$ & $0.1 \mu \mathrm{g} / \mathrm{L}$ & $96-102$ & EPA 1996e \\
\hline $\begin{array}{l}\text { Water (As }(I I I) \\
\text { As(V), MMA, } \\
\text { and DMA) }\end{array}$ & $\begin{array}{l}\text { Cryogenic GC, Digestion } \\
\text { with } 6 \mathrm{M} \mathrm{HCl} \text {; reduction to } \\
\text { arsine with sodium boro- } \\
\text { hydride; cold trap and } \\
\text { desorption into quartz } \\
\text { furnace }\end{array}$ & $\begin{array}{l}\text { EPA Method } \\
1632 \text { appendix; } \\
\text { HGAAS }\end{array}$ & $3 \mathrm{ng} / \mathrm{L}$ & No data & EPA 1998I \\
\hline Water & $\begin{array}{l}\text { Reduction to arsines; cold } \\
\text { trap and selectively warm to } \\
\text { separate arsine species }\end{array}$ & AAS & $2 \mathrm{ng} / \mathrm{L}$ & $91-109$ & $\begin{array}{l}\text { Andreae } \\
1977\end{array}$ \\
\hline Water & $\begin{array}{l}\text { Reduction of MMA, DMA } \\
\text { and inorganic } \mathrm{As}(\text { control } \mathrm{pH} \\
\left.\text { to select } \mathrm{As}^{+3} \text { or } \mathrm{As}^{+5}\right) \text { to } \\
\text { arsines with sodium tetra- } \\
\text { hydroborate; cold trap and } \\
\text { selectively warm to separate } \\
\text { arsine species }\end{array}$ & HGAAS & $0.019-0.061 \mathrm{ng}$ & No data & $\begin{array}{l}\text { Comber and } \\
\text { Howard } \\
1989\end{array}$ \\
\hline Water/soil & $\begin{array}{l}\text { Extraction with sodium } \\
\text { bicarbonate; reduction of } \\
\text { inorganic arsenic, MMA and } \\
\text { DMA to hydrides with } \\
\text { sodium borohydride; cold } \\
\text { trap arsines in n-heptane }\end{array}$ & $\begin{array}{l}\text { HG-HCT/GC- } \\
\text { MID }\end{array}$ & $0.2-0.4 \mu \mathrm{g} / \mathrm{L}$ & $97-102$ & $\begin{array}{l}\text { Odanaka et } \\
\text { al. } 1983\end{array}$ \\
\hline
\end{tabular}

AAS = atomic absorption spectrophotometry; APDC = ammonium pyrrolidine dithiocarbamate; $A S V=$ anodic stripping voltammetry; DMA = dimethylarsinate; EPA = Environmental Protection Agency; GC-MID = gas chromatography-multiple ion detection; GFAAS = graphite furnace atomic absorption spectrometry; HGAAS=hydride generation-atomic absorption spectroscopy; HG-HCT = hydride generation-heptane cold trap; HPLC = high performance liquid chromatography; ICP-AES = inductively coupled plasma-atomic emission spectrometry; ICP-MS = inductively coupled plasma-mass spectrometry; IEC = ion exchange chromatography; MMA = monomethylarsonate; NAA = neutron activation analysis; $\mathrm{NIOSH}=$ National Institute of Occupational Safety and Health; PTFE = polytetrafluoroethylene; SDDC = silver diethyldithiocarbamate; SRM = standard reference material; XRF = x-ray fluorescence 
The following categories of possible data needs have been identified by a joint team of scientists from ATSDR, NTP, and EPA. They are defined as substance-specific informational needs that if met would reduce the uncertainties of human health assessment. This definition should not be interpreted to mean that all data needs discussed in this section must be filled. In the future, the identified data needs will be evaluated and prioritized, and a substance-specific research agenda will be proposed.

\subsubsection{Identification of Data Needs}

Methods for Determining Biomarkers of Exposure and Effect. The most common biomarker for arsenic exposure is analysis of total arsenic in urine (Hughes 2006). Existing methods are sufficiently sensitive to measure background levels of arsenic in various tissues and biological fluids for average persons, and to detect increases as a result of above-average exposure (Agahian et al. 1990; Clyne et al. 1989; Curatola et al. 1978; Foà et al. 1984; Gebel et al. 1998b; Landsberger and Simsons 1987; Mushak et al. 1977; Pinto et al. 1976; Valentine et al. 1979; Versieck et al. 1983). The precision and accuracy of these methods are documented. Methods are also available that can distinguish nontoxic forms of arsenic (arsenobetaine) from inorganic and organic derivatives that are of health concern (Braman et al. 1977; Dix et al. 1987; Johnson and Farmer 1989; Norin and Vahter 1981; Tam et al. 1982). Further efforts to improve accuracy, reduce interferences, and detect multiple species using a single analysis would be valuable. Arsenic is believed to act by inhibition of numerous cellular and molecular processes. However, these effects are not specific to arsenic, and most can only be measured in tissue extracts.

\section{Methods for Determining Parent Compounds and Degradation Products in Environmental}

Media. Arsenic is ubiquitous in the environment. It is found in air, water, soil, sediments, and food in several inorganic and organic forms. Analytical methods exist for the analysis of arsenic species in all of these environmental media, and these methods have the sensitivity to measure background levels and to detect elevated concentrations due to emissions from sources such as smelters, chemical plants, or hazardous waste sites (APHA 1977, 1989c; EPA 1982b, 1983b, 1983c, 1983d, 1991, 1994b, 1994c, 1996f, 1996h, 1998j, 2000c; NIOSH 1994a, 1994b, 2003). However, further research to reduce chemical and matrix interferences may improve the speed and accuracy of the analyses.

Le et al. (2004) pointed out that there is a need for the development of certified reference materials (CRMs) for speciation analysis. A shortcoming of many CRMs is that they are only certified for the total concentration of arsenic, and only limited information is available on the identity and concentrations of specific arsenic species in some CRMs. 
Continued improvement of the methods for determination of the particular species of arsenic, rather than just the total arsenic concentration, present in foods, especially seafood, is needed since different arsenic species poses different hazards to individuals consuming these foods.

\subsubsection{Ongoing Studies}

The information in Table 7-3 was found as a result of a search of the Federal Research in Progress database (FEDRIP 2006). 


\section{Table 7-3. Ongoing Studies on Analytical Methods for Arsenic in Environmental and Biological Samples}

\begin{tabular}{|c|c|c|c|}
\hline Investigator & Affiliation & Research description & Sponsor \\
\hline Styblo, M & $\begin{array}{l}\text { University of North } \\
\text { Carolina Chapel Hill, } \\
\text { Chapel Hill, North } \\
\text { Carolina }\end{array}$ & $\begin{array}{l}\text { Optimized hydride } \\
\text { generation system for } \\
\text { arsenic analysis }\end{array}$ & $\begin{array}{l}\text { Fogarty International } \\
\text { Center }\end{array}$ \\
\hline Dietze, WT & $\begin{array}{l}\text { Tracedetect, Inc., Seattle, } \\
\text { Washington }\end{array}$ & $\begin{array}{l}\text { A continuous monitor for } \\
\text { arsenic in drinking water }\end{array}$ & NIEHS \\
\hline Dasgupta, PK & $\begin{array}{l}\text { Texas Tech University, } \\
\text { Department of Chemistry, } \\
\text { Lubbock, Texas }\end{array}$ & $\begin{array}{l}\text { A green fieldable } \\
\text { analyzer for arsenic }\end{array}$ & NSF \\
\hline
\end{tabular}

NIEHS = National Institute of Environmental Health Sciences; NSF = National Science Foundation

Source: FEDRIP 2006 


\section{REGULATIONS AND ADVISORIES}

The international and national regulations and guidelines pertaining to arsenic and its metabolites in air, water, and other media are summarized in Table 8-1.

ATSDR has not derived inhalation MRLs or an intermediate-duration oral MRL for inorganic arsenic, or any MRLs for organic arsenic, due to lack of suitable data.

ATSDR has derived an acute-duration oral MRL for inorganic arsenic of $0.005 \mathrm{mg} \mathrm{As} / \mathrm{kg} /$ day based on a LOAEL of $0.05 \mathrm{mg} \mathrm{As} / \mathrm{kg} /$ day for gastrointestinal effects and facial edema in Japanese people who ingested arsenic-contaminated soy sauce for 2-3 weeks (Mizuta et al. 1956). An uncertainty factor of 10 (10 for use of a LOAEL and 1 for human variability) was applied.

ATSDR has derived a chronic-duration oral MRL of $0.0003 \mathrm{mg} / \mathrm{kg} /$ day for inorganic arsenic based on a NOAEL of $0.0008 \mathrm{mg} \mathrm{As} / \mathrm{kg} /$ day for dermal effects in a Taiwanese farming population exposed to arsenic in well water (Tseng 1977; Tseng et al. 1968). An uncertainty factor of 3 (for human variability) was applied.

EPA (IRIS 2007) has derived a chronic oral reference dose (RfD) of $0.0003 \mathrm{mg} \mathrm{As} / \mathrm{kg} /$ day for inorganic arsenic, based on a NOAEL of $0.0008 \mathrm{mg} \mathrm{As} / \mathrm{kg} /$ day for dermal effects and possible vascular complications in a Taiwanese farming population exposed to arsenic in well water (Tseng 1977; Tseng et al. 1968). An uncertainty factor of 3 (to account for the lack of reproductive data and uncertainty in whether the NOAEL accounts for all sensitive individuals) was applied. No reference concentration (RfC) for chronic inhalation exposures to arsenic was reported. EPA is currently revising the assessment for inorganic arsenic.

The Department of Health and Human Services (DHHS) has determined that inorganic arsenic is known to be a human carcinogen (NTP 2005). The EPA has determined that inorganic arsenic is a human carcinogen and has assigned it the cancer classification, Group A (IRIS 2007). EPA's quantitative estimates of carcinogenic risk from oral exposures include a cancer slope factor of $1.5 \mathrm{mg} / \mathrm{kg} /$ day and a drinking water unit risk of $5 \times 10^{-5} \mu \mathrm{g} / \mathrm{L}$. The inhalation unit risk for cancer is $0.0043 \mu \mathrm{g} / \mathrm{m}^{3}$ (IRIS 2007). 


\section{Table 8-1. Regulations and Guidelines Applicable to Arsenic and Arsenic Compounds}

\begin{tabular}{|c|c|c|c|}
\hline Agency & Description & Information & Reference \\
\hline \multicolumn{4}{|c|}{ INTERNATIONAL } \\
\hline \multicolumn{4}{|c|}{ Guidelines: } \\
\hline IARC & $\begin{array}{l}\text { Carcinogenicity classification for arsenic and } \\
\text { arsenic compounds }\end{array}$ & Group $1^{a}$ & IARC 2004 \\
\hline \multirow[t]{2}{*}{$\mathrm{WHO}$} & Air quality guidelines & $1.5 \times 10^{-3}$ unit risk $^{b}$ & WHO 2000 \\
\hline & Drinking water quality guidelines for arsenic & $0.01 \mathrm{mg} / \mathrm{L}^{\mathrm{c}}$ & WHO 2004 \\
\hline \multicolumn{4}{|c|}{ NATIONAL } \\
\hline \multicolumn{4}{|c|}{ Regulations and Guidelines: } \\
\hline \multicolumn{4}{|c|}{ a. Air } \\
\hline ACGIH & TLV (TWA) for arsenic and inorganic compounds & $0.01 \mathrm{mg} / \mathrm{m}^{3}$ & ACGIH 2004 \\
\hline EPA & $\begin{array}{l}\text { Hazardous air pollutant (arsenic and inorganic } \\
\text { compounds, including arsine) }\end{array}$ & Yes & $\begin{array}{l}\text { EPA } 2004 b \\
42 \text { USC } 7412\end{array}$ \\
\hline \multirow[t]{2}{*}{$\mathrm{NIOSH}$} & $\begin{array}{l}\text { REL (15-minute ceiling limit) for arsenic and } \\
\text { inorganic compounds }\end{array}$ & $0.002 \mathrm{mg} / \mathrm{m}^{3}$ & NIOSH 2005a \\
\hline & IDLH for arsenic and inorganic compounds ${ }^{d}$ & $5 \mathrm{mg} / \mathrm{m}^{3}$ & \\
\hline \multirow[t]{4}{*}{ OSHA } & $\begin{array}{l}\text { PEL (8-hour TWA) for general industry for } \\
\text { arsenic organic compounds }\end{array}$ & $0.5 \mathrm{mg} / \mathrm{m}^{3}$ & $\begin{array}{l}\text { OSHA 2005d } \\
29 \text { CFR } 1910.1000\end{array}$ \\
\hline & $\begin{array}{l}\text { PEL (8-hour TWA) for general industry for } \\
\text { arsenic inorganic compounds }\end{array}$ & $10 \mu \mathrm{g} / \mathrm{m}^{3}$ & $\begin{array}{l}\text { OSHA 2005c } \\
29 \text { CFR } 1910.1018\end{array}$ \\
\hline & $\begin{array}{l}\text { PEL (8-hour TWA) for construction industry for } \\
\text { arsenic organic compounds }\end{array}$ & $0.5 \mathrm{mg} / \mathrm{m}^{3}$ & $\begin{array}{l}\text { OSHA 2005b } \\
29 \text { CFR } 1926.55\end{array}$ \\
\hline & $\begin{array}{l}\text { PEL (8-hour TWA) for shipyard industry for } \\
\text { arsenic organic compounds }\end{array}$ & $0.5 \mathrm{mg} / \mathrm{m}^{3}$ & $\begin{array}{l}\text { OSHA 2005a } \\
29 \text { CFR } 1915.1000\end{array}$ \\
\hline \multicolumn{4}{|l|}{ b. Water } \\
\hline \multirow[t]{9}{*}{ EPA } & $\begin{array}{l}\text { Designated as hazardous substances in } \\
\text { accordance with Section } 311(\mathrm{~b})(2)(\mathrm{A}) \text { of the } \\
\text { Clean Water Act }\end{array}$ & Yes & $\begin{array}{l}\text { EPA 2005d } \\
40 \text { CFR } 116.4\end{array}$ \\
\hline & $\begin{array}{l}\text { Arsenic pentoxide, arsenic trioxide, calcium } \\
\text { arsenate, and sodium arsenite }\end{array}$ & & \\
\hline & $\begin{array}{l}\text { Drinking water standards and health advisories } \\
\text { for arsenic }\end{array}$ & & EPA 2004a \\
\hline & DWEL & $0.01 \mathrm{mg} / \mathrm{L}$ & \\
\hline & $\begin{array}{l}\text { National primary drinking water standards for } \\
\text { arsenic }\end{array}$ & & EPA 2002a \\
\hline & MCLG & Zero & \\
\hline & $\mathrm{MCL}$ & $0.01 \mathrm{mg} / \mathrm{L}^{\mathrm{e}}$ & \\
\hline & $\begin{array}{l}\text { Reportable quantities of hazardous substances } \\
\text { designated pursuant to Section } 311 \text { of the Clean } \\
\text { Water Act }\end{array}$ & & $\begin{array}{l}\text { EPA } 2005 e \\
40 \text { CFR } 117.3\end{array}$ \\
\hline & $\begin{array}{l}\text { Arsenic pentoxide, arsenic trioxide, calcium } \\
\text { arsenate, sodium arsenite }\end{array}$ & 1 pound & \\
\hline
\end{tabular}


Table 8-1. Regulations and Guidelines Applicable to Arsenic and Arsenic Compounds

\begin{tabular}{|c|c|c|c|}
\hline Agency & Description & Information & Reference \\
\hline \multicolumn{4}{|c|}{ NATIONAL (cont.) } \\
\hline \multirow[t]{3}{*}{ EPA } & $\begin{array}{l}\text { Water quality criteria for human health } \\
\text { consumption of arsenic: }\end{array}$ & & EPA 2002b \\
\hline & Water + Organism & $0.018 \mu \mathrm{g} / \mathrm{L}^{f}$ & \\
\hline & Organism only & $0.14 \mu \mathrm{g} / \mathrm{L}^{\mathrm{f}}$ & \\
\hline \multicolumn{4}{|l|}{ c. Food } \\
\hline \multirow[t]{7}{*}{ EPA } & Tolerances for residues & & \\
\hline & Dimethylarsinic acid & & EPA 2005i \\
\hline & Cotton (undelinted seed) & $2.8 \mathrm{ppm}$ & 40 CFR 180.311 \\
\hline & Methanearsonic acid & & EPA 2005j \\
\hline & Cotton (undelinted seed) & $0.7 \mathrm{ppm}$ & 40 CFR 180.289 \\
\hline & Cotton, hulls & 0.9 ppm & \\
\hline & Fruit, citrus & 0.35 ppm & \\
\hline FDA & Bottled drinking water & $0.01 \mathrm{mg} / \mathrm{L}$ & $\begin{array}{l}\text { FDA } 2005 \\
21 \text { CFR } 165.110\end{array}$ \\
\hline USDA & $\begin{array}{l}\text { Nonsynthetic substances prohibited for use in } \\
\text { organic crop production }\end{array}$ & Arsenic & $\begin{array}{l}\text { USDA } 2004 \\
7 \text { CFR } 205.602\end{array}$ \\
\hline \multicolumn{4}{|l|}{ d. Other } \\
\hline \multirow[t]{2}{*}{ ACGIH } & $\begin{array}{l}\text { Carcinogenicity classification for arsenic and } \\
\text { arsenic compounds }\end{array}$ & $\mathrm{A} 1^{\mathrm{g}}$ & ACGIH 2004 \\
\hline & $\begin{array}{l}\text { Biological exposure indices for inorganic arsenic } \\
\text { plus methylated metabolites in urine at the end of } \\
\text { the workweek }\end{array}$ & $35 \mu \mathrm{g} \mathrm{As} / \mathrm{L}$ & \\
\hline \multirow[t]{11}{*}{ EPA } & Carcinogenicity classification & Group A & IRIS 2007 \\
\hline & Oral slope factor & 1.5 per mg/kg/day & \\
\hline & Inhalation unit risk & $4.3 \times 10^{-3}$ per $\mu \mathrm{g} / \mathrm{m}^{3}$ & \\
\hline & $\operatorname{RfC}$ & No data & \\
\hline & RfD & $3 \times 10^{-4} \mathrm{mg} / \mathrm{kg} / \mathrm{day}$ & \\
\hline & $\begin{array}{l}\text { Superfund, emergency planning, and community } \\
\text { right-to-know }\end{array}$ & & \\
\hline & Designated CERCLA hazardous substance & & EPA $2005 f$ \\
\hline & Reportable quantity & & 40 CFR 302.4 \\
\hline & Arsenic & Not applicable & \\
\hline & $\begin{array}{l}\text { Arsenic acid, arsenic pentoxide, arsenic } \\
\text { trioxide, calcium arsenate, dimethylarsinic } \\
\text { acid, and sodium arsenite }\end{array}$ & 1 pound & \\
\hline & $\begin{array}{l}\text { Effective date of toxic chemical release } \\
\text { reporting for arsenic }\end{array}$ & $01 / 01 / 87$ & $\begin{array}{l}\text { EPA 2005h } \\
40 \text { CFR } 372.65\end{array}$ \\
\hline
\end{tabular}




\section{Table 8-1. Regulations and Guidelines Applicable to Arsenic and Arsenic Compounds}

\begin{tabular}{|c|c|c|c|}
\hline Agency & Description & Information & Reference \\
\hline \multicolumn{4}{|c|}{ NATIONAL (cont.) } \\
\hline \multirow[t]{7}{*}{ EPA } & $\begin{array}{l}\text { Superfund, emergency planning, and community } \\
\text { right-to-know }\end{array}$ & & \multirow{7}{*}{$\begin{array}{l}\text { EPA } 2005 \mathrm{~g} \\
40 \text { CFR } 355 \text {, } \\
\text { Appendix A }\end{array}$} \\
\hline & Extremely hazardous substances & & \\
\hline & Reportable quantity & & \\
\hline & $\begin{array}{l}\text { Arsenic pentoxide, calcium arsenate, and } \\
\text { sodium arsenite }\end{array}$ & 1 pound & \\
\hline & Threshold planning quantities & & \\
\hline & Arsenic pentoxide & $100 / 10,000$ pounds & \\
\hline & Calcium arsenate and sodium arsenite & $500 / 10,000$ pounds & \\
\hline NTP & Carcinogenicity classification & $\begin{array}{l}\text { Known human } \\
\text { carcinogen }\end{array}$ & NTP 2005 \\
\hline
\end{tabular}

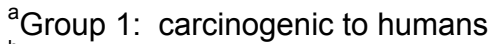

${ }^{\mathrm{b}}$ Cancer risk estimates for lifetime exposure to a concentration of $1 \mu \mathrm{g} / \mathrm{m} 3$.

${ }^{\mathrm{c}}$ Provisional guideline value: as there is evidence of a hazard, but the available information on health effects is limited. ${ }^{\mathrm{d}} \mathrm{NIOSH}$ potential occupational carcinogen

${ }^{\mathrm{e}} \mathrm{MCL}$ will become effective on 01/23/06.

${ }^{f}$ This criterion is based on carcinogenicity of $10^{-6}$ risk.

${ }^{9} \mathrm{~A} 1$ : confirmed human carcinogen

${ }^{\mathrm{h}} \mathrm{A}$ 3: confirmed animal carcinogen with unknown relevance to humans

'Group A: known human carcinogen

${ }^{\mathrm{j}}$ Indicates that no reportable quantity is being assigned to the generic or broad class.

ACGIH = American Conference of Governmental Industrial Hygienists; CERCLA = Comprehensive Environmetnal Response, Compensation, and Liability Act; CFR = Code of Federal Regulations; DWEL = drinking water equivalent level; EPA = Environmental Protection Agency; FDA = Food and Drug Administration; IARC = International Agency for Research on Cancer; IDLH = immediately dangerous to life or health; IRIS = Integrated Risk Information System; $\mathrm{MCL}=$ maximum contaminant level; $\mathrm{MCLG}=$ maximum contaminant level goal; NAS/NRC = National Academy of Sciences/National Research Council; NIOSH = National Institute for Occupational Safety and Health; NTP = National Toxicology Program; OSHA = Occupational Safety and Health Administration; PEL = permissible exposure limit; $\mathrm{REL}$ = recommended exposure limit; $\mathrm{RfC}=$ inhalation reference concentration; $\mathrm{RfD}=$ oral reference dose;

TLV = threshold limit values; TWA = time-weighted average; USC = United States Code; USDA = United States Department of Agriculture; WHO = World Health Organization
} 
EPA is currently revising the assessment for inorganic arsenic. The International Agency for Research on Cancer (IARC) cites sufficient evidence of a relationship between exposure to arsenic and human cancer. IARC classification of arsenic is Group 1 (IARC 2004). The American Conference of Governmental Industrial Hygienists (ACGIH) classifies arsenic (elemental and inorganic compound) as a confirmed human carcinogen, cancer category A1 (ACGIH 2004).

ATSDR has derived an intermediate-duration oral MRL of $0.1 \mathrm{mg}$ MMA/kg/day for MMA based on a $\mathrm{BMDL}_{10}$ of $12.38 \mathrm{mg} \mathrm{MMA} / \mathrm{kg} /$ day for diarrhea observed in rats exposed to MMA in the diet for 13 weeks (Arnold et al. 2003) and an uncertainty factor of 100 (10 for animal to human extrapolation and 10 for human variability).

ATSDR has derived a chronic-duration oral MRL of $0.01 \mathrm{mg} \mathrm{MMA} / \mathrm{kg} /$ day for MMA based on a $\mathrm{BMDL}_{10}$ of $1.09 \mathrm{mg} \mathrm{MMA} / \mathrm{kg} /$ day for increased incidence of progressive nephropathy in male mice exposed to MMA in the diet for 2 years (Arnold et al. 2003) and an uncertainty factor of 100 (10 for animal to human extrapolation and 10 for human variability).

ATSDR has derived a chronic-duration oral MRL of $0.02 \mathrm{mg}$ DMA $/ \mathrm{kg} /$ day for DMA based on a $\mathrm{BMDL}_{10}$ of $1.80 \mathrm{mg} \mathrm{DMA} / \mathrm{kg} /$ day for increased vacuolization of the urothelium in the urinary bladder of female mice exposed to DMA in the diet for 2 years (Arnold et al. 2006) and an uncertainty factor of 100 (10 for animal to human extrapolation and 10 for human variability).

EPA has not derived RfD values for organic arsenicals (IRIS 2007). 
This page is intentionally blank. 


\section{REFERENCES}

Abernathy CO, Chappell WR, Meek ME, et al. 1996. Is ingested inorganic arsenic a "threshold" carcinogen? Fundam Appl Toxicol 29(2):168-175.

ACGIH. 2004. Arsenic. Threshold limit values for chemical substances and physical agents and biological exposure indices. Cincinnati, $\mathrm{OH}$ : American Conference of Governmental Industrial Hygienists.

*Ademuyiwa O, Elsenhans B, Nguyen PT, et al. 1996. Arsenic-copper interaction in the kidney of the rat: Influence of arsenic metabolites. Pharmacol Toxicol 78(3):154-160.

Adinolfi M. 1985. The development of the human blood-CSF-brain barrier. Dev Med Child Neurol 27(4):532-537.

Adlercreutz H. 1995. Phytoestrogens: Epidemiology and a possible role in cancer protection. Environ Health Perspect Suppl 103(7):103-112.

Adobe Lumber. 2002. Consumer information sheet for CCA pressure treated lumber. Adobe Lumber. http://www.adobelumber.com/ccaconsumer.shtml. August 27, 2007.

Adonis M, Martinez V, Marin P, et al. 2005. CYP1A1 and GSTM1 genetic polymorphisms in lung cancer populations exposed to arsenic in drinking water. Xenobiotica 35(5):519-530.

Agahian B, Lee JS, Nelson JH, et al. 1990. Arsenic levels in fingernails as a biological indicator of exposure to arsenic. Am Ind Hyg Assoc J 51(12):646-651.

Agency for Toxic Substances and Disease Registry. 1989. Agency for Toxic Substances and Disease Registry. Decision guide for identifying substance-specific data needs related to toxicological profiles; Notice. Federal Register 54(174):37618-37634.

Agency for Toxic Substances and Disease Registry. 1990a. Arsenic toxicity. Case studies in environmental medicine. Atlanta, GA: Agency for Toxic Substances and Disease Registry, U.S. Department of Health \& Human Services, Public Health Services. HE20.7917:5 504-U.

Agency for Toxic Substances and Disease Registry. 1990b. Biomarkers of organ damage or dysfunction for the renal, hepatobiliary, and immune systems. Atlanta, GA: Subcommittee on Biomarkers of Organ Damage and Dysfunction, Agency for Toxic Substances and Disease Registry.

Aguilar MV, Martínez-Para MC, Gonzalez MJ. 1997. Effects of arsenic (V)-chromium (III) interaction on plasma glucose and cholesterol levels in growing rats. Ann Nutr Metab 41(3):189-195.

Ahmad S, Anderson WL, Kitchin KT. 1999a. Dimethylarsinic acid effects on DNA damage and

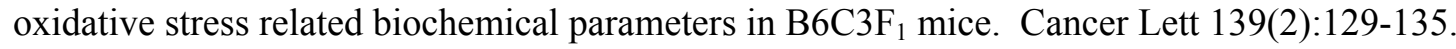

* Not cited in profile 
Ahmad SA, Bandaranayake D, Khan AW, et al. 1997. Arsenic contamination in ground water and arsenicosis in Bangladesh. Int J Environ Health Res 7(4):271-276.

Ahmad SA, Sayed MH, Barua S, et al. 2001. Arsenic in drinking water and pregnancy outcomes. Environ Health Perspect 109(6):629-631.

Ahmad SKA, Sayed MHSU, Hadi SA, et al. 1999b. Arsenicosis in a village in Bangladesh. Int J Environ Health Res 9(3):187-195.

Ahsan H, Chen Y, Parvez F, et al. 2006. Arsenic exposure from drinking water and risk of premalignant skin lesions in Bangladesh: Baseline results from the health effects of arsenic longitudinal study. Am J Epidemiol 163(12):1138-1148.

Ahsan H, Perrin M, Rahman A, et al. 2000. Associations between drinking water and urinary arsenic levels and skin lesions in Bangladesh. J Occup Environ Med 42(12):1195-1205.

Akkari KH, Frans RE, Lavy TL. 1986. Factors affecting degradation of MSMA in soil. Weed Sci 34:781-787.

Alain G, Tousignant J, Rozenfarb E. 1993. Chronic arsenic toxicity. Int J Dermatol 32(12):899-901.

Albores A, Cebrian ME, Bach PH, et al. 1989. Sodium arsenite induced alterations in bilirubin excretion and heme metabolism. J Biochem Toxicol 4(2):73-78.

Albores A, Koropatnick J, Cherian MG, et al. 1992. Arsenic induces and enhances rat hepatic metallothionein production in vivo. Chem Biol Interact 85:127-140.

Almela C, Algora S, Benito V, et al. 2002. Heavy metal, total arsenic, and inorganic arsenic contents of algae food products. J Agric Food Chem 50:918-923.

Altman PK, Dittmer DS. 1974. In: Biological handbooks: Biology data book. Vol. III, $2^{\text {nd }}$ ed. Bethesda, MD: Federation of American Societies for Experimental Biology, 1987-2008, 2041.

Amacher DE, Paillet SC. 1980. Induction of trifluorothymidine-resistant mutants by metal ions in L5178Y/TK ${ }^{* /-}$ cells. Mutat Res 78:279-288.

American Wood Preservers Association. 2007. FAQ's: Treated wood uses. American Wood Preserver's Association. http://www.awpa.com/references/faq.asp\#uses. August 28, 2007.

Andersen ME, Krishnan K. 1994. Relating in vitro to in vivo exposures with physiologically-based tissue dosimetry and tissue response models. In: Salem H, ed. Animal test alternatives: Refinement, reduction, replacement. New York: Marcel Dekker, Inc., 9-25.

Andersen ME, Clewell HJ, Gargas ML, et al. 1987. Physiologically-based pharmacokinetics and the risk assessment process for methylene chloride. Toxicol Appl Pharmacol 87:185-205.

Anderson LCD, Bruland KW. 1991. Biogeochemistry of arsenic in natural waters: The importance of methylated species. Environ Sci Technol 25:420-427.

Anderson HA, Falk C, Hanrahan L, et al. 1998. Profiles of Great Lakes critical pollutants: A sentinel analysis of human blood and urine. Environ Health Perspect 106(5):279-289. 
Andreae MO. 1977. Determination of arsenic species in natural waters. Anal Chem 49(6):820-823.

APHA. 1977. Tentative method of analysis for arsenic content of atmospheric particulate matter. In: Kate M, ed. Methods of air sampling and analysis. Washington, DC: American Public Health Association, 435-438.

APHA. 1989a. Arsenic. In: Clesceri LS, Greenberg AE, Trussell RR, eds. Standard methods for the examination of water and wastewater. 17th ed. Washington, DC: American Public Health Association, 3-74-3-78.

APHA. 1989b. Metals by electrothermal atomic absorption spectrometry. In: Clesceri LS, Greenberg AE, Trussell RR, eds. Standard methods for the examination of water and wastewater. 17th ed. Washington, DC: American Public Health Association, 3-32-3-50.

APHA. 1989c. Metals by hydride generation/atomic absorption spectrometry. In: Clesceri LS, Greenberg AE, Trussell RR, eds. Standard methods for the examination of water and wastewater. 17th ed. Washington, DC: American Public Health Association, 3-43-3-50.

APHA. 1989d. Metals by plasma emission spectrometry. In: Clesceri LS, Greenberg AE, Trussell RR, eds. Standard methods for the examination of water and wastewater. 17th ed. Washington, DC: American Public Health Association, 3-53-3-78.

Aposhian HV. 1989. Biochemical toxicology of arsenic. Rev Biochem Toxicol 10:265-299.

*Aposhian HV. 1998. Mobilization of mercury and arsenic in humans by sodium 2,3-dimercapto-1propane sulfonate (DMPS). Environ Health Perspect Suppl 106(4):1017-1025.

Aposhian HV, Arroyo A, Cebrian ME, et al. 1997. DMPS-arsenic challenge test. I: Increased urinary excretion of monomethylarsonic acid in humans given dimercaptopropane sulfonate. J Pharmacol Exp Ther 282(1):192-200.

Aposhian HV, Gurzau ES, Le XC, et al. 2000a. Occurrence of monomethylarsonous acid in urine of humans exposed to inorganic arsenic. Chem Res Toxicol 13(8):693-697.

Aposhian HV, Zheng B, Aposhian MM, et al. 2000b. DMPS-arsenic challenge test. 11. Modulation of arsenic species, including monomethylarsonous acid (MMA)(111), excreted in human urine. Toxicol Appl Pharmacol 165(1):74-83.

*Apostoli P, Alessio L, Romeo L, et al. 1997. Metabolism of arsenic after acute occupational arsine intoxication. J Toxicol Environ Health 52(4):331-342.

Apostoli P, Bartoli D, Alessio L, et al. 1999. Biological monitoring of occupational exposure to inorganic arsenic. Occup Environ Med 56(12):825-832.

Aranyi C, Bradof JN, O'Shea WJ, et al. 1985. Effects of arsenic trioxide inhalation exposure on pulmonary antibacterial defenses in mice. J Toxicol Environ Health 15:163-172.

*Arbouine MW, Wilson HK. 1992. The effect of seafood consumption on the assessment of occupational exposure to arsenic by urinary arsenic speciation measurements. J Trace Elem Electrolytes Health Dis 6(3):153-160. 
9. REFERENCES

Arenas V, Stoeppler M, Bergerhoff G. 1988. Arsenic determination in the ultratrace range by atomic absorption spectrometry after preconcentration of the hydride. Fresenius Z Anal Chem 332:447-452.

*Armbrust KL, Bridges DC. 2002. Dissipation of monosodium methane arsonate (MSMA) on peanuts. J Agric Food Chem 50(7):1959-1963

Armienta MA, Rodriguez R, Cruz O. 1997. Arsenic content in hair of people exposed to natural arsenic polluted groundwater at Zimapan, Mexico. Bull Environ Contam Toxicol 59:583-589.

Armstrong CW, Stroube RB, Rubio T, et al. 1984. Outbreak of fatal arsenic poisoning caused by contaminated drinking water. Arch Environ Health 39(4):276-279.

Arnold LL, Cano M, St. John M, et al. 1999. Effects of dietary dimethylarsinic acid on the urine and urothelium of rats. Carcinogenesis 20(11):2171-2179.

Arnold LL, Eldan M, Nyska A, et al. 2006. Dimethylarsinic acid: Results of chronic toxicity/oncogenicity studies in F344 rats and B6C3F1 mice. Toxicology 223(1-2):82-100.

Arnold LL, Eldan M, van Gemert M, et al. 2003. Chronic studies evaluating the carcinogenicity of monomethylarsonic acid in rats and mice. Toxicology 190:197-219.

Aschengrau A, Zierler S, Cohen A. 1989. Quality of community drinking water and the occurrence of spontaneous abortion. Arch Environ Health 44(5):283-290.

Aurillo AC, Mason RP, Hemond HF. 1994. Speciation and fate of arsenic in three lakes of the Aberjona Watershed. Environ Sci Tech 28(4):577-585.

Axelson O, Dahlgren E, Jansson C-D, et al. 1978. Arsenic exposure and mortality: A case referent study from a Swedish copper smelter. Br J Ind Med 35:8-15.

Azcue JM, Nriagu JO. 1994. Arsenic: Historical perspectives. In: Nriagu JO, ed. Arsenic in the Environment, part 1: Cycling and characterization. New York, NY: John Wiley and Sons, Inc., 1-15.

Babich H, Martin-Alguacil N, Borenfreund E. 1989. Arsenic-selenium interactions determined with cultured fish cells. Toxicol Lett 45:157-164.

Bagla P, Kaiser J. 1996. India's spreading health crisis draws global arsenic experts. Science 274(5285):174-175.

Baker BA, Topliff AR, Messing RB, et al. 2005. Persistent neuropathy and hyperkeratosis from distant arsenic exposure. J Agromedicine 10(4):43-54.

*Baker EL, Hayes CG, Landrigan PJ, et al. 1977. A nationwide survey of heavy metal absorption in children living near primary copper, lead, and zinc smelters. Am J Epidemiol 106(4):261-273.

Baker LW, Fitzell DL, Seiber JN, et al. 1996. Ambient air concentrations of pesticides in California. Environ Sci Tech 30(4):1365-1368.

*Barbaud A, Mougeolle JM, Schmutz JL. 1995. Contact hypersensitivity to arsenic in a crystal factory worker. Contact Dermatitis 33(4):272-273. 
Barbey JT, Pezzullo JC, Soignet SL. 2003. Effect of arsenic trioxide on QT interval in patients with advanced malignancies. J Clin Oncol 21(19):3609-15.

Barnes DG, Dourson M. 1988. Reference dose (RfD): Description and use in health risk assessments. Regul Toxicol Pharmacol 8:471-486.

Baroni C, van Esch GJ, Saffiotti U. 1963. Carcinogenic tests of two inorganic arsenicals. Arch Environ Health 7:668-674.

Bartolome B, Cordoba S, Nieto S, et al. 1999. Acute arsenic poisoning: Clinical and histopathological features. Br J Dermatol 141:1106-1109.

Barton EN, Gilbert DT, Raju K, et al. 1992. Arsenic: The forgotten poison? West Indian Med J 41(1):36-38.

Bashir S, Sharma Y, Irshad M, et al. 2006. Arsenic induced apoptosis in rat liver following repeated 60 days exposure. Toxicology 217(1):63-70.

Basu A, Ghosh P, Das JK, et al. 2004. Micronuclei as biomarkers of carcinogen exposure in populations exposed to arsenic through drinking water in West Bengal, India: A comparative study in three cell types. Cancer Epidemiol Biomarkers Prev 13(5):820-827.

Bates MN, Rey OA, Biggs ML, et al. 2004. Case-control study of bladder cancer and exposure to arsenic in Argentina. Am J Epidemiol 159(4):381-389.

*Bates MN, Smith AH, Cantor KP. 1995. Case-control study of bladder cancer and arsenic in drinking water. Am J Epidemiol 141(6):523-530.

Battista G, Bartoli D, Iaia TE, et al. 1996. Art glassware and sinonasal cancer: Report of three cases. Am J Ind Med 30(1):31-35.

Baxley MN, Hood RD, Vedel GC, et al. 1981. Prenatal toxicity of orally administered sodium arsenite in mice. Bull Environ Contam Toxicol 26:749-756.

Beane Freeman LE, Dennis LK, Lynch CF, et al. 2004. Toenail arsenic content and cutaneous melanoma in Iowa. Am J Epidemiol 160(7):679-687.

Beauchemin D, Bednas ME, Berman SS, et al. 1988. Identification and quantitation of arsenic species in a dogfish muscle reference material for trace elements. Anal Chem 60:2209-2212.

Beaudoin AR. 1974. Teratogenicity of sodium arsenate in rats. Teratology 10:153-158.

Beck BD, Boardman PD, Hook GC, et al. 1995. Response to Smith et al. Arsenic risk assessment. Environ Health Perspect 103:15-17.

Beckett WS, Moore JL, Keogh JP, et al. 1986. Acute encephalopathy due to occupational exposure to arsenic. Br J Ind Med 43:66-67.

Beckman L, Nordenson I. 1986. Interaction between some common genotoxic agents. Hum Hered 36:397-401. 


\section{REFERENCES}

Beckman G, Beckman L, Nordenson I. 1977. Chromosome aberrations in workers exposed to arsenic. Environ Health Perspect 19(5-6):145-146.

Beerman DH. 1994. Growth regulators (animal). In: Kroschwitz JI, Howe-Grant M, eds. Kirk-Othmer encyclopedia of chemical technology. Vol. 12. New York, NY: John Wiley and Sons, 795-815.

Bekemeier H, Hirschelmann R. 1989. Reactivity of resistance blood vessels ex vivo after administration of toxic chemicals to laboratory animals: Arteriolotoxicity. Toxicol Lett 49:49-54.

Bencko V. 2005. Hair and exposure to environmental pollutants. In: Tobin DJ, eds. Hair in toxicology: An important bio-marker. Cambridge, MA: RSC Publishing, 159-174.

Bencko V, Symon K. 1977. Health aspects of burning coal with a high arsenic content 1. Arsenic in hair, urine, and blood in children residing in a polluted area. Environ Res 13:378-385.

Bencko V, Franek P, Rames J. 2005. Non-melanoma skin and lung cancer incidence in relation to arsenic exposure: 20 years of observation. In: Edler L, Kitsos CP, eds. Recent advances in quantitative methods in cancer and human health risk assessment. West Sussex, England: John Wiley \& Sons, 383394.

Bencko V, Geist T, Arbetová D, et al. 1986. Biological monitoring of environmental pollution and human exposure to some trace elements. J Hyg Epidemiol Microbiol Immunol 30(1):1-10.

Bencko V, Symon K, Chlader V, et al. 1977. Health aspects of burning coal with a high arsenic content II. Hearing changes in exposed children. Environ Res 13:386-395.

Bencko V, Symon K, Stalnik L, et al. 1980. Rate of malignant tumor mortality among coal burning power plant workers occupationally exposed to arsenic. J Hyg Epidemiol Microbiol Immunol 24(3):278284.

Bencko V, Wagner V, Wagnerová M, et al. 1988. Immunological profiles in workers of a power plant burning coal rich in arsenic content. J Hyg Epidemiol Microbiol Immunol 32:137-146.

*Bennett BG. 1986. Exposure assessment for metals involved in carcinogenesis. IARC Sci Publ 71(8):115-127.

Benramdane L, Accominotti M, Fanton L, et al. 1999a. Arsenic speciation in human organs following fatal arsenic trioxide poisoning--a case report. Clin Chem 45(2):301-306.

Benramdane L, Bressolle F, Vallon J-J. 1999b. Arsenic speciation in humans and food products: A review. J Chromatogr Sci 37:330-344.

Benson AA. 1989. Arsonium compounds in algae. Proc Natl Acad Sci USA 86:6131-6132.

Berg M, Tran HC, Nguyen TC, et al. 2001. Arsenic contamination of groundwater and drinking water in Vietnam: A human health threat. Environ Sci Technol 35(13):2621-2626.

Berger GS. 1994. Epidemiology of endometriosis. In: Berger GS, ed. Endometriosis: Advanced management and surgical techniques. New York, NY: Springer-Verlag, 3-7. 
*Berry MR, Johnson LS, Jones JW, et al. 1997. Dietary characterizations in a study of human exposures in the lower Rio Grande Valley: I. Foods and beverages. Environ Int 23(5):675-692.

Berti PR, Receveur O, Chan HM, et al. 1998. Dietary exposure to chemical contaminants from traditional food among adult dene/métis in the western Northwest territories, Canada. Environ Res A76:131-142.

Bertolero F, Pozzi G, Sabbioni E, et al. 1987. Cellular uptake and metabolic reduction of pentavalent to trivalent arsenic as determinants of cytotoxicity and morphological transformation. Carcinogenesis 8:803-808.

Bettley FR, O'Shea JA. 1975. The absorption of arsenic and its relation to carcinoma. Br J Dermatol 92:563-568.

Bhumbla DK, Keefer RF. 1994. Arsenic mobilization and bioavailability in soils. In: Nriagu JO, ed. Arsenic in the environment, part 1: Cycling and characterization. New York, NY: John Wiley \& Sons, Inc., 51-82.

Bickley LK, Papa CM. 1989. Chronic arsenicism with vitiligo, hyperthyroidism, and cancer. N J Med 86(5):377-380.

*Biggs ML, Kalman DA, Moore LE, et al. 1997. Relationship of urinary arsenic to intake estimates and a biomarker of effect, bladder cell micronuclei. Mutat Res 386(3):185-195.

*Binder S, Forney D, Kaye W, et al. 1987. Arsenic exposure in children living near a former copper smelter. Bull Environ Contam Toxicol 39:114-121.

Bird MJ, MacIntosh DL, Williams PL. 2004. Occupational exposures during routine activities in coalfueled power plants. J Occup Environ Hyg 1:403-413.

Biswas BK, Dhar RK, Samanta G, et al. 1998. Detailed study report of Samta, one of the arsenicaffected villages of Jessore District, Bangladesh. Curr Sci 74(2):134-145.

Biswas S, Talukder G, Sharma A. 1999. Prevention of cytotoxic effects of arsenic by short-term dietary supplementation with selenium in mice in vivo. Mutat Res 441:155-160.

Blakley BR. 1987. Alterations in urethan-induced adenoma formation in mice exposed to selenium and arsenic. Drug Nutr Interact 5:97-102.

Bloch P, Shapiro IM. 1986. An x-ray fluorescence technique to measure in situ the heavy metal burdens of persons exposed to these elements in the workplace. J Occup Med 28(8):609-614.

Blom S, Lagerkvist B, Linderholm H. 1985. Arsenic exposure to smelter workers: Clinical and neurophysiological studies. Scand J Work Environ Health 11:265-269.

Bodwell JE, Gosse JA, Nomikos AP, et al. 2006. Arsenic disruption of steroid receptor gene activation: Complex dose-response effects are shared by several steroid receptors. Chem Res Toxicol 19:1619-1629. 
Bodwell JE, Kingsley LA, Hamilton JW. 2004. Arsenic at very low concentrations alters glucocorticoid receptor (GR)-mediated gene activation but not GR-mediated gene repression: Complex dose-response effects are closely correlated with levels of activated GR and require a functional GR DNA binding domain. Chem Res Toxicol 17:1064-1076.

Bolla-Wilson K, Bleecker ML. 1987. Neuropsychological impairment following inorganic arsenic exposure. J Occup Med 29(6):500-503.

Bolliger CT, van Zijl P, Louw JA. 1992. Multiple organ failure with the adult respiratory distress syndrome in homicidal arsenic poisoning. Respiration 59(1):57-61.

Bonnevie NL, Huntley SL, Found BW, et al. 1994. Trace metal contamination in surficial sediments from Newark Bay, New Jersey. Sci Total Environ 144:1-16.

Borgoño JM, Greiber R. 1972. Epidemiological study of arsenicism in the city of Antofagasta. Trace Subst Environ Health 5:13-24.

Borgoño JM, Venturino H, Vicent P. 1980. [Clinical and epidemiological study of arsenism in northern Chile.] Rev Med Chile 108:1039-1048. (Spanish).

*Börzsönyi M, Bereczky A, Rudnai P, et al. 1992. Epidemiological studies on human subjects exposed to arsenic in drinking water in southeast Hungary [letter]. Arch Toxicol 66(1):77-78.

Bourrain JL, Morin C, Béani JC, et al. 1998. Airborne contact dermatitis from cacodylic acid. Contact Dermatitis 38(6):364-365.

Boutwell RK. 1963. A carcinogenicity evaluation of potassium arsenite and arsanilic acid. Agric Food Chem 11:381-385.

Braman RS, Foreback CC. 1973. Methylated forms of arsenic in the environment. Science 182:12471249.

Braman RS, Johnson DL, Foreback CC, et al. 1977. Separation and determination of nanogram amounts of inorganic arsenic and methylarsenic compounds. Anal Chem 49(4):621-625.

*Braun W. 1958. Carcinoma of the skin and the internal organs caused by arsenic. German Med Monthly 3:321-324.

*Brenard R, Laterre PF, Reynaert M, et al. 1996. Increased hepatocytic mitotic activity as a diagnostic marker of acute arsenic intoxication: A report of two cases. J Hepato 25(2):218-220.

Brender JD, Suarez L, Felkner M, et al. 2006. Maternal exposure to arsenic, cadmium, lead, and mercury and neural tube defects in offspring. Environ Res 101(1):132-139.

Breslin VT, Adler-Ivanbrook L. 1998. Release of copper, chromium and arsenic from CCA-C treated lumber in estuaries. Estuarine Coastal Shelf Sci 46:111-125.

Brooks KM. 1996. Evaluating the environmental risks associated with the use of chromated copper arsenate-treated wood products in aquatic environments. Estuaries 19(2A):296-305. 
Broomhall J, Kovar IZ. 1986. Environmental pollutants in breast milk. Rev Environ Health 6(1-4):311337.

Brouwer OF, Onkenhout W, Edelbroek PM, et al. 1992. Increased neurotoxicity of arsenic in methylenetetrahydrofolate reductase deficiency. Clin Neurol Neurosurg 94(4):307-310.

Brown CC, Chu KC. 1982. Environmental epidemiology: Risk assessment: Proceedings of a conference. In: Prentice RL, Whittemore AS, eds. Philadelphia, PA: Society for Industrial and Applied Mathematics, 94-106.

Brown CC, Chu KC. 1983a. A new method for the analysis of cohort studies: Implications of the multistage theory of carcinogenesis applied to occupational arsenic exposure. Environ Health Perspect 50:293-308.

Brown CC, Chu KC. 1983b. Implications of the multistage theory of carcinogenesis applied to occupational arsenic exposure. J Natl Cancer Inst 70(3):455-463.

Brown EJ, Button DK. 1979. A simple method of arsenic speciation. Bull Environ Contam Toxicol 21:37-42.

Brown JL, Kitchin KT. 1996. Arsenite, but not cadmium, induces ornithine decarboxylase and heme oxygenase activity in rat liver: Relevance to arsenic carcinogenesis. Cancer Lett 98:227-231.

Brown KG, Guo H-R, Greene HL. 1997a. Uncertainty in cancer risk at low doses of inorganic arsenic. Hum Ecol Risk Assess 3(3):351-362.

Brown KG, Guo H-R, Kuo T-L, et al. 1997b. Skin cancer and inorganic arsenic: Uncertainty-status of risk. Risk Anal 17(1):37-42.

Brown JL, Kitchin KT, George M. 1997c. Dimethylarsinic acid treatment alters six different rat biochemical parameters: Relevance to arsenic carcinogenesis. Teratog Carcinog Mutagen 17:71-84.

Brown LM, Pottern LM, Blot WJ. 1984. Lung cancer in relation to environmental pollutants emitted from industrial sources. Environ Res 34:250-261.

Brown MM, Rhyne BC, Goyer RA, et al. 1976. Intracellular effects of chronic arsenic administration on renal proximal tubule cells. J Toxicol Environ Health 1:505-514.

Brown RM, Newton D, Pickford CJ, et al. 1990. Human metabolism of arsenobetaine ingested with fish. Hum Exp Toxicol 9:41-46.

Bruce BW, McMahon PB. 1996. Shallow ground-water quality beneath a major urban center: Denver, Colorado, USA. J Hydrol 186:129-151.

Brumbaugh WG, Ingersoll CG, Kemble NE, et al. 1994. Chemical characterization of sediments and pore water from the upper Clark Fork River and Milltown Reservoir, Montana. Environ Toxicol Chem 13(12):1971-1983.

* Brune O, Nordberg G, Wester PO. 1980. Distribution of 23 elements in kidney, liver and lung of a control group in Northern Sweden and of exposed workers from a smelter and refinery. Sci Total Environ 16:13-35. 
Buchancová J, Klimentova G, Knizkova M, et al. 1998. Health status of workers of a thermal power station exposed for prolonged periods to arsenic and other elements from fuel. Cent Eur J Public Health 6(1):29-36.

Buchet JP, Lauwerys R. 1985. Study of inorganic arsenic methylation by rat liver in vitro: Relevance for the interpretation of observations in man. Arch Toxicol 57:125-129.

Buchet JP, Lauwerys R. 1987. Study of factors influencing the in vivo methylation of inorganic arsenic in rats. Toxicol Appl Pharmacol 91:65-74.

Buchet JP, Lauwerys R. 1988. Role of thiols in the in vitro methylation of inorganic arsenic by rat liver cytosol. Biochem Pharmacol 37(16):3149-3153.

Buchet JP, Lison D. 1998. Mortality by cancer in groups of the Belgian population with a moderately increased intake of arsenic. Int Arch Occup Environ Health 71(2):125-130.

Buchet JP, Lauwerys R, Mahieu P, et al. 1982. Inorganic arsenic metabolism in man. Arch Toxicol Suppl 5:326-327.

Buchet JP, Lauwerys R, Roels H. 1981a. Comparison of the urinary excretion of arsenic metabolites after a single oral dose of sodium arsenite, monomethylarsonate or dimethylarsinate in man. Int Arch Occup Environ Health 48:71-79.

Buchet JP, Lauwerys R, Roels H. 1981b. Urinary excretion of inorganic arsenic and its metabolites after repeated ingestion of sodium metaarsenite by volunteers. Int Arch Occup Environ Health 48:111-118.

*Buchet JP, Lison D, Ruggeri M, et al. 1996b. Assessment of exposure to inorganic arsenic, a human carcinogen, due to the consumption of seafood. Arch Toxicol 70(11):773-778.

Buchet JP, Pauwels J, Lauwerys R. 1994. Assessment of exposure to inorganic arsenic following ingestion of marine organisms by volunteers. Environ Res 66(1):44-51.

*Buchet JP, Staessen J, Roels H, et al. 1996a. Geographical and temporal differences in the urinary excretion of inorganic arsenic: A Belgian population study. Occup Environ Med 53(5):320-327.

Budavari S, O'Neil MJ, Smith A, et al., eds. 2001. The Merck index an encyclopedia of chemicals, drugs and biologicals. 13th ed. Whitehouse Station, NJ: Merck \& Co., Inc., 440, 462.

Bulbulyan MA, Jourenkova NJ, Boffetta P, et al. 1996. Mortality in a cohort of Russian fertilizer workers. Scand J Work Environ Health 22(1):27-33.

Burgdorf W, Kurvink K, Cervenka J. 1977. Elevated sister chromatid exchange rate in lymphocytes of subjects treated with arsenic. Hum Genet 36:69-72.

Burger J, Dixon C, Shukla T, et al. 2003. Metals in horseshoe crabs from Delaware Bay. Arch Environ Contam Toxicol 44:36-42.

Burger J, Gaines KF, Boring CS, et al. 2002. Metal levels in fish from the Savannah River: Potential hazards to fish and other receptors. Environ Res 89:85-97. 


\section{REFERENCES}

*Burger J, Gochfeld M, Rooney AA, et al. 2000. Metals and metalloids in tissues of American alligators in three Florida lakes. Arch Environ Contam Toxicol 38:501-508.

Burkhard EG, Dutkiewicz VA, Husain L. 1994. A study of $\mathrm{SO}_{2}, \mathrm{SO}_{4}{ }^{2}$ and trace elements in clear air and clouds above the midwestern United States. Atmos Environ 28(8):1521-1533.

Burlo F, Guijarro I, Carbonell-Barrachina AA, et al. 1999. Arsenic species: Effects on and accumulation by tomato plants. J Agric Food Chem 47:1247-1253.

Burns LA, Munson AE. 1993. Gallium arsenide selectively inhibits T cell proliferation and alters expression of CD25 (IL-2R/P55). J Pharmacol Exp Ther 265(1):178-186.

Butler ECV. 1988. Determination of inorganic arsenic species in aqueous samples by ion-exclusion chromatography with electrochemical detection. J Chromatogr 450:353-360.

Butte W, Heinzow B. 2002. Pollutants in house dust as indicators of indoor contamination. Rev Environ Contam Toxicol 175:1-46.

Byrd DM, Roegner ML, Griffiths JC, et al. 1996. Carcinogenic risks of inorganic arsenic in perspective. Int Arch Occup Environ Health 68(6):484-494.

Byron WR, Bierbower GW, Brouwer JB, et al. 1967. Pathologic changes in rats and dogs from two-year feeding of sodium arsenite or sodium arsenate. Toxicol Appl Pharmacol 10:132-147.

*Caffery-Nolan RE, McCoy KL. 1998. Direct exposure to gallium arsenide upregulates costimulatory activity of murine macrophages. Toxicol Appl Pharmacol 151:330-339.

Calderón J, Navarro ME, Jimenez-Capdeville ME, et al. 2001. Exposure to arsenic and lead an neuropsychological development in Mexican children. Environ Res 85(Section A):69-76.

Calderon RL, Hudgens E, Le CX, et al. 1999. Excretion of arsenic in urine as a function of exposure to arsenic in drinking water. Environ Health Perspect 107:663-667.

Campbell JP, Alvarez JA. 1989. Acute arsenic intoxication. Am Fam Physician 40(6):93-97.

Cannon JR, Edmonds JS, Francesconi KA, et al. 1981. Isolation, crystal structure and synthesis of arsenobetaine, a constituent of the western rock lobster, the dusty shark, and some samples of human urine. Aust J Chem 34:787-798.

Cannon JR, Saunders JB, Toia RF. 1983. Isolation and preliminary toxicological evaluation of arsenobetaine- the water-soluble arsenical constituent from the hepatopancreas of the western rock lobster. Sci Total Environ 31:181-185.

Capar SG, Cunningham WC. 2000. Element and radionuclide concentrations in food: FDA Total Diet Study 1991-1996. J AOAC Int 83:157-177.

Carapella SC. 1992. Arsenic and arsenic alloys. In: Kroschwitz JI, Howe-Grant M, eds. Kirk-Othmer encyclopedia of chemical technology. Vol. 3. New York, NY: John Wiley and Sons, 624-633.

Carbonell-Barrachina AA, Burló F, Valero D, et al. 1999. Arsenic toxicity and accumulation in turnip as affected by arsenic chemical speciation. J Agric Food Chem 47:2288-2294. 


\section{REFERENCES}

Carbonell-Barrachina AA, Jugsujinda A, Burlo F, et al. 2000. Arsenic chemistry in municipal sewage sludge as affected by redox potential and $\mathrm{pH}$. Water Res 34:216-224.

Carlson-Lynch H, Beck BD, Boardman PD. 1994. Arsenic risk assessment. Environ Health Perspect 102(4):354-356.

Carpenter SJ. 1987. Developmental analysis of cephalic axial dysraphic disorders in arsenic-treated hamster embryos. Anat Embryol 176:345-365.

Casto BC, Meyers J, DiPaolo JA. 1979. Enhancement of viral transformation for evaluation of the carcinogenic or mutagenic potential of inorganic metal salts. Cancer Res 39:193-198.

Cebrián ME, Albores A, Aguilar M, et al. 1983. Chronic arsenic poisoning in the north of Mexico. Hum Toxicol 2:121-133.

Chaineau E, Binet S, Pol D, et al. 1990. Embryotoxic effects of sodium arsenite and sodium arsenate on mouse embryos in culture. Teratology 41:105-112.

Chakraborti D, Hussam A, Alauddin M. 2003b. Arsenic: Environmental health aspects with special reference to groundwater in South Asia. J Environ Sci Health Part A 38(1):xi-xv.

Chakraborti D, Mukherjee SC, Pati S, et al. 2003c. Arsenic groundwater contamination in Middle Ganga Plain, Bihar, India: A future danger? Environ Health Perspect 111:1194-1201.

Chakraborti D, Mukherjee SC, Saha KC, et al. 2003a. Arsenic toxicity from homeopathic treatment. J Toxicol Clin Toxicol 41(7):963-967.

Chakraborty AK, Saha KC. 1987. Arsenical dermatosis from tubewell water in west Bengal. Indian J Med Res 85:326-334.

Chan PC, Huff J. 1997. Arsenic carcinogenesis in animals and in humans: Mechanistic, experimental, and epidemiological evidence. J Environ Health Sci, C15(2):83-122.

Chan TYK. 1994. The prevalence use and harmful potential of some Chinese herbal medicines in babies and children. Vet Hum Toxicol 36(3):238-240.

Chang CC, Ho SC, Tsai SS, et al. 2004. Ischemic heart disease mortality reduction in an arseniasisendemic area in southwestern Taiwan after a switch in the tap-water supply system. J Toxicol Environ Health A 67(17):1353-1361.

Chao CC. 1996. Inhibition by arsenite of anticancer drug cis-diamminedichloroplatinum(II) induced DNA repair and drug resistance in HeLa cells. Environ Toxicol Pharmacol 1(3):199-205.

Chappell WR, Beck BD, Brown KG, et al. 1997. Inorganic arsenic: A need and an opportunity to improve risk assessment. Environ Health Perspect 105(10):1060-1067.

Charbonneau SM, Spencer K, Bryce F, et al. 1978. Arsenic excretion by monkeys dosed with arseniccontaining fish or with inorganic arsenic. Bull Environ Contam Toxicol 20:470-477. 
Chattopadhyay S, Ghosh S, Debnath J, et al. 2001. Protection of sodium arsenite-induced ovarian toxicity by coadministration of L-ascorbate (vitamin C) in mature wistar strain rat. Arch Environ Contam Toxicol 41(1):83-89.

ChemIDplus. 2007. ChemIDplus. Bethesda, MD: U.S. National Library of Medicine. http://sis.nlm.nih.gov/chemical.html. August 28, 2007.

Chen CJ, Wang CJ. 1990. Ecological correlation between arsenic level in well water and age-adjusted mortality from malignant neoplasms. Cancer Res 50:5470-5474.

${ }^{*}$ Chen CL, Whanger PD. 1994. Interaction of selenium and arsenic with metallothionein: Effect of vitamin $B_{12}$. J Inorg Biochem 54(4):267-276.

Chen CJ, Chen CW, Wu MM, et al. 1992. Cancer potential in liver, lung, bladder and kidney due to ingested inorganic arsenic in drinking water. Br J Cancer 66(5):888-892.

Chen CJ, Chiou HY, Chiang MH, et al. 1996. Dose-response relationship between ischemic heart disease mortality and long-term arsenic exposure. Arterioscler Thromb Vasc Biol 16(4):504-510.

Chen CJ, Chuang YC, Lin TM, et al. 1985. Malignant neoplasms among residents of a blackfoot disease-endemic area in Taiwan: High-arsenic artesian well water and cancers. Cancer Res 45:58955899.

Chen CJ, Chuang YC, You SL, et al. 1986. A retrospective study on malignant neoplasms of bladder, lung and liver in blackfoot disease endemic area in Taiwan. Br J Cancer 53:399-405.

Chen CJ, Hsu LI, Shih WL, et al. 2005. Biomarkers of exposure, effect, and susceptibility of arsenicinduced health hazards in Taiwan. Toxicol Appl Pharmacol 206(2):198-206.

Chen CJ, Hsueh YM, Lai MS, et al. 1995. Increased prevalence of hypertension and long-term arsenic exposure. Hypertension 25(1):53-60.

Chen CJ, Kuo T-L, Wu MM. 1988a. Arsenic and cancers [Letter]. Lancet (February 20):414-415.

Chen CJ, Wu MM, Lee SS, et al. 1988b. Atherogenicity and carcinogenicity of high-arsenic artesian well water: Multiple risk factors and related malignant neoplasms of blackfoot disease. Arteriosclerosis $8: 452-460$.

*Chen KS, Huang CC, Liaw CC, et al. 1988c. [Multiple primary cancers in blackfoot endemic area: Report of a case.] J Formosan Med Assoc 87:1125-1128. (Chinese)

Chen M, Ma LQ, Harris WG. 1999. Baseline concentrations of 15 trace elements in Florida surface soils. J Environ Qual 28:1173-81.

Chen Y, Ahsan H, Parvez F, et al. 2004. Validity of a food-frequency questionnaire for a large prospective cohort study in Bangladesh. Br J Nutr 92(5):851-859.

Chen YC, Guo YL, Su HJ, et al. 2003. Arsenic methylation and skin cancer risk in southwestern Taiwan. J Occup Environ Med 45(3):241-248. 
Chernoff N, Setzer W, Miller DB, et al. 1990. Effects of chemically induced maternal toxicity on prenatal development in the rat. Teratology 42:651-658.

Chhuttani PN, Chawla LS, Sharma TD. 1967. Arsenical neuropathy. Neurology 17:269-274.

*Ch'i I, Blackwell RQ. 1968. A controlled retrospective study of blackfoot disease, an endemic peripheral gangrene disease in Taiwan. Am J Epidemiol 88(1):7-24.

Chiang CE, Luk HN, Wang TM, et al. 2002. Prolongation of cardiac repolarization by arsenic trioxide. Blood 100(6):2249-2252.

*Chiang HS, Hong CL, Guo HR, et al. 1988. Comparative study on the high prevalence of bladder cancer in the blackfoot disease endemic area in Taiwan. J Formosan Med Assoc 87(11):1074-1080.

Chiba M, Masironi R. 1992. Toxic and trace elements in tobacco and tobacco smoke. Bull WHO 70(2):269-275.

Chicago Flameproof. 2000. Pressure-treated Supatimber. Montgomery, IL: Chicago Flameproof. http://www.chicagoflameproof.com/supatimber.html. July 29, 2005.

Chilvers DC, Peterson PJ. 1987. Global cycling of arsenic. In: Hutchinson TC, Meema KM, eds. Lead, mercury, cadmium and arsenic in the environment. New York, NY: John Wiley \& Sons, 279-301.

Chiou HY, Chiou ST, Hsu YH, et al. 2001. Incidence of transitional cell carcinoma and arsenic in drinking water: A follow-up study of 8,102 residents in an arseniasis-endemic area in northeastern Taiwan. Am J Epidemiol 153(5):411-418.

Chiou HY, Hsueh YM, Liaw KF, et al. 1995. Incidence of internal cancers and ingested inorganic arsenic: A seven-year follow-up study in Taiwan. Cancer Res 55(6):1296-1300.

Chiou HY, Huang WI, Su CL, et al. 1997. Dose-response relationship between prevalence of cerebrovascular disease and ingested inorganic arsenic. Stroke 28(9):1717-1723.

Chirenje T, Ma LQ, Clark C, et al. 2003a. Cu, Cr and As distribution in soils adjacent to pressure-treated decks, fences and poles. Environ Pollut 124(3):407-417.

Chirenje T, Ma LQ, Szulczewski M, et al. 2003b. Arsenic distribution in Florida urban soils:

Comparison between Gainsville and Miami. J Environ Qual 32:109-119.

${ }^{*}$ Chiu HF, Ho SC, Wang LY, et al. 2004. Does arsenic exposure increase the risk for liver cancer? J Toxicol Environ Health A 67(19):1491-1500.

Choucair AK, Ajax ET. 1988. Hair and nails in arsenical neuropathy. Ann Neurol 23(6):628-629.

Chow NH, Guo YL, Lin JSN, et al. 1997. Clinicopathological features of bladder cancer associated with chronic exposure to arsenic. Br J Cancer 75(11):1708-1710.

Chowdhury UK, Biswas BK, Chowdhury TR, et al. 2000. Groundwater arsenic contamination in Bangladesh and West Bengel, India. Environ Health Perspect 108(5):393-397. 
Christian JW, Hopenhayn C, Centeno JA, et al. 2006. Distribution of urinary selenium and arsenic among pregnant women exposed to arsenic in drinking water. Environ Res 100(1):115-122.

Chun JS, Killeen JC. 1989a. Salmonella/mammalian-microsome plate incorporation mutagenicity assay (Ames test) with and without metabolic activation with methanearsonic acid (MAA). Fermenta ASC Corporation. Submitted to the U.S. Environmental Protection Agency. MRID41651902.

Chun JS, Killeen JC. 1989b. In vitro chromosomal aberration assay in Chinese hamster ovary (CBO) cells with methanarsonic acid (MAA). Fermenta ASC Corporation. Submitted to the U.S. Environmental Protection Agency. MRID41651903.

Chun JS, Killeen JC. 1989c. L5178Y TK ${ }^{* /-}$ mouse lymphoma mutagenesis assay with methanearsonic acid. Fermenta ASC Corporation. Submitted to the U.S. Environmental Protection Agency. MRID41651904.

Chun JS, Killeen JC. 1989d. Unscheduled DNA synthesis assay in rat primary hepatocytes with methanearsonic acid (MAA). Fermenta ASC Corporation. Submitted to the U.S. Environmental Protection Agency. MRID41651905.

Chung JS, Kalman DA, Moore LE, et al. 2002. Family correlations of arsenic methylation patterns in children and parents exposed to high concentrations of arsenic in drinking water. Environ Health Perspect 110(7):729-733.

Cikrt M, Mravcová A, Malátová I, et al. 1988. Distribution and excretion of ${ }^{74} \mathrm{As}$ and ${ }^{75} \mathrm{Se}$ in rats after their simultaneous administration: The effect of arsenic, selenium and combined pretreatment. J Hyg Epidemiol Microbiol Immunol 32(1):17-29.

Civantos DP, Rodríguez AL, Aguado-Borruey JM, et al. 1995. Fulminant malignant arrhythmia and multiorgan failure in acute arsenic poisoning. Chest 108(6):1774-1775.

Clewell HJ, Andersen ME. 1985. Risk assessment extrapolations and physiological modeling. Toxicol Ind Health 1(4):111-131.

Clewell HJ, Thomas RS, Gentry PR, et al. 2007. Research toward the development of a biologically based dose response assessment for inorganic arsenic carcinogenicity: A progress report. Toxicol Appl Pharmacol 222(3):388-398.

Clyne N, Ericsson F, Lins L, et al. 1989. Plasma trace elements in pre-dialytic uremic patients determined by energy dispersive X-ray fluorescence. Trace Elem Med 6(1):37-40.

Cohen SM, Arnold LL, Eldan M, et al. 2006. Methylated arsenicals: The implications of metabolism and carcinogenicity studies in rodents to human risk assessment. Crit Rev Toxicol 36(2):99-133.

Cohen SM, Yamamoto S, Cano M, et al. 2001. Urothelial cytotoxicity and regeneration induced by dimethylarsinic acid in rats. Toxicol Sci 59(1):68-74.

Cöl M, Cöl C, Soran A, et al. 1999. Arsenic-related Bowen's disease, Palmer keratosis, and skin cancer. Environ Health Perspect 107(8):687-689.

Cole RH, Frederick RE, Healy RP, et al. 1984. Preliminary findings of the priority pollutant monitoring project of the nationwide urban runoff program. J Water Pollut Control Fed 56:898-908. 
Coles DG, Ragaini RC, Ondov JM, et al. 1979. Chemical studies of stack fly ash from a coal-fired power plant. Environ Sci Tech 13(4):455-459.

Comber SDW, Howard AG. 1989. Arsenic speciation by hydride generation atomic absorption spectrometry and its application to the study of biological cycling in the coastal environment. Anal Proc 26:20-22.

Concha G, Nermell B, Vahter M. 1998a. Metabolism of inorganic arsenic in children with chronic high arsenic exposure in northern Argentina. Environ Health Perspect 106(6):355-359.

Concha G, Vogler G, Lezcano D, et al. 1999. Exposure to inorganic arsenic metabolites during early human development. Toxicol Sci 44:185-190.

Concha G, Vogler G, Nermell B, et al. 1998b. Low-level arsenic excretion in breast milk of native Andean women exposed to high levels of arsenic in the drinking water. Int Arch Occup Environ Health 71(1):42-46.

Concha G, Vogler G, Nermell B, et al. 2002. Intra-individual variation in the metabolism of inorganic arsenic. Int Arch Occup Environ Health 75:576-580.

Cooper PA. 1991. Leaching of CCA from treated wood: pH effects. For Prod J 41(1):30-32.

Cooper CM, Gillespie WB. 2001. Arsenic and mercury concentrations in major landscape components of an intensively cultivated watershed. Environ Pollut 111:67-74.

Cordier S, Thériault G, Iturra H. 1983. Mortality patterns in a population living near a copper smelter. Environ Res 31:311-322.

Costa M, Zhitkovich A, Harris M, et al. 1997. DNA-protein cross-links produced by various chemicals in cultured human lymphoma cells. J Toxicol Environ Health 50(5):433-449.

Crecelius EA. 1977. Changes in the chemical speciation of arsenic following ingestion by man. Environ Health Perspect 19:147-150.

Crecelius EA. 1978. Modification of the arsenic speciation technique using hydride generation. Anal Chem 50(6):826-827.

Crown S, Kenan G, Nyska A, et al. 1987. Cacodylic acid toxicity in dietary administration to rats for 13 weeks: A preliminary study. Luxembourg Industries (Pamol) Ltd. Submitted to the U.S. Environmental Protection Agency. MRID42767701.

Crown S, Nyska A, Waner T. 1990. Methanearsonic acid combined chronic feeding and oncogenicity study in the rat. Luxembourg Industries (Pamol) Ltd. Submitted to the U.S. Environmental Protection Agency. MRID41669001.

Csanaky I, Gregus Z. 2002. Species variations in the biliary and urinary excretion of arsenate, arsenite and their metabolites. Comp Biochem Physiol C 131(3):355-365. 
*Cullen WR. 1998. Arsenic in the environment. In: Bunnett JF, Mikolajczyk M, eds. Arsenic and old mustard: Chemical problems in the destruction of old arsenical and 'mustard' munitions. Netherlands: Kluwer Academic Publishers, 123-134.

Cullen NM, Wolf LR, St Clair D. 1995. Pediatric arsenic ingestion. Am J Emerg Med 13(4):432-435.

Curatola CJ, Grunder FI, Moffitt AE. 1978. Hydride generation atomic absorption spectrophotometry for determination of arsenic in hair. Am Ind Hyg Assoc Journal 39:933-938.

Cuzick J, Sasieni P, Evans S. 1992. Ingested arsenic, keratoses, and bladder cancer. Am J Epidemiol 136(4):417-421.

*Czarnecki GL, Baker DH, Garst JE. 1984. Arsenic-sulfur amino acid interactions in the chick. J Anim Sci 59:1573-1581.

Dabeka RW, Lacroix GMA. 1987. Total arsenic in foods after sequential wet digestion, dry ashing, coprecipitation with ammonium pyrrolidine dithiocarbamate, and graphite-furnace atomic absorption spectrometry. J Assoc Off Anal Chem 70(5):866-870.

Dabeka RW, McKenzie AD, Lacroix GM, et al. 1993. Survey of arsenic in total diet food composites and estimation of the dietary intake of arsenic by Canadian adults and children. J AOAC Int 76(1):14-25.

Dallaire L, Beliveau R. 1992. Phosphate transport by capillaries of the blood-brain barrier. J Biol Chem 267(31):22323-22327.

Danan M, Dally S, Conso F. 1984. Arsenic-induced encephalopathy. Neurology 34:1524.

Darland JE, Inskeep WP. 1997. Effects of $\mathrm{pH}$ and phosphate competition on the transport of arsenate. J Environ Qual 26(4):1133-1139.

Das T, Roychoudhury A, Sharma A, et al. 1993. Modification of clastogenicity of three known clastogens by garlic extract in mice in vivo. Environ Mol Mutagen 21(4):383-388.

Datta S, Talukder G, Sharma A. 1986. Cytotoxic effects of arsenic in dietary oil primed rats. Sci Cult 52(6):196-198.

Davey JC, Bodwell JE, Gosse JA, et al. 2007. Arsenic as an endocrine disruptor: Effects of arsenic on estrogen receptor-mediated gene expression in vivo and in cell culture. Toxicol Sci 98(1):75-86.

Davidson CI, Goold WD, Mathison TP, et al. 1985. Airborne trace elements in Great Smoky Mountains, Olympic, and Glacier National Parks. Environ Sci Technol 19:27-35.

Davis A, Ruby MV, Bergstrom PD. 1992. Bioavailability of arsenic and lead in soils from the Butte, Montana, mining district. Environ Sci Technol 26(3):461-468.

Davis A, Ruby MV, Bloom M, et al. 1996. Mineralogic constraints on the bioavailability of arsenic in smelter-impacted soils. Environ Sci Technol 30(2):392-399.

Dekundt GH, Leonard A, Arany J, et al. 1986. In vivo studies in male mice on the mutagenic effects of inorganic arsenic. Mutagenesis 1(1):33-34. 
de la Rosa ME, Magnusson J, Ramel C, et al. 1994. Modulating influence of inorganic arsenic on the recombinogenic and mutagenic action of ionizing radiation and alkylating agents in Drosophila melanogaster. Mutat Res 318(1):65-71.

de Peyster A, Silvers JA. 1995. Arsenic levels in hair of workers in a semiconductor fabrication facility. Am Ind Hyg Assoc J 56(4):377-383.

Dhar P, Jaitley M, Kalaivani M, et al. 2005. Preliminary morphological and histochemical changes in rat spinal cord neurons following arsenic ingestion. Neurotoxicology 26(3):309-320.

DHHS. 1995. Report to Congress on workers' home contamination study conducted under the workers' family protection act (29 U.S.C. 671a). Cincinnati, OH: U.S. Department of Health and Human Services, Public Health Service, Centers for Disease Control and Prevention, National Institute for Occupational Safety and Health. September 1995. Pub no. 95-123. PB96192000.

*Díaz-Barriga F, Batres L, Calderón J, et al. 1997. The El Paso smelter 20 years later: Residual impact on Mexican children. Environ Res 74(1):11-16.

Dieke SH, Richter CP. 1946. Comparative assays of rodenticides on wild Norway rats. Public Health Rep 61:672-679.

DiPaolo JA, Casto BC. 1979. Quantitative studies of in vitro morphological transformation of Syrian hamster cells by inorganic metal salts. Cancer Res 39:1008-1013.

*Diwan A, Ward JM, Waalkes MP. 2002. Multiorgan transplacental carcinogenesis by arsenic in mice. Proc Am Assoc Cancer Res 43:1032.

Dix K, Cappon CJ, Toribara TY. 1987. Arsenic speciation by capillary gas-liquid chromatography. J Chromatogr Sci 25:164-169.

Dolan SP, Nortrup DA, Bolger PM, et al. 2003. Analysis of dietary supplements for arsenic, cadmium, mercury, and lead using inductively coupled plasma mass spectrometry. J Agric Food Chem 51(5):13071312.

*Domingo JL, Bosque MA, Piera V. 1991. meso-2,3-Dimercaptosuccinic acid and prevention of arsenite embryotoxicity and teratogenicity in the mouse. Fundam Appl Toxicol 17:314-320.

Dong JT, Luo XM. 1993. Arsenic-induced DNA-strand breaks associated with DNA-protein crosslinks in human fetal lung fibroblasts. Mutat Res 302(2):97-102.

Dong JT, Luo XM. 1994. Effects of arsenic on DNA damage and repair in human fetal lung fibroblasts. Mutat Res 315(1):11-15.

Dopp E, Hartmann LM, Florea AM, et al. 2004. Uptake of inorganic and organic derivatives of arsenic associated with induced cytotoxic and genotoxic effects in Chinese hamster ovary (CHO) cells. Toxicol Appl Pharmacol 201(2):156-165.

Driesback RH, ed. 1980. Arsenic and arsine. In: Handbook of poisoning: Prevention, diagnosis and treatment. 11th ed. Los Altos, CA: Lange Medical Publications, 241-245. 
Dueñas C, Pérez-Alvarez JC, Busteros JI, et al. 1998. Idiopatic portal hypertension and angiosarcoma associated with arsenical salts therapy. J Clin Gastroenterol 26(4):303-305.

*Dulout FN, Grillo CA, Seoane AI, et al. 1996. Chromosomal aberrations in peripheral blood lymphocytes from native Andean women and children from northwestern Argentina exposed to arsenic in drinking water. Mutat Res 370(3-4):151-158.

Dunlap LG. 1921. Perforations of the nasal septum due to inhalation of arsenous oxide. JAMA 76(9):568-569.

*Durant JL, Chen J, Hemond HF, et al. 1995. Elevated incidence of childhood leukemia in Woburn, Massachusetts: NIEHS Superfund basic research program searches for causes. Environ Health Perspect 103(Suppl 6):93-98.

Dutkiewicz T. 1977. Experimental studies on arsenic absorption routes in rats. Environ Health Perspect 19:173-177.

Ebdon L, Fisher A, Roberts NB, et al. 1999. Determination of organoarsenic species in blood plasma by HPLC-ICP MS. Appl Organomet Chem 13:183-187.

Edelman GM. 1992. Morphoregulation. Dev Dynamics 193:2-10.

Edmonds JS, Francesconi KA. 1987. Transformations of arsenic in the marine environment. Experientia 43:553-557.

Edmonds MS, Baker DH. 1986. Toxic effects of supplemental copper and roxarsone when fed alone or in combination to young pigs. J Anim Sci 63:533-537.

Eguchi N, Kuroda K, Endo G. 1997. Metabolites of arsenic induced tetraploids and mitotic arrest in cultured cells. Arch Environ Contam Toxicol 32(2):141-145.

Eisler R. 1994. A review of arsenic hazards to plants and animals with emphasis on fishery and wildlife. In: Nriagu JO, ed. Arsenic in the environment: Part II: Human health and ecosystem effects. New York, NY: John Wiley \& Sons, Inc, 185-259.

Eitzer BD, Iannucci-Berger WA, Mark G, et al. 1997. Fate of toxic compounds during composting. Bull Environ Contam Toxicol 58(6):953-960.

Ellenhorn MJ. 1997. Ellenhorn's medical toxicology. Diagnosis and treatment of human poisoning. Baltimore, MD: Williams \& Wilkins, 1538-1542.

Ellenhorn MJ, Barceloux DG. 1988. Medical toxicology: Diagnosis and treatment of human poisoning. New York, NY: Elsevier, 1012-1048.

Endo G, Kuroda K, Okamoto A, et al. 1992. Dimethylarsenic acid induces tetraploids in Chinese hamster cells. Bull Environ Contam Toxicol 48(1):131-137.

Engel RR, Receveur O. 1993. Re: "Arsenic ingestion and internal cancers: A review”. Am J Epidemiol 138(10):896-897. 
Engel RR, Smith AH. 1994. Arsenic in drinking water and mortality from vascular disease: An ecologic analysis in 30 counties in the United States. Arch Environ Health 49(5):418-427.

Enterline PE, Marsh GM. 1982. Cancer among workers exposed to arsenic and other substances in a copper smelter. Am J Epidemiol 116(6):895-911.

Enterline PE, Day R, Marsh GM. 1995. Cancers related to exposure to arsenic at a copper smelter. Occup Environ Med 52(1):28-32.

Enterline PE, Henderson VL, Marsh GM. 1987a. Exposure to arsenic and respiratory cancer: A reanalysis. Am J Epidemiol 125(6):929-938.

Enterline PE, Marsh GM, Esmen NA, et al. 1987b. Some effects of cigarette smoking, arsenic, and $\mathrm{SO}_{2}$ on mortality among U.S. copper smelter workers. J Occup Med 29(10):831-838.

Environment Canada. 1993. Canadian environmental protection act priority substances list assessment report: Arsenic and its compounds. Ottawa, Canada: Canada Communication Group.

EPA. 1977a. Selected non-carcinogenic effects of industrial exposure to inorganic arsenic. Washington, DC: U.S. Environmental Protection Agency. PB276988. EPA560677018.

*EPA. 1977b. The evaluation of the acute inhalation toxicology of technical grade organoarsenical pesticides. Research Triangle Park, NC: U.S. Environmental Protection Agency, Health Effects Research Laboratory.

EPA. 1979. Water-related environmental fate of 129 priority pollutants: Vol. I. Introduction and technical background, metals and inorganics, pesticides and PCBs. Washington, DC: U.S. Environmental Protection Agency, Office of Water Planning and Standard. EPA440479029a.

EPA. 1980a. Ambient water quality criteria for arsenic. Washington, DC: U.S. Environmental Protection Agency, Office of Water Regulations and Standards. EPA440580021.

*EPA. 1980b. Lung cancer/mortality in proximity to a pesticide plant. Washington, DC: U.S. Environmental Protection Agency. PB80207376.

*EPA. 1980c. STORET. Washington, DC: U.S. Environmental Protection Agency, Monitoring and Data Support Division.

*EPA. 1980d. U.S. Environmental Protection Agency. Fed Regist 45:33132-33133.

*EPA. 1980e. U.S. Environmental Protection Agency: Part V. Fed Regist 45:79325-79327.

EPA. 1980f. Failure to produce arsenic neurotoxicity in the rat. An experimental study. Washington, DC: U.S. Environmental Protection Agency, Office of Toxic Substances. EPA5601180022.

*EPA. 1980g. Fate of toxic and hazardous materials in the air environment. Research Triangle Park, NC: U.S. Environmental Protection Agency, U.S. Environmental Sciences Research Lab. PB80221948.

*EPA. 1980h. Lung cancer mortality in proximity to a pesticide plant. Washington, DC: American Public Health Association and U.S. Environmental Protection Agency, Office of Toxic Substances. PB80207376. 
*EPA. 1981a. Effluent guidelines and standards. Toxic pollutants. U.S. Environmental Protection Agency. Code of Federal Regulations. 40 CFR 401.

EPA. 1981b. Community health associated with arsenic in drinking water in Millard County, Utah. Cincinnati, OH: U.S. Environmental Protection Agency, Health Effects Research Laboratory. EPA600181064. PB82108374.

EPA. 1981c. The carcinogen assessment group's final risk assessment on arsenic. Washington, DC: U.S. Environmental Protection Agency, Office of Health and Environmental Assessment. EPA600681002. PB81206013.

*EPA. 1981d. Effluent guidelines and standards. Timber products processing point source category. U.S. Environmental Protection Agency. Code of Federal Regulations. 40 CFR 429.

*EPA. 1981e. Assessment of exposure to arsenic from CCA-treated wood: Final report. Washington, DC: U.S. Environmental Protection Agency, Office of Health and Environmental Assessment.

*EPA. 1982a. Effluent guidelines and standards. Inorganic chemicals manufacturing point source category. U.S. Environmental Protection Agency. Code of Federal Regulations. 40 CFR 415.

EPA. 1982b. Exposure and risk assessment for arsenic. Washington, DC: U.S. Environmental Protection Agency, Office of Water Regulations and Standards. PB85221711. EPA440485005. 1.14.68 .

EPA. 1982c. Inductively coupled plasma-atomic emission spectrometric method for trace element analysis of water and wastes - method 200.7. Cincinnati, OH: U.S. Environmental Protection Agency, Environmental Monitoring and Support Laboratory.

*EPA. 1982d. Arsenic. In: Intermedia priority pollutant guidance documents. Washington, DC: U.S. Environmental Protection Agency, Office of Pesticides and Toxic Substances.

*EPA. 1982e. Speciation of arsenic compounds in water supplies. Cincinnati, OH: U.S. Environmental Protection Agency, Health Effects Research Laboratory. EPA6005182010.

*EPA. 1983a. Effluent guidelines and standards. Electrical and electronic components point source category. U.S. Environmental Protection Agency. Code of Federal Regulations. 40 CFR 469.

EPA. 1983b. Method 206.2: atomic absorption, furnace technique. In: Methods for chemical analysis of water and wastes. Cincinnati, OH: U.S. Environmental Protection Agency, Environmental Monitoring and Support Laboratory. EPA600479020.

EPA. 1983c. Method 206.3: atomic absorption-gaseous hydride. In: Methods for chemical analysis of water and wastes. Cincinnati, OH: U.S. Environmental Protection Agency, Environmental Monitoring and Support Laboratory. EPA600479020.

EPA. 1983d. Method 206.4: spectrophotometric - SDDC. In: Methods for chemical analysis of water and wastes. Cincinnati, OH: U.S. Environmental Protection Agency, Environmental Monitoring and Support Laboratory. EPA600479020. 
EPA. 1983e. Method 206.5: sample digestion prior to total arsenic analysis by silver diethyldithiocarbamate or hydride procedures. In: Methods for chemical analysis of water and wastes. Cincinnati, OH: U.S. Environmental Protection Agency, Environmental Monitoring and Support Laboratory. EPA600479020.

*EPA. 1983f. Treatability manual: Vol. I: Treatability data. Washington, DC: U.S. Environmental Protection Agency, Office of Research and Development, I.4.2.1-I.4.2.6. EPA600282001a.

EPA. 1983g. Feasibility study to resolve questions on the relationship of arsenic in drinking water to skin cancer. Washington, DC: U.S. Environmental Protection Agency, Office of Research and Development.

*EPA. 1983h. Compliance monitoring data from federal reporting data system. U.S. Environmental Protection Agency, Office of Drinking Water, State Programs Division.

EPA. 1984a. Health Assessment Document for Arsenic. Research Triangle Park, NC: U.S. Environmental Protection Agency. EPA600823021F.

*EPA. 1984b. Health assessment document for inorganic arsenic. Final report. Research Triangle Park, NC: U.S. Environmental Protection Agency, Environmental Criteria and Assessment Office.

EPA600883021F. 2-1-3-22, 9-1-9-4.

*EPA. 1984c. Health effects assessment for arsenic. Washington, DC: U.S. Environmental Protection Agency, Office of Emergency and Remedial Response. EPA540186020.

*EPA. 1984d. Occurrence of arsenic in drinking water, food and air. Washington, DC: U.S. Environmental Protection Agency, Office of Water Regulations and Standards.

*EPA. 1986a. Evaluation of the potential carcinogenicity of arsenic (7940-38-2) in support of reportable quantity adjustments pursuant to CERCLA Section 102. Washington, DC: U.S. Environmental Protection Agency, Office of Health and Environmental Assessment. OHEA C-073-5.

*EPA. 1986b. Inorganic arsenic risk assessment for primary and secondary zinc smelters, primary lead smelters, zinc oxide plants, cotton gins, and arsenic chemical plants. Research Triangle Park, NC: U.S. Environmental Protection Agency, Strategies and Air Standards Division. PB86245255.

*EPA. 1986c. Method 7060 - Arsenic: atomic absorption, furnace technique. In: Test methods for evaluating solid waste. 3rd ed. SW-846. Washington, DC: U.S. Environmental Protection Agency, Office of Solid Waste and Emergency Response.

*EPA. 1986d. National emission standard for inorganic arsenic emissions from arsenic trioxide and metallic arsenic production facilities. U.S. Environmental Protection Agency. Code of Federal Regulations. 40 CFR 61, Subpart P.

*EPA. 1986e. Creosote, pentachlorophenol, and inorganic arsenicals: Amendment of notice to cancel registrations. U.S. Environmental Protection Agency. Fed Regist 51(7):1334-1348.

EPA. 1986f. National emission standards for household air pollutants; Standards for inorganic arsenic. U.S. Environmental Protection Agency: Part II. Fed Regist 51(149):27956-27960. 
9. REFERENCES

*EPA. 1986g. National emission standard for inorganic arsenic emissions from primary copper smelters. U.S. Environmental Protection Agency. Code of Federal Regulations. 40 CFR 61, Subpart O.

*EPA. 1987a. U.S. Environmental Protection Agency. Fed Regist 52:12278.

*EPA. 1987b. U.S. Environmental Protection Agency: Part II. Fed Regist 52(77):13396-13398, 13402.

*EPA. 1987c. List (phase 1) of hazardous constituents for ground-water monitoring. U.S. Environmental Protection Agency: Part II. Fed Regist 52(131):25942-25953.

*EPA. 1987d. Land disposal restrictions for certain "California list" hazardous wastes and modifications to the framework. U.S. Environmental Protection Agency: Part V. Fed Regist 52(130):25760-25763.

EPA. 1987e. The risk assessment guidelines of 1986. Washington, DC: U.S. Environmental Protection Agency. EPA600887045.

*EPA. 1987f. Carcinogenic effects of arsenic compounds in drinking water. Cincinnati, OH: U.S. Environmental Protection Agency, Health Effects Research Laboratory. PB87232542. EPA 600187007.

*EPA. 1988a. Inorganic arsenicals; Intent to cancel registrations for pesticide products registered for non-wood preservative use; conclusion of special review. U.S. Environmental Protection Agency. Fed Regist 53(126):24787-24796.

*EPA. 1988b. U.S. Environmental Protection Agency: Part II. Fed Regist 53:31138-31222.

*EPA. 1988c. U.S. Environmental Protection Agency: Part II. Fed Regist 53:4500-4501.

EPA. 1988d. Special report on ingested inorganic arsenic: Skin cancer; nutritional essentiality. Washington, DC: U.S. Environmental Protection Agency, Risk Assessment Forum. EPA625387013F. PB89125975

EPA. 1988e. Reference physiological parameters in pharmacokinetic modeling. Washington, DC: U.S. Environmental Protection Agency. EPA600688004. PB88196019.

*EPA. 1989a. Guidance for the reregistration of wood preservative pesticide products containing arsenic, chromium, and chromated arsenical compounds as the active ingredient. Washington, DC: U.S. Environmental Protection Agency, Office of Pesticide Programs. PB89102842.

EPA. 1989b. Interim methods for development of inhalation reference doses. Washington, DC: U.S. Environmental Protection Agency, Office of Health and Environmental Assessment. EPA600888066F. PB90145723.

*EPA. 1989c. U.S. Environmental Protection Agency: Part II. Fed Regist 54:35988-35992.

*EPA. 1989d. Reportable quantity adjustments; Digesting of ammonium trisulfate. U.S. Environmental Protection Agency: Part V. Fed Regist 54(155):33426, 33450, 33453-33454, 33456, 33463-33465, 33470.

EPA. 1989e. Recognition and management of pesticide poisonings. U.S. Environmental Protection Agency. EPA540988001. PB91145656, 54-62 
*EPA. 1990a. Effluent guidelines and standards. Nonferrous metals manufacturing point source category. U.S. Environmental Protection Agency. Code of Federal Regulations. 40 CFR 421.

*EPA. 1990b. Calcium arsenate; Revocation of tolerances. U.S. Environmental Protection Agency. Fed Regist 55(158):33332-33335.

*EPA. 1990c. Land disposal restrictions for third scheduled wastes. U.S. Environmental Protection Agency: Part II. Fed Regist 55(106):22520-22536, 22683-22714.

*EPA. 1990d. Notice of intent to remove certain active ingredients from list B and to those pesticides containing those ingredients. U.S. Environmental Protection Agency: Part V. Fed Regist 55(147):31164-31174.

EPA. 1990e. Pollutant concentration estimates from the National Sewage Sludge Survey: Arsenic. U.S. Environmental Protection Agency: Part III. Fed Regist 55(218):47229.

*EPA. 1990f. Toxics in the community: 1988: National and local perspectives. Washington, DC: U.S. Environmental Protection Agency, Office of Toxic Substances.

*EPA. 1990g. National emissions standard for inorganic arsenic emissions from glass manufacturing plants. U.S. Environmental Protection Agency. Code of Federal Regulations. 40 CFR 61. Subpart N.

*EPA. 1990h. Standards of performance for volatile organic compounds (VOC) emissions from synthetic organic chemical manufacturing industry (SOCMI) distillation operation. U.S. Environmental Protection Agency. Code of Federal Regulations. 40 CFR 60.667.

EPA. 1990i. Interim methods for development of inhalation reference concentrations. Washington, DC: U.S. Environmental Protection Agency, Office of Health and Environmental Assessment, Office of Research and Development, Environmental Criteria and Assessment Office. EPA600890066A.

EPA. 1991. Method 200.8 - Determination of trace elements in waters and wastes by inductively coupled plasma - mass spectrometry. Cincinnati, OH: U.S. Environmental Protection Agency, EPA monitoring and support laboratory.

*EPA. 1993a. Arsenic: Supply, demand, and the environment. In: Mercury and arsenic wastes: removal, recovery, treatment, and disposal. U.S. Environmental Protection Agency Pollution Technology Review No. 214, 67-71.

EPA. 1993b. Standards for the management of hazardous waste and specific types of hazardous waste facilities. Health-based limits for exclusion of waste-dericed residues. U.S. Environmental Protection Agency. Code of Federal Regulations. 40 CFR 266; Subpart H; Appendix VII.

EPA. 1994a. Method 6020 - Inductively coupled plasma - mass spectrometry. In: Test methods for evaluating sold waste. $3^{\text {rd }}$ ed. SW-846. Washington DC: U.S. Environmental Protection Agency, Office of Solid Waste and Emergency Response.

EPA. 1994b. Method 7060A- Arsenic: atomic absorption, furnace technique. In: Test methods for evaluating sold waste. $3^{\text {rd }}$ ed. SW-846. Washington DC: U.S. Environmental Protection Agency, Office of Solid Waste and Emergency Response. 
EPA. 1994c. Method 200.7 - Inductively coupled plasma-atomic emission spectrometric method for trace element analysis of water and wastes. Revision 4.4, EMMC version. Cincinnati, OH: U.S. Environmental Protection Agency, Environmental Monitoring and Support Laboratory. http://www.nemi.gov/. April 6, 2005.

*EPA. 1995a. Designation, reportable quantities, and notification. List of hazardous substance and reportable quantities. U.S. Environmental Protection Agency. Code of Federal Regulations. 40 CFR 302.4 .

*EPA. 1995b. Determination of reportable quantities for hazardous substances. U.S. Environmental Protection Agency. Code of Federal Regulations. 40 CFR 117.

EPA. 1995c. Effluent guidelines and standards. Pesticide chemicals. Metallo-organic pesticide chemicals manufacturing subcategory. U.S. Environmental Protection Agency. Code of Federal Regulations. 40 CFR 455, Subpart B.

*EPA. 1995d. Toxic chemical release reporting: Community right-to-know. Specific toxic chemical listings. U.S. Environmental Protection Agency. Code of Federal Regulations. 40 CFR 372.65.

*EPA. 1995e. Maximum contaminant levels for inorganic chemicals. U.S. Environmental Protection Agency. Code of Federal Regulations. 40 CFR 141, Subpart B.

*EPA. 1995f. Determination of background concentrations of inorganics in soil and sediments at hazardous waste sites. Washington, DC: U.S. Environmental Protection Agency, Office of Research and Development. EPA540S96500.

*EPA. 1996a. Effluent guidelines and standards. Pesticide chemicals. List of appropriate pollution control technologies. U.S. Environmental Protection Agency. Code of Federal Regulations. 40 CFR 455, Table 10 .

EPA. 1996b. Proposed guidelines for carcinogen risk assessment. U.S. Environmental Protection Agency. Fed Regist 61(79):17960-18011.

*EPA. 1996c. Superfund, emergency planning, and community right-to-know programs. Designation, reportable quantities, and notification. Emergency planning and notification. The list of extremely hazardous substances and their threshold planning quantities. U.S. Environmental Protection Agency. Code of Federal Regulations. 40 CFR 355, Appendix A.

EPA. 1996d. Method 6010B- Inductively coupled plasma-atomic emission spectrometry. In: Test methods for evaluating sold waste. $3^{\text {rd }}$ ed. SW-846. Washington DC: U.S. Environmental Protection Agency, Office of Solid Waste and Emergency Response.

EPA. 1996e. Method 7063- Arsenic in aqueous samples and extracts by anodic stripping voltammetry (ASV). In: Test methods for evaluating solid waste. $3^{\text {rd }}$ ed. SW-846. Washington DC: U.S. Environmental Protection Agency, Office of Solid Waste and Emergency Response.

EPA. 1996f. Land disposal restrictions. Prohibitions on storage. U.S. Environmental Protection Agency. Code of Federal Regulations. 40 CFR 268, Subpart E. 


\section{REFERENCES}

*EPA. 1996g. Land disposal restrictions. Metal bearing wastes prohibited from dilution in a combustion unit according to 40 CFR 268.3(c). U.S. Environmental Protection Agency. Code of Federal Regulations. 40 CFR 268, Appendix XI.

EPA. 1996h. Method 1632: Inorganic arsenic in water by hydride generation quartz furnace atomic absorption. U.S. Environmental Protection Agency, Office of Water, Engineering and Analysis Division.

*EPA. 1997a. Identification and listing of hazardous waste. Hazardous constituents. U.S. Environmental Protection Agency. Code of Federal Regulations. 40 CFR 261.

*EPA. 1997b. Standards for owners and operators of hazardous waste treatment, storage, and disposal facilities. Ground-water monitoring list. U.S. Environmental Protection Agency. Code of Federal Regulations. 40 CFR 264, Appendix IX.

*EPA. 1997c. National emission standards for wood furniture manufacturing operations. U.S. Environmental Protection Agency. Code of Federal Regulations. 40 CFR 63, Subpart JJ.

*EPA. 1997d. Land disposal restrictions Phase IV: Treatment standards for wood preserving wastes, paperwork reduction and streamlining, exemptions from RCRA for certain processed materials; and miscellaneous hazardous waste provision. U.S. Environmental Protection Agency. Fed Regist 62(91)25998.

*EPA. 1997e. Fish and wildlife advisory database. U.S. Environmental Protection Agency. http://www.epa.gov/ost/fishadvice/. March 31, 2005.

*EPA. 1997f. Addition of facilities in certain industry sectors; Revised interpretation of otherwise use; Toxic release inventory reporting; Community right-to-know. U.S. Environmental Protection Agency. Fed Regist 62(84):23834.

EPA. 1997g. Relative bioavailability of arsenic in mining wastes. Denver, CO: U.S. Environmental Protection Agency. Document control no. 4500-88-AORH.

EPA. 1997h. Special report on environmental endocrine disruption: An effects assessment and analysis. Washington, DC: U.S. Environmental Protection Agency, Risk Assessment Forum. EPA630R96012.

*EPA. 1997i. Report on the expert panel on arsenic carcinogenicity: Review and workshop. Washington, DC: U.S. Environmental Protection Agency.

*EPA. 1998a. List of pesticides banned and severely restricted in the U.S. U.S. Environmental Protection Agency. http://www.epa.gov.oppfead1/international/piclist.htm. March 31, 2005.

*EPA. 1998b. Effluent limitations guidelines, pretreatment standards and new source performance standards for the industrial waste combustor subcategory of the waste combustors point source category. U.S. Environmental Protection Agency. Code of Federal Regulations. 40 CFR 444. Fed Regist 63(25)6392.

*EPA. 1998c. National emission standards for hazardous air pollutants; proposed standards for hazardous air pollutants emissions for the Portland cement manufacturing industry. U.S. Environmental Protection Agency. Code of Federal Regulations. 40 CFR 63. Fed Regist 63(56)14182. 
*EPA. 1998d. National emissions standards for hazardous air pollutants for primary lead smelters. U.S. Environmental Protection Agency. Code of Federal Regulations. 40 CFR 63. Fed Regist 63(74)19200.

*EPA. 1998e. National emission standards for hazardous air pollutants for source categories: national emission standards for primary copper smelters. U.S. Environmental Protection Agency. Fed Regist 63(75)19582.

*EPA. 1998f. Land disposal restrictions phase IV: final rule promulgating treatment standards for metal wastes and mineral processing wastes; mineral processing secondary materials and bevill exclusion issues; treatment standards for hazardous soils, and exclusion of recycled wood preserving wastewaters. U.S. Environmental Protection Agency. Fed Regist 63(100)28556.

EPA. 1998g. Listing of fish and wildlife advisories - 1997. Washington, DC: U.S. Environmental Protection Agency, Office of Water/Office of Science and Technology. EPA823C98001, version 3.

*EPA. 1998h. U.S. Environmental Protection Agency. Code of Federal Regulations. 40 CFR 116.4.

*EPA. 1998i. U.S. Environmental Protection Agency. Code of Federal Regulations. 40 CFR 401.15.

EPA. 1998j. Method 6020A- Inductively coupled plasma - mass spectrometry. In: Test methods for evaluating solid waste. $3^{\text {rd }}$ ed. SW-846. Washington, DC: U.S. Environmental Protection Agency, Office of Solid Waste and Emergency Response.

EPA. 1998k. Notice of filing of pesticide petitions. U.S. Environmental Protection Agency. Fed Regist 63:40273-40279.

EPA. 19981. Method 1632. Chemical speciation of arsenic in water and tissue by hydride generation quartz furnace atomic absorption spectrometry. Revision A. U.S. Environmental Protection Agency. http://www.epa.gov/waterscience/methods/method/files/1632.pdf. August 27, 2007.

*EPA. 1999a. U.S. Environmental Protection Agency. Code of Federal Regulations. 40 CFR 372.65.

*EPA. 1999b. U.S. Environmental Protection Agency. Code of Federal Regulations. 40 CFR 141.11.

*EPA. 1999c. U.S. Environmental Protection Agency. Code of Federal Regulations. 40 CFR 302.4.

*EPA. 1999d. U.S. Environmental Protection Agency. Code of Federal Regulations. 40 CFR 261.33.

*EPA. 1999e. National Recommended Water Quality Criteria-Correction. U.S. Environmental Protection Agency, Office of Water. EPA822Z99001.

*EPA. 1999f. U.S. Environmental Protection Agency. Code of Federal Regulations. 40 CFR 180.180.

*EPA. 1999g. U.S. Environmental Protection Agency. Code of Federal Regulations. 40 CFR 180.3.

EPA. 1999h. International Pesticide Notice: EPA cancels the last agricultural use of arsenic acid in the United States. U.S. Environmental Protection Agency. http://www.epa.gov/oppfead1/17b/arsenic.htm. November 3, 1999.

*EPA. 1999i. Pesticide chemicals classified as known, probable or possible human carcinogens. Environmental Protection Agency. http://www.epa.gov/pesticides/carlist/table.htm. November 3, 1999. 
*EPA. 2000a. NCOD query results. Arsenic. Drinking water data. National Contaminant Occurrence Database. Public Water Systems. U.S. Environmental Protection Agency. http://www.epa.gov/ncod/. March 31, 2005.

*EPA. 2000b. National primary drinking water regulations; Arsenic and clarifications to compliance and new source contaminants monitoring; Proposed rule. U.S. Environmental Protection Agency. Fed Regist 65:38888.

EPA. 2000c. Method 6010C - Inductively coupled plasma-atomic emission spectrometry. U.S. Environmental Protection Agency, Office of Solid Waste. http://www.nemi.gov/. April 6, 2005.

EPA. 2001. National primary drinking water regulations; arsenic and clarifications to compliance and new source contaminants monitoring. U.S. Environmental Protection Agency. Fed Regist 66(14):69767066.

EPA. 2002a. National primary drinking water regulations. Washington, DC: Office of Ground Water and Drinking Water, U.S. Environmental Protection Agency. EPA816F02013. http://www.epa.gov/safewater/mcl.html. February 15, 2005.

EPA. 2002b. National recommended water quality criteria. Washington, DC: Office of Water, Office of Science and Technology, U.S. Environmental Protection Agency. EPA822R02047. http://www.epa.gov/waterscience/pc/revcom.pdf. February 15, 2005.

EPA. 2003a. Response to requests to cancel certain chromated copper arsenate (CCA) wood preservative products and amendments to terminate certain uses of other CCA products. Fed Regist 68 (68):17366-17372.

EPA. 2003b. Technical summary of information available on the bioaccumulation of arsenic in aquatic organisms. Washington, DC: U.S. Environmental Protection Agency. EPA822R03032. http://www.epa.gov/waterscience/criteria/arsenic/tech-sum-bioacc.pdf. August 27, 2007

EPA. 2004a. Drinking water standards and health advisories. Washington, DC: Office of Water, U.S. Environmental Protection Agency. EPA822R04005.

http://www.epa.gov/waterscience/drinking/standards/dwstandards.pdf. February 15, 2005.

EPA. 2004b. Hazardous air pollutants. Washington, DC: U.S. Environmental Protection Agency. United States Code. 42 USC 7412. http:/www.epa.gov/ttn/atw/orig189.html. February 15, 2005.

EPA. 2004c. National fish tissue study. Washington, DC: U.S. Environmental Protection Agency. http://www.epa.gov/waterscience/fishstudy/. April 1, 2005.

*EPA. 2004d. Risk assessment guidance for Superfund. Volume I: Human health evaluation manual. Part E: Supplemental guidance for dermal risk assessment. Washington, DC: U.S. Environmental Protection Agency. EPA540R99005. OSWER9285702EP. PB99963312, 3-16, 4-6, 6-2, R-1, R-5, B-20.

EPA. 2005b. 1996. Emissions of arsenic compounds. Technology Transfer Network. National Air Toxics Assessment. Pollutant-Specific Data Tables. U.S. Environmental Protection Agency. http://www.epa.gov/ttn/atw/nata/tablemis.html. March 31, 2005. 
EPA. 2005a. Chromated copper arsenate (CCA). U.S. Environmental Protection Agency. http://www.epa.gov/oppad001/reregistration/cca/. February 25, 2005.

EPA. 2005d. Designated as hazardous substances in accordance with Section 311(b)(2)(A) of the Clean Water Act. U.S. Environmental Protection Agency. Code of Federal Regulations. 40 CFR 116.4. http://www.epa.gov/ttn/atw/orig189.html. February 15, 2005.

EPA. 2005e. Reportable quantities of hazardous substances designated pursuant to Section 311 of the Clean Water Act. U.S. Environmental Protection Agency. Code of Federal Regulations 40 CFR 117.3. http://www.epa.gov/epacfr40/chapt-I.info/chi-toc.htm. February 16, 2005.

EPA. 2005c. STORET data warehouse. U.S. Environmental Protection Agency. http://www.epa.gov/storet/dw_home.html. April 8, 2005.

EPA. 2005f. Superfund, emergency planning, and community right-to-know programs. Designation, reportable quantities, and notifications. Code of Federal Regulations. 40 CFR 302.4. U.S.

Environmental Protection Agency. http:/www.epa.gov/epacfr40/chapt-I.info/chi-toc.htm. February 15, 2005.

EPA. 2005g. Superfund, emergency planning, and community right-to-know programs. Extremely hazardous substances and their threshold planning quantities. U.S. Environmental Protection Agency. Code of Federal Regulations. 40 CFR 355, Appendix A. http:/www.epa.gov/epacfr40/chapt-I.info/chitoc.htm. February 15, 2005.

EPA. 2005h. Superfund, emergency planning, and community right-to-know programs. Toxic chemical release reporting. U.S. Environmental Protection Agency. Code of Federal Regulations. 40 CFR 372.65. http://www.epa.gov/epacfr40/chapt-I.info/chi-toc.htm. February 16, 2005.

EPA. 2005i. Tolerances and exemptions from tolerances for pesticide chemicals in food. U.S. Environmental Protection Agency. Code of Federal Regulations. 40 CFR 180.311. http://www.epa.gov/epacfr40/chapt-I.info/chi-toc.htm. February 15, 2005.

EPA. 2005j. Tolerances and exemptions from tolerances for pesticide chemicals in food. U.S. Environmental Protection Agency. Code of Federal Regulations. 40 CFR 180.289.

http://www.epa.gov/epacfr40/chapt-I.info/chi-toc.htm. February 15, 2005.

EPA. 2005k. Toxic chemical release inventory reporting forms and instructions: Revised 2004 version. Section 313 of the Emergency Planning and Community Right-to-Know Act (Title III of the Superfund Amendments and Reauthorization Act of 1986). U.S. Environmental Protection Agency. Office of Environmental Information. EPA260B05001.

*EPA. 20051. Guidelines for carcinogen risk assessment. Washington, DC: U.S. Environmental Protection Agency. EPA630P03001F.

EPA. 2006. Revised reregistration eligibility decision for MSMA, DSMA, CAMA, and cacodylic acid, August 10, 2006. Washington, DC: U.S. Environmental Protection Agency. http://www.epa.gov/oppsrrd1/REDs/organic_arsenicals_red.pdf. September 12, 2006.

EPA. 2007a. Chromated copper arsenate (CCA): Consumer awareness program (CAP). U.S. Environmental Protection Agency. http://www.epa.gov/oppad001/reregistration/cca/cap.htm. January $16,2007$. 
9. REFERENCES

EPA. 2007b. Framework for metals risk assessment. U.S. Environmental Protection Agency. EPA120R07001. http://www.epa.gov/osa/metalsframework/pdfs/metals-risk-assessment-final.pdf. August 30, 2007.

Erdogan S, Celik S, Erdogan Z. 2004. Comparison of selected toxic elements in cow serum and milk samples from industrial and rural regions. Bull Environ Contam Toxicol 72(5):931-936.

Erry BV, Macnair MR, Meharg AA, et al. 1999. Seasonal variation in dietary and body organ arsenic concentrations in wood mice Apodemus sylvaticus and bank voles Clethrionomys glareolus. Bull Environ Contam Toxicol 63:567-574.

*Falk H, Caldwell GG, Ishak KG, et al. 1981a. Arsenic-related hepatic angiosarcoma. Am J Ind Med 2:43-50.

Falk H, Herbert JT, Edmonds L, et al. 1981b. Review of four cases of childhood hepatic angiosarcomaelevated environmental arsenic exposure in one case. Cancer 47:382-391.

Fan S, Ho I, Yeoh FL, et al. 1996. Squalene inhibits sodium arsenite-induced sister chromatid exchanges and micronuclei in Chinese hamster ovary-K1 cells. Mutat Res 368(3-4):165-169.

Farag AM, Woodward DF, Goldstein JN, et al. 1998. Concentrations of metal associated with mining waste in sediments, biofilm, benthic macroinvertebrates, and fish from the Coeur d'Alene River basin, Idaho. Arch Environ Contam Toxicol 34:119-127.

Farago ME. 1997. Arsenic in the marine environment. In: Gianguzza A, Pelizzetti E, Sammartano S, eds. Marine chemistry. Netherlands: Kluwer Academic Publishers, 275-291.

FDA. 2000. FDA approves arsenic trioxide for leukemia treatment in record time for a cancer drug development program. Food and Drug Administration.

http://www.fda.gov/bbs/topics/ANSWERS/ANS01040.html. August 30, 2007.

FDA. 2005. Beverages. Bottled water. Final Rule. Food and Drug Administration. Code of Federal Regulations. 21 CFR 165.110. Fed Regist 70:33694-33701.

http://www.fda.gov/OHRMS/DOCKETS/98fr/05-11406.pdf. September 1, 2005.

FEDRIP. 2006. Arsenic. Federal Research in Progress database. Springfield, VA: National Technical Information Service.

FEDRIP. 2007. Arsenic. Federal Research in Progress database. Springfield, VA: National Technical Information Service.

Feldman RG, Niles CA, Kelly-Hayes M, et al. 1979. Peripheral neuropathy in arsenic smelter workers. Neurology 29:939-944.

Feng X, Melander AP, Klaue B. 2000. Contribution of municipal waste incineration to trace metal deposition on the vicinity. Water Air Soil Pollut 119:295-316.

Ferm VH, Carpenter SJ. 1968. Malformations induced by sodium arsenate. J Reprod Fertil 17:199-201. 
*Ferm VH, Hanlon DP. 1985. Constant rate exposure of pregnant hamsters to arsenate during early gestation. Environ Res 37:425-432.

Ferm VH, Saxon A, Smith BM. 1971. The teratogenic profile of sodium arsenate in the golden hamster. Arch Environ Health 22:557-560.

*Ferreccio CR, Gonzalez CW, Solari JS, et al. 1995. [Lung cancer in workers exposed to arsenic. A case control study.] Rev Med Chil 124:119-123. (Spanish)

Ferreccio CR, Gonzalez CW, Solari JS, et al. 1996. [Bronchopulmonary cancer in workers exposed to arsenic: A case control study.] Rev Med Chil 124(1):119-123. (Spanish)

Ferreccio C, Gonzalez Psych C, Milosavjlevic Stat V, et al. 1998. Lung cancer and arsenic exposure in drinking water: A case-control study in northern Chile. Cad Saude Publica 14(Suppl 3):193-198.

Ferreccio C, Gonzalez C, Milosavjlevic V, et al. 2000. Lung cancer and arsenic concentrations in drinking water in Chile (Erratum in: Epidemiology 12(2):283). Epidemiology 11(6):673-679.

Fincher R, Koerker RM. 1987. Long-term survival in acute arsenic encephalopathy: Follow-up using newer measures of electrophysiologic parameters. Am J Med 82:549-552.

Flora SJS, Tripathi N. 1998. Hepatic and renal metallothionein induction following single oral administration of gallium arsenide in rats. Biochem Mol Biol Int 45(6):1121-1127.

*Flora SJS, Dube SN, Pant SC, et al. 1994. Effects of multiple gallium arsenide exposure on some biochemical alterations in rat brain [letter]. Ind Health 32(4):247-252.

Flora SJS, Dube SN, Vijayaraghavan R, et al. 1997a. Changes in certain hemetological and physiological variables following single gallium arsenide exposure in rats. Biol Trace Elem Res 58:197208.

Flora SJS, Kumar P, Kannan GM, et al. 1998. Acute oral gallium arsenide exposure and changes in certain hematological, hepatic, renal and immunological indices at different time intervals in male Wistar rats. Toxicol Lett 94:103-113.

Flora SJS, Pant SC, Malhotra PR, et al. 1997b. Biochemical and histopathological changes in arsenicintoxicated rats coexposed to ethanol. Alcohol 14(6):563-568.

Florea AM, Yamoah EN, Dopp E. 2005. Intracellular calcium disturbances induced by arsenic and its methylated derivatives in relation to genomic damage and apoptosis induction. Environ Health Perspect 113(6):659-664.

FNB/IOM. 2001. Arsenic, boron, nickel, silicon, and vanadium. Dietary reference intakes for vitamin A, vitamin $\mathrm{K}$, arsenic, boron, chromium, copper, iodine, iron, manganese, molybdenum, nickel, silicon, vanadium, and zinc (2000). A Report of the Panel on Micronutrients, subcommittees on upper reference levels of nutrients and of interpretation and uses of dietary reference intakes, and the standing committee on the scientific evaluation of dietary reference intakes. Food and Nutrition Board. Institute of Medicine. Washington, DC: National Academy Press, 502-553. http://books.nap.edu/openbook.php?record_id=10026\&page=R2. August 30, 2007. 
9. REFERENCES

Foà V, Colombi A, Maroni M, et al. 1984. The speciation of the chemical forms of arsenic in the biological monitoring of exposure to inorganic arsenic. Sci Total Environ 34:241-259.

Foà V, Colombi A, Maroni M, et al. 1987. Study of kidney function of workers with chronic low level exposure to inorganic arsenic. In: DeRosa E, Bartolucci GB, Foa V, eds. Occupational and environmental chemical hazards: Cellular and biochemical indices for monitoring toxicity. Horwood, NY: Halsted Press, 362-367.

Fomon SJ. 1966. Body composition of the infant: Part I: The male "reference infant". In: Falkner F, ed. Human development. Philadelphia, PA: WB Saunders, 239-246.

Fomon, SJ, Haschke F, Ziegler EE, et al. 1982. Body composition of reference children from birth to age 10 years. Am J Clin Nutr 35:1169-1175.

*Fowler BA, Woods JS. 1977. The transplacental toxicity of methyl mercury to fetal rat liver mitochondria: Morpho-metric and biochemical studies. Lab Invest 36:122-130.

Fowler BA, Woods JS. 1979. The effects of prolonged oral arsenate exposure on liver mitochondria of mice: Morphometric and biochemical studies. Toxicol Appl Pharmacol 50:177-187.

Fowler BA, Woods JS, Schiller CM. 1977. Ultrastructural and biochemical effects of prolonged oral arsenic exposure on liver mitochondria of rats. Environ Health Perspect 19:197-204.

Foy HM, Tarmapai S, Eamchan P, et al. 1992. Chronic arsenic poisoning from well water in a mining area in Thailand. Asia Pac J Public Health 6(3):150-152.

Francesconi KA, Edmonds JS, Morita M. 1994. Determination of arsenic and arsenic species in marine environmental samples. In: Nriagu JO, ed. Arsenic in the environment: Part II: Human health and ecosystem effects. New York, NY: John Wiley \& Sons, Inc., 185-259.

Francis CW, White GH. 1987. Leaching of toxic metals from incinerator ashes. J Water Pollut Control Fed 59(11):979-986.

Franklin M, Bean WB, Harden RC. 1950. Fowler's solution as an etiologic agent in cirrhosis. Am J Med Sci 219:589-596.

Franzblau A, Lilis R. 1989. Acute arsenic intoxication from environmental arsenic exposure. Arch Environ Health 44(6):385-390.

Freeman GB, Johnson JD, Killinger JM, et al. 1993. Bioavailability of arsenic in soil impacted by smelter activities following oral administration in rabbits. Fundam Appl Toxicol 21(1):83-88.

Freeman GB, Schoof RA, Ruby MV, et al. 1995. Bioavailability of arsenic in soil and house dust impacted by smelter activities following oral administration in cynomolgus monkeys. Fundam Appl Toxicol 28(2):215-222.

Frey MM, Edwards MA. 1997. Surveying arsenic occurrence. J Am Water Works Assoc 89(3):105-117. 
*Frost DV. 1987. Interrelationships of selenium and arsenic in biology. In: Combs GF, Levander OA, Spallholz JE, et al., eds. Selenium in biology and medicine: Part A: Proceedings of the Third International Symposium, Beijing, China, May 27-June 1, 1984. New York, NY: Van Nostrand Reinhold Company, 308-323.

Frost F, Harter L, Milham S, et al. 1987. Lung cancer among women residing close to an arsenic emitting copper smelter. Arch Environ Health 42(2):148-152.

Frost FJ, Muller T, Petersen HV, et al. 2003. Identifying US populations for the study of health effects related to drinking water arsenic. J Expo Anal Environ Epidemiol 13(3):231-239.

*Fujino Y, Guo X, Liu J, et al. 2004. Mental health burden amongst inhabitants of an arsenic-affected area in Inner Mongolia, China. Soc Sci Med 59(9):1963-1973.

Gailer J, George GN, Pickering IJ, et al. 2002. Biliary excretion of [(GS)2AsSe]- after intravenous injection of rabbits with arsenite and selenate. Chem Res Toxicol 15:1466-1471.

Gaines TB. 1960. The acute toxicity of pesticides to rats. Toxicol Appl Pharmacol 2:88-99.

Gallagher RE. 1998. Arsenic--new life for old potion. N Engl J Med 339(19):1389-1391.

Gao S, Burau RG. 1997. Environmental factors affecting rates of arsine evolution from and mineralization of arsenicals in soils. J Environ Qual 26(3):753-763.

Garbarino JR, Bednar AJ, Rutherford DW, et al. 2003. Environmental fate of roxarsone in poultry litter. I. Degradation of roxarsone during composting. Environ Sci Technol 37:1509-1514.

Garcia F, Ortega A, Domingo JL, et al. 2001. Accumulation of metals in autopsy tissues of subjects living in Tarragona county, Spain. J Environ Sci Health Part A 36(9):1767-1786.

García-Vargas GG, Hernández-Zavala A. 1996. Urinary porphyrins and heme biosynthetic enzyme activities measured by HPLC in arsenic toxicity. Biomed Chromatogr 10(6):278-284 .

*García-Vargas GG, Del Razo LM, Cebrián ME, et al. 1994. Altered urinary porphyrin excretion in a human population chronically exposed to arsenic in Mexico. Hum Exp Toxicol 13(12):839-847.

*Garland M, Morris JS, Rosner BA, et al. 1993. Toenail trace element levels as biomarkers: Reproducibility over a 6-year period. Cancer Epidemiol Biomarkers Prev 2(5):493-497.

Gartrell MJ, Craun JC, Podrebarac DS, et al. 1986. Pesticides, selected elements, and other chemicals in adult total diet samples, October 1980-March 1982. J Assoc Off Anal Chem 69(1):146-161.

Garvey GJ, Hahn G, Lee RV, et al. 2001. Heavy metal hazards of Asian traditional remedies. Int J Environ Health Res 11(1):63-71.

*Gebel T. 1997. Arsenic and antimony: Comparative approach on mechanistic toxicology. Chem Biol Interact 107:131-144.

Gebel T, Birkenkamp P, Luthin S, et al. 1998a. Arsenic(III), but not antimony(III), induces DNA-protein crosslinks. Anticancer Res 18:4253-4258. 
Gebel T, Christensen S, Dunkelberg H. 1997. Comparative and environmental genotoxicity of antimony and arsenic. Anticancer Res 17(4a):2603-2607.

Gebel TW, Suchenwirth RHR, Bolten C, et al. 1998b. Human biomonitoring of arsenic and antimony in case of an elevated geogenic exposure. Environ Health Perspect 106(1):33-39.

*Geiszinger A, Goessler W, Pedersen SN, et al. 2001. Arsenic biotransformation by the brown macroalga, Fucus serratus. Environ Toxicol Chem 20(10):2255-2262.

Gentry PR, Covington TR, Mann S, et al. 2004. Physiologically based pharmacokinetic modeling of arsenic in the mouse. J Toxicol Environ Health A 67(1):43-71.

Gerhardsson L, Brune D, Nordberg IGF, et al. 1985. Protective effect of selenium on lung cancer in smelter workers. Br J Ind Med 42:617-626.

Gerhardsson L, Brune D, Nordberg IGF, et al. 1988. Multielemental assay of tissues of deceased smelter workers and controls. Sci Total Environ 74:97-110.

*Gerhardt RE, Hudson JB, Rao RN, et al. 1978. Chronic renal insufficiency from cortical necrosis induced by arsenic poisoning. Arch Intern Med 138:1267-1269.

*Germolec DR, Spalding J, Boorman GA, et al. 1997. Arsenic can mediate skin neoplasia by chronic stimulation of keratinocyte-derived growth factors. Mutat Res 86(3):209-218.

Germolec DR, Spalding J, Yu H, et al. 1998. Arsenic enhancement of skin neoplasia by chronic stimulation of growth factors. Am J Pathol 153(6):1775-1785.

Gerr F, Letz R, Ryan PB, et al. 2000. Neurological effects of environmental exposure to arsenic in dust and soil among humans. Neurotoxicology 21(4):475-87.

Geubel AP, Mairlot MC, Buchet JP, et al. 1988. Abnormal methylation capacity in human liver cirrhosis. Int J Clin Pharmacol Res 8(2):117-122.

Ghosh P, Basu A, Mahata J, et al. 2006. Cytogenic damage and genetic variants in the individuals susceptible to arsenic-induced cancer through drinking water. Int J Cancer 118(10):2470-2478.

*Gianessi LP, Anderson JE. 1995a. Cacodylic acid. Pesticide use in U.S. crop production: National data report. Washington DC: National Center for Food and Agricultural Policy.

*Gianessi LP, Anderson JE. 1995b. Pesticide use in U.S. crop production. Washington, DC: National Center for Food and Agricultural Policy.

Gibson DP, Brauninger R, Shaffi HS, et al. 1997. Induction of micronuclei in Syrian hamster embryo cells: Comparison of results in the SHE cell transformation assay for national toxicology program test chemicals. Mutat Res 392(1-2):61-70.

Giwercman A, Carlsen E, Keiding N, et al. 1993. Evidence for increasing incidence of abnormalities of the human testis: A review. Environ Health Perspect Suppl 101(2):65-71.

Glazener FS, Ellis JG, Johnson PK. 1968. Electrocardiographic findings with arsenic poisoning. Calif Med 109(2):158-162. 
Goddard MJ, Tanhehco JL, Dau PC. 1992. Chronic arsenic poisoning masquerading as Landry-GuillainBarre syndrome. Electromyogr Clin Neurophysiol 32(9):419-423.

Goebel HH, Schmidt PF, Bohl J, et al. 1990. Polyneuropathy due to acute arsenic intoxication: Biopsy studies. J Neuropathol Exp Neurol 49(2):137-149.

Goering PL, Maronpot RR, Fowler BA. 1988. Effect of intratracheal gallium arsenide administration on -aminolevulinic acid dehydratase in rats: Relationship to urinary excretion of aminolevulinic acid. Toxicol Appl Pharmacol 92:179-193.

Goldfrank RL, Flomenbaum NE, Lewin NA, et al., eds. 1998. Goldfrank's toxicologic emergencies. $6^{\text {th }}$ ed. Stamford, CT: Appleton and Lange, 1261-1273.

Goldsmith JR, Deane M, Thom J, et al. 1972. Evaluation of health implications of elevated arsenic in well water. Water Res 6:1133-1136.

Goldsmith S, From AHL. 1986. Arsenic-induced atypical ventricular tachycardia. N Engl J Med 303:1096-1097.

Golomb D, Ryan D, Eby N, et al. 1997. Atmospheric deposition of toxics onto Massachusetts Bay-I. Metals. Atmos Environ 31(9):1349-1359.

*Gonsebatt ME, Vega L, Salazar AM, et al. 1997. Cytogenetic effects in human exposure to arsenic. Mutat Res 386(3):219-228.

Gonzalez MJ, Aguilar MV, Martínez Para MC. 1995. Gastrointestinal absorption of inorganic arsenic (V): The effect of concentration and interactions with phosphate and dichromate. Vet Human Toxicol 37:131-136.

Grandjean P, Weihe P, Needham LL, et al. 1995. Relation of a seafood diet to mercury, selenium, arsenic, and polychlorinated biphenyl and other organochlorine concentrations in human milk. Environ Res 71(1):29-38.

*Greenberg SA. 1996. Acute demyelinating polyneuropathy with arsenic ingestion. Muscle Nerve 19(12):1611-1613.

Groen K, Vaessen HAMG, Kliest JJG, et al. 1993. Bioavailability of inorganic arsenic from bog orecontaining soil in the dog. Environ Health Perspect 102(2):182-184.

Guerin T, Astruc A, Astruc M. 1999. Speciation of arsenic and selenium compounds by HPLC hyphenated to specific detectors: A review of the main separation techniques. Talanta 50:1-24.

Guha Mazumder DN. 1996. Treatment of chronic arsenic toxicity as observed in West Bengal. J Indian Med Assoc 94(2):41-42.

Guha Mazumder DN. 2005. Effect of chronic intake of arsenic-contaminated water on liver. Toxicol Appl Pharmacol 206(2):169-175.

Guha Mazumder DN, Chakraborty AK, Ghose A, et al. 1988. Chronic arsenic toxicity from drinking tubewell water in rural west Bengal. Bull WHO 66(4):499-506. 
9. REFERENCES

Guha Mazumder DN, Das Gupta J, Santra A, et al. 1998a. Chronic arsenic toxicity in west Bengal--the worst calamity in the world. J Indian Med Assoc 96(1):4-7.

Guha Mazumder DN, Ghoshal UC, Saha J, et al. 1998b. Randomized placebo-controlled trial of 2,3dimercaptosuccinic acid in therapy of chronic arsenicosis due to drinking arsenic-contaminated subsoil water. Clin Toxicol 36(7):683-690.

Guha Mazumder DN, Haque R, Ghosh N, et al. 1998c. Arsenic levels in drinking water and the prevalence of skin lesions in West Bengal, India. Int J Epidemiol 27:871-877.

Guha Mazumder DN, Steinmaus C, Bhattacharya P, et al. 2005. Bronchiectasis in persons with skin lesions resulting from arsenic in drinking water. Epidemiology 16(6):760-765.

Gunderson EL. 1995a. Dietary intake of pesticides, selected elements, and other chemicals: FDA total diet study, June 1984-April 1986. J AOAC Int 78(4):910-921.

Gunderson EL. 1995b. FDA total diet study, July 1986-April 1991, dietary intakes of pesticides, selected elements, and other chemicals. J AOAC Int 78(6):1353-63.

Gunn TM, Juriloff DM, Harris MJ. 1992. Further genetic studies of the cause of exancephaly in SELH mice. Teratology 45:679-686.

Guo HR. 2004. Arsenic level in drinking water and mortality of lung cancer (Taiwan). Cancer Causes Control 15:171-177.

Guo HR, Tseng YC. 2000. Arsenic in drinking water and bladder cancer: Comparison between studies based on cancer registry and death certificates. Environ Geochem Health 22: 83-91.

Guo HR, Yu HS, Hu H, et al. 2001b. Arsenic in drinking water and skin cancers: Cell-type specificity (Taiwan, R.O.C.). Cancer Causes Control 12(10):909-916.

Guo T, Baasner J, Tsalev DL. 1997. Fast automated determination of toxicologically relevant arsenic in urine by flow injection-hydride generation atomic absorption spectrometry. Analytica Chimica Acta 349(1-3):313-318.

Guo X, Fujino Y, Kaneko S, et al. 2001a. Arsenic contamination of groundwater and prevalence of arsenical dermatosis in the Hetao plain area, Inner Mongolia, China. Mol Cell Biochem 222(1-2):137140.

Gur E, Nyska A. 1990. Acute oral toxicity study in rats. Luxembourg Industries (Pamol) Ltd. Submitted to the U.S. Environmental Protection Agency. MRID45405601.

Gur E, Nyska A, Pirak M, et al. 1989a. Cacodylic acid oncogenicity study in the mouse. Luxembourg Industries (Pamol) Ltd. Submitted to the U.S. Environmental Protection Agency. MRID41914601.

Gur E, Nyska A, Waner T, et al. 1989b. Cacodylic acid combined chronic feeding and oncogenicity study in the rat. Luxembourg Industries (Pamol) Ltd. Submitted to the U.S. Environmental Protection Agency. MRID41862101. 
Gur E, Pirak M, Waner T. 1991. Methanearsonic acid oncogenicity study in the mouse. Luxembourg Industries (Pamol) Ltd. Submitted to the U.S. Environmental Protection Agency. MRID42173201.

Gutleb AC, Helsberg A, Mitchell C. 2002. Heavy metal concentrations in fish from a pristine rainforest valley in Peru: A baseline study before the start of oil-drilling activities. Bull Environ Contam Toxicol 69:523-529.

Guzelian PS, Henry CJ, Olin SS, eds. 1992. Similarities and differences between children and adults: Implications for risk assessment. Washington, DC: International Life Sciences Institute Press.

Haddad LM, Winchester JF. 1990. Clinical management of poisoning and drug overdose. 2nd ed. Philadelphia, PA: W.B. Saunders Company, 1024-1027, 18, 92, 97, 98.

*Hall JC, Harruff R. 1989. Fatal cardiac arrhythmia in a patient with interstitial myocarditis related to chronic arsenic poisoning. South Med J 82(12):1557-1560.

Hamel SC, Buckley B, Lioy PJ. 1998. Bioaccessibility of metals in soils for different liquid to solid ratios in synthetic gastric fluid. Environ Sci Tech 32(3):358-362.

Hanlon DP, Ferm VH. 1977. Placental permeability of arsenate ion during early embryogenesis in the hamster. Experientia 33(9):1221-1222.

Hanlon DP, Ferm VH. 1986. Teratogen concentration changes as the basis of the heat stress enhancement of arsenate teratogenesis in hamsters. Teratology 34:189-193.

*Hanlon DP, Ferm VH. 1987. The concentration and chemical status of arsenic in the early placentas of arsenate-dosed hamsters. Environ Res 42:546-552.

Hantson P, Verellen-Dumoulin C, Libouton JM, et al. 1996. Sister chromatid exchanges in human peripheral blood lymphocytes after ingestion of high doses of arsenicals. Int Arch Occup Environ Health 68:342-344.

Hanusch K, Grossmann H, Herbst K, et al. 1985. Arsenic and arsenic compounds. In: Gerhartz W, Yamamoto YS, Campbell FT, et al., eds. Ullman's encyclopedia of industrial chemistry. Weinham, Germany: VCH Verlagsgesellschaft, 113-141

Haque R, Mazumder DN, Samanta S, et al. 2003. Arsenic in drinking water and skin lesions: Doseresponse data from West Bengal, India. Epidemiology 14(2):174-182.

Harrington JM, Middaugh JP, Morse DL, et al. 1978. A survey of a population exposed to high concentrations of arsenic in well water in Fairbanks, Alaska. Am J Epidemiol 108(5):377-385.

Harrisson JWE, Packman EW, Abbott DD. 1958. Acute oral toxicity and chemical and physical properties of arsenic trioxides. Arch Ind Health 17:118-123.

Hartmann A, Speit G. 1994. Comparative investigations of the genotoxic effects of metals in the single cell gel (SCG) assay and the sister chromatid exchange (SCE) test. Environ Mole Mutagen 23(4):299305.

Hartmann A, Speit G. 1996. Effect of arsenic and cadmium on the persistence of mutagen-induced DNA lesions in human cells. Environ Mol Mutagen 27(2):98-104. 
*Hartwell TW, Handy RW, Harris BS, et al. 1983. Heavy metal exposure in populations living around zinc and copper smelters. Arch Environ Health 38(5):284-295.

Hartwig A, Gröblinghoff UD, Beyersmann D, et al. 1997. Interaction of arsenic(III) with nucleotide excision repair in UV-irradiated human fibroblasts. Carcinogenesis 18(2):399-405.

Hartwig A, Mullenders L, Asmuá M, et al. 1998. Disruption of DNA repair process by carcinogenic metal compounds. Fresenius J Anal Chem 361:377-380.

Haupert TA, Wiersma JH, Goldring JM. 1996. Health effects of ingesting arsenic-contaminated groundwater. Wis Med J 95(2):100-104.

Hayakawa T, Kobayashi Y, Cui X, et al. 2005. A new metabolic pathway of arsenite: Arseniteglutathione complexes are substrates for human arsenic methyltransferase Cyt19. Arch Toxicol 79:183191.

Hayashi H, Kanisawa M, Yamanaka K, et al. 1998. Dimethylarsinic acid, a main metabolite of inorganic arsenics, has tumorigenecity and progression effects in the pulmonary tumors of $\mathrm{A} / \mathrm{J}$ mice. Cancer Lett 125:83-88.

HazDat. 2006. Arsenic. ATSDR's Hazardous substance release and health effects database. Agency for Toxic Substances and Disease Registry. http://www.atsdr.cdc.gov/hazdat.html. October 16, 2006.

He X, Logan TJ, Traina SJ. 1995. Physical and chemical characteristics of selected US municipal solid waste compost. J Environ Qual 24:543-552.

Healy SM, Casarez EA, Ayala-Fierro F, et al. 1998. Enzymatic methylation of arsenic compounds. V. Arsenite methyltransferase activity in tissues of mice. Toxicol Appl Pharmacol 148(1):65-70.

Heck JE, Gamble MV, Chen Y, et al. 2007. Comsumption of folate-related nutrients and metabolism of arsenic in Bangladesh. Am J Clin Nutr 85:1367-1374.

Hei TK, Liu SX, Waldren C. 1998. Mutagenicity of arsenic in mammalian cells: Role of reactive oxygen species. Proc Natl Acad Sci U S A 95:8103-8107.

Heiny JS, Tate CM. 1997. Concentration, distribution, and comparison of selected trace elements in bed sediment and fish tissue in the South Platte River basin, USA, 1992-1993. Arch Environ Contam Toxicol 32(3):246-259.

Heit M, Klusek C, Baron J. 1984. Evidence of deposition of anthropogenic pollutants in remote Rocky Mountain lakes. Water Air Soil Pollut 22:403-416.

Heitkemper DT, Kaine LA, Jackson DS, et al. 1994. Practical applications of element-specific detection by inductively coupled plasma atomic emission spectroscopy and inductively coupled plasma mass spectrometry to ion chromatography of foods. J Chromatogr A 671(1-2):101-108.

Helgesen H, Larsen EH. 1998. Bioavailability and speciation of arsenic in carrots grown in contaminated soil. Analyst 123(5):791-796. 
9. REFERENCES

Helleday T, Nilsson R, Jenssen D. 2000. Arsenic [111] and heavy metal ions induce intrachromosomal homologous recombination in the hprt gene V79 Chinese hamster cells. Environ Mol Mutagen 35(2):114-122.

Hemond HF, Solo-Gabriele HM. 2004. Children's exposure to arsenic from CCA-treated wooden decks and playground structures. Risk Anal 24(1):51-64.

Hernández-Zavala A, Del Razo LM, Aguilar C, et al. 1998. Alteration in bilirubin excretion in individuals chronically exposed to arsenic in Mexico. Toxicol Lett 99:79-84.

Hershey JW, Oostdyk TS, Keliher PN. 1988. Determination of arsenic and selenium in environmental and agricultural samples by hydride generation atomic adsorption spectrometry. J Assoc Off Anal Chem 71(6):1090-1093.

Hertz-Picciotto I, Smith AH. 1993. Observations on the dose-response curve for arsenic exposure and lung cancer. Scand J Work Environ Health 19(4):217-226.

*Hessl SM, Berman E. 1982. Severe peripheral neuropathy after exposure to monosodium methyl arsonate. J Toxicol Clin Toxicol 19:281-287.

Hewitt DJ, Millner GC, Nye AC, et al. 1995. Investigation of arsenic exposure from soil at a Superfund site. Environ Res 68(2):73-81.

Heydorn K. 1970. Environmental variation of arsenic levels in human blood determined by neutron activation analysis. Clin Chim Acta 28:349-357.

Heyman A, Pfeiffer JB, Willett RW, et al. 1956. Peripheral neuropathy caused by arsenical intoxication: A study of 41 cases with observations on the effects of BAL (2,3 dimercapto-propanol). N Engl J Med 254(9):401-409.

Heywood R, Sortwell RJ. 1979. Arsenic intoxication in the Rhesus monkey. Toxicol Lett 3:137-144.

Hill CH. 1975. Interrelationships of selenium with other trace elements. Fed Proc 34(11):2096-2100.

Hindmarsh JT, McCurdy RF. 1986. Clinical and environmental aspects of arsenic toxicity. CRC Crit Rev Clin Lab Sci 23:315-347.

Hindmarsh JT, McLetchie OR, Heffernan LPM, et al. 1977. Electromyographic abnormalities in chronic environmental arsenicalism. J Anal Toxicol 1:270-276.

Hingston JA, Collins CD, Murphy RJ, et al. 2001. Leaching of chromated copper arsenate wood preservatives: A review. Environ Pollut 111(1):53-66.

Hirata M, Hisanaga A, Tanaka A, et al. 1988. Glutathione and methylation of inorganic arsenic in hamsters. Appl Organomet Chem 2:315-321.

Historic Pesticide Contamination Task Force. 1999. Findings and recommendations for the remediation of historic pesticide contamination. New Jersey Department of Environmental Protection. Historic Pesticide Contamination Task Force. http://www.state.nj.us/dep/special/hpctf/final/hpctf99.pdf. August $27,2007$. 
Hochadel JF, Waalkes MP. 1997. Sequence of exposure to cadmium and arsenic determines the extent of toxic effects in male Fischer rats. Toxicology 116:89-98.

Hoel DG, Davis DL, Miller AB, et al. 1992. Trends in cancer mortality in 15 industrialized countries, 1969-1986. J Natl Cancer Inst 84(5):313-320.

Hoff RM, Strachan WMJ, Sweet CW, et al. 1996. Atmospheric deposition of toxic chemicals to the Great Lakes: A review of data through 1994. Atmos Environ 30(20):3505-3527.

Holland JW. 1904. Arsenic. In: Peterson F, Haines WS, eds. A textbook of legal medicine and toxicology. Philadelphia, PA: WB Saunders and Co., 404-433.

Holland RH, McCall MS, Lanz HC. 1959. A study of inhaled arsenic-74 in man. Cancer Res 19:11541156.

Holmberg RE, Ferm VH. 1969. Interrelationships of selenium, cadmium and arsenic in mammalian teratogenesis. Arch Environ Health 18:873-877.

Holmqvist I. 1951. Occupational arsenical dermatitis: A study among employees at a copper ore smelting work including investigations of skin reactions to contact with arsenic compounds. Acta Derm Venerol 31(Suppl 26):26-29, 44-45, 110-112, 195-204.

Holson JF, Stump DG, Clevidence KJ, et al. 2000. Evaluation of the prenatal developmental toxicity of orally administered arsenic trioxide in rats. Food Chem Toxicol 38:459-466.

Holson JF, Stump DG, Ulrich CE, et al. 1999. Absence of prenatal developmental toxicity from inhaled arsenic trioxide in rats. Toxicol Sci 51:87-97.

Hood RD. 1985. Cacodylic acid: Agricultural uses, biological effects and environmental fate.

Washington, DC: Agent Orange Projects Office. Veterans Administration Central Office, 1-2, 22-24, 3549, 95-106.

*Hood RD. 1998. Developmental effects of methylated arsenic metabolites in mice. Bull Environ Contam Toxicol 61:231-238.

Hood RD, Bishop SL. 1972. Teratogenic effects of sodium arsenate in mice. Arch Environ Health 24:62-65.

Hood RD, Harrison WP. 1982. Effects of prenatal arsenite exposure in the hamster. Bull Environ Contam Toxicol 29:671-678.

*Hood RD, Harrison WP, Vedel JC. 1982. Evaluation of arsenic metabolites for prenatal effects in the hamster. Bull Environ Contam Toxicol 29:679-687.

Hood RD, Thacker GT, Patterson BL, et al. 1978. Prenatal effects of oral versus intraperitoneal sodium arsenate in mice. J Environ Pathol Toxicol 1:857-864.

Hood RD, Vedel GC, Zaworotko MJ, et al. 1988. Uptake distribution and metabolism of trivalent arsenic in the pregnant mouse. J Toxicol Environ Health 25:423-434. 
Hood RD, Vedel-Macrander GC, Zaworotko MJ, et al. 1987. Distribution, metabolism and fetal uptake of pentavalent arsenic in pregnant mice following oral or intraperitoneal administration. Teratology 35:19-25.

Hopenhayn C, Bush HM, Bingcang A, et al. 2006. Asociation between arsenic exposure from drinking water and anemia during pregnancy. J Occup Environ Hyg 48(6):635-643.

Hopenhayn C, Ferreccio C, Browning SR, et al. 2003a. Arsenic exposure from drinking water and birth weight. Epidemiology 14(5):593-602.

Hopenhayn C, Huang B, Christian J, et al. 2003b. Profile of urinary arsenic metabolites during pregnancy. Environ Health Perspect 111(16):1888-1891.

Hopenhayn-Rich C, Biggs ML, Fuchs A, et al. 1996a. Bladder cancer mortality associated with arsenic in drinking water in Argentina. Epidemiology 7(2):117-124.

Hopenhayn-Rich C, Biggs ML, Smith AH, et al. 1996b. Methylation study of a population environmentally exposed to arsenic in drinking water. Environ Health Perspect 104(6):620-628.

Hopenhayn-Rich C, Biggs ML, Smith AH. 1998. Lung and kidney cancer mortality associated with arsenic in drinking water in Córdoba, Argentina. Int J Epidemiol 27:561-569.

Hopenhayn-Rich C, Browning SR, Hertz-Picciotto I, et al. 2000. Chronic arsenic exposure and risk of infant mortality in two areas of Chile. Environ Health Perspect 108(7):667-673.

Howell GO, Hill CH. 1978. Biological interaction of selenium with other trace elements in chicks. Environ Health Perspect 25:147-150.

HSDB. 2007. Arsenic. Hazardous Substances Data Bank. National Library of Medicine. http://toxnet.nlm.nih.gov. January 16, 2007.

*Hsieh LL, Chen HJ, Hsieh JT, et al. 1994. Arsenic-related Bowen's disease and paraquat-related skin cancerous lesions show no detectable ras and p53 gene alterations. Cancer Lett 86:59-65.

Hsu CH, Yang SA, Wang JY, et al. 1999. Mutational spectrum of p53 gene in arsenic-related skin cancers from the blackfoot disease endemic area of Taiwan. Br J Cancer 80(7):1080-1086.

Hsu YH, Li SY, Chiou HY, et al. 1997. Spontaneous and induced sister chromatid exchanges and delayed cell proliferation in peripheral lymphocytes of Bowen's disease patients and matched controls of arseniasis-hyperendemic villages in Taiwan. Mutat Res 386(3):241-251.

Hsueh YM, Cheng GS, Wu MM, et al. 1995. Multiple risk factors associated with arsenic-induced skin cancer: Effects of chronic liver disease and malnutritional status. Br J Cancer 71(1):109-14.

Hsueh YM, Huang YL, Huang CC, et al. 1998a. Urinary levels of inorganic and organic arsenic metabolites among residents in an arseniasis-hyperendemic area in Taiwan. J Toxicol Environ Health 54(6):431-444.

Hsueh YM, Lin P, Chen HW, et al. 2005. Genetic polymorphisms of oxidative and antioxidant enzymes and arsenic-related hypertension. J Toxicol Environ Health A 68(17-18):1471-1484. 
Hsueh YM, Wu WL, Huang YL, et al. 1998b. Low serum carotene level and increase risk of ischemic heart disease related to long-term arsenic exposure. Atherosclerosis 141:249-257.

$\mathrm{Hu}$ Y, Su L, Snow ET. 1998. Arsenic toxicity is enzyme specific and its effects on ligation are not caused by the direct inhibition of DNA repair enzymes. Mutat Res 408:203-218.

Huang H, Huang CF, Huang JS, et al. 1992. The transition from late $\mathrm{G}_{1}$ to early $\mathrm{S}$ phase is most vulnerable to the coclastogenic effect of ultraviolet radiation plus arsenite. Int J Radiat Biol 61(1):57-62.

Huang H, Huang CF, Wu DR, et al. 1993. Glutathione as a cellular defense against arsenite toxicity in cultured Chinese hamster ovary cells. Toxicology 79(3):195-204.

Huang RN, Ho IC, Yih LH, et al. 1995. Sodium arsenite induces chromosome endoreduplication and inhibits protein phosphatase activity in human fibroblasts. Environ Mol Mutagen 25(3):188-196.

*Huang SY, Chang CS, Tang JL, et al. 1998. Acute and chronic arsenic poisoning associated with treatment of acute promyelocytic leukaemia. Br J Haematol 103:1092-1095.

Huang YZ, Qian XC, Wang GQ, et al. 1985. Endemic chronic arsenism in Xinjiang. Chin Med J 98(3):219-222.

Huggett DB, Khan IA, Allgood JC, et al. 2001. Organochlorine pesticides and metals in select botanical dietary supplements. Bull Environ Contam Toxicol 66:150-155.

Hughes MF. 2002. Arsenic toxicity and potential mechanisms of action. Toxicol Lett 133(1):1-16.

Hughes MF. 2006. Biomarkers of exposure: A case study with inorganic arsenic. Environ Health Perspect 114:1790-1796.

*Hughes MF, Kenyon EM. 1998. Dose-dependent effects on the disposition of monomethylarsonic acid and dimethylarsinic acid in the mouse after intravenous administration. J Toxicol Environ Health A53(2):95-112.

Hughes MF, Del Razo LM, Kenyon EM. 2000. Dose-dependent effects on tissue distribution and metabolism of dimethylarsinic acid in the mouse after intravenous administration. Toxicology 143(2):155-166.

Hughes MF, Devesa V, Adair BM, et al. 2005. Tissue dosimetry, metabolism and excretion of pentavalent and trivalent monomethylated arsenic in mice after oral administration. Toxicol Appl Pharmacol 208:186-197.

Hughes MF, Kenyon EM, Edwards BC, et al. 2003. Accumulation and metabolism of arsenic in mice after repeated oral administration of arsenate. Toxicol Appl Pharmacol 191(3):202-210.

Hughes MF, Menache M, Thompson DJ. 1994. Dose-dependent disposition of sodium arsenate in mice following acute oral exposure. Fundam Appl Toxicol 22(1):80-89.

*Hullinger G, Sangster L, Colvin B, et al. 1998. Bovine arsenic toxicosis from ingestion of ashed copper-chrome-arsenate treated timber. Vet Hum Toxicol 40(3):147-148. 
Hwang YH, Chen SC. 2000. Monitoring of low level arsenic exposure during maintenance of ion implanters. Arch Environ Health 55(5):347-354.

Hwang YH, Bornschein RL, Grote J, et al. 1997a. Environmental arsenic exposure of children around a former copper smelter site. Environ Res 72(1):72-81.

Hwang YH, Bornschein RL, Grote J, et al. 1997b. Urinary arsenic excretion as a biomarker of arsenic exposure in children. Arch Environ Health 52(2):139-147.

IARC. 1980. Arsenic and arsenic compounds. IARC monographs on the evaluation of the carcinogenic risk of chemicals to humans. Vol. 23. Some metals and metallic compounds. Lyon, France:

International Agency for Research on Cancer, 39-141.

IARC. 2004. Overall evaluations of carcinogenicity to humans: As evaluated in IARC Monographs volumes 1-82 (at total of 900 agents, mixtures and exposures). Lyon, France: International Agency for Research on Cancer. http://www-cie.iarc.fr/monoeval/crthall.html. March 08, 2006.

Ide CW, Bullough GR. 1988. Arsenic and old glass. J Soc Occup Med 38:85-88.

Ihrig MM, Shalat SL, Baynes C. 1998. A hospital-based case-control study of stillbirths and environmental exposure to arsenic using and atmospheric dispersion model linked to a geographical information system. Epidemiology 9(3):290-294.

Inoue Y, Kawabata K, Takahashi H, et al. 1994. Determination of arsenic compounds using inductively coupled plasma mass spectrometry with ion chromatography. J Chromatog A675(1-2):149-154.

IRIS. 2007. Arsenic. Integrated Risk Information System. Washington, DC: U.S. Environmental Protection Agency. http://www.epa.gov/iris/subst/index.html. August 28, 2007.

Irvine L, Boyer IJ, DeSesso JM. 2006. Monomethylarsonic acid and dimethylarsenic acid:

Developmental toxicity studies with risk assessment. Birth Defects Res B Dev Reprod Toxicol 77:53-68.

*Ishinishi N, Kodama Y, Nobutomo K, et al. 1977. Preliminary experimental study on carcinogenicity of arsenic trioxide in rat lung. Environ Health Perspect 19:191-196.

Ishinishi N, Yamamoto A, Hisanaga A, et al. 1983. Tumorigenicity of arsenic trioxide to the lung in Syrian golden hamsters by intermittent instillations. Cancer Lett 21:141-147.

ITA. 2007a. U.S. trade quick-reference tables: December 2005 imports. 280480: Arsenic. International Trade Association, U.S. Department of Commerce. http://hqtpisweb.ita.doc.gov/portal/page/portal/rptsforms/p_hsmthly?p_year=2005\&p_hs $=2804800000 \& p \_m o n t h$ $="$ December"\&p_flow="imports"\&p_table=ita.hs_10dig_mcons_mth_Dec_2005. February 07, 2007.

ITA. 2007b. U.S. trade quick-reference tables: December 2005 imports. 281129: Arsenic trioxide. International Trade Association, U.S. Department of Commerce. http://hqtpisweb.ita.doc.gov/portal/page/portal/rptsforms/p_hsmthly?p_year=2005\&p_hs=2811291000\&p_month ="December"\&p_flow="imports"\&p_table=ita.hs_10dig_mcons_mth_Dec_2005. February 07, 2007.

*Ivankovic S, Eisenbrand G, Preussmann R. 1979. Lung carcinoma induction in BD rats after a single intratracheal instillation of an arsenic-containing pesticide mixture formerly used in vineyards. Int $\mathrm{J}$ Cancer 24:786-788. 
*Jacobson-Kram D, Montalbano D. 1985. The reproductive effects assessment group's report on the mutagenicity of inorganic arsenic. Environ Mutagen 7:787-804.

Jager JW, Ostrosky-Wegman P. 1997. Arsenic: A paradoxical human carcinogen. Mutat Res 386(3):181-184.

Jaghabir MTW, Abdelghani A, Anderson AC. 1988. Oral and dermal toxicity of MSMA to New Zealand white rabbits, Oryctalagus cuniculus. Bull Environ Contam Toxicol 40:119-122.

Jaghabir MTW, Abdelghani AA, Anderson AC. 1989. Histopathological effects of monosodium methanearsonate (MSMA) on New Zealand white rabbits (Oryctalagus cuniculus). Bull Environ Contam Toxicol 42:289-293.

Jain A, Loeppert RH. 2000. Effect of competing anions on the adsorption of arsenate and arsenite by ferrihydrite. J Environ Qual 29:1422-1430.

Jain A, Raven KP, Loeppert RH. 1999. Arsenite and arsenate adsorption on ferrihydrite: Surface charge reduction and net $\mathrm{OH}^{-}$release stoichiometry. Environ Sci Technol 33:1179-1184.

Jan KY, Huang RY, Lee TC. 1986. Different modes of action of sodium arsenite, 3-aminobenzamide and caffeine on the enhancement of ethyl methanesulfonate clastogenicity. Cytogenet Cell Genet 41:202208.

Janssen RPT, Peijnenburg WJGM, Posthuma L, et al. 1997. Equilibrium partitioning of heavy metals in Dutch field soils: I. Relationship between metal partition coefficients and soil characteristics. Environ Toxicol Chem 16(12):2470-2478.

Järup L, Pershagen G. 1991. Arsenic exposure, smoking, and lung cancer in smelter workers - a casecontrol study. Am J Epid 134(6):545-551.

Järup L, Pershagen G, Wall S. 1989. Cumulative arsenic exposure and lung cancer in smelter workers: A dose-response study. Am J Ind Med 15:31-41.

Jelinek CF, Corneliussen PE. 1977. Levels of arsenic in the United States food supply. Environ Health Perspect 19:83-87.

Jenkins RB. 1966. Inorganic arsenic and the nervous system. Brain 89:479-498.

Jensen GE, Hansen ML. 1998. Occupational arsenic exposure and glycosylated haemoglobin. Analyst 123(1):77-80.

Jensen GE, Olsen ILB. 1995. Occupational exposure to inorganic arsenic in wood workers and taxidermists-air sampling and biological monitoring. J Environ Sci Health A30(4):921-938.

Jha AN, Noditi M, Nilsson R, et al. 1992. Genotoxic effects of sodium arsenite on human cells. Mutat Res 284(2):215-221.

*Jiang JQ. 2001. Removing arsenic from groundwater for the developing world--a review. Water Sci Technol 44(6):89-98. 
*Jianhua N, Guoqiang C, Zhiziang S, et al. 1998. Pharmacokinetics of intravenous arsenic trioxide in the treatment of acute promyelocytic leukemia. Chin Med J 111(12):1107-1110.

Jin Y, Xi S, Li X, et al. 2006. Arsenic speciation transported through the placenta from mother mice to their newborn pups. Environ Res 101(3):349-355.

Johansen MG, McGowan JP, Tu SH, et al. 1984. Tumorigenic effect of dimethylarsinic acid in the rat. Proc West Pharmacol Soc 27:289-291.

Johanson CE. 1980. Permeability and vascularity of the developing brain: Cerebellum vs cerebral cortex. Brain Res 190:3-16.

Johnson LR, Farmer JG. 1989. Urinary arsenic concentrations and speciation in Cornwall residents. Environ Geochem Health 11:39-44.

Jones CA, Inskeep WP, Neuman DR. 1997. Arsenic transport in contaminated mine tailings following liming. J Environ Qual 26(2):433-439.

*Jonnalagadda SB, Nenzou G. 1996. Studies on arsenic rich mine dumps: III. Effect on the river water. J Environ Sci Health A31(10):2547-2555.

Kachinskas DJ, Qin Q, Phillips MA, et al. 1997. Arsenate suppression of human keratinocyte programming. Mutat Res 386(3):253-261.

Kaise T, Watanabe S, Itoh K. 1985. The acute toxicity of arsenobetaine. Chemosphere 14(9):13271332.

Kaise T, Yamauchi H, Horiguchi Y, et al. 1989. A comparative study on acute toxicity of methylarsonic acid, dimethylarsinic acid and trimethylarsine oxide in mice. Appl Organomet Chem 3:273-277.

Kala SV, Neely MW, Kala G, et al. 2000. The MRP2/cMOAT transporter and arsenic-glutathione complex formation are required for biliary excretion of arsenic. J Biol Chem 275(43):33404-33408.

Kalbitz K, Wennrich R. 1998. Mobilization of heavy metals and arsenic in polluted wetland soils and its dependence on dissolved organic matter. Sci Total Environ 209:27-39.

Kalman DA. 1987. Dietary contributions to arsenic species in urine. In: Trace elements in human health and disease: Extended abstracts from the second Nordic symposium, Odense, 17-21 August 1987. Copenhagen: World Health Organization, 136-139, 230-233.

Kamijo Y, Soma K, Asari Y, et al. 1998. Survival after massive arsenic poisoning self-treated by high fluid intake. Clin Toxicol 36(1-2):27-29.

Kannan GM, Tripathi N, Dube SN, et al. 2001. Toxic effects of arsenic (III) on some hematopoietic and central nervous system variables in rats and guinea pigs. J Toxicol Clin Toxicol 37(7):675-682.

Karagas MR, Morris JS, Weiss JE, et al. 1996. Toenail samples as an indicator of drinking water arsenic exposure. Cancer Epidemiol Biomarkers Prev 5(10):849-852.

Karagas MR, Stukel TA, Morris JS, et al. 2001. Skin cancer risk in relation to toenail arsenic concentrations in a US population-based case-control study. Am J Epidemiol 153(6):559-565. 
Karagas MR, Tosteson TD, Blum J, et al. 1998. Design of an epidemiologic study of drinking water arsenic exposure and skin and bladder cancer risk in a U.S. population. Environ Health Perspect Suppl 106(4):1047-1050.

Karagas MR, Tosteson TD, Morris JS, et al. 2004. Incidence of transitional cell carcinoma of the bladder and arsenic exposure in New Hampshire. Cancer Causes Control 15:465-472.

Kashiwada E, Kuroda K, Endo G. 1998. Aneuploidy induced by dimethylarsinic acid in mouse bone marrow cells. Mutat Res 413:33-38.

Kasper ML, Schoenfield L, Strom RL, et al. 1984. Hepatic angiosarcoma and bronchioloalveolar carcinoma induced by Fowler's solution. JAMA 252(24):3407-3408.

Kato K, Hayashi H, Hasegawa A, et al. 1994. DNA damage induced in cultured human alveolar (L-132) cells by exposure to dimethylarsinic acid. Environ Health Perspect 102(Suppl 3):285-8.

*Katsnelson BA. 1998. Re: Arsenic carcinogenicity testing. Environ Health Perspect 106(12):582.

Kavanagh PJ, Farago ME, Thornton I, et al. 1997. Bioavailability of arsenic in soil and mine wastes of the Tamar Valley, SW England. Chem Speciat Bioavail 9(3):77-81.

Kavlock RJ, Chernoff N, Rogers EH. 1985. The effect of acute maternal toxicity on fetal development in the mouse. Teratog Carcinog Mutagen 5:3-13.

Kawaguchi K, Oku N, Rin K, et al. 1996. Dimethylarsenics reveal DNA damage induced by superoxide anion radicals. Biol Pharm Bull 19(4):551-553.

Kennedy S, Rice DA, Cush PF. 1986. Neuropathology of experimental 3-nitro-4-hydroxyphenylarsonic acid toxicosis in pigs. Vet Pathol 23:454-461.

*Kenyon EM, Hughes MF. 2001. A concise review of the toxicity and carcinogenicity of dimethylarsinic acid. Toxicology 160(1-3):227-236.

Kenyon EM, Del Razo LM, Hughes MF. 2005. Tissue distribution and urinary excretion of inorganic arsenic and its methylated metabolites in mice following acute oral administration of arsenate. Toxicol Sci 85(1):468-475.

*Kenyon EM, Hughes MF, Levander OA. 1997. Influence of dietary selenium on the disposition of arsenate in the female B6C3F mouse. J Toxicol Environ Health 51(3):279-299.

Kerckaert GA, Brauninger R, LeBoeuf RA, et al. 1996. Use of the Syrian hamster embryo cell transformation assay for carcinogenicity prediction of chemicals currently being tested by the national toxicology program in rodent bioassays. Environ Health Perspect 104(Suppl. 5):1075-1084.

Kerkvliet NI, Steppan LB, Koller LD, et al. 1980. Immunotoxicology studies of sodium arsenate effects of exposure on tumor growth and cell-mediated tumor immunity. J Environ Pathol Toxicol 4:6579.

Kerr HD, Saryan LA. 1986. Arsenic content of homeopathic medicines. Clin Toxicol 24(5):451-459. 
Kerr KB, Cavett JW, Thompson OL. 1963. The toxicity of an organic arsenical, 3-nitro-4-hydroxylphenylarsonic acid. I. Acute and subacute toxicity. Toxicol Appl Pharmacol 5:507-525.

Kersjes MP, Maurer JR, Trestrail JH, et al. 1987. An analysis of arsenic exposures referred to the Blodgett Regional Poison Center. Vet Hum Toxicol 29(1):75-78.

Kessel M, Liu SX, Xu A, et al. 2002. Arsenic induces oxidative DNA damage in mammalian cells. Mol Cell Biochem 234-235(1-2):301-308.

Khan AH, Tarafdar SA, Ali M, et al. 1989. The status of trace and minor elements in some Bangladeshi foodstuffs. J Radioanalyt Nuc Chem 134(2):367-381.

Kidwell JM, Phillips LJ, Birchard GF. 1995. Comparative analyses of contaminant levels in bottom feeding and predatory fish using the national contaminant biomonitoring program data. Bull Environ Contam Toxicol 54(6):919-923.

Kim MJ, Nriagu J, Haack S. 2002. Arsenic species and chemistry in groundwater of southeast Michigan. Environ Pollut 120(2):379-390.

Kingston RL, Hall S, Sioris L. 1993. Clinical observations and medical outcome in 149 cases of arsenate ant killer ingestion. J Toxicol Clin Toxicol 31(4):581-591.

Kinney PL, Chillrud SN, Ramstrom S, et al. 2002. Exposures to multiple air toxics in New York City. Environ Health Perspect 110(Suppl 4):539-546.

Kitamura M, Kuroda K, Endo Y, et al. 2002. Cysteine enhances clastogenic activity of dimethylarsinic acid. Appl Organomet Chem 16:391-396.

Kitchin KT. 2001. Recent advances in arsenic carcinogenesis: Modes of action, animal model systems, and methylated arsenic metabolites. Toxicol Appl Pharmacol 172(3):249-261.

*Kitchin KT, Ahmad S. 2003. Oxidative stress as a possible mode of action for arsenic carcinogenesis. Toxicol Lett 137(1-2):3-13.

Klemmer HW, Leitis E, Pfenninger K. 1975. Arsenic content of house dusts in Hawaii. Bull Environ Contam Toxicol 14(4):449-452.

Ko Y. 1986. A critical review of epidemiologic studies on blackfoot disease. J Voeh 8(3)339-353.

Kochhar TS, Howard W, Hoffman S, et al. 1996. Effect of trivalent and pentavalent arsenic in causing chromosome alterations in cultured Chinese hamster ovary (CHO) cells. Toxicol Lett 84(1):37-42.

Koh E, Kondoh N, Kaihara H, et al. 1989. Ureteral tumor with multiple Bowen's disease forty-two years after exposure to arsenic. Eur Urol 16:398-400.

*Kollmer WE. 1992. Arsenic in induced hair of the rat and its relation to the content in various organs during chronic exposure. J Trace Elem Electrolytes Health Dis 6(1):11-14.

Komori M, Nishio K, Kitada M et al. 1990. Fetus-specific expression of a form of cytochrome P-450 in human livers. Biochemistry 29:4430-4433. 
Kraus RJ, Ganther HE. 1989. Synergistic toxicity between arsenic and methylated selenium compounds. Biol Trace Elem Res 20:105-113.

Kraus T, Quidenus G, Schaller KH. 2000. Normal values for arsenic and selenium concentrations in human lung tissue. Arch Environ Contam Toxicol 38:384-389.

Kreiss K, Zack MM, Feldman RG, et al. 1983. Neurologic evaluation of a population exposed to arsenic in Alaskan well water. Arch Environ Health 38(2):116-121.

Kreppel H, Bauman JW, Liu J, et al. 1993. Induction of metallothionein by arsenicals in mice. Fundam Appl Toxicol 20:184-189.

Kreppel H, Liu J, Liu Y, et al. 1994. Zinc induced arsenite tolerance in mice. Fundam Appl Toxicol 23:32-37.

Kreppel H, Reichl FX, Kleine A, et al. 1995. Antidotal efficacy of newly synthesized dimercaptosuccinic acid (DMSA) monoesters in experimental arsenic poisoning in mice. Fundam Appl Toxicol 26(2):239-245.

Krishnan K, Andersen ME. 1994. Physiologically-based pharmacokinetic modeling in toxicology. In: Hayes AW, ed. Principles and methods of toxicology. 3rd ed. New York, NY: Raven Press, Ltd., 149188.

Krishnan K, Andersen ME, Clewell HJ, et al. 1994. Physiologically based pharmacokinetic modeling of chemical mixtures. In: Yang RSH, ed. Toxicology of chemical mixtures: Case studies, mechanisms, and novel approaches. San Diego, CA: Academic Press, 399-437.

Kroemer G, de Thé H. 1999. Arsenic trioxide, a novel mitochondriotoxic anticancer agent? J Natl Cancer Inst 91(9):743-745.

Kroes R, van Logten MJ, Berkvens JM, et al. 1974. Study on the carcinogenicity of lead arsenate and sodium arsenate and on the possible synergistic effect of diethylnitrosamine. Food Cosmet Toxicol 12(56):671-679.

Kumana CR, Au WY, Lee NSL, et al. 2002. Systemic availability of arsenic from oral arsenic-trioxide used to treat patients with hematological malignancies. Eur J Clin Pharmacol 58:521-526.

Kumaresan M, Riyazuddin P. 2001. Overview of speciation chemistry of arsenic. Curr Sci 80(7):837846.

Kuroda K, Yoshida K, Yoshimura M, et al. 2004. Microbial metabolite of dimethylarsinic acid is highly toxic and genotoxic. Toxicol Appl Pharmacol 198(3):345-353.

Kurokawa Y, Takahashi M, Maekawa A, et al. 1989. Promoting effect of metal compounds on liver, stomach, kidney, pancreas, and skin carcinogenesis. J Am Coll Toxicol 8(7)1235-1239.

Kurttio P, Komulain H, Hakala E, et al. 1998. Urinary excretion of arsenic species after exposure to arsenic present in drinking water. Arch Environ Contam Toxicol 34:297-305.

Kurttio P, Pukkala E, Kahelin H, et al. 1999. Arsenic concentrations in well water and risk of bladder and kidney cancer in Finland. Environ Health Perspect 107(9):705-710. 
Kwon E, Zhang H, Wang Z, et al. 2004. Arsenic on the hands of children after playing in playgrounds. Environ Health Perspect 112(14):1375-1380.

Kyle RA, Pease GL. 1965. Hematologic aspects of arsenic intoxication. N Engl J Med 273(1):18-23.

LaForce MJ, Fendorf SE, Li GC, et al. 1998. A laboratory evaluation of trace element mobility from flooding and nutrient loading of Coeur d'Alene River sediments. J Environ Qual 27:318-328.

Lagerkvist BJ, Zetterlund B. 1994. Assessment of exposure to arsenic among smelter workers: A fiveyear follow-up. Am J Ind Med 25(4):477-488.

Lagerkvist B, Linderholm H, Nordberg GF. 1986. Vasospastic tendency and Raynaud's phenomenon in smelter workers exposed to arsenic. Environ Res 39:465-474.

Lagerkvist BEA, Linderholm H, Nordberg GF. 1988. Arsenic and Raynaud's phenomenon: Vasospastic tendency and excretion of arsenic in smelter workers before and after the summer vacation. Int Arch Occup Environ Health 60:361-364.

Lai MS, Hsueh YM, Chen CJ, et al. 1994. Ingested inorganic arsenic and prevalence of diabetes mellitus. Am J Epidemiol 139(5):484-492.

*Lakso JU, Peoples SA. 1975. Methylation of inorganic arsenic by mammals. J Agric Food Chem 23:674-676.

*Lambert TW, Lane S. 2004. Lead, arsenic, and polycyclic aromatic hydrocarbons in soil and house dust in the communities surrounding the Sydney, Nova Scotia, tar ponds. Environ Health Perspect 112(1):3541.

*Lamm SH, Kruse MB. 2004. Ingested arsenic and cancer. What's new? Presentation of the US Senate Committee on the environment and public works. http://www.ruralwater.org/arsenic/lamm1.ppt. May 09, 2007.

Lamm SH, Engel A, Kruse MB, et al. 2004. Arsenic in drinking water and bladder cancer mortality in the United States: An analysis based on 133 U.S. counties and 30 years of observation. J Occup Environ Med 46(3):298-306.

Lander JJ, Stanley RJ, Sumner HW, et al. 1975. Angiosarcoma of the liver associated with Fowler's solution (potassium arsenite). Gastroenterology 68(6):1582-1586.

Landolph JR. 1994. Molecular mechanisms of transformation of C3H/10T1/2 C1 8 mouse embryo cells and diploid human fibroblasts by carcinogenic metal compounds. Environ Health Perspect 102(Suppl3):119-125.

*Landrigan PJ. 1981. Arsenic-state of the art. Am J Ind Med 2:5-14.

Landsberger S, Simsons A. 1987. Chromium, nickel, and arsenic determinations in human samples by thermal and epithermal neutron activation analyses. Biol Trace Elem Res 13:357-362. 
Landsberger S, Wu D. 1995. The impact of heavy metals from environmental tobacco smoke on indoor air quality as determined by Compton suppression neutron activation analysis. Sci Total Environ 173174(1-6):323-337.

Lantz RC, Hays AM. 2006. Role of oxidative stress in arsenic-induced toxicity. Drug Metab Rev 38:791-804.

Lantzsch H, Gebel T. 1997. Genotoxicity of selected metal compounds in the SOS chromotest. Mutat Res 389(2-3):191-197.

Lanz H, Wallace PC, Hamilton JG. 1950. The metabolism of arsenic in laboratory animals using $\mathrm{As}^{74}$ as a tracer. Univ California Publ Pharmacol 2(29):263-282.

Laparra JM, Velez D, Montoro R, et al. 2003. Estimation of arsenic bioaccessibility in edible seaweed by an in vitro digestion method. J Agric Food Chem 51:6080-6085.

*Larochette N, Decaudin D, Jacotot E, et al. 1999. Rapid communication: Arsenite induces apoptosis via a direct effect on the mitochondrial permeability transition pore. Exp Cell Res 249:413-421.

Larramendy ML, Popescu NC, DiPaolo J. 1981. Induction by inorganic metal salts of sister chromatid exchanges and chromosome aberrations in human and Syrian hamster strains. Environ Mutagen 3:597606.

Larsen EH, Moseholm L, Nielsen MM. 1992. Atmospheric deposition of trace elements around point sources and human health risk assessment: II. Uptake of arsenic and chromium by vegetables grown near a wood preservation factory. Sci Total Environ 126(3):263-275.

Lasky T, Sun W, Kadry A, et al. 2004. Mean total arsenic concentrations in chicken 1989-2000 and estimated exposures for consumers of chicken. Environ Health Perspect 112(1):18-21.

Le XC, Lu X, Li XF. 2004. Arsenic speciation. Anal Chem 76:27A-33A.

Lebow S, Williams RS, Lebow P. 2003. Effect of simulated rainfall and weathering on release of preservative elements from CCA treated wood. Environ Sci Technol 37:4077-4082.

Lebow ST, Lebow PT, Foster DO, et al. 2000. Environmental impact of preservative-treated wood in a wetland boardwalk. U.S. Department of Agriculture, Forest Service. FPL-RP-582.

Lee AM, Fraumeni JF. 1969. Arsenic and respiratory cancer in man: An occupational study. J Natl Cancer Inst 42:1045-1052.

Lee DC, Roberts JR, Kelly JJ, et al. 1995. Whole-bowel irrigation as an adjunct in the treatment of radiopaque arsenic [letter]. Am J Emerg Med 13(2):244-245.

Lee MY, Bae ON, Chung SM, et al. 2002. Enhancement of platelet aggregation and thrombus formation by arsenic in drinking water: A contributing factor to cardiovascular disease. Toxicol Appl Pharmacol 179(2):83-88.

Lee TC, Ho IC. 1994. Differential cytotoxic effects of arsenic on human and animal cells. Environ Health Perspect 102(Suppl 3):101-105. 
Lee TC, Oshimura M, Barrett JC. 1985. Comparison of arsenic-induced cell transformation, cytotoxicity, mutation and cytogenetic effects in Syrian hamster embryo cells in culture. Carcinogenesis 6(10):1421-1426.

Lee TC, Tanaka N, Lamb PW, et al. 1988. Induction of gene amplification by arsenic. Science 241:7981.

Lee TC, Wang-Wuu S, Huang RY, et al. 1986. Differential effects of pre- and posttreatment of sodium arsenite on the genotoxicity of methyl methanesulfonate in Chinese hamster ovary cells. Cancer Res 46:1854-1857.

Lee-Chen SF, Gurr JR, Lin IB, et al. 1993. Arsenite enhances DNA double-strand breaks and cell killing of methyl methanesulfonate-treated cells by inhibiting the excision of alkali-labile sites. Mutat Res 294(1):21-28.

Lee-Chen SF, Yu CT, Jan KY. 1992. Effect of arsenite on the DNA repair of UV-irradiated Chinese hamster ovary cells. Mutagenesis 7(1):51-55.

Lee-Chen SF, Yu CT, Wu DR, et al. 1994. Differential effects of luminol, nickel, and arsenite on the rejoining of ultraviolet light and alkylation-induced DNA breaks. Environ Mol Mutagen 23(2):116-120.

Lee-Feldstein A. 1983. Arsenic and respiratory cancer in man: Follow-up of an occupational study. In: Lederer W, Fensterheim R, eds. Arsenic: Industrial, biomedical and environmental perspectives. New York, NY: Van Nostrand Reinhold, 245-265.

Lee-Feldstein A. 1986. Cumulative exposure to arsenic and its relationship to respiratory cancer among copper smelter employees. J Occup Med 28(4):296-302.

Leeder JS, Kearns GL. 1997. Pharmacogenetics in pediatrics: Implications for practice. Pediatr Clin North Am 44:55-77.

Léonard A, Lauwerys RR. 1980. Carcinogenicity, teratogenicity and mutagenicity of arsenic. Mutat Res 75:49-62.

Le Quesne PM, McLeod JG. 1977. Peripheral neuropathy following a single exposure to arsenic. J Neurol Sci 32:437-451.

Lerda D. 1994. Sister-chromatid exchange (SCE) among individuals chronically exposed to arsenic in drinking water. Mutat Res 312(2):111-120.

Lerman BB, Ali N, Green D. 1980. Megaloblastic, dyserythropoietic anemia following arsenic ingestion. Ann Clin Lab Science 10(6):515-517.

Lerman S, Clarkson TW, Gerson RJ. 1983. Arsenic uptake and metabolism by liver cells is dependent on arsenic oxidation state. Chem Biol Interact 45:401-406.

Leung HW. 1993. Physiologically-based pharmacokinetic modelling. In: Ballantine B, Marro T, Turner P, eds. General and applied toxicology. Vol. 1. New York, NY: Stockton Press, 153-164.

*Leung PL, Huang HM. 1997. Analysis of trace elements in the hair of volunteers suffering from nasopharyngeal cancer. Biol Trace Elem Res 57(1):19-25. 
Levander OA. 1977. Metabolic interrelationships between arsenic and selenium. Environ Health Perspect 19:159-164.

Levander OA, Baumann CA. 1966. Selenium metabolism: VI. Effect of arsenic on the excretion of selenium in the bile. Toxicol Appl Pharm 9:106-115.

Levin-Scherz JK, Patrick JD, Weber FH, et al. 1987. Acute arsenic ingestion. Ann Emerg Med 16(6):702-704.

Lewis DR, Southwick JW, Ouellet-Hellstrom R, et al. 1999. Drinking water in Utah: A cohort mortality study. Environ Health Perspect 107(5):359-365.

*Lewis TA, Hartman CB, McCoy KL. 1998a. Gallium arsenide differentially affects processing of phagolysosmal targeted antigen by macrophages. J Leukoc Biol 63:321-330.

*Lewis TA, Hartmann CB, McCoy KL. 1998b. Gallium arsenide modulates proteolytic cathepsin activities and antigen processing by macrophages. J Immunol 161:2151-2157.

Li JH, Rossman TG. 1989. Inhibition of DNA ligase activity by arsenite: A possible mechanism of its comutagenesis. Mol Toxicol 2:1-9.

Li JH, Rossman TG. 1991. Comutagenesis of sodium arsenite with ultraviolet radiation in Chinese hamster V79 cells. Biol Met 4:197-200.

Li W, Chou IN. 1992. Effects of sodium arsenite on the cytoskeleton and cellular glutathione levels in cultured cells. Toxicol Appl Pharmacol 114(1):132-139.

Li W, Wanibuchi H, Salim EI, et al. 1998. Promotion of the NCI-Black-Reiter male rat bladder carcinogenesis by dimethylarsinic acid an organic arsenic compound. Cancer Lett 134(1):29-36.

*Li W, Wei C, Zhang C, et al. 2003. A survey of arsenic species in Chinese seafood. Food Chem Toxicol 41(8):1103-1110.

*Li YM, Broome JD. 1997. Apoptosis induced in premyelocytic leukemia cells by arsenic and proteasome inhibitors. Blood 90(10):203b.

*Li YM, Broome JD. 1999. Arsenic targets tubulins to induce apoptosis in myeloid leukemia cells. Cancer Res 59:776-780.

Lianfang W, Jianzhong H. 1994. Chronic arsenism from drinking water in some areas of Xinjiang, China. In: Nriagu JO, ed. Arsenic in the environment: Part II: Human health and ecosystem effects. New York, NY: John Wiley and Sons, Inc., 159-172.

Liao WT, Chang KL, Chen GS, et al. 2004. Arsenic induces human keratinocyte apoptosis by the FAS/FAS ligand pathway, which correlates with alterations in nuclear factor- $\mathrm{kB}$ and activator protein1 activity. J Invest Dermatol 122(1):125-129.

Liebscher K, Smith H. 1968. Essential and nonessential trace elements: A method of determining whether an element is essential or nonessential in human tissue. Arch Environ Health 17:881-890. 
Lin JK, Tseng S. 1992. Chromosomal aberrations and sister-chromatid exchanges induced by $N$-nitroso2-acetylaminofluorene and their modifications by arsenite and selenite in Chinese hamster ovary cells. Mutat Res 265(2):203-210.

*Lin TH, Huang Y, Tseng W. 1995. Arsenic and lipid peroxidation in patients with blackfoot disease. Bull Environ Contam Toxicol 54(4):488-493.

Lindgren A, Danielsson BRG, Dencker L, et al. 1984. Embryotoxicity of arsenite and arsenate: Distribution in pregnant mice and monkeys and effects on embryonic cells in vitro. Acta Pharmacol Toxicol 54:311-320.

Lindgren A, Vahter M, Dencker L. 1982. Autoradiographic studies on the distribution of arsenic in mice and hamsters administered ${ }^{74}$ As-arsenite or -arsenate. Acta Pharmacol Toxicol 51:253-265.

Liou SH, Lung JC, Chen YH, et al. 1999. Increased chromosome-type chromosome aberration frequencies as biomarkers of cancer risk in a blackfoot endemic area. Cancer Res 59(7):1481-1484.

Lithner G, Holm K, Borg H. 1995. Bioconcentration factors for metals in humic waters at different $\mathrm{pH}$ in the Röbbskär area (N. Sweden). Water, Air and Soil Pollution 85:785-790.

Little RE, Kay GN, Cavender JB, et al. 1990. Torsade de pointes and T-U wave alternans associated with arsenic poisoning. PACE 13:164-170.

Liu YC, Huang H. 1997. Involvement of calcium-dependent protein kinase $\mathrm{C}$ in arsenite-induced genotoxicity in Chinese hamster ovary cells. J Cell Biochem 64(3):423-433.

Liu YT, Chen Z. 1996. A retrospective lung cancer mortality study of people exposed to insoluble arsenic and radon. Lung Cancer 14(Suppl 1):137-148.

Liu J, Liu Y, Goyer RA, et al. 2000. Metallothionein-I/II null mice are more sensitive than wild-type mice to the hepatotoxic and nephrotoxic effects of chronic oral or injected inorganic arsenicals. Toxicol Sci 55(2):460-467.

*Liu J, Xie Y, Ducharme DM, et al. 2006. Global gene expression associated with hepatocarcinogenesis in adult male mice induced by in utero arsenic exposure. Environ Health Perspect 114(3):404-411.

Liu J, Zheng B, Aposhian HV, et al. 2002. Chronic arsenic poisoning from burning high-arseniccontaining coal in Guizhou, China. Environ Health Perspect 110(2):119-122.

Livingston AL. 1978. Forage plant estrogens. J Toxicol Environ Health 4:301-324.

Loebenstein JR. 1994. The materials flow of arsenic in the United States. Washington, DC: Bureau of Mines, Division of Mineral Commodities, United States Department of the Interior. BUMINES-IC-9382.

Loffredo CA, Aposhian HV, Cebrian ME, et al. 2003. Variability in human metabolism of arsenic. Environ Res 92(2):85-91.

Löfroth G, Ames BN. 1978. Mutagenicity of inorganic compounds in Salmonella typhimurium: arsenic, chromium, and selenium. Mutat Res 53:65-66. 
López-Gonzálvez MA, Gómez MM, Cámara C, et al. 1994. On-line microwave oxidation for the determination of organoarsenic compounds by high-performance liquid chromatography-hydride generation atomic absorption spectrometry. J Anal Atom Spectrom 9(3):291-295.

Lovell MA, Farmer JG. 1985. Arsenic speciation in urine from humans intoxicated by inorganic arsenic compounds. Hum Toxicol 4:203-214.

Lowney YW, Ruby MV, Wester RC, et al. 2005. Percutaneous absorption of arsenic from environmental media. Toxicol Ind Health 21(1-2):1-14.

Lu FJ, Shih SR, Liu TM, et al. 1990. The effect of fluorescent humic substances existing in the well water of blackfoot disease endemic areas in Taiwan on prothrombin time and activated partial thromboplastin time in vitro. Thromb Res 57:747-753.

Lu M, Wang H, Li XF, et al. 2004a. Evidence of hemoglobin binding to arsenic as a basis for the accumulation of arsenic in rat blood. Chem Res Toxicol 17(12):1733-1742.

*Lu SN, Chow NH, Wu WC, et al. 2004b. Characteristics of hepatocellular carcinoma in a high arsenicism area in Taiwan: A case-control study. J Occup Environ Med 46(5):437-441.

*Lubin JH, Fraumeni JF. 2000. Re: "Estimates of the annual number of clinically recognized pregnancies in the United States, 1981-1991". Am J Epidemiol 152(3):297-293.

Lubin JH, Pottern LM, Stone BJ, et al. 2000. Respiratory cancer in a cohort of copper smelter workers: Results from more than 50 years of follow-up. Am J Epidemiol 151(6):554-565.

Lüchtrath H. 1983. The consequences of chronic arsenic poisoning among Moselle wine growers: Pathoanatomical investigations of post-mortem examinations performed between 1960 and 1977. J Cancer Res Clin Oncol 105:173-182.

Lugo G, Cassady G, Palmisano P. 1969. Acute maternal arsenic intoxication with neonatal death. Am J Dis Child 117:328-330.

Luong KVQ, Nguyen LTH. 1999. Organic arsenic intoxication from bird's nest soup. Am J Med Sci 317(4):269-271.

Luten JB, Riekwel-Booy G, Rauchbaar A. 1982. Occurrence of arsenic in plaice (Pleuronectes platessa), nature of organo-arsenic compound present and its excretion by man. Environ Health Perspect 45:165170.

Lynn S, Lai HT, Gurr JR, et al. 1997. Arsenite retards DNA break rejoining by inhibiting DNA ligation. Mutagenesis 12(5):353-358.

Mabuchi K, Lilienfeld AM, Snell LM. 1979. Lung cancer among pesticide workers exposed to inorganic arsenicals. Arch Environ Health 34:312-320.

MacIntosh DL, Williams PL, Hunter DJ, et al. 1997. Evaluation of a food frequency questionnaire-food composition approach for estimating dietary intake of inorganic arsenic and methylmercury. Cancer Epidemiol Biomarkers Prev 6(12):1043-1050. 
Maehashi H, Murata Y. 1986. Arsenic excretion after treatment of arsenic poisoning with DMSA or DMPS in mice. Jpn J Pharmacol 40:188-190.

Mahaffey KR, Fowler BA. 1977. Effects of concurrent administration of lead, cadmium, and arsenic in the rat. Environ Health Perspect 19:165-171.

Mahaffey KR, Capar SG, Gladen BC, et al. 1981. Concurrent exposure to lead, cadmium and arsenic. Effects on toxicity and tissue metal concentrations in the rat. J Lab Clin Med 98(1):463-481.

*Mahajan SK, Aggarwal HK, Wig N, et al. 1992. Arsenic induced neuropathy. J Assoc Physicians India 40(4):268-269.

Mahata J, Basu A, Ghosal S, et al. 2003. Chromosomal aberrations and sister chromatid exchanges in individuals exposed to arsenic through drinking water in West Bengel, India. Mutat Res 534(1-2):133143.

Maher WA. 1989. Some observations on the determination of total arsenic in biological tissues. Microchem J 40:132-135.

*Mahieu P, Buchet J, Lauwerys R. 1987. Evolution clnique et biologique d'une intoxication orale aiguë par l'anhydride arsénieux et considérations sur l'attitude thérapeutique. J Toxicol Clin Exp 7:273-278.

Maiorino RM, Aposhian HV. 1985. Dimercaptan metal-binding agents influence the biotransformation of arsenite in the rabbit. Toxicol Appl Phamacol 77:240-250.

Maitani T, Saito N, Abe M, et al. 1987a. Chemical form-dependent induction of hepatic zinc-thionein by arsenic administration and effect of co-administered selenium in mice. Toxicol Lett 39:63-70.

*Maitani T, Uchiyama S, Saito Y. 1987b. Hydride generation-flame atomic-absorption spectrometry as an arsenic detector for high-performance liquid chromatography. J Chromatogr 391:161-168.

*Man ACK, Zheng YH, Mak PK. 1996. Trace elements in scalp hair of professional drivers and university teachers in Hong Kong. Biol Trace Elem Res 53(1-3):241-247.

Mandal BK, Suzuki KT. 2002. Arsenic around the world: A review. Talanta 58:201-235.

Mandal BK, Ogra Y, Suzuki KT. 2001. Identification of dimethylarsinous and monomethylarsonous. Chem Res Toxicol 14(4):371-378.

Mandal BK, Ogra Y, Suzuki KT. 2003. Speciation of arsenic in human nail and hair from arsenicaffected area by HPLC-inductively coupled argon plasma mass spectrometry. Toxicol Appl Pharmacol 189(2):73-83.

Mann S, Droz PO, Vahter M. 1996a. A physiologically based pharmacokinetic model for arsenic exposure. I. Development in hamsters and rabbits. Toxicol Appl Pharmacol 137(1):8-22.

Mann S, Droz PO, Vahter M. 1996b. A physiologically based pharmacokinetic model for arsenic exposure. II. Validation and application in humans. Toxicol Appl Pharmacol 140(2):471-486.

Mappes R. 1977. [Experiments on excretion of arsenic in urine.] Int Arch Occup Environ Health 40:267-272. (German) 
Marafante E, Vahter M. 1984. The effect of methyltransferase inhibition on the metabolism of $\left[{ }^{74}\right.$ As $]$ arsenite in mice and rabbits. Chem Biol Interact 50:49-57.

Marafante E, Vahter M. 1986. The effect of dietary and chemically induced methylation deficiency on the metabolism of arsenate in the rabbit. Acta Pharmacol Toxicol 59(Suppl 7):35-38.

Marafante E, Vahter M. 1987. Solubility, retention and metabolism of intratracheally and orally administered inorganic arsenic compounds in the hamster. Environ Res 42:72-82.

Marafante E, Lundborg M, Vahter M, et al. 1987a. Dissolution of two arsenic compounds by rabbit alveolar macrophages in vitro. Fundam Appl Toxicol 8:382-388.

*Marafante E, Vahter M, Dencker L. 1984. Metabolism of arsenocholine in mice, rats and rabbits. Sci Total Environ 34:223-240.

Marafante E, Vahter M, Envall J. 1985. The role of the methylation in the detoxication of arsenate in the rabbit. Chem Biol Interact 56:225-238.

Marafante E, Vahter M, Norin H, et al. 1987b. Biotransformation of dimethylarsinic acid in mouse, hamster and man. J Appl Toxicol 7(2):111-117.

Marcus WL, Rispin AS. 1988. Threshold carcinogenicity using arsenic as an example. In: Cothern CR, Mehlman MA, Marcus WL, eds. Advances in modern environmental toxicology. Vol. XV: Risk assessment and risk management of industrial and environmental chemicals. Princeton, NJ: Princeton Scientific Publishing Co., 133-158.

Margitich DJ, Ackerman LJ. 1991a. Caccdylic acid 21 day toxicity study in rabbit. Luxembourg Industries (Pamol) Ltd. Submitted to the U.S. Environmental Protection Agency. MRID41872801.

Margitich DJ, Ackerman LJ. 1991b. Methanearsonic acid 21 day dermal toxicity study in rabbits. Luxembourg Industries (Pamol) Ltd. Submitted to the U.S. Environmental Protection Agency. MRID41872701.

Mariner PE, Holzmer FJ, Jackson RE, et al. 1996. Effects of high pH on arsenic mobility in a shallow sandy aquifer and on aquifer permeability along the adjacent shoreline, Commencement Bay Superfund Site, Tacoma, Washington. Environ Sci Tech 30(5):1645-1651.

* Marlowe M, Cossairt A, Moon C, et al. 1985. Main and interaction effects of metallic toxins on classroom behavior. J Abnorm Child Psychol 13(2):185-198.

Martin DS, Willis SE, Cline DM. 1990. N-Acetylcysteine in the treatment of human arsenic poisoning. J Am Board Fam Pract 3:293-296.

Martínez V, Creus A, Venegas W, et al. 2004. Evaluation of micronucleus induction in a Chilean population environmentally exposed to arsenic. Mutat Res 564(1):65-74.

Martínez V, Crues A, Venegas W, et al. 2005. Micronuclei assessment in buccal cells of people environmentally exposed to arsenic in northern Chile. Toxicol Lett 155:319-327. 
Mason RP, Laporte JM, Andres S. 2000. Factors controlling the bioaccumulation of mercury, methylmercury, arsenic, selenium, and cadmium by freshwater invertebrates and fish. Arch Environ Contam Toxicol 38:283-97.

Mason RW, Edwards IR, Fisher LC. 1989. Teratogenicity of combinations of sodium dichromate, sodium arsenate and copper sulphate in the rat. Comp Biochem Physiol 93(2):407-411.

Matanoski G, Landau E, Tonascia J, et al. 1981. Cancer mortality in an industrial area of Baltimore. Environ Res 25:8-28.

Matschullat J. 2000. Arsenic in the geosphere - a review. Sci Total Environ 249:297-312.

Matsui M, Nishigori C, Toyokuni S, et al. 1999. The role of oxidative DNA damage in human arsenic carcinogenesis: Detection of 8-hydroxy-2'-deoxyguanosine in arsenic-related Bowen's disease. J Invest Dermatol 113:26-31.

Matthews EJ, Spalding JW, Tennant RW. 1993. Transformation of BALB/c-3T3 cells: V. Transformation responses of 168 chemicals compared with mutagenicity in Salmonella and carcinogenicity in rodent bioassays. Environ Health Perspect 101(Suppl 2):347-482.

May TW, Wiedmeyer RH, Gober J, et al. 2001. Influence of mining-related activities on concentrations of metals in water and sediment from streams of the Black Hills, South Dakota. Arch Environ Contam Toxicol 40:1-9.

Mayr U, Butsch A, Schneider S. 1992. Validation of two in vitro test systems for estrogenic activities with zearalenone, phytoestrogens and cereal extracts. Toxicology 74:135-149.

Mazumdar S, Redmond CK, Enterline PE, et al. 1989. Multistage modeling of lung cancer mortality among arsenic-exposed copper-smelter workers. Risk Anal 9(4):551-563.

McDorman EW, Collins BW, Allen JW. 2002. Dietary folate deficiency enhances induction of micronuclei by arsenic in mice. Environ Mol Mutagen 40(1):71-77.

McFall TL, Richards S, Matthews G. 1998. Rehabilitation in an individual with chronic arsenic poisoning: Medical, psychological, and social implications. J Spinal Cord Med 21(2):142-147.

McGeehan SL. 1996. Arsenic sorption and redox reactions: Relevance to transport and remediation. J Environ Sci Health A31(9):2319-2336.

McGeehan SL, Fendorf SE, Naylor DV. 1998. Alteration of arsenic sorption in flooded-dried soils. Soil Sci Soc Am J 62:828-833.

McLaren RG, Naidu R, Smith J, et al. 1998. Fractionation and distribution of arsenic in soils contaminated by cattle dip. J Environ Qual 27:348-354.

Meacher DM, Menzel DB, Dillencourt MD, et al. 2002. Estimation of multimedia inorganic arsenic intake in the U.S. population. Hum Ecol Risk Assess 8(7):1697-1721.

Meharg AA, Rahman M. 2003. Arsenic contamination of Bangladesh paddy field soils: Implications fro rice contribution to arsenic consumption. Environ Sci Technol 37:229-234. 
9. REFERENCES

Meister RT, ed. 1987. Farm chemicals handbook. Willoughby, OH: Meister Publishing. Section C:21, 46-47, 101, 177-178, 230-231.

Meister RT, ed. 1999. Farm chemicals handbook '99. Willoughby, OH: Meister Publishing Company.

Meister RT, Sine C, Sharp DT, et al. 2006. Crop protection handbook 2006. Willohughby, OH: Meister Media Worldwide, D 65.

Meng Z. 1993a. Effects of arsenic on DNA synthesis in human lymphocytes stimulated by phytohemagglutinin. Biol Trace Elem Res 39(1):73-80.

Meng Z. 1993b. Effects of arsenic on DNA synthesis in human lymphocytes. Arch Environ Contam Toxicol 25(4)525-528.

Meng Z. 1994. Effects of arsenic on DNA synthesis in human lymphocytes. In: Nraigu JO, ed. Arsenic in the environment, part II: Human health and ecosystem effects. New York, NY: John Wiley \& Sons, Inc., 133-142.

Meng Z, Hsie AW. 1996. Polymerase chain reaction-based deletion analysis of spontaneous and arsenite-enhanced gpt mutants in CHO-As s2 $_{5}$ cells. Mutat Res 356(2)255-259.

Menzel DB, Rasmussen RE, Lee E, et al. 1998. Human lymphocyte heme oxygenase 1 as a response biomarker to inorganic arsenic. Biochem Biophys Res Commun 250:653-656.

Menzel DB, Ross M, Oddo SV, et al. 1994. A physiologically based pharmacokinetic model for ingested arsenic. Environ Geochem Health 16:209-218.

Merwin I, Pruyne PT, Ebel JG, et al. 1994. Persistence, phytotoxicity, and management of arsenic, lead and mercury residues in old orchard soils of New York State. Chemosphere 29(6):1361-1367.

*Meyer I, Heinrich J, Lippold U. 1999. Factors affecting lead, cadmium, and arsenic levels in house dust in a smelter town in eastern Germany. Environ Res 81:32-44.

Michaud DS, Wright ME, Cantor KP, et al. 2004. Arsenic concentrations in prediagnostic toenails and the risk of bladder cancer in a cohort study of male smokers. Am J Epidemiol 160(9):853-859.

Miles AK, Tome MW. 1997. Spatial and temporal heterogeneity in metallic elements in industrialized aquatic bird habitats. Environ Pollut 95(1):75-84.

Milham S, Strong T. 1974. Human arsenic exposure in relation to a copper smelter. Environ Res 7:176182.

Miller M. 1998. Scientists explore use of arsenic in therapy. J Natl Cancer Inst 90(24):1866-1867.

Milton AH, Rahman M. 2002. Respiratory effects and arsenic contaminated well water in Bangladesh. Int J Environ Health Res 12(2):175-179.

Milton AH, Hasan Z, Shahidullah SM, et al. 2004. Association between nutritional status and aresnicosis due to chronic arsenic exposure in Bangladesh. Int J Environ Health Res 14(2):99-108. 
Milton AH, Smith W, Rahman B, et al. 2005. Chronic arsenic exposure and adverse pregnancy outcomes in Bangladesh. Epidemiology 16(1):82-86.

Mirkes PE, Cornel L. 1992. A comparison of sodium arsenite- and hyperthermia-induced stress responses

*Mitra K, Kundu SN, Khuda Bukhsh AR. 1999. Efficacy of a potentized homoeopathic drug (Arsenic Album-30) in reducing toxic effects produced by arsenic trioxide in mice: II. On alterations in body weight and total protein. Complement Ther Med 7:24-34.

Mitra SR, Mazumder DN, Basu A, et al. 2004. Nutritional factors and susceptibility to arsenic-caused skin lesions in West Bengal, India. Environ Health Perspect 112(10):1104-1109.

Miyazaki K, Ushijima K, Kadono T, et al. 2003. Negative correlation between urinary selenium and arsenic levels of the residents living in an arsenic-contaminated area in Bangladesh. J Health Sci 49(3):239-242.

Mizuta N, Mizuta M, Ito F, et al. 1956. An outbreak of acute arsenic poisoning caused by arseniccontaminated soy-sauce (shoyu): A clinical report of 220 cases. Bull Yamaguchi Med Sch 4(2-3):131149.

Mohamed KB. 1998. Occupational contact dermatitis from arsenic in a tin-smelting factory. Contact Dermatitis 38:224-225.

Mok WM, Wai CM. 1994. Mobilization of arsenic in contaminated river waters. In: Nriagu JO, ed. Arsenic in the environment, part 1: Cycling and characterization. New York, NY: John Wiley \& Sons, Inc., 99-117.

Mok WM, Riley JA, Wai CM. 1988. Arsenic speciation and quality of groundwater in a lead-zinc mine, Idaho. Water Res 22(6):769-774.

Moore DF, O'Callaghan CA, Berlyne G, et al. 1994a. Acute arsenic poisoning: Absence of polyneuropathy after treatment with 2,3-dimercaptopropanesulphonate (DMPS). J Neurol Neurosurg Psychiatry 57(9):1133-1135.

Moore JN, Ficklin WH, Johns C. 1988. Partitioning of arsenic and metals in reducing sulfidic sediments. Environ Sci Technol 22:432-437.

Moore LE, Lu M, Smith AH. 2002. Childhood cancer incidence and arsenic exposure in drinking water in Nevada. Arch Environ Health 57(3):201-206.

Moore LE, Smith AH, Hopenhayn-Rich C, et al. 1995. Increased bladder cells micronuclei in two populations environmentally exposed to arsenic in drinking water. Clin Chem 41(12 Part 2)1915-1917.

Moore LE, Smith AH, Hopenhayn-Rich C, et al. 1997a. Micronuclei in exfoliated bladder cells among individuals chronically exposed to arsenic in drinking water. Cancer Epidemiol Biomarkers Prev 6(1)3136.

Moore LE, Warner ML, Smith AH, et al. 1996. Use of the fluorescent micronucleus assay to detect the genotoxic effects of radiation and arsenic exposure in exfoliated human epithelial cells. Environ Mol Mutagen 27(3):176-184. 
Moore MM, Harrington-Brock K, Doerr CL. 1994b. Genotoxicity of arsenic and its methylated metabolites. Environ Geochem Health 16:191-198.

*Moore MM, Harrington-Brock K, Doerr CL. 1997b. Relative genotoxic potency of arsenic and its methylated metabolites. Mutat Res 386(3):279-290.

*Moreira JC. 1996. Threats by heavy metals: Human and environmental contamination in Brazil. Sci Total Environ 188(Suppl 1):S61-S71.

Morris JS, Schmid M, Newman S, et al. 1974. Arsenic and noncirrhotic portal hypertension. Gastroenterology 66(1):86-94.

Morrissey RE, Mottet NK. 1983. Arsenic-induced exencephaly in the mouse and associated lesions occurring during neurulation. Teratology 28:399-411.

Moriss-Kay GM, Wood H, Chen W. 1994. Normal neurulation in mammals. In: Ciba Foundation Symposium, neural tube defects. New York: John Wiley \& Sons, 51-69.

Morse DL, Harrington JM, Housworth J, et al. 1979. Arsenic exposure in multiple environmental media in children near a smelter. Clin Toxicol 14(4):389-399.

Morselli PL, Franco-Morselli R, Bossi L. 1980. Clinical pharmacokinetics in newborns and infants: Age-related differences and therapeutic implications. Clin Pharmacokin 5:485-527.

Morton W, Starr G, Pohl D, et al. 1976. Skin cancer and water arsenic in Lane County, Oregon. Cancer 37:2523-2532.

Morton WE, Caron GA. 1989. Encephalopathy: An uncommon manifestation of workplace arsenic poisoning? Am J Ind Med 15:1-5.

Mottet NK, Ferm VH. 1983. The congenital teratogenicity and perinatal toxicity of metals. In: Clarkson TW, Nordberg GF, Sager PR, eds. Reproductive and developmental toxicity of metals. New York, NY: Plenum Press, 93-125.

Moxon AL, Paynter CR, Halverson AW. 1945. Effect of route of administration on detoxication of selenium by arsenic. J Pharmacol Exp Ther 84:115-119.

Mukherjee S, Das D, Darbar S, et al. 2003. Dietary intervention affects arsenic-generated nitric oxide and reactive oxygen intermediate toxicity in islet cells of rats. Curr Sci 85(6):786-793.

Mukherjee S, Das D, Darbar S, et al. 2004. Arsenic trioxide generates oxidative stress and islet cell toxicity in rabbit. Curr Sci 86(6):854-857.

Mumford JL, Wu K, Xia Y, et al. 2007. Chronic arsenic exposure and cardiac repolarization abnormalities with QT interval prolongation in a population-based study. Environ Health Perspect 115(5):690-694.

Mumma RO, Raupach DC, Waldman JP, et al. 1984. National survey of elements and other constituents in municipal sewage sludges. Arch Environ Contam Toxicol 13:75-83. 
Murai T, Iwata H, Otoshi T, et al. 1993. Renal lesions induced in F34/DuCrj rats by 4-weeks oral administration of dimethylarsinic acid. Toxicol Lett 66:53-61.

*Murata K, Iwazawa T, Takayama T, et al. 1994. Quadruple cancer including Bowen's disease after arsenic injections 40 years earlier: Report of a case. Surg Today 24(12):1115-1118.

Mure K, Uddin AN, Lopez LC, et al. 2003. Arsenite induces delayed mutagenesis and transformation in human osteosarcoma cells at extremely low concentrations. Environ Mol Mutagen 41:322-331.

Murphy MJ, Lyon LW, Taylor JW. 1981. Subacute arsenic neuropathy: Clinical and electrophysiological observations. J Neurol Neurosurg Psychiatry 44:896-900.

Mushak P, Crocetti AF. 1995. Risk and revisionism in arsenic cancer risk assessment. Environ Health Perspect 103(7-8):684-689.

Mushak P, Dessauer K, Walls EL. 1977. Flameless atomic absorption (FAA) and gas-liquid chromatographic studies in arsenic bioanalysis. Environ Health Perspect 19:5-10.

Muzi G, dell'Omo M, Madeo G, et al. 2001. Arsenic poisoning caused by Indian ethnic remedies. J Pediatr 139(1):169.

Nagai N, Usui T, Asahi A, et al. 1956. Comparison of biochemical findings and clinical pictures on subacute or chronic arsenic poisoning of infants due to arsenic containing powdered milk. NTIS no. PB258710-T.

Nagymajtényi L, Selypes A, Berencsi G. 1985. Chromosomal aberrations and fetotoxic effects of atmospheric arsenic exposure in mice. J Appl Toxicol 5(2):61-63.

Nakadaira H, Endoh K, Katagiri M, et al. 2002. Elevated mortality from lung cancer associated with arsenic exposure for a limited duration. J Occup Environ Med 44(3):291-299.

Nakamuro K, Sayato Y. 1981. Comparative studies of chromosomal aberrations induced by trivalent and pentavalent arsenic. Mutat Res 88:73-80.

NAS. 1977a. Medical and biologic effects of environmental pollutants: Arsenic. Washington, DC: National Academy of Sciences. http://www.nap.edu/books/0309026040/html/index.html. May 12, 2005.

NAS. 1977b. Arsenic. Drinking water and health. Washington, DC: National Academy of Sciences, 316-344, 428-430.

NAS/NRC. 1989. Report of the oversight committee. In: Biologic markers in reproductive toxicology. Washington, DC: National Academy of Sciences, National Research Council, National Academy Press.

Navas-Acien A, Sharrett AR, Silbergeld EK, et al. 2005. Arsenic exposure and cardiovascular disease: A systematic review of the epidemiologic evidence. Am J Epidemiol 162(11):1037-1049.

*Navas-Acien A, Silbergeld EK, Streeter RA, et al. 2006. Arsenic exposure and type 2 diabetes: A systematic review of the experimental and epidemiological evidence. Environ Health Perspect 114(5):641-648. 
Neiger RD, Osweiler GD. 1989. Effect of subacute low level dietary sodium arsenite on dogs. Fundam Appl Toxicol 13:439-451.

Neiger RD, Osweiler GD. 1992. Arsenic concentrations in tissues and body fluids of dogs on chronic low level dietary sodium arsenite. J Vet Diagn Invest 4:334-337.

*Nelson WC, Lykins MH, Mackey J, et al. 1973. Mortality among orchard workers exposed to lead arsenate spray: A cohort study. J Chronic Dis 26:105-118.

Nemec MD, Holson JF, Farr CH, et al. 1998. Developmental toxicity assessment of arsenic acid in mice and rabbits. Reprod Toxicol 12(6):647-658.

Newman JA, Archer VE, Saccomanno G, et al. 1976. Histologic types of bronchogenic carcinoma among members of copper-mining and smelting communities. Ann N Y Acad Sci 271:260-268.

Nielson KK, Sanders RW. 1983. Multielement analysis of unweighed biological and geological samples using backscatter and fundamental parameters. Advances in X-ray Analysis 26:385-390.

NIOSH. 1984. Arsenic trioxide, as As - method 7901. In: NIOSH manual of analytical methods. 3rd ed. Vol. 1. Cincinnati, OH: U.S. Department of Health and Human Services, Public Health Service, Centers for Disease Control, National Institute for Occupational Safety and Health, DHHS (NIOSH) Publication no. 84-100. PB85179018.

NIOSH. 1994a. Method 7900. Arsenic and compounds, as As (except $\mathrm{AsH}_{3}$ and $\mathrm{As}_{2} \mathrm{O}_{3}$ ). In: NIOSH manual of analytical methods (NMAM). 4th edition. National Institute for Occupational Safety and Health. http://www.cdc.gov/niosh/nmam/pdfs/7900.pdf. August 10, 2007.

NIOSH. 1994b. Method 7901. Arsenic trioxide, as As. In: NIOSH manual of analytical methods (NMAM). 4th edition. National Institute for Occupational Safety and Health.

http://www.cdc.gov/niosh/nmam/pdfs/7901.pdf. August 10, 2007.

NIOSH. 1994c. Method 5022. Arsenic, organo-. In: NIOSH manual of analytical methods (NMAM). 4th edition. National Institute for Occupational Safety and Health. August 1994.

http://www.cdc.gov/niosh/nmam/pdfs/5022.pdf. August 10, 2007.

NIOSH. 1994d. Method 6001. Arsine. In: NIOSH manual of analytical methods (NMAM). 4th edition. National Institute for Occupational Safety and Health.

http://www.cdc.gov/niosh/nmam/pdfs/6001.pdf. August 10, 2007.

*NIOSH. 1994e. NIOSH Manual of Analytical Methods. 4th ed., August 15, 1994. Cincinnati, OH: U.S. Department of Health and Human Services, Public Health Service, Centers for Disease Control, National Institute for Occupational Safety and Health, DHHS. Method 7300 - Elements by ICP; Method 7900 - Arsenic; method 5022 - Arsenic, organo; Method 7901 - Arsenic trioxide.

NIOSH. 2003. Method 7300. Elements by ICP (nitric/perchloric acid ashing). NIOSH manual of analytical methods (NMAM). 4th ed. Cincinnati, OH: National Institute for Occupational Safety and Health. DHHS (NIOSH) Publication 94-113. http://www.cdc.gov/niosh/nmam/pdfs/7300.pdf. March 21, 2006. 
NIOSH. 2005a. Arsenic. NIOSH pocket guide to chemical hazards. Atlanta, GA: National Institute for Occupational Safety and Health, Centers for Disease Control and Prevention.

http://www.cdc.gov/niosh/npg/npgdname.html. February 15, 2004.

*NIOSH. 2005b. International Chemical Safety Cards (ICSCs): U.S. National Version. National Institute for Occupational Safety and Health. http://www.cdc.gov/niosh/ipcs/nicstart.html. April 7, 2005.

Nishioka H. 1975. Mutagenic activities of metal compounds in bacteria. Mutat Res 31:185-189.

NOES. 1990. National Occupational Exposure Survey, National Institute for Occupational Safety and Health, Cincinnati, OH. July 16, 1990.

Nordenson I, Beckman G, Beckman L, et al. 1978. Occupational and environmental risks in and around a smelter in northern Sweden: II. Chromosomal aberrations in workers exposed to arsenic. Hereditas $88: 47-50$.

Nordenson I, Salmonsson S, Brun E, et al. 1979. Chromosome aberrations in psoriatic patients treated with arsenic. Hum Genet 48:1-6.

Nordenson I, Sweins A, Beckman L. 1981. Chromosome aberrations in cultured human lymphocytes exposed to trivalent and pentavalent arsenic. Scand J Work Environ Health 7:277-281.

Nordström S, Beckman L, Nordenson I. 1978a. Occupational and environmental risks in and around a smelter in northern Sweden. I. Variations in birthweight. Hereditas 88:43-46.

Nordström S, Beckman L, Nordenson I. 1978b. Occupational and environmental risks in and around a smelter in northern Sweden. III. Frequencies of spontaneous abortion. Hereditas 88:51-54.

Nordström S, Beckman L, Nordenson I. 1979a. Occupational and environmental risks in and around a smelter in northern Sweden. V. Spontaneous abortion among female employees and decreased birth weight in their offspring. Hereditas 90:291-296.

Nordström S, Beckman L, Nordenson I. 1979b. Occupational and environmental risks in and around a smelter in northern Sweden. VI. Congenital malformations. Hereditas 90:297-302.

Norin H, Vahter M. 1981. A rapid method for the selective analysis of total urinary metabolites of inorganic arsenic. Scand J Work Environ Health 7:38-44.

Norin H, Christakopoulos A, Rondahl L, et al. 1987. Identification and quantification of arsenocholine and acetylarsenocholine in trace amounts in biological material by use of pyrolysis gas chromatography/mass spectrometry. Biomed Environ Mass Spectrom 14:117-125.

NPIRS. 2007. Chemical ingredients: Arsenic. National Pesticide Information Retrieval System. http://ppis.ceris.purdue.edu/htbin/epachem.com. February 7, 2007.

NRC. 1993. Pesticides in the diets of infants and children. National Research Council. Washington, DC: National Academy Press.

NRC. 1999. Arsenic in drinking water. National Research Council. Washington, DC: National Academy Press. 
NRC. 2001. Arsenic in drinking water. 2001 Update. National Research Council. Washington, DC: National Academy Press.

*Nriagu JO. 1988. A silent epidemic of environmental metal poisoning? Environ Pollut 50:139-161.

Nriagu JO, Lin T. 1995. Trace metals in wild rice sold in the United States. Sci Total Environ 172(23):223-228.

Nriagu JO, Pacyna JM. 1988. Quantitative assessment of worldwide contamination of air, water and soils by trace metals. Nature 333:134-139.

*NTP. 1989a. National Toxicology Program. Research Triangle Park, NC: U.S. Department of Health and Human Services, Public Health Service, National Toxicology Program. NTP 89-239.

NTP. 1989b. National Toxicology Program - technical report series no. 345. Toxicology and carcinogenesis studies of roxarsone (CAS No. 121-19-7) in F344/N rats and B6C3F1 mice (feed studies). Research Triangle Park, NC: U.S. Department of Health and Human Services, Public Health Service, National Institutes of Health. NTP-TR-345. NIH pub no. 89-2800.

NTP. 2005. Report on carcinogens. 11 ed. Research Triangle Park, NC: U.S. Department of Health and Human Services, Public Health Service, National Toxicology Program. http://ntpserver.niehs.nih.gov/ntp/roc/toc11.html. February 15, 2004.

Nygren O, Nilsson CA, Lindahl R. 1992. Occupational exposure to chromium, copper and arsenic during work with impregnated wood in joinery shops. Ann Occup Hyg 36(5)509-517.

Oberly TJ, Hoffman WP, Garriott ML. 1996. An evaluation of the twofold rule for assessing a positive response in the L5178Y TK ${ }^{* /}$ mouse lymphoma assay. Mutat Res 369 (3-4):221-232.

Oberly TJ, Piper CE, McDonald DS. 1982. Mutagenicity of metal salts in the L5178Y mouse lymphoma assay. J Toxicol Environ Health 9:367-376.

Odanaka Y, Matano O, Goto S. 1980. Biomethylation of inorganic arsenic by the rat and some laboratory animals. Bull Environ Contam Toxicol 24:452-459.

Odanaka Y, Tsuchiya N, Matano O, et al. 1983. Determination of inorganic arsenic and methylarsenic compounds by gas chromatography and multiple ion detection mass spectrometry after hydride generation-heptane cold trap. Anal Chem 55:929-932.

*O’Day PA, Carroll SA, Waychunas GA. 1998. Rock-water interactions controlling zinc, cadmium, and lead concentrations in surface waters and sediments, U.S. Tri-State Mining District. 1. Molecular identification using x-ray absorption spectroscopy. Environ Sci Technol 32(7):943-955.

Offergelt JA, Roels H, Buchet JP, et al. 1992. Relation between airborne arsenic trioxide and urinary excretion of inorganic arsenic and its methylated metabolites. Br J Ind Med 49(6)387-393.

*Ohnishi Y, Murakami S, Ohtsuka H, et al. 1997. Merkel cell carcinoma and multiple Bowen's disease: Incidental association or possible relationship to inorganic arsenic exposure? J Dermatol 24(5):310-316. 
Ohyama S, Ishinishi N, Hisanaga A, et al. 1988. Comparative chronic toxicity, including tumorigenicity, of gallium arsenide and arsenic trioxide intratracheally instilled into hamsters. Appl Organomet Chem 2:333-337.

Okada S, Yamanaka K. 1994. Induction of lung-specific DNA damage by methylarsenics via the production of free radials. In: Nriagu JO, ed. Arsenic in the environment. New York, NY: John Wiley \& Sons, Inc., 143-157.

*O'Kamura S, Inoue N. 1999. [Hematological disturbances caused by arsenic poisoning.] Fukuoka Igaku Zasshi 90(2):33-38. (Japanese)

Okui T, Fujiwara Y. 1986. Inhibition of human excision DNA repair by inorganic arsenic and the comutagenic effect in V79 Chinese hamster cells. Mutat Res 172:69-76.

O'Rourke MK, Rogan SP, Jin S, et al. 1999. Spatial distributions of arsenic exposure and mining communities from NHEXAS Arizona. National Human Exposure Assessment Survey. J Expo Anal Environ Epidemiol 9(5):446-455.

OSHA. 2005a. Air contaminants. Occupational safety and health standards for shipyard employment. Occupational Safety and Health Administration. Code of Federal Regulations. 29 CFR 1915.1000. http://www.osha.gov/comp-links.html. February 15, 2005.

OSHA. 2005b. Gases, vapors, fumes, dusts, and mists. Safety and health regulations for construction. Occupational Safety and Health Administration. Code of Federal Regulations. 29 CFR 1926.55, Appendix A. http://www.osha.gov/comp-links.html. February 15, 2005.

OSHA. 2005c. Inorganic arsenic. Occupational safety and health standards. Occupational Safety and Health Administration. Code of Federal Regulations. 29 CFR 1910.1018. http://www.osha.gov/complinks.html. March 24, 2005.

OSHA. 2005d. Limits for air contaminants. Occupational safety and health standards. Washington, DC: Occupational Safety and Health Administration. Code of Federal Regulations. 29 CFR 1910.1000. http://www.osha.gov/comp-links.html. February 15, 2005.

O’Shaughnessy E, Kraft GH. 1976. Arsenic poisoning: Long-term follow-up of a nonfatal case. Arch Phys Med Rehabil 57:403-406.

OTA. 1990. Neurotoxicity: Identifying and controlling poisons of the nervous system. Washington, DC: Office of Technology Assessment. OTA-BA-438.

Ott MG, Holder BB, Gordon HL. 1974. Respiratory cancer and occupational exposure to arsenicals. Arch Environ Health 29:250-255.

Owen GM, Brozek J. 1966. Influence of age, sex and nutrition on body composition during childhood and adolescence. In: Falkner F, ed. Human development. Philadelphia, PA: WB Saunders, 222-238.

Oya-Ohta Y, Kaise T, Ochi T. 1996. Induction of chromosomal aberrations in cultured human fibroblasts by inorganic and organic arsenic compounds and the different roles of glutathione in such induction. Mutat Res 357(1-2)123-129. 
Øygard JK, Lundebye A, Julshamin K. 1999. Determination of inorganic arsenic in marine food samples by hydrochloric acid distillation and flow-injection hydride-generation atomic absorption spectrometry. J AOAC Int 82(5):1217-1223.

Pacyna JM. 1987. Atmospheric emissions of arsenic, cadmium, lead and mercury from high temperature processes in power generation and industry. In: Hutchinson TC, Meema KM, eds. Lead, mercury, cadmium and arsenic in the environment. New York: John Wiley \& Sons Ltd., 69-87.

Pacyna JM, Scholtz MT, Li Y. 1995. Global budget of trace metal sources. Environ Rev 3(2):145-159.

Page BJ, Loar GW. 1993. Chromium compounds. In: Kroschwitz JI, Howe-Grant M, eds. KirkOthmer encyclopedia of chemical technology. Vol. 6. New York, NY: John Wiley and Sons, 297-298.

Page GW. 1981. Comparison of groundwater and surface water for patterns and levels of contamination by toxic substances. Environ Sci Technol 15(12):1475-1481.

Pantsar-Kallio M, Manninen PKG. 1997. Speciation of mobile arsenic in soil samples as a function of pH. Sci Total Environ 204(2):193-200.

Park J, Presley BJ. 1997. Trace metal contamination of sediments and organisms from the Swan Lake area of Galveston Bay. Environ Pollut 98(2):209-221.

Paschal DC, DiPietro ES, Phillips DL, et al. 1989. Age dependence of metals in hair in a selected US population. Environ Res 48:17-28.

Pascoe GA, Blanchet RJ, Linder G. 1994. Bioavailability of metals and arsenic to small mammals at a mining waste-contaminated wetland. Arch Environ Contam Toxicol 27:44-50.

Pedersen GA, Mortensen GK, Larsen EH. 1994. Beverages as a source of toxic trace element intake. Food Addit Contam 11(3):351-363.

*Pellizzari ED, Smith DJ, Clayton CA, et al. 2001. An assessment of the data quality for NHEXAS-Part I: Exposure to metals and volatile organic chemicals in Region 5. J Expo Anal Environ Epidemiol 11:140-154.

Peoples SA, Maddy KT, Peifer WR, et al. 1979. Occupational exposures to pesticides containing organoarsenicals in California. Vet Hum Toxicol 21:417-421. (Retrieval in progress)

Permapost. 2000. Chromated copper arsenate, CCA-C. Permapost Products Co., Hillsboro, Oregon. http://www.permapost.com/cca.htm. March 3, 2005.

Perry K, Bowler RG, Buckell HM, et al. 1948. Studies in the incidence of cancer in a factory handling inorganic compounds of arsenic--II: Clinical and environmental investigations. Br J Ind Med 5:6-15.

Pershagen G. 1985. Lung cancer mortality among men living near an arsenic-emitting smelter. Am J Epidemiol 122(4):684-694.

Pershagen G, Björklund NE. 1985. On the pulmonary tumorigenicity of arsenic trisulfide and calcium arsenate in hamsters. Cancer Lett 27:99-104. 
Pershagen G, Bergman F, Klominek J, et al. 1987. Histological types of lung cancer among smelter workers exposed to arsenic. Br J Ind Med 44:454-458.

Pershagen G, Nordberg G, Bjorkland NE. 1984. Carcinomas of the respiratory tract in hamsters given arsenic trioxide and/or benzo[a]pyrene by the pulmonary route. Environ Res 34:227-241.

Pershagen G, Wall S, Taube A, et al. 1981. On the interaction between occupational arsenic exposure and smoking and its relationship to lung cancer. Scand J Work Environ Health 7:302-309.

Pesch B, Ranft U, Jakubis P, et al. 2002. Environmental arsenic exposure from a coal-burning power plant as a potential risk factor for nonmelanoma skin carcinoma: Results from a case-control study in the district of Prievidza, Slovakia. Am J Epidemiol 155(9):798-809.

*Peters RA, Thompson RH, King AJ, et al. 1944. The treatment of post-arsphenamine jaundice with sulphur-containing amino acids. Q J Med 14:35-56.

Peters SC, Blum JD, Klaue B, et al. 1999. Arsenic occurrence in New Hampshire drinking water. Environ Sci Technol 33:1328-1333.

Peterson RG, Rumack BH. 1977. D-penicillamine therapy of acute arsenic poisoning. J Pediatr 91(4):661-666.

Petres J, Baron D, Hagedorn M. 1977. Effects of arsenic cell metabolism and cell proliferation: Cytogenic and biochemical studies. Environ Health Perspect 19:223-227.

Petrick JS, Ayala-Fierro F, Cullen WR, et al. 2000. Monomethylarsenous acid (MMA III) is more toxic than arsenite in Chang human hepatocytes. Toxicol Appl Pharmacol 163:203-207.

Petrick JS, Jagadish B, Mash EA, et al. 2001. Monomethylarsenous acid (MMA ${ }^{\mathrm{III}}$ ) and arsenite: LD $_{50}$ in hamsters and in vitro inhibition of pyruvate dehydrogenase. Chem Res Toxicol 14(6):651-656.

Philp RB, Leung FY, Bradley C. 2003. A comparison of the metal content of some benthic species from coastal waters of the Florida panhandle using high-resolution inductively coupled plasma mass spectrometry (ICP-MS) analysis. Arch Environ Contam Toxicol 44:218-223.

Pi J, He Y, Bortner C, et al. 2005. Low level, long-term inorganic arsenite exposure causes generalized resistance to apoptosis in cultured human keratinocytes: Potential role in skin co-carcinogenesis. Int $\mathrm{J}$ Cancer 116:20-26.

*Pi J, Yamauchi H, Kumagai Y, et al. 2002. Evidence for induction of oxidative stress caused by chronic exposure of Chinese residents to arsenic contained in drinking water. Environ Health Perspect 110:331336.

Pinto SS, McGill CM. 1953. Arsenic trioxide exposure in industry. Ind Med Surg 22(7):281-287.

Pinto SS, Enterline PE, Henderson V, et al. 1977. Mortality experience in relation to a measured arsenic trioxide exposure. Environ Health Perspect 19:127-130.

Pinto SS, Henderson V, Enterline PE. 1978. Mortality experience of arsenic-exposed workers. Arch Environ Health 33:325-331. 
Pinto SS, Varner MO, Nelson KW, et al. 1976. Arsenic trioxide absorption and excretion in industry. J Occup Med 18(10):677-680.

Piontek M, Hengels KJ, Borchard F, et al. 1989. [Noncirrhotic liver fibrosis after chronic arsenic poisoning.] Dtsch Med Wochenschr 114:1653-1657. (German)

Pirrone N, Keeler GJ. 1996. A preliminary assessment of the urban pollution in the Great Lakes region. Sci Total Environ 189/190:91-98.

Pitten F, Müller G, König P, et al. 1999. Risk assessment of former military base contaminated with organoarsenic-based warfare agents: Uptake of arsenic by terrestrial plants. Sci Total Environ 226:237245.

Poddar S, Mukherjee P, Talukder G, et al. 2000. Dietary protection by iron against clastogenic effects of short-term exposure to arsenic in mice in vivo. Food Chem Toxicol 38(8):735-737.

Polissar L, Lowry-Coble K, Kalman DA, et al. 1990. Pathways of human exposure to arsenic in a community surrounding a copper smelter. Environ Res 53:29-47.

Poma K, Degraeve N, Kirsch-Volders M, et al. 1981. Cytogenetic analysis of bone marrow cells and spermatogonia of male mice after in vivo treatment with arsenic. Experientia 37:129-130.

Poma K, Degraeve N, Susanne C. 1987. Cytogenetic effects in mice after chronic exposure to arsenic followed by a single dose of ethylmethane sulfonate. Cytologia 52:445-449.

Pomroy C, Charbonnaeu SM, McCullough RS, et al. 1980. Human retention studies with ${ }^{74}$ As. Toxicol Appl Pharm 53:550-556.

Pongratz R. 1998. Arsenic speciation in environmental samples of contaminated soil. Sci Total Environ 224:133-141.

Pouschat P, Zagury GJ. 2006. In vitro gastrointestinal bioavailability of arsenic in soils collected near CCA-treated utility poles. Environ Sci Technol 40(13):4317-4323.

Prasad GVR, Rossi NF. 1995. Arsenic intoxication associated with tubulointerstitial nephritis. Am J Kidney Dis 26(2)373-376.

Prier RF, Nees PO, Derse PH. 1963. The toxicity of an organic arsenical, 3-nitro-4-hydroxyphenylarsonic acid. II. Chronic toxicity. Toxicol Appl Pharmacol 5:526-542.

Prukop JA, Savage NL. 1986. Some effects of multiple, sublethal doses of monosodium methanearsonate (MSMA) herbicide on hematology, growth, and reproduction of laboratory mice. Bull Environ Contam Toxicol 36:337-341.

PSA. 2000. Excalibur atomic fluorescence: Measurements of arsenic, selenium, antimony, bismuth and tellurium. Orpington, England: PS Analytical Ltd. http://www.banian.com. March 31, 2005.

Qiao Y, Taylor PR, Yao S, et al. 1997. Risk factors and early detection of lung cancer in a cohort of Chinese tin miners. Ann Epidemiol 7:533-541. 
Quatrehomme G, Ricq O, Lapalus P, et al. 1992. Acute arsenic intoxication: Forensic and toxicologic aspects (an observation). J Forensic Sci 37(4)1163-1171.

Rabano ES, Castillo NT, Torre KJ, et al. 1989. Speciation of arsenic in ambient aerosols collected in Los Angeles. J Air Pollut Control Assoc 39:76-80.

*Rahman M, Axelson O. 1995. Diabetes mellitus and arsenic exposure: A second look at case-control data from a Swedish copper smelter. Occup Environ Med 52(11)773-774.

Rahman FA, Allan DL, Rosen CJ, et al. 2004. Arsenic availability from chromated copper arsenate (CCA)-treated wood. J Environ Qual 33(1):173-180.

Rahman M, Tondel M, Ahmad SA, et al. 1998. Diabetes mellitus associated with arsenic exposure in Bangladesh. Am J Epidemiol 148(2):198-203.

*Rahman M, Wingren G, Axelson O. 1996. Diabetes mellitus among Swedish art glass workers-an effect of arsenic exposure? Scand J Work Environ Health 22(2):146-149.

Rahman M, Tondel M, Ahmad SA, et al. 1999. Hypertension and arsenic exposure in Bangladesh. Hypertension 33:74-78.

Raie RM. 1996. Regional variation in $\mathrm{As}, \mathrm{Cu}, \mathrm{Hg}$, and $\mathrm{Se}$ and interaction between them. Ecotoxicol Environ Saf 35:248-252.

Ramirez P, Eastmond DA, Laclette JP, et al. 1997. Disruption of microtubule assembly and spindle formation as a mechanism for the induction of aneuploid cells by sodium arsenite and vanadium pentoxide. Mutat Res 386(3):291-298.

Ramos-Morales P, Rodríguez-Arnaiz R. 1995. Genotoxicity of two arsenic compounds in germ cells and somatic cells of Drosophila melanogaster. Environ Mol Mutagen 25(4):288-299.

Ramsey JC, Andersen ME. 1984. A physiologically based description of the inhalation pharmacokinetics of styrene in rats and humans. Toxicol Appl Pharmacol 73:159-175.

Rasmussen RE, Menzel DB. 1997. Variation in arsenic-induced sister chromatid exchange in human lymphocytes and lymphoblastoid cell lines. Mutat Res 386(3)299-306.

Rastogi SC, Pritzl G. 1996. Migration of some toxic metals from crayons and water colors. Bull Environ Contam Toxicol 56(4)527-533.

Reddy PRK, Reddy SJ. 1997. Elemental concentrations in medicinally important leafy materials. Chemosphere 34(9-10):2193-2212.

Redman AD, Macalady DL, Ahmann D. 2002. Natural organic matter affects arsenic speciation and sorption onto hematite. Environ Sci Technol 36:2889-2896.

Regelson W, Kim U, Ospina J, et al. 1968. Hemangioendothelial sarcoma of liver from chronic arsenic intoxication by Fowler's solution. Cancer 21(3):514-522.

*Reichl F, Hunder G, Liebl B, et al. 1995. Effect of DMPS and various adsorbents on the arsenic excretion in guinea-pigs after injection with $\mathrm{As}_{2} \mathrm{O}_{3}$. Arch Toxicol 69(10):712-717. 
Reimann C, De Caritat P, Halleraker JH, et al. 1997. Rainwater composition in eight arctic catchments in Northern Europe (Finland, Norway and Russia). Atmos Environ 31(2):159-170.

Reimer KJ. 1989. The methylation of arsenic in marine sediments. Appl Organomet Chem 3:475-490.

Reitz RH, Mendrala AL, Corley RA, et al. 1990. Estimating the risk of liver cancer associated with human exposures to chloroform using physiologically based pharmacokinetic modeling. Toxicol Appl Pharm 105:443-459.

Reymann F, Mller R, Nielsen A. 1978. Relationship between arsenic intake and internal malignant neoplasms. Arch Dermatol 114:378-381.

Rhine ED, Phelps CD, Young LY. 2006. Anaerobic arsenite oxidation by novel denitrifying isolates. Environ Microbiol 8(5):899-908.

Rhoads K, Sanders CL. 1985. Lung clearance, translocation and acute toxicity of arsenic, beryllium, cadmium, cobalt, lead, selenium, vanadium, and ytterbium oxides following deposition in rat lung. Environ Res 36:359-378.

Rice DA, Kennedy S, McMurray CH, et al. 1985. Experimental 3-nitro-4-hydroxyphenylarsonic acid toxicosis in pigs. Res Vet Sci 39:47-51.

Rin K, Kawaguchi K, Yamanaka K, et al. 1995. DNA-strand breaks induced by dimethylarsinic acid, a metabolite of inorganic arsenics, are strongly enhanced by superoxide anion radicals. Biol Pharm Bull 18(1):45-48.

Rivara MIZ, Cebrián MG, Corey G, et al. 1997. Cancer risk in an arsenic contaminated area of Chile. In: International congress on hazardous waste: Impact on human and ecological health. Hazardous waste, impacts on human ecological health: Proceedings of the 2nd International Congress on Hazardous Waste, Impact on Human and Ecological Health. Princeton, New Jersey: Princeton Scientific Pub. Co., 408-426.

Roberts SM, Munson JW, Lowney YW, et al. 2007. Relative oral bioavailablity of arsenic from contaminated soils measured in the cynomolgus monkey. Toxicol Sci 95(1):281-288.

Roberts SM, Weimar WR, Vinson JRT, et al. 2002. Measurement of arsenic bioavailability in soil using a primate model. Toxicol Sci 67:303-310.

Robertson FN. 1989. Arsenic in ground-water under oxidizing conditions, southwest United States. Environ Geochem Health 11:171-185.

Rodriguez RR, Basta NT, Casteel SW, et al. 1999. An in vitro gastrointestinal method to estimate bioavailable arsenic in contaminated soils and solid media. Environ Sci Technol 33:642-649.

Rodriguez VM, Carrizales L, Jimenez-Capdeville ME, et al. 2001. The effects of sodium arsenite exposure on behavioral parameters in the rat. Brain Res Bull 55(2):301-308.

Rodriguez VM, Carrizales L, Mendoza MS, et al. 2002. Effects of sodium arsenite exposure on development and behavior in the rat. Neurotoxicol Teratol 24(6):743-750. 
*Rogers CE, Tomita AV, Trowbridge PR, et al. 1997. Hair analysis does not support hypothesized arsenic and chromium exposure from drinking water in Woburn, Massachusetts. Environ Health Perspect 105(10):1090-1097.

Rogers EH, Chernoff N, Kavlock RJ. 1981. The teratogenic potential of cacodylic acid in the rat and mouse. Drug Chem Toxicol 4(1):49-61.

Roper JM, Cherry DS, Simmers JW, et al. 1996. Bioaccumulation of toxicants in the zebra mussel, Dreissena polymorpha, at the Times Beach confined disposal facility, Buffalo, New York. Environ Pollut 94(2):117-129.

Rosen BP. 2002. Biochemistry of arsenic detoxification. FEBS Lett 529:86-92.

Rosenbaum AS, Axelrad DA, Woodruff TJ, et al. 1999. National estimates of outdoor air toxics concentrations. J Air Waste Manage Assoc 49:1138-1152.

Rosenberg HG. 1974. Systemic arterial disease and chronic arsenicism in infants. Arch Pathol 97:360365.

*Rosner MH, Carter DE. 1987. Metabolism and excretion of gallium arsenide and arsenic oxides by hamsters following intratracheal instillation. Fundam Appl Toxicol 9:730-737.

Rossman TG. 2003. Mechanism of arsenic carcinogenesis: An integrated approach. Mutat Res 533:3765 .

Rossman TG, Uddin AN. 2004. Selenium prevents spontaneous and arsenite-induced mutagenesis. Int Congr Ser 1275:173-179.

Rossman TG, Stone D, Molina M, et al. 1980. Absence of arsenite mutagenicity in E coli and Chinese hamster cells. Environ Mut 2:371-379.

Rossman TG, Uddin AN, Burns FJ. 2004. Evidence that arsenite acts as a cocarcinogen in skin cancer. Toxicol Appl Pharmacol 198(3):394-404.

Rössner P, Bencko V, Havránková H. 1977. Effect of the combined action of selenium and arsenic on suspension culture of mice fibroblasts. Environ Health Perspect 19:235-237.

Roth F. 1957. The sequelae of chronic arsenic poisoning in Moselle Vintners. Ger Med Mon 2:172-175.

Roy P, Saha A. 2002. Metabolism and toxicity of arsenic: A human carcinogen. Curr Sci 82(1):38-45.

RTECS. 2007. Arsenic. Registry of Toxic Effects on Chemical Substances. National Institute of Occupational Safety and Health. MDL Information Systems, Inc. May 8, 2007

Rubin Y, Gal N, Nyska A, et al. 1989. Cacodylic acid two generation reproduction study in the rat. Luxembourg Industries (Pamol) Ltd. Submitted to the U.S. Environmental Protection Agency. MRID41059501.

Ruby MV, Davis A, Schoof R, et al. 1996. Estimation of lead and arsenic bioavailability using a physiologically based extraction test. Environ Sci Technol 30(2):422-430. 


\section{REFERENCES}

Ruby MV, Schoof R, Brattin W, et al. 1999. Advances in evaluating the oral bioavailability of inorganics in soil for use in human health risk assessment. Environ Sci Technol 33(21):3697-3705.

*Rudnai P, Borzonyi M. 1980. Carcinogenic effect of arsenic trioxide in transplacentally and neonatally treated CFLP mice. Nat Sci 2:11-18.

Rupa DS, Schuler M, Eastmond DA. 1997. Detection of hyperdiploidy and breakage affecting the 1cen$1 \mathrm{q} 12$ region of cultured interphase human lymphocytes treated with various genotoxic agents. Environ Mole Mutagen 29(2):161-167.

Rutherford DW, Bednar AJ, Garbarino JR, et al. 2003. Environmental fate of roxarsone in poultry litter. Part II. Mobility of arsenic in soils amended with poultry litter. Environ Sci Technol 37:1515-1520.

Ryan PB, Scanlon KA, MacIntosh DL. 2001. Analysis of dietary intake of selected metals in the NHEXAS-Maryland investigation. Environ Health Perspect 109(2):121-128.

Saady JJ, Blanke RV, Poklis A. 1989. Estimation of the body burden of arsenic in a child fatally poisoned by arsenite weedkiller. J Anal Toxicol 13:310-312.

Saha KC, Poddar D. 1986. Further studies on chronic arsenical dermatosis. Indian J Dermatol 31:29-33.

Salim EI, Wanibuchi H, Morimura K, et al. 2003. Carcinogenicity of dimethylarsinic acid in p53 heterozygous knockout and wild-type C57BL/6J mice. Carcinogenesis 24(2):335-342.

Samsøe-Petersen L, Larsen EH, Larsen PB, et al. 2002. Uptake of trace elements and PAHs by fruit and vegetables from contaminated soils. Environ Sci Technol 36:3057-3063.

Sanders JG, Riedel GF, Osmann RW. 1994. Arsenic cycling and its impact in estuarine and coastal marine ecosystems. In: Nriagu JO, ed. Arsenic in the environment, part I: Cycling and characterization. New York, NY: John Wiley \& Sons, Inc., 289-308.

Sandstrom AIM, Wall SGI, Taube A. 1989. Cancer incidence and mortality among Swedish smelter workers. Br J Ind Med 46:82-89.

Sanok WJ, Ebel JG, JR, Manzell KL, et al. 1995. Residues of arsenic and lead in potato soils on long island. Chemosphere 30(4):803-806.

Santra A, Maiti A, Das S, et al. 2000. Hepatic damage caused by chronic arsenic toxicity in experimental animals. J Toxicol Clin Toxicol 38(4):395-405.

Sardana MK, Drummond GS, Sassa S, et al. 1981. The potent heme oxygenase inducing action of arsenic in parasiticidal arsenicals. Pharmacology 23:247-253.

*Sarin SK, Sharma G, Banerjee S, et al. 1999. Hepatic fibrogenesis using chronic arsenic ingestion studies in a murine model. Indian J Exp Biol 37:147-151.

Sarkar D, Datta R. 2004. Arsenic fate and bioavailability in two soils contaminated with sodium arsenate pesticide: An incubation study. Bull Environ Contam Toxicol 72(2):240-247.

Sass U, Grosshans E, Simonart JM. 1993. Chronic arsenicism: Criminal poisoning or drug-intoxication? Report of two cases. Dermatology 186(4)303-305. 
Schmitt CJ, Zajicek JL, May TW, et al. 1999. Organochlorine residues and elemental contaminants in U.S. freshwater fish, 1976-1986: National contaminant biomonitoring program. Rev Environ Contam Toxicol 162:43-104.

Schoenwolf GC, Smith JL. 1990. Mechanisms of neurulation: Traditional viewpoint and recent advances. Development 109:243-270.

Schoof RA, Eickhoff J, Yost LJ, et al. 1999a. Dietary exposure to inorganic arsenic. In: Chappell WR, Abernathy CO, Calderon RL, eds. Arsenic exposure and health effects. Amsterdam: Elsevier Science, 81-88.

Schoof RA, Yost LJ, Crecelius K, et al. 1998. Dietary arsenic intake in Taiwanese districts with elevated arsenic in drinking water. Hum Ecol Risk Assess 4(1):117-135.

Schoof RA, Yost LJ, Eickhoff J, et al. 1999b. A market basket survey of inorganic arsenic in food. Food Chem Toxicol 37:839-846.

Schrauzer GN. 1987. Effects of selenium antagonists on cancer susceptibility: New aspects of chronic heavy metal toxicity. J UOEH 9(Suppl):208-215.

Schrauzer GN, Ishmael D. 1974. Effects of selenium and of arsenic on the genesis of spontaneous mammary tumors in inbred $\mathrm{C}_{3} \mathrm{H}$ mice. Ann Clin Lab Sci 4(6):441-447.

Schrauzer GN, White DA, McGinness JE, et al. 1978. Arsenic and cancer: Effects of joint administration of arsenite and selenite on the genesis of adenocarcinoma in inbred female $\mathrm{C}_{3} \mathrm{H} / \mathrm{St}$ mice. Bioinorg Chem 9:245-253.

Schrauzer GN, White DA, Schneider CJ. 1976. Inhibition of the genesis of spontaneous mammary tumors in $\mathrm{C}_{3} \mathrm{H}$ mice: Effects of selenium and of selenium-antagonistic elements and their possible role in human breast cancer. Bioinorg Chem 6:265-270.

*Schroeder HA, Balassa JJ. 1966. Abnormal trace metals in man: Arsenic. J Chron Dis 19:85-106.

Schroeder HA, Balassa JJ. 1967. Arsenic, germanium, tin and vanadium in mice: Effects on growth, survival and tissue levels. J Nutr 92:245-252.

Schroeder HA, Mitchener M. 1971. Toxic effects of trace elements on the reproduction of mice and rats. Arch Environ Health 23:102-106.

Schroeder HA, Kanisawa M, Frost DV, et al. 1968. Germanium, tin and arsenic in rats: Effects on growth, survival, pathological lesions and life span. J Nutr 96:37-45.

Schroeder RE. 1994. A two-generation reproduction study in rats with methanearsonic acid (MAA). Luxembourg Industries (Pamol) Ltd. Submitted to the U.S. Environmental Protection Agency. MRID43178301.

Schroeder WH, Dobson M, Kane DM, et al. 1987. Toxic trace elements associated with airborne particulate matter: A review. J Air Pollut Control Assoc 37(11):1267-1285. 
Schulz H, Nagymajtenyi L, Institoris L, et al. 2002. A study on behavioral, neurotoxicological, and immunotoxicological effects of subchronic arsenic treatment in rats. J Toxicol Environ Health A 65(16):1181-1193.

Scott N, Hatlelid KM, MacKenzie NE, et al. 1993. Reactions of arsenic (III) and arsenic (V) species with glutathione. Chem Res Toxicol 6:102-106.

Scudlark JR, Church TM. 1988. The atmospheric deposition of arsenic and association with acid precipitation. Atmos Environ 22(5):937-943.

Scudlark JR, Conko KM, Church TM. 1994. Atmospheric wet deposition of trace elements to Chesapeake Bay: CBAD study year 1 results. Atmos Environ 28(8):1487-1498.

Senesi GS, Baldassarre G, Senesi N, et al. 1999. Trace element inputs into soils by anthropogenic activities and implications for human health. Chemosphere 39(2):343-377.

Setchell BP, Waites GMH. 1975. The blood-testis barrier. In: Creep RO, Astwood EB, Geiger SR, eds. Handbook of physiology: Endocrinology V. Washington, DC: American Physiological Society.

Shahin U, Yi SM, Paode RD, et al. 2000. Long-term elemental dry deposition fluxes measured around Lake Michigan with an automated dry deposition sampler. Environ Sci Technol 34:1887-1892.

Shalat SL, Solo-Gabriele HM, Fleming LE, et al. 2006. A pilot study of children's exposure to CCAtreated wood from playground equipment. Sci Total Environ 367(1):80-88.

Shannon RL, Strayer DS. 1989. Arsenic-induced skin toxicity. Hum Toxicol 8:99-104.

Sheehy JW, Jones JH. 1993. Assessment of arsenic exposures and controls in gallium arsenide production. Am Ind Hyg Assoc J 54(2):61-69.

Shen J, Wanibuchi H, Salim EI, et al. 2003. Induction of glutathione S-transferase placental form positive foci in liver and epithelial hyperplasia in urinary bladder, but no tumor development in male epithelial hyperplasia in urinary bladder, but no tumor development in male Fischer 344 rats treated with monomethylarsonic acid for 104 weeks. Toxicol Appl Pharmacol 193(3):335-345.

*Shen Z, Chen G, Ni J, et al. 1997. Use of arsenic trioxide $\left(\mathrm{As}_{2} \mathrm{O}_{3}\right)$ in the treatment of acute promyelocytic leukemia (APL): II. Clinical efficacy and pharmacokinetics in relapsed patients. Blood 89(9):3354-3360.

*Shi H, Shi X, Liu KJ. 2004. Oxidative mechanism of arsenic toxicity and carcinogenesis. Mol Cell Biochem 255(1-2):67-78.

*Shibata A, Ohneseit PF, Tsai YC, et al. 1994. Mutational spectrum in the p53 gene in bladder tumors from the endemic area of blackfoot disease in Taiwan. Carcinogenesis 15(6):1085-1087.

Shirachi DY, Johansen MG, McGowan JP, et al. 1983. Tumorigenic effect of sodium arsenite in rat kidney. Proc West Pharmacol Soc 26:413-415.

Shum S, Whitehead J, Vaughn L, et al. 1995. Chelation of organoarsenate with dimercaptosuccinic acid. Vet Hum Toxicol 37(3):239-242. 
9. REFERENCES

Siewicki TC. 1981. Tissue retention of arsenic in rats fed witch flounder or cacodylic acid. J Nutr 111:602-609.

Sikorski EE, McCay JA, White KL Jr, et al. 1989. Immunotoxicity of the semiconductor gallium arsenide in female B6C3F1 mice. Fundam Appl Toxicol 13:843-858.

Silver AS, Wainman PL. 1952. Chronic arsenic poisoning following use of an asthma remedy. JAMA 150(6):584-585.

Singh I. 1983. Induction of reverse mutation and mitotic gene conversion by some metal compounds in Saccharomyces cerevisiae. Mutat Res 117:149-152.

Skeaff JM, Dubreuil AA. 1997. Calculated 1993 emission factors of trace metals for Canadian nonferrous smelters. Atmos Environ 31(10):1449-1457.

Slayton TM, Beck BD, Reynolds KA, et al. 1996. Issues in arsenic cancer risk assessment. Environ Health Perspect 104 (10):1012-1018.

Smith AH, Biggs ML, Hopenhayn-Rich C, et al. 1995. Correspondence: Arsenic risk assessment. Environ Health Perspect 103(1): http://www.ehponline.org/docs/1995/103-1/correspondence.html. August 30, 2007.

Smith AH, Goycolea M, Haque R, et al. 1998. Marked increase in bladder and lung cancer mortality in a region of northern Chile due to arsenic in drinking water. Am J Epidemiol 147(7):660-669.

Smith AH, Hopenhayn-Rich C, Bates MN, et al. 1992. Cancer risks from arsenic in drinking water. Environ Health Perspect 97:259-267.

Smith AH, Marshall G, Yuan Y, et al. 2006. Increased mortality from lung cancer and bronchiectasis in young adults after exposure to arsenic in utero and in early childhood. Environ Health Perspect 114(8):1293-1296.

Smith CJ, Livingston SD, Doolittle DJ. 1997. An international literature survey of "IARC group I carcinogens" reported in mainstream cigarette smoke. Food Chem Toxicol 35(10-11):1107-1130.

Smith E, Naidu R, Alston AM. 1999. Chemistry of arsenic in soils: I. Sorption of arsenate and arsenite by four Australian soils. J Environ Qual 28:1719-1726.

Smith E, Naidu R, Alston AM. 2002. Chemistry of inorganic arsenic in soils: II. Effect of phosphorus, sodium, and calcium on arsenic sorption. J Environ Qual 31(2):557-563.

Smith RA, Alexander RB, Wolman MG. 1987. Water-quality trends in the nation's rivers. Science 235:1607-1615.

Smith TJ, Crecelius EA, Reading JC. 1977. Airborne arsenic exposure and excretion of methylated arsenic compounds. Environ Health Perspect 19:89-93.

Sobel W, Bond GG, Baldwin CL, et al. 1988. An update of respiratory cancer and occupational exposure to arsenicals. Am J Ind Med 13:263-270. 
Sofuni T, Honma M, Hayashi M, et al. 1996. Detection of in vitro clastogens and spindle poisons by the mouse lymphoma assay using the microwell method: Interim report of an international collaborative study. Mutagenesis 11(4):349-355.

*Soignet SL, Maslak P, Wang Z, et al. 1998. Complete remission after treatment of acute promyelocytic leukemia with arsenic trioxide. N Engl J Med 339(19):1341-1348.

Sommers SC, McManus RG. 1953. Multiple arsenical cancers of the skin and internal organs. Cancer 6:347-359.

Somogyi A, Beck H. 1993. Nurturing and breast-feeding: Exposure to chemicals in breast milk. Environ Health Perspect 101(Suppl 2):45-52.

Sotera JJ, Dulude GR, Stux RL. 1988. Determination of toxic elements in biological materials by furnace atomic absorption spectrometry. Sci Total Environ 71:45-48.

SRI. 2006. Directory of chemical producers. Menlo Park, CA: SRI Consulting, 469, 533, 654, 784, 787.

Steinmaus C, Carrigan K, Kalman D, et al. 2005a. Dietary intake and arsenic methylation in a U.S. population. Environ Health Perspect 113(9):1153-1159.

Steinmaus C, Moore LE, Shipp M, et al. 2007. Genetic polymorphisms in MTHFR 677 and 1298, GSTM1 and T1, and metabolism of arsenic. J Toxicol Environ Health A 70:159-170.

Steinmaus C, Yuan Y, Bates MN, et al. 2003. Case-control study of bladder cancer and drinking water arsenic in the western United States. Am J Epidemiol 158(12):1193-1201.

*Steinmaus C, Yuan Y, Kalman D, et al. 2005b. Intraindividual variability in arsenic methylation in a U.S. population. Cancer Epidemiol Biomarkers Prev 14(4):919-924.

Steinnes E, Rambaek JP, Hanssen JK. 1992. Large scale multi-element survey of atmospheric deposition using naturally growing moss as a biomonitor. Chemosphere 25(5):735-752.

Sternowsky HJ, Moser B, Szadkowsky D. 2002. Arsenic in breast milk during the first 3 months of lactation. Int J Hyg Environ Health 205(5):405-409.

Stevens JT, DiPasquale LC, Farmer JD. 1979. The acute inhalation toxicology of the technical grade organoarsenical herbicides, cacodylic acid and disodium methanearsonic acid; a route comparison. Bull Environ Contam Toxicol 21:304-311.

Stevens JT, Hall LL, Farmer JD, et al. 1977. Disposition of ${ }^{14} \mathrm{C}$ and/or ${ }^{74}$ As-cacodylic acid in rats after intravenous, intratracheal or peroral administration. Environ Health Perspect 19:151-157.

Stilwell DE, Graetz TJ. 2001. Copper, chromium, and arsenic levels in soil near highway traffic sound barriers built using CCA pressure-treated wood. Bull Environ Contam Toxicol 67:303-308.

Storelli MM, Marcotrigiano GO. 2001. Total, organic, and inorganic arsenic in some commercial species of crustaceans from the Mediterranean Sea (Italy). J Food Prot 64(11):1858-1862. 
Storer RD, McKelvey TW, Kraynak AR, et al. 1996. Revalidation of the in vitro alkaline elution/rat hepatocyte assay for DNA damage: Improved criteria for assessment of cytotoxicity and genotoxicity and results for 81 compounds. Mutat Res 368(2):59-101.

Stump DG, Holson JF, Fleeman TL, et al. 1999. Comparative effects of single intraperitoneal or oral doses of sodium arsenate or arsenic trioxide during in utero development. Teratology 60:283-291.

Stutz DR, Janusz SJ. 1988. Hazardous materials injuries: A handbook for pre-hospital care. 2nd ed. Beltsville, MD: Bradford Communications Corporation, 208-209.

Styblo M, Thomas DJ. 2001. Selenium modifies the metabolism and toxicity of arsenic in primary rat hepatocytes. Toxicol Appl Pharmacol 172(1):52-61.

Sweins A. 1983. Protective effect of selenium against arsenic-induced chromosomal damage in cultured human lymphocytes. Hereditas 98:249-252.

Szinicz L, Forth W. 1988. Effect of $\mathrm{As}_{2} \mathrm{O}_{3}$ on gluconeogenesis. Arch Toxicol 61:444-449.

Szuler IM, Williams CN, Hindmarsh JT, et al. 1979. Massive variceal hemorrhage secondary to presinusoidal portal hypertension due to arsenic poisoning. Can Med Assoc J 120:168-171.

Tabacova S, Hunter ES, Gladen BC. 1996. Developmental toxicity of inorganic arsenic in whole embryo culture: Oxidation state, dose, time, and gestational age dependence. Toxicol Appl Pharmacol 138(2):298-307.

Takahashi K, Yamauchi H, Yamato N, et al. 1988. Methylation of arsenic trioxide in hamsters with liver damage induced by long-term administration of carbon tetrachloride. Appl Organomet Chem 2:309-314.

Tam GKH, Lacroix G. 1982. Dry ashing, hydride generation atomic absorption spectrometric determination of arsenic and selenium in foods. J Assoc Off Anal Chem 65(3):647-650.

*Tam GK, Charbonneau SM, Lacroix G, et al. 1979a. Confirmation of inorganic arsenic and dimethylarsenic acid in urine and plasma of dog by ion-exchange and TLC. Bull Environ Contam Toxicol 21:371-374.

Tam GKH, Charbonneau SM, Bryce F, et al. 1979b. Metabolism of inorganic arsenic $\left({ }^{74} \mathrm{As}\right)$ in humans following oral ingestion. Toxicol Appl Pharmacol 50:319-322.

Tam GKH, Charbonneau SM, Bryce F, et al. 1982. Excretion of a single oral dose of fish-arsenic in man. Bull Environ Contam Toxicol 28:669-673.

Tamaki S, Frankenberger WT. 1992. Environmental biochemistry of arsenic. Rev Environ Contam Toxicol 124:79-110.

Tao SS, Bolger PM. 1999. Dietary intakes of arsenic in the United States. Food Addit Contam 16:465472.

Taubeneck MW, Daston GP, Rogers JM, et al. 1994. Altered maternal zinc metabolism following exposure to diverse developmental toxicants. Reprod Toxicol 8(1):25-40. 
Tay C, Seah C. 1975. Arsenic poisoning from anti-asthmatic herbal preparations. Med J Aust 2:424428.

Taylor HE, Antweiler RC, Roth DA, et al. 2001. The occurrence and distribution of selected trace elements in the upper Rio Grande and tributaries in Colorado and northern New Mexico. Arch Environ Contam Toxicol 41:410-426

Taylor PR, Qiao YL, Schatzkin A, et al. 1989. Relation of arsenic exposure to lung cancer among tin miners in Yunnan Province. Br J Ind Med 46:881-886.

Teräsahde P, Pantsar-Kallio M, Manninen PKG. 1996. Simultaneous determination of arsenic species by ion chromatography-inductively coupled plasma mass spectrometry. J Chromatog A750(1-2):83-88.

Tezuka M, Hanioka K, Yamanaka K, et al. 1993. Gene damage induced in human alveolar type II (L132) cells by exposure to dimethylarsinic acid. Biochem Biophys Res Commun 191(3):1178-1183.

Thomas DC, Whittemore AS. 1988. Methods for testing interactions, with applications to occupational exposures, smoking, and lung cancer. Am J Ind Med 13:131-147.

Thomas DJ, Li J, Waters SB, et al. 2007. Arsenic (+3 oxidation state) methyltransferase and the methylation of arsenicals. Exp Biol Med 232(1):3-13.

Thomas K, Colborn T. 1992. Organochlorine endocrine disruptors in human tissue. In: Colborn T, Clement $\mathrm{C}$, eds. Chemically induced alterations in sexual and functional development: The wildlife/human connection. Princeton, NJ: Princeton Scientific Publishing, 365-394.

Thomas P, Sniatecki K. 1995. Inductively coupled plasma mass spectrometry: Application to the determination of arsenic species. Fresenius J Anal Chem 351 (4-5):410-414.

Thomas DJ, Waters SB, Styblo M. 2004. Elucidating the pathway for arsenic methylation. Toxicol Appl Pharmacol 198(3):319-326.

Thomas KW, Pellizzari ED, Berry MR. 1999. Population-based dietary intakes and tap water concentrations for selected elements in the EPA Region V National Human Exposure Assessment Survey (NHEXAS). J Expo Anal Environ Epidemiol 9:402-413.

Thompson TS, Le MD, Kasick AR, et al. 1999. Arsenic in well water supplies in Saskatchewan. Bull Environ Contam Toxicol 63:478-483.

Tian D, Ma H, Feng Z, et al. 2001. Analyses of micronuclei in exfoliated epithelial cells from individuals chronically exposed to arsenic via drinking water in inner Mongolia, China. J Toxicol Environ Health A 64(6):473-484.

Tice RR, Yager JW, Andrews P, et al. 1997. Effect of hepatic methyl donor status on urinary excretion and DNA damage in B6C3F1 mice treated with sodium arsenite. Mutat Res 386(3):315-334.

Tintinalli JE, Ruiz E, Krone RL, eds. 1996. Emergency medicine. A comprehensive study. American College of Emergency Physicians. $4^{\text {th }}$ ed. New York, NY: The McGraw-Hill Companies, Inc.

Tokunaga H, Roychowdhury T, Chandraskaran N, et al. 2002. Urinary arsenic species in an arsenicaffected area of West Bengal, India. Appl Organomet Chem 16:406-414. 
9. REFERENCES

Tollestrup K, Daling JR, Allard J. 1995. Mortality in a cohort of orchard workers exposed to lead arsenate pesticide spray. Arch Environ Health 50(3):221-229.

Tondel M, Rahman M, Magnuson A, et al. 1999. The relationship of arsenic levels in drinking water and the prevalence rate of skin lesions in Bangladesh. Environ Health Perspect 107(9):727-729.

*Trepka MJ, Heinrich J, Schulz C, et al. 1996. Arsenic burden among children in industrial areas of eastern Germany. Sci Total Environ 180(2):95-105.

TRI04. 2006. TRI explorer: Providing access to EPA's toxics release inventory data. Washington, DC: Office of Information Analysis and Access. Office of Environmental Information. U.S. Environmental Protection Agency. Toxics Release Inventory. http://www.epa.gov/triexplorer/. January 31, 2006.

Trocine RP, Trefry JH. 1996. Metal concentrations in sediment, water and clams from the Indian River Lagoon, Florida. Mar Pollut Bull 32(10):754-759.

Tsai M, Chien R, Hsieh S, et al. 1998a. Primary hepatic angiosarcoma: Report of a case involving environmental arsenic exposure. Chang Keng I Hsueh Tsa Chih 21(4):469-474.

Tsai S, Chou H, The H, et al. 2003. The effects of chronic arsenic exposure from drinking water on the neurobehavioral development in adolescence. Neurotoxicology 24:747-753.

*Tsai S, Wang T, Ko Y. 1998b. Cancer mortality trends in a blackfoot disease endemic community of Taiwan following water source replacement. J Toxicol Environ Health 55:389-404.

Tsai SM, Wang TN, Ko YC. 1999. Mortality for certain diseases in areas with high levels of arsenic in drinking water. Arch Environ Health 54(3):186-193.

Tseng W. 1977. Effects and dose-response relationships of skin cancer and blackfoot disease with arsenic. Environ Health Perspect 19:109-119.

Tseng W. 1989. Blackfoot disease in Taiwan: A 30-year follow-up study. Angiology 40(6):547-558.

Tseng CH, Chong CK, Chen CJ, et al. 1995. Abnormal peripheral microcirculation in seemingly normal subjects living in blackfoot-disease-hyperendemic villages in Taiwan. Int J Microcirc Clin Exp 15(1):2127.

Tseng CH, Chong C, Chen CJ, et al. 1996. Dose-response relationship between peripheral vascular disease and ingested inorganic arsenic among residents in blackfoot disease endemic villages in Taiwan. Atherosclerosis 120:125-133.

Tseng $\mathrm{CH}$, Chong $\mathrm{CK}$, Tseng CP, et al. 2003. Long-term arsenic exposure and ischemic heart disease in arseniasis-hyperendemic villages in Taiwan. Toxicol Lett 137(1-2):15-21.

Tseng CH, Huang YK, Huang YL, et al. 2005. Arsenic exposure, urinary arsenic speciation, and peripheral vascular disease in blackfoot disease-hyperendemic villages in Taiwan. (Erratum in: Toxicol Appl Pharmacol 211(2):175). Toxicol Appl Pharmacol 206(3):299-308. 
Tseng CH, Tai TY, Chong CK, et al. 2000. Long-term arsenic exposure and incidence of non-insulin dependent diabetes mellitus: A cohort study in arseniasis-hyperendemic villages in Taiwan. Environ Health Perspect 108(9):847-851.

Tseng WP, Chu HM, How SW, et al. 1968. Prevalence of skin cancer in an endemic area of chronic arsenicism in Taiwan. J Natl Cancer Inst 40:453-463.

Tsuda T, Babazono A, Yamamoto E, et al. 1995a. Ingested arsenic and internal cancer: A historical cohort study followed for 33 years. Am J Epidemiol 141(3):198-209.

Tsuda T, Indue T, Kojima M, et al. 1995b. Market basket and duplicate portion estimation of dietary intakes of cadmium, mercury, arsenic, copper, manganese, and zinc by Japanese adults. J AOAC Int 78(6):1363-1368.

Tsuda T, Kume Y, Yamamoto M, et al. 1987. An epidemiological study on cancer in certified arsenic poisoning patients in Toroku. Jpn J Ind Health 29:496-497.

Tsuji JS, Van Kerkhove MD, Kaetzel RS, et al. 2005. Evaluation of exposure to arsenic in residential soil. Environ Health Perspect 113(12):1735-1740.

Tsuruta D, Hamada T, Mochida K, et al. 1998. Merkel cell carcinoma, Bowen's disease and chronic occupational arsenic poisoning. Br J Dermatol 139:291-294.

Uddin AN, Burns FJ, Rossman TG. 2005. Vitamin E and organoselenium prevent the cocarcinogenic activity of arsenite with solar UVR in mouse skin. Carcinogenesis 26(12):2179-2186.

Ueda H, Kuroda K, Endo G. 1997. The inhibitory effect of selenium on induction of tetraploidy by dimethylarsinic acid in Chinese hamster cells. Anticancer Res 17(3c):1939-1943.

Uede K, Furukawa F. 2003. Skin manifestations in acute arsenic poisoning from the Wakayama currypoisoning incident. Br J Dermatol 149(4):757-762.

Ulitzur S, Barak M. 1988. Detection of genotoxicity of metallic compounds by the bacterial bioluminescence test. J Biolumin Chemilumin 2:95-99.

Ulman C, Gezer S, Anal O, et al. 1998. Arsenic in human and cow's milk: A reflection of environmental pollution. Water Air Soil Pollut 101(1-4):411-416.

Unnikrishnan D, Dutcher JP, Varshneya N, et al. 2001. Torsades de pointes in 3 patients with leukemia treated with arsenic trioxide. Blood 97(5):1514-1516.

Ursitti F, Vanderlinden L, Watson R, et al. 2004. Assessing and managing exposure from arsenic in CCA-treated wood play structures. Can J Public Health 95(6):429-433.

U.S. Bureau of Mines. 1988. Mineral commodity summaries. Washington, DC: U.S. Bureau of Mines, 14-15.

U.S. Bureau of Mines. 1990. Mineral commodity summaries. Washington, DC: U.S. Bureau of Mines, 22-23. 


\section{REFERENCES}

*U.S. Congress. 1990. Clean Air Act. Title III. Section 112. National emission standards for hazardous air pollutants. U.S. Congress. 42 USC 7412. Revised by Public Law 101-549. November 15, 1990.

USDA. 2004. Nonsynthetic substances prohibited for use in organic crop production. U.S. Department of Agriculture. Code of Federal Regulations 7 CFR 205.602. http://www.access.gpo.gov/nara/cfr/waisidx_04/7cfr205_04.html. February 15, 2005.

USDA/USDT. 2000. Assessment of the Environmental effects associated with wooden bridges preserved with creosote, pentachlorophenol, or chromated copper arsenate. U.S. Department of Agriculture. United States Department of Transportation. FPL-RP-587. http://www.fpl.fs.fed.us/documnts/fplrp/fplrp587.pdf. August 27, 2007.

USGS. 1984. Element concentrations in soils and other surficial materials on of the conterminous United States. U.S. Geological Survey. Professional Paper 1270.

USGS. 1987. Composition, distribution, and hydrologic effects of contaminated sediments resulting from the discharge of gold milling wastes to Whitewood Creek at Lead and Deadwood, South Dakota. Water-Resources Investigation Report 87-4051. U.S. Geological Survey. http://pubs.er.usgs.gov/usgspubs/wri/wri874051 August 27, 2007.

USGS. 1998. Arsenic. U.S. Geological Survey - Mineral Information - 1998. U.S. Geological Survey. http://minerals.usgs.gov/minerals/pubs/commodity/arsenic/160498.pdf.

*USGS. 1999a. Mineral Commodity Summaries 1999. Arsenic. U.S. Geological Survey. http://minerals.usgs.gov/minerals/pubs/commodity/arsenic/index.html.

USGS. 1999b. Arsenic in ground water of the Willamette Basin, Oregon. U.S. Geological Survey. http://or.water.usgs.gov/pubs_dir/Online/Pdf/98-4205.pdf. April 7, 2005.

USGS. 1999c. Arsenic-rich pyrite in the Mississippian Marshall Sandstone: Source of anomalous arsenic in southeastern Michigan ground water. U.S. Geological Survey. http://minerals.usgs.gov/east/midwest/abstract7.html. August 23,1999.

USGS. 2006a. Arsenic. 2005 Minerals yearbook. U.S. Department of the Interior, U.S. Geological Survey.:7.1-7.4, tables 1-3. http://minerals.usgs.gov/minerals/pubs/commodity/arsenic/arsenmyb05.pdf. September 12, 2006.

USGS. 2006b. Arsenic. Mineral commodity studies. U.S. Geological Survey, 26-27. http://minerals.usgs.gov/minerals/pubs/commodity/arsenic/arsenmcs06.pdf. September 12, 2006.

USGS. 2007a. Arsenic in ground water in the United States. U.S. Geological Survey. http://water.usgs.gov/nawqa/trace/pubs/geo_v46n11/fig2.html. August 29, 2007.

USGS. 2007b. The national geochemical survey-database and documentation. U.S. Geological Survey. http://tin.er.usgs.gov/geochem/doc/home.htm. August 29, 2007.

Vaessen HA, van Ooik A. 1989. Speciation of arsenic in Dutch total diets: Methodology and results. Z Lebensm Unters Forsch 189:232-235.

Vahter M. 1981. Biotransformation of trivalent and pentavalent inorganic arsenic in mice and rats. Environ Res 25:286-293. 
9. REFERENCES

Vahter M. 1983. Metabolism of arsenic. In: Fowler BA, ed. Biological and environmental effects of arsenic. New York, NY: Elsevier Science Publishers, 171-198.

Vahter M. 1986. Environmental and occupational exposure to inorganic arsenic. Acta Pharmacol Toxicol 59:31-34.

Vahter M. 2002. Mechanisms of arsenic biotransformation. Toxicology 181-182:211-217.

Vahter M, Envall J. 1983. In vivo reduction of arsenate in mice and rabbits. Environ Res 32:14-24.

Vahter M, Marafante E. 1983. Intracellular interaction and metabolic fate of arsenite and arsenate in mice and rabbits. Chem Biol Interact 47:29-44.

Vahter M, Marafante E. 1985. Reduction and binding of arsenate in Marmoset monkeys. Arch Toxicol 57:119-124.

Vahter M, Marafante E. 1987. Effects of low dietary intake of methionine, choline or proteins on the biotransformation of arsenite in the rabbit. Toxicol Lett 37:41-46.

Vahter M, Norin H. 1980. Metabolism of ${ }^{74}$ As-labeled trivalent and pentavalent inorganic arsenic in mice. Environ Res 21:446-457.

*Vahter M, Concha G, Nermell B, et al. 1995. A unique metabolism of inorganic arsenic in native Andean women. Eur J Pharmacol 293(4):455-462.

Vahter M, Friberg L, Rahnster B, et al. 1986. Airborne arsenic and urinary excretion of metabolites of inorganic arsenic among smelter workers. Int Arch Occup Environ Health 57:79-91.

*Vahter M, Marafante E, Dencker L. 1983. Metabolism of arsenobetaine in mice, rats and rabbits. Sci Total Environ 30:197-211.

Vahter M, Marafante E, Dencker L. 1984. Tissue distribution and retention of ${ }^{74}$ As-dimethylarsinic acid in mice and rats. Arch Environ Contam Toxicol 13:259-264.

Vahter M, Marafante E, Lindgren A, et al. 1982. Tissue distribution and subcellular binding of arsenic in Marmoset monkeys after injection of ${ }^{74}$ As-arsenite. Arch Toxicol 51:65-77.

Valentine JL, Campion DS, Schluchter MD, et al. 1981. Arsenic effects on human nerve conduction. In: Howell JC, Gawthorne JM, White L, eds. Trace element metabolism in man and animals - TEMA 4. Proceedings of the Fourth International symposium on Trace Elements or Man and Animals. Canberra: Australian Academy of Science, 409-411.

Valentine JL, He S, Reisbord LS, et al. 1992. Health response by questionnaire in arsenic-exposed populations. J Clin Epidemiol 45(5):487-94.

Valentine JL, Kang HK, Spivey G. 1979. Arsenic levels in human blood, urine and hair in response to exposure via drinking water. Environ Res 20:24-32. 
Valentine JL, Reisbord LS, Kang HK, et al. 1985. Arsenic effects on population health histories. In: Mills CF, Bremner I, Chesters JK, eds. Trace elements in man and animals - TEMA 5: Proceedings of the Fifth International Symposium on Trace Elements in Man and Animals. Slough, UK:

Commonwealth Agricultural Bureaux, 289-294.

Vallee BL, Ulmer DD, Wacker WE. 1960. Arsenic toxicology and biochemistry. Arch Ind Health 21:132-151.

van Netten C, Cann SAH, Morley DR, et al. 2000. Elemental and radioactive analysis of commercially available seaweed. Sci Total Environ 255:169-175.

Vantroyen B, Heilier JF, Meulemans A, et al. 2004. Survival after a lethal dose of arsenic trioxide. J Toxicol Clin Toxicol 42(6):889-895.

*Vargas M, Hamadeh H, Lee E, et al. 1998. Activation of transcription factors by sodium arsenite in human lymphocytes. Toxicologist 42(1-S):323-324.

Vega L, Gonsebatt ME, Ostrosky-Wegman P. 1995. Aneugenic effect of sodium arsenite on human lymphocytes in vitro: An individual susceptibility effect detected. Mutat Res 334(3):365-373.

Versieck J, Hoste J, Vanballenberghe L, et al. 1983. Trace element measurements in serum by neutron activation analysis. In: Harling, OK, Clark L, Von der Hardt P, eds. Use and development of low and medium flux research reactors. Proceedings of the International Symposium on the use and development of low and medium flux research reactors, Massachusetts Institute of Technology, Cambridge, Massachusetts, U.S.A., October 16-19, 1983. Munchen: Karl Thiemig, 717-723.

Vieira I, Sonnier M, Cresteil T. 1996. Developmental expression of CYP2E1 in the human liver: Hypermethylation control of gene expression during the neonatal period. Eur J Biochem 238:476-483.

*Vienna A, Capucci E, Wolfsperger M, et al. 1995. Heavy metal concentration in hair of students in Rome. Anthropol Anz 53(1):27-32.

Vig BK, Figueroa ML, Cornforth MN, et al. 1984. Chromosome studies in human subjects chronically exposed to arsenic in drinking water. Am J Ind Med 6:325-338.

Vinas P, Lopez-Garcia I, Merino-Merono B, et al. 2003. Stability of arsenobetaine levels in manufactured baby foods. J Food Prot 66(12):2321-2324.

Viren JR, Silvers A. 1994. Unit risk estimates for airborne arsenic exposure: An updated view based on recent data from two copper smelter cohorts. Regul Toxicol Pharmacol 20(2):125-138.

Viren J, Silvers A. 1999. Nonlinearity in the lung cancer dose-response for airborne arsenic: Apparent confounding by year of hire in evaluating lung cancer risks from arsenic exposure in Tacoma smelter workers. Regul Toxicol Pharmacol 30:117-129.

von Ehrenstein OS, Guha Mazumder DN, Hira-Smith M, et al. 2006. Pregnancy of outcomes, infant mortality, and arsenic in drinking water in West Bengal, India. Am J Epidemiol 163(7):662-669.

von Ehrenstein OS, Mazumder DN, Yuan Y, et al. 2005. Decrements in lung function related to arsenic in drinking water in West Bengal, India. Am J Epidemiol 162(6):533-541. 
von Ehrenstein O, Poddar S, Yuan Y, et al. 2007. Children's intellectual function in relation to arsenic exposure. Epidemiology 18:44-51.

Von Endt DW, Kearney PC, Kaufman DD. 1968. Degradation of monosodium methanearsonic acid by soil microorganisms. J Agric Food Chem 16(1):17-20.

Vuyyuri SB, Ishaq MKuppala D, Grover P, et al. 2006. Evaluation of micronucleus frequencies and DNA damage in glass workers exposed to arsenic. Environ Mol Mutagen 47:562-570.

Waalkes MP, Harvey MJ, Klaassen CD. 1984. Relative in vitro affinity of hepatic metallothionein for metals. Toxicol Lett 20:33-39.

Waalkes MP, Liu J, Ward JM, et al. 2004a. Mechanisms underlying arsenic carcinogenesis: Hypersensitivity of mice exposed to inorganic arsenic during gestation. Toxicology 198(1-3):31-38.

Waalkes MP, Liu J, Ward JM, et al. 2004b. Animal models for arsenic carcinogenesis: Inorganic arsenic is a transplacental carcinogen in mice. Toxicol Appl Pharmacol 198(3):377-384.

Waalkes MP, Liu J, Ward JM, et al. 2006a. Enhanced urinary bladder and liver carcinogenesis in male CD1 mice exposed to transplancental inorganic arsenic and postnatal diethylstilbestrol or tamoxifen. Toxicol Appl Pharmacol 215(3):295-305.

Waalkes MP, Liu J, Ward JM, et al. 2006b. Urogenital carcinogenesis in female CD1 mice induced by in utero arsenic exposure is exacerbated by postnatal diethylstilbestrol treatment. Cancer Res 66(3):13371345.

Waalkes MP, Ward JM, Diwan BA. 2004c. Induction of tumors of the liver, lung, ovary and adrenal in adult mice after brief maternal gestational exposure to inorganic arsenic: Promotional effects of postnatal phorbol ester exposure on hepatic and pulmonary, but not dermal cancers. Carcinogenesis 25(1):133-141.

Waalkes MP, Ward JM, Liu J, et al. 2003. Transplacental carcinogenicity of inorganic arsenic in the drinking water: Induction of hepatic, ovarian, pulmonary, and adrenal tumors in mice. Toxicol Appl Pharmacol 186(1):7-17.

Wade HJ, Frazer ES. 1953. Toxipathic hepatitis due to Fowler's solution; A case treated with dimercaprol. Lancet (February):269-271.

Wadge A, Hutton M. 1987. The leachability and chemical speciation of selected trace elements in fly ash from coal combustion and refuse incineration. Environ Pollut 48:85-99.

Wagner SL, Maliner JS, Morton WE, et al. 1979. Skin cancer and arsenical intoxication from well water. Arch Dermatol 115:1205-1207.

Wahlberg JE, Boman A. 1986. Contact sensitivity to arsenical compounds: Clinical and experimental studies. Derm Beruf Umwelt 34:10-12.

Wakao N, Koyatsu H, Komai Y, et al. 1988. Microbial oxidation of arsenite and occurrence of arseniteoxidizing bacteria in acid mine water from a sulfur-pyrite mine. Geomicrobiol J 6:11-24.

Walker S, Griffin S. 1998. Site-specific data confirm arsenic exposure predicted by the U.S. Environmental Protection Agency. Environ Health Perspect 106(3):133-139. 
Wall S. 1980. Survival and mortality pattern among Swedish smelter workers. Int J Epidemiol 9(1):7387.

Walton FS, Waters SB, Jolley SL, et al. 2003. Selenium compounds modulate the activity of recombinant rat As111-methyltransferase and the methylation of arsenite by rat and human hepatocytes. Chem Res Toxicol 16(3):261-265.

Wan B, Christian RT, Soukup SW. 1982. Studies of cytogenetic effects of sodium arsenicals on mammalian cells in vitro. Environ Mutagen 4:493-498.

Waner T, Nyska A. 1988. Data evaluation report: Methanearsonic acid fifty-two week chronic oral toxicity study in beagle dogs. Permenta Plant Protection Company. Submitted to the U.S. Environmental Protection Agency. MRID40546101.

Wang ZY. 2001. Arsenic compounds as anticancer agents. Cancer Chemother Pharmacol 48(Suppl 1):S72-S76.

Wang TS, Huang H. 1994. Active oxygen species are involved in the induction of micronuclei by arsenite in XRS-5 cells. Mutagenesis 9(3):253-257.

Wang CH, Jeng JS, Yip PK, et al. 2002. Biological gradient between long-term arsenic exposure and carotid atherosclerosis. Circulation 105:1804-1809.

Wang SH, Wang ZH, Cheng XT, et al. 2007. Arsenic and fluoride exposure in drinking water: Children's IQ and growth in Shanyin County, Shanxi Province, China. Environ Health Perspect 115(4):643-647.

Wang SL, Chiou JM, Chen CJ, et al. 2003. Prevalence of non-insulin-dependent diabetes mellitus and related vascular diseases in southwestern arseniasis-endemic and nonendemic areas in Taiwan. Environ Health Perspect 111(2):155-59.

Wang TS, Shu YF, Liu YC, et al. 1997. Glutathione peroxidase and catalase modulate the genotoxicity of arsenite. Toxicology 121(3):229-237.

Wang Z, Kown E, Zhang H, et al. 2005. Arsenic on the hands of children: Wang et al. Respond. Environ Health Perspect 113(6):364-365.

Wanibuchi H, Yamamoto S, Chen H, et al. 1996. Promoting effects of dimethylarsinic acid on N-butylN-(4-hydroxybutyl)nitrosamine-induced urinary bladder carcinogenesis in rats. Carcinogenesis 17(11):2435-2439.

*Warner ML, Moore LE, Smith MT, et al. 1994. Increased micronuclei in exfoliated bladder cells of individuals who chronically ingest arsenic-contaminated water in Nevada. Cancer Epidemiol Biomarkers Prev 3(7):583-590.

Washington State. 2006. What is area-wide soil contamination? In: Area-Wide Soil Contamination Project. Washington State. http://www.ecy.wa.gov/programs/tcp/area_wide/AW/toolbox_chap1.html. August 27, 2007. 
Wasserman GA, Liu X, Parvez F, et al. 2004. Water arsenic exposure and children's intellectual function in Araihazar, Bangladesh. Environ Health Perspect 112(13):1329-1333.

Wasserman GA, Liu X, Parvez F, et al. 2007. Water arsenic exposure and intellectual function in 6-yearold children in Arailhazar, Bangladesh. Environ Health Perspect 115(2):285-289.

Watanabe C, Inaoka T, Kadono T, et al. 2001. Males in rural Bangladeshi communities are more susceptible to chronic arsenic poisoning than females: Analyses based on urinary arsenic. Environ Health Perspect 109(12):1265-1270.

Watrous RM, McCaughey MB. 1945. Occupational exposure to arsenic: In the manufacture of arsphenamine and related compounds. Ind Med 14(8):639-646.

Webb DR, Sipes IG, Carter DE. 1984. In vitro solubility and in vivo toxicity of gallium arsenide. Toxicol Appl Pharmacol 76:96-104.

Webb DR, Wilson SE, Carter DE. 1986. Comparative pulmonary toxicity of gallium arsenide, gallium (III) oxide, or arsenic (III) oxide intratracheally instilled into rats. Toxicol Appl Pharmacol 82:405-416.

Webb DR, Wilson SE, Carter DE. 1987. Pulmonary clearance and toxicity of respirable gallium arsenide particulates intratracheally instilled into rats. Am Ind Hyg Assoc J 48(7):660-667.

Wedepohl KH. 1991. The composition of the upper earth's crust and the natural cycles of selected metals. Metals in natural raw materials. Natural resources. In: Merian E, ed. Metals and their compounds in the environment. Occurrence, analysis, and biological relevance. New York, NY: VCH, $3-17$.

Wei M, Wanibuchi H, Morimura K, et al. 2002. Carcinogenicity of dimethylarsinic acid in male F344 rats and genetic alterations in induced urinary bladder tumors. Carcinogenesis 23(8):1387-1397.

Wei M, Wanibuchi H, Yamamoto S, et al. 1999. Urinary bladder carcinogenicity of dimethylarsinic acid in male F344 rats. Carcinogenesis 20(9):1873-1876.

Weis JS, Weis P, Proctor T. 1998. The extent of benthic impacts of CCA-treated wood structures in Atlanic coast estuaries. Arch Environ Contam Toxicol 34(4):313-322.

*Weis P, Weis JS, Lores E. 1993. Uptake of metals from chromated-copper-arsenate (CCA)-treated lumber by Epibiota. Mar Pollut Bull 26(8):428-430.

Welch AH, Lico MS, Hughes JL. 1988. Arsenic in groundwater of the western United States. Ground Water 26(3):333-347.

Welch K, Higgins I, Oh M, et al. 1982. Arsenic exposure, smoking and respiratory cancer in copper smelter workers. Arch Environ Health 37(6):325-335.

Wendt PH, Van Dolah RF, Bobo MY, et al. 1996. Wood preservative leachates from docks in an estuarine environment. Arch Environ Contam Toxicol 31(1):24-37.

Werner MA, Knobeloch LM, Erbach T, et al. 2001. Use of imported folk remedies and medications in the Wisconsin Hmong community. Wis Med J 100(7):32-34. 


\section{REFERENCES}

West JR, Smith HW, Chasis H. 1948. Glomerular filtration rate, effective renal blood flow, and maximal tubular excretory capacity in infancy. J Pediatrics 32:10-18.

Wester RC, Maibach HI, Sedik L, et al. 1993. In vivo and in vitro percutaneous absorption and skin decontamination of arsenic from water and soil. Fundam Appl Toxicol 20(3):336-340.

Westhoff DD, Samaha RJ, Barnes A. 1975. Arsenic intoxication as a cause of megaloblastic anemia. Blood 45(2):241-246.

Whitman FT. 1994. Subchronic (90-day) inhalation toxicity study in rats with cacodylate 3.25 (MRD92-416). Luxembourg Industries (Pamol) Ltd. Submitted to the U.S. Environmental Protection Agency. MRID44700301.

WHO. 1981. Environmental health criteria 18: Arsenic. IPCS International Programme on Chemical Safety. Geneva, Switzerland: World Health Organization.

WHO. 2000. Air quality guidelines. 2nd ed. Geneva, Switzerland: World Health Organization. http://www.euro.who.int/air/Activities/20050104_1. February 15, 2005.

WHO. 2001. Arsenic and arsenic compounds. Environmental Health Criteria 224. Geneva: United Nations Environment Programme. International Labour Organisation. World Health Organization. http://www.inchem.org/documents/ehc/ehc/ehc224.htm. August 27, 2007.

WHO. 2004. Guidelines for drinking-water quality. 3rd ed. Geneva, Switzerland: World Health Organization. http://www.who.int/water_sanitation_health/dwq/gdwq3/en/. February 15, 2005.

*Wicklund KG, Daling JR, Allard J, et al. 1988. Respiratory cancer among orchardists in Washington state, 1968-1980. J Occup Med 30:561-564.

Wicks MJ, Archer VE, Auerbach O, et al. 1981. Arsenic exposure in a copper smelter as related to histological type of lung cancer. Am J Ind Med 2:25-31.

Widdowson EM, Dickerson JWT. 1964. Chemical composition of the body. In: Comar CL, Bronner F, eds. Mineral metabolism: An advanced treatise. Volume II: The elements Part A. New York: Academic Press.

Wiencke JK, Yager JW. 1992. Specificity of arsenite in potentiating cytogenetic damage induced by the DNA crosslinking agent diepoxybutane. Environ Mol Mutagen 19(3):195-200.

Wiencke JK, Yager JW, Varkonyi A, et al. 1997. Study of arsenic mutagenesis using the plasmid shuttle vector pZ189 propagated in DNA repair proficient human cells. Mutat Res 386(3):335-344.

Willhite CC. 1981. Arsenic-induced axial skeletal (dysraphic) disorders. Exp Mol Pathol 34:145-158.

Willhite CC, Ferm VH. 1984. Prenatal and developmental toxicology of arsenicals. Adv Exp Med Biol 177:205-228.

Williams L, Schoof RA, Yager JW, et al. 2006. Arsenic bioaccumulation in freshwater fishes. Hum Ecol Risk Assess 12:904-923. 
Williams TM, Rawlins BG, Smith B, et al. 1998. In-vitro determination of arsenic bioavailability in contaminated soil and mineral beneficiation waste from Ron Phibun, Southern Thailand: A basis for improved human risk assessment. Environ Geochem Health 20(4):169-177.

Wingren G, Axelson O. 1993. Epidemiologic studies of occupational cancer as related to complex mixtures of trace elements in the art glass industry. Scand J Work Environ Health 19(Suppl 1):95-100.

Wlodarczyk BJ, Bennett GD, Calvin JA, et al. 1996. Arsenic-induced neural tube defects in mice: Alterations in cell cycle gene expression. Reprod Toxicol 10(6):447-454.

Wolz S, Fenske RA, Simcox NJ, et al. 2003. Residential arsenic and lead levels in an agricultural community with a history of lead arsenate use. Environ Res 93:293-300.

Wong O, Whorton MD, Foliart DE, et al. 1992. An ecologic study of skin cancer and environmental arsenic exposure. Int Arch Occup Environ Health 64(4):235-241.

Wong SS, Tan KC, Goh CL. 1998a. Cutaneous manifestations of chronic arsenicism: Review of seventeen cases. J Am Acad Dermatol 38:179-185.

Wong ST, Chan HL, Teo SK. 1998b. The spectrum of cutaneous and internal malignancies in chronic arsenic toxicity. Singapore Med J 39(4):171-173.

Woods JS, Fowler BA. 1977. Effects of chronic arsenic exposure on hematopoietic function in adult mammalian liver. Environ Health Perspect 19:209-213.

Woods JS, Fowler BA. 1978. Altered regulation of mammalian hepatic heme biosynthesis and urinary porphyrin excretion during prolonged exposure to sodium arsenate. Toxicol Appl Pharmacol 43:361-371.

Woods JS, Southern MR. 1989. Studies on the etiology of trace metal-induced porphyria: Effects of porphyrinogenic metals on coproporphyrinogen oxidase in rat liver and kidney. Toxicol Appl Pharmacol 97:183-190.

Woolson EA, Kearney PC. 1973. Persistence and reactions of ${ }^{14} \mathrm{C}$-cacodylic acid in soils. Environ Sci Technol 7:47-50.

*Woollons A, Russel-Jones R. 1998. Chronic endemic hydroarsenicism. Br J Dermatol 139:1092-1096.

Woolson EA. 1976. Generation of dimethyl arsine from soil. 1976 Meeting of the Weed Science Society of America. February 3-5, 1976, No. 218.

WSDOE. 2005. Tacoma Shelter Plume: South King County mainland soil study. Washington State Department of Ecology. http://www.ecy.wa.gov/programs/tcp/sites/tacoma_smelter/soil_study.htm. July 14, 2005.

*Wu MM, Chiou HY, Wang TW, et al. 2001. Association of blood arsenic levels with increased reactive oxidants and decreased antioxidant capacity in a human population of northeastern Taiwan. Environ Health Perspect 109(10):1011-1017.

Wu MM, Kuo TL, Hwang Y, et al. 1989. Dose-response relation between arsenic concentration in well water and mortality from cancers and vascular diseases. Am J Epidemiol 130(6):1123-1132. 
Wulff M, Högberg U, Sandstrom A. 1996. Cancer incidence for children born in a smelting community. Acta Oncologica (Stockholm) 35(2):179-183.

Wuyi W, Linsheng Y, Shaofan H, et al. 2001. Prevention of endemic arsenism with selenium. Curr Sci 81(9):1215-1218.

Wyatt CJ, Fimbres C, Romo L, et al. 1998b. Incidence of heavy metal contamination in water supplies in Northern Mexico. Environ Res A76:114-119.

Wyatt CJ, Quiroga VL, Acosta RTO, et al. 1998a. Excretion of arsenic (As) in urine of children, 711 years, exposed to elevated levels of As in the city water supply in Hermosillo, Sonora, Mexico. Environ Res A78:19-24.

Xuan XZ, Lubin JH, Li JY, et al. 1993. A cohort study in southern China of tin miners exposed to radon and radon decay products. Health Phys 64(2):120-131.

Yager JW, Wiencke JK. 1993. Enhancement of chromosomal damage by arsenic: Implications for mechanism. Environ Health Perspect 101(Suppl 3):79-82.

Yager JW, Wiencke JK. 1997. Inhibition of poly(ADP-ribose) polymerase by arsenite. Mutat Res 386(3):345-351.

Yager JW, Hicks JB, Fabianova E. 1997. Airborne arsenic and urinary excretion of arsenic metabolites during boiler cleaning operations in a Slovak coal-fired power plant. Environ Health Perspect 105(8):836-842.

Yamamoto A, Hisanaga A, Ishinishi N. 1987. Tumorigenicity of inorganic arsenic compounds following intratracheal instillations to the lungs of hamsters. Int J Cancer 40:220-223.

Yamamoto S, Konishi Y, Matsuda T, et al. 1995. Cancer induction by an organic arsenic compound, dimethylarsinic acid (cacodylic acid), in F344/DuCrj rats after pretreatment with five carcinogens. Cancer Res 55(6):1271-1276.

*Yamamoto S, Wanibuchi H, Hori T, et al. 1997. Possible carcinogenic potential of dimethylarsinic acid as assessed in rat in vivo models: A review. Mutat Res 386(3):353-361.

Yamanaka K, Okada S. 1994. Induction of lung-specific DNA damage by metabolically methylated arsenics via the production of free radicals. Environ Health Perspect 102(Suppl 3)37-40.

Yamanaka K, Hasegawa A, Sawamura R, et al. 1989a. Dimethylated arsenics induce DNA strand breaks in lung via the production of active oxygen in mice. Biochem Biophys Res Commun 165(1):43-50.

Yamanaka K, Hayashi H, Kato K, et al. 1995. Involvement of preferential formation of apurinic/apyrimidinic sites in dimethylarsenic-induced DNA strand breaks and DNA-protein crosslinks in cultured alveolar epithelial cells. Biochem Biophys Res Commun 207(1):244-249.

Yamanaka K, Hayashi H, Kato K, et al. 1997. DNA single-strand breaks in L-132 cells resulting from inhibition of repair polymerization shortly after exposure to dimethylarsinic acid. Biol Pharm Bull 20(2):163-167. 
9. REFERENCES

Yamanaka K, Ohba H, Hasegawa A, et al. 1989b. Mutagenicity of dimethylated metabolites of inorganic arsenics. Chem Pharm Bull 37(10):2753-2756.

*Yamanaka K, Ohtsubo K, Hasegawa A, et al. 1996. Exposure to dimethylarsinic acid, a main metabolite of inorganic arsenics, strongly promotes tumorigenesis initiated by 4-nitroquinoline 1-oxide in the lungs of mice. Carcinogenesis 17(4):767-770.

Yamanaka K, Takabayashi F, Mizoi M, et al. 2001. Oral exposure of dimethylarsinic acid, a main metabolite of inorganic arsenics, in mice leads to an increase in 8-oxo-2'-deoxyguanosine level, specifically in the target organs for arsenic carcinogenesis. Biochem Biophys Res Commun 287(1):6670.

Yamanaka K, Tezuka M, Kato K, et al. 1993. Crosslink formation between DNA and nuclear proteins by in vivo and in vitro exposure of cells to dimethylarsinic acid. Biochem Biophys Res Commun 191(3):1184-1191.

Yamauchi H, Yamamura Y. 1984. Metabolism and excretion of orally administered dimethylarsinic acid in the hamster. Toxicol Appl Pharmacol 74:134-140.

Yamauchi H, Yamamura Y. 1985. Metabolism and excretion of orally administered arsenic trioxide in the hamster. Toxicology 34:113-121.

Yamauchi H, Kaise T, Yamamura Y. 1986. Metabolism and excretion of orally administered arsenobetaine in the hamster. Bull Environ Contam Toxicol 36:350-355.

Yamauchi H, Takahashi K, Mashiko M, et al. 1989. Biological monitoring of arsenic exposure of gallium arsenide- and inorganic arsenic-exposed workers by determination of inorganic arsenic and its metabolites in urine and hair. Am Ind Hyg Assoc J 50(11):606-612.

Yamauchi H, Yamato N, Yamamura Y. 1988. Metabolism and excretion of orally and intraperitoneally administered methylarsonic acid in the hamster. Bull Environ Contam Toxicol 40:280-286.

Yan-Chu H. 1994. Arsenic distribution in soils. In: Nriagu JO, ed. Arsenic in the environment, part 1: Cycling and characterization. New York, NY: John Wiley \& Sons, Inc., 17-49.

Yang CY, Chang CC, Tsai SS, et al. 2003. Arsenic in drinking water and adverse pregnancy outcome in an arseniasis-endemic area in northeastern Taiwan. Environ Res 91(1):29-34.

Yang JL, Chen MF, Wu CW, et al. 1992. Posttreatment with sodium arsenite alters the mutational spectrum induced by ultraviolet light irradiation in Chinese hamster ovary cells. Environ Mol Mutagen 20(3)156-164.

Yang L, Wang W, Hou S, et al. 2002. Effects of selenium supplementation on arsenism: An intervention trial in inner Mongolia. Environ Geochem Health 24:359-374.

Yee-Chien L, Haimei H. 1996. Lowering extracellular calcium content protects cells from arseniteinduced killing and micronuclei formation. Mutagenesis 11(1):75-78.

Yih LH, Ho IC, Lee TC. 1997. Sodium arsenite disturbs mitosis and induces chromosome loss in human fibroblasts. Cancer Res 57(22):5051-5059. 
Yokel J, Delistraty DA. 2003. Arsenic, lead, and other trace elements in soils contaminated with pesticide residues at the Hanford site (USA). Environ Toxicol 18(2):104-114.

Yoshida K, Inoue Y, Kuroda K, et al. 1998. Urinary excretion of arsenic metabolites after long-term oral administration of various arsenic compounds to rats. J Toxicol Environ Health A 54:179-192.

Yoshida K, Kuroda K, Inoue Y, et al. 2001. Metabolism of dimethylarsinic acid in rats: Production of unidentified metabolites in vivo. Appl Organomet Chem 15:539-547.

Yost LJ, Schoof RA, Aucoin R. 1998. Intake of inorganic arsenic in the North American diet. Human Ecol Risk Assess 4(1):137-152.

Yost LJ, Tao SH, Egan SK, et al. 2004. Estimation of dietary intake of inorganic arsenic in U.S. children. Hum Ecol Risk Assess 10:473-483.

Yu D. 1998a. A realistic risk assessment of inorganic arsenic. J Environ Sci Health Part A 33(6):11491170.

Yu D. 1998b. Uncertainties in a pharmacokinetic modeling for inorganic arsenic. J Environ Sci Health Part A 33(7):1369-1390.

Yu D. 1999a. A physiologically based pharmacokinetic model of inorganic arsenic. Regul Toxicol Pharmacol 29:128-141.

Yu D. 1999b. A pharmacokinetic modeling of inorganic arsenic: A short-term oral exposure model for humans. Chemosphere 39(15):2737-2747.

Yu HS, Chang KL, Wang CM, et al. 1992. Alterations of mitogenic responses of mononuclear cells by arsenic in arsenical skin cancers. J Dermatol 19(11):710-714.

Yu HS, Sheu HM, Ko SS, et al. 1984. Studies on blackfoot disease and chronic arsenism in southern Taiwan: With special reference to skin lesions and fluorescent substances. J Dermatol 11:361-370.

Zadorozhnaja TD, Little RE, Miller RK, et al. 2000. Concentrations of arsenic, cadmium, copper, lead, mercury, and zinc in human placentas from two cities in Ukraine. J Toxicol Environ Health A 61:255263.

Zakharyan R, Wu Y, Bogdan GM, et al. 1995. Enzymatic methylation of arsenic compounds: Assay, partial purification, and properties of arsenite methyltransferase and monomethylarsonic acid methyltransferase of rabbit liver. Chem Res Toxicol 8(8):1029-1038.

Zakharyan RA, Wildfang E, Aposhian HV. 1996. Enzymatic methylation of arsenic compounds. III. The marmoset and tamarin, but not the Rhesus, monkeys are deficient in methyltransferases that methylate inorganic arsenic. Toxicol Appl Pharmacol 140(1):77-84.

Zaldívar R. 1974. Arsenic contamination of drinking water and foodstuffs causing endemic chronic poisoning. Beitr Pathol 151:384-400.

Zaldívar R. 1977. Ecological investigations on arsenic dietary intake and endemic chronic poisoning in man: Dose-response curve. Zentralbl Bakteriol Hyg 164:481-484. 
Zaldívar R, Guillier A. 1977. Environmental and clinical investigations on endemic chronic arsenic poisoning in infants and children. Zentralbl Bakteriol Hyg 165:226-234.

Zaldívar R, Prunés L, Ghai G. 1981. Arsenic dose in patients with cutaneous carcinomata and hepatic haemangio-endothelioma after environmental and occupational exposure. Arch Toxicol 47:145-154.

Zanzoni F, Jung EG. 1980. Arsenic elevates the sister chromatid exchange (SCE) rate in human lymphocytes in vitro. Arch Dermatol Res 267:91-95.

Zartarian VG, Xue J, Ozkaynak H, et al. 2006. A probabilistic arsenic exposure assessment for children who contact CCA-treated playsets and decks, Part 1: Model methodology, variability results, and model evaluation. Risk Anal 26(2):515-531.

*Zhang X, Cornelis R, De Kimpe J, et al. 1996. Accumulation of arsenic species in serum of patients with chronic renal disease. Clin Chem 42(8 Pt 1):1231-1237.

*Zhang X, Cornelis R, De Kimpe J, et al. 1997. Speciation of arsenic in serum, urine, and dialysate of patients on continuous ambulatory peritoneal dialysis. Clin Chem 43(2):406-408.

Zheng Y, Wu J, Ng JC, et al. 2002. The absorption and excretion of fluoride and arsenic in humans. Toxicol Lett 133(1):77-82.

Ziegler EE, Edwards BB, Jensen RL, et al. 1978. Absorption and retention of lead by infants. Pediatr Res 12:29-34.

Zierler S, Theodore M, Cohen A, et al. 1988. Chemical quality of maternal drinking water and congenital heart disease. Int J Epidemiol 17(3):589-594.

Zierold KM, Knobeloch L, Anderson H. 2004. Prevalence of chronic diseases in adults exposed to arsenic-contaminated drinking water. Am J Public Health 94(11):1936-1937.

Zomber G, Nyska A, Waner T, et al. 1989. Cacodylic acid 52-week oral toxicity study in beagle dogs. Luxembourg Industries (Pamol) Ltd. Submitted to the U.S. Environmental Protection Agency. MRID41490901. 


\section{GLOSSARY}

Absorption-The taking up of liquids by solids, or of gases by solids or liquids.

Acute Exposure-Exposure to a chemical for a duration of 14 days or less, as specified in the Toxicological Profiles.

Adsorption-The adhesion in an extremely thin layer of molecules (as of gases, solutes, or liquids) to the surfaces of solid bodies or liquids with which they are in contact.

Adsorption Coefficient $\left(\mathbf{K}_{\mathbf{o c}}\right)$ - The ratio of the amount of a chemical adsorbed per unit weight of organic carbon in the soil or sediment to the concentration of the chemical in solution at equilibrium.

Adsorption Ratio (Kd) — The amount of a chemical adsorbed by sediment or soil (i.e., the solid phase) divided by the amount of chemical in the solution phase, which is in equilibrium with the solid phase, at a fixed solid/solution ratio. It is generally expressed in micrograms of chemical sorbed per gram of soil or sediment.

Benchmark Dose (BMD) - Usually defined as the lower confidence limit on the dose that produces a specified magnitude of changes in a specified adverse response. For example, a BMD 10 would be the dose at the $95 \%$ lower confidence limit on a $10 \%$ response, and the benchmark response (BMR) would be $10 \%$. The BMD is determined by modeling the dose response curve in the region of the dose response relationship where biologically observable data are feasible.

Benchmark Dose Model-A statistical dose-response model applied to either experimental toxicological or epidemiological data to calculate a BMD.

Bioconcentration Factor (BCF) - The quotient of the concentration of a chemical in aquatic organisms at a specific time or during a discrete time period of exposure divided by the concentration in the surrounding water at the same time or during the same period.

Biomarkers-Broadly defined as indicators signaling events in biologic systems or samples. They have been classified as markers of exposure, markers of effect, and markers of susceptibility.

Cancer Effect Level (CEL) - The lowest dose of chemical in a study, or group of studies, that produces significant increases in the incidence of cancer (or tumors) between the exposed population and its appropriate control.

Carcinogen-A chemical capable of inducing cancer.

Case-Control Study-A type of epidemiological study that examines the relationship between a particular outcome (disease or condition) and a variety of potential causative agents (such as toxic chemicals). In a case-controlled study, a group of people with a specified and well-defined outcome is identified and compared to a similar group of people without outcome.

Case Report-Describes a single individual with a particular disease or exposure. These may suggest some potential topics for scientific research, but are not actual research studies. 
Case Series-Describes the experience of a small number of individuals with the same disease or exposure. These may suggest potential topics for scientific research, but are not actual research studies.

Ceiling Value-A concentration of a substance that should not be exceeded, even instantaneously.

Chronic Exposure-Exposure to a chemical for 365 days or more, as specified in the Toxicological Profiles.

Cohort Study-A type of epidemiological study of a specific group or groups of people who have had a common insult (e.g., exposure to an agent suspected of causing disease or a common disease) and are followed forward from exposure to outcome. At least one exposed group is compared to one unexposed group.

Cross-sectional Study-A type of epidemiological study of a group or groups of people that examines the relationship between exposure and outcome to a chemical or to chemicals at one point in time.

Data Needs-Substance-specific informational needs that if met would reduce the uncertainties of human health assessment.

Developmental Toxicity-The occurrence of adverse effects on the developing organism that may result from exposure to a chemical prior to conception (either parent), during prenatal development, or postnatally to the time of sexual maturation. Adverse developmental effects may be detected at any point in the life span of the organism.

Dose-Response Relationship-The quantitative relationship between the amount of exposure to a toxicant and the incidence of the adverse effects.

Embryotoxicity and Fetotoxicity-Any toxic effect on the conceptus as a result of prenatal exposure to a chemical; the distinguishing feature between the two terms is the stage of development during which the insult occurs. The terms, as used here, include malformations and variations, altered growth, and in utero death.

Environmental Protection Agency (EPA) Health Advisory-An estimate of acceptable drinking water levels for a chemical substance based on health effects information. A health advisory is not a legally enforceable federal standard, but serves as technical guidance to assist federal, state, and local officials.

Epidemiology-Refers to the investigation of factors that determine the frequency and distribution of disease or other health-related conditions within a defined human population during a specified period.

Genotoxicity-A specific adverse effect on the genome of living cells that, upon the duplication of affected cells, can be expressed as a mutagenic, clastogenic, or carcinogenic event because of specific alteration of the molecular structure of the genome.

Half-life-A measure of rate for the time required to eliminate one half of a quantity of a chemical from the body or environmental media.

Immediately Dangerous to Life or Health (IDLH) - The maximum environmental concentration of a contaminant from which one could escape within 30 minutes without any escape-impairing symptoms or irreversible health effects. 
Immunologic Toxicity-The occurrence of adverse effects on the immune system that may result from exposure to environmental agents such as chemicals.

Immunological Effects—Functional changes in the immune response.

Incidence-The ratio of individuals in a population who develop a specified condition to the total number of individuals in that population who could have developed that condition in a specified time period.

Intermediate Exposure-Exposure to a chemical for a duration of 15-364 days, as specified in the Toxicological Profiles.

In Vitro-Isolated from the living organism and artificially maintained, as in a test tube.

In Vivo-Occurring within the living organism.

Lethal Concentration $(\mathbf{L O})\left(\mathbf{L C}_{\mathbf{L O}}\right)$ - The lowest concentration of a chemical in air that has been reported to have caused death in humans or animals.

Lethal Concentration ${ }_{(50)}\left(\mathbf{L C}_{\mathbf{5 0}}\right)$ - A calculated concentration of a chemical in air to which exposure for a specific length of time is expected to cause death in $50 \%$ of a defined experimental animal population.

Lethal Dose $\mathbf{L}_{(\mathbf{L O})}\left(\mathbf{L D}_{\mathbf{L o}}\right)$ - The lowest dose of a chemical introduced by a route other than inhalation that has been reported to have caused death in humans or animals.

Lethal Dose $\mathbf{( 5 0 )}_{(\mathbf{L})}\left(\mathbf{L D}_{\mathbf{5 0}}\right)$ - The dose of a chemical that has been calculated to cause death in $50 \%$ of a defined experimental animal population.

Lethal Time $\mathbf{( 5 0}_{\mathbf{5}}\left(\mathbf{L T}_{\mathbf{5 0}}\right)-\mathrm{A}$ calculated period of time within which a specific concentration of a chemical is expected to cause death in $50 \%$ of a defined experimental animal population.

Lowest-Observed-Adverse-Effect Level (LOAEL) - The lowest exposure level of chemical in a study, or group of studies, that produces statistically or biologically significant increases in frequency or severity of adverse effects between the exposed population and its appropriate control.

Lymphoreticular Effects-Represent morphological effects involving lymphatic tissues such as the lymph nodes, spleen, and thymus.

Malformations-Permanent structural changes that may adversely affect survival, development, or function.

Minimal Risk Level (MRL) — An estimate of daily human exposure to a hazardous substance that is likely to be without an appreciable risk of adverse noncancer health effects over a specified route and duration of exposure.

Modifying Factor (MF) - A value (greater than zero) that is applied to the derivation of a Minimal Risk Level (MRL) to reflect additional concerns about the database that are not covered by the uncertainty factors. The default value for a MF is 1 .

Morbidity—State of being diseased; morbidity rate is the incidence or prevalence of disease in a specific population. 
Mortality-Death; mortality rate is a measure of the number of deaths in a population during a specified interval of time.

Mutagen-A substance that causes mutations. A mutation is a change in the DNA sequence of a cell's DNA. Mutations can lead to birth defects, miscarriages, or cancer.

Necropsy - The gross examination of the organs and tissues of a dead body to determine the cause of death or pathological conditions.

Neurotoxicity-The occurrence of adverse effects on the nervous system following exposure to a chemical.

No-Observed-Adverse-Effect Level (NOAEL) - The dose of a chemical at which there were no statistically or biologically significant increases in frequency or severity of adverse effects seen between the exposed population and its appropriate control. Effects may be produced at this dose, but they are not considered to be adverse.

Octanol-Water Partition Coefficient $\left(\mathbf{K}_{\mathbf{o w}}\right)$ - The equilibrium ratio of the concentrations of a chemical in $n$-octanol and water, in dilute solution.

Odds Ratio (OR) - A means of measuring the association between an exposure (such as toxic substances and a disease or condition) that represents the best estimate of relative risk (risk as a ratio of the incidence among subjects exposed to a particular risk factor divided by the incidence among subjects who were not exposed to the risk factor). An OR of greater than 1 is considered to indicate greater risk of disease in the exposed group compared to the unexposed group.

Organophosphate or Organophosphorus Compound-A phosphorus-containing organic compound and especially a pesticide that acts by inhibiting cholinesterase.

Permissible Exposure Limit (PEL) - An Occupational Safety and Health Administration (OSHA) allowable exposure level in workplace air averaged over an 8-hour shift of a 40-hour workweek.

Pesticide-General classification of chemicals specifically developed and produced for use in the control of agricultural and public health pests.

Pharmacokinetics - The dynamic behavior of a material in the body, used to predict the fate (disposition) of an exogenous substance in an organism. Utilizing computational techniques, it provides the means of studying the absorption, distribution, metabolism, and excretion of chemicals by the body.

Pharmacokinetic Model-A set of equations that can be used to describe the time course of a parent chemical or metabolite in an animal system. There are two types of pharmacokinetic models: data-based and physiologically-based. A data-based model divides the animal system into a series of compartments, which, in general, do not represent real, identifiable anatomic regions of the body, whereas the physiologically-based model compartments represent real anatomic regions of the body.

Physiologically Based Pharmacodynamic (PBPD) Model-A type of physiologically based doseresponse model that quantitatively describes the relationship between target tissue dose and toxic end points. These models advance the importance of physiologically based models in that they clearly describe the biological effect (response) produced by the system following exposure to an exogenous substance. 
Physiologically Based Pharmacokinetic (PBPK) Model-Comprised of a series of compartments representing organs or tissue groups with realistic weights and blood flows. These models require a variety of physiological information: tissue volumes, blood flow rates to tissues, cardiac output, alveolar ventilation rates, and possibly membrane permeabilities. The models also utilize biochemical information, such as air/blood partition coefficients, and metabolic parameters. PBPK models are also called biologically based tissue dosimetry models.

Prevalence-The number of cases of a disease or condition in a population at one point in time.

Prospective Study - A type of cohort study in which the pertinent observations are made on events occurring after the start of the study. A group is followed over time.

$\mathbf{q}_{1}{ }^{*}$-The upper-bound estimate of the low-dose slope of the dose-response curve as determined by the multistage procedure. The $\mathrm{q}_{1} *$ can be used to calculate an estimate of carcinogenic potency, the incremental excess cancer risk per unit of exposure (usually $\mu \mathrm{g} / \mathrm{L}$ for water, $\mathrm{mg} / \mathrm{kg} / \mathrm{day}$ for food, and $\mu \mathrm{g} / \mathrm{m}^{3}$ for air).

Recommended Exposure Limit (REL) —A National Institute for Occupational Safety and Health (NIOSH) time-weighted average (TWA) concentration for up to a 10-hour workday during a 40-hour workweek.

Reference Concentration (RfC)—An estimate (with uncertainty spanning perhaps an order of magnitude) of a continuous inhalation exposure to the human population (including sensitive subgroups) that is likely to be without an appreciable risk of deleterious noncancer health effects during a lifetime. The inhalation reference concentration is for continuous inhalation exposures and is appropriately expressed in units of $\mathrm{mg} / \mathrm{m}^{3}$ or $\mathrm{ppm}$.

Reference Dose (RfD) — An estimate (with uncertainty spanning perhaps an order of magnitude) of the daily exposure of the human population to a potential hazard that is likely to be without risk of deleterious effects during a lifetime. The RfD is operationally derived from the no-observed-adverse-effect level (NOAEL, from animal and human studies) by a consistent application of uncertainty factors that reflect various types of data used to estimate RfDs and an additional modifying factor, which is based on a professional judgment of the entire database on the chemical. The RfDs are not applicable to nonthreshold effects such as cancer.

Reportable Quantity (RQ) - The quantity of a hazardous substance that is considered reportable under the Comprehensive Environmental Response, Compensation, and Liability Act (CERCLA). Reportable quantities are (1) 1 pound or greater or (2) for selected substances, an amount established by regulation either under CERCLA or under Section 311 of the Clean Water Act. Quantities are measured over a 24-hour period.

Reproductive Toxicity-The occurrence of adverse effects on the reproductive system that may result from exposure to a chemical. The toxicity may be directed to the reproductive organs and/or the related endocrine system. The manifestation of such toxicity may be noted as alterations in sexual behavior, fertility, pregnancy outcomes, or modifications in other functions that are dependent on the integrity of this system. 
Retrospective Study-A type of cohort study based on a group of persons known to have been exposed at some time in the past. Data are collected from routinely recorded events, up to the time the study is undertaken. Retrospective studies are limited to causal factors that can be ascertained from existing records and/or examining survivors of the cohort.

Risk-The possibility or chance that some adverse effect will result from a given exposure to a chemical.

Risk Factor-An aspect of personal behavior or lifestyle, an environmental exposure, or an inborn or inherited characteristic that is associated with an increased occurrence of disease or other health-related event or condition.

Risk Ratio-The ratio of the risk among persons with specific risk factors compared to the risk among persons without risk factors. A risk ratio greater than 1 indicates greater risk of disease in the exposed group compared to the unexposed group.

Short-Term Exposure Limit (STEL) - The American Conference of Governmental Industrial Hygienists (ACGIH) maximum concentration to which workers can be exposed for up to 15 minutes continually. No more than four excursions are allowed per day, and there must be at least 60 minutes between exposure periods. The daily Threshold Limit Value-Time Weighted Average (TLV-TWA) may not be exceeded.

Standardized Mortality Ratio (SMR) —A ratio of the observed number of deaths and the expected number of deaths in a specific standard population.

Target Organ Toxicity-This term covers a broad range of adverse effects on target organs or physiological systems (e.g., renal, cardiovascular) extending from those arising through a single limited exposure to those assumed over a lifetime of exposure to a chemical.

Teratogen-A chemical that causes structural defects that affect the development of an organism.

Threshold Limit Value (TLV)—An American Conference of Governmental Industrial Hygienists (ACGIH) concentration of a substance to which most workers can be exposed without adverse effect. The TLV may be expressed as a Time Weighted Average (TWA), as a Short-Term Exposure Limit (STEL), or as a ceiling limit (CL).

Time-Weighted Average (TWA) —An allowable exposure concentration averaged over a normal 8-hour workday or 40-hour workweek.

Toxic Dose $\left.\mathbf{( 5 0 )}_{\mathbf{5 0}} \mathbf{T D}_{50}\right)$-A calculated dose of a chemical, introduced by a route other than inhalation, which is expected to cause a specific toxic effect in $50 \%$ of a defined experimental animal population.

Toxicokinetic - The absorption, distribution, and elimination of toxic compounds in the living organism. 
10. GLOSSARY

Uncertainty Factor (UF) - A factor used in operationally deriving the Minimal Risk Level (MRL) or Reference Dose (RfD) or Reference Concentration (RfC) from experimental data. UFs are intended to account for (1) the variation in sensitivity among the members of the human population, (2) the uncertainty in extrapolating animal data to the case of human, (3) the uncertainty in extrapolating from data obtained in a study that is of less than lifetime exposure, and (4) the uncertainty in using lowestobserved-adverse-effect level (LOAEL) data rather than no-observed-adverse-effect level (NOAEL) data. A default for each individual UF is 10; if complete certainty in data exists, a value of 1 can be used; however, a reduced UF of 3 may be used on a case-by-case basis, 3 being the approximate logarithmic average of 10 and 1 .

Xenobiotic-Any chemical that is foreign to the biological system. 
This page is intentionally blank. 


\section{APPENDIX A. ATSDR MINIMAL RISK LEVELS AND WORKSHEETS}

The Comprehensive Environmental Response, Compensation, and Liability Act (CERCLA) [42 U.S.C. 9601 et seq.], as amended by the Superfund Amendments and Reauthorization Act (SARA) [Pub. L. 99499], requires that the Agency for Toxic Substances and Disease Registry (ATSDR) develop jointly with the U.S. Environmental Protection Agency (EPA), in order of priority, a list of hazardous substances most commonly found at facilities on the CERCLA National Priorities List (NPL); prepare toxicological profiles for each substance included on the priority list of hazardous substances; and assure the initiation of a research program to fill identified data needs associated with the substances.

The toxicological profiles include an examination, summary, and interpretation of available toxicological information and epidemiologic evaluations of a hazardous substance. During the development of toxicological profiles, Minimal Risk Levels (MRLs) are derived when reliable and sufficient data exist to identify the target organ(s) of effect or the most sensitive health effect(s) for a specific duration for a given route of exposure. An MRL is an estimate of the daily human exposure to a hazardous substance that is likely to be without appreciable risk of adverse noncancer health effects over a specified duration of exposure. MRLs are based on noncancer health effects only and are not based on a consideration of cancer effects. These substance-specific estimates, which are intended to serve as screening levels, are used by ATSDR health assessors to identify contaminants and potential health effects that may be of concern at hazardous waste sites. It is important to note that MRLs are not intended to define clean-up or action levels.

MRLs are derived for hazardous substances using the no-observed-adverse-effect level/uncertainty factor approach. They are below levels that might cause adverse health effects in the people most sensitive to such chemical-induced effects. MRLs are derived for acute (1-14 days), intermediate (15-364 days), and chronic (365 days and longer) durations and for the oral and inhalation routes of exposure. Currently, MRLs for the dermal route of exposure are not derived because ATSDR has not yet identified a method suitable for this route of exposure. MRLs are generally based on the most sensitive chemical-induced end point considered to be of relevance to humans. Serious health effects (such as irreparable damage to the liver or kidneys, or birth defects) are not used as a basis for establishing MRLs. Exposure to a level above the MRL does not mean that adverse health effects will occur. 
MRLs are intended only to serve as a screening tool to help public health professionals decide where to look more closely. They may also be viewed as a mechanism to identify those hazardous waste sites that are not expected to cause adverse health effects. Most MRLs contain a degree of uncertainty because of the lack of precise toxicological information on the people who might be most sensitive (e.g., infants, elderly, nutritionally or immunologically compromised) to the effects of hazardous substances. ATSDR uses a conservative (i.e., protective) approach to address this uncertainty consistent with the public health principle of prevention. Although human data are preferred, MRLs often must be based on animal studies because relevant human studies are lacking. In the absence of evidence to the contrary, ATSDR assumes that humans are more sensitive to the effects of hazardous substance than animals and that certain persons may be particularly sensitive. Thus, the resulting MRL may be as much as 100 -fold below levels that have been shown to be nontoxic in laboratory animals.

Proposed MRLs undergo a rigorous review process: Health Effects/MRL Workgroup reviews within the Division of Toxicology and Environmental Medicine, expert panel peer reviews, and agency-wide MRL Workgroup reviews, with participation from other federal agencies and comments from the public. They are subject to change as new information becomes available concomitant with updating the toxicological profiles. Thus, MRLs in the most recent toxicological profiles supersede previously published levels. For additional information regarding MRLs, please contact the Division of Toxicology and Environmental Medicine, Agency for Toxic Substances and Disease Registry, 1600 Clifton Road NE, Mailstop F-32, Atlanta, Georgia 30333. 


\section{MINIMAL RISK LEVEL (MRL) WORKSHEET}

$\begin{array}{ll}\text { Chemical Name: } & \text { Inorganic Arsenic } \\ \text { CAS Number: } & 7440-38-2 \\ \text { Date: } & \text { August 2007 } \\ \text { Profile Status: } & \text { Post-Public Comment, Final Draft } \\ \text { Route: } & \text { [] Inhalation [X] Oral } \\ \text { Duration: } & \text { [X] Acute [] Intermediate [] Chronic } \\ \text { Graph Key: } & 29 \\ \text { Species: } & \text { Human }\end{array}$

Minimal Risk Level: 0.005 [X] mg/kg/day [ ] ppm

Reference: Mizuta N, Mizuta M, Ito F, et al. 1956. An outbreak of acute arsenic poisoning caused by arsenic-contaminated soy-sauce (shōyu): A clinical report of 220 cases. Bull Yamaguchi Med Sch 4(2-3):131-149.

Experimental design: Mizuta et al. (1956) summarized findings from 220 poisoning cases associated with an episode of arsenic contamination of soy sauce in Japan. The soy sauce was contaminated with approximately $0.1 \mathrm{mg} \mathrm{As} / \mathrm{mL}$, probably as calcium arsenate. Arsenic intake in the cases was estimated by the researchers to be $3 \mathrm{mg} /$ day $(0.05 \mathrm{mg} / \mathrm{kg} /$ day, assuming $55 \mathrm{~kg}$ average body weight for this Asian population). Duration of exposure was 2-3 weeks in most cases. Clinical symptoms were recorded. Seventy patients were examined opthalmologically. Laboratory tests were performed on some patients and included hematology, urinalysis, fecal exam, occult blood in gastric and duodenal juice, biochemical examination of blood, liver function tests, electrocardiograph, and liver biopsy.

Effects noted in study and corresponding doses: The primary symptoms were edema of the face, and gastrointestinal and upper respiratory symptoms initially, followed in some patients by skin lesions and neuropathy. Other effects included mild anemia and leukopenia, mild degenerative liver lesions and hepatic dysfunction, abnormal electrocardiogram, and ocular lesions. For derivation of the acute oral MRL, facial edema and gastrointestinal symptoms (nausea, vomiting, diarrhea), which were characteristic of the initial poisoning and then subsided, were considered to be the critical effects.

Dose and end point used for MRL derivation: $0.05 \mathrm{mg} \mathrm{As} / \mathrm{kg} / \mathrm{day}$

\section{[ ] NOAEL [X] LOAEL}

Uncertainty factors used in MRL derivation:
[ ] 1 [ ] 3 [X] 10 (for use of a LOAEL)
[ ] 1 [ ] 3 [ ] 10 (for extrapolation from animals to humans)
[X]1 [ ] 3 [ ] 10 (for human variability)

Was a conversion factor used from $\mathrm{ppm}$ in food or water to a $\mathrm{mg} /$ body weight dose? Not applicable.

If an inhalation study in animals, list conversion factors used in determining human equivalent dose: Not applicable.

Was a conversion used from intermittent to continuous exposure? Not applicable. 
Other additional studies or pertinent information that lend support to this MRL: The MRL is supported by the case of a man and wife in upstate New York who experienced gastrointestinal symptoms (nausea, diarrhea, abdominal cramps) starting almost immediately after beginning intermittent consumption of arsenic-tainted drinking water at an estimated dose of $0.05 \mathrm{mg} \mathrm{As} / \mathrm{kg} / \mathrm{day}$ (Franzblau and Lilis 1989). Gastrointestinal symptoms have been widely reported in other acute arsenic poisoning reports as well, although in some cases, the doses were higher and effects were severe, and in other cases, dose information was not available. The UF of 1 for intrahuman variability reflects the fact that the database includes persons of various ethnicities and age groups, including infants.

Agency Contact (Chemical Manager): Selene Chou, Ph.D and Carolyn Harper, Ph.D. 


\section{MINIMAL RISK LEVEL (MRL) WORKSHEET}

$\begin{array}{ll}\text { Chemical Name: } & \text { Inorganic Arsenic } \\ \text { CAS Number: } & 7440-38-2 \\ \text { Date: } & \text { August 2007 } \\ \text { Profile Status: } & \text { Post-Public Comment, Final Draft } \\ \text { Route: } & \text { [ ] Inhalation [X] Oral } \\ \text { Duration: } & \text { [ ] Acute [ ] Intermediate [X] Chronic } \\ \text { Graph Key: } & 134 \\ \text { Species: } & \text { Human }\end{array}$

Minimal Risk Level: $0.0003[\mathrm{X}] \mathrm{mg} / \mathrm{kg} /$ day $\quad[$ ] ppm

References: Tseng, WP, Chu HM, How SW, et al. 1968. Prevalence of skin cancer in an endemic area of chronic arsenicism in Taiwan. J Natl Cancer Inst 40:453-463.

Tseng, WP. 1977. Effects and dose-response relationships of cancer and Blackfoot disease with arsenic. Environ Health Perspect 19:109-119.

Experimental design: Tseng et al. (1968) and Tseng (1977) investigated the incidence of Blackfoot disease and dermal lesions (hyperkeratosis and hyperpigmentation) in a large number of poor farmers (both male and female) exposed to high levels of arsenic in well water in Taiwan. A control group consisting of 17,000 people was identified. The authors stated that the incidence of dermal lesions increased with dose, but individual doses were not provided. However, incidence data were provided based on stratification of the exposed population into low $(<300 \mu \mathrm{g} / \mathrm{L})$, medium $(300-600 \mu \mathrm{g} / \mathrm{L})$, or high $(>600 \mu \mathrm{g} / \mathrm{L})$ exposure levels. Doses were calculated from group mean arsenic concentrations in well water, assuming the intake parameters described by Abernathy et al. (1989). Accordingly, the control, low-, medium-, and high-exposure levels correspond to doses of $0.0008,0.014,0.038$, and $0.065 \mathrm{mg}$ As/kg/day, respectively. The NOAEL identified by Tseng (1977) (0.0008 $\mathrm{mg} \mathrm{As/kg/day)} \mathrm{was} \mathrm{limited} \mathrm{by}$ the fact that the majority of the population was $<20$ years of age and the incidence of skin lesions increased as a function of age, and because the estimates of water intake and dietary arsenic intake are highly uncertain. Schoof et al. (1998) estimated that dietary intakes of arsenic from rice and yams may have been 15-211 $\mu \mathrm{g} /$ day (mean $61 \mu \mathrm{g} /$ day), based on arsenic analyses of foods collected in Taiwan in 1993-1995. Use of the $50 \mu \mathrm{g} /$ day estimate would result in an approximate doubling of the NOAEL $(0.0016 \mathrm{mg} / \mathrm{kg} /$ day $)$.

Effects noted in study and corresponding doses: A clear dose-response relationship was observed for characteristic skin lesions:

$0.0008 \mathrm{mg} \mathrm{As} / \mathrm{kg} /$ day $\quad=$ control group $(\mathrm{NOAEL})$

$0.014 \mathrm{mg} \mathrm{As} / \mathrm{kg} /$ day $\quad=$ hyperpigmentation and keratosis of the skin (less serious LOAEL)

0.038-0.065 $\mathrm{mg} \mathrm{As} / \mathrm{kg} /$ day = increased incidence of dermal lesions

Dose and end point used for MRL derivation: $0.0008 \mathrm{mg} \mathrm{As} / \mathrm{kg} /$ day

\section{[X] NOAEL [ ] LOAEL}

Uncertainty factors used in MRL derivation:

[ ] 1 [ ] 3 [ ] 10 (for use of a LOAEL)

[ ] 1 [ ] 3 [ ] 10 (for extrapolation from animals to humans) 


\section{[ ] 1 [x] 3 [ ] 10 (for human variability)}

Was a conversion factor used from $\mathrm{ppm}$ in food or water to a $\mathrm{mg} /$ body weight dose? The arithmetic mean concentration of arsenic in well water for the control group $(0.009 \mathrm{mg} / \mathrm{L})$ was converted to a NOAEL of $0.0008 \mathrm{mg} \mathrm{As} / \mathrm{kg} /$ day as described below:

$$
\left[\left(\frac{0.009 m g}{L} \times \frac{4.5 L}{\text { day }}\right)+\frac{0.002 m g}{\text { day }}\right] \div 55 \mathrm{~kg}=0.0008 m g \text { As } / \mathrm{kg} / \text { day }
$$

This NOAEL conversion assumed a water intake of $4.5 \mathrm{~L} /$ day and a body weight of $55 \mathrm{~kg}$, and includes an estimation of arsenic intake of $0.002 \mathrm{mg} \mathrm{As} / \mathrm{kg} / \mathrm{day}$ from food. These assumptions are detailed in Abernathy et al. (1989). This approach to deriving a chronic oral MRL is identical to EPA's approach to deriving a chronic oral RfD.

If an inhalation study in animals, list conversion factors used in determining human equivalent dose: Not applicable.

Was a conversion used from intermittent to continuous exposure? Not applicable.

Other additional studies or pertinent information that lend support to this MRL: The MRL is supported by a number of well conducted epidemiological studies that identify reliable NOAELs and LOAELs for dermal effects. EPA (1981b) identified a NOAEL of 0.006-0.007 mg As/ $\mathrm{kg} /$ day for dermal lesions in several small populations in Utah. Harrington et al. (1978) identified a NOAEL of $0.003 \mathrm{mg} \mathrm{As} / \mathrm{kg} / \mathrm{day}$ for dermal effects in a small population in Alaska. Guha Mazumder et al. (1988) identified a NOAEL of $0.009 \mathrm{mg} \mathrm{As} / \mathrm{kg} /$ day and a LOAEL of $0.006 \mathrm{mg} \mathrm{As} / \mathrm{kg} /$ day for pigmentation changes and hyperkeratosis in a small population in India. Haque et al. (2003) identified a LOAEL of $0.0043 \mathrm{mg} \mathrm{As} / \mathrm{kg} / \mathrm{day}$ for hyperpigmentation and hyperkeratosis in a case-control study in India. Cebrían et al. (1983) identified a NOAEL of $0.0004 \mathrm{mg} \mathrm{As} / \mathrm{kg} /$ day and a LOAEL of $0.022 \mathrm{mg} \mathrm{As} / \mathrm{kg} /$ day in two regions in Mexico. Borgoño and Greiber (1972) and Zaldívar (1974) identified a LOAEL of $0.02 \mathrm{mg} \mathrm{As} / \mathrm{kg} /$ day for abnormal skin pigmentation in patients in Chile, and Borgoño et al. (1980) identified a LOAEL of $0.01 \mathrm{mg}$ $\mathrm{As} / \mathrm{kg} /$ day for the same effect in school children in Chile. Valentine et al. (1985) reported a NOAEL of $0.02 \mathrm{mg} \mathrm{As} / \mathrm{kg} /$ day for dermal effects in several small populations in California. Collectively, these studies indicate that the threshold dose for hyperpigmentation and hyperkeratosis is approximately $0.002 \mathrm{mg} \mathrm{As} / \mathrm{kg} /$ day. 


\section{MINIMAL RISK LEVEL (MRL) WORKSHEET}

$\begin{array}{ll}\text { Chemical Name: } & \text { Monomethylarsonic acid (MMA) } \\ \text { CAS Number: } & 124-58-3 \\ \text { Date: } & \text { August 2007 } \\ \text { Profile Status: } & \text { Post-Public Comment, Final Draft } \\ \text { Route: } & \text { [ ] Inhalation [X] Oral } \\ \text { Duration: } & \text { [ ] Acute [X] Intermediate [] Chronic } \\ \text { Graph Key: } & 12 \\ \text { Species: } & \text { Rat }\end{array}$

Minimal Risk Level: $0.1[\mathrm{X}] \mathrm{mg} \mathrm{MMA} / \mathrm{kg} /$ day $\quad[$ ] ppm

References: Arnold LL, Eldan M, van Gemert M, et al. 2003. Chronic studies evaluating the carcinogenicity of monomethylarsonic acid in rats and mice. Toxicology 190:197-219.

Crown S, Nyska A, Waner T. 1990. Methanearsonic acid: Combined chronic feeding and oncogenicity study in the rat. Conducted by Life Science Research Israel Ltd., Ness Ziona Israel. Submitted to EPA Office of Pesticide Programs (MRID 41669001).

Experimental design: Groups of 60 male and 60 female Fischer 344 rats were exposed to 0, 50, 400, or $1,300 \mathrm{ppm}$ MMA in the diet for 104 weeks. Using the average doses for weeks $1-50$ reported in an unpublished version of this study (Crown et al. 1990), doses of 0, 3.5, 30.2, and 106.9 $\mathrm{mg} \mathrm{MMA} / \mathrm{kg} / \mathrm{day}$ and $0,4.2,35.9$, and $123.3 \mathrm{mg} \mathrm{MMA} / \mathrm{kg} /$ day were calculated for males and females, respectively. Body weights, food consumption, and water intake were monitored regularly. Blood was taken at 3,6, and 12 months for clinical chemistry measurements, and urine samples were collected at the same interval.

Effects noted in study and corresponding doses: Mortality was increased in high-dose males and females during the first 52 weeks of the study. Body weights were decreased in the mid- and high-dose groups of both sexes; however, at 51 weeks, only the body weight for the high-dose males was $<10 \%$ of the control weight (14.5\%). Food and water consumption was increased in the mid- and high-dose groups. Diarrhea was observed in $100 \%$ of the high-dose males and females and in 16.7 and $40 \%$ of the mid-dose males and females during the first 52 weeks of exposure. Diarrhea first occurred after 3 weeks of exposure to the high dose and 4 weeks of exposure to the mid-dose group; the severity of the diarrhea was doserelated. The gastrointestinal system was the primary target in animals dying early; numerous macroscopic and histological alterations were observed.

Dose and end point used for MRL derivation: Benchmark dose analysis of the dose-response data (Table A-1) for diarrhea in male and female rats exposed to MMA in the diet for 1-52 weeks (incidence data reported in Crown et al. 1990) was conducted. All available dichotomous models in EPA's Benchmark Dose Software (version 1.4.1) were fit to the data. Predicted doses associated with a 10\% extra risk were calculated. As assessed by the chi-square goodness-of-fit statistic, all models, with the exception of the quantal linear model for male incidence data and the quantal linear model for female incidence data, provided an adequate fit $\left(X^{2} p>0.1\right)$ (Table A-2). Comparing across models, a better fit is generally indicated by a lower Akaike's Information Criteria (AIC). As assessed by AIC, the gamma model for the males (Figure A-1) and the 2-degree polynomial multi-stage model for the females (Figure A-2) provide the best fit to the data. The predicted $\mathrm{BMD}_{10}$ and $\mathrm{BMDL}_{10}$ are $28.25 \mathrm{mg}$ MMA/kg/day and $22.99 \mathrm{mg} \mathrm{MMA} / \mathrm{kg} /$ day for the male rat incidence data and $16.17 \mathrm{mg} \mathrm{MMA} / \mathrm{kg} / \mathrm{day}$, and $12.38 \mathrm{mg} \mathrm{MMA} / \mathrm{kg} /$ day for the female rat incidence data. 
Table A-1. Incidences of Diarrhea in Rats Exposed to MMA in the Diet for 1-52 Weeks

\begin{tabular}{ccl}
\hline Dietary concentration $(\mathrm{ppm})$ & Dose $(\mathrm{mg} \mathrm{MMA} / \mathrm{kg} /$ day $)$ & Incidence \\
\hline Male rats & 0 & \\
0 & 3.5 & $2 / 60$ \\
50 & 30.2 & $0 / 60$ \\
400 & 106.9 & $10 / 60$ \\
1,300 & & $60 / 60$ \\
Female rats & 0 & \\
0 & 4.2 & $0 / 60$ \\
50 & 35.9 & $0 / 60$ \\
400 & 123.3 & $24 / 60$ \\
1,300 & & $60 / 60$ \\
\hline
\end{tabular}

Sources: Arnold et al. 2003; Crown et al. 1990

Table A-2. Modeling Predictions for the Incidence of Diarrhea in Rats Exposed to MMA in the Diet for 1-52 Weeks

\begin{tabular}{lcccc}
\hline Model & $\begin{array}{l}\mathrm{BMD}_{10} \\
(\mathrm{mg} \mathrm{MMA} / \mathrm{kg} / \text { day })\end{array}$ & $\begin{array}{l}\mathrm{BMDL}_{10} \\
(\mathrm{mg} \mathrm{MMA} / \mathrm{kg} / \text { day })\end{array}$ & $\mathrm{x}^{2} p$-value & AIC \\
\hline Male rats & & & & \\
Gamma $^{\mathrm{a}}$ & $\mathbf{2 8 . 2 5}$ & $\mathbf{2 2 . 9 9}$ & $\mathbf{0 . 3 6}$ & $\mathbf{7 8 . 4 1}$ \\
Logistic $^{\text {Log-logistic }}{ }^{\mathrm{b}}$ & 24.60 & 20.19 & 0.16 & 79.59 \\
Multi-stage $^{\mathrm{c}}$ & 29.32 & 24.73 & 0.15 & 80.41 \\
Probit $_{\text {Log-probit }}^{\mathrm{b}}$ & 25.74 & 19.90 & 0.35 & 78.51 \\
Quantal linear $_{\text {Weibull }}^{\mathrm{a}}$ & 23.11 & 18.67 & 0.11 & 80.02 \\
Female rats $^{\text {Gamma }}$ & 28.79 & 24.47 & 0.15 & 80.41 \\
Logistic $^{\mathrm{a}}$ & 6.317 & 5.079 & 0.00 & 123.06 \\
Log-logistic $^{\mathrm{b}}$ & 27.99 & 20.66 & 0.15 & 80.41 \\
Multi-stage $^{\mathrm{c}}$ & & & & \\
Probit $^{\text {Log-probit }}$ & 26.81 & 15.18 & 1.00 & 84.76 \\
Quantal linear $_{\text {Weibull }}^{\mathrm{a}}$ & 32.85 & 21.49 & 1.00 & 84.76 \\
\hline
\end{tabular}

Sources: Arnold et al. 2003; Crown et al. 1990

${ }^{a}$ Restrict power $\geq 1$.

${ }^{\mathrm{b}}$ Slope restricted to $>1$.

${ }^{\mathrm{C}}$ Restrict betas $\geq 0$; lowest degree polynomial with an adequate fit is reported; degree of polynomial=3.

${ }^{d}$ Restrict betas $\geq 0$; lowest degree polynomial with an adequate fit is reported; degree of polynomial=2. 
Figure A-1. Predicted and Observed Incidence of Diarrhea in Male Rats Exposed to MMA in the Diet for 1-52 Weeks*

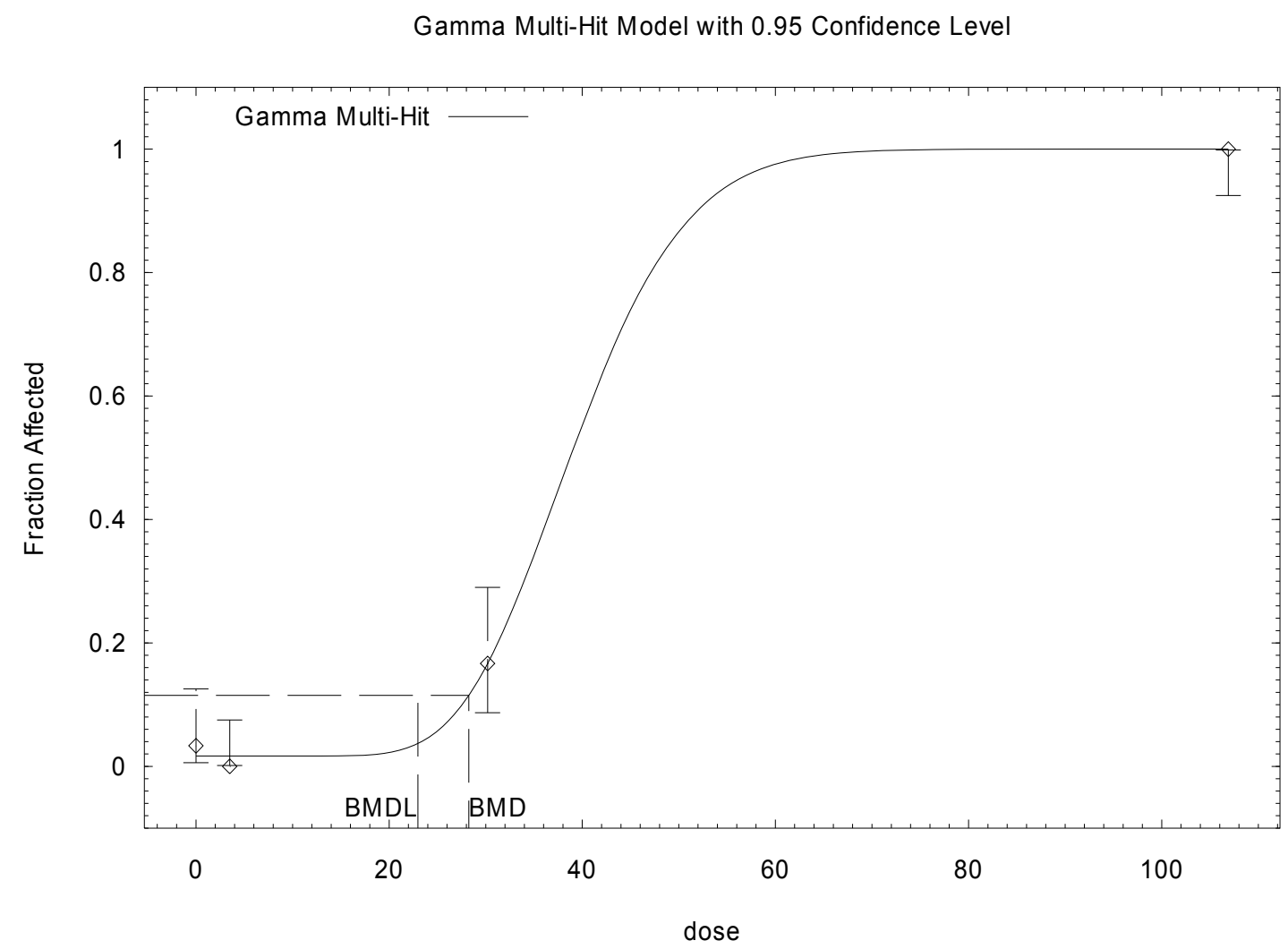

09:50 08/03 2007

*BMDs and BMDLs indicated are associated with a $10 \%$ extra risk change from the control, and are in units of mg MMA $/ \mathrm{kg} / \mathrm{day}$. 
Figure A-2. Predicted and Observed Incidence of Diarrhea in Female Rats Exposed to MMA in the Diet for 52 Weeks*

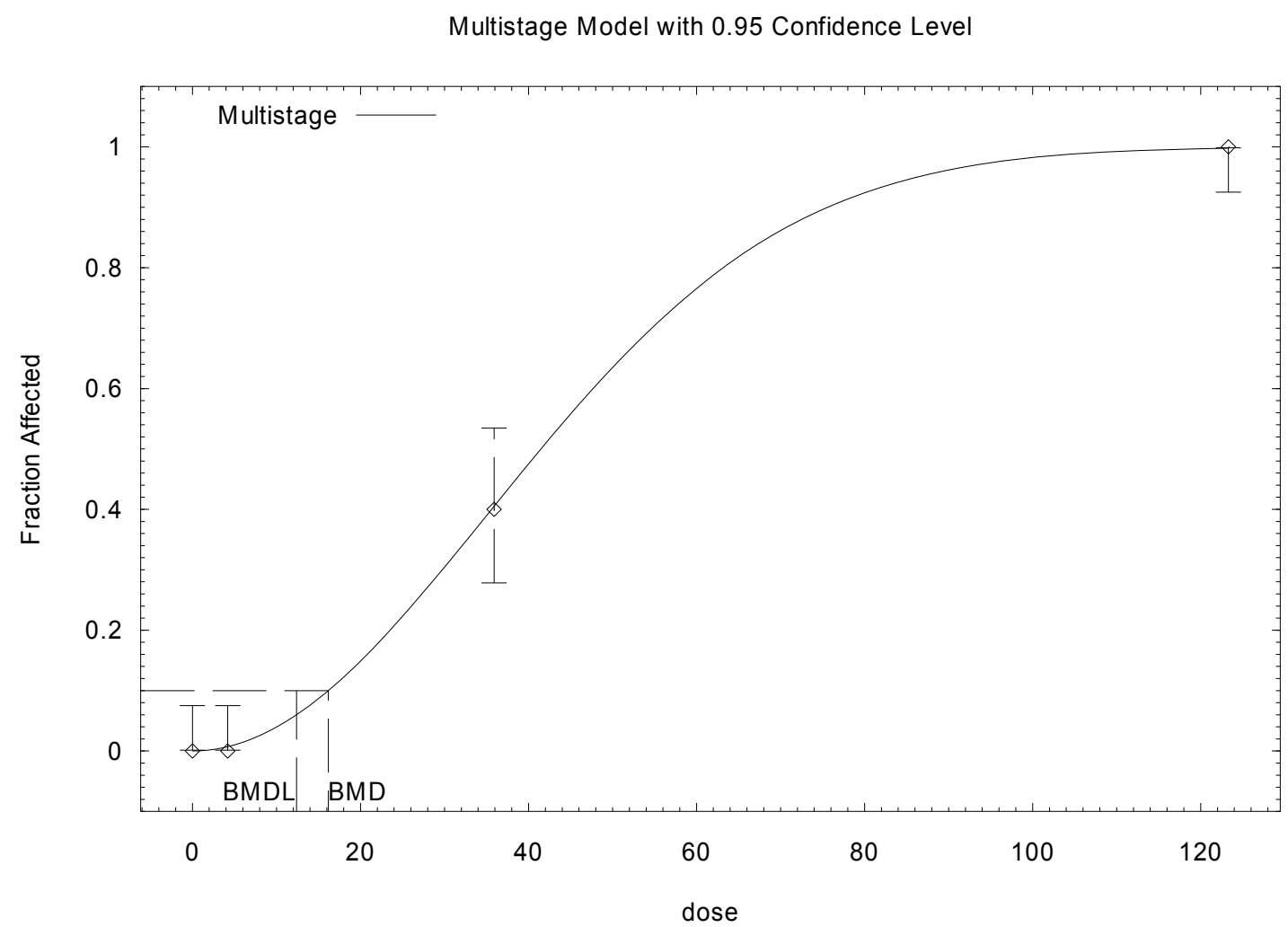

$10: 42$ 08/03 2007

${ }^{*} \mathrm{BMDs}$ and BMDLs indicated are associated with a $10 \%$ extra risk change from the control, and are in units of $\mathrm{mg} \mathrm{MMA} / \mathrm{kg} / \mathrm{day}$.

The $\mathrm{BMDL}_{10}$ of $12.38 \mathrm{mg} \mathrm{MMA} / \mathrm{kg} /$ day for female rats was selected as the point of departure for deriving the intermediate-duration oral MRL because it was lower than the $\mathrm{BMDL}_{10}(22.99 \mathrm{mg} \mathrm{MMA} / \mathrm{kg} / \mathrm{day})$ calculated using the male incidence data.

[] NOAEL [ ] LOAEL [X] BMDL

Uncertainty factors used in MRL derivation: 100

[ ] 10 for use of a LOAEL

[X] 10 for extrapolation from animals to humans

[X] 10 for human variability

Was a conversion factor used from $\mathrm{ppm}$ in food or water to a $\mathrm{mg} /$ body weight dose? Doses calculated using the average of the achieved doses for weeks 1-50 reported in Crown et al. (1990): 0, 3.5, 30.2, and $106.9 \mathrm{mg} \mathrm{MMA} / \mathrm{kg} /$ day for males and 0, 4.2,35.9, and $123.3 \mathrm{mg} \mathrm{MMA} / \mathrm{kg} /$ day for females.

If an inhalation study in animals, list conversion factors used in determining human equivalent dose: Not applicable.

$\underline{\text { Was a conversion used from intermittent to continuous exposure? }}$ Not applicable. 
Other additional studies or pertinent information that lend support to this MRL: Increases in the incidence of diarrhea has also been observed in dogs administered via capsule $2 \mathrm{mg} \mathrm{MMA} / \mathrm{kg} /$ day for 52 weeks (Waner and Nyska 1988); the increased incidence of diarrhea started during weeks 25-28. At $35 \mathrm{mg} \mathrm{MMA} / \mathrm{kg} /$ day, vomiting was also observed in the dogs. Diarrhea has also been observed in rats and mice exposed to MMA for 2 years (Arnold et al. 2003); the LOAELs are 25.7 and $67.1 \mathrm{mg}$ $\mathrm{MMA} / \mathrm{kg} /$ day, respectively.

Agency Contacts (Chemical Managers): Selene Chou, Ph.D and Carolyn Harper, Ph.D. 


\section{MINIMAL RISK LEVEL (MRL) WORKSHEET}

$\begin{array}{ll}\text { Chemical Name: } & \text { Monomethylarsonic acid (MMA) } \\ \text { CAS Number: } & 124-58-3 \\ \text { Date: } & \text { August 2007 } \\ \text { Profile Status: } & \text { Post-Public Comment, Final Draft } \\ \text { Route: } & \text { [ ] Inhalation [X] Oral } \\ \text { Duration: } & \text { [ ] Acute [] Intermediate [X] Chronic } \\ \text { Graph Key: } & 21 \\ \text { Species: } & \text { Mouse }\end{array}$

Minimal Risk Level: $0.01[\mathrm{X}] \mathrm{mg} \mathrm{MMA} / \mathrm{kg} /$ day $\quad[$ ] ppm

References: Arnold LL, Eldan M, van Gemert M, et al. 2003. Chronic studies evaluating the carcinogenicity of monomethylarsonic acid in rats and mice. Toxicology 190:197-219.

Gur E, Piraic H, Waner T. 1991. Methanearsonic acid: Combined oncogenicity study in the mouse. Conducted by Life Science Research Israel Ltd., Ness Ziona Israel. Submitted to EPA Office of Pesticide Programs (MRID 42173201).

Experimental design: Groups of 52 male and 52 female $\mathrm{B}_{6} \mathrm{C}_{3} \mathrm{~F}_{1}$ mice were exposed to $0,10,50,200$, or $400 \mathrm{ppm}$ of MMA in the diet for 104 weeks. The reported MMA doses were 0, 1.2, 6.0, 24.9, and $67.1 \mathrm{mg}$ MMA/kg/day (males) and 0, 1.4, 7.0, 31.2, and $101 \mathrm{mg} \mathrm{MMA} / \mathrm{kg} /$ day (females). Body weights, food consumption, and water intake were monitored regularly. Blood was taken at 3,6,12,18, and 24 months for white cell counts. At sacrifice, complete necropsies were performed, including histological examination of at least 13 organs.

Effects noted in study and corresponding doses: No treatment-related increases in mortality were observed. Significant decreases in body weights were observed in males and females exposed to 32.2 or $48.5 \mathrm{mg} \mathrm{As} / \mathrm{kg} /$ day, respectively; at week 104, the males weighed $17 \%$ less than controls and females weighed $23 \%$ less. Food consumption was increased in females exposed to $101 \mathrm{mg} \mathrm{MMA} / \mathrm{kg} / \mathrm{day}$, and water consumption was increased in $67.1 \mathrm{mg} \mathrm{MMA} / \mathrm{kg} / \mathrm{day}$ males and 31.2 and $101 \mathrm{mg} \mathrm{MMA} / \mathrm{kg} /$ day females. Loose and mucoid feces were noted in mice exposed to 67.1/101 mg MMA/kg/day. No changes were seen in white cell counts of either sex. Small decreases in the weights of heart, spleen, kidney, and liver were seen in some animals, but the decreases were not statistically significant. Squamous metaplasia of the cecum, colon, and rectum was observed at $67.1 / 101 \mathrm{mg} \mathrm{MMA} / \mathrm{kg} /$ day. The incidences of metaplasia in the cecum, colon, and rectum were 29/49, 14/49, and 39/49 in males and 38/52, 17/52, and 42/52 in females; metaplasia was not observed in other groups of male or female mice. An increased incidence of progressive glomerulonephropathy (incidence of 25/52, 27/52, 38/52, 39/52, and 46/52 in the $0,1.2,6.0,24.9$, and $67.1 \mathrm{mg} \mathrm{MMA} / \mathrm{kg} / \mathrm{day}$ ) was observed in males; the incidence was significantly higher (Fisher Exact Test) than controls at $\geq 6.0 \mathrm{mg} \mathrm{MMA} / \mathrm{kg} /$ day. Significant increases in the incidence of nephrocalcinosis was observed in the males at 24.9 and $67.1 \mathrm{mg} \mathrm{MMA} / \mathrm{kg} /$ day (Fisher Exact Test) (incidence of 25/52, 30/52, 30/52, 45/522 45/51 and 0/52, 1/52, 1/52, 2/52, and 5/52 in males and females, respectively). The investigators noted that the kidney lesions were consistent with the normal spectrum of spontaneous renal lesions and there was no difference in character or severity of the lesions between groups. A reduction in the incidence of cortical focal hyperplasia in the adrenal gland of male mice exposed to $67.1 \mathrm{mg} \mathrm{MMA} / \mathrm{kg} /$ day was possibly related to MMA exposure; the toxicological significance of this effect is not known.

Dose and end point used for MRL derivation: Benchmark dose analysis of the dose-response data (Table A-3) for progressive glomerulonephropathy in male mice exposed to MMA in the diet for 2 years 
(incidence data reported in Gur et al. 1991) was conducted. All available dichotomous models in EPA's Benchmark Dose Software (version 1.4.1) were fit to the data. Predicted doses associated with a 10\% extra risk were calculated. As assessed by the chi-square goodness-of-fit statistic, all models, with the exception of the log-probit model, provided an adequate fit $\left(X^{2} p>0.1\right)$ (Table A-4). Comparing across models, a better fit is generally indicated by a lower Akaike's Information Criteria (AIC). As assessed by AIC, the log-logistic model (Figure A-3) provided the best fit to the data. The predicted BMD 10 and $\mathrm{BMDL}_{10}$ for the incidence data are 2.09 and $1.09 \mathrm{mg} \mathrm{MMA} / \mathrm{kg} /$ day.

Table A-3. Incidence of Progressive Glomerulonephropathy in Male Mice Exposed to MMA in the Diet for 2 Years

\begin{tabular}{rcl}
\hline Dietary Concentration $(\mathrm{ppm})$ & Dose $(\mathrm{mg} \mathrm{MMA} / \mathrm{kg} /$ day $)$ & Incidence \\
\hline & 0 & \\
0 & 1.2 & $25 / 52$ \\
10 & 6.0 & $27 / 52$ \\
50 & 24.9 & $38 / 52$ \\
200 & 67.1 & $39 / 52$ \\
400 & $46 / 52$ \\
\hline
\end{tabular}

Sources: Arnold et al. 2003; Gur et al. 1991

Table A-4. Modeling Predictions for the Incidence of Progressive Glomerulonephropathy in Male Mice Exposed to MMA in the Diet for 2 Years

\begin{tabular}{lcccc}
\hline Model & $\begin{array}{l}\mathrm{BMD}_{10} \\
(\mathrm{mg} \mathrm{MMA} / \mathrm{kg} / \text { day })\end{array}$ & $\begin{array}{l}\mathrm{BMDL}_{10} \\
(\mathrm{mg} \mathrm{MMA} / \mathrm{kg} / \text { day })\end{array}$ & $\mathrm{x}^{2}$-value & AIC \\
\hline Gamma $^{\mathrm{a}}$ & 4.60 & 3.15 & 0.18 & 309.33 \\
Logistic $^{\text {Log-logistic }}$ & 6.09 & 4.45 & 0.13 & 310.15 \\
Multi-stage $^{\mathrm{c}}$ & $\mathbf{2 . 0 9}$ & $\mathbf{1 . 0 9}$ & $\mathbf{0 . 3 8}$ & $\mathbf{3 0 7 . 4 7}$ \\
Probit $^{\text {Log-probit }}$ & 4.60 & 3.15 & 0.18 & 309.33 \\
Quantal linear $^{\mathrm{b}}$ & 6.62 & 5.00 & 0.11 & 310.43 \\
Weibull $^{\mathrm{a}}$ & 8.54 & 5.50 & 0.08 & 311.11 \\
\hline
\end{tabular}

Sources: Arnold et al. 2003; Gur et al. 1991

${ }^{\mathrm{a}}$ Restrict power $\geq 1$.

${ }^{\mathrm{b}}$ Slope restricted to $>1$.

${ }^{\mathrm{c}}$ Restrict betas $\geq 0$; lowest degree polynomial with an adequate fit is reported; degree of polynomial=1. 


\section{Figure A-3. Predicted and Observed Incidence of Progressive Glomerulonephropathy in Male Mice Exposed to MMA*}

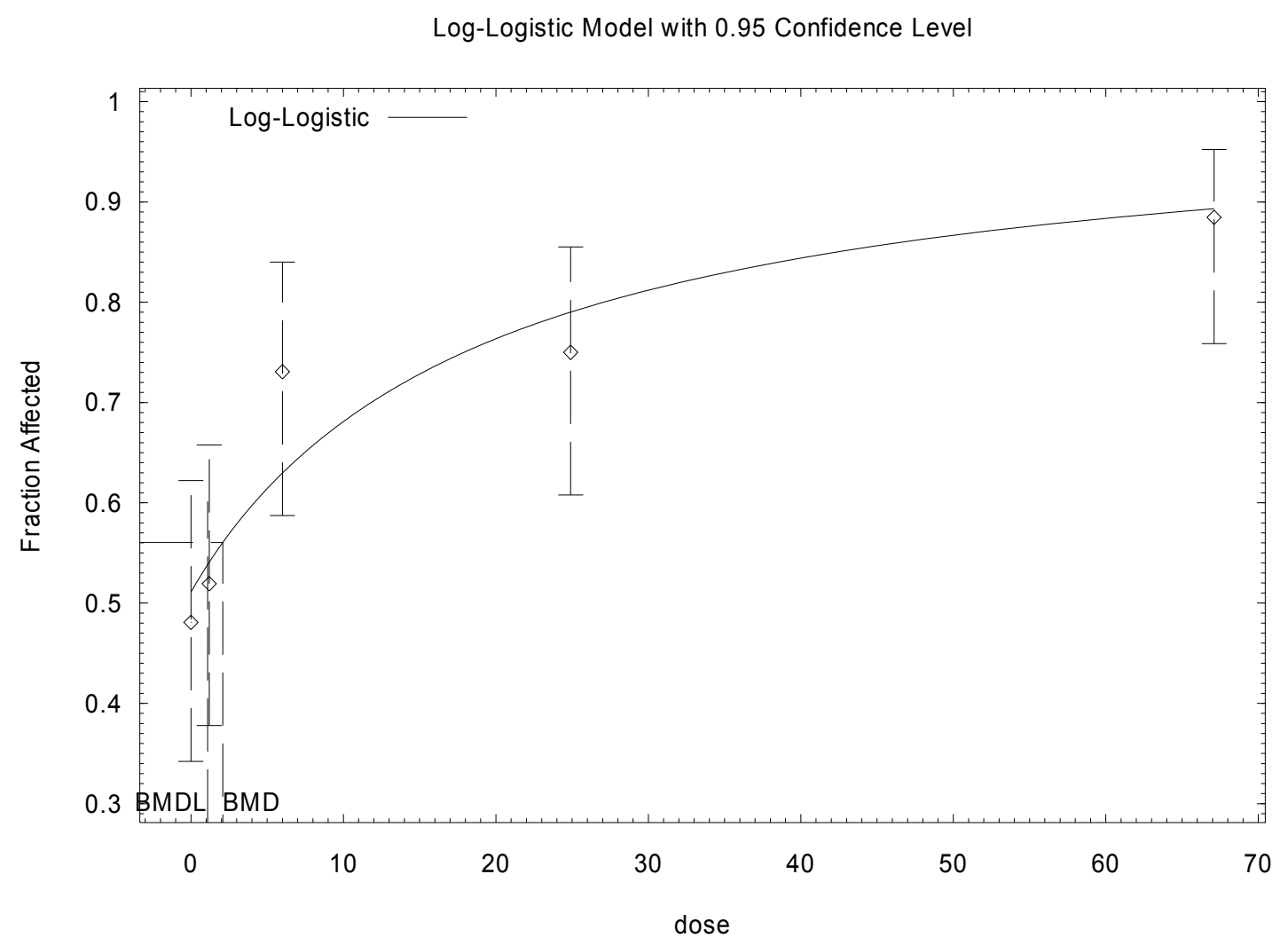

$10: 5708 / 032007$

${ }^{*} \mathrm{BMDs}$ and BMDLs indicated are associated with a $10 \%$ extra risk change from the control, and are in units of mg MMA/kg/day.

The $\mathrm{BMDL}_{10}$ of $1.09 \mathrm{mg} \mathrm{MMA} / \mathrm{kg} /$ day for male mice was selected as the point of departure for deriving the chronic-duration oral MRL.

[] NOAEL [ ] LOAEL [X] BMDL

Uncertainty factors used in MRL derivation: 100

[ ] 10 for use of a LOAEL

[X] 10 for extrapolation from animals to humans

[X] 10 for human variability

Was a conversion factor used from $\mathrm{ppm}$ in food or water to a $\mathrm{mg} /$ body weight dose? Doses calculated using the average of the achieved doses reported in Gur et al. (1991): 0, 1.2, 6.0, 24.9, and 67.1 $\mathrm{mg}$ MMA/kg/day for males and 0, 1.4, 7.0,31.2, and $101 \mathrm{mg} \mathrm{MMA} / \mathrm{kg} /$ day for females.

If an inhalation study in animals, list conversion factors used in determining human equivalent dose: Not applicable.

$\underline{\text { Was a conversion used from intermittent to continuous exposure? }}$ Not applicable. 
Other additional studies or pertinent information that lend support to this MRL: An exacerbation of chronic progressive nephropathy (an increase in the severity of the nephropathy) has also been observed in rats exposed to $\geq 33.9 \mathrm{mg}$ MMA/kg/day for 2 years (Arnold et al. 2003).

Agency Contacts (Chemical Managers): Selene Chou, Ph.D and Carolyn Harper, Ph.D. 


\section{MINIMAL RISK LEVEL (MRL) WORKSHEET}

$\begin{array}{ll}\text { Chemical Name: } & \text { Dimethylarsinic acid (DMA) } \\ \text { CAS Number: } & 75-60-5 \\ \text { Date: } & \text { August 2007 } \\ \text { Profile Status: } & \text { Post-Public Comment, Final Draft } \\ \text { Route: } & \text { [ ] Inhalation [X] Oral } \\ \text { Duration: } & \text { [ ] Acute [] Intermediate [X] Chronic } \\ \text { Graph Key: } & 35 \\ \text { Species: } & \text { Mouse }\end{array}$

Minimal Risk Level: $0.02[\mathrm{X}] \mathrm{mg} \mathrm{DMA} / \mathrm{kg} /$ day $\quad[$ ] ppm

References: Arnold LL, Eldan M, Nyska A, et al. 2006. Dimethylarsinic acid: Results of chronic toxicity/oncogenicity studies in $\mathrm{F} 344$ rats and $\mathrm{B}_{6 \mathrm{C} 3 \mathrm{~F}_{1}}$ mice. Toxicology 223:82-100.

Gur E, Nyska A, Pirak M, et al. 1989b. Cacodylic acid: Oncogenicity study in the mouse. Conducted by Life Science Research Israel Ltd., Ness Ziona Israel. Submitted to EPA Office of Pesticide Programs (MRID 41914601).

Experimental design: Groups of 56 male and 56 female $\mathrm{B} 6 \mathrm{C}_{3} \mathrm{~F}_{1}$ mice were exposed to $0,8,40,200$, or 500 ppm DMA in the diet for 2 years. The investigators reported the dietary doses were equivalent to approximately $0,1.3,7.8,37$, and $94 \mathrm{mg} \mathrm{DMA} / \mathrm{kg} /$ day. The following parameters were used to assess toxicity: clinical observations, body weight, food consumption, water consumption, differential leukocyte levels measured at 12, 18, and 24 months in mice in the control and $94 \mathrm{mg} \mathrm{DMA} / \mathrm{kg} /$ day groups, organ weights (brain, kidneys, liver, and testes), and histopathological examination of major tissues and organs.

Effects noted in study and corresponding doses: No deaths were observed. Decreases in body weight gain were observed in the male mice exposed to $94 \mathrm{mg}$ DMA/kg/day; the difference was $<10 \%$ and was not considered adverse. An increase in water consumption was observed in males exposed to $94 \mathrm{mg}$ DMA $/ \mathrm{kg} /$ day during weeks 60-96. No treatment-related clinical signs were observed. In the female mice exposed to $94 \mathrm{mg} \mathrm{DMA} / \mathrm{kg} /$ day, a statistically significant decrease in lymphocytes and increase in monocytes were observed at 24 months. Treatment related nonneoplastic alterations were observed in the urinary bladder and kidneys. In the urinary bladder, increases in the vacuolization of the superficial cells of the urothelium were observed in males exposed to 37 or $94 \mathrm{mg}$ DMA/kg/day $(0 / 44,1 / 50,0 / 50,36 / 45$, 48/48) and in females exposed to 7.8, 37, or $94 \mathrm{mg}$ DMA/kg/day $(1 / 45,1 / 48,26 / 43,47 / 47,43 / 43)$; incidence data reported in Gur et al. (1989b). An increased incidence of progressive glomerulonephropathy was observed in males at $37 \mathrm{mg} \mathrm{DMA} / \mathrm{kg} /$ day $(16 / 44,22 / 50,17 / 50,34 / 45,30 / 50)$ and an increased incidence of nephrocalcinosis was also observed in male mice at $94 \mathrm{mg} \mathrm{DMA} / \mathrm{kg} / \mathrm{day}$ $(30 / 44,25 / 50,27 / 50,29 / 50,45 / 50)$. Neoplastic alterations were limited to an increased incidence of fibrosarcoma of the skin in females exposed to $94 \mathrm{mg} \mathrm{DMA} / \mathrm{kg} /$ day; the incidence was $3 / 56,0 / 55,1 / 56$, $1 / 56$, and $6 / 56$ in the $0,1.3,7.8,37$, and $94 \mathrm{mg} \mathrm{DMA} / \mathrm{kg} /$ day groups, respectively; however it was concluded that this lesion was not related to DMA exposure.

Dose and end point used for MRL derivation: Benchmark dose analysis of the dose-response data (Table A-5) for vacuolization of the urothelium in the urinary bladder in female mice exposed to DMA in the diet for 2 years (incidence data reported in Gur et al. 1989b) was conducted. All available dichotomous models in EPA's Benchmark Dose Software (version 1.4.1) were fit to the data. Predicted doses associated with a $10 \%$ extra risk were calculated. As assessed by the chi-square goodness-of-fit statistic, all models, with the exception of the quantal linear model, provided an adequate fit $\left(\mathrm{X}^{2} p>0.1\right)$ (Table A-6). Comparing across models, a better fit is generally indicated by a lower AIC. The AIC 
values were similar for the logistic, multi-stage, and probit models; of these three models, the multi-stage had the lowest $\mathrm{BMD}_{10}$ and was selected for the analysis (see Figure A-4). The predicted $\mathrm{BMD}_{10}$ and $\mathrm{BMDL}_{10}$ for the incidence data are 2.68 and $1.80 \mathrm{mg} \mathrm{DMA} / \mathrm{kg} /$ day.

\section{Table A-5. Incidence of Vacuolization of Urotheium in Urinary Bladder of Female Mice Exposed to DMA in the Diet for 2 Years}

\begin{tabular}{lcl}
\hline Dietary concentration $(\mathrm{ppm})$ & Dose $(\mathrm{mg} \mathrm{DMA} / \mathrm{kg} /$ day $)$ & Incidence \\
\hline & 0 & $1 / 45$ \\
8 & 1.3 & $1 / 48$ \\
40 & 7.8 & $26 / 43$ \\
200 & 37 & $47 / 47$ \\
500 & 94 & $43 / 43$ \\
\hline
\end{tabular}

Sources: Arnold et al. 2006; Gur et al. 1989b

Table A-6. Modeling Predictions for the Incidence of Vacuolization in of Urothelium in Urinary Bladder of Female Mice Exposed to DMA in the Diet for 2 Years

\begin{tabular}{|c|c|c|c|c|}
\hline Model & $\begin{array}{l}\mathrm{BMD}_{10} \\
\text { (mg DMA/kg/day) }\end{array}$ & $\begin{array}{l}\mathrm{BMDL}_{10} \\
\text { (mg DMA/kg/day) }\end{array}$ & $x^{2} p$-value & $\mathrm{AIC}$ \\
\hline Gamma $^{a}$ & 5.01 & 1.85 & 1.00 & 83.03 \\
\hline Logistic & 3.66 & 2.78 & 0.95 & 81.37 \\
\hline Log-logistic $^{b}$ & 6.23 & 2.34 & 1.00 & 83.03 \\
\hline Multi-stage $^{c}$ & 2.68 & 1.80 & 0.90 & 81.69 \\
\hline Probit & 3.20 & 2.46 & 0.89 & 81.60 \\
\hline Log-probit $^{\mathrm{b}}$ & 5.03 & 2.00 & 1.00 & 83.03 \\
\hline Quantal linear & 0.98 & 0.76 & 0.07 & 91.75 \\
\hline Weibull $^{\mathrm{a}}$ & 4.77 & 1.88 & 1.00 & 83.03 \\
\hline
\end{tabular}

Sources: Arnold et al. 2006; Gur et al. 1989b

${ }^{a}$ Restrict power $\geq 1$.

${ }^{\mathrm{b}}$ Slope restricted to $>1$.

${ }^{\mathrm{c}}$ Restrict betas $\geq 0$; lowest degree polynomial with an adequate fit is reported; degree of polynomial=2. 


\section{Figure A-4. Predicted and Observed Incidence of Vacuolization of Urothelium in Urinary Bladder of Female Mice*}

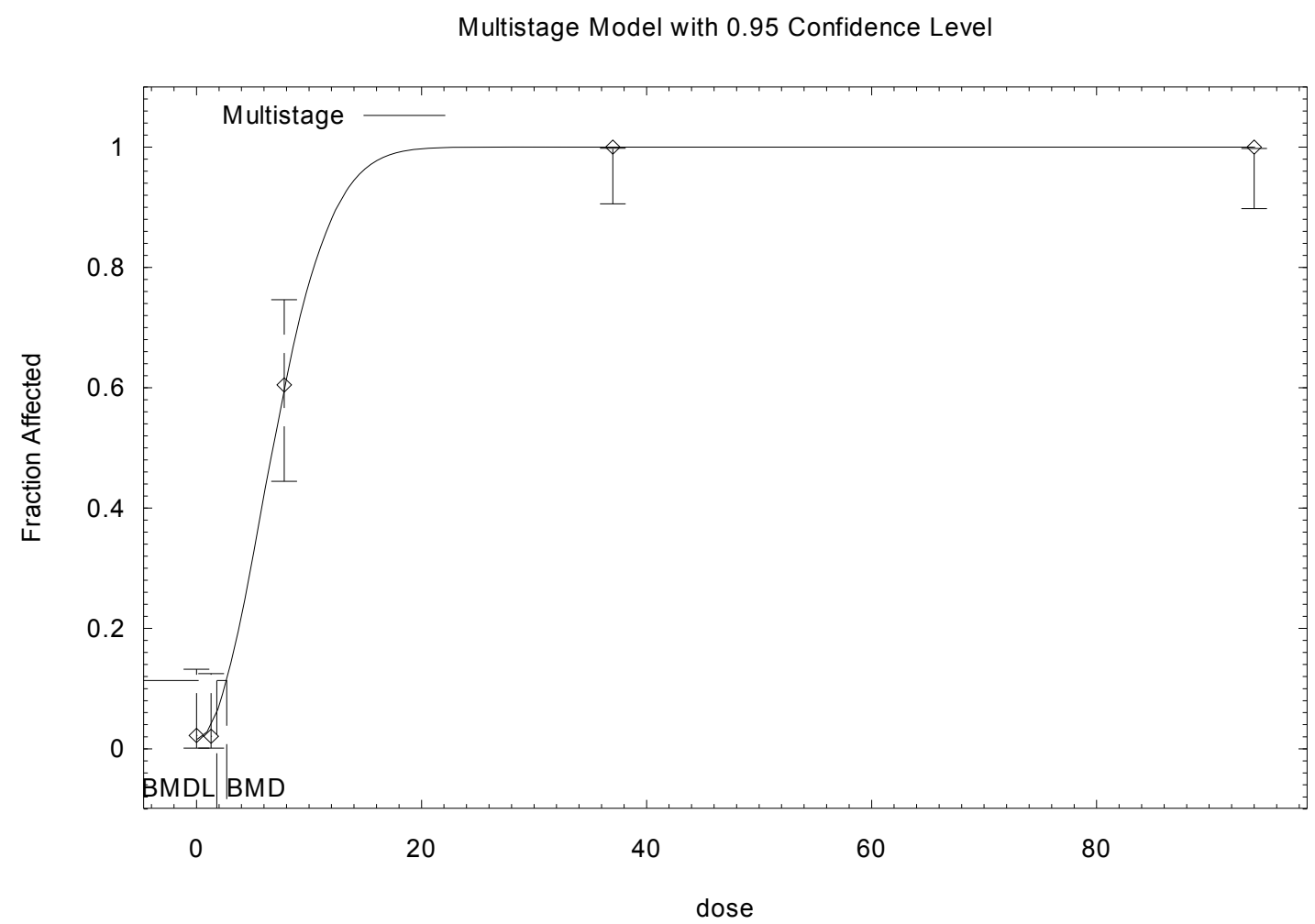

$13: 2508 / 032007$

Source: Arnold et al. 2006

${ }^{*}$ BMDs and BMDLs indicated are associated with a $10 \%$ extra risk change from the control, and are in units of mg $\mathrm{DMA} / \mathrm{kg} /$ day.

The $\mathrm{BMDL}_{10}$ of $1.80 \mathrm{mg} \mathrm{DMA} / \mathrm{kg} /$ day for female mice was selected as the point of departure for deriving the chronic-duration oral MRL.

[] NOAEL [ ] LOAEL [X] BMDL

Uncertainty factors used in MRL derivation: 100

[ ] 10 for use of a LOAEL

[X] 10 for extrapolation from animals to humans

[X] 10 for human variability

Was a conversion factor used from $\mathrm{ppm}$ in food or water to a $\mathrm{mg} /$ body weight dose? Doses reported in Gur et al. (1989b): 0, 1.3, 7.8, 37, and $94 \mathrm{mg}$ DMA/kg/day.

If an inhalation study in animals, list conversion factors used in determining human equivalent dose: Not applicable.

Was a conversion used from intermittent to continuous exposure? Not applicable. 
Other additional studies or pertinent information that lend support to this MRL: One other study has investigated the chronic toxicity of DMA in species other than rats. In this study, administration of $16 \mathrm{mg}$ $\mathrm{DMA} / \mathrm{kg} /$ day via a capsule for 52 weeks resulted in increases in the incidence of diarrhea; no histological alterations were observed (Zomber et al. 1989).

Agency Contacts (Chemical Managers): Selene Chou, Ph.D and Carolyn Harper, Ph.D. 
This page is intentionally blank. 


\section{APPENDIX B. USER'S GUIDE}

\section{Chapter 1}

\section{Public Health Statement}

This chapter of the profile is a health effects summary written in nontechnical language. Its intended audience is the general public, especially people living in the vicinity of a hazardous waste site or chemical release. If the Public Health Statement were removed from the rest of the document, it would still communicate to the lay public essential information about the chemical.

The major headings in the Public Health Statement are useful to find specific topics of concern. The topics are written in a question and answer format. The answer to each question includes a sentence that will direct the reader to chapters in the profile that will provide more information on the given topic.

\section{Chapter 2}

\section{Relevance to Public Health}

This chapter provides a health effects summary based on evaluations of existing toxicologic, epidemiologic, and toxicokinetic information. This summary is designed to present interpretive, weightof-evidence discussions for human health end points by addressing the following questions:

1. What effects are known to occur in humans?

2. What effects observed in animals are likely to be of concern to humans?

3. What exposure conditions are likely to be of concern to humans, especially around hazardous waste sites?

The chapter covers end points in the same order that they appear within the Discussion of Health Effects by Route of Exposure section, by route (inhalation, oral, and dermal) and within route by effect. Human data are presented first, then animal data. Both are organized by duration (acute, intermediate, chronic). In vitro data and data from parenteral routes (intramuscular, intravenous, subcutaneous, etc.) are also considered in this chapter.

The carcinogenic potential of the profiled substance is qualitatively evaluated, when appropriate, using existing toxicokinetic, genotoxic, and carcinogenic data. ATSDR does not currently assess cancer potency or perform cancer risk assessments. Minimal Risk Levels (MRLs) for noncancer end points (if derived) and the end points from which they were derived are indicated and discussed.

Limitations to existing scientific literature that prevent a satisfactory evaluation of the relevance to public health are identified in the Chapter 3 Data Needs section.

\section{Interpretation of Minimal Risk Levels}

Where sufficient toxicologic information is available, ATSDR has derived MRLs for inhalation and oral routes of entry at each duration of exposure (acute, intermediate, and chronic). These MRLs are not 
meant to support regulatory action, but to acquaint health professionals with exposure levels at which adverse health effects are not expected to occur in humans.

MRLs should help physicians and public health officials determine the safety of a community living near a chemical emission, given the concentration of a contaminant in air or the estimated daily dose in water. MRLs are based largely on toxicological studies in animals and on reports of human occupational exposure.

MRL users should be familiar with the toxicologic information on which the number is based. Chapter 2, "Relevance to Public Health," contains basic information known about the substance. Other sections such as Chapter 3 Section 3.9, "Interactions with Other Substances," and Section 3.10, "Populations that are Unusually Susceptible" provide important supplemental information.

MRL users should also understand the MRL derivation methodology. MRLs are derived using a modified version of the risk assessment methodology that the Environmental Protection Agency (EPA) provides (Barnes and Dourson 1988) to determine reference doses (RfDs) for lifetime exposure.

To derive an MRL, ATSDR generally selects the most sensitive end point which, in its best judgement, represents the most sensitive human health effect for a given exposure route and duration. ATSDR cannot make this judgement or derive an MRL unless information (quantitative or qualitative) is available for all potential systemic, neurological, and developmental effects. If this information and reliable quantitative data on the chosen end point are available, ATSDR derives an MRL using the most sensitive species (when information from multiple species is available) with the highest no-observed-adverse-effect level (NOAEL) that does not exceed any adverse effect levels. When a NOAEL is not available, a lowest-observed-adverse-effect level (LOAEL) can be used to derive an MRL, and an uncertainty factor (UF) of 10 must be employed. Additional uncertainty factors of 10 must be used both for human variability to protect sensitive subpopulations (people who are most susceptible to the health effects caused by the substance) and for interspecies variability (extrapolation from animals to humans). In deriving an MRL, these individual uncertainty factors are multiplied together. The product is then divided into the inhalation concentration or oral dosage selected from the study. Uncertainty factors used in developing a substance-specific MRL are provided in the footnotes of the levels of significant exposure (LSE) tables.

\section{Chapter 3}

\section{Health Effects}

\section{Tables and Figures for Levels of Significant Exposure (LSE)}

Tables and figures are used to summarize health effects and illustrate graphically levels of exposure associated with those effects. These levels cover health effects observed at increasing dose concentrations and durations, differences in response by species, MRLs to humans for noncancer end points, and EPA's estimated range associated with an upper- bound individual lifetime cancer risk of 1 in 10,000 to 1 in 10,000,000. Use the LSE tables and figures for a quick review of the health effects and to locate data for a specific exposure scenario. The LSE tables and figures should always be used in conjunction with the text. All entries in these tables and figures represent studies that provide reliable, quantitative estimates of NOAELs, LOAELs, or Cancer Effect Levels (CELs).

The legends presented below demonstrate the application of these tables and figures. Representative examples of LSE Table 3-1 and Figure 3-1 are shown. The numbers in the left column of the legends correspond to the numbers in the example table and figure. 


\section{LEGEND}

\section{See Sample LSE Table 3-1 (page B-6)}

(1) Route of Exposure. One of the first considerations when reviewing the toxicity of a substance using these tables and figures should be the relevant and appropriate route of exposure. Typically when sufficient data exist, three LSE tables and two LSE figures are presented in the document. The three LSE tables present data on the three principal routes of exposure, i.e., inhalation, oral, and dermal (LSE Tables 3-1, 3-2, and 3-3, respectively). LSE figures are limited to the inhalation (LSE Figure 3-1) and oral (LSE Figure 3-2) routes. Not all substances will have data on each route of exposure and will not, therefore, have all five of the tables and figures.

(2) Exposure Period. Three exposure periods - acute (less than 15 days), intermediate (15364 days), and chronic (365 days or more) - are presented within each relevant route of exposure. In this example, an inhalation study of intermediate exposure duration is reported. For quick reference to health effects occurring from a known length of exposure, locate the applicable exposure period within the LSE table and figure.

(3) Health Effect. The major categories of health effects included in LSE tables and figures are death, systemic, immunological, neurological, developmental, reproductive, and cancer. NOAELs and LOAELs can be reported in the tables and figures for all effects but cancer. Systemic effects are further defined in the "System" column of the LSE table (see key number 18).

(4) Key to Figure. Each key number in the LSE table links study information to one or more data points using the same key number in the corresponding LSE figure. In this example, the study represented by key number 18 has been used to derive a NOAEL and a Less Serious LOAEL (also see the two "18r" data points in sample Figure 3-1).

(5) Species. The test species, whether animal or human, are identified in this column. Chapter 2, "Relevance to Public Health," covers the relevance of animal data to human toxicity and Section 3.4, "Toxicokinetics," contains any available information on comparative toxicokinetics. Although NOAELs and LOAELs are species specific, the levels are extrapolated to equivalent human doses to derive an MRL.

(6) Exposure Frequency/Duration. The duration of the study and the weekly and daily exposure regimens are provided in this column. This permits comparison of NOAELs and LOAELs from different studies. In this case (key number 18), rats were exposed to "Chemical x" via inhalation for 6 hours/day, 5 days/week, for 13 weeks. For a more complete review of the dosing regimen, refer to the appropriate sections of the text or the original reference paper (i.e., Nitschke et al. 1981).

(7) System. This column further defines the systemic effects. These systems include respiratory, cardiovascular, gastrointestinal, hematological, musculoskeletal, hepatic, renal, and dermal/ocular. "Other" refers to any systemic effect (e.g., a decrease in body weight) not covered in these systems. In the example of key number 18, one systemic effect (respiratory) was investigated.

(8) NOAEL. A NOAEL is the highest exposure level at which no harmful effects were seen in the organ system studied. Key number 18 reports a NOAEL of $3 \mathrm{ppm}$ for the respiratory system, 
which was used to derive an intermediate exposure, inhalation MRL of $0.005 \mathrm{ppm}$ (see footnote "b").

(9) LOAEL. A LOAEL is the lowest dose used in the study that caused a harmful health effect. LOAELs have been classified into "Less Serious" and "Serious" effects. These distinctions help readers identify the levels of exposure at which adverse health effects first appear and the gradation of effects with increasing dose. A brief description of the specific end point used to quantify the adverse effect accompanies the LOAEL. The respiratory effect reported in key number 18 (hyperplasia) is a Less Serious LOAEL of $10 \mathrm{ppm}$. MRLs are not derived from Serious LOAELs.

(10) Reference. The complete reference citation is given in Chapter 9 of the profile.

(11) CEL. A CEL is the lowest exposure level associated with the onset of carcinogenesis in experimental or epidemiologic studies. CELs are always considered serious effects. The LSE tables and figures do not contain NOAELs for cancer, but the text may report doses not causing measurable cancer increases.

(12) Footnotes. Explanations of abbreviations or reference notes for data in the LSE tables are found in the footnotes. Footnote "b" indicates that the NOAEL of 3 ppm in key number 18 was used to derive an MRL of $0.005 \mathrm{ppm}$.

\section{LEGEND}

\section{See Sample Figure 3-1 (page B-7)}

LSE figures graphically illustrate the data presented in the corresponding LSE tables. Figures help the reader quickly compare health effects according to exposure concentrations for particular exposure periods.

(13) Exposure Period. The same exposure periods appear as in the LSE table. In this example, health effects observed within the acute and intermediate exposure periods are illustrated.

(14) Health Effect. These are the categories of health effects for which reliable quantitative data exists. The same health effects appear in the LSE table.

(15) Levels of Exposure. Concentrations or doses for each health effect in the LSE tables are graphically displayed in the LSE figures. Exposure concentration or dose is measured on the log scale "y" axis. Inhalation exposure is reported in $\mathrm{mg} / \mathrm{m}^{3}$ or $\mathrm{ppm}$ and oral exposure is reported in $\mathrm{mg} / \mathrm{kg} /$ day.

(16) NOAEL. In this example, the open circle designated $18 \mathrm{r}$ identifies a NOAEL critical end point in the rat upon which an intermediate inhalation exposure MRL is based. The key number 18 corresponds to the entry in the LSE table. The dashed descending arrow indicates the extrapolation from the exposure level of $3 \mathrm{ppm}$ (see entry 18 in the table) to the MRL of $0.005 \mathrm{ppm}$ (see footnote "b" in the LSE table).

(17) CEL. Key number $38 \mathrm{~m}$ is one of three studies for which CELs were derived. The diamond symbol refers to a CEL for the test species-mouse. The number 38 corresponds to the entry in the LSE table. 
(18) Estimated Upper-Bound Human Cancer Risk Levels. This is the range associated with the upperbound for lifetime cancer risk of 1 in 10,000 to 1 in 10,000,000. These risk levels are derived from the EPA's Human Health Assessment Group's upper-bound estimates of the slope of the cancer dose response curve at low dose levels $\left(\mathrm{q}_{1}{ }^{*}\right)$.

(19) Key to LSE Figure. The Key explains the abbreviations and symbols used in the figure. 


\section{SAMPLE}

Table 3-1. Levels of Significant Exposure to [Chemical x] - Inhalation

\begin{tabular}{|c|c|c|c|c|c|c|c|c|c|}
\hline & & \multirow[b]{2}{*}{$\begin{array}{l}\text { Key to } \\
\text { figure }^{a}\end{array}$} & \multirow[b]{2}{*}{ Species } & \multirow{2}{*}{$\begin{array}{l}\text { Exposure } \\
\text { frequency/ } \\
\text { duration }\end{array}$} & \multirow[b]{2}{*}{ System } & \multirow[b]{2}{*}{$\begin{array}{l}\text { NOAEL } \\
\text { (ppm) }\end{array}$} & \multicolumn{2}{|l|}{ LOAEL (effect) } & \multirow[b]{2}{*}{ Reference } \\
\hline & & & & & & & $\begin{array}{l}\text { Less serious } \\
(\mathrm{ppm})\end{array}$ & Serious (ppm) & \\
\hline \multirow{2}{*}{\multicolumn{2}{|c|}{$\rightarrow$}} & \multicolumn{8}{|c|}{ INTERMEDIATE EXPOSURE } \\
\hline & & & 5 & 6 & 7 & 8 & 9 & & 10 \\
\hline 3 & $\rightarrow$ & Systemic & $\downarrow$ & $\downarrow$ & $\downarrow$ & $\downarrow$ & $\downarrow$ & & $\downarrow$ \\
\hline \multirow{7}{*}{\multicolumn{2}{|c|}{4}} & 18 & Rat & $\begin{array}{l}13 \mathrm{wk} \\
5 \mathrm{~d} / \mathrm{wk} \\
6 \mathrm{hr} / \mathrm{d}\end{array}$ & Resp & $3^{b}$ & 10 (hyperplasia) & & Nitschke et al. 1981 \\
\hline & & CHRONIC & XPOSUR & & & & & & \\
\hline & & Cancer & & & & & 11 & & \\
\hline & & & & & & & $\downarrow$ & & \\
\hline & & 38 & Rat & $\begin{array}{l}18 \mathrm{mo} \\
5 \mathrm{~d} / \mathrm{wk} \\
7 \mathrm{hr} / \mathrm{d}\end{array}$ & & & 20 & $\begin{array}{l}\text { (CEL, multiple } \\
\text { organs) }\end{array}$ & Wong et al. 1982 \\
\hline & & 39 & Rat & $\begin{array}{l}89-104 \mathrm{wk} \\
5 \mathrm{~d} / \mathrm{wk} \\
6 \mathrm{hr} / \mathrm{d}\end{array}$ & & & 10 & $\begin{array}{l}\text { (CEL, lung tumors, } \\
\text { nasal tumors) }\end{array}$ & NTP 1982 \\
\hline & & 40 & Mouse & $\begin{array}{l}79-103 \mathrm{wk} \\
5 \mathrm{~d} / \mathrm{wk} \\
6 \mathrm{hr} / \mathrm{d}\end{array}$ & & & 10 & $\begin{array}{l}\text { (CEL, lung tumors, } \\
\text { hemangiosarcomas) }\end{array}$ & NTP 1982 \\
\hline
\end{tabular}




\section{SAMPLE}

13

Figure 3-1. Levels of Significant Exposure to [Chemical X] - Inhalation
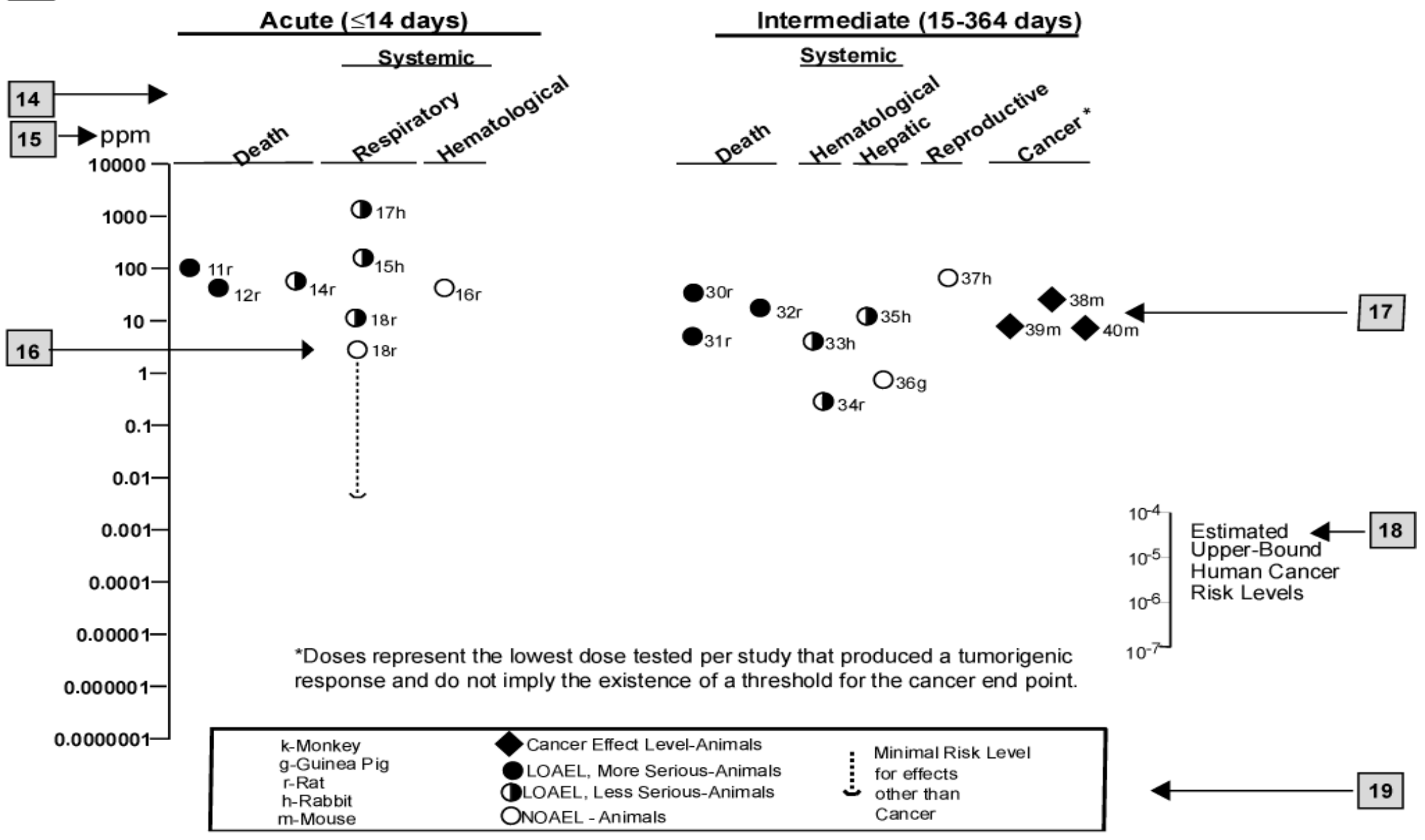
APPENDIX B

This page is intentionally blank. 


\section{APPENDIX C. ACRONYMS, ABBREVIATIONS, AND SYMBOLS}

\begin{tabular}{|c|c|}
\hline ACGIH & American Conference of Governmental Industrial Hygienists \\
\hline ACOEM & American College of Occupational and Environmental Medicine \\
\hline ADI & acceptable daily intake \\
\hline ADME & absorption, distribution, metabolism, and excretion \\
\hline AED & atomic emission detection \\
\hline AFID & alkali flame ionization detector \\
\hline AFOSH & Air Force Office of Safety and Health \\
\hline ALT & alanine aminotransferase \\
\hline AML & acute myeloid leukemia \\
\hline AOAC & Association of Official Analytical Chemists \\
\hline AOEC & Association of Occupational and Environmental Clinics \\
\hline AP & alkaline phosphatase \\
\hline APHA & American Public Health Association \\
\hline AST & aspartate aminotransferase \\
\hline atm & atmosphere \\
\hline ATSDR & Agency for Toxic Substances and Disease Registry \\
\hline AWQC & Ambient Water Quality Criteria \\
\hline BAT & best available technology \\
\hline $\mathrm{BCF}$ & bioconcentration factor \\
\hline BEI & Biological Exposure Index \\
\hline BMD & benchmark dose \\
\hline BMR & benchmark response \\
\hline BSC & Board of Scientific Counselors \\
\hline $\mathrm{C}$ & centigrade \\
\hline CAA & Clean Air Act \\
\hline CAG & Cancer Assessment Group of the U.S. Environmental Protection Agency \\
\hline CAS & Chemical Abstract Services \\
\hline $\mathrm{CDC}$ & Centers for Disease Control and Prevention \\
\hline CEL & cancer effect level \\
\hline CELDS & Computer-Environmental Legislative Data System \\
\hline CERCLA & Comprehensive Environmental Response, Compensation, and Liability Act \\
\hline CFR & Code of Federal Regulations \\
\hline $\mathrm{Ci}$ & curie \\
\hline $\mathrm{CI}$ & confidence interval \\
\hline $\mathrm{CL}$ & ceiling limit value \\
\hline CLP & Contract Laboratory Program \\
\hline $\mathrm{cm}$ & centimeter \\
\hline CML & chronic myeloid leukemia \\
\hline CPSC & Consumer Products Safety Commission \\
\hline CWA & Clean Water Act \\
\hline DHEW & Department of Health, Education, and Welfare \\
\hline DHHS & Department of Health and Human Services \\
\hline DNA & deoxyribonucleic acid \\
\hline DOD & Department of Defense \\
\hline DOE & Department of Energy \\
\hline DOL & Department of Labor \\
\hline DOT & Department of Transportation \\
\hline
\end{tabular}




\begin{tabular}{|c|c|}
\hline $\begin{array}{l}\text { DOT/UN/ } \\
\text { NA/IMCO }\end{array}$ & $\begin{array}{l}\text { Department of Transportation/United Nations/ } \\
\text { North America/Intergovernmental Maritime Dangerous Goods Code }\end{array}$ \\
\hline DWEL & drinking water exposure level \\
\hline ECD & electron capture detection \\
\hline ECG/EKG & electrocardiogram \\
\hline EEG & electroencephalogram \\
\hline EEGL & Emergency Exposure Guidance Level \\
\hline EPA & Environmental Protection Agency \\
\hline $\mathrm{F}$ & Fahrenheit \\
\hline $\mathrm{F}_{1}$ & first-filial generation \\
\hline FAO & Food and Agricultural Organization of the United Nations \\
\hline FDA & Food and Drug Administration \\
\hline FEMA & Federal Emergency Management Agency \\
\hline FIFRA & Federal Insecticide, Fungicide, and Rodenticide Act \\
\hline FPD & flame photometric detection \\
\hline fpm & feet per minute \\
\hline FR & Federal Register \\
\hline FSH & follicle stimulating hormone \\
\hline $\mathrm{g}$ & gram \\
\hline GC & gas chromatography \\
\hline $\operatorname{gd}$ & gestational day \\
\hline GLC & gas liquid chromatography \\
\hline GPC & gel permeation chromatography \\
\hline HPLC & high-performance liquid chromatography \\
\hline HRGC & high resolution gas chromatography \\
\hline HSDB & Hazardous Substance Data Bank \\
\hline IARC & International Agency for Research on Cancer \\
\hline IDLH & immediately dangerous to life and health \\
\hline ILO & International Labor Organization \\
\hline IRIS & Integrated Risk Information System \\
\hline $\mathrm{Kd}$ & adsorption ratio \\
\hline $\mathrm{kg}$ & kilogram \\
\hline $\mathrm{kkg}$ & metric ton \\
\hline $\mathrm{K}_{\mathrm{oc}}$ & organic carbon partition coefficient \\
\hline $\mathrm{K}_{\mathrm{ow}}$ & octanol-water partition coefficient \\
\hline $\mathrm{L}$ & liter \\
\hline LC & liquid chromatography \\
\hline $\mathrm{LC}_{50}$ & lethal concentration, $50 \%$ kill \\
\hline $\mathrm{LC}_{\mathrm{Lo}}$ & lethal concentration, low \\
\hline $\mathrm{LD}_{50}$ & lethal dose, $50 \%$ kill \\
\hline $\mathrm{LD}_{\mathrm{LO}}$ & lethal dose, low \\
\hline LDH & lactic dehydrogenase \\
\hline LH & luteinizing hormone \\
\hline LOAEL & lowest-observed-adverse-effect level \\
\hline LSE & Levels of Significant Exposure \\
\hline $\mathrm{LT}_{50}$ & lethal time, $50 \%$ kill \\
\hline $\mathrm{m}$ & meter \\
\hline MA & trans,trans-muconic acid \\
\hline MAL & maximum allowable level \\
\hline $\mathrm{mCi}$ & millicurie \\
\hline MCL & maximum contaminant level \\
\hline
\end{tabular}




\begin{tabular}{|c|c|}
\hline MCLG & maximum contaminant level goal \\
\hline MF & modifying factor \\
\hline MFO & mixed function oxidase \\
\hline $\mathrm{mg}$ & milligram \\
\hline $\mathrm{mL}$ & milliliter \\
\hline $\mathrm{mm}$ & millimeter \\
\hline $\mathrm{mmHg}$ & millimeters of mercury \\
\hline $\mathrm{mmol}$ & millimole \\
\hline mppcf & millions of particles per cubic foot \\
\hline MRL & Minimal Risk Level \\
\hline MS & mass spectrometry \\
\hline NAAQS & National Ambient Air Quality Standard \\
\hline NAS & National Academy of Science \\
\hline NATICH & National Air Toxics Information Clearinghouse \\
\hline NATO & North Atlantic Treaty Organization \\
\hline NCE & normochromatic erythrocytes \\
\hline NCEH & National Center for Environmental Health \\
\hline NCI & National Cancer Institute \\
\hline ND & not detected \\
\hline $\begin{array}{l}\text { NFPA } \\
\text { ng }\end{array}$ & $\begin{array}{l}\text { National Fire Protection Association } \\
\text { nanogram }\end{array}$ \\
\hline NHANES & National Health and Nutrition Examination Survey \\
\hline NIEHS & National Institute of Environmental Health Sciences \\
\hline NIOSH & National Institute for Occupational Safety and Health \\
\hline NIOSHTIC & NIOSH's Computerized Information Retrieval System \\
\hline NLM & National Library of Medicine \\
\hline $\mathrm{nm}$ & nanometer \\
\hline nmol & nanomole \\
\hline NOAEL & no-observed-adverse-effect level \\
\hline NOES & National Occupational Exposure Survey \\
\hline NOHS & National Occupational Hazard Survey \\
\hline NPD & nitrogen phosphorus detection \\
\hline NPDES & National Pollutant Discharge Elimination System \\
\hline NPL & National Priorities List \\
\hline NR & not reported \\
\hline NRC & National Research Council \\
\hline NS & not specified \\
\hline NSPS & New Source Performance Standards \\
\hline NTIS & National Technical Information Service \\
\hline NTP & National Toxicology Program \\
\hline ODW & Office of Drinking Water, EPA \\
\hline OERR & Office of Emergency and Remedial Response, EPA \\
\hline $\mathrm{OHM} / \mathrm{TADS}$ & Oil and Hazardous Materials/Technical Assistance Data System \\
\hline OPP & Office of Pesticide Programs, EPA \\
\hline OPPT & Office of Pollution Prevention and Toxics, EPA \\
\hline OPPTS & Office of Prevention, Pesticides and Toxic Substances, EPA \\
\hline OR & odds ratio \\
\hline OSHA & Occupational Safety and Health Administration \\
\hline OSW & Office of Solid Waste, EPA \\
\hline OTS & Office of Toxic Substances \\
\hline OW & Office of Water \\
\hline
\end{tabular}




$\begin{array}{ll}\text { OWRS } & \text { Office of Water Regulations and Standards, EPA } \\ \text { PAH } & \text { polycyclic aromatic hydrocarbon } \\ \text { PBPD } & \text { physiologically based pharmacodynamic } \\ \text { PBPK } & \text { physiologically based pharmacokinetic } \\ \text { PCE } & \text { polychromatic erythrocytes } \\ \text { PEL } & \text { permissible exposure limit } \\ \text { pg } & \text { picogram } \\ \text { PHS } & \text { Public Health Service } \\ \text { PID } & \text { photo ionization detector } \\ \text { pmol } & \text { picomole } \\ \text { PMR } & \text { proportionate mortality ratio } \\ \text { ppb } & \text { parts per billion } \\ \text { ppm } & \text { parts per million } \\ \text { ppt } & \text { parts per trillion } \\ \text { PSNS } & \text { pretreatment standards for new sources } \\ \text { RBC } & \text { red blood cell } \\ \text { REL } & \text { recommended exposure level/limit } \\ \text { RfC } & \text { reference concentration } \\ \text { RfD } & \text { reference dose } \\ \text { RNA } & \text { ribonucleic acid } \\ \text { RQ } & \text { reportable quantity } \\ \text { RTECS } & \text { Registry of Toxic Effects of Chemical Substances } \\ \text { SARA } & \text { Superfund Amendments and Reauthorization Act } \\ \text { SCE } & \text { sister chromatid exchange } \\ \text { SGOT } & \text { serum glutamic oxaloacetic transaminase } \\ \text { SGPT } & \text { serum glutamic pyruvic transaminase } \\ \text { SIC } & \text { standard industrial classification } \\ \text { SIM } & \text { selected ion monitoring } \\ \text { SMCL } & \text { secondary maximum contaminant level } \\ \text { SMR } & \text { standardized mortality ratio } \\ \text { SNARL } & \text { suggested no adverse response level } \\ \text { SPEGL } & \text { Short-Term Public Emergency Guidance Level } \\ \text { STEL } & \text { short term exposure limit } \\ \text { STORET } & \text { Storage and Retrieval } \\ \text { TD } & \text { toxic dose, 50\% specific toxic effect } \\ \text { TLV } & \text { threshold limit value } \\ \text { TOC } & \text { total organic carbon } \\ \text { TPQ } & \text { threshold planning quantity } \\ \text { TRI } & \text { Toxics Release Inventory } \\ \text { TSCA } & \text { Toxic Substances Control Act } \\ \text { TWA } & \text { time-weighted average } \\ \text { UF } & \text { uncertainty factor } \\ \text { U.S. } & \text { United States } \\ \text { USDA } & \text { United States Department of Agriculture } \\ \text { USGS } & \text { United States Geological Survey } \\ \text { VOC } & \text { volatile organic compound } \\ \text { WBC } & \text { white blood cell } \\ \text { WHO } & \text { World Health Organization } \\ & \end{array}$




$\begin{array}{ll}> & \text { greater than } \\ \geq & \text { greater than or equal to } \\ = & \text { equal to } \\ < & \text { less than } \\ \leq & \text { less than or equal to } \\ \% & \text { percent } \\ \alpha & \text { alpha } \\ \beta & \text { beta } \\ \gamma & \text { gamma } \\ \delta & \text { delta } \\ \mu \mathrm{m} & \text { micrometer } \\ \mu \mathrm{g} & \text { microgram } \\ \mathrm{q}_{1}{ } & \text { cancer slope factor } \\ - & \text { negative } \\ + & \text { positive } \\ (+) & \text { weakly positive result } \\ (-) & \text { weakly negative result }\end{array}$


APPENDIX C

This page is intentionally blank. 


\section{APPENDIX D. INDEX}

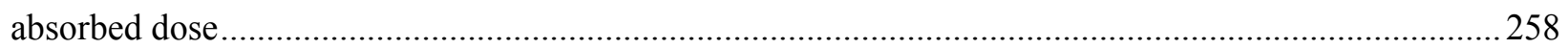

adenocarcinoma

adenocarcinomas

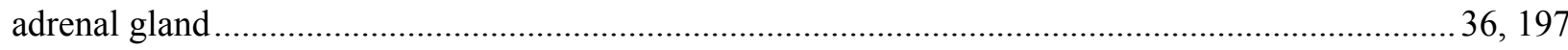

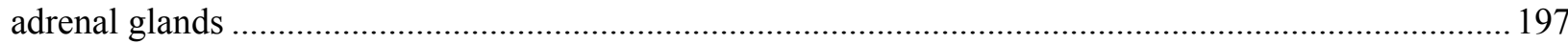

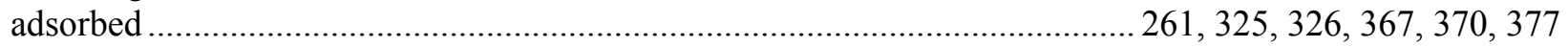

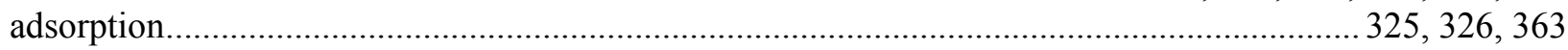

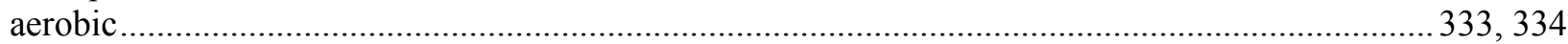

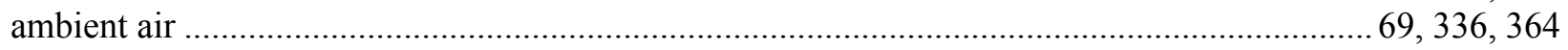

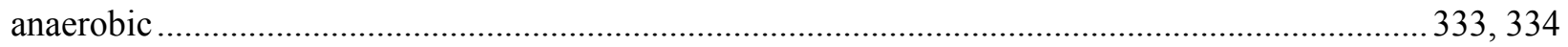

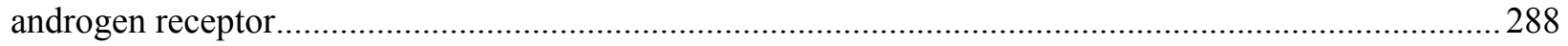

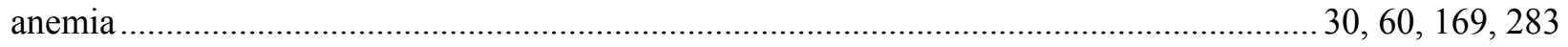

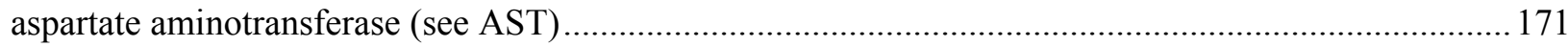

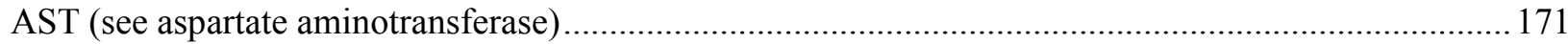

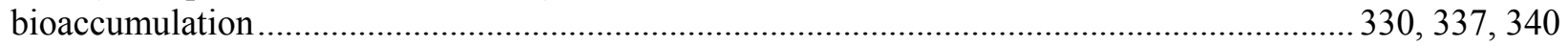

bioavailability ……........................................ 213, 214, 215, 333, 335, 363, 364, 365, 370, 373, 380

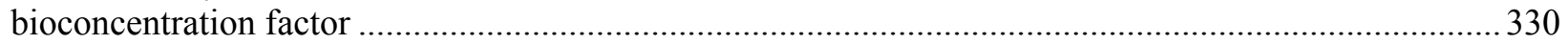

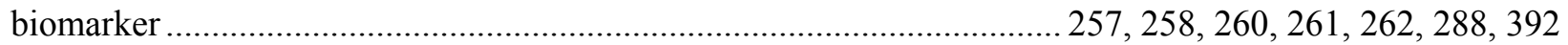

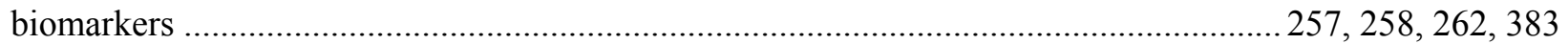

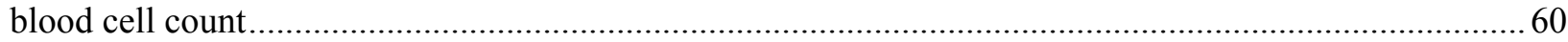

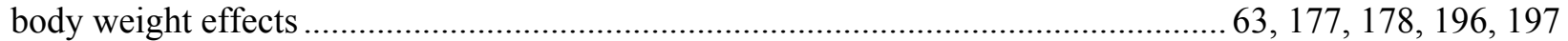

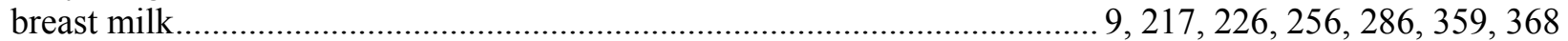

cancer …............................................... 7, 8, 17, 18, 19, 22, 23, 42, 56, 64, 68, 69, 70, 71, 72, 73, 74,

$176,185,188,189,190,191,192,193,198,199,211,248,250$,

$253,254,255,262,263,265,270,277,278,280,282,286,287,288,289,395,399$

carcinogen .....

$7,18,23,187,192,193,395,398,399$

carcinogenic ........................................... 7, 26, 29, 41, 42, 44, 192, 194, 250, 276, 277, 395, 398

carcinogenicity ................................ 28, 44, 189, 192, 193, 194, 231, 248, 249, 250, 251, 277, 289, 398

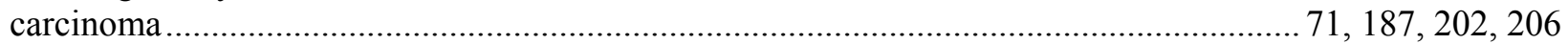

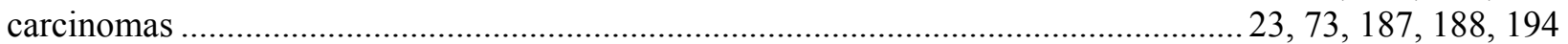

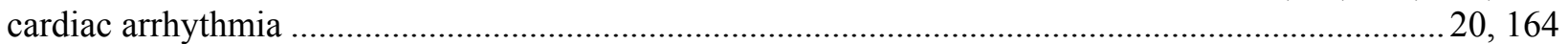

cardiovascular ................................... 17, 18, 19, 20, 31, 56, 58, 59, 74, 164, 166, 173, 196, 254, 282

cardiovascular effects........................................................... 17, 20, 58, 59, 163, 164, 166, 196

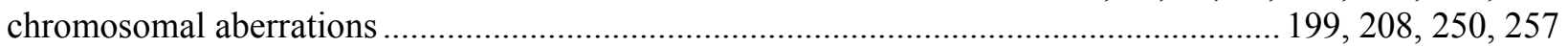

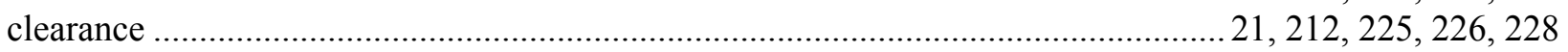

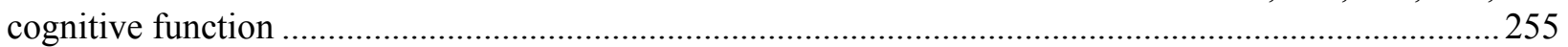

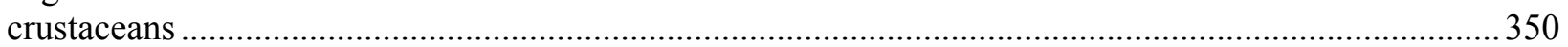

death..................................... 7, 9, 23, 41, 56, 73, 74, 162, 163, 185, 186, 191, 194, 216, 248, 251, 256

deoxyribonucleic acid (see DNA) ..................................................................2 205, 207, 208, 210

dermal effects.............................................................. 17, 19, 31, 62, 175, 176, 197, 273, 275, 395

DNA (see deoxyribonucleic acid)........................................... 199, 200, 201, 202, 203, 204, 205, 206, $207,208,209,210,249,250,251,258,269,278,285$

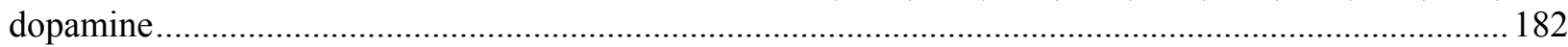

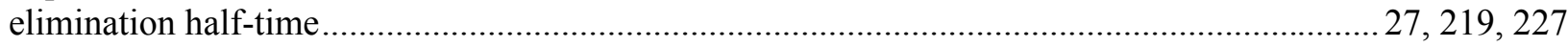

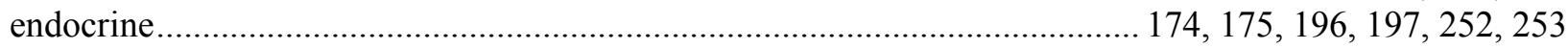

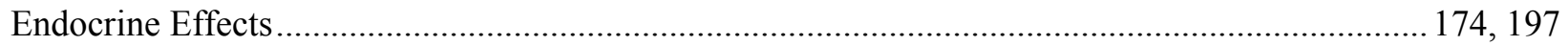

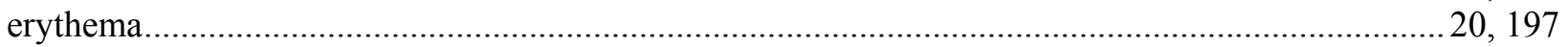


estrogen receptor

fetal tissue .....

fetus.

$187,217,253,280,372$

gastrointestinal effects

general population.

genotoxic.

$21,24,26,28,33,37,59,60,74,166,168,274,395$
$\ldots \ldots \ldots \ldots \ldots . .15,57,165,224,257,364,374,375,378$

genotoxicity

$21,24,26,28,33,37,59,60,74,166,168,274,395$
$\ldots \ldots \ldots \ldots \ldots . .15,57,165,224,257,364,374,375,378$

groundwater

$199,208,250,278$

growth retardation

$4,10,16,313,315,322,323,326,331,337,338$, $340,341,342,343,356,369,375,380,381$

half-life.

$227,258,331,375$

hematological effects

$60,169,196$

hepatic effects

homeopathic

.61, 170, 171, 196, 262

hydrolysis... 354

hydroxyl radical

331,334

immune system

250

immunological

64,281

immunological effects

$29,41,64,179,198$

$\mathrm{K}_{\mathrm{ow}}$ 29

$\mathrm{LD}_{50}$

297, 298, 299, 300, 301, 376

leukemia

$32,39,162$

leukopenia

lymphoreticular

$15,17,20,287,311$

melanoma.

30,168

micronuclei

milk

64, 179, 198

mucociliary

99, 208, 250

musculoskeletal effects

$217,256,359,361,368,369$

neonatal

$217,256,359,361,368,369$

neoplastic

61,170

neurobehavioral

norepinephrine

nuclear.

$185,256,280,286$

$25,26,27,28,193$

ocular effects

odds ratio

251,384

pancytopenia

$63,177,197$

partition coefficients

$64,183,191$

pharmacodynamic

273

pharmacokinetic.

pharmacokinetics

243

photolysis

.. 228

placenta

253,286

placental barrier

217,256

rate constant

217,256

renal effects

$232,243,246$

retention

$228,229,230,234,235,236,237,239,240$

salivation....

$61,62,172,173,174,196$

sarcoma $224,264,309,363$

sodium pump

182

solubility .... 

$92,93,94,95,96,97,98,99,100,101,102,103,104,105,106,107,108,109,110,111$, $112,113,114,115,116,125,126,127,128,129,130,131,132,137,138,139,140$, …….......................... 141, 142, 143, 144, 145, 146, 147, 148, 152, 153, 154, 155, 156, 157, 158, 195

thyroid $25,26,35,175,194,197,251$

toxicokinetic. $17,27,32,41,43,71,211,221,259,263,278,282,284,286$ tremors $28,39,65,274$ tumors $18,23,26,27,28,73,187,190,191,192,193,194,199,251,256,262,278$ volatilization 
